$\lim _{1}(\mathrm{CPO}) \mathrm{l}$

(3) (c)

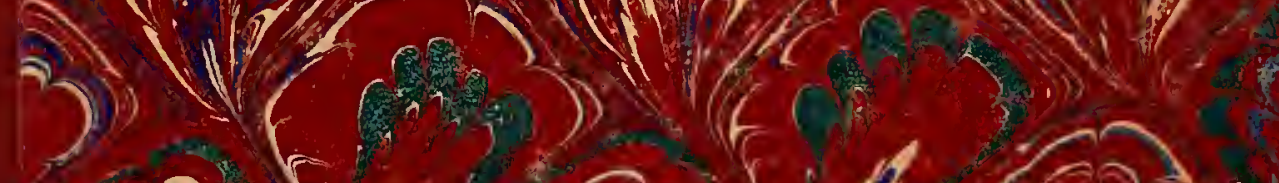
3) (m) ह( (m) हरित)

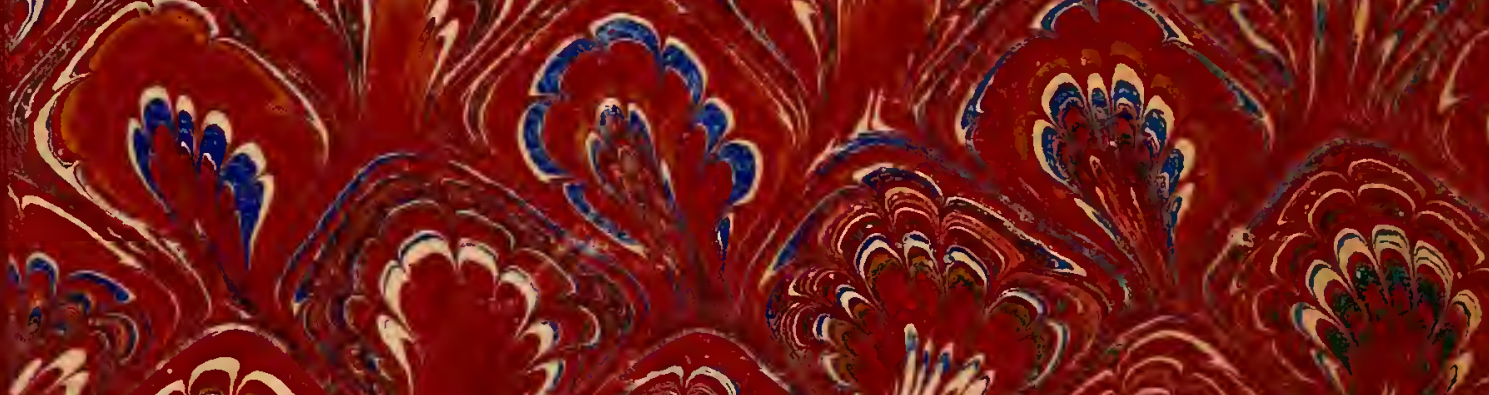

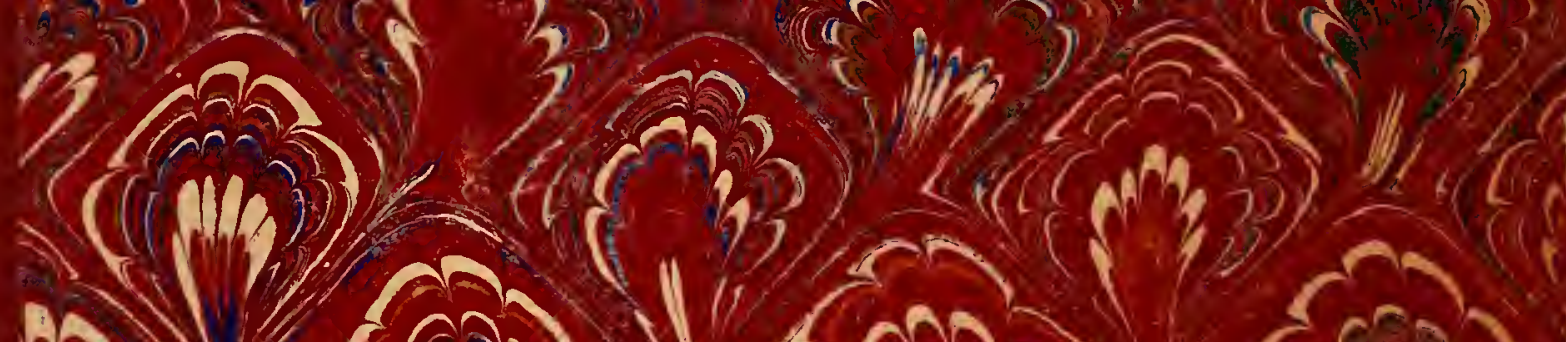
m. (sin) 1

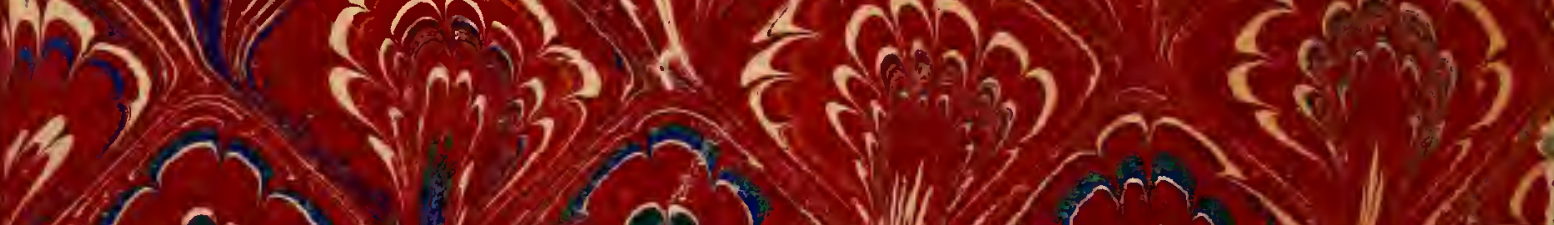

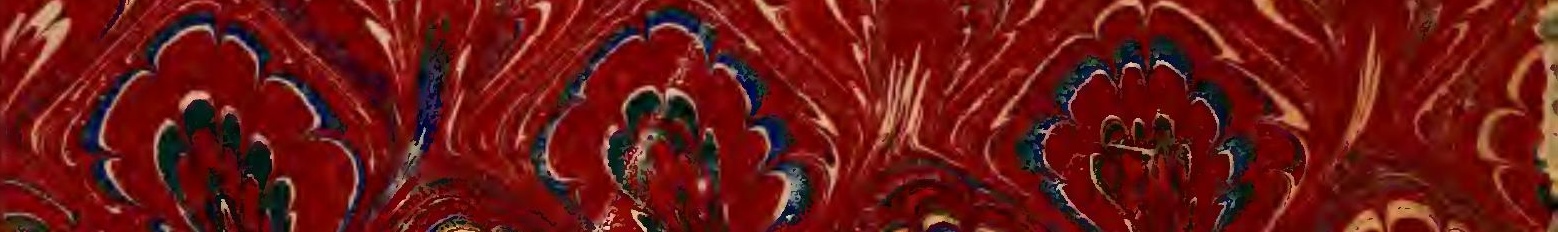

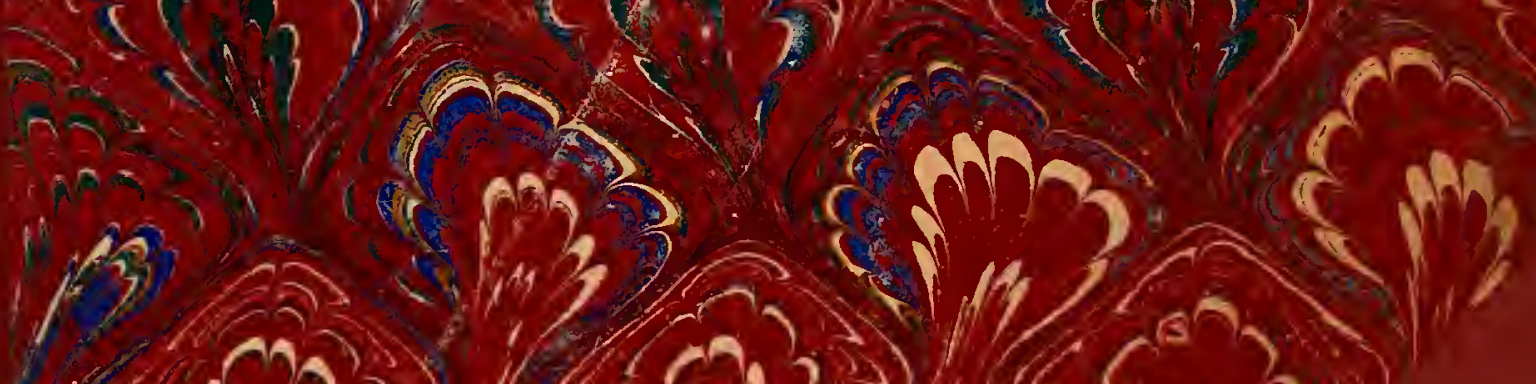
(n)

दूरी

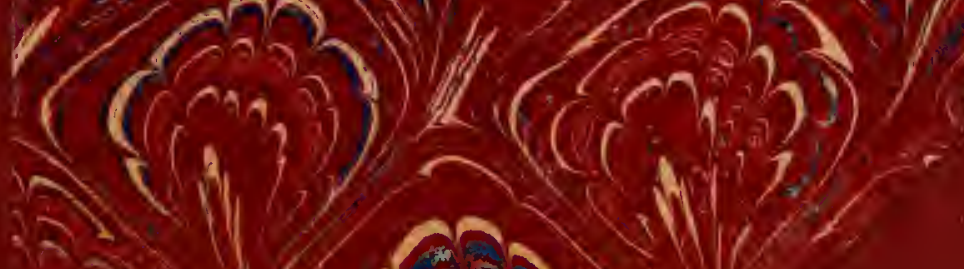



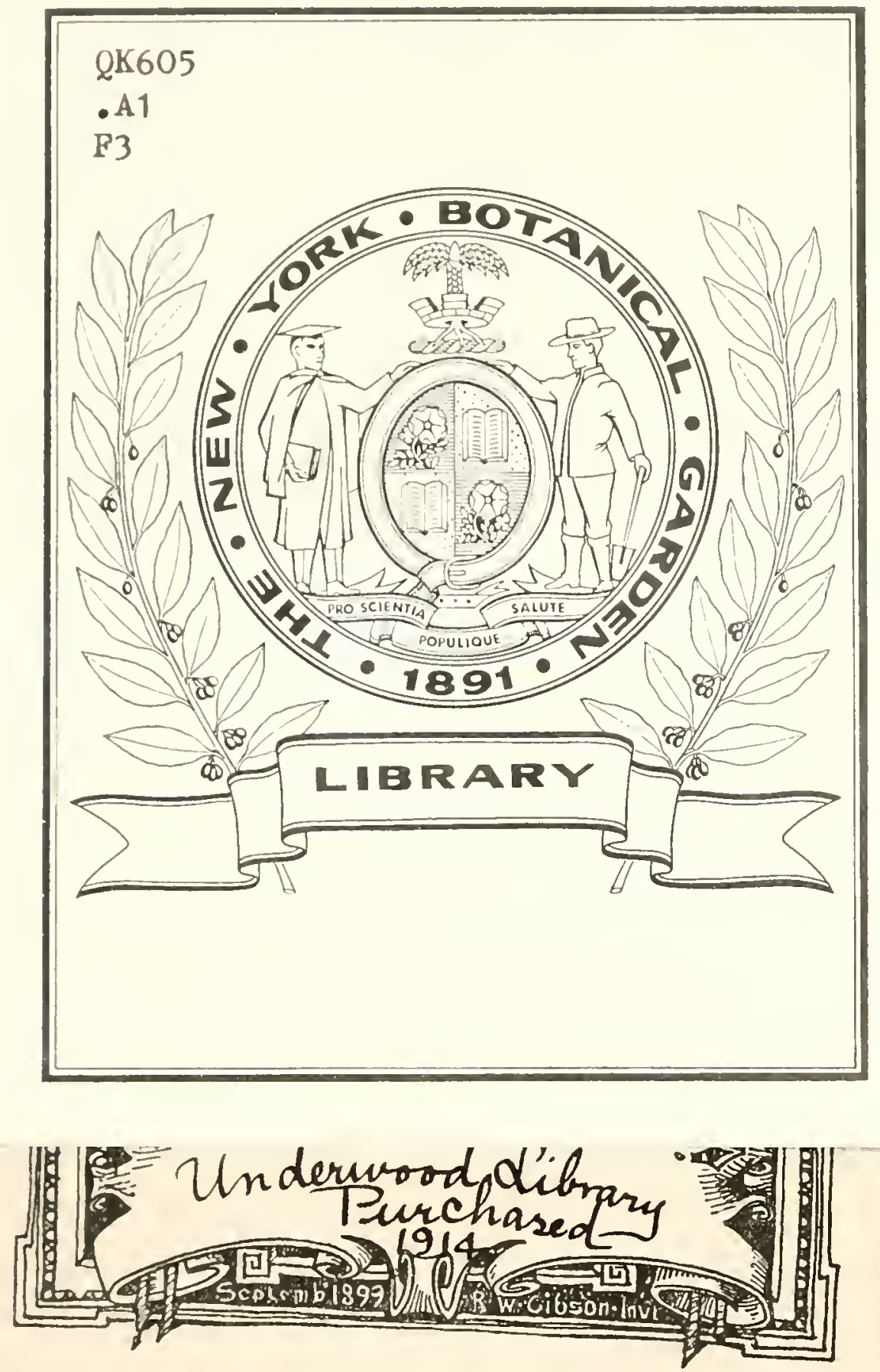




L. M. Unchrurrog 



\section{A PROVISIONAL HOST-INDEX}

OF THE

\section{Fungi of the United STATES :}

W. G. FARLOW AND A. B. SEYMOUR

PART I.

POLYPETALAE 



\section{INTRODUCTION}

A Host 1NDEx is a necessity in the study of North American fungi at the present day and everyone who has attempted to determine our species with any prospect of success has been obliged to prepare such an index for himself. The result is that a number of persons, some of whom are not well provided with mycological works except those which have been published within the last few years, are all spending considerable time and labor in an effort to do the same thing. To make a complete host-index is, for several reasons, quite out of the question but, believing that an approximately complete list of our parasitic species and their hosts would aid materially in the advance toward a more accurate study of our mycological flora and would tend to lessen the amount of indiscriminate species making which has already become al serions evil, the present index, the result of work extending over several years, has been prepared for publication.

The basis of the present record is the "List of Works on North American Fungi" by Farlow and Trelease, issued in I $\$ 97$, and the "Supplement" issued the present year. The species of fungi mentioned in the works whose titles are given in the two papers just mentioned are here classified and arranged under their recorded hosts. It has seemed best to give only those hosts which have already been recorded in print, for to go beyond that and record other hosts of the fungi of unpublished collections might learl to confusion or might be considered an unwarranted anticipation of the proposed publications of other botanists. It should not be supposed, however, that we have recorded the hosts mentioned in all the papers included in the "List of Works on North American Fungi." Sone of the works there mentioned are so clearly inaccurate and unscientific that probably all respectable mycologists would agree that their contents had hetter be ignored. As it is, by far the greater part of the works mentioned in the "List" are here included and it is probable that, if we have erred, it has been by including too many rather than too few of them.

In the nomenclature of the hosts themselves we have merely aimed at adopting the names given in standard worls, such as Gray's Manual, the Synoptical Flora, Watson's Index, the Flora of California, etc., which are in general use and accessible to anyone, avoiding any attempt to settle the recondite and disputed points which concern the experts in Phaenogamic Botany. For mycological purposes it is of slight consequence whether a phaenogamic species, as here given, had better be spijt up into two closely related species or, on the other hand, united with another nearly related species. The fact remains that the same parasitic fungi are apt to occur on closely related Phaenogams and the present index will still serve as a general guide, which is all that it purports to be in any case, even if the specific limitations of the hosts be hereafter changed by experts in that branch of botany. All we can expect 
to do is to qunte the m:mucs uriven in the treatises most widely known at the present dily. For Polyctalar, Watson's Index has generally been followed.

Where alle screral difliculties which arise in attempting to give the hosts accurately. 'Whe collector is not always well informed about the Phacnogams of the region in which he collects and it often happens that the infested planthas neither flowers nor fruit, in which case an cxict determination is often impossible. Perplexing errors hatre not infrepuently arisen as, for instance. in the case of the fungus named by Schweinity Cacoma Chclidoniz, which, as an examination of the original specimens shows, occurred not on Chelidonimm but on Osmorrhiza, while another species mentioncel hy the sime anthor on Smilax really grew on Rosa. One is surprised at the mumber of similar enors on the part cren of good hotanists, and onc will fail to find in this part of the index a number of familiar names of fungi, for the reason that we have discovered erro!s in the determination of the hosts, which make it necessary to remove them from Polyptalae to other divisions which follow. Futhermore, when we consider how little is known ahout the Phaenogams of a large part of our country, it is not to be expected that errors conld well be aroided by collectors and travellers. Again, the practice of giving common and popular instead of scientific names causcs grealt confusion. The same common name is applied to widely different plants in different parts of the combry. and it is not unfrequently impossible to say what species really is meant. The matter is malle still worse by the fact that a good many of our fungi have been described or have been included in worlis by foreign botanists and in forcign languages, and ludicrous errors have crept in. Sage-brush, for instance, is not unfrequently translated Salvia instcal of Artemisia, and this cror. transparent as it is to botanists in this combry, ma at length become fixed in mycological literature until. at last, we shall find ourselves scarching in Labiatae for what reallybelongs in Compositac. It is not asking too much to insist upon the necessity of grving the scientific rather than the common name and, even when the collector is not sure of the specific name, it would often be of great help to know the generic name. It would have simplified matters for us in several cases hald the collector, instead of saying "poplar," said Populns or Liriodendron, as the case might be. So far as possible, we have here referred popular to scientific names, but it is probalble that we have erred in some cases.

With regard to the nomenclaturc of cultivated plants authorities differ widely, but although the names we have adopted may not meet the approval of many phaenoomic botanists. mycologists will prohably have little difficulty in recognizing the plints intended. In some doubtful cases we have given the common names.

The nomenclature of the species of fungi is that generally accepted by European mycologists, the name of the original describer being given in parenthesis, followed hy the name of the author by whom the species was first placed in the accepted crenus. This method, although widely aldopted by writers on fungi, does not secm to us to be so satisfactory as that of those phaenogamic botanists who do not use the parcnthesis, but this is not the place to institute a change in the prevailing practice. Where the number of species on a given host is small, they are arranged alphabetically, regardless of their systematic position, and the eye will quickly calch the upecies of a particular order if onc has any knowledge of the genera of fungi. If he hro unt this knowledere a host-index would be of little use ao matter on whalt plan it 
vere arranged. When the number of species on a single host is large, they are arranged alphabetically under the proper orders and, where there are many species of some orders and but fow of others, the latter are placed under the general heading, Miscellanea. In many cases fungi were originally described as growing on a certain genus without mention of the species and, hence, in searching for the fungi to be found on a given species, one should also eximine the list of fungi placed under the general heading, sp. indet., at the enci of diferent genera. In some cases, like Nyssa and Lagerstroemia, it is very probable that the species here placed under sp. indet. really betong under $N$. maltiflora and $L$. Indica, but, as cases of other senera are doubtful, we have thought best to adhere to the indefiniteness of the original publications.

It s'rould be borne distinctly in mind that the index purports to include only those species of parasitic fungi which have been recorded as occurring in extratropical North America. The word parasitic is here used to include many saprophytes, properly speaking, but ubiquitons specic's, like some moulds, have not been included. The fungi inclucled have many synonyms, but, as a rule, we have given only those synonyms which have appeared in frint in connection with the recurd of the occurence of the species on particular hosts in North America, omitting other synonyms which have been used in works on the fungi of other countries. In a few cases, where confusion might otherwise arise. a synonym not, as yet, used in works on North American fungi is given in italics. Synomyms are indicated by braces, and are given, with some exceptions, in chronological order, but it, of course, does not follow that the latest name is always to be preferred to others, although that is more frequently the case. In the index, as elsewhere, discretion has to be used, and in cases of what we knew positively to be errors in the determination of the hosts or the fungi themselves we have inserted corrections, and iu other doubtful cases we have called attention to disputed points by means of interrogation marks. An interogation before a name signifies that it is doubtful whether the fungus in question really occurs on the host named; one after the name means thit the determination of the fungus is uncertinin; one after a name in a brace, that it is doubtiul whether it is really synonymous with the other names in the same brace. Names of undescribed species from lists and catalogues have usually been omitted except where necessary to complete the synonymy. The abbreviation, Auct. Amer., his been used in a number of cases in which names have frequently been used by writers on North American fungi without proper authority, although the species referred to in these cases are well known.

In the calse of fungi to whose different stages different names have been given, the names of secondiry stages (conidia, pycnidia, etc.), when it was thought necessary to give them at all, have been placed in alphabetical order and, in the hosts infested by a large number of fungi, under the orders to which so-called form-genera are usually assigned. The complications of Uredineae are so great that we have not attempted to show here the connection of diferent forms which can be learned only from special works on that subject. In most instances the uredo name is not given unless it differs widely from that of the tcleutosporic form or unless, for some special reason, to avoid confusion in the synonymy. Aecidial forms have been kept distinct on the sime principle. 
Some funci which are finum on a considerable number of hosts of the sane order, as (istopus candidus, mighte perhatps, better have hecu placed together in a group finst under the name of the order. and it was originally intended to adopt such ani armingement, for it is evident that the frequent repetion of the name of a common species tends to interfere with rather than facilitate the use of at host-index. But we found it difficult to mantige such an arrangement without spending more time than was at our command. It is to be regretted that we were not able to indicate in some way whether the fungi oceur on the leaves, stems, or fruits. But, in many cases. the facts could not be ascertaned without much additional labor, while, in others. the hathitat call readily be inferred and, in still others, the fungi maly be expected to oecur on any of the organs. As it is, the index is sufficiently complicated, and it was found impossible to add other distinguishing characters except at considcrable extra expense.

As the printing of the first part has required considerable time, there are sereral additions from current literature which could not be included. It is hoped that the remander of the index will be issued during the coming winter, and it is intended to have additions and corrections of the present part given in an appendix to the second part. By an error in paiging, the fungi on Magnolia sp. indet. were not placed in the proper order. They are here given on a supplementary sheet, and will be included in their proper place in the appendix of the following part of the index.

Cambritge. $7413^{1}, 1 S S S$.

W. G. FARLOW 


\section{PHANEROGAMS.}

\section{RANUNCULACEAE.}

Actaea alba, Bigelow.

Aecidium Actaeae, (Opiz) Wallr.

Ramularia Actaeae, Ell. \& Holw.

Actaea spicata, L.

f Lêptostroma lineare, Pk.

$\{$ Leptostroma lineatum, Sacc.

Anemone acutiloba, Dawson.

Caeoma (Aecidium) hepaticatum, S.

Aecidium punctatum, $P$.

Gloeosporium Hepaticae, Pk.

Protomyces fuscus, Pk.

Urocystis Anemones, (P.).

$\{$ Urocystis pompholygodes, (Schl.) Rabh.

Polycystis Ranunculacearum, Desm.

Anemone cylindrica, Gray. $/ \& 2$

Dothidea Anemones, Fr.

$\{$ Puccinia Anemones-Virginianae, S.

Puccinia solida, S.

Anemone dichotoma, L.1/2

Aecidium Ranunculacearum, DC.

Didymaria Ungeri, Cda.

+Peronospora pygmaea, Ung.

$\int$ Urocystis Anemones, (P.).

$\{$ Urocystis poinpholygodes, (Schl.) Rabh. Polycystis Ranunculacearum, Desm.

Anemone Hepatica, L. $1 \leqslant 2$

Aecidium punctatum, $P$.

Caeoma (Aecidium) hepaticatum, S.

Gloeosporium Hepaticae, Pk.

+ Peronspora pygmaea, Ung.

Urocystis Anemones, (P.).

Anemone narcissiflora, L.

Puccinia vesiculosa, Ehrenb.

Anemone nemorosa, L. 162

f Aecidium Anemones, P.

- $\{$ Aecidium leucospermum, DC.

$\{$ Aecidium punctatum, $\mathrm{P}$.

$\{$ Aecidium quadrifidum, DC.

Aecidium Ranunculacearum, DC.

Caeoma (Uredo) Anemones, S.

Dothidea Anemones, $\mathrm{Fr}$.

+ Peronospora pygmaea, Ung.
( Puccinia Anemones, P.

Puccinia fusca, (Relh.) Wint.

Synchytrium Anemones, (DC.) Wor.

\{ Urocystis Anemones, (P.).

Orocystis poinpholygodes, (Schl.) Rabh.

Anemone patens, L.

Puccinia Anemones-Virginianae, S.

Anernone patens, L., var. Nuttalliana, Gray.

Puccinia fusca, (Relh.) Wint.

Anemone Virginiana, L. / 5

Aecidium Ranunculacearum, DC.

Dothidea Anemones, Fr.

-Erysiphe communis, (Wallr.) Schl.

$\{$ Puccinia Anemones-Virginianae, S.

\{ Puccinia solida, $\mathrm{S}$.

\{ Ramularia didyma, Ung.

\{ Didymaria Ungeri, Cda.

Sclerotium Anemones, S.

Sphaeria platypus, S.

Anemonella thalictroides, Spach.

Aecidium Thalictri-flavi, (DC.)

- Erysiphe communis, (WVallr.) Schl.

Urocystis pompholygodes, (Sch1.) Rabl.

Anemone, sp. indet.

Phleospora Anemones, Ell. \& Kell.

Aquilegiá vulgaris, L.

Septoria Aquilegiae, Ell. \& Kell.

Aquilegia, sp. indet.

Helminthosporium tenuissimum, Lk.

Caltha leptosepala, DC.

Puccinia Zopfii, Wint.

Caltha palustris, L.

Aecidium Calthae, Grev.

Puccinia Calthae, Lk.

Cimicifuga racemosa, Nutt.

Aecidium Actaeae, (Opiz) Wallr.

Aecidium cimicifugatum, S.

Sphaeria Ogilviensis, B. \& Br.

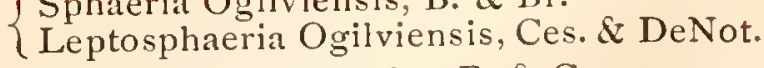

$\{$ Thecrphora carcinodes, B. \& C.

Urocystis carcinodes, Fisch. Wald.

Xyloma Actaeae, S.

$\{$ Leptostroma vulgare, Fr.

Leptostrmoa Actaeae, S. 

Silonia aftlattum, S.
lictortroma : thlantum, Fr.
1) uthidea altlata. S.

Cimicituga, sp. indet.

Phoma Cimicituerac. 13. A C.

Sphacria Cimiciunac. S.

Clematis ligusticitolia, Nutt. I \&z

Aceidinu Clematidis, I) C.

1)idrmaria Clematidis, Cke. N llark.

Clematis Virginiana, L.if -

(Aecidium Clematidis, D) C.

Decidium Clematits. 5 .

Iecidium clematititum, S.

Cercospora squalidula, Pk.

Eintyloma Mhalictri, Schrt.

Fistrloma Ranunculi, (Bon.) Schrt.. forma Tinalictri, Farl.

Cylindrosporium Ranunculi. (Bon.) Sacc., forma Thalictri, Ell. E Ev.

Cylindrosporium Clematidis, Ell. \& Ev.

- Eryiphe communis, (Wallr.) Schl.

- Erysplic tortilis. (Walle) Fr.

Clematis, sp. indet.

C'rcospora rubigo, Cke. S Hark.

Puccinia stromatica, B. S C.

Vormicularia Dematium, (P.) Fr.

Coptis trifolia, Salisb. ,

Sieptoria Coptidis, B. \& C.

I Sphaeria Coptis, S.

(Spluarella Coptis. Farl.

Vermicularia coptina, Pk.

Delphinium elatum, L.

- Erysiphe communis, (TVallr.) Schl., var. Delphinii, Ell.

Delphimiun, sp. indet.

Ciratophoma myriospora, Che.

Isopyrum biternatum, Torr. $\mathbb{S} \mathrm{Gr}$.

Aecidium Ranunculacearum, DC.

Paeonia Moutan, Sims.

Discosia urammita, B. S C.

Pueolia ofticinalis, DC. $/ \times 2$

Cercospora varicolor, Wint.

Paconia, sp.indet.182

-1-pererillus cimmerius, B. AC C

Clado-poriun Paenniac, Sacc.

l'ericonial brisoides, P.

1 Sphacria thaccida, A. A S.

( Cronurlium Haccidum, Wint.

Ranunculus abortivus. L. 182

( Jecidium Ranumculacearum, DC.

Accidium Ranumculi, S.

(Cacoma (Accidium) ranunculaceatum, Lk.

- l: "riphe communis, (Walln.) Schl.

Ranunculus acris, L. 182

- Erysiphe commmis, (Wallr.) Schl.

F'еmonospora Ficariac, '[ul.

septocyliudrimu Ranunculi. l'k.
Ranunculus bulbosus, L.

+ Peronospora Ficariae. 'Tul.

Ranunculus Californicus, Bentl.

Stigmatea Ranunculi, Fr.

Ranunculus Cymbalaria, Pursh./82

Accidium Ranunculi.S.

Ranunculus fascicularis, Muhl.

Didymaria Ungeri, Cda.

Entyloma microsporum, (Ung.) Schrt.

Ranunculus Pennsylvanicus, L.

\{Ramularia didyma, Unger.

S Didymaria Ungeri. Cda.

Placidium Ranunculi, Desm.

Ranunculus recurvatus, Poir.

Didymaria Ungeri, Cda.

fPeronospora Ficariae, Tu.

Ramularia Ranunculi, Pk.

Ranunculus repens, L. $/ \& 2$

Cercospora Ranunculi, Ell. \& Holw.

Entyloma microsporum, (Ung.) Schrt.

+ Peronospora Ficariae, Tul.

Puccinia Ranunculi, Seymour.

Ranunculus repens, L., var. hispidus, Torr. \& $\mathrm{Gr} \cdot 132$

Peziza singulasia, Pk.

Ranunculus sceleratus, L. 189

Phacidium Ranunculi, Desm.

Ranunculus, sp. indet. $/ \& 3$

$\{$ Excipula conglutinata, Ell. \& Ev.

\{ Excipulina conglutinata, Sacc.

Phacidium Ranunculi, Desm.

Uromyces Ficariae, Lev.

Uromyces Jonesii, Pk.

Thalictrlm Cornuti, L. 183

Aecidium Thalictri, Grev.

Caeoma (Uredo) Thalictri, S.

Diplodia herbarum, Lev.

-Erysiphe communis, (Wallr.) Schl.

Peziza Thalictri, $\mathrm{Pk}$.

$\{$ Puccinia Anemones, P.

Puccinia Thalictri, Chev.

Thalictrum dioicum, L.

Aecidium Thalictri, Grev.

Entyloma Thalictri, Schrt.

Entyloma Ranunculi, (Bon.) Schrt., forma Thalictri, Farl.

$\{$ Puccinia Anenones, P.

Puccina Thalictri, Chev.

Sphaerella septorioides, $\mathrm{Pk}$.

Sphaerclla Thalictri, Ell. \& Ev.

Thalictrum Fendleri, Engelm. / sक्ष

Aecidium Thalictri, Grev.

Tlalictrum Fendleri, Engelm, var. poly. carpum, Torr.

Leptostroma vulgare, $\mathrm{Fr}$.

Phoma nebulosum, (P.) Mont. 
Thalictrum purpurascens, L. 18

Aecidium Thalictri, Grev.

(Entyloma Thalictri, Schrt.

$\{$ Cylindrosporium Ranunculi, (Bon.) Sacc., forma Thalictri, Ell. \& Ev.

-Erysiphe communis. (Wallr.) Schl.

Trollius, sp. indet.

Urocystis pompholygodes, (Schl.) Rabh.

\section{CALYCANTHACEAE.}

Calycanthus, sp. indet.

Cytospora laxa, B. S C.

Didymosporium Calycanthi, S.

Patellaria nigrocinnabarina, S.

Phoma Calycanthi, S.

S Sphaeria Calycanthi, S.

\{ Botryosphaeria Calycanthi, Sacc.

\section{MAGNOLIACEAE.}

\section{Liriodendron Tulipifera, L. 184}

\section{MYXOMYCETES.}

\{ Trichia clavata, P.

Heniarcyria clavata, Rostf.

f Licea circumscissa, P.

Perichaena corticalis, (Batsch.) Rostf.

f Trichia botrytis, P.

\{ Trichia fragilis, (Sow.) Rostf.

\section{PYRENOMYCETES.}

Apiosporium salicinum, Kze.

Capnodium elongatum, B. S. Desm.

Diatrype plagia, B. \& C.

Didymosphaeria phyllogena, Wint.

S Xyloma Liriodendri, S.

$\{$ Ectostroma Liriodendri, Fr.

Dothidea Liriodendri, S.

- Erysiphe Liriodendri, S.

Glonium stellatum, Muhl.

$\{$ Sphaeria rufa, P.

$\{$ Hypocrea rufa, Fr.

Hypoderma virgultorum, DC., var, petiolare, Cke.

Hypoxylon investiens, (S.) B.

$\{$ Hysterium maculare, $\mathrm{Fr}$.

\{ Lophodermium maculare, DeNot.

\{ Melogramma Liriodendri, Cke.

\{ Botryosphaeria Liriodendri, Sacc.

Nectria coccinea, (P.) Fr.

Ostropa cinerascens, S.

$\{$ Phyllactinia guttata, Lev.

$\{$ Phyllactinia suffulta, (Reb.) Sacc.

Sphaerella Liriodendri, Cke.

Sphaeria ditopa, Fr.

Sphaeria divergens, S.

$\{$ Valsa divergens, Cke.

Cryptosporella divergens, Berl. \& Vogl.
Sphaeria faginea, $P$.

\{ Sphaeria Liriodendri, S.

$\{$ Diatrypella Liriodendri, Sacc.

\{ Sphaeria microloncha, B. \& C.

$\{$ Pleosphaeria microloncha, Sacc.

Sphaeria millegrana, S.

Sphaeria mutila, S.

Sphacria ovina, $\mathrm{P}$.

Sphaeria personata, $\mathrm{Fr}$.

$\{$ Sphaeria stictoides, B. \& C.

$\{$ Leptosphaeria stictoides, Sacc.

S Sphneria subiculata, S.

\{ Rosellinia subiculata. Sacc.

Sphaeria transversalis, S.

Sphaeria tubaeformis, Tode.

$\int$ Sphaeria turgida, $\mathrm{P}$.

$\{$ Valsa turgida, Fr.

Anthostoma turgidum, Nits.

Valsa nirea, Hoffin.

Valsa platgia, B. \& C.

Valsa quaternata, Fr.

Sphreria mucronata, S.

$\{$ Cordyceps mucronata, M. A. Curtis.

Xylaria mucronata, Sacc.

SPHAEROPSIDEAE.

Asteroma Liriodendri, Cke.

f Sphaeria Tulipiferae, S.

\{ Depazea Tulipiferae, M. A. Curtis.

Dothiorella Liriodendri, Sacc.

Excipula nitidula, S.

Hendersonia panciseptata, B. \& C.

Leptothyrium Liriodendri, Cke.

Phoma mixtum, B. \& C.

Phoma Tulipiferae, S.

Phyllosticta circumvallata, Wint.

$\{$ Phyllosticta Liriodendri, Cke.

Phyllosticta liriodendrica, Sacc.

Septoria Liriodendri, B. \& C.

Vermicularia albomaculata, S.

$$
\text { MELANCONIEAE. }
$$

Cylindrosporium cercosporoides, Ell. \& Ev.

Gloeosporium Liriodendri, Ell. \& Ev.

$\{$ Melanconium coloratum, Pk.

Myxosporium coloratum, Sacc.

\section{HYPHOMYCETES.}

Cercospora Liriodendri, Ell. \& Hark.

Monilia aurea, Lk.

Oospora Tulipiferae, Ell. \& Martin.

Septonema caespitosum, B. \& C.

Septonema circinatum, B. S C.

Septonema punctiforme, B. \& C.

Sporidesmium sarcinula, B. \& C.

Sporotrichum incarnatum, $\mathrm{S}$.

Trichoderma globosum, S. 
DISCU.MCLTES.

Patellaria nigrocinnabarina. S.

Peziza aurelia, P.

Peziza Erinaceus. S.

Pezizavillosa, P.

Peziza vinosa, A. S S.

Stictis hrsterina, Fr.

TREMELLIN゙J。

Tremella dependens, B. \& C.

Tremella moriformis, $B$.

HYMEXOMYCETES.

Agaricus glandulosus, Bull.

Agaricus ulmarius, Bubl.

Hrdnum ciliolatum, B. \& C.

Hydnum cirrhatum, P.

Hydnum Halei, B. \& C.

Irpex pallescens, Fr.

(Boletus Tulipiferae, S.

$\{$ Polyporus corticola, Fr.

Irpex Tulipiferae, S.

f Agaricus strigosus, S.

\{Lentinus strigosus, Fr.

Polyporus chartaceus, B. \& C.

Polyporus cinereus, $\mathrm{S}$.

Polyporus fulvus, $\mathrm{Fr}$.

Colyporus hirsutus, Fr.

Boletus nigromarginatus, $\mathrm{S}$.

Polyporus nigromarginatus, $\mathrm{S}$.

Polyporus Neelgerhensis, Mont.

PoIyporus scarrosus, B. \& C.

Polyporus tephroleucus, Fr.

Porothelium pezizoides, S.

( Agaricus alneus, L.

TSchizophyllum commune, Fr.

Magnolia acuminata, L.

Phyllactinia guttata, Lev.

Sphaeronema Magnoliae, Pk.

Sporidesmium fusus, B. \& C.

Steganosporium formosum, Ell. \& Ev.

Magnolia cordata, Michx.

fDiscella Magnoliae, B. \& C.

\{ Discula Magnoliae, Sacc.

Magnolia Fraseri, Walt.

Helminthosporium macrocarpon, Grev.

Magnolia glauca, L. 184

\section{PIREXOMICETES.}

Antennaria scmiorata, B. \& Br.

Apiosporium ? erysiphoides, Sacc. \& Ell.

Asterina comata, B. \& Rav.

Capnodium pelliculosum, B. \& Rav.

Diatrype asteromata, B. \& C.

Diatrype disciformis, (Hoffm.) Fr., var. Americanum, Thm.
(Diatrype disciformis, (Hofin.) Fr., var. Magnoliae, Thm.

Diatrype tremellophora, Ell.

Diatrype Duriaei, Mont.

Siatrype hypophlaea, B. \& Rav.

Nummularia hypophlaea, Cke.

Anthostoma hypophlaeum, Sacc.

Diatrype minima, Ell. \& Ev.

Diatrype sphaerophora, Ell. \& Ev.

$\{$ Dothidea excavata. C. \& E.

Curreya excavata, Sacc.

Gnomonia Nagnoliae, Ell.

Hypocrea corticiicola, Ell. \& Ev.

Hypoxylon epiphaeum, B. \& C.

Nectria aureofulva, C. \& E.

Nectria coccinea, (P.) Fr.

$\{$ Nectria conigena, Ell. \& Ev.

$\{$ Dialonectria conigena, Ell. \& Ev.

$\{$ Nectria microspora, C. \& E.

$\{$ Nectriella microspora, Sacc.

Nectria rubicarpa, Cke.

Sphaeria discreta, S.

\{ Nummularia discreta, Tul.

Hypoxylon discretum, B. \& C.

Saccardia Martinii, Ell. \& Sacc., var. major, E11.

Sphaerella glauca, Cke.

Sphaeria discreta, S.

Sphaeria Magnoliae, S.

Sphaerella Magnoliae, Ell.

Laestadia Magnoliae, Sacc.

Sphaerostilbe flammea, Tul.

Sphaerostilbe flammea, 'Tul., var. pallida, Sacc.

f Valsa binoculata, Ell.

(Diaporthe binoculata, Sacc.

$\{$ Valsa monticulosa, B. \& C.

\{ Eutypella monticulosa, Sacc.

Venturia applanata, Ell. \& Martin.

Venturia saccardioides, Ell. \& Martin.

\section{SPHAEROPSIDEAE.}

Actinothyrium Nagnoliae, S.

Discosia artocreas, (Tode) Fr.

(Eustegia Magnoliae, Rav.

$\{$ Stegia Magnoliae. Rav.

Protostegia Magnoliae, Rav.

Phoma mamillaris, B. \& C.

Septoria glauca, Cke.

Phyllosticta glauca, Cke.

Depazea glauca, Sacc.

\{ Sphaeronema rufum, Fr.

Sphaeronemella rufa, Sacc.

\{ Sphaeropsis macrospora, B. \& C.

\{ Dothiorella macrospora, Sacc.

MELANCONIEAE.

\{ Stilbospora monotospora, Cke.

$\{$ Nelanconium Monotospora, Sacc. 
IIYPIIOMYCETES.

ANONACEAE.

Atractium pallidum, B. \& C.

Cercospora Magnoliae, Ell. \& l Hark.

Cladosporium clelectum, C. \& E.

Cladosporium fasciculatum. Cda.

Dictyosporium elegans, Cda.

Helminthosporium arbuscula, B. \& C.

Helminthosporium trichellum, Sacc.

Macrosporium Martindalei, Ell. \& Martin.

Menispora ciliata, Cda.

Septosporium maculatum, C. \& E.

$\{$ Sporidesmium ellipticum, Che.

\{ Sirodesmium ellipticum, Sacc.

Sporidesmium maculare, B. \& C.

Sporidesmium rude, Ell.

DISCOMYCETES.

Cenangium Magnoliae, B. \& C.

Ombrophila albofusca, Ell.

Peziza albopileata, Cke.

Peziza protrusa, B. \& C.

Rhytisma Magnoliae, $\mathrm{S}$.

HYMENOMYCETES.

Corticium subgiganteum, B.

Hydnum ciliolatum, B. \& C.

Hydnum fuscoatrum, Fr.

Irpex Tulipiferae, S.

Polyporus Salleanus, B.

Radulum Magnoliae, B. \& C.

Magnolia grandiflora, L. If

Asterina comata, B. \& Rav.

Campsotrichum circinatum, B. \& C.

Campsotrichum tenue, B. St C.

Diatrype hypophlaea, B. \& Rav.

Diplodia punctipetiolae, Cke.

Discosia ocellata, B. \& C.

Hypochnus albocinctus, Mont.

Marasmius bombycirhiza, B. S Cke.

Meliola amphitricha, Fr.

Pestalozzia Guepini, Desm.

Phyllosticta Magnoliae, Sacc.

$\{$ Eustegia Magnoliae, Rav.

\{ Protostegia Magnoliae, Rav.

Septoria Magnoliae, Cke.

Septoria niphostoma, B. \& C.

Sphaeria fructuosa, B. d C.

\{ Sporidesmium punctiphyllum, Cke.

\{Sirodesmium stictophyllum, Sacc.

Vermicularia carbonacea, B. \& C.

Magnolia macrophylla, Michx.

\{ Sphaeria Berenıce, B. \& C.

\{ Acanthostigma Berenice, Sacc.

Magnolia Umbrella, Lam.

Discosia artocreas, (Tode) Fr.

Phoma melalencum, B. \& C.
A simina triloba, Dunal. /85

Capnodium Fuligo, B. \& Desm.

Cercospora Asiminae, Ell. \& Kell.

CXvloma Annonae, S.

$\int$ Ectostroma Annonae, Fr.

(Dothidea Annonae, S.

Phleospora Asiminae, Ell. \& Morg.

Phyllosticta Asiminae, Ell. \& Kell.

Sphaeria myriadea, DC.

Vermicularia albomaculata, S.

Asimina, sp. indet.

Hirneola scutelliformis, B. S C.

Polyporus contiguus, P.?

Anonaceae, sp. indet.

Phoma helvolum. B. \& C.

MENISPERMACEAE.

Menispermum Canadense, L.183-

Entyloma Menispermi, Farl. \& Trelease.

IIjcrosphaera Menispermi, Howe.

Phoma Menispermi, Pk.

Phyllosticta abortiva, Ell. \& Kell.

f Sphaeria sarmentorum, Fr.

\{ Diplodia sarmentorum, Fr

Sphaeropsis Menispermi, Pk.

Tubercularia Menispermi, Fr.

Valsa Menispermi, Ell. \& Holw.

\section{BERBERIDACEAE.}

Berberis Aquifolium, Pursh.

Puccinia Berberidis, Mont.

Puccinia mirabilissima, $\mathrm{Pk}$.

Uromyces sanguineus, $\mathrm{Pk}$.

Berberis Canadensis, Pursh.

$\{$ Aecidium Berberidis, Gmel.

$\{$ Caeoma (Aecidium) berberidatum, Lk.

$\{$ Dothidea Berberidis, (Wahl.) De Not.

$\{$ Plowrightia Berberidis, Sacc.

Sphaeria aequilinearis, S.

$\{$ Diatrype aequilinearis, M. A. Curtis.

\{ Sphaeria Berberidis, P.

\{ Cucurbitaria Berberidis, Gray.

Valsaria Farlowiana, Sacc.

Berberis pinnata, Lagasca.

Phacidium coronatum, Fr.

Berberis repens, Lindl. $/ 65$

Puccinia mirabilissima, $\mathrm{Pk}$.

Berberis, sp. indet.

Phoma melaleucum, B. \& C.

- Phyllactinia guttata, Lev.

S Sphaeria velata, $P$.

Diaporthe velata, Nits.

Caulophyllum thalictroides, Michx.

Cercospora Caulophylli, Pk. 
\{ Polyactis streptothrix, C. SE E.

Botrytis streptothrix, Sncc.

Podophyllum peltatum, L. 165

$\{$ Accidium Podophylli, S.

\{ Cacoma (Aecidium) podophyllatum, S.

$\{$ Ascospora Porlophylli, M. A. Curtis.

Phyllosticta Podophylli, Wint.

$\{$ Puccinia Podophylli, s.

$\{$ Puccinia aculeati, Lk.

Septoria podophyllina, Pk.

\section{NYMPHAEACEAE.}

Nymphaea odorata, Ait.

Cercospora nymphaeacea. C. \& E.

SARRACENIACEAE.

Sarracenia flava, L. 185

Peziza atrata, P.

Sphaeria Sarraceniae, S.

Sarracenia purpurea, L. 165

Peckin Sarracenine, Pk. \& Clinton.

$\{$ Sphaeria Sarraceniae, S.

\{ Sphaerella Sarraceniac, Sacc.

\{ Spharia scapophila, Pk.

\{Leptosphaeria scapophilit, Sacc.

Sarracenia rubra, Walt.

$\{$ Sphaeria Sarraceniae, S.

Sphaerella Sarraceniae, Sacc.

Sarracenia variolaris, Michx.

Splacria Sarraceniae, S.

\section{PAPAVERACEAE.}

Argemone platyceras, Link \& Otto.185

Gloeosporium Argemonis, Ell. \& Ev.

Bocconia cordata, Willd. /85

$\{$ Sphacria Bocconiae, C. \& E.

\{Leptosphacria Bocconiae, Sacc.

Dendromecon rigidum, Benth.

Massaria cleistotheca, Hark.

Pleospora argyrospora, Hark.

Splaterella Dendromeconis, Cke. \& Hark.

Eschscholtzia Californica, Cham./85

Entvloma Eschscholtziac, Hark.

Ifeterosporium Eschscholtziac, Hark.

Pcziza Eschscholtziac, Phil. \& Hark.

Papaver nudicaule, L.185

Pleospora herbarum, (P.) Rabh.

Sanguinaria Canadensis, L.

Cercospora Sanguinariae, Pk.

Cylindrosporium circinans, Wint.

Phyllonticta Sanguinariae, Wint.

FUMARIACEAE. Corydalis aurea, Willd., var. occidentalis, 185
Gray.

Aecidium bicentrae, Trelease.

+ Peronospora Corydalis, DBy.
Corydalis Brandegei, Watson.

Puccinia Brandegei, $\mathrm{Pk}$.

Dicentra Canadensis, DC.

+ Peronospora Corydalis DBy.

Dicentra Cucullaria, DC.

Aecidium Dicentrae, Trelease.

+ Peronospora Corydalis, DBy.

\section{CRUCIFERAE.}

\section{Arabis Holboellii, Hornem. 186}

Aecidium monoicum, $\mathrm{Pk}$.

Arabis Lyallii, IVatson.

Puccinia aberrans, $\mathrm{Pk}$.

Arabis, sp. indet.

Aecidium Bahiae, B. \& C.

+Cystopus candidus; (P.) Lev.

Pyrenophora depressa, $\mathrm{Pk}$.

Barbarea vulgaris, R. Br. $\%$

Macrosporium caudatum, C. \& E.

Brassica nigra, Koch.

+Cystopus candidus, (P.) Lev.

+ Peronospora parasitica, (P.) Tul.

Pleospora media, Niessl.

Brassica oleracea, L. $/ 80$

Cladosporium atrum, Lk.

\& Cystopus candidus, (P.) Lev.

Fusidium torulosum, B. \& C.

$\{$ Helminthosporium Brassicola, S.

Helminthosporium folliculatum, Cda.

Macrosporium Brassicae, B.

Macrosporium Cheiranthi, Fr., var. circinans, B. \& C.

Mucor Beaumontii, B. \& C.

$\{$ Nectria Brassicae. Ell. \& Sacc.

\{Dialonectria Brassicae, Ell. \& Er.

$\{$ Botrytis parasitica, $P$.

\{ Peronospora parasitica, Tul.

Peziza brassicaecola, B.

Polyactis cana, B.

Sphaerella brassicaecola, (Duby) Ces.\& DeNot.

Stilbum annulatum, B. \& C.

Stilbum Spraguei, B. \& C.

Tubercularia liceoides, Fr.

Brassica Rapa, L.

+ Cystopus candidus, (P.) Lev.

Brassica, sp. indet. 186

Alternaria tenuis, Nees.

Aspergillus purpureofuscus, $S$.

Botrytis lateritia, S.

Dacryom yces capitatus, S.

Gibbera pulicaris, Fr.

Polyactis rulgaris, Lk.

Sarea brassicaecola, S.

Sphaeria Brassicae, S.

$\{$ Sphacria alericola, B. \& C.

\{ Leptosphaeria alericola, Sacc. 
Sporotrichum sporulosum, Lk.

Stachylidium fulvum, S.

Capsella Bursa-pastoris, Moench.

Cylindrosporium Capsellae, Ell. \& Ev.

tCystopus candidus, (P.) Lev.

+ Peronospora parasitica, (P.) Tul.

Cardamine rhomboidea, DC. 186

* Peronospora parasitica, (P.) Tul.

Cheiranthus asper, Cham. \& Schl.

Pleospora vulgaris, Niessl.

Cheiranthus Menziesii, Benth. \& Hook.

Aecidium auriellım, Pk.

Cheiranthus, sp. indet.

Pleospora quadriseptata, Cke. \& Hark.

Sphaeria Cruciferarum, Fr.

f Uredo Cheiranthi, P.

$\Psi$ Cystopus candidus, (P.) Ler.

Dentaria diphylla, Michx.

千Cystopus candidus, (P.) Ler.

Septoria Dentariae, Pk.

Dentaria laciniata, Muhl.

Chaetomella Stevensonii, Ell.

+Peronospora parasitica, (P.) Tul.

Septoria Sisymbrii, Ell.

Draba alpina, L. 186

Sphaerella Stellarinearum, Karst.

Draba Caroliniana, Walt.

+ Peronospora parasitica, (P.) Tul.

Draba, sp. indet.

Phoma Drabae, Fckl.

Pleospora hispida, Niessl.

Lepidium campestre, $\mathrm{R}$. Br.

Cercospora Lepidii, Pk.

Lepidium intermedium, Gray. $/ \mathrm{fb}$

+ Peronospora parasitica, (P.) Tul.

Lepidium Virginicum, L. If

\& Cystopus candidus, (P.) Lev.

* Peronospora parasitica, (P.) Tul.

Septoria lepidiicola, Ell. \& Martin.

S Sphaeria Virginica, C. \& E.

Leptosphaeria Virginica, Sacc.

Matthiola incana, R. Br.

Pleospora quadriseptata, Cke. \& Hark.

Nasturtium Armoracia, Fr.

\& Cystopus candidus, (P.) Lev.

Macrosporium herculeum, Ell. \& Martin.

* Peronospora parasitica, (P.) Tul.

Ramularia Armoraciae, Fckl.

Nasturtium palustre, DC. 166

\& Cystopus candidus, (P.) Lev.

* Peronospora parasifica (P.) Tul.

Raphanus sativus, L./fG

Cercospora Cruciferarum, Ell. \& EV.

+ Cy'stopus candidus, (P.) Lev.

* Peronospora parasitica, (P.) Tul.
Sisymbrium canescens, Nutt.186

* Cystopus candidus, (P.) Ler.

Sisymbrium officinale, scop. $16 \%$

+Cystopus candidus, (P.) Lev.

Septoria Sisymbrii, Ell.

Smelowskia calycina, Meyer.

Aecidium Parryi, Pk.

Puccinia aberrans, Pk.

Thlaspi, sp. indet.

f Uredo candicla, P.

+ Cystopus candidus, Ler.

Cruciferae, sp. indet.

Pleospora Drabae, Schrt.

Puccinia Barbareae, Cke.

CAPPARIDACEAE.

Isomeris arborea, Nutt.

Aecidium Anisacanthi, Pk.

Aecidium isomerinum, $\mathrm{Pk}$.

RESEDACEAE.

Reseda odorata, L.

Cercospora Resedae, Fckl.

\section{CISTACEAE.}

Hudsonia tomentosa, Nutt.

Cucurbitaria Coremae, Ell. \& Ev.

Diaporthe Conradii, Ell.

PITTOSPORACEAE.

Pittosporum, sp. indet.

Diplodia Pittospori, Cke. \& Hark.

Gibberella Saubinetii, (Mont.) Sacc.

Phoma Pittospori, Cke. \& Hark.

Sphaerella Pittospori, Cke.

Stictis monilifera, Phil. \& Hark.

\section{VIOLACEAE.}

Ionidium concolor, Benth. \& Hook.

Cercospora Columbiensis, Ell. \& Ev.

Viola blanda, willd. $18 \$$

Cercospora Violae, Sacc.

Puccinia Violae, (Schum.) DC.

Viola canina, L.

Puccinia Violae, (Schum.) DC.

Viola cucullata, Ait.

Aecidium Mariae-IVilsoni, Pk.

$\{$ Aecidium Violae, Schum.

\{ Caeoma (Ecidium) violatum, Lk.

Cercospora granuliformis, Ell. \& Holw.

Cercospora murina, Ell. \& Kell.

Cercospora Violae, Sacc.

Phyllosticta Violae, Desm. 
Puccinia Violac, DC.

Septorna Violae, Westd.

Viola delphinifolia, Nutt.

Accidium Petersii, B. \& C.

Aecidimin Violine, Schum.

Puccinia Violae (Sihmm.) DC.

Viola hastata, Michx./y?

$\{$ Aecidium Violate Schmm.

\{ Cacoma (Aecidium) violatum, Lk.

Puccinia hastata, Cke.

Viola lanceolata, L.

Puccisia Violac, (Schum.) DC.

Septoria Violac, Westd.

Viola odorata, L./57

Cercospora Violae, Sacc.

Viola pedata, L.

Cacoma (Aecidium) pedatatum, S.

Accidium Violae, Schum.

Viola primulaefolia, L.

Puccinia hastata, Cke.

Viola pubescens, Ait./6?

Aecidium Violae, Schum.

Ascochyta Violae, Sacc. \& Speg.

Darluca filum, Cast.

(Puccinia Violae, (Schum.) DC.

\{ Puccinia Violarum, Lk.

Septoria Violae, Westd.

Viola renifolia, Gray.

Aecidium Violne, Schum.

Viola rostrata, Mubl.

Puccinia Violae, (Sclum.) DC.

Viola rotundifolia, Michx.

Puccinia Violae, (Schum.) DC.

$\{$ Vermicularia concentrica, Pk. \& Clinton.

\{Vermicularia Peckii, Sacc.

Viola sagittata, Ait.

Caeoma (Aecidium) sagittatum, $\mathrm{S}$.

Viola striata, Ait.

Puccinia Violae, (Schum.) DC.

Viola tricolor, L., var. arvensis, Ging.

Peronospora Violae, DBy.

Viola, sp. iudet. 187

Uredo Violarum, DC.

Urocystis Violac, B. \& Br.

\section{POLYGALACEAE.}

Polygala cruciata, L.

Cercospora grisea, C. \& E.

Polygala grandiflora, Walter.

$\{$ Dothidea Polygalac, S.

\{Phyllachora Polygalac, Sacc.

Polygala lutea, L.

$\{$ Cercospora grisea, C. \& E.

\{ Cercospora minuta, C. $\mathbb{E}$ E.
Polygala paucifolia, Willd.

Puccinia Pyrolae, Cke.

Septoria Polygalae. Pk. \& Clinton.

Polygala Senega, L.

Aecidium polygalinum, $\mathrm{Pk}$.

Septoria consocia, Pk.

Polygala, sp. indet. / 87

Gibbera Saubinetii, Sacc.

Phoma Polygalae, Cke. \& Hark.

Phomatospora Berkeleyi, Sacc.

\section{CARYOPHYLLACEAE.}

Arenaria verna, L.

Puccinia hysteriformis, $\mathrm{Pk}$.

Cerastium nutans, Raf.

Aecidium Cerastii, Wint.

Cerastium oblongifolium, Torr.

Septoria Cerastii, Rob. \& Desm.

Cerastium viscosum, $\mathrm{L}$.

Isariopsis pusilla, Fres.

Septoria Cerastii, Rob. \& Desm.

Cerastium vulgatum, L.

Septoria Cerastii, Rob. \& Desm.

Cerastium, sp. indet.

Uredo Caryophyllacearum, Johnst.

Dianthus barbatus, L.

I Puccinia Dianthi, DC.

$\{$ Puccinia Lychnidearum, Lk.

-Septoria Dianthi, Desm.

Dianthus Chinensis, L.

Vermicularia subeffigurata, S., var. dianthicola,

Dianthus, sp. indet.

\{ Sphaeria Dianthi, A. \& S.

\{ Ascochyta Dianthi, Berk.

Asteroma Dianthi, Cke. \& Hark.

Botryosporium prorumpens, S.

Polyactis vulgaris, Lk.

\{ Sphaerella caryophyllea, Cke. \& Hark.

\{Laestadia caryophyllea, Berl. \& Vogl.

Lychnis Flos-cuculi, L.

Leptothyrium Lychnidis, B. \& C.

Lychnis vespertina, Sibth.

Leptoria Melandrii, Pass.

Lychnis, sp. indet.

Hymenula Lychnidis, Pk.

Ustilago antherarum, Fr.

Saponaria officinalis, L.

Macrosporium Saponariae, Pk.

Saponaria, sp. indet.

\{ Sphacria permunda, Cke.

\{ Pleospora permunda, Sacc.

Silene gallica, L.

Pleospora oligomera, Sacc. \& Speg. 
Silene noctiflora, L.

Septoria Silenes, Ell. \& Martin.

Septoria noctiflorae, Ell. \& Kell.

Silene stellata, Ait.

f Septoria Silenes, Ell. \& Martin.

$\left\{\begin{array}{l}\text { Septoria silenicola, Sacc. } \\ \text { Siles }\end{array}\right.$

Silene, sp. indet.

+Peronospora Arenariae, (B.) DBy., var. macrospora, Farl.

Stellaria longipes, Goldie.

Sphaerella Stellarinearum, Karst.

Stellaria, sp. indet.

Darluca interseminata, Wint.

\section{PARONYCHIEAE.}

Scleranthus annuus, L.

Septoria Scleranthi, Desm.

PORTULACACEAE.

Calandrinia Leana, Porter.

Uromyces unitus, $\mathrm{Pk}$.

Claytonia Carjliniana, Michx.

Caeoma (Aecidium) claytoniatum, Schw.

Puccinia Mariae-Wilsoni, Clinton.

Uromyces Claytoniae, C. \& P.

Claytonia Virginica, L.

Caeoma (Aecidium) claytoniatum, S.

+ Peronospora Claytoniae, Farl.

Puccinia Mariae-IVilsoni, Clinton.

Portulaca oleracea, L.

$\{$ Cystopus Bliti, (Bivon) Lev.

$+\{$ Cystopus Portulacae, (DC.) Lev.

Portulaca, sp. indet.

Phoma stigma, Cke. S Hark.

Spraguea umbellata, Torr.

Uromyces Spragueae, Hark.

\section{TAMIARISCINEAE.}

Tamarix Gallica, L.

fDothidea Tamaricis, Cke.

$\left\{\begin{array}{l}\text { Bagnisiella Tamaricis, Sacc. } \\ \text { Bathe }\end{array}\right.$

\section{IIY PERICACEAE.}

Elodes Virginica, Nutt.

Uromyces IIyperici, M. A. Curtis.

$\{$ Uromyces Hyperici-frondosi, Arth.

Uromyces triquetra, Cke.

Hypericum aureum, Barton.

Aecidium Hyperici-frondosi, S.

Caeoma hypericatum, Lk.

Aecidium hypericatum, Auct.

Aecidium oblongum, Bon.

Didymosporium complanatum, Nees.

Fusarium effusum, S.
Hypericum corymbosum, Muhl.

Septoria sphaerelloides, Ell. \& Kell.

Uromyces Hyperici. M. A. Curtis.

$\{$ Trichobasis Hyperici, Gerard.

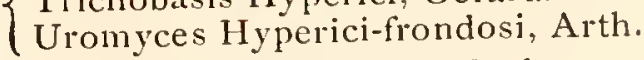

Hypericum ellipticum, Hook.

Aecidium hypericatum. Anct.

Hypericum inutilum, L.

Aecidium hypericatum, Auct.

$\{$ Aecidium minutissimum, Gerard.

Caeoma (Uredo) Hyperici, S.

Uromyces Hyperici, M. A. Curtis.

Uromyces Hyperici-frondosi, Arth.

Uromyces triquetra, Cke.

Hypericum perforatum, L.

Aecidium hypericaturn, Auct.

Leptostroma Hyperici, S.

Uromyces triquetra, Cke.

Hypericum prolificum, L.

Botryosphaeria Hypericorum, Cke.

Sphaerella hypericina, Ell.

Sphaeria Hyperici, S.

Hypericum pyramidatum, Ait.

Aecidium hypericatum, S.

Uromyces IIyperici, M. A. Curtis.

$\{$ Uromyces Hyperici-frondosi, Arth.

Hyperioum Sarothra, Michx.

Peziza atrocinerea, Cke.

Hypericum, sp. indet.

Sphaeria Hyperici, S.

\{ Sphaeria hypericina, B. \& C.

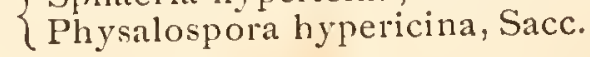

\section{TERNSTROEMIACEAE.}

Gordonia lasiantha, L.

Cystospora Gordoniae, B.

Meliola cryptocarpa, E11. \& Martin.

Phyllosticta Gordoniae, Ell. \& Martin.

Gordonia, sp. indet.

Sphaerella Gordoniae, Cke.

\section{MALVACEAE.}

Abutilon Avicennae, Gaertn.

Cercospora althaeina, Sacc.

Fusisporium roseum, Lk.

Abutilon crispum, Don.

Puccinia heterospora, B. \& C.

Abutilon holosericeum, Scheele.

Uromyces pulcherrimus, B. \& C.

Abutilon Texense, Torr. \& Gr.

$\{$ Puccinia heterospora, B. \& C.

$\{$ Uromyces pulcherrimus, B. \& C.

Althaea rosea, L.

Cercospora athaeina, Sacc.

Puccinia Malvacearum, Mont. 
Callirrhoe involucrata, Gray. Aecidium Callirrhoes, Ell. \& Kell. Cercospora althaeina, Sacc.

Gossypium album, Ham.

Sphaeria subconnata, S.

$\{$ Thuemenia valsarioides, Rehm.

Botryosphaeria subconnata, Cke.

Gossypium herbaceum, $L$.

Fusisporium aurantiacum, Lk.

Macrosporium gossypinum, Thm.

Gossypium, sp. indet.

Cercospora gossypina, Cke.

Diplodia gossypina, Cke.

Diplodia herbarum, (Cda.) Lev., rar.Gossypii, Sacc.

Dothiorella botryosphaerioides, Sacc.

$\{$ Melogramma horizontale, B. \& C.

$\{$ Botryosphaeria horizontalis, Sacc.

Phoma Gossypii, Sacc.

Phoma subconnata, B. \& C.

Phyllosticta gossypina, Ell. \& Martin.

Rhinotrichum macrosporum, Farl.

Septoria arcuata, (B.) Sacc., var. Gossypii,

$\{$ Sacc.

Phlyctaena Gossypii, Sacc.

Septoria gossypina, Cke.

\{ Sphaeria Gossypii, S.

\{Hyponectria ?'Gossypii, Sacc.

Sphaeria millegrana, $\mathrm{S}$.

Valsa gossypina, Cke.

Hibiscus esculentus, L.

Macrosporium abruptum, C. \& E.

Macrosporinm canificans, Thm.

Macrosporium hibiscinum, Thm.

Macrosporium spadiceum, Thm.

Mystrosporium polytrichum, Cke.

Phoma okra, Cke.

Hibiscus militaris, Cav.

$\{$ Caeoma (Aecidium) hibisciatum, S.

$\{$ Aecidium hibisciatum, Auct.

$\{$ Dothidea hibiscicola, S.

$\{$ Phyllachora hibiscicola, Sacc.

Sphaeria hibiscicola, S.

Hibiscus Moscheutos, L.

$\{$ Aecidium hibisciatum, Auct.

$\{$ Aecidium Hibisci, M. A. Curtis.

Sphaeria hibiscicola, S.

Hibiscus rosens, 'Thor.

Dothidea atra, $\mathrm{Fr}$.

$\{$ Sphaeria Hibisci, S.

Botryosphaeria Hibisci, Sacc.

Sphaeria tenella, S.

Hibiscus Syriacus, L.

Fusarium Berkelei, Mont.

Gibbera pulicaris, Fr.

$\{$ Nectria offuscata, B. St C.

$\{$ Nectriella offuscata, Sacc.
Peziza alboviolascens, A. S S.

\{ Sphaeria Hibisci, S.

\{ Melogramma Hibisci, B. \& C.

Sphaerostilbe gracilipes, Tul.

Torula brevissima, B. \& C.

Hibiscus, sp. indet.

Diaporthe ophites, Sacc.

Diplodia hibiscina. C. \& E.

Fusarium Berkelei, Mont.

Hendersonia collapsa, C. \& E.

Nectria offuscata, B. \& C.

Peziza variecolor, Fr.

Stilbum cinereorubrum, B. \& C.

Lavatera assurgentifolia, Kellogg.

Valsa Lavaterae, Cke. \& Hark.

Lavatera, sp. indet.

Torula quaternata, B. \& C.

Malva Alcea, L.

Sphaeria malvicola, S.

Malva borealis, Wallm.

(Puccinia Malvacearum, Mont., var. Malvastri, Farl.

Puccinia Malvastri, $\mathrm{Pk}$.

Malva rotundifolia, $\mathrm{L}$.

$\{$ Cercospora althaeina, Sacc.

$\{$ Cercospora malvicola, Ell. \& Martin.

$\{$ Septoria destruens, Auct. Amer.

$\{$ Septoria heterochroa, Desm.

Septoria malvicola, Eli. \& Martin.

Malva, sp. indet.

Capillaria Malvacearum, S.

Malvastrum coccineum, Gray.

(Puccinia Malvacearum, Mont., var. Malvas-

$\left\{\begin{array}{l}\text { tri, Farl. } \\ \text { Puccinial }\end{array}\right.$

Puccinia Malvastri, $\mathrm{Pk}$.

Roestelia interveniens, $\mathrm{Pk}$.

Malvastrum marrubioides, Dur. \& Hilg.

$\{$ Puccinia Malvacearum, Mont., var. Malvas-

$\{$ tri, Farl.

Puccinia Malvastri, $\mathrm{Pk}$.

Malvastrum Thurberi, Gray.

Roestelia interveniens, $\mathrm{Pk}$.

Malvaviscus Drummondi, Torr. \& Gr.

Puccinia heterospora, B. \& C.

Napaea dioica, $\mathrm{L}$.

Aecidium Napaeae, Arth. \& Holw.

- Erysiphe Cichoracearum, DC.

Sida lepidota, Gray.

Puccinia lobata, B. \& C.

Sida physocalyx, Gray.

Puccinia heterospora, B. \& C.

Sida spinosa, L.

Phyllosticta spinosa, Ell. \& Kell.

$\{$ Puccinia heterospora, B. \& C.

\{ Puccinia Thwaitesii, (B. \& Br.) Wint.

Sida supina, L'Her.

Puccinia heterospora, B. \& C. 
Sidalcea malvaeflora, Gray. Aecidium roestelioides, E11. \& Ev.

Septoria destruens, Auct. Amer.

Sidalcea, sp. indet.

Ramularia Sidalceae, Ell. \& Ev.

Malvaceae, sp. indet.

Periconia byssoides, $\mathrm{P}$.

\section{STERCULIACEAE.}

Fremontia Californica, Torr. Ascochyta Fremontine, Hark.

\section{TILIACEAE.}

Tilia Americana, L.

Agaricus aurivellus, Batsch.

Agaricus coloreus, $\mathrm{Pk}$.

Agaricus tiliophilus, $\mathrm{Pk}$.

\{ Cercospora microsora, Sacc.

$\{$ Cercospora Tiliae, Pk.

Corticium laeve, $\mathrm{P}$.

Cryptospora Tiliae, Pk.

Dendrophoma Tiliae, $\mathrm{Pk}$.

$\{$ Diatrype angularis, $\mathrm{Pk}$.

$\{$ Valsaria angularis, Sacc.

$\{$ Diatrype tiliacea, Ell.

$\{$ Hercospora tiliacea, Sacc.

Exosporum Tiliae. Lk.

$\{$ Coryneum pulvinatum, Kze. \& Schm.

Helminthosporium Tiliae, Fr.

Fusarium erubescens, B. \& C.

S Sphaeria Tiliae, P.

$\{$ Hercospora Tiliae, Tul.

Hypoxylon anthracodes, (Fr.) Mont.

Hypoxylon atropurpureum, Fr.

Massaria Curreyi, Tul.

Merulius sulcatus, Pk.

Naematelia nucleata, Fr.

Peziza Tiliae, Pk.

Solenia anomala, (P.) Fckl.

\{ Sphaeria citrispora, B. \& C.

\{Physalospora citispora, Sacc.

Sphaeria maculaeformis, $\mathrm{P}$.

$\{$ Sphaeria melanostyla, DC.

$\{$ Gnomoniella melanostyla, Sacc.

Sphaeria nobilis, B. S C.

\{ Sphaeria phileura, C. \& P.

\{Amphisphaeria phileura, Sacc.

Sphaeronema Robiniae, B. \& C.

Sphaeropsis tiliacea, $\mathrm{Pk}$.

Stemonitis ferruginea, Ehrb.

- Uncinula Clintonii, Pk.

Tilia Americana, L.var., pubescens, Loud.

Discosia minima, B. \& C.

Tilia, sp. indet.

Agaricus eximius, $\mathrm{Pk}$.
Coryne Ellisii, B.

$\{$ Diatrype Texensis, Ell. S Ev.

\{'Thyrdaria Texensis, Berl. \& Vogl.

Epidochium nigricans, Fr.

Gomphidius viscidus, Fr.

Merulius haedinus, B. I C.

Naematelia atrata, $\mathrm{Pk}$.

Oedemium atrum, Lk.

Pestalozzia stictica, B. \& C.

Radulum Bennettii, B. \& C.

Sphaerella sparsa, (Wallr.) Awd.

Sphaeria barbata, $\mathrm{P}$.

\{ Sphaeria Clypeus, S.

\{ Nummularia Clypeus, Cke.

Sphaeria furfuracea, Fr.

$\{$ Valsa furfuracea, Fr.

Diaporthe furfuracea, Sacc.

\{ Sphaeria incanescens, S.

\{ Sphaerella incanescens, Cke.

\{ Sphaeria variolaria, S.

\{ Valsa variolaria, Cke.

$\{$ Sphaeria velata, $\mathrm{P}$.

$\{$ Diaportne velata, Nits.

S Stemonites ovata, $\mathrm{P}$.

$\{$ Stemonites obtusata, Fr.

( Comatricha Friesiana, (DBy.) Rostf.

CAMELLIACEAE.

Camellia Japonica, L.

Hendersonia subalbicans, Gerard.

Pestalozzia Guepini, Desm.

Thea Bohea, L.

Sphaeria orthogramma, B. \& C.

LINACEAE.

Linum Breweri, Gray.

I. congestum, Gray.

L. drymarioides, Curran.

Melampsora Lini, (P.) Desm.

Linum sulcatum, Riddel.

tPeronospora Lini, Ell. \& Kell.

Linum Virginianum, L.

Cercospora Lini, Ell. \& Ev.

\section{GERANIACEAE.}

Erodium cicutarium, L'Her.

Synchytrium papillatum, Farl.

Geranium Carolinianum, L.

Botrytis pellucida, S.

Caeoma (Aecidium) geraniatum, Lk.

* Peronospora Geranii, Pk.

Pestalozziella subsessilis, Sacc. \& Ell.

Phyllosticta Geranii, Ell. \& Ev.

Septoria expansa, Niessl.

Stigmatea Geranii, Fr. 
Geranium maculatum, L.

S Aecidium Geranii, DC.

$\{$ Aecidium Geranii-maculati, S.

Caeoma (Aecidinm) geraniatum, Lk.

Botrytis pellucida, $\mathrm{S}$.

- Erysiphe communis. (Walls.) Fr.

$+\{$ Peronospora Geranii, Pk.

$+\{$ Peronospora nivea, Ung., var. Geranii, Farl.

Ramularia Geranii, Fckl.

- Sphaerotheca Castagnei, Lev.

Geranium Robertianum, L.

f Dothidea Robertiani, Fr.

Stigmatea Robertiani, Fr.

+ Peronospora Geranii, Pk.

Impatiens Balsamina, L.

Septoria nolitangeris, Ger.

Vermicularia Balsamitae, S.

Impatiens fulva, Nutt.

$\{$ Aecidium Impatientis, S.

$\{$ Caeoma (Aecidium) impatientatum, S.

(Dothidea Impatientis, S.

Asteroma Impatientis, M. A. Curtis.

Phyllachora Impatientis, Sacc.

tPeronospora obducens, Schrt.

Puccinia nolitangeris, Cda.

\{ Puccinia argentata, (Schultz) Wint.

Ramularia Impatientis, $\mathrm{Pk}$.

Septoria nolitangeris, Gerard.

Sphaerella Impatientis, Pk. S Clinton.

Vermicularia subefifgurata, S., var. Impatientis, Sacc.,

Impatiens pallida, Nutt.

$\{$ Aecidium Impatientis, S.

$\{$ Caeoma (Aecidium) impatientatum, S.

HPeronospora obducens, Schrt.

S Puccinia nolitangeris. Cda.

$\{$ Puccinia argentata, (Schultz) IVint.

Ramularia Impatientis, Pk.

Septoria nolitangeris, Gerard.

Oxalis Acetosella, L.

Didymium oxalinum, $\mathrm{Pk}$.

Ramularia Oxillidis, Farl.

Oxalis corniculata, L.

Phyllachora oxalina, Ell. \& Ev.

Oxalis corniculata, L., var. stricta, Sav.

- Microsphaera Russellii, Clinton.

Oxalis violacea, L.

Aecidium Oxalidis, Thm.

Pelargonium zonale, Willd.

Amerosporium Geranii, Cke. \& Hark.

Diaporthe elephantina, Cke. \& Hark.

Diaporthe Geranii, Cke \& Hark.

Leptosphaeria subcaespitosa, Cke. S Hark.

Physalospora Geranii, Cke. \& Hark.

Tubercularia Geranii, Cke. \& Hark.

Tubercularia sphaeroidea, Cke. \& Hark.
Tropaeolum minus, L.

\{ Sphaeria sarmenti, Cke. \& Hark.

\{ Didymosphacria sarmenti, Berl. \& Vogl.

\section{RUTACEAE.}

Citrus aurantium, L.

Capnodium Citri, B. \& Desm.

f Oidium fasciculatum, Berk.

\{Oospora fasciculata, Sacc. \& Vog\}.

Citrus, sp. indet.

Capnodium elongatum, B. \& Desm.

f Discella Citri, Cke.

\{ Discula Citri, Sacc.

Pestalozzia Guepini, Desm.

Peziza lecideola, Fr.

\{ Torula pallida, B. \& Rav.

\{ Oospora pallida, Sacc. \& Vogl.

Ptelea trifoliata, L.

Aecidium Pteleae, B. \& C.

Cercospora afflata. Wint.

- Cercospora Pteleae, Wint.

Xanthoxylum Americanum, Mill.

Aecidium Xanthoxyli, Pk.

Dermatea Xanthoxyli, Pk.

$\{$ I.ophiostoma obtectum, Pk.

\{Lophidium obtectum, Sacc.

Septoria pachyspora, Ell. \& Holw.

Salsa Xanthoxyli, Ph.

$\{$ Pseudoralsa Xanthoxyli, Sacc.

Fenestella Xanthoxyli. Sacc.

Xanthoxylum Clava-Herculis, L.

Cercospora Xanthoxyli. Cke.

Septoria pachyspora, Ell. \& Holw.

Xanthoxylum, sp. indet.

i Valsa stellulata, $\mathrm{Fr}$.

Z Eutypella stellulata. Sacc.

SIMARUBEAE.

Ailanthus glandulosus, L.

$\{$ Badhamia hyalina, (P.) B.

$\{$ Didymium simulans, Howe.

Cercospora glandulosa, Ell. \& Kell.

Cladosporium delectum, C. \& E.

Cucurbitaria Ravenelii, Cke. \& Mass.

Cytospora Ailanthi, B. \& C.

$\{$ Diderma albulum, Howe.

\{ Chondrioderma albulum, Cke.

$\{$ Diplodia Ailanthi, Cke.

\{ Botryodiplodia Ailanthi, Sacc.

Fusarium lateritium, Nees.

$\{$ Hendersonia Berkeleyana, Ler.

\{ Camarosporium Berkeleyanum, Sacc.

$\{$ Leptostroma petiolorum, Cke.

\{eptothyrium petiolorum, Sacc.

Phoma glandulosum, Cke.

Polyporus contiguus, (P.) Fr. 
Sphaerella Ailanthi, Cke.

$\{$ Sphaeropsis glandulosa, Cke.

\{Dothiorella glandulosa, Sacc.

Sphaeropsis tertia, Cke.

Phoma tertia, Sacc.

Nacrophoma tertia, Berl. \& Vogl.

Tubercularia Ailanthi, Cke.

Typhula gyrans, (Batsch.) Fr.

Valsa glandulosa, Cke.

$\{$ Valsa ventriosa, C. \& E.

$\{$ Eutypella ventriosa, Sacc.

\section{MELIACEAE.}

\section{Melia Azedarach, L.}

Agaricus corticola, P.

Agaricus meliigena, B. \& Cke.

Cercospora Meliae, Ell. \& Ev.

Corticium comedens, Fr.

Cucurbitaria cupularis, (P.) Cke.

Diatrype Azedarachtae, Cke.

Fusisporium azedarachinum, 'Thm.

Hypoxylon rubricosum, (Fr.) Mont.

Hysterium rufulum, Spreng.

Patellaria nigrocinnabarina, S.

Tryblidiella rufula, Sacc.

Hysterium nigrocinnabarinum, Rehm.

Sphaeria Meliae, S.

Selogramma Meliae, B. \& C.

Melogramma fuliginosum, Ell.p.p.

Botryosphaeria Meliae, Sacc.

Melogramma Meliae, Herb. M. A. Curtis.

Nectria coccinea, (P.) Fr.

Sphaeria Cucurbitula, Tode.

$\{$ Nectria Cucurbitula, Fr.

Chilonectria Cucurbitula, Sacc.

Nectria ditissima, Tul.

$\{$ Sphaeria verrucosa, S.

$\{$ Nectria verrucosil, Sacc.

Pachnocybe rosella, B. \& C,

Phoma petiolorum, Desm.

Sphaeria appendiculata, P.

Sphaerostilbe gracilipes, Tul.

\{Valsa aculeans, (S.) B. \& C.

$\{$ Calospora aculeans, Sacc.

?Valsal stilbostoma, Fr.

Vermicularia rectispora, Cke.

\section{ILICINEAE.}

Ilex Dahoon, Walt.

Asterina orbicularis, B. \& C.

Phyllosticta terminalis, Ell. \& M Iartin.

Ilex decidua, Walt.

$\{$ Sphaeria friabilis, $P$.

$\{$ Diatrype friabilis, M. A. Curtis.

Fusarium helotioides, B. \& C.
- Microsphaera Alni, (DC.) Wint.

- Phyllactinia suffulta, (Reb.) Sacc.

f Xyloma Ilicincola, S.

\{ Rhytisma Ilicincola, $\mathrm{Fr}$.

SXyloma velatum, $\mathrm{S}$.

$\{$ Rhytisma velatum, $\mathrm{Fr}$.

Tympanis Ravenelii, B. \& C.

Ilex glabra, Gray.

Asterina Ilicis, Ell.

Capnodium elongatum, B. \& Desm.

Cercospora Ilicis, Ell.

Dermatea olivacea, Ell.

$\{$ Diatrype Badhami, Curr.

$\{$ Diaporthe Badhami, Sacc.

$\{$ ? Valsa ocularia, C. \& E.

$\{$ ? Diaporthe ocularia, Sacc.

Nectria punicea, (Schm.) Fr.

Phacidium sphaeroideum, C. \& E.

Sphaerella Ilicis, Ell.

Sphaerella Prini, Cke.

\{ Sphaeronema stellatum, Ell.

\{ Sphaerographium stellatum, Sacc.

Ilex laevigata, Gray.

Tympanis Ravenelii, B. \& C.

Ilex Iucida, Torr. \& Gr.

$\{$ Asterina orbicularis, B. \& C.

$\{$ Dimerosporium orbiculare, Martin.

Asterina pelliculosa, B.

Capnodium elongatum, B. \& Desm.

Patellaria cyanea, E11. S Martin, nec Cke.

Ilex mollis, Gray.

Ramularia Prini, Pk.

\{ Xyloma Prini, S.

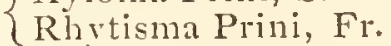

Ilex opaca, Ait.

Asterina cuticulosa, Cke.

$\{$ Asterina orbicularis, B. \& C.

\{ Dimerosporium orbiculare, Martin.

Cenangium microspermum, Sacc. \& Ell.

Cercospora pulvinula, C. S E.

Chaetophoma ilicifolia, Cke.

$\{$ Valsa cercophora, E1l.

\{ Diaporthe cercophora, Sacc.

f Valsa angulata, Fr.

\{ Diatrypella nigroannulata, (Grev.) Nits.

$\{$ Diatrype opaca, Cke.

$\{$ Diatry pella opaca, Cke.

Diplodia ilicicola, Desm.

$\{$ Sphaeria Ilicis, Fr.

$\{$ Diplodia Ilicis, Fr.

Discosia deflectens, Sacc.

$\{$ Dothidea Ilicis, Cke.

$\{$ Bagnisiella Ilicis, Sacc.

IIypoxylon fuscum, (P.) Fr.

Leptothyrium ? foraminulatum, Sacc. \& Ell.

Sphaeropsis phacidioides, C. \& E.

$\{$ Phoma phacidiella, Sacc.

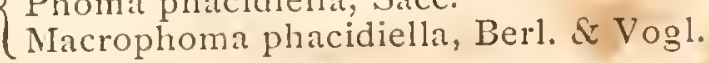


Pestalozzia stellata, B. \& C.

Peziza aquifoliae, C. S E.

$\{$ Phacidium multivalve, (DC.) Schn.

\{ Phacidium Ilicis, Lib.

Phoma Ilicis, Desm., var. Ilicis-opacae, Sacc.

Pilacre Petersii, B. \& C.

Polyporus dibaphus, B. S C.

Polyporus ilicincola, B. \& C.

Rhytisma Curtisii, B. \& Rav.

Sphaerella ilicella, Cke.

Sphaeria atrovirens, A. \& S.

\{ Sphaeria stemmatea, $\mathrm{Fr}$.

\{ Septoria stemmatea, B.

S Sphaeria Ilicis, Ell.

\{ Physalospora Ilicis, Sacc.

Tympanis picastra, B. S C.

Valsa didymospora, Ell.

Ilex verticillata, Gray.

Cordyceps pistillariaeformis, B. \& Br.

\{ Torrubia clavulata, $\mathrm{Pk}$.

Diatrype Badhami, Curr.

Glenospora Curtisii, B. \& Desm.

Hypoxylon callostroma, (S.) B.

Ramularia Prini, Pk.

Rhytisma Ilicis-Canadensis, S.

Rhytisma Prini, (S.) Fr.

Sphaeria prinicola, B. \& C.

$\{$ Rosellinia prinicola, Sacc.

Sphaeronema caespitosum, Pk.

Tympanis stictica, B. \& C.

Ilex, sp. indet.

\{ Excipula microspora, C. E E.

$\{$ Amerosporium microsporum, Sacc.

(Sphaeria nigrotecta, B. \& Rav.

\{ Anthostomella nigrotecta, Sacc.

Corticium cremoricolor, B. \& C.

Cytospora leucosperma, (P.) Fr.

Diplodia ilicicola, Desm.

Discosia minima, B. \& C.

Nacrosporium fasciculatum. C. \& E.

Mystrosporium orbiculare, C. \& E.

Pestalozzia annulata, B. SC.

Phacidium elegantissimum, B. \& C.

$\{$ Sphaeropsis ilicicola, C. \& E.

\{ Phoma ilicicola, Sacc.

Sphaeria philoprina. B. \& C.

T Physalospora philoprina, Sacc.

Septoria examinans, B. \& C.

Septoria ilicifolia, C. \& E.

Trichothecium roseum, Lk.

Dactylium roseum, (Lk.) B.

Valsa ceratophora, Tul.

Talsa subcuticularis, C. S E.

Nemopanthes Canadensis, DC.

- Microsphaera Nemopanthis, Pk.

Ramularia Nemopanthis. C. \& P.
Rhytisma Ilicis-Canadensis, S.

Rhytisma Canadensis, Pk.

Tympanis Nemopanthis, $\mathrm{Pk}$.

YValsa oxyspora. Pk.

Diaporthe oxyspora, Sacc.

Venturia curviseta, $\mathrm{Pk}$.

\section{CYRILLEAE.}

Cyrilla racemiflora, $\mathrm{L}$.

Phyllostict Cyrillae, Ell. \& Martin.

\section{CELASTRACEAE.}

Celastrus scandens, L.

Asterina Celastri, E11. \& Kell.

Corticium comedens, (Nees.) Fr.

$\{$ Sphaeria subconfluens, S.

Diatrype subconfluens, Cke.

Didymosporium erumpens, S.

fDiplodia Celastri, Cke.

\{ Botryodiplodia Celastri, Sacc.

Exidia lurida, S.

fHendersonia abnormalis, Pk.

\{ Canarosporium abnorme, Sacc.

Leptothyrium Celastri, B. \& C.

S Sphaeria Celastri. S.

$\{$ Nectria Celastri, Pk.

$\{$ Nectria inaurata, B. \& Br.

Aponectria inaurata, Sacc,

Phoma pallens, B. \& C.

- Phyllactinia guttata, Lev.

\{ Phyllactinia suffulta, (Reb.) Sacc.

( Ramularia Celastri, Ell. \& MIartin.

Ramularia Celastri, Pk.

Sphaeria mamillana, Fr.

Sphaeria sarmentorum, Fr.

Sphaeropsis celastrina, Pk.

Sphaeria propullans, S.

$\{$ cf. Sphaeropsis propullans, Pk.

cf. Botryosphaeria propullans, Cke.

Tubercularia Celastri, S.

S Sphaeria dolosa. Fr.

\{ Talsa dolosa, Nits.

Euonymus Americanus, L.

Cercospora Euonymi, Ell.

Euonymus atropurpureus, Jacq.

- Microsphaera Alni, (DC.) Wint.

Ramularia Euonymi, Ell. \& Kell.

Enonymus Japonicus, Thunb.

Cercospora destructiva, Rav.

\{ Cytospora foliicola. Lib., var.

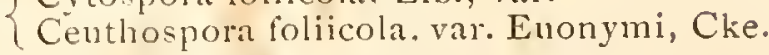

Cicinnobolus Cesatii, DBy.

Cladosporium fasciculatum, Cda., var, densum, Cke.

Cladosporium punctulatum, Sacc. \& Ell.

Diplodia Euonymi, West. 
Gibberella pulicaris, (Fr.) Sacc.

S Pestalozzia Planimi, Vize.

\{ Pestalozzia Enonymi, Vize.

Euonymus occilentalis, Nutt.

Ramularia Euonymi, Ell. \& Kell.

Euonymus vulgaris, Scop.

Cercospora Euonymi, Ell.

Euonymus, sp. indet.

Cucurbitaria seriata, Pk.

Otthia seriata, Sacc.

Sphaeria (Leptosphaeria) Californica, Cke. \& Hark.

- Microsphaera comata, Lev.

$\{$ Sporidesmium Fumago, Cke.

\{ Sirodesmium Fumago, Sacc.

? Pachystima Canbyi, Gray.

Melasmia alnea, Lev.

\section{RHAMNACEAE.}

\section{Berchemia volubilis, DC.}

f Aecidium crassum, P.

$\{$ Aecidium pulcherrimum, Rav.

f Valsa Berchemiae, Cke.

Eutypella Berchemiae, Sacc.

Helminthosporium lanceolatum, Cke.

\{ Hysterium medium, Cke.

$\{$ Glonium medium. Sacc.

? Peziza earoleuca, Rav. Fung. Am.

STriblidium rufulum, Spr.

Triblidiella rufula, Sacc.

Ceanothus Americanus, L.

$\{$ Discella microspora, B. \& C.

Discula microspora, Sacc.

(Erysiphe Ceanothi, S.

- Microsphaera Alni, (DC.) Wint.

Microsphaera Ceanothi, Pk.

S Spharia ceanothina, Pk.

Physalospora ceanothina, Sacc.

Ceanothus ovatus, Desf.

Aecidium Ceanothi, Ell. \& Kell.

Ceanothus, sp. indet.

Cytospora Ceanothi, S.

Diatrype Ceanothi, Cke. \& Hark.

Didymosphaeria Ceanothi. Cke. \& Hark.

\{Hysterium Ceanothi, Phil. \& Hark.

Hysterographium Ceanothi, Berl. \& Vogl.

Leptosphaeria Ceanothi, Cke. \& Hark.

f Spineria Ceanothi, S.

Valsa Ceanothi, Cke.

Rhamnus alnifolia, L'Her.

f Aecidium Rluamni, Gmel.

$\{$ Aecidium crassum, P.

Rhamnus Californica, Esch.

Capnodium Rhamni, Cke. \& Hark.

Diplodia Frangulae, Fckl.

Phoma communis, Roh.

Phoma rhamnicola, Cke. \& Hark.
Pleospora Frangulae, Fckl.

Tympanis Frangulae, Fr.

Rhamnus cathartica, L.

Aecidium crassum, P.

Rhamnus crocea, Nutt.

$\{$ Cytospora foliicola, Lib.

\{ Ceuthospora foliicola, (Lib.).

Puccinia digitata, Ell. \& Hark.

Rhamnus lanceolata, Pursh.

Aecidim crassum, P.

Dacryonyces deliquescens, (Bull.) Duby.

Polyporus subspadiceus, Fr.

$R$ hamnus, sp. indet.

Cercospora aeruginosa, Cke,

Cercospora Rhamni, Fckl.

Nectria Cucurbitula, (Tode) Fr.

Sphaeropsis Rhamni, Cke.

\section{VI'TACEAE.}

Ampelopsis quinquefolia, Michx.

Cercospora Ampelopsidis, Pk.

Cercospora pustula, Cke.

Sphaeropsis Ampelopsidis. C. E E.

$\{$ Phoma Ampelopsidis, Sacc.

(Macrophoma Ampelopsidis, Berl. \& Vogl.

+Peronospora viticola, (B. \& C.) DBy.

Phoma pallens, B. \& C.

(Phoma uvicola, B. \& C.

Phyllosticta viticola, Thm.

Phyllosticta Ampelopsidis, Ell. \& Martin.

Podosporium rigidum, S.

Spharonema Ampelopsidis, B. \& C.

(Uncinula spiralis, B. \& C.

$\rightarrow$ Uncinula Ampelopsidis, Pk.

Uncinula subfusca, B. \& C.

- Uncinula Wallrothii, Lev.

SValsa Ampelopsidis. Ell.

\{ryptosporella Ampelopsidis, Sacc.

Spliaeria capsularis, $\mathrm{P}$.

$\{$ Valsa capsularis, (P.).

(Valsa ambiens, (P.) Fr.

Vitis aestivalis, Michx.

Shelephora viticola, $\mathrm{Fr}$.

Corticiun viticolum, Fr.

Corticium crocicreas, B. \& C.

$\{$ Diplodia viticola, Desm.

$\{$ ? Diplodia Vitis, Desm.

Discosia artocreas, ('Iode) Fr., var. viticola, Cke.

f Dothidea picea, B. \& C.

\{ Phyllachora picea, Sacc.

Excipula viticola, S.

Hendersonia ampelina, Thm.

Hydnum luteopallidum, S.

(Leptothyrium longisporum, Thm.

Phoma longispora, Cke.

Nacrophoma longispora, Berl. \& Vogl. 
\$Peronospora viticola. (B. \& C.) DBy.

Pestalozzia pezizoides. DeNot.

$\{$ Coniothyrium ampelinum, Cke.

$\{$ Phoma ampelogena, Sacc.

Phoma ustulata, B. \& C.

Phoma uvicola, B. \& C.

Rhytisma monogramme, B. \& C.

Rhytisma Vitis, S.

Septoria ampelina, B. \& C.

Sphaeria pruina, S.

Sporocybe byssoides, (P.) Fr.

-Uncinula spiralis, B. \& C.

Vitis arborea, L.

Podosporium rigidum, S.

Vitis Calkornica, Benth.

\{ Septosporium Fuckelii, Thm.

\{ Cercospora Roesleri, (Catt.) Sacc.

$\{$ Cladosporium viticolum, Ces.

$\{$ Cercospora viticola, Sacc.

+Peronospora viticola, (B. \& C.) DBy.

Phoma Vitis, Bon.

Vitis cordifolia, Lam.

Ascochyta ampelina, Sacc.

Cercospora viticola, (Ces.) Sacc.

Corticium calceum, (P.) Fr.

+Peronspora viticola, (B. \& C.) DBy.

Phoma ustulata, B. \& C. (?)

$\{$ Phoma uvicola, B. \& C.

\{ Phyllosticta Labruscae, Thm.

Septoria Kellermaniana, Thm.

- \{Uncinula spiralis, B. \& C.

\{ Uncinula Americana, Howe.

Vitis indivisa, Willd.

Cercospora truncata, Ell. \& Ev.

Vitis Labrusca, L.

\section{PERONOSPOREAE.}

$\{$ Botrytis viticola, B. \& C.

$+\{$ Peronospora viticola, DBy.

PYREXOIIYCETES.

Acrospermum compressum, Tode.

Acrospermum Ravenelii, B. \& C.

Erysiphe necator, S.

$\{$ Hysterium australe, Duby.

\{ Gloniopsis australis, Sacc.

\{Hysterinm commune, Fr.

II ypoderma commune, Duby.

$\{$ Hysterium Rubi, P.

\{Hypoderma Rubi, DeNot.

\{ Hysterium viticolum, C. \& P.

\{Ilysterographium viticolum, Rehm.

Leptostroma sphaeroides, Fr.

SLophiostoma sexnucleatum, Cke.

$\{$ Lophiotrema sexnucleatum, Sacc., var. Peckianum, Sacc.

$\{$ Dothidea viticula, S.

\{ Phyllachora viticola, Sacc.
Sphaeria subfasciculata, S.

$\left\{\begin{array}{c}\text { Psilosphaeria (Melanopsamma) sufascicu- } \\ \text { lata Che. }\end{array}\right.$

\{ Sphaeria sentina, Fr.

Sphaerella sentina, $(\mathrm{Fr}$. $)$.

Sphaeria ampelos, S.

Uncinula spiralis, B. \& C.

- Uncinula Ampelopsidis, Pk.

Uncinula Americana, Howe.

Uncinula subfusca, B. \& C.

- Uncinula spiralis, B. \& C., var. racemorum, Thm.

Sphaeria Vitis, S.

? Diatrype Vitis, B. \& C.

Valsa Vitis, B. \& C.

Sphaeria propagata, Plow.

Eutypa viticola, Sacc.

Cryptosphaeria propagata, Sacc.

Sphaeria insitiva, Tode.

$\{$ Diatrype insitiva, $\mathrm{Fr}$.

Valsaria insitiva, Ces. \& DeNot.

SPHAEROPSIDEAE.

Ascochyta Ellisii, Thm.

Cytospora coryneoides, B. \& C.

Diplodia viticola, Desm.

(Sphaeropsis Peckiana, Thm.

$\{$ Phoma Peckiana, Sacc.

Macrophoma Peckiana, Berl. \& Vogl.

Phoma ampelina, B. \& C.

Naemaspora ampelicida, Engelm.

Phoma uvicola, B. \& C.

Depazea Labruscae, Engelm.

Septoria viticola, B. \& C.

Phoma uvicola, B. \& C., var. Labruscae, Thin.

Phyllosticta viticola, Thm.

Phyllosticta Labruscae, Thm.

Sacidium viticolum, Cke.

Phyllosticta Ampelopsidis, Ell. \& Martin.

HYPHOMYETES.

Alternaria tenuis, Nees.

Cladosporium viticolum, Ces.

Cladosporium ampelinum, Pass.

Graphim clavisporum, B. \& C.

Helminthosporium Vitis, Pirotta.

Cercospora viticola, Sacc.

Isariopsis clavispora, Sacc.

Epochnium monilioides, Lk.

Gonytrichum caesium, Nees.

Myrothecium convexum, B. \& C.

Sporotrichum alutaceum, S.

Sporotrichum viticolum, S.

Tubercularia subdiaphana, S.

Volutella ciliata, (A. \& S.) Fr.

DISCOMYCETES.

Calloria vinosa, (A. \& S.) $\mathrm{F}$ :

Seziza viticola, S.

$\{$ Tympanis viticola, Fr.

Cenangium viticolım, Fckl. 
Helotium pullatum, Gerard.

Peziza ascoboloidea, S.

Peziza penicillata, S.

Peziza viticola, P., nec S.

Peziza Vitis, S.

Stictis prominula, S.

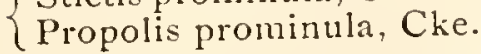

\section{HYMENOMYCETES.}

Thelephora albidocarnea, S.

$\{$ Corticium albidocarneum, Rav.

f Peziza villosa, P.

\{Cyphella villosa, Karst.

Marasmius viticolus, B. \& C.

Merulius Corium, Fr.

Boletus papyraceus, $\mathrm{S}$.

Polyporus vaporarius, (P.) Fr., var. b.

Poiyporus papyraceus, $S$.

Polyporus vaporarius, (P.) Fr.,

( var. papyraceus, Thm.

Polyporus viticolus, $\mathrm{S}$.

\section{MISCELLANEA.}

Cyathus striatus, (Huds.) Hoffin.

Dacryomyces viticolus, S.

Ditiola sulcata, (Tode) Fr.

Pestalozzia pezizoides, DeNot.

\section{Vitis riparla, Michx.}

f Hysterium flexuosum, S.

$\{$ Hysterographium flexuosum, Rehm.

+Peronospora viticola, (B. \& C.) DBy.

Pestalozzia Jefferisii, Ell.

Pestalozzia pezizoides, DeNot.

Phyllosticta spermoides, Pk.

Septoria Kellermaniana, Thm.

\section{Vitis vinifera. L.}

$\{$ Peziza rinosa, A. $\mathcal{S}$ S.

$\{$ Calloria rinosa, Fr.

Cenangium viticolum, Fckl.

$\{$ Helminthosporium Vitis, Pirotta.

$\{$ Cercospora riticola, (Ces.) Sacc.

Cytospora Vitis, Mont.

Diplodia viticola, Desm.

Helminthosporium siliquosum, B. \& C.

Hendersonia longipes, B. \& C.

$\{$ Hysterium viticolum, C. \& P.

$\{$ Hysterographium viticolum, Rehm.

Nectria viticola, B. \& C.

4 Peronospora viticola, (B. \& C.) DBy.

Pestalozzia pezizoides, DeNot.

Peziza penicillata, S.

Peziza viticola, P., nec S.

Phoma ampelina, B. \& C.

Phoma confluens, B. \& C.

$\{$ Phoma uricola, B. \& C.

$\{$ Septoria viticola, B. \& C.
Sphaeria uvaesarmenti, Cke.

$\{$ Physalospora uvaesarmenti, Sacc.

Septoria ampelina, B. \& C.

f Septoria falx, B. \& C.

Rhabdospora falx, Sacc.

Sphaeronema viticolum, B. \& C.

$\{$ Sphaeria antiqua, Ell. \& Ev.

Thyridium antiquum, Ell. \& Ev.

Uredo Vitis, Thm.

S Valsa Vitis, B. \& C.

\{ Eutypa viticola, Sacc.

Vermicularia compacta, C. \& E.

Vitis vulpina, L.

Cercospora vulpina, Ell. \& Kell.

Helminthosporium siliquosum, B. \& C.

Hysterium flexuosum, S.

Hysterium vulvatum, $\mathrm{S}$.

Patellaria atrofusca, B. \& C.

+ Peronospora viticola, (B. \& C.) DBy.

Peziza cinereofusca, S.

f Sphaeropsis uvarum, B. \& C.

$\{$ Phoma uvarum, Sacc.

\{ Phoma uvicola, B. \& C.

Phyllosticta viticola, Thm.

Septoria ampelina, B. \& C.

$\{$ Valsa Vitis, B. \& C.

Eutypa viticola, Sacc.

Sphaeria viticola, $\mathrm{S}$.

$\{$ Diatrype viticola, B. \& C.

Valsaria viticola, Sacc.

\{Xerotus lateritius, B. \& Rav., nec B. \& C.

\{Xerotus viticola, B. \& C.

Vitis, sp. indet.

\section{MYXOMYCETES.}

Lamproderma arcyrioides, (Sommf.) Rostf., var. iridea, Cke.

\section{PYREYOMYCETES.}

Capnodium elongatum, B. \& Desm.

$\{$ Nectria viticola, B. \& C.

$\{$ Dialonectria viticola, Cke.

Didymosphaeria bacchans, Pass.

$\{$ Hysterium Curtisii, Duby.

$\{$ Gloniella Curtisii, Sacc.

Hysterium pulicare, Fr.

Lophiostoma stenostomum, Ell. \& Ev.

S Sphaeria Bidwellii, Ell.

\{ Physalospora Bidwellii, Sacc.

\section{SPHAEROPSIDEAE.}

Coniothyrium Diplodiella, (Speg.) Sacc.

Cytospora coryneoides, B. \& C.

(Dichomera viticola, Cke. \& Hark.

\{ Camarosporium riticolum, Sacc.

Diplodia ampelina, Cke.

Discella albomaculans, $\mathrm{Pk}$.

Discosia artocreas, (Tode) $\mathrm{Fr}_{\mathrm{r}}$. 
Greeneria fuliginea, Scribner \& Viala.

Hendersonia corticalis, EH. \& Ev.

Hendersonia sarmentorum, Westd.

S Sphaeropsis viticola, Cke.

$\{$ Phoma viticola. Sacc.

Macrophoma viticola, Berl. \& Vogl.

Phoma longipes, B. \& C.

Phoma pallens, B. \& C.

Septoria Vitis, B. \& C.

\section{MELANCONIEAE.}

\{ Gloeosporium ampelophagum, Pass.

\{ Sphaceloma ampelinum, DBy.

Steganosporium viticolum, E11. \& Ev.

\section{HYPHOMYCETES.}

Botrytis acinorum, $\mathrm{P}$.

Cladosporium Fumago, Lk.

Dendryphium Harknessii, Ell., var. leptaleum, Ell.

Fusarium Schweinitzii, Ell. \& Hark.

$\{$ Helminthosporium oosporum, Cda.

\{ Brachysporium oosporum, Sacc.

? Oidium Tuckeri, B. \& Br.

Sporidesmium antiquum, Cda.

Sporidesmium Rauii, Ell. \& Hark.

Tubercularia ciliata, Lk.

\section{DISCOMYCETES.}

$\{$ Peziza occulta, Rehm.

\{ Calloria occulta, Rehm.

Helotium sarmentorum, DeNot.

Helotium vitigenum, DeNot.

Peziza cinerea, Batsch.

Peziza scutula, P.

Vibrissea hypogaea, Thm. \& Pass.

\section{HYMENOMYCETES.}

Agaricus applicatus, Batsch, var. sarmenticius, Sacc.

(Corticinm ceraceum, B. \& Rav.

Corticium molle, B. \& C.

Corticium armeniacum, Thm.

Corticium confluens, Fr.

Corticium hypopyrrhinum, B. \& C.

Cyphella virgultorum, Cke.

Hydnum udum, Fr.

\{ Hydnum mucidum, Gmel, var. viticolum, S.

$\{$ Hydnum viticolum, S.

Hydnum stipatum, Fr.

Irpex viticolus, C. \& P.

Marasmius Vaillantii, (P.) Fr.

$\{$ Odontia fimbriata, $\mathrm{P}$.

\{ Hydnum fimbriatum, Fr.

Polyporus barbaeformis, B. \& C.
Polyporus viticolus, S.

Stereum cristatum, B. \& C.

Stereum Leveilleanım, B. \& C.

\section{SAPINDACEAE.}

\section{Acer dasycarpum, Ehrh.}

Alternaria tenuis, Nees, var. condensata, Sacc.

Gloeosporium acerinum, Westd.

f Melanconis Everhartii, Ell.

\{Melanconis dasycarpa, Eil. \& Kell.

f Septoria Aceris, (Lib.) B. \& Br.

$\{$ Phleospora Aceris, Sacc.

Phyllosticta acericola, C. \&E.

$\{$ Rhytisma acerinum, (P.) Fr.

\{ Rhytisma Aceris-eriocarpae, S.

Septoria acerina, $\mathrm{Pk}$.

Stilbospora piriforme, Hoffim.

$\{$ Stilbospora ovata, P.

Steganosporium piriforme, Cda.

-Uncinula circinata, C. \& P.

Acer masrophyllum, Pursh.

f Ceratostoma tinctum, Ell. \& Ev.

$\{$ Rhynchostoma tinctum, Berl. \& Vogl.

Chaetophoma atriella, Cke. \& Hark.

Diatrypella Frostii, Pk.

Diplodia extensa, Cke. \& Hark.

Hypocrea contorta, (S.) B. \& C.

Hypoxylon concurrens, B. \& C.

Melanconium magnum, B.

$\{$ Stilbospora Monotospora, Cke.

\{Melanconium Monotospora, Sacc.

Myriococcum sparsum, Hark.

Sphaerella maculiformis, (P.) Awd.

$\{$ Stilbospora ovata, $\mathrm{P}$.

\{ Steganosporium piriforme, (Hoffm.) Cda.

Trichaegum opacum, Cke. \& Hark.

Valsa exigua, Nits.

Acer Pennsylvanicum, L.

$\{$ Melogramma subaquilum, B. \& C.

$\{$ Diaporthe subaquila, Sacc.

$\{$ Sphaeria sphaerellula, $\mathrm{Pk}$.

\{Didymella sphaerellula, Sacc.

$\{$ Discella obscura, B. \& C.

\{Discula obscura, Sacc.

$\{$ Hysterium rufilabrum, B. \& C.

\{Hypoderma rufilabrum, Duby.

Hypoxylon cohaerens, (P.) Fr.

Lentinus haematopus. B.

Leptostroma acerinum, $\mathrm{S}$.

Macrosporium concinnum. B. \& Br.

Phyllosticta acericola, C. \& E.

$\{$ Xyloma punctatum, $\mathrm{P}$

Rhytisma punctatum, Fr.

Septoria acerina, $\mathrm{Pk}$.

Sphaeria corticis, Fr. 
Trichia reniformis, $\mathrm{Pk}$.

$\rightarrow$ Uncinula circinata, C. \& P.

Acer pseudoplatanus, L.

Steganosporium cellulosum, Cda.

Acer rubrum, L.

\section{PYRENOMYCETES.}

$\{$ Diatrype sphendamnina, B. \& C.

\{Diaporthe sphendammina, Sacc.

Sphaeria Hystrix, Tode.

\{ Diatrype Hystrix, Fr.

Diatrype platystoma, (S.) B.

Sphaeria quadrata, S.

Diatrype obesa, B. \& C.

Diatrype quadrata, $B$.

Valsaria quadrata, Sacc.

$\{$ Valsa corynostoma, B. S Rav.

Eutypella corynostoma, Sacc.

f Valsa rugiella, C. S E.

$\{$ Eutypella rugiella, Sacc.

(Hypociea scutellaeformis, B. \& Rav.

Hypocrea Ravenelii, B.

Broomella Ravenelii, Sacc.

Hypoxylon florideum, B. \& C.

Hypoxylon jecurinum, B. \& Rav.

Hypoxylon serpens, (P.) Fr.

$\{$ Hysterium flexuosum, S.

\{ Hysterographium flexuostum, Rehm.

(Depazea brunnea, B. \& C.

$\{$ Sphaerella brunnea, Cke.

Laestadia brunnea, Sacc.

$\{$ Sphaerella glaucescens, Cke.

\{ Laestadia glaucescens, Sacc.

$\{$ Massaria sudans, B. \& C.

\{ Massariella sudans, Sacc.

Massaria vomitoria, B. \& C.

$\{$ Sphaeria Clypeus, S.

\{Nummularia Clypeus, Cke.

$\{$ Hypoxylon nummularium, Bull.

\{ Nummularia Bulliardi, Tul.

Sphaeria myriocarpa, Fr.

Sphaerostilbe flammea, Tul.

- Uncinula circinata, C. \& P.

Valsa grisea, Pk.

Valsa pauperata, C. E E.

S Sphaeria stellulata, Fr.

$\{$ Valsa stellulata, Fr.

Xylaria corniformis, Fr.

SPHAEROPSIDEAE.

Cytospora hyalosperma, Fr.

$\{$ Diplodia stenospora, B. \& C.

\{ Diplodina stenospora, Sacc.

$\{$ Discella rugosa, B. \& C.

$\{$ Discula rugosa, Sacc.

$\{$ Phoma albifructua, $\mathrm{Pk}$.

\{ Macrophoma albifructua, Berl. \& Vogl.

Sphaeropsis minima, B. \& C.

$\{$ Phyllosticta acericola, C. \& E.

Phoma minima, Sacc.
$\{$ Sphaeronema acerinum, $\mathrm{Pk}$.

S Sphaeronema nigripes, Ell.

\{ Sphaeronema Radula, B. \& C.

\{ Rhynchophoma Radula, Sacc.

\section{HYPIOMYCETES.}

Cheiromyces Beaumontii, B. \& C. in Ellis.

Glenospora Curtisii, B. \& Desm.

Helicosporium auratum, Ell.

Helminthosporium arbusculum, B. \& C.

Helminthosporium persistens, Cke.

\{Hymenella rhabdophora, B. \& Rav.

$\{$ Hymenula rhabdophora, Sacc.

\{ Dematium salicinum. P.

Sporotrichum salicinum, Fr.

DISCOMYCETES.

Dermatea carnea, C. \& E.

Pezicula acericola, Pk., var. gregaria, Pk.

Peziza miniopsis, Ell.

Peziza turbinulata, S.

$\{$ Xyloma acerinum, P.

Rhytisma acerinum, Fr.

Tympanis picastra, B. \& C.

MISCELLANEA.

\{ Corticium aschistum, B.

$\{$ Peniophora aschista, Cke.

Gloeosporium Aceris, Cke.

$\{$ Steremm neglectum, Pk.

\{ Peniophora neglecta, Pk.

$\{$ Stilbospora ovata, $P$.

\{ Steganosporium piriforme, (Hoffm.) Cda.

Acer' saccharinum, Wang.

Agaricus saccharinophilus, $\mathrm{Pk}$.

? Antennaria pinophila, Nees.

Asteroma Aceris, Rob.

Capnodium expansum, B. \& Desm.

Corticium subgiganteum, B.

$\int$ Sphaeria spinosa, $\mathrm{P}$.

$\{$ Sphaeria horrida, S.

Eutypa spinosa, Tul.

Hydnum nudum, B. \& C.

Hymenochaete corrugata, (Fr.) Lev.

Hymenochaete insularis, $\mathrm{B}$.

$\{$ Diatrype cercidicola, B. \& C.

$\{$ Hypoxylon suborbiculare, Pk.

Irpex tabacinus, B. \& C.

Lophodermium petiolicolum, Fckl., var. acerinum, $\mathrm{Pk}$.

Patellaria leptosperma, $\mathrm{Pk}$.

$\{$ Septoria Aceris, (Lib.) B. \& Br.

$\{$ Phleospora Aceris, Sacc.

Phyllosticta saccharina, Ell. \& Martin.

Polyporus bombycinus, Fr.

Polyporus connatus, $F_{r}$.

Polyporus purpureus, Fr. 
$\{$ Polyporus radiatus, (Sow.) Fr.

$\{$ Polyporus glomeratus, $\mathrm{Pk}$.

Polyporus sinuosus, Fr.

Polyporus versicolor, (L.) Fr.

Polyporus violaceus. Fr.

Rhytisma acerinum, (P.) Fr.

Rhytisma punctatum, (P.) Fr.

Septoria Salliae, Gerard.

Steganosporium cellulosum, Cda.

- Uncinula circinata, C. \& P.

Valsa leucostomoides, Pk.

Acer saccharinum, Wang., var. nigrum,

T. \& G.

Massaria vomitoria, B. \& C.

Polyporus applanatus, (P.) Wallr.

Polyporus resinosus, (Schrad.) Fr.

Acer spicatum, Lam.

Agaricus variabilis, $P$.

Ascomyces letifer, $\mathrm{Pk}$.

Capnodium expansum, B. \& Desm.

(Valsa acerina, $\mathrm{Pk}$.

Valsa albocincta, C. \& P.

Diaporthe acerina, Sacc.

Diaporthe albocincta, Sacc.

f Valsa myinda, C. \& E.

$\{$ Diaporthe myinda, Sacc.

Discella obscura, B. \& C.

$\{$ Sphaeria callista, B. \& C.

Fracchiaea callista, Sacc.

$\{$ Sphaeria petiolophila, $\mathrm{Pk}$.

$\{$ Gnomonia petiolophila, Berl. \& Vogl,

Hydnum sulphurellum, $\mathrm{Pk}$.

Hysterium virgultorum, (DC.), var. Aceris, Desm.

Sodularia acericola, Pk.

Dermatea acericola, Cke.

Pezicula acericola, $\mathrm{Pk}$.

Polyporus semipileatus, $\mathrm{Pk}$.

Rhytisma punctatum, (P.) Fr.

Sphaeria albidostoma, $\mathrm{Pk}$.

Sphaeria peliospora, B. \& C.

- Uncinula circinata, C.\& P.

Valsella Laschii, (Nits.) Sacc.

Acer, sp. indet.

\section{PYRENOMYCETES.}

Anthostoma saprophilum, Ell. \& Ev.

$\{$ Sphaeria trames, B. \& C.

$\{$ Botryosphaeria trames, Sacc.

$\{$ Sphaeria caminata, C. \& E.

$\{$ Ceratostoma caminatum, Sacc.

f Sphaeria barbirostris, Dufour.

\{ Ceratostomella barbirostris, Sacc.

$\{$ Sphaeria rostrata, $\mathrm{Fr}$.

\{ Ceratostomella rostrata, Sacc.

Chaetomium velutinum, Ell. \& Ev.

Cryptovalsa eutypaeformis, Sacc.

Dialonectria (Nectriella) vulpina, Cke.
$\{$ Diaporthe Aceris, Nits.

\{ Valsa Aceris. Cke.

Diatrype albopruinosa, (S.) C. \& E.

$\{$ Sphaeria Stigma, Hofim.

\{ Diatrype Stigma, Fr.

Hypocrea gelatinosa, ('Tode) Fr.

\{ Melogramma Aceris, C. \& E.

$\{$ Hypoxylon Aceris, Sacc.

Hypoxylon multiforme, Fr.

Hysterium acerinum, Frost.?

Hysterium ellipticum, Fr.

Hysterium Gerardi, C. \& P.

Hysterium stictoideum, C. \& E.

Hysterium vulvatum, $\mathrm{S}$.

\{ Sphaeria Pezizula, B. \& C.

$\{$ Lasiosphaeria Pezizula, Sacc.

Lophiostoma macrostoma, (Tode) Ces. \& DeNot.

$\{$ Lophiostoma microstoma, C. \& E.

$\{$ Lophiostoma scelestum, C. \& E.

$\{$ Lophiostoma tingens, Ell.

$\{$ Lophidium tingens, Sacc.

S Hysterium xylomoides, Chev.

Lophodermium hysterioides, (P.) Sacc.

S Sphaeria inquinans, Tode.

\{ Massaria inquinans, Fr.

\{ Valsa stilbostoma, Fr.

\{Melanconis stilbostoma, Tul.

S Sphaeria Papilla, S.

Mlelanopsamma Papilla, Sacc.

Melogranmma campylosporum, Fr.

\{ Nectria Cucurbitula, (Tode) Fr.

$\{$ Chilonectria Cucurbitula, Sacc.

Nectria cinnabarina, (Tode) Fr.

Nectria coccinea, (P.) Fr.

Nectria sanguinea, (Sihth.) Fr.

(Sphaeria microtheca, C. \& E.

$\{$ Calosphaeria microtheca, Sacc.

Physalospora microtheca, Sacc.

f Massaria seiridia, B. \& C.

\{Pseudovalsa profusa, (Fr.) Cke.

( Sphaeria megalocarpa, Plow.

\{ Rosellinia megalocarpa, Sacc.

\{ Sphaeria obtusissima, B. \& C.

Rosellinia obtusissima, Sacc.

$\{$ Sphaeria subiculata, S.

\{Rosellinia subiculata, Sacc.

Sordaria lutea, Ell. \& Ev.

Sphaeria pilulifera, Fr.

$\{$ Sphaeria vetusta, E11.

$\{$ Teichospora vetusta, Sacc.

Sphaeria culcitella, B. \& Rav.

Sphaeria acanthostroma, Mont.

Trichosphaeria acanthostroma, Sacc.

Byssosphaeria acanthostroma, Cke.

Valsa decorticans, Fr.

Valsa magnispora, Ell. \& Ev.

f Sphaeria Pugillus, S.

\{ Valsa Pugillus, Cke. 
Valsa quaternata, Fr.

Valsa thelebola. Fr.

Xylaria Hypoxylon, (L.) Grev.

Sphaeria atriella, C. \& E.

$\{$ Zignoella atrella, Sacc.

\section{SPIIAEROPSIDEAE.}

Dinemasporium acerinum, $\mathrm{Pk}$.

f Hendersonia collapsa, C. \& E.

$\{$ Stagonospora collapsa, Sacc.

f Phoma consorta, C. \& E.

$\{$ Ploma consors, Sacc. Syll.

Sphaeropsis Clintonii, Pk.

\section{MELANCONIEAE.}

Melanconium magnum, $\mathrm{B}$.

Naemaspora crocea, Pers.

Stilbospora piriformis, Hoffm.

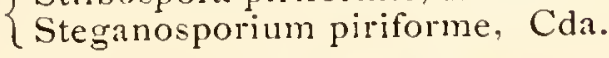

HYPHONYCETES.

Arthrobotryum robustum, C. S E.

Atractium flammeum, B. \& Rav., var. minor, Rav.

Bactridium Ellisii, B.

Botrytis fuliginosa, C. \& E.

f Chromosporium lateritium, Cke. \& Hark.

\{ Chromosporium Cookei, Sacc.

Coniothecium effusum, Cda.

\{ Sporidesmium Lepraria, B.

Dendryphium Ellisi, Cke.

\{ Helicoma Mülleri, Cda.

$\{$ Helicosporium Milleri, Sacc.

? Helminthosporium attenuatum, C. \& P.

Helminthosporium brachytrichum, C. \& E.

Helminthosporium macrocarpon, Grev.

Helminthosporium septemseptatum, Pk.

Septonema breviusculum, B. \& C.

Septosporium velutinum, C. \& E.

$\{$ Synphraguidium effusum, $\mathrm{Pk}$.

Speira effusa, Sacc.

Sporidesmium antiquum, Cda.

Sporidesmium aurantiacum, B. \& C.

Sporidesmium hysterioideum, C. $d$ E.

$\{$ Epochnium macrosporoideum, B. \& Br.

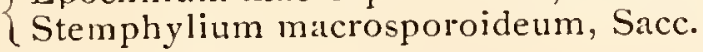

Stilbum aleuriatum, B. \& C.

Stilbum cinnabarinum, Mont.

Stilbum giganteum, Pk.

$\{$ Graphium giganteum, Sacc.

Stilbum parvulum, C. \& E.

$\{$ Torula uniformis, $\mathrm{Pk}$.

$\{$ Hormiscium uniforme, Sacc.

Tubercularia confluens, $\mathrm{P}$.

\section{DISCOMYCETES.}

Agyrium rufum, (P.) Fr.

Helotium fraternum, $\mathrm{Pk}$.
Helotium naviculaesporum, Ell.

Patellaria cylindrospora, Ell.

Patellaria nigrovirens, Ell. \& Sacc.

Peziza acerina, C. \& E.

Peziza aeruginosa, $P$.

Peziza atrovirens, $P$.

Peziza aurata, B. $\mathbb{E}$ Rav.

Peziza Erinaceus, S.

Peziza compressa, P.

Peziza floriformis, Pk.

Peziza paulupuncta, C. \& E:

Peziza rubella, $P$.

Peziza simulata. Ell.

Peziza vinosa, A. \& S., var. minor, C. \& E.

Propolis lobata, C. $\&$ E.

Stictis linearis, C. E E.

Tympanis acerina, $\mathrm{Pk}$.

Tympanis bicolor, Ell.

Tympanis rhabdospora, B. $\mathbb{S} \mathrm{C}$.

HYMENOMYCETES.

Agaricus albocrenulatus. $\mathrm{Pk}$

Agaricus atrocaeruleus, Fr.

Agaricus bombycinus, Schaeff.

Agaricus fulvotomentosus, Pk.

Agaricus lyerbarum, $\mathrm{Pk}$.

Agaricus hirtosquamulosus, $\mathrm{Pk}$.

Agaricus lignatilus, Fr.

Agaricus squarrosoides, Pk.

Artocreas Micheneri, B. \& C.

Calocera cornea, (Batsch) Fr.

Corticium confluens, Fr.

Corticium Oakesii, B. \& C.

Cortinarius maculatus, Jolınson.

Hydnum alutaceum, $\mathrm{Fr}$.

Hydnum chlorinum, Cke.

Leutinus haematopus, $B$.

Marasmius spondoleucus, B. \& Br.

Panus stipticus, (Bull.) Fr.

$\{$ Sistotrema violaceum, P.

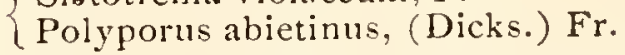

Polyporus contiguus. (P.) Fr.

Polyporus fimbriatellus, $\mathrm{Pk}$.

Polyporus frondosus, Fr.

$\{$ Boletus gilvus, $\mathrm{S}$.

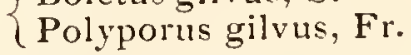

Polyporus semitinctus, Pk.

Polyporus vaporarius, Fr.

Polyporus vulgaris, Fr.

Stereum acerinum, (P.) Fr.

Stereum complicatum, Fr.

\section{MISCELLANEA.}

Dacryomyces moriformis, Fr.

Rhizoctonia aurantiaca, Ell. \& Ev. 
Aesculus Californica, Nutt.

Ascomyces deformans, $B$.

Diaporthe Aesculi, Cke. \& Hark.

Diatrype asterostoma, B. \& C.

Gloeosporium carpigenum, Cke.

Nectria cinnabarina, (Tode) Fr.

Nectria Zealandica, Cke.

Valsaria majuscula, Cke. \& Hark.

Aesculus flava, Ait.

$\{$ Boletus Aesculi-flavae, S.

\{ Polyporus Aesculi, Fr.

Aesculus glabra, Willd.

Aecidium Aesculi, Ell. \& Kell.

Phyllosticta Aesculi, Ell. \& Martin.

Plyyllusticta sphaeropsoidea, Ell. \& Ev.

Septoria Aesculi, (Lib.) Westd.

-Uncinula flexuosa, $\mathrm{Pk}$.

Aesculus Hippocastanum, L.

Agaricus comosus. Fr., var. albus, $\mathrm{Pk}$.

Agaricus sapidus, Kalchb.

Laestadia Aesculi, Pk.

Phoma Clintonii, Pk.

Phyllosticta sphaeropsoidea, Ell. \& Ev.

Septoria Hippocastani, B. \& Br.

-Uncinula flexuosa, $\mathrm{Pk}$.

Aesculus, sp. indet.

Agaricus salignus, P.

Diatrype disciformis, (Hoffin.) Fr.

\{ Sphaeria Saubinetii, Mont.

\{ Gibberella Saubinetii, (Mont.) Sacc.

\{ Arcyria globosa, S.

\{ Lach nobolus globosus, Rostf.

$\{$ ?Leotia circinans, P.

\{ Vib issea lutea, Pk.

Patellaria congregata, B. \& C.

Peziza furfuracea, Rotls.

Sphaeria aesculicola, Fr.

Stereum complicatum, Fr.

Trichia varia, $P$.

Vermicularia involucri, S.

Vermicularia petiolorum, S.

Negundo aceroides, Moench.

Badhamia hyalina, (P.) B.

Comatricha pulchella, (Bab.) Rostf.

Coryneum Negundinis, B. \& C.

\{ Fusisporium cinnabarinum, B. \& C.

Fusarium cinnabarinum, Sacc.

\{ Labrella scripta. S.

\{Leptostroma scriptum, Fr.

Sphaeria cristata, P.

$\{$ Sphaeria crenata, $P$.

(Lophiotrema crenatum, Sacc.

$\{$ Septoria Aceris, (Lib.) B. \& Br.

Phieospora Aceris, Sacc.

Phoma negundinicola, Thm.

Physarum polymorphum, (Mont.) Rostf.

Septoria acerella, Sacc.
Sphaeronema nitidum, B. \& C.

Stilbum aleuriatum, B. \& C.

Negundo Californicum, Torr. \& Gr.

Phoma Lebiseyi, Sacc.

Negundo, sp. indet.

$\{$ Sphaeria excipuliformis, $\mathrm{Fr}$.

Lophiostoma excipuliforme, Ces. \& DeNot.

Sapindus marginatus, Willd.

Sphaerella Sapindi, E11. \& Ev.

$\{$ Sphaeria appendiculosa, B. \& C.

Anthostomella confusa, Sacc.

Staphylea trifolia, L.

Cenangium Staphyleae, S.

$\{$ Stemonitis oblonga, Fr.

Comatricha Persoonii, Rostf.

Coniothyrium Staphyleae, Pk.

Diaporthe Robergeana, (Desm.) Niessl.

Hendersonia Staphyleae, Ell. \& Ev.

Leptosphaeria rubrotincta, E11. \& E、.

Lophiostoma roseotinctum, Ell. \& Ev.

$\{$ Sphaeria staphylina. Pk.

Metasphaeria staphylina, Sacc.

$\{$ Sphaeria atrofusca, $S$.

$\{$ Nectria atrofusca, Ell. \& Ev.

Ophiobolus staphylinus, Ell. \& Ev.

Oidium irregulare, $\mathrm{Pk}$.

Orularia isarioides, Sacc.

Ovularia irregularis, Trelease.

Ramularia isarioides, Ell. \& Ev.

Sacidium vegetans, $\mathrm{S}$.

Septoria cirrhosa, Wint.

Sphaerella staphylina, Ell. \& Ev.

Sphaeria Staphyleae, S.

$\{$ Periconia gracilis, S.

\{ Sporocybe gracilis, Sacc.

Stilbospora Staphyleae, S.

Vermicularia Staphyleae, S.

\section{ANACARDIACEAE.}

Datisca glomerata, Benth. \& Hook.

Phomatospora Datiscae, Hark.

Pistacia Mexicana, HBK.

Sphaerella Pistaciae, Cke.

Rhus aromatica, Ait.

Pileolaria effusa, $\mathrm{Pk}$.

Rhus aromatica, Ait., var. triloba, Gray.

\{ Dichomera rhoina, Cke. \& Hark.

\{ Camarosporium rhoinum, Sacc.

Diplodia resurgens, Cke. E Hark.

Diplodia rhoina, Cke. \& Hark.

Rhus copallina, L.

\section{PYRENONICETES.}

$\{$ Nelogramma ambiguum, (S.) Rav.

$\{$ Botryosphaeria ambigua, Sacc.

$\{$ Valsa albovelata, B. \& C.

$\{$ Diaporthe albovelata, Sacc. 
Syloma Rhois, S.

Dothidea Rhois, Fr.

Phyllachorat Rlıois, Sacc.

-Sphaerotheca pruinosa, C. \& P.

Sphaeria aculeans, S.

\{Valsa aculeans, B.

Sphaeria quadrata, S.

$\{$ Diatrype quadrata, B.

Valsaria quadrata, Sacc.

\{ Sphaeria thoina. Ell. \& Ev.

$\{$ Winteria rhoina, Berl. \& Vogl.

Xylaria cornuformis, $\mathrm{Fr}$.

\section{MISCELLANEA.}

Cercospora copallina, Cke.

Cercospora rhoina, C. \& E.

Eurotium lierbariorum, Lk.

Gonatobotrys maculicola, Wint.

Hainesia rhoina, Ell. \& Sacc.

Helminthosporium Arbuscula, B. \& C.

Hendersonia hyalopus, B. \& C.

Hydnum Rhois, S.

Sphaeropsis pulchella, B. \& C.

$\{$ Phoma pulchella, Sacc.

Polyporus cupulaeformis, B. \& C.

Septoria irregularis, $\mathrm{Pk}$.

Sphateronema Rhois, B. \& C.

\{ Sporocybe Rhois, B. \& C.

\{Stilbum Rhois, B. \& C.

Ascomyces deformans, B., var. purpurascens, Ell. \& Ev.

Taphrina purpurascens, Robinson.

Rhus cotinoides, Nutt.

Septoria Rhois, B. \& C.

Septoria rhoina, Sacc.

Rhus diversiloba, Torr. \& Gr.

Botryosphaeria ambigua, (S.) Sacc.

Diatrype Rhois, (S.) Cke.

Diplodia resurgens, Cke. \& Hark.

Diplodia rhoina, Cke. \& Hark.

Ophiodothis tarda, Hark.

f Tryblidium rufulum, (Spreng.) Cke.

$\{$ Tryblidiella rufula, Sacc.

Uromyces punctato-striatus, Cke. \& Hark.

Rhus glabra, L.

\section{PYRENOMYCETES.}

Melogramma atrofuscum, B. \& C.

Fuckelia atrofusca, Herb. Berk.

Diatrype atrofusca, Cke.

Anthostoma atrofuscum, Berl. \& Vogl.

Sphaeria Sumachi, S.

$\{$ Sphaeropsis Suınachi, C. \& E.

Botryosphaeria Sumachi, Cke.

Sphaeria rufescens, S.

Sphaeria aculeans, S.

Valsa rufescens. M. A. Curtis.

Valsa aculeans, $\mathrm{B}$.

Valsa rhoiphila, C. \& E.

Calospora aculeans, Sacc.
S Sphaeria elevans. S.

\{ Eutypa elevans, Cke.

Microthyrium microscopicum, Desm.

S Xyloma Rhois, S.

Dothidea Rhois, Fr.

Phyllachora Rhois, Sacc.

\{Sphaeria subsimplex, S.

\{ Physalospora subsimplex, Sacc.

Sphaeria quaternata, P., var. Americana, Fr.

\{ Quaternaria Persoonii, Tul.,var. Americana.

$\{$ Sphaeria nigredo. S.

\{ Sphaerella nigredo, Cke.

Sphaeria Aucupariae, var. g., S.

Sphaeria rhoina, S.

- Sphaerotheca pruinosa, C. \& P.

MISCELLANEA.

Cenangium Rhois, S.

Ceratosporium fuscescens, S.

Cercospora rhoina, C. \& E.

Exidia applanata, S.

Hendersonia glabra, Cke.

Leptostroma rhoinum, S.

\{ Sphaeropsis pulchella, B. \& C.

\{Phoma pulchella, Sacc.

SSeptoria Rhois B. \& C.

\{ Septoria rhoina, Sacc.

Sphaeronema pruinosum, Pk.

Sphaeropsis Frostii, B. \& C.

Stemphylium Fuligo, B. \& C.

f Sporocybe Rhois, B. \& C.

\{ Stilbum Rhois. B. \& C.

Rhus Toxicodendron, L.

Cercospora Toxicodendri, Ell.

Diaporthe sparsa, $\mathrm{Pk}$.

Gloeosporium Toxicodendri, Ell. \& Martin.

Phyllosticta toxica, Ell. \& Martin.

Phyllosticta Toxicodendri, Thm.

$\{$ Polyporus dryadeus, S.

$\{$ Polyporus scruposus, Fr.

\{ Sphaeria Aquila, Fr.

\{Rosellinia Aquila, DeNot.

Septoria irregularis, $\mathrm{Pk}$.

Septoria Toxicodendri, M. A. Curtis.

Tubercularia vulgaris, Tode.

(Pileolaria Terebinthi, (DC.) Cast.

Pileolaria brevipes, B. \& Rav.

Uredo Toxicodendri, B. \& Rav.

Trichobasis Toxicodendri, B. \& Rav.

Uromyces Toxicodendri, B. \& Rav.

Uromyces Terebinthi, (DC.) Wint.

Xerotus nigrita, Lev.

Rhus Toxicodendron, L., var. radicans,

Torr.

S Sphaeria ambigua, S.

$\{$ Melogramma ambiguum, Rav.

Botryosphaeria ambigua, Sacc.

f Sphaeria Rhois, S.

\{ Diatrype Rhois, Cke.

Dothidea rhoina, $\mathrm{S}$. 
S Xyloma Toxicodendri. S.

$\{$ Dothidea Toxici, S.

Ectostroma toxici, M. A. Curtis.

Hydnum Rhois, S.

Lophiostoma radicans, Ell. \& Ev.

Melanconium cellulosum, B. S C.

Podosporinm rigidum, S.

Poly porus Floridanus, B.

Sphaerostjlbe cinnabarina, Tul.

$\{$ Hysterium rufulum, Spreng.

\{riblidiella rufula, Sacc.

$\{$ Valsa gemmata, B. S C.

Calospora gemmata, Sacc.

Sphaeria Toxici, S.

Valsa Toxici, B.

$\{$ Talsaria Toxici. Sacc.

Melogramma Toxici, Cke.

Rhus typhina, L.

Ceratosporium fuscescens, $\mathrm{S}$.

Cytospora grandis, Pk.

$\{$ Sphaeria eutypa, Fr.

Eutypa Acharii. Tul.

Hysterium Rhois, S.

Septoria Rlois, B. \& C.

Septoria thoina, Sacc.

Sphaeria mucida, $\mathrm{Fr}^{r}$, var. rostellata, S.

Sphaeropsis pallida, Pk.

\{ Sporucy be Rhois, B. \& C.

$\{$ Stilbum Rhois, B. $\mathbb{E}$ C.

Tubercularia floccosa, Lk.

Sphaeria aculeans, S.

Valsa aculeans, B.

Calosporat aculeans, Sacc.

$\{$ Valsa leptasca, Pk. \& Clinton.

\{ Cryptosporella leptasca, Sacc.

Rhus venenata, DC.

(Dothidea venenata, C. \& E.

$\{$ Melogramma fuliginosum, Ell p. p.

Botryosphaeria venenata, Sacc.

Corticium colliculosum, B. \& C..

(Dermatea pallida, Cke. in N. A. F.

$\{$ Dermatea carnea, C. \& E., var. pallida, Ell.

Dermatea pallidula, Cke.

Diatrype Duriaej, Mont.

\{ Diatrype rhoina, C. S E.

\{ Calospora thoira, Sacc.

\{ Hypoxylon Morsei, B. \& C.

\{ Fuckelia Morsei, Cke.

Peziza theiodea, C. $\mathrm{A}$ E.

\{ Phoma fibriseda, C.S E.

Apiosphaeria fibriseda, Sacc.

Scoriomyces Cragini, Ell. \& Sacc.

Sphaeronema pruinosum, $\mathrm{Pk}$.

Sphaeropsis fibriseda, C. \& E.

Valsa rufescens, (S.) MI. A. Curtis.

$\{$ Talsa rhoiphila, C. E E.

Valsa aculeans, (S.) $B$.

$\{$ Valsa goniostoma, (S.) M. A. Curtis.

Eutypella goniostoma, Sacc.
Zygodesmus rudis, Ell.

Rhus, sp. indet.

Cytospora rhoina, Fr.

Sphaesia pruinosa, $\mathrm{Fr}^{\circ}$.

\{ Dendrophoma pruinosa, Sacc.

Didymosporium corticale, S.

$\{$ Diplodia compressa, Cke.

\{ Botryodiplodia compressa, Sacc.

$\{$ Naemaspora Rhois, B. \& C.

$\{$ Myxosporium Rhois, Sacc.

$\{$ Nectria inaurata, B. \& Br.

\{ Aponectria inaurata, Sacc.

$\{$ Nectria Cucurbitula. (Tode) Fr.

\{ Chilonectria Cucurbitula, Sacc.

Peziza triformis, $\mathrm{Fr}_{r}$.

Sphaeria subsolitaria, S.

$\{$ Diplodia subsolitaria. Curr.

Physalospora subsolitaria, Sacc.

$\{$ Sistotrema digitatum, $\mathrm{P}$.

Radulum orbiculare, Fr.

Sphaeria livida, P.

Thyridium lividum, Sacc.

Tubercularia dubia, Lk.

CORIARIEAE.

Coriaria inyrtifolia L.

$\{$ Sphaeropsis macrospora, B. $\&$ C.

Dothiorella macrospora, Sacc.

LEGUMINOSAE.

Papilionaceae.

Amorpha Californica, Nutt.

Puccinia Amorphae, M. A. Curtis.

Amorpha callescens, Nutt.

Cercospora passaloroides, Wint.

Puccinia Amorphae, M. A. Curtis.

Amorpha fruticosa, L.

Cercospora passaloroides, Wint.

f Aecidium Amorphae, Cke.

I Puccinia Amorphae, M. A. Curtis.

Sphaeria dissepta, Fr.

Valsa dissepta, Fr.

Quaternaria dissepta, Tul.

Amorpha herbacea, Walt.

$\{$ Puccinia Amorphae, M. A. Curtis.

Uropyxis Amorphae, Schrt.

Cladosporium Amorphae, Thm.

Amorpha microphylla, Pursh.

Puccinia Amorphae, M. A. Curtis.

Amphicarpaea monoica, Elliott.

Aecidium leguminosatum, Lk.

Aecidium Orobi, P.

Cercospora inonoica, Ell. \& Holw.

$\{$ Erysiphe communis, (Wallr.) Schl.

- Erysiphe Martii, Lev.

Xyloma scandentium, S.

Leptostroma scandentium, S. 
Stilbum candidum, Pk.

\{tilbum Peckii, Sacc.

C Uredo Leguminosarum, Herb. Curt.

Uredo Fabae, Herb. Curt.

Uredo aecidioides, Pk.

Uredo Peckii, Thm.

Synchytrium fulgens, var. decipiens, Farl.

Synchytrium decipiens, Farl.

Uromyces appendiculatus, (P.) Lev.

Uromyces Phaseoli, Wint.

Apios tuberosa, Moench.

Aecidium leguminosatum, Lk.

\{ Cercospora tuberosa, Ell. \& Kell.

$\{$ Cercospora glaucescens, Wint.

Uromyces Phaseoli, Wint.

Arachis hypogaea, $\mathrm{L}$.

\{Cladosporium personatum, B. \& C

$\{$ Cercospora personata, Ell. \& Ev.

Astragalus bisulcatus, Gray.

S Sphaerella megastoma, Pk.

\{ Physalospora megastoma, Sacc.

Astragalus Canadensis, L.

$-\{$ Erysiphe coinmunis, (Wallr.) Schl.

Erysiphe Martii, Lev.

APeronospora Trifoliorum, DBy.

$\{$ Ramularia Astragali, Ell. \& Holw.

\{Didymaria Astragali, Sacc.

Astragalus Cooperi, Gray.

$-\{$ Microsphaera Astragali, (DC.) Trer.

$\checkmark$ Microsphaera holosericea, (IVallr.) Lev.

Astragalus Drummondii, Dougl.

\{ Sorosporium Astragali, Pk.

$\{$ Thecaphora Astragal, Tor.

Astrágalus Menziesii, Gray.

Phoma Astragali, Cke. S Hark.

Astragalus, sp. indet.

Phyllosticta Astragali, Pk.

S Sphaeria Astragali, Curr.

Sphaerella Astragali, Cke.

Septoria astragalicola, Pl.

S Fusidium Ravenelianum, Thm.

$\left\{\begin{array}{l}\text { Ramularia Desmodii, Cke., var. epiphylla, } \\ \text { Ell. }\end{array}\right.$

Baptisia australis, R. Br.

Cercospora velutina, Ell. \& Kell.

Baptisia leucantha, Torr. \& Gr.

$\{$ Sphaeria baptisiaecola, Cke.

Sphaerella baptisiaecola, Sacc.

Baptisia leucophaea, Nutt.

Aecidium amphigenum, Ell. \& Kell.

Sphaerella leucophaea, Ell. \& Kell.

Baptisia perfoliata, R. Br.

Macrosporium Baptisiae, Thm.

Phoma Baptisiae, Cke.

Septoria Baptisine, Cke.

\{ Sphaeria Baptisiae, Cke.

Anthostomella Baptisiae, Sacc.

Sphaeropsis Baptisiae, Thm.
Baptisia tinctoria, R. Br.

- Erysiphe Martii, Lev.

\{ Sphaeria comatella, C. \& E.

\{eptosphaeria comatella, Sacc.

Sphaerella baptisiaecola, Cke.

Sphaerella granulata, E11. \& Ev.

Colutea arborescens, L.

$\{$ Hendersonia Coluteae, Pk. \& Clinton.

$\{$ Camarosporium Coluteae, Sacc.

Colutea, sp. indet.

Dothidea Coluteae, B. \& C.

Crotalaria sagittalis, L.

Cercospora Demetrioniana, Wint.

Crotalaria verrucosa, $\mathrm{L}$.

$\{$ Sporidesmium polymorphum, Cda.

Stemphylium polymorphum, Thm.

Cytisus scoparius, Lk.

Diaporthe Sarothamni, Nits.

Diplodia Sarothamni, Cke. \& Hark.

Pestalozzia polychaeta. Cke. \& Hark.

Phoma Sarothamni, Cke.

Sphaeria (Leptosphaeria) Californica, Cke. \& Hark.

Dalea laxiflora, Pursh.

Cercospora Daleae, Ell. \& Kall.

Desmanthus brachylobus, Benth.

(Cercospora condensata, Ell. \& Kell., var.

Desmanthi, Ell. \& Kell.

Cercospora Desmanthi, Ell. \& Kell.

Desmodium acuminatum, DC.

Cercospora Desmodii, Ell. \& Kell.

$\{$ Dothidea perisporioides, B. \& C.

Parodiella perisporioides, Speg.

$\{$ Sorosporium Desmodii, Pk.

$\{$ Thecaphora Desmodii, Wor.

Desmodium Canadense, DC.

- Microsphaera diffusa, C. \& P.

Ramularia Desmodii, Cke.

Sphaeria perisporioides, B. \& C.

Dothidea perisporioides, B. \& C.

Dothidea seminata, B. \& Rav.

Stigmatea seminata, Sacc.

Parodiella perisporioides, Speg.

Phyllachora perisporioides, $\mathrm{Pk}$.

Parodiella grammodes, (Kze.) Cke.

Uromyces Hedysari-paniculati, (S.) Farl.

Desmodium canescens, DC.

- Microsphaera diffusa, C. \& P.

$\{$ Dothidea perisporioides, B. \& C.

$\{$ Parodiella perisporioides, Speg.

Sphaerella Desmodii, Wint.

SUromyces Desmodii, Thm.

Uromyces Hedysari-paniculati, (S.) Farl.

Desmodium ciliare, DC.

Ramularia Desmodii, Cke.

$\{$ Uromyces Desmodii, Thm.

\{ Uromyces Hedysari-paniculati, (S.) Farl. 
Desmodium cuspidatum, Hook.

- Microsphaera diffusa, C. \& P.

Uromyces Hedysari-paniculati, (S.) Farl.

Desmodium Dillenii, Darling.

$-\{$ Microsphaera Mougeotii, Lev.

\{ Microsphaera Lycii, (Lasch) Sacc. \& Roum.

Desmodium Iineatum, B. \& C.

Fusisporium pubescens, B. \& C.

Ramularia Desmodii, Cke.

Fusidium Ravenelianum, Thm.

Cercosporella Raveneliana, Sacc.

Dothidea simillima, B. \& Rav.

J Dothidea Desmodii, M. A. Curtis.

Phyllachora simillima, Sacc.

Parodiella simillima, Cke.

Desmodium nudiflorum, DC.

Cercospora Desmodii, Ell. \& Kell.

Sorosporium Desmodii, Pk.

$\{$ Thecaphora Desmodii, Wor.

Vermicularia uncinata, B. \& C.

Desmodium paniculatum, DC.

- Microsphaera diffusa, C. \& P.

Puccinia Hedysari-paniculati, S.

Phragmidium Hedrsari, S.

Uromyces solida, B. \& C.

Uromyces Desmodii, Thm.

Uromyces Hedysari-paniculati, Farl.

Desmodium rotundifolium, DC.

Sclerotium Desmodii, Thm.

Uromyces solida, B. \& C.

$\{$ Uromyces Des̀modii, Thm.

Uromyces Hed ysari-paniculati, (S.) Farl.

Desmodium sessilifolium, Torr. \& Gr.

Uromyces Hedysari-paniculati, (S.) Farl.

Desmodium strictum, DC.

Cladosporium infuscans, Thm.

\{ Didymosporium Hedysari, S.

\{ Coryneum Hedysari, S.

Hypoderma virgultorum, DC.

$\{$ Sphaeria comatella, C. \& E.

$\{$ Leptosphaeria comatella, Sacc.

$\{$ Sphaeria dissiliens, C. \& E.

\{Metasphaeria dissiliens, Sacc.

Pistillaria clavulata, Ell.

Uromyces solida, B. \& C.

$\{$ Uromyces Desmodii, Cke.

Uromyces Hedysari-paniculati, (S.) Farl.

Desmodium, sp. indet.

Aecidium Orobi, P.

$\{$ Sphaeria desmodiana, C. \& E.

Diaporthe desmodiana, Sacc.

\{ Sphaeria Desmodii, $\mathrm{Pk}$.

\{ Diaporthe Desmodii, Sacc.

Sphaeria distributa, C. \& E.

$\{$ Leptosphaeria distributa, Sacc.

Patellaria ferruginea, C. \& E.

Peziza atrocinerea, Cke.

$\{$ Sphaeria phomopsis, C. \& E.

$\{$ Physalospora phomopsis, Sacc.
Sphaeria (Didymosphaeria) cupula, Ell.

Vermicularia compacta, C. \& E.

Dolichos melanophthalmus, DC.

Septoria Melanopthalmi, B. \& C.

Dolichos, sp. indet.

Acrospermum compressum, Tode.

Cercospora cruenta, Sacc.

Helminthosporium molle, B. \& C.

Septoria Dolichi, B. \& C.

$\{$ Sphaeria phlyctanoides, B. \& C.

$\{$ Physalospora phlyctanoides, Sacc.

Erythrina Crista-galli, L.

Cercospora Erythrinae, Ell. \& Ev.

Erythrina herbacea, L.

Sporocybe byssoides, (P.) Fr.

Eysenhardtia amorphoides, HBK.

? Uromyces apiculatus, (Str.) Lev.

Galactia pilosa, Elliott.

Uromyces Phaseoli, Wint.

Genista tinctoria, L.

Dothidea tetraspora, B. \& Br.

Glycyrrhiza lepidota, Pursh.

Cylindrosporium Glycyrrhizae, Hark.

Uromyces Trifolii, (Hedw. f.) Lev.

Hedysarum boreale, Nutt.

Hedysarum Mackenzii, Richard.

Uromyces borealis, $\mathrm{Pk}$.

Hedysarum, sp. indet.

Sphaeria Aegopodii, P.

Hosackia glabra, Torr.

Pestalozzia Moorei, Hark.

Phoma Hosackiae, Cke. \& Hark.

Hosackia Purshiana, Benth.

Pleospora Leguminum, (Wallr.) Rabh.

Hosackia strigosa, Nutt.

Septoria Hosackiae, Hark.

Hosackia, sp. indet.

\{ Sphaerella (?) Hosackiae, Cke. \& Hark.

i Ditopella Hosackiae, Sacc.

Indigofera Caroliniana, Walt.

$\{$ Dothidea perisporioides, B. \& C.

$\{$ Parodiella perisporioides, Speg.

Lathyrus latifolius, L.

Sphaeria lathyrina, B. \& C.

$\{$ Sphaerella lathyrina, Cke.

Didymella lathyrina, Sacc.

Lathyrus maritimus, Bigel.

Septoria Astragali, Rob.

Lathyrus ochroleucus, Hook.

- Microsphaera diffusa, C. \& P.

Septoria Astragali, Rob.

$\{$ Uromyces Fabae, (P.) DBy.

\{Uromyces Orobi, Fckl.

Uromyces polymorphus, Pk. \& Clinton.

Lathyrus paluster, $\mathrm{L}$.

Ascochyta Pisi, Lib. 
Microsphaera Ravenelii, B.

Septoria emaculata, Pk. \& Clinton.

$\{$ Uromyces Fabae, (P.) DBy.

$\{$ Úromyces Orobi, Fckl.

Lathyrus venosus, Muhl.

Aecidium porosum, $\mathrm{Pk}$.

Erysiphe communis, (Wallr.) Schl.

$\{$ Sphaeria Vizeana, Cke.

$\{$ Didymosphaeria Vizeana, Sacc.

$\{$ Uromyces Fabae, (P.) DBy.

$\{$ Uromyces Orobi, Fckl.

Uromyces polymorphus, Pk. \& Clinton.

Lathyrus, sp. indet.

Pleococcum pezizoideum, Cke.

Torula herbarum, Lk.

Lespedeza angustifolia, Elliott.

$\{$ Aecidium leucostictum, B. \& C.

$\{$ Aecidium Orobi, P., var. leucostictum, B.

Lespedeza capitata, Michx.

(Sphaeria Trifolii, S. Car.

Sphaeria Lespedezae, S.

Dothidea Lespedezae, B.

Phyllachora Lespedezae, Sacc.

$\{$ Uromyces macrosporus, B. \& C.

$\{$ Uromyces Lespedezae, Pk.

Lespedeza hirta, Elliott.

- Microsphaera diffusa, C. \& P.

$\{$ Puccinia Lespedezae-polystachyae, S.

\{ Uromyces Lespedezae, Pk.

Lespedeza leptostachya, Engelm.

Uromyces Lespedezae, $\mathrm{Pk}$.

Lespedeza repens, Barton.

Aecidium leucostictum, B. \& C.

Phyllachora Lespedezae, (S.) Sacc.

Puccinia Lespedezae-procumbentis, S.

Uromyces Lespedezae-procumbentis,

M. A. Curtis.

Uromyces Lespedezae, Pk.

Lespedeza striata, Hook. \& Arn.

- Microsphaera diffusa, C. \& P.

Lespedeza violacea, $\mathrm{P}$.

- Microsphaera diffusa, C. \& P.

Puccinia Lespedezae-procumbentis, S.

Puccinia Lespedezae-polystachyae, S.

Puccinia Lespedezae-violaceae, S.

Uromyces Lespedezae-violaceae, M. A. Curtis.

Uromyces Lespedezae-procumbentis, M. A. Curtis.

Uromyces macrosporus, B. \& C.

Uromyces Lespedezae, $\mathrm{Pk}$.

Uredo Lespedezae, Thm.

Lespedeza, sp. indet.

Darluca Filum, Cast.

$\{$ Dothidea perisporioides, B. \& C.

\{ Parodiella perisporioides, Speg.

Lupinus arboreus, Sims.

Diaporthe Lupini, Hark.

Diplodia Lupini, Cke. \& Hark.

Hymenula Lupini, Cke. \& Hark.
\{ Phoma Lupini, Cke. \& Hark.

Macrophoma Lupini, Berl. \& Vogl.

Metasphaeria Lathyri, Sacc.

$\{$ Dialonectria depallens, Cke. \& Hark.

$\{$ Nectria depallens, Berl. \& Vogl.

Pleospora Leguminum, (Wallr.) Rabh.

Sordaria lanuginosa, Sacc.

Sphaeria (Didymella) Lupini, Cke. \& Hark.

Sphaeropsis Lupini, Cke. \& Hark.

Stictis decipiens, Karst.

Stictis Lupini, Phil. \& Hark.

Valsa Lupini, C'ke. \& Hark.

Lupinus Chamissonis, Esch.

Hendersonia Lupini, Cke. \& Hark.

Lupinus densiflorus, Benth.

Septoria Lupini, Hark.

Lupinus diffusus, Nutt.

Cercospora Lupini, Cke.

Septosporium Lupini, Thm.

Lupinus Douglasii, Agardh.

$\{$ Uredo Lupini, B. \& C.

$\{$ Uromyces Lupini, B. \& C.

Lupinus Kingii, Watson.

Tuberculina Lupini, Farl.

Lupinus perennis, L.

Cercospora filispora, $\mathrm{Pk}$.

Cercospora longispora, $\mathrm{Pk}$.

- Erysiphe Martii, Lev.

Lupinus polyphyllus, Lindl.

Uromyces Orobi, Fckl.

Lupinus villosus, Willd.

Cercospora longispora, $\mathrm{Pk}$.

Lupinus, sp. indet.

Cryptosporium Lupini, Cke.

Nectria coccinea, (P.) Fr.

Nectria Peziza, (Tode) Fr.

? Phoma Lupini, Ell. \& Ev.

Pleospora amplispora, Ell. \& Ev.

Medicago denticulata, Willd.

Uromyces oblonga, Vize.

Medicago Iupulina, L.

Uromyces striatus, Schrt.

$\left\{\begin{array}{l}\text { Uromyces Medicaginis-falcatae, (DC.) } \\ \text { Wint. }\end{array}\right.$

Medicago sativa, L.

Gloeosporium Medicaginis, Ell. \& Kell.

$\{$ Ascobolus Trifolii, Bernh.

\{ Phacidium Trifolii, Boud.

$\{$ Septoria allantoidea, B. \& C.

$\{$ Rhabdospora allantoidea, Sacc.

Medicago, sp. indet.

Phacidium Medicaginis, Lib.

Melilotus alba, Lam.

Gloeosporium (Marsonia) Meliloti, Trelease.

Melilotus, sp. indet.

$\{$ Excipula lanuginosa, $\mathrm{Pk}$.

\{ Cyphina lanuginosa, Sacc. 
\{ Sphaeria viridella, Pk.

$\{$ Leptosphaeria viridella, Sacc.

Macrosporium Meliloti, Pk.

Periconia pycnospora, Fres.

Oxytropis Lamberti, Pursh.

Trichobasis Oxytropi, Pk.

Petalostemon violaceus, Michx.

Púccinia Petalostemonis, Farl.

Phaseolus diversifolius, $\mathrm{P}$.

Phyllosticta phaseolina, Sacc.

Uredo appendiculata, P.

Puccinia Phaseoli-trilobi, S.

Uromyces appendiculatus, Lev.

Uromyces Phaseolorum, (DC.) Tul.

Phaseolus helvolus, L.

Uromyces appendiculatus, (P.) Lev.

Phaseolus lanatus, L.

Cercospora canescens, Ell. \& Martin.

Cladosporium herbarum, (P.) Lk.

Epicoccum Duriaeanum, Mont.

Fusarium roseum, Lk.

Vermicularia polytricha, Cke.

Phaseolus pauciflorus, Benth.

Uromyces Phaseoli, Wint.

Phaseolus perennis, Walt.

- Nicrosphaera diffusa, C. \& P.

Phaseolus vulgaris, L.

Gloeosporium Lindemuthianum, Sacc. \& Magn.

Phyllosticta phaseolina, Sacc.

Uromyces appendiculatus, (P.) Lev.

Phaseolus Wrightii, Gray.

$\{$ Dothidea perisporioides, B. \& C.

\{ Parodiella perisporioides, Speg.

Phaseolus, sp. indet.

Cercospora cruenta, Sacc.

Cercospora Phaseolorum, Cke.

Dothidea Phaseolorum, C. \& E.

Epicoccum micropus, Cda.

Fusarium oxysporum, Schl.

\{Graphium laxum, Ell.

Isariopsis laxa, Sacc.

\{ Sphaeria comatella, C. \& E.

\{Leptosphaeria comatella, Sacc.

Macrosporium fasciculatum, C. \& E.

Mystrosporium polytrichum, Cke.

Nectria umbrina, (P.) Fr.

Polyactis vulgaris, Lk.

Sphaeria (Diaporthe) Phaseolorum, C. \& E.

Trichobasis rubrum, Bon.

Typhula tenuissima, M. A. Curtis.

Vermicularia truncata, S.

Pisum sativum, L.

$\therefore$ Erysiphe communis, (Wallr.) Schl.

$\{$ Erysiphe Martii, Lev.

$\{$ Sphneria Pisi, Sow.

$\{$ Pleospora Pisi, Fckl.
$\{$ Uredo appendiculata. P.

$\{$ Uromyces appendiculatus, Lev.

Psoralea argophylla, Pursh.

Aecidium porosum, $\mathrm{Pk}$.

Cercospora latens, Ell. \& Ev.

Septoria argophylla, Ell. \& Kell.

Uromyces Argophyllae, Seymour.

Psoralea lanceolata, Pursh.

Aecidium Psoraleae, Pk.

Uromyces Psoraleae, Pk.

Psoralea macrostachys, DC.

Calloria myriospora, Phil. \& Hark.

Psoralea Onobrychis, Nutt.

Aecidium Onobrychidis, Burrill.

Psoralea orbicularis, Lindl.

Leptosphaeria Doliolum, (P.) DeNot.

Psoralea physodes, Dougl.

Uromyces Psoraleae, $\mathrm{Pk}$.

Psoralea tenuiflora, Pursh.

Aecidium Orobi, P.

Aecidium Psoraleae, Pk.

Dothidea perisporioides, B. \& C.

$\{$ Parodiella perisporioiles, Speg.

Psoralea, sp. indet.

Phoma Psoraleae, Che.

Rhynchosia tomentosa, Hook \& Arn.

\{ Depazea Rhynchosiae, Thm.

Ascochyta Rhynchosiae, Sacc.

Nectria Peziza, (Tode) Fr.

Sphaeria perisporioides, B. \& C.

$\{$ Dothidea perisporioides, B. \& C.

Parodiella perisporioides, Speg.

Rhynchosia, sp. indet.

Graphium hyalosporum, B. \& Rav.

Graphium Leguminum, Cke.

Robinia Pseudacacia, L.

? Agaricus spectabilis, Fr.

$\{$ Sphaeria elongata, Fr.

$\{$ Cucurbitaria elongata, Grev.

$\{$ Naemaspora leucosperma, $P$.

$\{$ Cytospora leucosperma, Fr.

Cytospora parva, B. \& C.

Valsa oncostoma, (Duby) Fckl.

Valsa personata, C. $\&$ E.

\{Diaporthe oncostoma, Fckl.

Diaporthe personata, Sacc.

Dinemasporium Robiniae, Gerard.

Diplodia profusa, DeNot.

Diplodia vulgaris, Lev.

\{Valsa venusta, Ell.

Eutypella venusta, Sacc.

Sphaeria pubens, S.

Hendersonia Robiniae, Westd.

Hendersonia pubens, Herb. Curtis.

Hendersonia subfenestrata, B. \& C.

Hendersonia Sartwellii, Herb. Curtis.

Camarosporium Robiniae, Sacc.

Camarosporium subfenestratum, Sacc.

Hysterium truncatum, $\mathrm{P}$. 
Pachnocybe rosella, B. \& C.

$\{$ Pachnocybe rosea, B. \& C.

Isaria rosea, Sacc.

Melanconium viscosum, S. ?

Naemaspora Russellii, B. \& C.

Myxosporium Russellii, Sacc.

Nectria cinnabarina, (Tode) Fr.

Nectria ditissima, Tul.

Phoma Leguminum, Westd.

Sphaeria profusa, Fr.

Valsa profusa, Fr.

Aglaospora profusa, DeNot.

Massaria seiridia, B. \& C.

Pseudovalsa profusa, Cke.

f Sphaeria intonsa, S.

Coniochaeta intonsa, Cke.

\{ Sphaeronema macrosporum, B. \& C.

\{ Cornularia macrospora, Sacc.

Stereum muscigenum, B. \& Br.

Sphaeria Robinine, S.

$\{$ Diatrype Robiniae, M. A. Curtis.

Valsaria Robiniae, Cke.

Robinia viscosa, Vent.

Melanconium viscosum, S.

Rhytisma Robiniae, S.

Sphaeria conspersa, S.

Sphaeria tuberculosa, S.

\{ Sphaeronema macrosporum, B. \& C.

Cornularia macrospora, Sacc.

Robinia, sp. indet.

\section{MISCELLANEA.}

$\{$ Cryptosporium Pseudacaciae, S.

Coniosporium Pseudacaciae, Sacc.

Cytospora orthospora, B. \& C.

Cytospora Robiniae, S.

Fusarium lateritium, Nees.

Fusarium scolecoides, Sacc. \& Ell.

Gloeosporium Leguminis, Cke. \& Hark.

Leotia acicularis, $P$.

Helotium aciculare, $P$.

Peziza acicularis, Fr.

Sarea acicularis, S.

Peziza clavata, $P$.

$\{$ Cytospora abnormis, B. \& C.

$\{$ Phoma abnormis, Sacc.

Phoma capsularum, Cke. \& Hark.

$\{$ Spumaria licheniformis, $\mathrm{S}$.

$\{$ Physarum lividum, Rostf.

Polyporus hirsutus, Fr.

Polyporus rimosus, B.

Polyporus versicolor, (L.) Fr.

Schizoxylon tuberculatum, S.

S Septoria breviuscula, B. \& C.

\{ Rhabdospora breviuscula, Sacc.

Sphaeronema Robiniae, B. \& C.

$\{$ Periconia Robiniae, S.

$\{$ Sporocybe Robiniae, Fr.
Trichothecium roseum, (P.) Lk.

$\{$ Myxormia convexula, C. \& E.

\{Trullula convexula, Sacc.

Tubercularia granulata, $P$.

Tubercularia vulgaris, Tode.

Volutella comata, E1l.

\section{PYRENONYCETES.}

Sphaeria applanata, Fr.

\{ Amphisphaeria applanata, Ces. \& DeNot.

Sphaeria lanuginosa, B. \& C.

$\{$ Melanopsamma lanuginosa, Sacc.

Byssosphaeria lanuginosa, Cke.

\{ Sphaeria stricta, P.

\{ Ceratostomella stricta, Sacc.

Sphaeria parasitans, S.

\{Cucurbitaria parasitans, Cke.

$\{$ Valsa enteroleucs, Fr.

$\{$ Diaporthe enteroleuca, Sacc.

Dothidea Robiniae, S.

S Sphaeria allostoma, S.

$\{$ Valsa allostoma, Cke.

Eutypa allostoma, Cke.

$\{$ Hysterium fibrisedum, Gerard.

\{ Gloniopsis fibriseda, Sacc.

Massaria vomitoria, B. \& C.

$\{$ Sphaeria anisometra, Cke. \& Hark.

$\{$ Metasphaeria anisometra, Sacc.

S Sphaeria convergens, Tode.

\{ Pseudovalsa convergens, Sacc.

Sphaerella petiolicola, (Desm.) Awd.

Sphaeria nigrobrunnea, S.

Sphaeria prorumpens, Fr.

\{ Sphaeria anomia, S., nec. Fr.?

\{ Valsa anomia, Cke.

Valsa ceratophora, Tul.

Valsa subscripta, (Fr.) Cke.

Sphaeria Notarisii, Mont.

$\{$ Valsa Notarisii, Mont.

Valsaria Notarisii, Sacc.

Sesbania vesicaria, Elliott.

Gloeosporium Glottidii, Ell. \& Martin.

Macrosporium Floridanum, Cke.

Phoma clitoricarpa, (Cke.) Sacc.

Phoma Leguminum, Westd.

Sesbania, sp. indet.

- Erysiphe Montagnei, Lev.

Sophora sericea, Nutt.

Uromyces hyalinus, $\mathrm{Pk}$.

Uromyces Sophorae, Pk.

Stylosanthes elatior, Swartz.

Cladosporium stenosporum, B. \& C.

Cercospora Stylosanthis, E1l. \& Ev.

$\{$ Sphaeria Gramma, S.

Dothidea Gramma, Fr.

Tephrosia hispidula, P.

Tephrosia spicata, Torr. \& Gr.

Ravenelia glandulaeformis, B. \& C. 
Tephrosia Virginiana, $\mathrm{P}$.

Hendersonia anomala, C. \& E.

\{ Sphaeria Tephrosiae, C. $\mathbb{E}$ E.

\{Leptosphaeria Tephrosiae, Sacc.

Sphaeria epiphylla, S.

J Ravenelia glandulosa, B.

Ravenelia glandulaeformis, B. \&.C.

Ravenelia sessilis, $B$.

Thermopsis Californica, Watson.

Stigmina Thermopsidis, Hark.

Trifolium agrarium, L.

Cercospora zebrina, Pass.

Trifolium Carolinianum, Michx.

$\{$ Aecidium elegans, B. \& C.

$\{$ Aecidium Orobi, P., var. elegans, B.

$\{$ Uromyces striatus, Schrt.

$\{$ Uromyces Medicaginis-falcatae, (DC.) Wint.

Trifolium incarnatum, $\mathrm{L}$.

Uromyces Trifolii, (Hedw. f.) Lev.

Trifolium pratense, L.

Gloeosporium Trifolii, Pk.

$\{$ Sphaeria bucera, C. \& E.

$\{$ Metasphaeria bucera, Sacc.

$\{$ Ophiobolus collapsus, Ell. \& Sacc.

S Sphaeria collapsa, Ell.

Peziza Sphaerella, Pk. \& Clinton.

Phacidium Trifolii, (Bernh.) Boud.

Phyllachora Trifolii, (P.) Fckl.

$\{$ Sphaeria denotata, C. \& E.

$\{$ Pleospora denotata, Sacc.

Polythrincium Trifolii, Kze.

$\{$ Septoria Trifolii, Ell.

$\{$ Rhabdospora Trifolii, Sacc.

Stictis pustulata, Ell.

Trifolium repens, L.

Aecidium Trifolii-repentis, Cast.

Cercospora helvola, Sacc.

$\{$ Dothidea Trifolii, (P.) Fr.

$\{$ Phyllachora Trifolii, Fckl.

Polythrincium Trifolii, Kze.

$\{$ Uromyces apiculatus, (Str.) Lev.

Uromyces Medicaginis-falcatae,(DC.) Wint.

Uromyces Trifolii, (A. \& S.) Wint.

Trifolium, sp. indet.

Actinothyrium Graminis, Kze.

Darluca Filum, Cast.

-Erysiphe Martii, Lev.

T-Peronospora Trifoliorum, DBy.

Vicia Americana, Muhl.

- Microsphaera Ravenelii, B.

\& Peronospora Viciae, (B.) DBy.

$\{$ Synchytrium Jonesii, Pk.

$\{$ Tuberculina Jonesii, Sacc.

Vicia Americana, Muhl, var. linearis, Watson.

Aecidium porosum, $\mathrm{Pk}$.

Vicia Faba, L.

Caeoma (Uredo) Leguminosarum, Lk.
\$Peronospora Viciae, (B.) DBy.

(Puccinia Fabae, Lk.

$\{$ Uromyces Fabae, (P.) DBy.

Uromyces Viciae-Fabae, (P.). Schrt.

Uromyces Orobi, Fckl.

Vicia sativa, L.

Cercospora Viciae, Ell. \& Holw.

Vicia, sp. indet.

Aecidium album, Clinton,

+ Peronospora Trifoliorum, DBy.

Septoria Astragali, Rob.

Uromyces appendiculatus, (P.) Lev.

Vigna luteola, Benth.

Cercospora canescens, Ell. \& Martin.

Cercospora Vignae, Ell. \& Ev.

Coscinaria Langloisii, Ell. \& Ey.

Wistaria Sinensis, DC.

Hendersonia Wistariae, Cke.

(Thuemenia Wistariae, Rehm.

$\{$ Melogramma Wistariae, Cke.

Botryosphaeria Wistariae, Sacc.

Sphaerella Wistariae, Cke.

Wistaria, Sp. indet.

Sphaeropsis fusiger, B. \& C.

$\{$ Phoma fusigera, Sacc.

Macrophoma fusigera, Berl. \& Vogl.

\section{Caesalpinieae.}

Cassia Chamaecrista, L.

Aecidium Cassiae, Ell. \& Kell.

Cercospora Chamaecrista, Ell. \& Kell.

Cassia Marylandica, L.

Cercospora simulata, Ell. \& Ev.

Helminthosporium Cassiae, Gerard.

Cassia occidentalis, L.

Cladosporium personatum, B. \& C., var.

Cercospora occidentalis, Cke.

Cladosporium personatum, B. \& C., var. Cassiae, Thm.

Cercospora personata, Sacc., var. Cassiaeoccidentalis, Sacc.

Macrosporium cassiaecolum, Thm.

Macrosporium commune, Rabh.

Macrosporium Leguminum, Cke.

Macrosporium Ravenelii, Thm.

Phoma Cassiae, Sacc.

Cassia Tora, L.

Cercospora atromaculans, Ell. \& Ev.

Cercospora nigricans, Cke.

Cassia, sp. indet.

Hypoderma commune, Duby.

Leptosphaeria cassiaecola, E1l. \& Ev.

Pleospora Cassiae, Ell. \& Ev.

Cercis Canadensis, L.

Acrospermum Ravenelii, B. \& C.

Cercospora cercidicola, Ell.

$\{$ Cercospora chionea, Ell. \& Kell.

$\{$ Cercosporella chionea, Sacc. 
Discosia atrocreas, (Tode) Fr.

$\{$ Dothidea Cercidis, Cke.

\{ Bagnisiella Cercidis, Berl. \& Vogl.

$\{$ Sphaeria durissima, S.

$\{$ Hypoxylon durissimum, $\mathrm{B}$.

$\{$ Corticium Leveillianum, B. \& C.

\{Stereum Leveillianum, B. \& C.

Sphaerella cercidicola, EIl. \& Kell.

Cercis, sp. indet.

\{ Nummularia discreta, (S.) Tul.

\{ Hypoxylon discretum, B. \& C.

$\{$ Peziza Lecideola, Fr.

$\{$ Patellaria Lecideola, Fr.

Peziza cinereofusca, S.

Gleditschia triacanthos, L.

(Sphaeria rhizogena, B.

$\{$ Melogramma rhizogenum, B.

Botryosphaeria rhizogena, Sacc.

Calloria rubrococcinea, Rehm \& IVint.

Cercospora condensata, Ell. \& Kell.

Helminthosporium olivaceum, B. \& Rav.

Cercospora Berkeleyi, Cke.

Cercospora Seymouriana, Wint.

Cercospora olivacea, Wint.

Didymium rugulosum, $\mathrm{B}$.

$\{$ Hysterium flexuosum, S.

\{Hysterographium flexuosum, Sacc.

$\{$ Hysterium Lesquereuxii, Duby.

Hysterographium Lesquereuxii, Sacc.

Libertella Gleditschiae, Wint.

$\{$ Calocladia Ravenelii, B.

- Microsphaera Ravenelii, B.

Nummularia discreta, (S.) Tul.

Pleonectria denigrata, Wint.

Polyporus albus, (Huds.) Fr.

Polyporus versicolor, (L.) Fr.

Schizophyllum commune, Fr.

Xylaria polymorpha, (P.) Grev.

Gleditschia, sp. indet.

Cladosporium Gleditschiae, Cke.

Cucurbitaria elongata, (Fr.) Grev.

Sphaeria Gleditschiae, S.

$\{$ Melogramma Gleditschiae, M. A. Curtis.

? Cucurbitaria Gleditschiae, B. \& C.

Botryosphaeria Gleditschiae, Sacc.

Dothidea Encoelium, S.

Fusarium anomalum, B. \& C.

$\{$ Hysterium lineare, B., nec Fr.

Hysterium lineariforme, Sacc.

Leptostroma hypophyllum, B. \& Rav.

Nectria nigrescens, Cke.

Peziza cinereofusca, $\mathrm{S}$.

Sphaeropsis Gleditschiae, Cke.

$\{$ Phoma Triacanthi, Sacc.

Macrophoma Triacanthi, Berl. \& Vogl.

Sacidium Gleditschiae, Lev.

Sphaeropsis gleditschiaecola, Cke.

$\{$ Sphaeropsis mamillaris, B. \& C.

\{ Phoma mamillaris, Sacc.
$\{$ Sphaeria amorphostoma, S.

$\{$ Valsa amorphostoma, Cke.

$\{$ Sphaeria conseptata, S.

$\{$ Valsa conseptata, Cke.

$\int$ Sphaeria Notarisii, Mont.

$\{$ Valsa Notarisii, Mont.

Valsaria Notarisii, Sacc.

Gymnocladus Canadensis, Lam.

Cercospora Gymnocladi, Ell. \& Kell.

Vermicularia albomaculata, S. ?

Mimosere.

Acacia anisophylla, Watson.

Ravenelia Indica, B.

Acacia crassifolia, Gray.

Ravenelia Indica, B.

Acacia Greggii, Gray.

Uromyces versatilis, Peck.

Acacia Julibrissin, Willd.

Pachnocybe subulata, (Nees) B.

Fusarium aurantiacum, Cda.

$\{$ Verticillium pulvinatum, B. \& C.

$\{$ Verticicladium pulvinatum, Sacc.

Acacia, sp. indet.

Coniothyrium decipiens, Cke. \& Hark.

Diaporthe gorgonoidea, Cke. \& Hark.

Diplodia decorticata. C. \& E.

Diplodia lata, Cke. \& Hark.

$\{$ Diplodia millegrana, Cke. \& Hark.

\{ Diplodiella millegrana, Sacc.

Diplodia mutila, (Fr.) MIont.

Diplodia phyllodiae, Cke. \& Hark.

Diplodia vulgaris, Lev.

Fusarium cataleptum, Cke. \& Hark.

Fusarium Acaciae, Cke. \& Hark.

Fusarium obtusisporum, Cke. \& Hark.

$\{$ Fusisporium Leguminum, Cke.

$\{$ Fusarium Leguminum, Sacc.

Helminthosporium atroolivaceum, Cke. \& Hark.

Hysterographium (Gloniopsis) insigne, Cke.

\& Hark.

Nectria ditissima, Tul.

Nectria infusaria, Cke. \& Hark.

Pachnocybe subulata, (Nees) B.

$\{$ Phoma librincola, Cke. \& Hark.

$\{$ Aposphaeria librincola, Sacc.

Phoma lignicola, Rabh.

Physarum nodulosum, Cke. \& Balf.

Rhabdospora decorticata, Cke. \& Hark.

Septosporium praelongum, Sacc.

Sphaeria (Wallrothiella) eunotiaespora, Cke. \& Hârk.

Psilosphaeria eunotiaespora, Cke.

Sphaerella Acaciae, Cke. \& Hark.

Sphaeria (Thyridium) personata, Cke. \& Hark. 
Strumella Acaciae, Cke. \& Hark.

Volutella ciliata, (A. S S.) Fr.

Prosopis, sp. indet.

(Discella Leguminum, Cke.

$\{$ Discula Leguminum, Sacc.

Gloeosporium Leguminum, Sacc.

Leguminosae, sp. indet.

Phoma punctulata, Cke.

Triphragmium degluhens, B. \& C.

Xylaria tentaculata, B. \& Br.

\section{ROSACEAE.}

Amy'gdaleae.

Prunus Americana, Marshall.

Capnodium pelliculosum, B. \& Rav.

Cenangium prunastri, (P.) Fr.

Cercospora prunicola, Ell. \& Ev.

Dematium fimbriatum, S.

$\{$ Sphaeria intermedia, S.

$\{$ Enslinia Schweinitzii, Cke.

$\{$ Hysterium stictoideum, C. \& E.

$\{$ Gloniopsis stictoidea, Sacc.

Melanconium effusum, Lk.

S Sphaeria morbosa, S.

Cucurbitaria morbosa, Ell.

Gibbera morbosa, Cke.

Plowrightia morbosa, Sacc.

Otthia morbosa, Cke.

- Podosphaera Oxyacanthae, (DC.) DBy.

Polyporus igniarius, (L.) Fr.

Propolis versicolor, Fr.

Puccinia Pruni-spinosae, $\mathrm{P}$.

Puccinia Pruni, DC.

Puccinia Prunorum, Lk.

Uromyces Prunorum, Fckl.

$\{$ Septoria cerasina, $\mathrm{Pk}$.

\{ Septoria Pruni, Ell.

Sphaeria rhytostoma, Fr.

\{ Sphaeronema Persicae, (S.) Ell.

$\{$ Cornicularia Persicae, Sacc.

$\{$ Exoascus Pruni, Fckl.

\{ Taphrina Pruni, Tul.

Prunus Amygdalus. Baill.

Diplodia Pruni, Fckl.

Phoma ramealis, Desm.

Prunus Armeniaca, L.

Diplodia Amygdali, Cke. \& Hark.

Puccinia Pruni-spinosae, $P$.

Prunus avium, L.

Micropera Drupacearum, Lev.

Prunus Caroliniana, Ait.

\{Asteroma Cerasi, Cke.

Asteroma cerasicolum, Sacc.

Ceuthospora Cookei, Thm.

$\{$ Cytospora leucophthalma, B. \& C.

$\{$ Cytospora nivosa, B. \& C.
Discosia artocreas, (Tode) Fr.

Septoria Ravenelii, Thm.

Sphaerella lenticula, Cke.

Prunus Cerasus, L.

Micropera Drupacearum, Lev.

Nectria coccinea, (P.) Fr.

Phlebia radiata, $\mathrm{Fr}$.

f Sphaeria morbosa, S.

¿ Plowrightia morbosa, Sacc.

- Podosphaera Cerasi, Lev.

- Podosphaera Kunzei, Lev.

- Podosphaera Oxyacanthae, (DC.) DBy.

Podosphaera tridactyla, (Wallr.) DBy.

Polyporus cinnabarinus, (Jacq.) Fr.

Polyporus sulfureus, (Bull.) Fr.

Sphaerella cinerascens, Fckl.

Sphaeria varia, $P$.

$\{$ Sporocybe Persicae, (S.) Fr.

\{ Sphaeronema Persicae, Ell,

Prunus Chicasa, Michx.

Capnodium pelliculosum, B. \& Rav.

$\{$ Sphaeria morbosa, S.

\{ Cucurbitaria morbosa, Ell.

$\{$ Puccinia Pruni-spinosae, $P$.

$\{$ Uromyces Prunorum, Fck 1 .

Prunus demissa, givalp.

Lophiostoma acervatum, Karst.

Plowrightia morbosa, (S.) Sacc.

(Podosphaera Kunzei, Lev.

- Podosphaera Oxyacanthae, (DC.) DBy.

Podosphaera tridactyla, (Wallr.) DBy.

Corticium evolvens, $\mathrm{Fr}$.

Prunus domestica, L.

Aspergillus ovalispermus, Lk.

Phyllosticta pirina, Sacc.

$\{$ Sphaeria morbosa, S.

$\{$ Plowrightia morbosa, Sacc.

$\{$ Puccinia Pruni-spinosae, P.

$\{$ Puccinia Prunorum, Lk.

Septoria cerasina, $\mathrm{Pk}$.

Taphrina Pruni, (Fckl.) Tul.

Prunus Lauro-Cerasus, L.

$\{$ Phoma cerasina, Cke.

\{ Macrophoma cerasina, Berl. \& Vogl.

Sphaerella cerasina, Cke.

Prunus Mahaleb, L.

(Dothidea Cerasi, C. \& E.

$\{$ Melogramma fuliginosum, Ell. p. p.

Botryosphaeria Cerasi, Sacc.

Diatrype Stigma, (Hoftim.) Fr.

Valsa stellulata, Fr.

$\{$ Valsa tetraploa, B. \& C.

Eutypella tetraploa, Sacc.

Valsa Mahaleb, C. \& E.

Prunus maritima, Wang.

$\{$ Sphaeria morbosa, S.

\{ Plowrightia morbosa, Sacc.

Taphrina Pruni, (Fckl.) Tul. 
Prunus Pennsylvanica, L. f.

$\{$ Cenangium Cerasi, (P.) Fr.

\{ Dermatea Cerasi, Fr.

Diaporthe cylindrospora, $\mathrm{Pk}$.

Phlebia radiata, Fr.

$\{$ Sphaeria morbosa, S.

\{ Plowrightia morbosa, Sacc,

Tympanis conspersa, Fr.

\{ Valsa Pennsylvanica, B. \& C.

\{ Calospora Pennsylvanica, Sacc.

Valsa prunicola, $\mathrm{Pk}$.

Prunus Persica, Jess.

(Sphaeria pulchella, P.

$\{$ Valsa pulchella, Fr.

Calosphaeria princeps, Tul.

Capnodium elongatum, B. \& Desm.

Sphaeria Putaminum, S.

$\{$ Caryospora Putaminum, DeNot.

Amphisphaeria Putaminum, Cke.

$\{$ Cercospora consobrina, Ell. \& Ev.

$\{$ Cercospora rubrotincta, Ell. \& Ev.

Cercospora persica, Sacc.

Ciboria fructicola, Wint.

Cytospora Persicae, S.

Fusarium sticticum, B. \& C.

Helminthosporium rhabdiferum, B.

\{ Hysterium Gerardi, C. \& P.

\{Hysterographium Gerardi, Sacc.

$\{$ Hysterium Putaminum, Cke.

Hysterographium Putaminum, Sacc.

Lichenopsis sphaeroboloidea, S.

Sphaeropsis persicina, B. \& C.

$\{$ Phoma persicina, Sacc.

Macrophoma persicina, Berl. \& Vogl.

$\{$ Monilia fructigena, P.

\{ Oidium fructigenum, Kze. \& Schm.

Pestalozzia Guepini, Desm.

$\{$ Boletus cervinus, S.

$\{$ Polyporus cervinus, Fr.

$\{$ Boletus conchatus, $\mathrm{P}$.

\{ Polyporus conchatus, Fr.

\{ Boletus marginatus, $P$.

$\{$ Pulyporus marginatus, Fr.

Polyporus supinus, Swartz.

\{ Massaria Seiridia, B. \& C.

\{ Psendovalsa profusa, (Fr.) Cke.

Puccinia Pruni-spinosae, $\mathrm{P}$.

Uredo prunastri, De.

Uromyces Prunorum, Fckl.

Uromyces Prunorum, Fckl., var. Amygdali, Vize.

Periconia Persicae, L.

Sporocybe Persicae, $\mathrm{Fr}$

Sphaeronema Persicae, Ell.

Cornularia Persicae, Sacc.

$\{$ Sphinctrina Cerasi, B. \& C.

Sphinctrina gummae, B. \& Mont.

Ascomyces deformans, B.

$\{$ Taphrina deformans, Tul.

Exoascus deformans, Fckl.
Tremella ustulata, Bull.

Sclerotium pirinum, Fr.

S Sphaeria leucostoma, P.

\{ Valsa leucostoma, Fr.

$\{$ Sphaeria microstoma, P.

\{Valsa microstoma, Fr.

S Sphaeria persicaria, S.

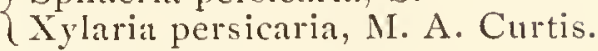

Prunus pumila, L.

$\{$ Exoascus Pruni, Fckl.

\{Taphrina Pruni, Tul.

Prunus serotina, Ehrh.

$\{$ Dothidea geographica, (DC.) Fr.

\{ Asteroma geographica, Desm.

$\{$ Peziza Cerasorum, S.

\{ Cenangium clavatum, Fr.

$\{$ Cercospora circumscissa, Sacc.

\{ Cercospora graphioides, E11.

Sphaeria exilis, A. \& S.

$\{$ Coelosphaeria exilis, Sacc.

Lasiosphaeria exilis, Cke.

Sphaeria Prunastri, P.

$\{$ Valsa Prunastri, Fr.

Eutypella Prunastri, Sacc.

Helminthosporium Pruni, B. it C.

Irpex lacteus, Fr.

Leptothyrium cinctum, Cke.

Oidium destruens, Pk.

Phoma prunicola, S.

Phyllosticta serotina, Cke.

Phyllosticta Treleasei, Berl. \& Vogl.

?Physalospora pustulata, Sacc.

$\{$ Sphaeria morbosa, S.

\{ Plowrightia morbosa, Sacc.

Polyporus pargamenus, Fr.

$\{$ Puccinia Pruni-spinosae, P.

$\{$ Puccinia Prunorum, Lk.

Sclerotium Prunorum, S.

Septoria cerasina, $\mathrm{Pk}$.

Septoria Ravenelii, Thm.

Sphaeronema minutissimum, $\mathrm{Pk}$.

\{ Sphinctrina Cerasi, B.\& C.

Sphinctrina gummae, B. \& Mont.

Stereum purpureum, $P$.

\{ Exoascus Pruni, Fck

\{ Taphrina Pruni, Tul.

Tubercularia granulata, P.

Valsa leucostoma, (P.) Fr.

(Sphaeria microstoma, P.

\{ Valsa microstoma, Fr.

Prunus subcordata, Benth.

$\{$ Ascomyces Pruni, (Fckl.) B. \& Br.

\{ Taphrina Pruni, Tul.

Prunus Virginiana, L.

Cercospora circumscissa, Sacc.

Cytospora leucosperma, (P.) Fr.

Gloeosporium prunicolum, Ell. \& Ev.

Helminthosporium Pruni, B. \& C. 
Melanconium cerasinum, $\mathrm{Pk}$.

Monilia Peckiana, Sacc. \& Vogl.,

var. angustior, Sacc.

Phoma Pruni, Pk.

Phoma prunicola, S.

Phoma Virginiana, Ell. \& Halsted.

f Sphaeria morbosa, S.

\{ Plowrightia morbosa, Sacc.

Podosphaera Kunzei, Lev.

Podosphaera Oxyacanthae. (DC.) DBy.

Podosphaera tridactyla, (Vallr.) DBy.

Puccinia Pruni-spinosae, $\mathrm{P}$.

Sclerotium Prunorum, S.

Sphaeria ocellata, Fr.

Sphaeria vibratilis, Fr.

Sphaeropsis cerasina, Pk.

Taphrina Pruni, (Fckl.) Tul.

Thelephora lilacina, $P$.

Prunus (Plum).

Cytospora carphosperma, Fr.

Micropera Drupacearum, Lev.

Monilia cinerea, Bon.

Solystigma rubrum, (P.) DC.

$\{$ Dothidea rubra, $\mathrm{Fr}$.

Valsa Prunastri, (P.)Fr.

$\{$ Diatrype quadrata, (S.) B.

\{ Valsaria quadrata, Sacc.

Prunzs (Cherry).

Badhamia hyalina, (P.) B.

$\{$ Valsa pulchella, (P.) Fr.

$\{$ Calosphaeria princeps, Tul.

Coniosporium atrum, $\mathrm{S}$.

Hendersonula morbosa, Sacc.

Hydnum amplissimum, B. \& C.

Micropera rubida, B. \& C.

Phyllachora Beaumontii, Cke.

Sphaeria epidermidis, Fr., var. microscopica,

Desm.

(Taphrina deformans, (B̈.) Tul.

$\{$ Exoascus Wiesneri, Rathay.

Sphaeria interstitialis, C. \& P.

$\{$ Teichospora interstitialis, Sacc.

Prunus, sp. indet.

?Amaurochaete minor, Sacc. \& Ell.

Auricularia sambucina, Mart.

Cytospora ocellata, Fckl.

Exidia glandulosa, (Bull.) Fr.

\{ Sistotrema Cerasi, P.

Irpex paradoxus, (Schrad.) Fr.

Irpex sinuosus, Fr.

(Peziza plicatocrenata, S.

$\{$ Tympanis plicatocrenata, Fr.

Phacidium plicatum, $\mathrm{Fr}$.

Podosporium rigidum, S.

Radulum obiculare, Fr.

Schizophyllum commune, Fr.
Sphaeropsis anomala, Pk.

$\{$ Sphaeropsis Peckii, Sacc.

PYRENOMYCETES.

Nectria Cucurbitula, (Tode) Fr.

$\{$ Chilonectria Cucurbitula, Sacc.

Sphaeria versatilis, Fr.

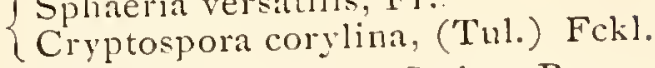

Sphaeria conjuncta, S. Am. Bor.

$\left\{\begin{array}{l}\text { Valsa hormospora. Herb. Berk. } \\ \text { Venesta hor }\end{array}\right.$

(Fenestella hormospora, Cke.

Hypocrea contorta, (S.) B. \& C.

Hypoxylon fuscum, (P.) Fr.

f Hysterium flexuosum, S.

Hysterographium flexuosmm, Rehm.

Sphaeria excavata, S.

Valsa ambiens, (P.) Fr.

S Sphaeria ciliata, $\mathrm{P}$.

Valsa ciliata, Fr.

Sphaeria monadelpha, Fr.

$\{$ Valsa monadelpha. Fr.

f Sphaeria scutellata, P.

$\{$ Valsa scutellata. Cke.

Sphneria vasculosa, Fr.

$\{$ Valsa vasculosa, Fr.

\section{Rosaceae propriae.}

Adenostoma, sp. indet.

Corticium scutellare, B. \& C.

Agrimonia Eupatoria, L.

Uredo Rosae, S. Syn. Car.

Caeoma (Uredo) Ágrimoniae, S.

Coleosporium ochraceum, Bon.

Uredo Agrimoniae-Eupatoriae, (DC.) Wint.

-Sphaerotheca Humuli, (DC.) Burrill.

Agrimonia parviflora, Ait.

Caeoma (Uredo) Agrimoniae, S.

$\{$ Coleosporium ochraceum, Bon.

Alchemilla vulgaris, L.

Uromyces intrusa, Lev.

Dryas octopetala, L.

Pleospora Dryadis, Fckl.

Fragaria Chilensis, Ehrh.

Ramularia Tulasnei, Sacc.

Fragaria vesca, L.

Ramularia Tulasnei, Sacc.

Sphaerella Earliana, Wint.

Fragaria Virginiana, Ehrh.

A scochyta colorata, Pk.

f Ramularia Tulasnei, Sacc.

Ramularia Fragariae, Pk.

Fragaria, sp. indet.

f Diacher elegans, Fr.

$\{$ Diachea leucopoda, (Bull.) Rostf.

Shysarum cinereum, (Batsch) P.

$\{$ Didymium cinereum, Fr., var. 
\{ Leptothyrium Fragariae, Lil,

Gloeosporium Fragariae, Mont.

Gloeosporium. Potentillae, (Desm.) Oud.

$\{$ Coremium fragariastrum, S.

\{raphiothecium Fragariastri, Sacc.

Peziza Earliana, Ell. \& Er.

Septoria aciculosa, Ell. \& Er.

Sphaerella Earliana, Wint.

Sphaerella Fragariae, (Tul.) Sacc.

Sphaeria Fragariae, S.

- Sphaerotheca Castagnei, Ler.

Geum album, Gmel.

*Peronospora Potentillae, DBy.

- Sphaerotheca Castagnei, Ler.

Geum macrophyllum, Willd.

+ Peronospora Potentillae, DBy.

Geum radiatum, Michx., var. Peckii, Gray.

Cylindrosporium Gei, Farl.

Geum rivale, L.

+ Peronospora Potentillae, DBy.

Geum Virginianım, L.

Septoria Gei, Rob.

Geum, sp. indet.

? Puccinia simplex, Pk.

Gillenia stipulacea, Nint.

Roestelia lacerata, (Sow.) Fr.

Heteromeles arbutifolia, Roemer.

Capnodium Heteromeles, Cke. \& Hark.

Cercospora Heteromeles. Hk.

Ceuthospora brevispora, Cke. \& Hark.

Hypoderma Heteromeles, Phil. \& Hark.

$\{$ Meliolopsis Heteromeles, Cke. \& Hark.

\{Meliola Heteromeles, Berl. \& Vogl.

Peziza Heteroneles, Phil. \& Hark.

Phacidium Heteromeles, Phil. \& Hark.

Phoma Heteromeles, Cke. \& Hark.

Phyllosticta Heteromeles, Cke. \& Hark.

Pleosporopsis Heteromeles, Cke. \& Hark.

Septoria Photiniae, B. \& C.

Kerria Japonica, DC.

$\{$ Cesatia turbinata, B. \& C.

\{ Trullula turbinata, Sacc.

Diplodia Corchori, (Desm.) Kickx.

(Sphaeria flavitecta, B. \& C.

Nectria auriger, B. \& Rav., var. flavitecta,

B. \& C.

(Calonectria flavitecta, Sacc.

Stictis glaucoma, B. \& C.

Valsa decorlicans, $\mathrm{Fr}_{\mathrm{r}}$.

Neillia op nlifolia, Benth. \& Hook.

$\{$ Sphaeria Closterium, B. \& C.

Anthostomella Closterium, Sacc.

Cytospora globifera, Fr.

$\left\{\begin{array}{l}\text { Dermea Spiraeae, S. } \\ \text { Cenangium Spiraeae, B. } \\ \text { Dermatea Spiraeae, Cke. }\end{array}\right.$
Diaporthe Neilliae, Pk.

Diaporthe spiculosa, (A. \& S.) Nits.

Excipula epidermidis, S.

\{ Marsonia Neilliae, Hark.

\{ Gloeosporium Neilliae, Ell. \& Ev.

\{Lophiostoma Spiraeae, Pk.

Lophiotrema Spiraeae, Sacc.

Pestalozzia monochaetoidea, Sacc. \& Ell.

Peziza Opulifoliae, S.

Ramularia Spiraeae, Pk.

Sphaeria crenata, P., var. Spiraeae, S.

Sphaeria saepincola, Fr.

- Sphaerotheca Castagnei, Lev.

$\{$ Sporidesmium nitens, S.

\{lasterosporium nitens, Sacc.

Valsa Opulifoliae, Pk.

Nuttallia cerasiformis, Torr. \& Gr.

\{ Septogloeum Nuttallii, Hark.

\{Gloeosporium Nuttallii, Ell. \& Ev.

Potentilla Anserina, L.

\{ Gloeosporium Potentillae, (Desm.) Oud.

\{ Marsonia Potentillae, Fisch.

Potentilla argentea, L.

Peziza Dehnii, Rabh.

Potentilla Canadensis, L.

Darluca Filum, Cast.

$\{$ Dothidea Potentillae, Fr.

\{ Stigmatea Potentillae, $F_{r}$.

Phragmidium obtusum, Wint.?

Uredo obtusa, Strauss.

Uredo Potentillarum, DC.

Aregma obtusatum, $\mathrm{Fr}$.

Phragmidium obtusum, Kze. \& Schm.

Uredo Alchemillae, S. Syn. Car.

Caeoma Potentillarum, Lk.

Phragmidium triarticulatum, (B. \& C.) Farl.

Phragmidium Potentillae, Wint.

S Sphaeria Potentillae, S.

$\{$ Phyllachora Potentillae, Pk.

Puccinia Potentillae, S.

Synchytrium Myosotidis, var. Potentillae, Schrt.

$\{$ Exoascus deformans, var. Potentillae, Farl.

Taphrina Potentillae, Johanson.

Potentilla fruticosa, L.

Phraginidium bulbosum, (Strauss) Schl.

Potentilla glandulosa, Lindl.

$\{$ Dothidea Potentillae, Fr.

\{ Stigmatea Potentillae, Fr.

Potentilla gracilis, Dougl.

$\{$ Phragnidium Fragariae, (DC.) Wint.

$\{$ Phragmidium Fragariastri, (DC.) Schit.

Potentilla Norvegica, L.

+Peronospora Potentillae, DBy.

Peziza Dehnii, Rabh.

Ramularia arrensis, Sacc.

Septoria purpurascens, Ell. \& Martin. 
Potentilla palustris, Scop.

Cercospora Comari, Pk.

Potentilla Pennsylvanica, L.

f Aregma triarticulatum, B. \& C.

\{ Phragmidium triarticulatum, Farl.

Poterium Canadense, Benth. \& Hook.

f Erysiphe fuliginea, Lk.

$\Rightarrow$ Sphaerotheca Castagnei, Lev.

Rosa acicularis, Lindl.

Rosa Arkansana, Porter.

Uredo miniata, P.

$\{$ Phragmidium subcorticium, (Schrank)

Rosa blanda, Ait.

Phragmidium speciosum, Fr.

Aregma mucronatum, Fr.

Phragmidium mucronatum, Auct. Amer.

Coleosporium miniatum, Bon.

Phragmidium subcorticium, (Schrank.)

l Wint.

Rosa Californica, Cham. \& Schl.

Asteroma Rosae, Lib.

Diaporthe Rosae, B. \& C.

\&Peronospora sparsa, $B$.

Rosa Carolina, L.

Cenangium Rosae, S.

Cercospora rosaecola, Pass.

Cytospora Rosae, S. Am. Bor.

Dacryomyces difformis, $\mathrm{S}$.

Phragmidium subcorticium, (Schrank) Wint.

Sphaeria convergens, Tode.

$\{$ Valsa convergens, Fr.

Pseudovalsa convergens, Sacc.

Sphaeria palitiolata, S.

f Sphaeria expers, S.

$\{$ Valsa expers, Cke.

Rosa cinnamomea, L.

Cercospora rosaecola, Pass.

Diaporthe simulans, Sacc.

Rosa humilis, Marsh.

$\{$ Aregma speciosum, Fr.

Phragmidium speciosum, Fr.

Uredo miniata, $\mathrm{P}$.

$\left\{\frac{\text { Phragmidium subcorticium, (Schrank) }}{\text { Wint. }}\right.$

- Sphaerotheca pannosa, (Wallr.) Lev.

Rosa laevigata, Michx.

Sphaeria inordinata, B. \& C.

Cryptosphaeria inordinata, Sacc.

Rosa Iucida, Ehrh.

Phragmidium speciosum, Fr.

Coleosporium miniatum, Bon.

$\{$ Phragmidium subcorticium, (Schrank)

Wint.

Sphaerotheca pannosa, (Wallr.) Lev.

$\boldsymbol{R}$ osa nitida, Willd.

f Aregma speciosum, $\mathrm{Fr}$.

I Phragmidium speciosum, Fr.
Rosa rubiginosa, L.

Asteroma Rosae, Lib.

Dothidea Rosae, S.

Actinonema Rosae, $\mathrm{Fr}$.

Phyllachora Rosae, Sacc.

Stictis glancoma, B. \& C.

Rosa Sayi, S.

Phragmidium subcorticium, (Schrank) Wint.

Rosa, sp. indet.

Asteroma geographica, Desm.

Asteroma punctiforme, B.

Caeoma (Peridermium) germinale, S.

Coniothyrium Rosarum, Cke. \& Hark.

Coryneum microstictum, B. \& Br.

\{ Sphaeria fissicula, C. \& E.

$\{$ Cryptosphaeria fissicola, Sacc.

$\{$ Sphaeria rostellata, Fr.

Diaporthe rostellata, Nits.

Diatrype incarcerata, B. \& Br.

Diplodia Rosae, B. \& C.

$\{$ Diplodia rosaecarpa, Cke.

$\{$ Diplodia rhodocarpa, Sacc.

Hendersonia Rosae, $\mathrm{Fr}_{\mathrm{r}}$.

Hendersonia longipes, B. \& C., forma Rosarum, Cke.

?Heydenia Americana, Sacc. \& Ell.

Hypoxylon epirhodium, B. \& Rav.

Leptothyrium Rosarum, Cke.

S Sphaeria brachytheca, B. \& C.

Metasphaeria brachytheca, Sacc.

S Sphaeria leiostega, Ell.

\{Metasphaeria leiostega, Sacc.

f Naemaspora erythraea, B. \& C.

\{ Naemaspora erythrella, B. \& C.

Perisporium speireum, Fr.

+Peronospora sparsa, $B$.

Pestalozzia Guepini, Desm.

Pestalozzia suffocata, Ell. \& Ev.

f Peziza umbilicata, B. \& C.

\{ Peziza Curtisii, Che.

Peziza Rosae, P.

Phragmidium mucronatum, var. Americanum, Pk.

\{ Sphaeria erratica, C. \& E.

\{ Physalospora erratica, Sacc.

Polyactis vulgaris, Lk.

Sphaerella rosigena, Ell. \& Ev.

Sphaeria clypeata, Nees.

Sphaeria fissa. P.?

Sphaeria (Didymella) Rauii, Ell. \& Ev.

Sphaeria Rosae, S.

Sphaeria saepincola, Fr.

Sphaeropsis Rosarum, C. \& E.

Valsa Rosarum, DeNot.

Rubus Canadensis, L.

Ascochyta Rubi, Lasch.

Caeoma (Uredo) Juminatum, Lk. 
Iendersonia platypus, Ell. \& Ev.

$\{$ Septoria Rubi, Westd.

\{ Septoria Rubi, B. \& C.

Septoria Rubi, Westd., var. alba, Pk.

f ?Sphaeria subclypeata, B. \& C.

\{ Valsa subclypeata, C. \& P.

Rubus Dalibarda, L.

\{ Dothidea Dalibardae, Pk.

\{ Phyllachora Dalibardae, Sacc.

Septoria Dalibardae, Pk.

Rubus deliciosus, James.

Lecythea deliciosa, $\mathrm{Pk}$.

Rubus hispidus, L.

Iacrosporium Rubi, Ell.

Rhytisma Blakei, M. A. Curtis.

Septoria Rubi, Westd., var. pallida, Ell. \& Holw.

- Sphaerotheca Castagnei, Lev.

$R u b u s$ Nutkanus, Mocino.

Diaporthe rostellata, (Fr.) Nits.

Peziza acutipila, Karst.

Peziza bicolor, Bull.

Peziza scabrovillosa, Phil.

Phacidium Rubi, Fr.

Phragmidium gracile, Auct. Amer.

Septoria Rubi, Westd.

$R u b u s$ occidentalis, L.

$\{$ Aecidium nitens, S.

\{ Caeoma (Uredo) luminatum, I.k.

\{ Sphaeria Hendersonia, Ell.

\{Clypeosphaeria Hendersoniae, Sacc.

Gloeosporium necator, Ell. \& Ev.

Macrosporium punctiforme, B.

(Phragmidium incrassatum, Lk., var. gracile, Farl.

PhragmidiumRubi-Idaei, (P.) Wint.

Sphaeria eriostega, C. \& E.

Physalospora eriostega, Sacc., var. glabrata, Sacc.

Puccinia Peckiana, Howe.

Septosporium praelongum, Sacc.

Sphaeropsis rubicola, C. \& E.

\section{Rubus odoratus, L.}

$\{$ Sphaeria melanostyla, DC.

Gnomonia melanostyla, Fckl.

Lophiostoma bicuspidatum, Cke.

Peziza lacerata, C. \& P.

Peziza subochracea, C. \& P.

Phragmidium gracile, Auct. Amer.

Phyllosticta variabilis, $\mathrm{Pk}$.

Rubus strigosus, Michx.

$\{$ Aecidium nitens, S.

\{ Caeoma (Uredo) luminatum, Lk.

Cytospora Rubi, S.

$\{$ Valsa obscura, Pk.

\{ Diaporthe obscura, Sacc.

Gloeosporium necator, Ell. \& Ev.
Graphium gracile, $\mathrm{Pk}$.

$\{$ Stilbum pruinosipes, $\mathrm{Pk}$.

\{ Graphium pruinosipes, Sacc.

C Hysterium Rubi, P.

Hy poderma virgultorum, DC.

Hysterium virgultorum, Rob. \& Desm.

Hypoderma Rubi, DeNot.

Melanconium griseum, S.

Peziza clandestina, Bull.

S Uredo gyrosa, Reb.

$\{$ Phragnidium gracile, Auct. Amer.

Phragmidium Rubi-Idaei, (P.) Wint.

Puccinia Peckiana, Howe.

Rhabdospora Rubi, Ell.

Septoria Rubi, Westd.

\{ Sphaeria caespitulans, S.

\{Coniochaeta caespitulans, Cke.

\{ Sphaeria fuscella, B. \& Br.

\{eptosphaeria fuscella, Ces. \& DeNot.

(Sphaeria Hendersonia, Ell.

$\{$ Sphaeria melantera, $\mathrm{Pk}$.

Clypeosphaeria Hendersoniae, Sacc.

Sphaeria Ruborum, S.

Sphaeropsis rubicola, C. \& E.

Sphaeria tumida, S.

Sphaeria syngenesia, Fr.

(Valsa syngenesia, Fr.

Vermicularia compacta, C. \& E.

Rubus triflorus, Richard.

Caeoma (Uredo) Iuminatum, Lk.

- Sphaerotheca Castagnei, Lev.

- Sphaerotheca pannosa, (Wallr.) Lev.

Rubus trivialis, Michx.

Cercospora Rubi, Sacc.

Meliola sanguinea, Ell. \& EV.

Tubercularia persicina, Ditm.

Rubus ursinus, Cham. \& Schl.

S Sphaeria rostellata, $\mathrm{Fr}$.

\{ Diaporthe rostellata, Nits.

(Hendersonia sarmentorum, Westd., var.

$\{$ Rubi, C. \& E.

Hendersonia Rubi, Sacc.

Libertella Rosae, Desm.

Rubus villosus, Ait.

$\{$ Aecidium nitens, $S$.

$\{$ Caeoma (Uredo) luminatum, Lk.

Cercospora rosaecola, Pass.

$\{$ Coleosporium Rubi, Ell. \& Holw.

Chrysomyxa albida, Kühn.

\{ Sphaeria rostellata, $\mathrm{Fr}$.

\{ Diaporthe rostellata, Nits.

Diplodia Rubi, Fr.

\{ Fusisporium ? Rubi, Wint.

\{Fusarium? Rubi, Berl. \& Vogl.

Hendersonia platypus, Ell. \& Ev.

Hypoderma Rubi, (P.) DeNot.

SHysterium viticolum. C. \& P. p.p.

$\{$ Hysterographium viticolum, Rehm, var. 
Isariopsis Graviana, Ell.

Lecythea tripustulata, Cke.

Patellaria rhabarbarina, B.

$\{$ Phragmidium bulhosum, (Strauss) Schl.

\{ Phragmidium Rubi, (P.) Wint.

f Puccinia Peckiani, Howe.

\{ Puccinia tripustulata, Pk.

Septoria Rubi, IVestd.

Septoria Rubi, Westd., var. alba, Pk.

Sphaeria (Diaporthe) gallophila, Ell.

Sphaeria obtusa, S.

Stictis Rubi, S.

\{ Valsa Rubi, Fckl.

\{ Valsa Rubi, Pk.

Rubus, sp. indet.

Asterina Pearsoni, E11. \& Ev.

Capnodium elongatum, B. \& Desm.

$\{$ Excipula Rubi, Fr.

\{enangium Rubi, Duby.

$\int$ ? Corticium Sambuci, P.

Corticium rubicola, B. \& C.

Corticium Rubi, B. \& C.

Cylindrosporium Rubi, Ell. E Morgan.

Hymenella nigra, Fr.

Hysterium confluens, S.

Sphaeria clypeata, Nees.

S Splraeria Doliolum, P.

$\{$ Leptosphaeria Doliolum, DeNot.

Leptostroma rulgare, $\mathrm{Fr}$.

SLophiostoma Jerdoni, B. \& Br.

Lophiotrema praemorsum, (Lasch) Sacc.

S Sphaeria anisometra, Cke. \& Hark.

Metasphaeria anisometra, Sacc.

Myxormia atroviridis, B. \& Br.

Peziza compressa, P., var. rubicola, C. \& E.

Peziza rufo-olivacea, A. \& S.

Peziza vinosa, A. \& S.

Sphaeria saepincola, Fr.

Stigmatea Chaetomium, (Kunze) Fr.

Tympanis conspersa, Fr.

Typhula rubicola, B. \& C.

$\{$ Spluaeria rubincola, S.

\{ Valsa rubincola, Cke.

Vermicularia compacta, C. S E., var. Ruborum, C. \& E.

Vermicularia effusa, $\mathrm{S}$.

Spiraea Douglasii, Hook., var. dumosa, Watson.

- Podosphaera Oxyacanthae, (DC.) DBy.

(Microsphaera fulvofulcra, Cke.

Spiraea salicifolia, L.

\{ Sphaeria Collinsii, S.

(Dimerosporium Collinsii, Thm.

C Podosphaera Kunzei, Lev.

- Podosphaera minor. Howe.

(Microsphaera fulvofulcra, Cke.

$\{$ Ascochyta Salicifoliae, Trelease.

Septoria Salicifoliae, E1l. \& Ev.
Spiraea tomentosa, L.

C Podosphaera Kunzei, Lev.

Podosphaera Oxyacanthae, (DC.) DBy.

- Podosphaera minor, Howe.

Microsphaera fulvofulcra, Cke.

Spiraea, sp. indet.

Cercospora Rubigo, Cke. \& Hark.

Leptostroma Spiraeae, Fr.

Pestalozzia monochnetoidea, Sacc. \& Ell.

Peziza Schumacheri, $\mathrm{Fr}$.

Ramularia Ulmariae, Cke.

- Sphaerotheca Castagnei, Lev.

Sporidesmium spiraeicolum, Cke.

Waldsteinia fragarioides, Tratt.

Puccinia Waldsteiniae, M. A. Curtis.

Septoria Waldsteiniae, Pk. \& Clinton.

Pomeae.

Amelanchier alnifolia, Nutt.

Sphaeria Collinsii, S.

$\{$ Dimerosporinm Collinsii, Thm.

Plowrightia phyllogona, Hark.

Roestelia lacerata, (Sow.) Fr.

Amelanchier Canadensis, Torr. \& Gr.

(Diatrype phaeosperma, Ell.

Anthostoma phaeospermum, Sacc.

Fuckelia phaeosperma, Cke.

Sphaeria Collinsii, S.

Sphaeria papilionacea, B. \& C.

Sphaeria Russellii: B. \& C.

Dimérosporium Collinsii, Thm.

Dothidea Collinsii, Ell.

Lasiosphaeria papilionacea, Sacc.

Lasiosphaeria Russellii, Sacc.

Plowrightia phyllogona, Hark.

Discella Canadensis, Pk.

$\{$ Sphaerella Peckii, Speg.

\{ Metasphaeria Peckii, Sacc.

Morthiera Mespili, (DC.) Fckl.

- Podosphaera Oxyacanthae, (DC.) DBy.

Roestelia aurantiaca, Pk.

(Caeoma (Roestelia) Botryapites, S.

$\{$ Roestelia Ellisii, Pk.

Roestelia Botryapites, B.

Roestelia cornuta, (Gmel.) Fr.

Roestelia lacerata, (Sow.) Fr.

$\{$ Roestelia penicillata, Anct. Amer.

$\{$ Roestelia pirata, (S.) Thaxter.

Sphaeronema pruinosum, Pk. Jo, tef

Stysanus Stemonitis, Cda.

Valsa ceratophora, Tul.

(Valsa punctostoma, E11.

$\{$ Diaporthe stictostoma, Sacc.

Valsa stictostoma, Cke.

$\{$ Valsa tuberculosa, Ell.

$\{$ Diaporthe tuberculosa, Sacc.

Amelanchier, sp. indet.

Asteroma atramentarium, $B$.

Cenangium Aucupariae, $\mathrm{Fr}$. 
\{ Lophiostoma pallidum, Ell.

\{Lophiotrema pallidum, Berl. \& Vogl.

Crataegus coccinea, L.

$\{$ Morthiera Thimenii, Cke.

\{ntomosporium Thumenii, Sacc.

\{ Podosphaera Kunzei, Lev.

\{ Podosphaera Oxyacanthae, (DC.) DBy.

Sporidesmium curvatum, B. \& C.

Hirudinaria macrospora, Ces.

Clasterisporium curvatum, Sacc.

Roestelia aurantiaca, $\mathrm{Pk}$.

Aecidium Crataegi, var. Oxyacanthae, S.

\{ Roestelia lacerata, (Sow.) Fr.

Crataegus cordata, Ait.

Botryosphaeria melathroa, B. \& C. in Herb. Berk.

Roestelia lacerata, (Sow.) Fr.

Crataegus Crus-galli, L.

Crepidotus rufolateritius, Bresadola.

Roestelia aurantiaca, Pk.

Roestelia lacerata, (Sow.) Fr.

f Roestelia penicillata, Auct. Amer.

Roestelia pirata, (S.) Thaxter.

Crataegus flava, Ait.

Morthiera Thümenii, Cke.

Entomosporium Thümenii, Sacc.

Crataegus Oxyacantha, L.

Roestelia aurantiaca, $\mathrm{Pk}$.

Roestelia lacerata, (Sow.) Fr.

Crataegus parvifolia, Ait.

$\{$ Daedalea confragosa, P.

\{Lenzites Crataegi, Berk.

Macrosporium stilbosporoideum, B. \& C.

Crataegus subvillosa, Schrader.

Roestelia lacerata, (Sow.) Fr.

Crataegus tomentosa, L.

$\{$ Cheilaria Crataegi, Cke.

\{ Phyllosticta? Crataegi, Sacc.

Diatrypella quercina, (P.) Nits.

Phyllosticta rubra, Pk.

SPodosphaera Oxyacanthae, (DC.) DBy.

- Podosphaera tridactyla, (Wallr.) DBy.

Roestelia lacerata, (Sow.) Fr.

Crataegus tomentosa, L., var. punctata, Gray.

Roestelia aurantiaca. $\mathrm{Pk}$.

Crataggus tomentosa, L., var, pyrifolia, Gray.

- Phyllactinia suffulta, (Reb.) Sacc.

Crataegus, sp. indet.

$\{$ Sphaeria Crataegi, S.

\{ Botryosphaeria Crataegi, Sacc.

Cenangium Crataegi, S.

Discosia artocreas, (Tode) Fr.

Exosporium melampsoroides, Sacc.

Patellaria nigrocinnabarina, $\mathrm{S}$.
Pestalozzia concentrica, B. \& Rav.

(Sphaeropsis fusiger, B. \& C.

$\{$ Plioma fusigera, Sacc.

(Macrophoma fusigera, Berl. \& Vogl.

Roestelia cornuta, (Gmel.) Fr.

Roestelia hyalina, Cke.

Sporidesmium Fumago, Cke.

Tubercularia difformis, S.

Valsa ceratophora. Tul., var. Crataegi, C. \& E.

\{Valsa tumidula, C. \& P.

\{Eutypella tumidula, Sacc.

Cydonia vulgaris, P.

Entomosporium maculatum, Lev.

- Podosphaera Oxyacanthae, (DC.) DBy.

Roestelia aurantiaca, $\mathrm{Pk}$.

Sphaeria Pomorum, S.

Cydonia, sp. indet.

$\{$ Dermea Cydoniae, S.

I Dermatea Cydoniae, Cke.

(Morthiera Mespili, (DC.) Fckl., var. Cydoniae, C. \& E.

Blastesis tridens, Zabriskie.

Entomosporium maculatum, Lev., var. Cydoniae, Sacc.

Erysiphe Syringae, S. ?

Hendersonia Cydoniae, C. \& E.

Roestelia cancellata, (Gmel.) Reb. ?

Sphaeropsis Cydoniae, C. \& E.

Tricothecium roseum, (P.) Lk.

Pirus Americana, DC.

Atractium flammeum, B. \& Rav.

Cenangium Aucupariae, Fr.

Cytospora rubescens, Fr.

Dothiora pyrenophora, B. an Fr.?

Graphium Sorbi, Pk.

$\{$ Melogramma superficialis, Pk. \& Clinton.

$\{$ Fenestella superficialis, Sacc.

\{ Sphaeria Semen, C. \& P.

\{ Metasphaeria Semen, Sacc.

Phyllosticta Sorbi, Westd.

Roestelia cornuta, (Gmel.) Fr.

Sphaeronema pallidum, Pk.

fValsa impulsa, C. St P.

(Diaporthe impulsa, Sacc.

Valsa Sorbi, Fr.

Pirus angustifolia, Ait.

Roestelia lacerata, (Sow.) Fr.

Roestelia penicillata, Auct. Amer.

Roestelia pirata, (S.) Thaxter.

Pirus arbutifolia, L.

Asteroma atramentarium, B.

Cercospora Piri, Farl.

Phyllosticta Arbutifolia, Ell. \& Martin.

Roestelia transformans, Ell.

Valsa excorians, C. \& E.

f Valsa pulchella, (P.) Fr.

$\{$ Calosphaeria princeps, Tul. 
Pirzs communis. L.

Acrospermum riridulum, B. \& C.

Capnodium elongatum, B. S Desm.

\{ Diatrype irregularis, C. \& E.

\{ Diatrypella irregularis, Sacc.

Diplodia manra, C. \& E.

f Discella effusa, B. S C.

$\{$ Discula effusa, Sicc.

Epochnium monilioides, Lk.

fIlelminthosporium Pirorum, Lib.

Cladosporium dendriticum, Wallr.

Fusicladium dendriticum. Fckl.

Fusicladium pirinum, Fckl.

Ilendersonia Cydoniae, C. \& E.

(Dacryounyces destructor, B, \& Rav.

\{Hypsilophora destructor, B.

Macrosporium Pirortm, Cke.

Marasmins pirinus, Ell.

Massaria Piri, Otth.

\{ Sphaeria pulris-pyrius, P.

$\{$ Melanomma pulvis-pyrius, Fckl.

Sphaeria subcutanea, C. \& E.

$\{$ Metasphaeria subcutanea, Sacc.

Conisphaeria subcutanea, Che.

\{ Morthiera Mespili, (DC.) Fckl.

\{ Blastesis tridens, Zabriskie.

Nucor amethysteus, Bon.

Myxosporium microsporum, Cke. \& Hark.

Oidium fructigenum, (P.) Kze. \& Schm.

Phoma Radula, B. \& Br.

Phyllosticta pirina. Sacc.

Phyllosticta Pirorum, Cke.

Polyporus versicolor, (L.) Fr.

$\{$ Aecidium cancellatum, Gmel.

$\{$ Roestelia cancellata. Reb.

Sphaerella sentina, ( $\mathrm{Fr}$. ).

Sphaeropsis Malorum, B.

\{ Sporidesinium Fumago, Cke.

$\{$ Sirodesmium Fumago, Sacc.

Pirus coronaria, L.

Cenangium pirinum, $\mathrm{S}$.

Fusicladium dendriticum, (Wallr.) Fckl.

Secidium piratum, S.

Caeoma (Roestelia) Cylindrites, var. Mali,S.

Roestelia penicillata, Auct. Amer.

Roestelia pirata, Thaxter.

Piras Malus, L.

\section{PYRENOMYCETES.}

$\{$ Splacra bisphaerica, C. S E:

\{Amphisphaeria bisphaerica, Sacc.

Capnodium Pomorum, B. \& C.

Dialonectria vulpina, Cke.

S Sphaeria Murrayi, B. \& C.

\{ Diaporthe Murrayi, Sacc.

Eutypa leioplaca, (Fr.) Cke.

$\{$ Sphaeria contorta. S.

\{llypocrea contorta, B. \& C.
Iypoxylon atramentosum, $\mathrm{Fr}$.

Lophiostoma scelestım, C. \& E.

Massaria Piri, Otth.

Sphaeria discincola, S. p.p.

Diatrype discincola, M. A. Curtis.

Anthostoma discincolum. Sacc.

Nummularia discincola, Cke.

Sphaeria discincola, S. p p.

Sphaeria discreta, S.

Nummularia discreta, Tul.

Diatrype discreta, M. A. Curtis.

Hypoxylon discretum, B. \& C.

$\{$ Dothidea fructigena, S.

\{ Phyllachora fructigena. Sacc.

(Dothidea pomigena, S.

Asteroma pomigena, $\mathrm{B}$.

(Phyllachora pomigena, Sacc.

Sphaeria viscosa, C. \& E.

$\{$ Melogramma fuliginosum, E11. p. P.

Physalospora viscosa, Sacc.

f Podosphaera Kunzei, Lev.

\{ Podosphaera tridactyla, (Wallr.) DBy.

Sphaeria collapsa, S.

Sphaeria excussa. S.

Sphaeria pirina, $F_{1}$.

Sphaeria pyramidalis, $\mathrm{S}$.

Splraeria subbullans, S.

Valsa ambiens, (P.) Fr.

Valsa leucostoma, (P.) Fr.

S Sphaeria melastoma, $\mathrm{Fr}^{\mathrm{r}}$.

$\{$ Valsa melastoma, $\mathrm{Fr}$.

S Sphaeria nivea, Hoftm.

\{ralsa nivea, Fr.

Talsa stellulata, Fr.

SPIIAEROPSIDEAE.

Cytospora carphosperma, Fr.

Diplodia maura, C. \& E.,

Diplodia Piri, Ell. \& Martin.

fDothiora pyrenophora, B. an Fr.?

$\{$ Diplodia pyrenophora, M. A. Curtis.

$\{$ Discella effusa, B. \& C.

$\{$ Discula effusa, Sacc.

Discosia maculaecola, Gerard.

Hendersonia Cydoniae, C. \& E.

fHendersonia prominula, B. \& C.

\{ Stagonospora prominula, Sacc.

SLabrella Pomi, Mont. \& Fr.

$\{$ Microsticta Pomi, Desm.

Leptothyrim Pomi, Sacc.

Leptostroma fructigenum, S.

Sphaeropsis Malorum, B.

Phoma Malorum, Sacc.

Macrophoma Malorum, Berl. \& Vogl.

Phyllosticta pirina. Sacc.

S Septoria Piri, Cast.

$\{$ Septoria piricola, Desm.

Sphaeronema conforme, $\mathrm{Pk}$. 
\{ Macroplodia cinerea, C. \& E.

\{ Sphaeropsis cinerea, Sacc.

\section{MELANCONIEAE.}

Gloeosporium versicolor, B. \& C.

$\{$ Pestalozzia concentrica, B. \& Rav.

$\{$ Pestalozzia rostrata, Zabriskie.

\section{IIYPHOMYCETES.}

Aspergillus ovalispermus, Lk.

Cercospora Piri, Farl.

Cheiromyces Beaumontii, B. \& C. in N. A. F.

Cladosporium stenosporum, B. \& C.

Epochnium monilioides, Lk.

$\{$ Fusarium pirinum, $S$.

$\{$ Fusarium apiogenum, Sacc.

Fusarium arcuatum, B. \& C.

Spilocaea fructigena, S. Am. Bor.

Spilocaea Pomi, Fr.

Helminthosporium Pirorum, Lib.

Fusicladium dendriticum, Fckl.

Oidium fructigenum, (P.) Kze. $\&$ Schm.

Rhinotrichum repens, Preuss.

\{ Sporidesmium melanopum, (Ach.) B. \& Br.

\{ Sporidesmium melanopodum, Sacc.

Sporidesmium moriforme, $\mathrm{Pk}$.

f Periconia truncata, C. \& P.

$\{$ Sporocybe truncata, Sacc.

$\{$ Sporotrichum luteoalbum, S. Am. Bor.

\{ Sporotrichum Thimenii, Sacc.

f Trichothecium roseum, (P.) Lk.

\{ Dactylium roseum, B.

Tubercularia nigricans, (Bull.) DC.

\section{DISCOMYCETES.}

$\left\{\begin{array}{c}\mathrm{C} \\ \mathrm{C} e n\end{array}\right.$ Cenangium Prunastri, (P.) Fr., var. rigidum, Fr.

Cenangium rigidum, S.

Dermatea fascicularis, (A. \& S.) Fr.

Peziza inquinans, Cke.

Peziza regalis, C. $\& \mathrm{E}$.

Tympanis conspersa, $\mathrm{Fr}$.

Tympanis gyrosa, B. \& C.

UREDINEAE.

Roestelia aurantiaca, Pk.

Aecidium cancellatum, Gmel.

? Roestelia cancellata, Reb.

Cacoma Roestelites, Lk. p. p.

$\{$ Aecidium laceratum, Sow.

Roestelia lacerate, $\mathrm{Fr}$.

Aecidium piratum, S.

Caeoma (Roestelia) Cylindrites, var. Mali, S.

Roestelia penicillata, Auct. Amer.

Roestelia pirata, Thaxter.

Roestelia transformans, Ell.

$\{$ Myxosporium colliculosum, B.

\{ Roestelia, sp.
TREMELLINI.

Exidia truncata, Fr.

Sremella frayiformis, S. Syn. Car.

Hormomyces aurantiacus, Sacc., nec Bon.

Hypsilophora fragiformis, Cke.

f Tremella violacea, Rell.

$\{$ Dacryomyces violacens, $F r$.

\section{HYMENOMYCETES.}

Agaricus corticola, P.

Agaricus pulvinatus, $P$.

Hydnum Schiedermayeri, Heufl.

Irpex obliquus, Fr.

Merulius porinoides, Fr.

Thelephora fusca, (P.) Fr.

$\{$ Polyporus populinus, S.

Trametes malicola, B. \& C.

Pirus prunifolia, Wiild.

Fusicladium dendriticum, (Wallr.) Fckl.

Pirus sambucifolia, Cham. \& Schl.

\{ Valsa impulsa, C. \& P.

$\{$ Diaporthe impulsa, Sacc.

Pirus, sp. indet.

Cenangium enteroxanthum, S.

Cladosporium stenosporum, B. \& C.

$\{$ Thelephora polygonia, P.

\{Corticium polygonium, $\mathrm{Fr}$.

Sphaeria subaffixa. S.

$\{$ Nummularia subaffixa, Sacc.

Diatrype subaffixa, Cke.

Monilia punctans, S.

Nectria cinnabarina, (Tode) $\mathrm{Fr}^{\mathrm{r}}$.

Polyporus connatus, Fr.

$\{$ Boletus marginatus, $P$.

\{ Polyporus marginatus, Fr.

Sclerotium pirinum, Fr.

$\{$ Septoria Oxyacanthae, Kze.

\{ Phloeospora Oxyacanthae, Wallr.

Sphaeronema piriforme, (P.) Fr.

Sporotrichum fructigenum. Lk.

\section{SAXIFRAGACEAE.}

Deutzia gracilis, Sieb. \& Zucc.

Cercospora Deutziae, Ell. \& Ev.

Fendlera rupicola, Engelm. \& Gr.

Aecidium Rusbyi, Gerard.

Heuchera Americana, L.

Cercospora Heucherae, Ell. \& Martin.

$\{$ Puccinia Saxifragae, Schl.

(Puccinia curtipes, Howe.

Uredo Heucherae, S.

$\{$ Caeoma Heucherae, Lk.

Puccinia spreta, Pk.

Heuchera hispida, Pursh.

Cercospora Heucherae, E. \& M.

Heuchera micrantha, Dougl.

Puccinia congregata, Ell. \& Hark. 
Heuchera villosa, Michx.

Uredo Heucherae, S.

Caeoma Heucherae, Lk.

Puccinia spieta, Pk.

Hydrangea arborescens, L.

Peziza fracta. B. \& C.

Uredo Hydrangeae, B. \& C.

Hydrangea, sp. indet.

Cenangium apertum, S.

\{ Sphaeria sphaerocephala, S.

\{ Cytospora sphaerocephala, M. A. Curtis.

Peziza Hydrangeae, S.

\{ Sphaeria Hydrangeae, S.

\{Ohleria Hydrangeae, Cke.

? Sphaeria Sclerotium, S.

Sphaerobolus sparsus, S.

Stictis Hydrangeae, $\mathrm{S}$.

\{ Torula pallida, B. \& Rav.

\{ospora pallida, Sacc. \& Vogl.

Mitella diphylla, L.

Phyllosticta Mitellae, Pk.

Puccinia spreta, $\mathrm{Pk}$.

Puccinia Tiarellae, B. \& C.

Ramularia Mitellae, Pk.

Mitella nuda, L.

Puccinia congregata, Ell. \& Hark.

Puccinia spreta, $\mathrm{Pk}$.

Puccinia Tiarellae, B. \& C.

Pamassia Caroliniana, Michx.

f Sphaeria Parnassiae, Pk.

\{Didymosphaeria Parnassiae, Sacc.

Philadelphus coronarius, L.

Cercospora angulata, Wint.

Philadelphus inodorus, L.

Stictis Philadelphi, S.

Philadelphus microphyllus, Gray.

Aecidium gracilens, $\mathrm{Pk}$.

Ribes aureum, Pursh.

Aecidium Grossulariae, DC.

Uredo ribicola, C. \& E.

Uredo Jonesii, Pk.

Caeoma Ribes-alpini, (P.) Wint.

Septoria Grossulariae, (Lib.) Westd.

$\{$ Sphaeria penicillata, $S$.

$\{$ Coniochaeta penicillata, Cke.

S Sphaeria concrescens, S.

L Diaporthe concresens, Che.

Ribes Cynosbati, L.

Aecidium Grossulariae, DC.

Septoria Ribis, Desm.

- S Sphaerotheca Mors-uvae, (S.) B. \& C.

- Sphaerotheca pannosa, var. Ribis, $\mathrm{Pk}$.

Ribes divaricatum, Dougl., var. irriguum, Gray.

- Sphaerotheca Mors-uvae, (S.) B. \& C. S Sphaerotheca pannosa, (Wallr.) Lev. Stictis pelvicula, $\mathrm{P}$.
Valsa agnostica Cke. \& Hark.

Ribes floridum, L'Her.

Aecidium Grossulariae, DC்.

Sphaeria ribesia, S.

Diatrype ribesia, MI. A. Curtis.

f Diatrype Strumella, Fr.

Diaporthe Strumella, Fckl.

Sphaeria ribesia. P.

Dothidea ribesia, Fr.

Plowrightia ribesia, Sacc.

Septoria Ribis, Desm.

Sphaeria recondita, S.

Sphaeropsis ribicola, C. \& E.

- Sphaerotheca Mora-uvae, (S.) B. \& C.

- Sphaerotheca pannosa, (Wallr.) Lev.

S Sphaeria vestita, Fr.

\{ Valsa vestita, Fr.

Ribes gracile, Michx.

Aecidium Grossulariae, DC.

- Sphaerotheca Mors-uvae, (S.) B. \& C.

Ribes lacustre, Poir., var. parvulum, Gray.

Aecidium Grossulariae, DC.

Ribes nigrum, L.

$\{$ Leptothyrium Ribis, Lib.

$\{$ Gloeosporium Ribis, Desm. \& Mont.

Sphaeria (Depazea) ribicola, Fr.

Ribes prostratum, L'Her.

Aecidium Grossulariae, DC.

Gloeospoi ium Ribis, (Lib.) Desm. \& Mont.

$\{$ Puccinia pulchella, Pk.

$\{$ Puccinia Grossulariae, Wint.

Septoria Ribis, Desm.

Septoria Siberica, Thm.

Ribes rotundifolium, Michx.

$\{$ Aecidium Grossulariae, DC.

$\{$ Caeoma (Aecidium) grossulariatum, Lk.

f Septoria ribicola, B. \& C.

$\{$ Rhabdospora ribicola, Sacc.

Septoria Ribis, Desm.

Erysiphe Mors-uvae, S.

- Sphaerotheca Mlors-uvae, B. \& C.

(Sphaerotheca pannosa, (Wallr.) Lev.

Ribes rubrum, L.

Aecidium Grossulariae, DC.

Sphaeropsis hyalina, B. \& C.

Phoma hyalina, Sacc.

( Macrophoma hyalina, Berl. \& Vogl.

$\{$ Puccinia Grossulariae, Wint.

\{ Puccinia Ribis, DC:

Nectria Ribis, Niessl.

Pleonectria Ribis, Karst.

Pleonectria Berolinensis, Sacc.

Nectria Berolinensis, Ell. \& Ev.

(Sphaeria Ribis, Tode.

Sphaeria appendiculata, S. Syn. Car.

Nectria Ribis, Oud.

Phyllosticta Grossulariae, Sacc.

Septoria Ribis, Desm.

Tubercularia vulgaris, Tode. 
Ribes sanguineum, Pursh.

Cucurbitaria Ribis, Niessl.

Ribes Uva-crispa, L.

Phyllosticta Grossulariae, Sacc.

$\{$ Erysiphe Mors-urae, S.

- Sphiaerotheca Mors-uvae, B. \& C.

Ribes (Gooseberry).

Nectria Ribis, (Tode) Oud.

Ribes, sp. indet.

Cenangium Ribis, Fr.

Cladosporium cubisporum, B. \& C.

\{ Sphaeria subcutanea, Wahl.

\{Eutypa subcutanea, Sacc.

f Fusidium Fumago, S.

\{ Coniosporium Fumago, Sacc.

- Microsphaera Grossulariae. Lev.

$\{$ Sphaeria decolorans. P.

$\{$ Nectria cinnabarina, (Tode) Fr.

Peziza vinosa, A. \& S.

- Phyllactinia guttata, Lev.

Polyporus Ribis, (Schum.) Fr.

Thelephora lactea, $\mathrm{Fr}$.

Thelephora ribesina, $\mathrm{Fr}$.

Tubercularia confluens, P.

Saxifraga peltata, Torr.

Peziza setigera, Phil.

Saxifraga Pennsylvanica, L.

$\{$ Puccinia Saxifragae, Schl.

$\{$ Puccinia curtipes, Howe.

Saxifraga Virginiensis, Michx.

$\{$ Puccinia Saxifragae, Schl.

$\{$ Puccinia curtipes, Howe.

Tiarella cordifolia, L.

Puccinia Tiarellae, B. \& C.

$\{$ Puccinia Tiarellae, Pk., nec. B. S C.

$\{$ Puccinia spreta, $\mathrm{Pk}$.

Tiarella, sp. indet.

Puccinia Saxifragae, Schl.

\section{CRASSULACEAE.}

Crassula, sp. indet.

Diplodia Crassulae, Cke. \& Hark.

Penthorum sedoides, L.

Cercospora sedoides, Ell. \& Ev.

Sedum Telephium, L.

$\{$ Hysterium commune, Fr.

$\{$ Hypoderma commune, Duby.

Sedum, sp. indet.

Diplodia sedicola, Cke. \& Hark.

HAMAMELACEAE.

\section{Fothergilla Gardeni, L.}

$\{$ Xyloma Fothergillae, S.

Dothidea Fothergillae, S.
Hamamelis Virginiana, L.

Diatrype platystoma, (S.) M. A. Curtis.

$\{$ Gonatobotrys maculicola, Wint.

\{ Gonatobotryum maculicolum, Sacc.

Haplographium apiculatum, Pk.

Patellaria Hamamelidis, $\mathrm{Pk}$.

Pestalozzia consocia, $\mathrm{Pk}$.

(Phyllosticta Hamamelidis, Cke.

Gloeosporium Hamamelidis, Cke.

Phyllosticta Hamamelidis, Pk.?

Podosphaera binuncinata, C. \& P.

Polyporus scutellatus, S.

Ramularia Hamamelidis, Pk.

Liquidambar Styraciflua, L.

PYRENOMYCETES.

$\{$ Sphaeria atrograna, C. $\mathbb{E}$ E.

Amphisphaeria atrograna, Sacc.

Melanconis amygdalina, Cke.

$\{$ Anthostoma amygdalinum, Sacc.

Fuckelia amygdalina, Cke.

$\{$ Nectria polythalama, B.

Calonectria polythalama, Sacc.

(Sphaeria caminata, C. \& E.

$\{$ Xylosphaeria caminata, Cke.

Ceratostoma caminatum, Sacc.

Sphaeria subcongregata, B. \& C.

Sphaeria subconnata, B. \& C.

Coelosphaeria subconnata, Sacc.

(Fracchiaea subconnata, Cke.

Sphaeria divergens, $\mathrm{S}$.

$\{$ Valsa divergens, M. A. Curtis.

Cryptosporella divergens, Berl. \& Vogl.

$\{$ Nectria rimincola, Cke.

$\{$ Dialonectria rimincola, Cke.

Eutypa limaeformis, (S.) B.

f Valsa stellulata, Fr.

\{Eutypella stellulata, Sacc.

$\{$ Sphaeria petiolorum, S.

\{ Gnomonia petiolorum, Cke.

Hypocrea contorta, (S.) B. \& C.

Sphaeria perforata, $\mathrm{S}$.

\{Hypoxylon perforatum, $\mathrm{Fr}$.

$\{$ Hysterium cinereum, S. Syn. Car.

\{ Stictis farinosa, Fr. El.

? Ostropa cinerea, Fr.

$\{$ Hysterium varium, $\mathrm{Fr}$.

Ostreichnion Americanum, Duby.

\{ Sphaeria Pezizula, B. \& C.

\{Lasiosphaeria Pezizula, Sacc.

$\{$ Sphaeria Racodium, P.

Lasiosphaeria Racodinm, Ces. \& DeNot.

Sypoxylon Walterianum, B. \& Rav.

$\{$ Hypoxylon gemmatum, B. \& Rav.

Melogramma (Valsaria) gemnatum, Cke.

Sphaeria gyrosa, S.

Endothia gyrosa, Fr.

Melogramma gyrosum, Tul.

f Sphaeria Aquila, Fr.

$\{$ Rosellinia Aquila, DeNot. 
Sphaeria millegrana, S.

\{ Rosellinia millegrana, Sacc.

\{ Sphaeria pulveracea, Ehrh.

$\{$ Rosellinia pulveracea, Fckl.

$\{$ Hypoxylon subiculosum, B.

Rosellinia subiculata, (S.) Sacc.

Splnaria pileata, Yode.

Sphaeria disseminata, B. \& C.

$\{$ Teichospora disseminata, Sacc.

Sypoxylon erinaceum, B. S Rav.

$\{$ Hypoxylon? Ravenelii, Sacc.

Valsa erinacea, Cke.

S Sphaeria Liquidambaris, S.

$\{$ Valsa Liquidambaris, M. A. Curtis.

Valsa salicina, (P.) Fr.

$\{$ Diatrype quadrata, (S.) B.

\{ Valsaria quadrata, Sacc.

S Sphaeria carpophila, P.

Tylaria carpophila, Fr.

\section{HYNEXOMYCETES.}

Agaricus niger, $\mathrm{S}$.

Clavaria Petersii, B. S C.

Corticium crocicreas, B. \& C.

Corticium laeve, Fr.

$\{$ Corticium miniatum, B.

$\{$ Corticium sanguineum, Fr.

Corticium siparium, B. \& C .

Daedalea confragosa, P.

Lenzites Crataegi, B.

Hydnum Cookei, B.

Hydnum ochracemn, P.

Hydnum setulosum. B. \& C.

Irpex obliquas, Fr.

Lenzites bicolor, $\mathrm{Fr}^{\text {r. }}$

Polyporus adustus, (Willd.) Fr.

$\{$ Polyporus biformis, Fr.

$\{$ Polyporus Caroliniensis, B. $\&$ C.

$\{$ Boletus cinnabarinus, Jacq.

$\{$ Polyporus cin nabarinus, Fr.

SBoletus gilvus, S.

$\{$ Polyporus gilvus, Fr.

Polyporus pargamenus, Fr.

(Boletus carpineus, var. b. S. Syı. Car.

$\{$ Polyporus rutilans, S. Am. Bor.

Polyporus scruposus, Fr.

$\{$ Peziza pruinata, S.

Solenia poriaeformis, (DC.) Fckl.

Stereum complicatum, Fr.

SThelephora Strraciflua, S.

$\{$ Stereum styracifluum, M. A. Curtis.

Thelephora bufonia, $\mathbf{P}$.

Typhula Grevillei, Fr.

\section{MSCELLANEA.}

Clasterosporium subulatum, C. $\mathbb{E}$ P. Dendryphium fasciculare, B. S. Rav. Harknessia affinis, Ell. \& Ev.
\{ Helicoma Berkeleyi, M. A. Curtis.

\{ Helicosporium Berkeleyi, Sacc.

Helminthosporium asterinum, Cke.

Hirneola scutelliformis, B. \& C.

Melampsora Liquidambaris, Cke.

f Peziza flammea. S. Syn. Car.

\{ Peziza cinnabarina, $\dot{S}$.

Peziza rubella, P.

Peziza saccharifera, $\mathrm{B}$.

\{ Peziza sanguinella, B. S C.

$\{$ Peziza vulgaris, Fr., var. sanguinella, B.

f Seiridium inarticulatum, MI. A. Curtis.

$\{$ Seiridium Iiquidambaris, B. \& C. .

Septoria Liquidambaris, C. S E.

Sporidesmium concinnum, B.

Tortula binale, C. $\&$ E.

\section{HALORAGEAE.}

Proserpinaca palustris, L.

Puccinia Proserpinacae, Farl.

Proserpinaca pectinata, Lam.

(Puccinia Epilobii, DC., var. Proserpinacae, Farl.

Puccinia Proserpinacae, Farl.

Proserpinaca, sp. indet.

Aecidium Proserpinacae, P. S C.

\section{MYRTACEAE.}

Eucalyptus globulus, Labill.

$\{$ Acrospermum corrugatum, Ell.

\{crospermum fultum, Hark.

Calloria Eucalypti, Phil. \& Hark.

Camposporium antennatum, IIark.

Chalara Montellica, Sacc.

f Fumago vagans, $P$.

\{ Cladosporum Fumago: Lk.

Cleistosoma purpureum, Hark.

Cryptosporium Eucalypti, Cke. \& Ilark.

Diaporthe Eucalypti, Hark.

Diatrype Eucalypti, Cke. S Hark.

(Sphaeria (Didymosphaeria) Cupula, E11.

\{Didymosphaeria circinans, Hark.

Eriosphaeria investans, (Cke.) Sacc.

f Ilarknessia Eucalypti, Cke.

$\{$ Harknessia uromycoides, Speg.

Peziza emergens, Phil. \& Hark.

Peziza subcornea, Phil. \& Hark.

Phoma Eucalypti, Cke. S Hark.

Sphaeropsis Nolleriana, Thm.

$\{$ Phoma Molleriana, Sacc.

(Macroplroma Molleriana, Berl. \& Vogl.

Sphaerella Molleriana, Thm.

Theclospora bifida, Hark.

Inzengaea erythrospora, Borzi.

Triposporium elegans, Cda. 
Valsa Eucalypti, Cke. \& Hark.

Eucalyptus odorata, Behr.

\{ Harknessia uromycoides, Speg.?

\{ Harknessia longipes, Hark.

Eucalyptus, sp. indet.

PYRENOMYETES.

Chaetosphaeria ornata, IIark.

Hypocrea consimilis. Ell.

Hypoderma Eucalypti, Cke. \& Hark.

Hysterium Eucalypti, Phil. \& Hark.

Hysterium pulicare, $\mathrm{P}$.

Lasiosphaeria orina, (P.) Ces. \& DeNot.

$\{$ Dialonectria Eucalypti, Cke. \& Hark.

\{Nectria Eucalypti, Berl. \& Vogl.

$\{$ Byssonectria chrysocoma, Cke. \& Hark.

$\{$ Nectria chrysocoma, Berl. \& Vogl.

Pleosphaeria modesta, Hark.

Rosellinia spinosa, Hark.

Sphaeria anisometra, Cke. \& Hark.

Sphaeria (Leptosphaeria) anisometra, Cke.?

Metasphaeria anisometra, Sacc.

Sphaeria ('Teichospora) Eucalypti, Cke. \& Hark.

Sphaeria mutila, var. Eucalypti, Cke. \& Hark.

Sphaeria (Metasphaeria) plagarum, Cke. \& Hark.

Valsaria Eucalypti, (Kalch. \& Cke.) Sacc.

SPIIAEROPSIDEAE.

Diplodia Eucalypti, Cke. \& Hark.

$\{$ Diplodia microspora, Sacc., nec B. \& C.

\{ Diplodia microsporella, Sacc.

Diplodia tenuis, Cke. \& Hark.

Hendersonia corynoidea, Cke. \& Hark.

Hendersonia Eucalypti, Cke. \& Hark.

$\left\{\begin{array}{c}\text { Cryptosporium ceutlosporoides, Cke. } \& \\ \text { Hark. }\end{array}\right.$

$\left\{\begin{array}{l}\text { Hark. } \\ \text { Septoria ceuthosporoides, Sacc. }\end{array}\right.$

Sphaeronema Encalypti, Cke. \& Hark.

\{ Sphaeropsis macrosperma, Cke. \& Hark.

A Aposphaeria macrosperma, Sacc.

\section{MELANCONIEAE.}

Gloeosporium capsularum, Cke. \& Hark.

Melanconium globosum, Cke. \& Hark.

Pestalozzia funerea, Desm.

Pestalozzia inquinans, Cke. \& Hurk.

Pestalozzia monochaeta, Desm.

f Pestalozzia truncata, Lev.

\{Pestalozzia truncatula, (Cda.) Fckl.

Stilbospora angustata, $\mathrm{P}$.

\section{HYPHOMYCETES.}

$\{$ Polyactis fusca, Cke. \& Hark.

$\{$ Botrytis fusca, Sacc.
Chaetopsis fusca, Cda.

Chalara fusidioides, Cda.

Circinotrichum maculiforme, Nees.

\{ Bactrodesmium clavatum, Cke. \& Ilark.

\{ Clasterosporium clavatum, Sacc.

Coremium grtaucum, Lk.

$\{$ Menispora hyalina, Cke. \& Hark.

$\{$ Cylindrocephalum hyalinum, Sacc.

Epidochium Eucalypti, Cke.

$\{$ Fusidium albidocarnenm, Cke. \& Hark.

$\{$ Fusarium albidocarneum, Sacc.

Fusarium Eucalyptorum, Cke. \& Hark.

Fusarium mesentericum, Cke. \& Hark.

$\{$ Helicoma Berkeleyi, M. A. Curtis.

\{ Helicosporiun Berkleyi, Sacc.

Helminthosporimm obovatum, B. \& Br.

Monilia viridiflava, Cke. \& Hark.

$\{$ Mystrosporium Curtisii, B.

\{ Mystrosporium Spraguei, B. \& C. in Herb.

Septonema multiplex, B. \& C.

Septosporium scyphophorum, Cke. \& Hark.

Speira toruloides, Cda.

Stysanus Stemonitis, (P.) Cda.

Tubercularia Eucalypti, Cke. \& Hark.

Volutella coronata, Cke. \& Hark.

DISCOMYCETES.

Dermatea Eucalypti, Cke. \& Hark.

Peziza carneorosea, Cke. \& Hark.

Peziza cerina, $P$.

Peziza luteorubella, Nyl.

Peziza rufoolivacea, A. \& S.

Propolis versicolor, $\mathrm{Fr}$.

Stictis radiata, P.

HYNENOMYCETES.

Agaricus sacchariferus, B. \& Br.

Corticium epiphyllum, (P.) Cke.

Pistillaria ovata, Fr.

Psidium, sp. indet.

Antennaria Guava, Cke.

\section{LYTHRACEAE.}

Cuphaea viscosissima, Jacq. Septoria maculosa, Gerard.

Lagerstroemia, sp. indet.

Hendersonia pauciseptata, B. \& C.

Pestalozzia Guepini, Desm.

Lythrum alatum, Pursh.

Lythrum Salicaria, L.

Septoria lythrina, Pk.

Nesaea verticillata, Нвк. Aecidium Nesaeae, Gerard.

Peziza cyathoidea, Bull. 
Peziza subatra, C. S P.

Phyllosticta Nesaeae, Pk.

\section{ONAGRACEAE.}

Roisduvalia densiflora, Watson.

Puccinia Oenotherae, Vize.

Boisduvalia Torreyi, Watson.

Puccinia Boisduvaliae, $\mathrm{Pk}$.

Circaea alpina, L.

Puccinia Circaeae, $\mathrm{P}$.

Circaea I,utetiana, L.

Puccinia Circaeae, P.

Circaea Pacifica, Aschers. \& Mag.

Puccinia Circaeae, $\mathrm{P}$.

Circaea, sp. indet.

(Uredo Circaeae, Schum.

$\{$ Caeoma (Uredo) Onagrarum, Lk.

Clarkia pulchella, Pursh.

Puccinia Clarkiae, Pk.

Clarkia rhomboidea, Dougl.

Puccinia Clarkiae, Pk.

Epilobium alpinum, L.

Aecidium Epilobii, DC.

Cercospora Epilobii, Schneid.

Doassansia Epilobii, Farl.

- Sphaerotheca Epilobii, (Lk.) DBy.

Epilobium coloratum, Muhl.

Melampsora Epilobii, (P.) Fckl.

Septoria Epilobii, Westd.

Epilobium hirsutum, L.

Uredo pustulata, $\mathrm{P}$.

Epilobium latifolium, L.

Pleospora arctica, Fckl.

Sphaeria nivalis, Fckl.

Epilobium palustre, L., yar. lineare, Gray.

Ielampsora Epilobii, (P.) Fckl.

Ejilobium paniculatum, Nutt.

Puccinia Epilobii, DC.

Epilobium spicatum, Lam.

f Dothidea Epilobii, Fr.

Asteroma Epilobii. Fr.

Clavaria typhuloides, $\mathrm{Pk}$.

I Sphaeria Racemula, C. \& P.

$\{$ Diaporthe Racemula, Sacc.

(Uredo pustulata, P.

\{ Melampsora Epilobii, (P.) Fckl.

Epilobium tetragonum, L.

Lecythea macrosora, $\mathrm{Pk}$.

Uredo Epilobii, DC.

Epilobium, sp. indet.

\{ Aecidium Epilobii, DC.

C. Caeoma (Aecidium) Epilobii, Schl.

Venturia Johnstoni, (B. \& Br.) Sacc.?

Fuchsia, sp. indet.

Didymella Fuchsiae, Cke \& Hark.

Diplodia Fuchsiae, Cke. S Hark.
Gaura coocinea, Nutt.

Aecidium gaurinum, $\mathrm{Pk}$.

Trichobasis gaurina, Pk.

Gaura parviflora, Dougl.

Septoria gaurina, Ell. \& Kell.

Gayophytum ramosissimum, Torr. \& Gr.

Aecidium Gayophrti, Vize.

Gayophytum, sp. indet.

Puccinia Gayophyti, Billings.

Jussiaea repens, L.

Septoria Jussiaeae, Ell. \& Kell.

Ludwigia alternifolia, L.

Aecidium Epilobii, DC.

Ludwigia palustris, Elliott.

Septoria Ludwigiae, Cke.

Ludwigia, sp. indet.

$\{$ Sphacria Ludwigiae, Cke.

$\{$ Physalospora Ludwigiae, Sacc.

Oenothera biennis, L.

Aecidium Epilobii, DC.

Aecidium Oenotherae, $\mathrm{Pk}$.

Ceratostoma subulatum, Ell.

-Erysiphe communis, (Wallr.) Schl.

S Sphaeria Doliolum, P.

$\{$ Leptosphaeria Doliolum, DeNot.

Sphaeria subconica, C. \& P.

$\{$ Leptosphaeria subconica, Sacc.

Leptothyrium protuberans, (Lev.) Sacc.

\{ Lophiostoma vermispora, Ell.

$\{$ Lophionema vermisporum, Sacc.

\{ Ophiotheca umbrina, B. \& C.

$\{$ Perichaena variabilis, Rostf.

XPeronospora Arthuri, Farl.

Peziza Oenotherae, C. \& E.

f Septoria Oenotherae, VVestd.

$\{$ Septoria Oenotherae, B. \& C.

Sphaerella Oenotherae, Ell. \& Ev.

Sphaeronema corneum, C. \& E.

Synchytrium fulgens, Schrt.

Oenothera caespitosa, Nutt.

Aecidium Oenotherae, Pk.

Uromyces plumbarius, $\mathrm{Pk}$.

Oenothera Fremonti, Watson.

Puccinia Oenotherae, Vize.

Uromyces Qenotherae, Burrill.

Oenothera linifolia, Nutt.

Uromyces Oenotherae, Burrill.

Oenothera ovata, Nutt.

Septoria Oenotherae, Westd.

Oenotherae serrulata, Nutt.

Aecidium Oenotherae, $\mathrm{Pk}$.

Septoria Oenotherae, Westd.

Oenothera sinuata, L.

Accidium Oenotherae, $\mathrm{Pk}$.

Sphaeria Oenotherae, B. \& C.

$\{$ Physalospora Oenotherae, Sacc.

Septoria Oenotherae, Westd. 
Oenothera strigulosa, Torr. \& Gr.

Puccinia Oenotherae, Vize.

Oenothera, sp. indet.

Sphaeria (Didymella) lophospora, (Sacc. \& Speg.) Ell.

Stenosiphon virgatus, Spach.

Septoria Stenosiphonis, Ell. \& Kell.

Zauschneria Californica, Presl.

S Synchytrium Jonesii, Pk.

$\{$ Tuberculina Jonesii, Sacc.

\section{LOASACEAE.}

Mentzelia nuda, Torr. \& Gr.

Mentzelia ornata, Torr. \& Gr.

Phyllosticta Mentzeliae, Ell. \& Kell.

Septoria Mentzeliae, Ell. \& Kell.

\section{PASSIFLORACEAE.}

\section{Passiflora lutea, I.}

Cercospora fuscovirens, Sacc.

Erineum Passiflora-luteae, S.

Passiflora, sp. indet.

Helminthosporium molle, B. \& C.

\{ Septoria fructicola, B. \& C.

$\{$ Septoria fructigena, B. \& C .

\section{CUC.URBITACEAE.}

Citrullus vulgaris, Schrad.

Cercospora citrullina, Cke.

Gloeosporium Lindemuthianum, Sacc. \& Magn.

Myrothecium Verrucaria, (A. \& S.) Dittm.

Pestalozzia torulosa, B. \& C.

\{ Phoma Citrulli, B. \&. C.

\{Macrophoma Citrulli, Berl. \& Vogl.

Phoma cucurbitalis, B. \& C.

Sphaeropsis seminalis, B. \& C.

Phoma seminalis, Sacc.

TMacrophoma seminalis, Berl. \& Vogl.

Cucumis Melo, L.

Nucur curtus, B. \& C.

Mucospurtus, B. \& C.

Cucurbita Pepo, L.

Epicoccum micropus, Cda.

Mucor inaequalis, $\mathrm{Pk}$.

Phyllosticta orbicularis, Ell. \& Ev.

Septoria Cucurbitacearum, Sacc.

Sphaeria Peponis, S.

Spilocaea conceutrica, S.

Cucurbita perennis, Gray.

Cercospora Cucurbitae, Ell. \& Ev.

Cucurbita verrucosa, L.

Verticillium verrucosum, B. \& C.

Cucurbita (Squash).

Aspergillus clavellus, $\mathrm{Pk}$.
Macrosporium Sarcinula, B.

Mucor inaequalis, Pk.

Peziza Cucurbitae, Gerd.

Phoma Cucurbita cearum, (Fr.) M. A. Curtis.

Phoma cucurbitale, B. \& C.

Rhopalomyces Cucurbitarum, B. \& Rav.

Cucurbita, sp. indet.

Botrytis cinerea, $\mathrm{P}$.

Coniothyrium Arthurianum, Sacc. \& Berl.

Nucor clavatus, Lk.

Nucor minimus, Lk.

Sphaeria mucosa, P.

Torula herbarum, Lk.

\{ Fusarium ciliatum, Lk.

\{Volutella ciliata, Fr.

Echinocystis lobata, Torr. S Gr.

Cercospora Echinocystis, Ell. \& Martin.

Septoria Sicyi, Pk.

Lagenaria vulgaris, Ser.

Fusarium aurantiacum, Cda.

$\{$ Fusisporium Lagenariae, $\mathrm{S}$.

Fusarium Lagenariae, Sacc.

Gloeosporium lagenarium, (Pass.) Sacc. \& Roum.

Sphaeria Cucurbitacearum, Fr.p. p.

$\{$ Sphaerella Cucurbitacearum, Cke.

Laestadia Cucurbitacearum, Sacc.

Nucor Cucurbitarum, B. \& C.

Sectria perpusilla, B. \& C.

$\{$ Nectria Peponum, B. \& C.

Dialonectria Peponum, Cke.

f Sphaeria Cucurbitacearum, Fr. p. p.

\{ Phoma Cucurbitacearum, M. A. Curtis.

Septoria vestita, B. \& C.

Torula Cucurbitarum, S.

Vermicularia Cucurbitae, Cke.

Megarrhiza Californica, Torr.

(Splraeria (Didymella) Megarrhizae, Cke. \& Hark.

(Didymella Cookeana, Berl. \& Vogl.

Didymella Megarrhizae, Cke. \& Hark.

Gibberella pulicaris, (Fr.) Sacc.

Hymenula Megarrhizae, Cke. \& Hark.

Stictis Megarhizae, Phil. \& Hark.

Vermicularia rectispora, Cke.

Megarrhiza Oregana, Torr.

Vermicularia rectispora, Cke.

Sicyos angulatus, L.

\& $\{$ Peronospora australis, Speg.

$\phi\{$ Peronospora sicyicola, Trelease.

Septoria Sicyi, Pk.

Spharella sicyicola, Ell. \& Ev.

Cucurbitaceae, sp. indet.

Asterina Wrightii, B. \& C.

Chondrom yces crocatus, B. \& C.

Sporocybe byssoides, (P.) Fr. 


\section{CACTACEAE.}

Cactus, sp. indet.

Asterina Wrightii, B. \& C.

Cereus giganteus, Engelm.

\{llendersonia Cerei, Pk.

\{ Camarosporium Cerei, Sacc.

Echinocactus, sp. indet.

Naemaspora pruinosa, B. \& C.

Opuntia Brasiliensis, Haw.

Gloeosporium Opuntiae, Ell. \& Er.

Opuntia Engelmanni, Salm.

Sphaeria Cacti, S.

Opuntia Rafinesquii, Engelm.

Perisporium Wrightii, B. \& C.

Opuntia, sp. indet.

Gibbera pulicaris, $\mathrm{Fr}$.

Rhytisma Cacti, S.

Sphaeria Cacti, S.

Vermicularia Dematium, (P.) Fr.

\section{FICOIDEAE.}

Mesembrianthemum aequilaterale, Haworth.

Camarosporinm ellipticum, Che. \& Hark.

Mesembrianthemum, sp. indet.

Gibberella Saubinetii, (Mont.) Sacc.

Torula herbarnm, Lk.

UMBELLIFERAE.

Angelica Breweri, Gray.

Phyllosticta Angelicae, Sacc.

Angelica genuflexa, Nutt

(Puccinia Pimpinellae, (Stranss) Lk:

$\{$ Puccinia Angelicae, Ell. \& Ev.

Angelica Iucida, L.

Sphaeria Angelicae-lucidae, S.

Capillaria caulincola, (P.) Lk.

Angelica, sp. indet.

Sphaeria chloromela, Fr.

Apium graveolens, L.

Cercospora Apii, Fres.

Archangelica atropurpurea, Hofim.

Dothidea stipata, $\mathrm{Fr}$.

Cladosporium depressum, B. \& Br.

Passalora depressa, Sacc.

Fusicladium depressum, Sacc.

S Puccinia bullata, (P.) Schrt.

Puccinia Umbelliferarum, DC.

Archangelica, sp. indet.

\{ Gloeosporium Angelicae, Cke.

Septogloem Angelicae, Sacc.

Chaerophyllum procumbens, Crantz.

(Puccinia Pimpinellae, (Stranss) Lk.

Caeoma (Uredo) Chaerophylli, S.

Puccinia Myrrhis, S.
Cicuta maculata, L.

Aecidium Pimpinellae, Kirchner.

Helminthosporinm interseminatum, B. \& Rav.

Peziza cyathoidea, Bill.

Septoria Sii, Rob.

Cryptotaenia Canadensis, DC.

$\int$ Puccinia enormis, Fckl.

$\{$ Puccinia Cryptotaeniae, Pk.

Precinia Astrantiae, B. \& C.

Septoria Cryptotaeniae, Ell. \& Rau.

Sphaeria Myrrhis. S.

Daucus Carota, L.

\{ Sphaeria comatella, C. \& E.

$\{$ Leptosphaeria comatella, Sacc.

Daucus, sp. indet.

\{ Sphaeria herbarum, P.

$\{$ Pleospora herbarum, Rabh.

Eryngium yuccaefolium, Michx.

Cylindrosporium Eryngii, E1l. \& Kell.

Ferula dissoluta, Watson.

Puccinia asperior, Ell. \& Ev.

Ferula multifida, Gray.

(Puccinia Pimpinellae, (Strauss) Lk.

$\{$ Puccinia Jonesii, $\overrightarrow{P k}$.

Foeniculum vulgare, Gaert.

Agaricus cyphellaeformis, B.

Cyphella Capula, (Holmsk.) Fr.

$\{$ Pleospora brunnea, Cke.

$\{$ Metasphaeria brunnea, Sacc.

Heraclemm lanatum, Michx.

Cylindrosporium Heraclei, EIl. \& Ev.

Diaporthe Arctii, (Lasch) Nits.

f Sphaeria rubella, $\mathrm{P}$.

OOphiobolus porphyrogonus, (Tode) Sacc.

Phyllachora Heracle1, (Fr.) Fckl.

Ramularia Heraclei, (Ond.) Sacc.

Septoria Heraclei, (Lib.) Desm.

Hydrocotyle Americana, L.

Septoria Hydrocotyles, Desm.

Hydrocotyle interrupta, Muhl.

Cercospora Hydrocotyles, Ell. \& Er.

Hydrocotyłe prolifera, Kellogg.

( Uredo Hydrocotyles, (Lk.) Mont.

Trichobasis Hydrocotyles, Cke.

Hydrocotyle repanda, $\mathrm{P}$.

Septoria Hlydrocotyles, Desm.

Hydrocotyle nmbellata, L.

Uredo Hydrocotyles, (Lk.) Mont.

Ligusticum Scoticum, L.

Aecidium Ligustici, Ell. \& Ev.

Oenanthe Californica, Watson.

Triphragmium echinatum, Lev.

Osmorrliza brevistylis, DC.

$\{$ Aecidium Pimpinellae, Kirchner.

\{ Aecidium Osmorthizae, Pk. 
(Puccinia Pimpinellac, (Strauss) Lk.

Cateoma (Uredo) Chaerophylli, S.

Cacoma (Uredo) Chelidonii, S.

Puccinia Myrrhis, S.

Puccinia Bupleuri, Auct. Amer.

Puccinia Osmorrhizac, C. \& P.

Osmorrhiza longistylis, DC.

Aecidium Pimpinellae, Kirchner.

Puccinia Pimpinellae, (Strauss) Lk.

$\{$ Puccinia Myrthis, $S$.

Puccinia Osmorrhizae, C. \& P.

Septoria Aegopodii, Desm.

Osmorrhiza nuda, Torr.

Puccinia Pimpinellae, (Strauss) Lk.

osmorrhiza, sp. indet.

$\{$ Sphaeria Dematium, P.

$\{$ Vermicularia Dematium, Fr.

Pastinaca sativa, L.

Capillaria caulincola, (P.) Lk.

Cercospora Apii, Fres., var. Pastinacae, Farl.

Diaporthe Arctii, Nits.

$\{$ Peziza pallescens, P.

\{Helotium pallescens, Fr.

$\{$ Peziza Pastinacae, S.

\{ Helotium pastinacum, Cke.

S Sphaeria Patella, (Tode) P.

Heterosphaeria Patella, Grcr.

$\{$ Sphaeria variabilis, $S$.

$\{$ Lophiostoma variabile, Sacc.

Peziza relicina, Fr.

Septoria pastinacina, Sacc.

Sphaeria chloromela, Fr.

Splueria Pastinacae, S.

Stictis Umbellatarum, S.

Stilbum carcinopthalmum, B. \& C.

Peucedanum simplex, Nutt.

Peucedanum Suksdorfii, Watson.

$\{$ Puccinia Pimpinellae, (Strauss; Lk.

$\{$ Puccinia Jonesii, Pk.

Pimpinella integerrima, Benth. \& Hook.

Cercospora platyspora, Ell. \& Holw.

$\{$ Passalora depressa, (B. \& Br.) Sacc.

$\{$ Fusicladium depressum, Sacc.

Fusicladium punctiforme, Wint.

?Puccinia Umbelliferarum, DC.

Polytaenia Nuttallii, DC.

Cercospora Polytaeniae, Ell. \& Kell.

Sanicula Canadensis, L.

Puccinia Saniculae, Grev.

Sanicula Marylandica, L.

Entyloma Saniculae, Pk.

Puccinia Saniculae, Grev.

Septoria Saniculae, Ell. \& Ev.

$\checkmark$ Uromyces pluriannulatus, B. \& C.

Synchytrium pluriannulatum, Farl.

Sanicula Menziesii, Hook. \& Arn.

Belonidium fuscum, Phil. \& Hark.
, Puccinia Saniculae, Hark.

Synchytrium pluriannulatum, Farl.

Sanicula, sp. indet.

Aecidium Saniculac, Carm.

\{ Puccinia bullata, (P.) Schrt.

Puccinia Bullaria, Lk.

Sium cicutaefolium, Gmel.

Accidium Sii-latifolii, (Fiedler) Wint.

Thaspium trifoliatum, Gray., var. apteraun, Gray.

Cercospora Ziziae, Ell. \& Ev.

Umbellifierae, sp. indet.

Dermosporium decolorans, S.

\{ Sphacria curirostra, Sow.

\{ Gnomonia? curvirostra, Sacc.

Helminthosporium delicatulum, B. \& Br.

tPeronospora niven, (Ung.) DBy.

Peziza Campanula, Nees.

Peziza extricata, B. \& C.

Splacria ampliata, S.

Sphaeria Umbellatarum, S.

Torula abbreviata, Cda.

Vermicularia hypodermia, (Lk.) S.

\section{ARALIACEAE.}

Aralia Californica, Watson.

Sphaerclla Araliae, Cke. \& Hark.

Sphaeria commanipula, B. \& Br.?

Araiia nudicaulis, L.

Triphragmium clavellosum, Berk.

Cercospora leptosperma, $\mathrm{Pk}$.

Aralia racemosa, L.

Acrospermum album, Pk.

Peziza cyathoidea, Bull.

Aralia spinosa, L.

$\{$ Melogramma Araliae, M. A. Curtis.

\{ Botryosphaeria Araliae, Cke.

Discosia artocreas, (Tode) $\mathrm{Fr}$.

Fusarium nitidum, B. \& C.

Fusarium roseum, Lk.

$\{$ Helicoma Berkeleyi, M. A. Curtis.

\{Helicosporium Berkeleyi, Sacc.

Hysterium variegatum, B. \& C.

Irpex lacteus, Fr.

Pestalozzia brevipes, Cke.

Phoma melaleucum, B. \& C.

f Dothidea nodicola, S.

\{ Phyllachora nodicola, Sacc.

Vermicularia maculans, Lk.

Aralia, sp. indet.

Leptostroma vulgare, Fr.

Peziza relicina, Fr.

Peziza setigera, Phil.

Tubercularia lutescens, Lk. 


\section{Hedera Helix, L.}

, Cladosporium brunneum, Cke. \& Ilark.

\{ Clidosporium brunneolum, Sacc.

Depazea hederaecola. Fr.?

Jiplodia IJederae, Desin.

Diplodia vulgaris, Ler.

S Sphacria hederaefolia, Cke.

Alasphaeria hederaefolia, Sacc.

Metasphaeria helicicola, (D)sm.) Sacc.

S Sphneria Eunotia, B. \& C.

\{ Physalospora Eunotia, Sacc.

Trochila Craterium, Fr.

Vermicularia trichella, Fr.

\section{CORNACEAE.}

\section{Cornus alternifolia, L. f.}

(Sphaeria callista, B. \& C.

Cucurbitaria callista, B. \& C.

Chilonectria callista, Pk.

Fracchiaea callista, Sacc.

- Nicrosphaera pulchra, C. \& P.

$\approx$ Microsphaera Alni, (DC.) Wint.

Myxosporium nitidum, B. \& C.

Septoria cornicola, Desm.

Sphaeria Pezizula, B. \& C.

f Sphaeronema aurantiacum, Pk.

$\left\{\begin{array}{l}\text { Sythia aurantiaca, Sacc. } \\ \text { Zyta }\end{array}\right.$

Cornus asperifolia, Michx.

Septoria cornicola, Desm.

\section{Cornus Canadensis, L.}

Glomerularia Corni, Pk.

Physarum luteolum, lk.

$\{$ Puccinia acuminata, $\mathrm{Pk}$.

$\left\{\begin{array}{l}\text { Puccinia porplyyrogenita, M. A. Curtis. } \\ \text { Pus }\end{array}\right.$

Cornus circinata, Liller.

$\{$ Sphaeropsis cornina, Pk.

\{ Phoma cornina, Pk.

Venturia Clintonii, Pk.

\section{Cornus florida, L.}

Corticium cinereum. Fr.

$\{$ Fusisporium miniatum, B. \& C.

\{ Fusarium miniatuin, Sacc.

$\{$ Helicoma Berkeleyi, M. A, Curtis.

$\{$ Helicosporium Berkeleyi, Sacc.

Helminthosporium apiculatum, Cia.

Ielminthosporium macrocarpon, Grer.

\{ Lichenopsis sphaeroboloidea, S.

$\{$ Stictis sphacroboloidea, $\mathrm{B}$.

$\{$ Perichaena populina, $\mathrm{Fr}$.

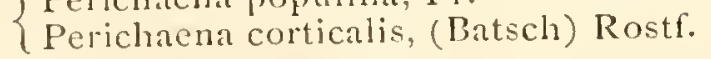

Peziza exidieila, B. \& C.

Peziza fascicularis, A. \& S.

Peziza miltophthalma, B. \& C.

Peziza roseoalba, S.

- P Phyllactinia guttata, Lev.

- Phyllactina sufulta, (Reb.) Sacc.

\{ Sphatia (Depazea) cornicola, (DC.) Fr.

$\{$ Sieptoria cornicola, Desm.
Septoria Corni-maris, Sacc.

Septosporium fuliginosum, Ell.

S Peziza pruinata, S.

\{Solenia poriaeformis, (DC.) Fckl.

Sphaeronema metulacforme, $B$.

Thelephora alhomarginata, S.

Tremellat virens, $\mathrm{S}$.

\section{PYRENOMYCETES.}

\{ Sphaeria exilis, A. \& S.

$\{$ Coelosphaeria exilis, Sacc.

$\{$ Sphacria atropurpurea, Fr.

Ilypoxylon atropurpureum, Fr.

Hypoxylon perforatum, Fr.

f Spluatia xestothele, B. \& C.

$\{$ Lasiosphaeria xestothelc, Sacc.

Sphaeria olivaespora, B. \& C.

$\{$ Leptosphateria olivaespora, Sacc.

Sphaeria Aquila, Fr.

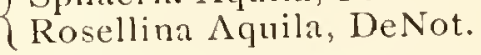

f Sphaeria Desmazierii, B. \& Br.

\{Rosellinia Desmazierii, Sacc.

\{ Splaeria Corni, S.

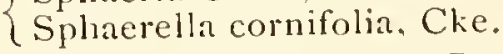

Sphaeria punctiformis, P.

Sphaerella punctiformis, $\mathrm{Fr}$.

\{ Sphaeria scutellata, P.

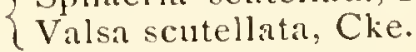

Cornus paniculata, L'Her.

Dimerosporium pulchrum, Sacc.

$\{$ Sphatria nidulans, $S$.

$\{$ Meliola nidulans, Cke.

Phyllosticta cornicola, Rabh.

Septoria cornicola, Desm.

Valsa cornina, Pk.

Cormus sericea, L.

Cytospora chloroglaea, B. \& C.

Dimerosporium pulchrum, Sacc.

\{ Iysterium flexuosum, S.

Alystcrographium flexuosum, Sacc.

Pestalozzia cornifolia, Ell. \& Ev.

Phyllosticta comicola, Rabh.

Septoria cornicola, Desm.

Stictis radiata, $P$.

Cornus stolonifera, Michs.

Iymenula hysterioides, Pk.

- Microsphaera Alni, (DC.) Wint.

Septoria cornicola, Desm.

Cornus Suecica, L.

Dimerosporium pulchrum, Sacc.

Patellaria applanata, B. \& C.

Patcllaria rhabarbarina, $B$.

Cornus, sp. indet.

\{ Corticium ceraceum, B. \& Rav.

$\{$ Corticium molle, 13. \& C.

Corticium incarnatum. Fr.

f Bacdalea confragosa, $P$.

Lenzites Cookeii, B. 
D.redilea torrugata, Klotzsch.

$\{$ I,en ites corrugatal, B. \& $\mathrm{C}$.

Dermatea Corni, Phil. \& Hark.

Dermatea flavocinerea, Phil.

fDiatrype aspera, Fr.

Diatrypella aspera, Nits.

- Erysiple tortilis, (IVallr.) Fr.

Fusarium Berkelei, Mont.

Ifelotium propinquum, Sacc. \& Ell.

Lichenopsis spluaroboloidea, S.

(Dothidea subcuticularis. S.

$\{$ Phyllachora subcuticularis, Sacc.

(Asteroma sp.? Cke.

Rhytisma maximum, $\mathrm{Fr}$.

flendersonia pallida, B. \& C.

$\{$ Stagonopsis pallida, Sacc.

Stictis Stigma, C. \& E.

Thelephora pedicellata, S.

Valsa munda, B. \& C.

Valsa scutellata, (P.) Cke.

f Valsa atomaespora, Cke.

Eutypella atomaespora, Sacc.

\section{Garrya elliptica, Dougl}

Aulographum lucens, Hark.

Cercospora Garryae, Hark.

Cercospora glomerata, Hark.

Ceuthospora foliicola, (Lib.) Cke.

Peziza tautilla, Phil. \& Hark.

Phillipsiella purpurea, Phil. \& Hark.

Phyllosticta Garryae, Cke. \& Hark.

Septosporium velutinum, C. \& E.

Sphaeria (Thyridium) Garryae, Cke. \& Hark.

Garrya, sp. indet.

Meliola amphitricha, $\mathrm{Fr}$.

Nyssa capitata, Walt.

Plyyllosticta Nyssae, Cke.

Nyssa muitiflora, Wang.

Asterina erysiphoides, B. \& C.

S Sphaeronema hispidulum, Ell.

$\{$ Cornularia hispidula, Sacc.

Diatrype asterostoma, B. \& C.

Diatrype Duriaei, Mont.

Sacodium ramosum, S.

Glenospora Curtisii, B. \& Desm.

Glenospora ramorum, B. \& C.

\{ Isothea Nyssae, B. \& C.

$\{$ Hypospila Nyssae, Che.

f Lophiostoma minus, Ell.

\{ Lophidium minus, Sacc.

Lophiostoma subcollapsum, Ell. \& Ev.

Peziza nyssaegena, Ell.

Sphaeria thuridonta, C. \& E.

Pleospora thuridonta, Sacc.

Sphaerella nyssaecola, Cke.

Stereun strictum, Fr.

Nyssa, sp. indet.

Agyrium sexdecimsporum, Fckl
Clavaria mucida, P.

Comatricha typhina, Rostf.

Cryptosporium Nyssae, C. $\mathbb{E}$ E.

Epicoccum scabrum, Cda.

Glonium lineare, (Fr.) DeNot.

\{ IIendersonia delicatula, C. S E.

\{ Stagonospora delicatula, Sacc.

Hyalopus griseus, B. S C.

Lichenopsis sphateroboloiden, S.

Merulius Corium, Fr.

f Oidinm megalospormm, B. \& C.

\{ Monilia megalospora, Sacc.

\{ Tremella nucleata, s.

$\{$ Namatelia nucleati, Fr.

?Panus alliaceus, B. \& C.

Polyporus delectans, Pk.

Radulum orbiculare, $\mathrm{Fr}$.

Radulum spinulosum, B. \& C.

Rhizoctonia moniliformis, Ell. \& Ev.

Sphaeropsis fibriseda, C. \& E.

\section{PYRENOMYCETES.}

f Dothidea geographica, (DC.) Fr.

Asteroma geographicum, Desm.

Sphaeria Avocetta, C. \& E.

Ceratostoma Arocetta, Sacc.

Sphaeria nyssaecola, B. \& C.

\{ Ceratostomella uyssaecola, Sacc.

(Nectria sanguinea, (Sibth.) Fr.

$\{$ Dialonectria sanguinea, Che.

$\{$ Valsa paulula, C. \& E.

\{ Diaporthe paulula, Sacc.

\{ Diatrypella olivacea, C. \& E.

\{ Diatrype olivacea, Cike.

$\{$ Diatrype subfulva, $B . \& C$.

$\{$ Diatrypella subfulva, Sacc.

\{ Sphaeria segna, C. \& E.

\{Didymella segna, Sacc.

Hypocrea lenta, (Tode) B.

Hypoxylon botryosum, (Fr.) B.

Hypoxylon cohaerens, (P.) Fr.

Hysterium stictoidemm, C. \& E.

I Sphaeria orthotricha, B. C.

\{ Lasiosphaeria orthotricha, Sacc.

Myriococcum Everhartii, Sacc. \& Ell.

Nectria Cucurbitula, (Tode) Fr.

Patellaria ligniota, Fr.

Peziza rubella, P.

Phlebia merismoides, Fr.

Phoma nyssaecarpa, Cke.

Sphaerella maculiformis. (P.) Awd.

Valsa ambiens, (P.) Fr.

Valsa Nyssae. Cke.

Valsa praestans, B. \& C.

Valsa salicina, (P.) Fr.?

Valsa apatosa, C. \& E.

$\{$ Valsaria apatosa, Sacc.

Pseudovalsa apatosa. Cke. 

Magnolia, sp. indet.

\section{PYRENOMYCETES.}

Cucurbitaria congesta, C. \& E.

$\{$ Dothidea Magnoliae, Cke.

\{ Homostegia Magnoliae, Sacc.

Dothidea scutula, B3. \& C.

Hypocrea minima, Sacc. \& Ell.

Iypoxylon annulatum. (S.) Mont., var. b.

Hypoxylon tinctor, (B.) Cke.

Lasiosphaeria subvelutina, Ell. \& Ev.

?Lophodermium exaridum, C. \& P.

$\{$ Hysterium foliicolum, Fr.

\{Lophodermium hysterioides, (P.) Sacc.

\{ Hysterium maculare, Fr.

Lophodermium maculare, DcNot.

Nectria Russellii, B. \& C.

Sphaerella granulata, Cke.

Xylaria corniformis, Fr.

Xylaria filiformis, (A. \& S.) Fr.

\section{SPHAEROPSIDEAE.}

Diplodia Ravenelii, Cke.

$\{$ Discosia alnea, (P.) B.

Discosia nitida, Lev.

(Phoma atomispora, Cke.

$\{$ Diplodia tephrospora, M. A. Curtis.

Sphaeropsis tephrospora, B. \& C.

\section{MELANCONIEAE.}

Melanconium ramulorum, Cda.

Naemaspora rufa, B. \& C.

\section{HYPIIOMYCETES.}

Acrothecium obovatum, C. \& E.

$\{$ Polyactis curta, B. \& C.

Botrytis curta, Sacc.

Dendrodochium rubellum, Sacc., var. macrosportum, Sacc.

Glenospora Curtisii, B. \& Desm.
Haplaria chlorina, Ell. \& Ev.

\{ Helicoma velutinum, EH.

\{Helicosporium velutinum, Sacc.

$\{$ Illosporium acaroides, Sacc.

$\{$ Volutellaria acaroides, Sacc.

?Illosporium Pezizula, Ell. \& Sacc.

Menispora glanconigra, C. \& E.

Ovularia monilioides, Ell. \& Martin.

Penicillium glaucum, Lk.

Penicillium repens, C. \& E.

Polyscytalum sericeum, Sacc.

Stachylidium fuscum, C. \& E.

Verticillium candelabrum, Bon.

Virgaria globiger, Sacc. \& Ell.

Zygodesmus laevisporus, Cke.

Zygodesmus pannosus, B. \& C.

DISCOMYCETES.

Chlorosplenium aeruginosum, (P.) DeNot.

Dermatea Magnoliac, B. \& C.

Peziza diaphanula, Cke.

Peziza fumigata, Ell. \& Ev.

Peziza Glenospora, Ell. \& Ev.

Peziza gracilipes, Cke.

Peziza incondita, Ell.

Peziza phlegmacea, Ell.

\section{IIYMENOMYCETES.}

Agaricus (Collybia) conigenoides, Ell.

Corticium laeve, Fr.

Hymenochate corticolor, B. \& Rav.

Hypochnus rubrocinctus, Ehr.

Irpex sinuosus, Fr.

Kneiffia setigera, Fr.

Polyporus adustus, Fr.

Polyporus cinnabarinus, Fr.

Polyporus hemileucus, B. \& C.

Polyporus spumeus, Fr.

Polyporus velutinus, Fr.

\section{ERRATA.}

Page I, left, I3th line from bottom, for Peronspora read Peronospora.

" I, right, bottom line, for Leptostrmoa read Leptostroma.

"3, right, $24_{\text {th }}$ line from bottom, for panciseptata read pauciseptata.

" 5 , left, bottom line, for melalencum read melaleucum.

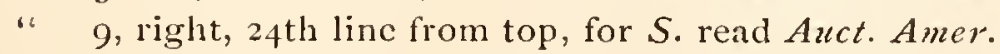

"II, left, $5^{\text {th }}$ line from bottom, for citispora read citrispora.

" I I, right, 24 th and 25 th lines from top, for Stemonites read Stemonitis.

" 15, right, 25th line from bottom, for Spharonema read Sphaeronema.

" 16, left, 26th line from top, for Peronspora read Peronospora.

“ 17, left, 27 th line from bottom, for Rehm read Sacc.

" 19, left, 32 dine from bottom, for Rehm read Sacc.

"33, right, i $4_{\text {th }}$ line from top, for geograpica read geographicum.

"34, left, ISth line from top, for $P$. read $S$.

"34, right, I4th line from top, for Rehm read Sacc. 



\section{A PROVISIONAL HOST-INDEX}

OF THE

Fungi of the United STATES

W. G. FARLOW AND A. B. SEYMOUR

PAR'T II.

GAMOPETALAE-APETALAE

CAMBRIDGE, SEPTEMBER, ISgo. 

Although it was originally intended that the second part of the Host Index should include everything after Polypetalae, it has seemed best to issue a second part including only Gamopetalae and Apetalae as those divisions cover an unexpectedly large number of pages. The remainder of the work will appear in November and the authors request that botanists having errors or omissions to report in the parts already issued would kindly inform them regarding such errors and omissions at an early date in order that the corrections and additions may be inserted in the forthcoming third part. It should be borne in mind that unpublished hosts are not included in the present Index. Also that in the case of some very common fungi occurring on many different hosts it has not been found practicable to include all known hosts unless the species are of economical interest. Since most Myxomycetes can hardly be said to be parasitic on particular species of Phaenogams, in the present part, the Myxomycetes have, as a rule, been omitted except where their rarity or some special peculiarity warranted referring to them in connection with certain hosts.

W. G. FARLOW.

Cambridge, Mass. 



\section{CAPRIFOLIACEAE.}

\section{Adoxa Moschatellina, L.}

Aecidium albescens, Grev.

Synchytrium anomalum, Schrt.

Diervilla trifida, Moench.

\{ Hysterium rimicolum, S.

\{Dothidea rimicola, $\mathrm{Pk}$.

Radulum? investiens, S.

Ramularia Diervillae, $\mathrm{Pk}$.

Septoria Diervillae, Eli. \& Ev.

$\{$ Septoria Diervillae, $\mathrm{Pk}$.

Septoria diervillicola, Berl. \& Vogl.

Tympanis turbinata, $\mathrm{S}$.

Linnaea borealis, Gronov.

$\{$ Dothidea Vittrockii, Erikss.

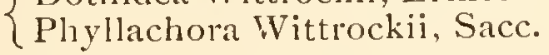

Sphaerella minor, Karst.

Venturia Dickiei, (B. \& Br.) Ces. \& DeNot.

Lonicera Caprifolium, L.

Diplodia Lonicerae, Fckl.

I Iormococcus nitidulus. Sacc.

Lonicera ciliata, Muhl.

Glomerularia Corni, Pk.

Phyllosticta Lonicerae, Desm.

Iıonicera conjugalis, Kellogg.

\{ Marsonia Lonicerae, Hark.

\{Gloeosporium Lonicerae, Ell. \& Ev.

Lonicera tlava, Sims.

Aecidium Periclymeni, Schum.

Cercospora antipus, E11. \& Holw.

Hendersonia Mariae, Clinton.

Hendersonia Peckii, Clintun.

$\{$ Microsphaera pulchra, C. \& P.

$\{$ Microsphaera Alni, (DC.) Wint.

Phoma Mariae, Clinton.

$\{$ Sphaeropsis Wilsoni, Clinton.

Sphaeropsis puncta, C. \& E.

Lonicera glanca, Hill.

\{ Microsphaera pulchra, C. \& E.

\{Microsphaera Alni, (DC.) Wint.

Microsphaera Dubyi, Lev.

Lonicsra hirsuta, Eaton.

Microsphaera Dubyi, Lev.

Lonicera hispidula, Dougl.

Lasiobotrys affinis, Hark.
Phoma Xylostei, Cke. \& Hark.

$\{$ Pleospora vitrispora, Cke. \& Hark.

$\{$ Peltosphaeria vitrispora, Berlese.

Lonicera involucrata, Banks.

Diaporthe cryptica, Nits.

Eutypa lata, (P.) Tul.

$\{$ Hysterium Lonicerae, Phil. \& Hark.

$\{$ Gloniopsis Lonicerae. Berl. \& Vogl.

Libertella Lonicerae, Cke. \& Hark.

f Sphaeria anisometra, Cke. \& Hark.

(Netasphaeria anisometra, Sacc.

Lonicera Japonica, Thunb.

Diatrypella ramularis, Ell. \& Ev.

Lonicera sempervirens, L.

Dothidea Lonicerae, Cke.

$\{$ Microsphaera penicillata, Lev.

\{ Microsphaera Alni, (DC.) Wint.

Sphaeria Lonicerae, Sorv.

r Sphaeria umbilicata, P.

(Valsa umbilicata, Fr.

Lonicera Sullivantii, Gray.

Aecidium Periclymeni, Schum.

Lonicera Tartarica, L.

Phoma Mariae, Clinton.

Lonicera, sp. indet.

Diatrypella pustulata, Ell. \& Ev.

(Diplodia quisquiliarum, B. \& C.

\{Diplodia paupercula, B. \& Br.

Dothidea ribesina, (P.) Fr.

Dothidea tetraspora, B. S Br.

Hypocrea subcarnea, Ell. \& Ev.

Lasiobotrys Lonicerae, Kze.

Lichenopsis sphaeroboloidea, S.

\{ Sphaeria aulica, C \& E.

$\{$ Metasphaeria aulica, Sacc.

$\{$ Pestalozzia concentrica, B. \& Rav.

\{ Pestalozzia rostrata, Zabriskie.

Peziza atrata, P.

Phoma Lonicerae, Cke.

$\{$ Sphaeria erratica, C. \& E.

$\{$ Physalospora erratica, Sacc.

\{ Pleospora vitrispora, Cke. \& Hark.

$\{$ Peltosphaeria vitrispora, Berlese.

Phyllosticta vulgaris, Desm.

\{ Septoria decipiens, B. \& C.

$\{$ Rhabdospora decipiens Sacc. 
Cryptosporium Lonicerae, C. \& E.

$\{$ Rhabdospora Lonicerae, Sacc.

Sphaeria (Leptosphaeria) dumetorum,

(Niessl) C. \& E.

Sphaeria saepincola, Fr.

Stictis annulata, Cke. \& Phil.

\section{Sambucus Canazensis, L.}

\{ Aecidium Sambuci, S

Caeoma (Aecidium) sambuciatum, S.

Cercospora depazeoides, (Desm.) Sacc.

$\{$ Cercospora sambucina, Ell. \& Kell.

Cercospora lateritia, Ell. \& Halsted.

\{ Corticium Sambuci, P.

\{Hypochnus Sambuci, Sacc. Syll.

S Splaeria sambucivora, S.

$\{$ Diatrype sambucivora, Cke.

Dinemasporium Pezizula, B.

Diplodia paupercula, B. \& Br.

\{ Sphaeria Sambuci, P.

Sothidea Sambuci, Fr.

\{ Hendersonia microspora, B. \& C.

\{ Stagonospora microspora, Sacc.

S Hysterium commune, Fr.

$\{$ Hypoderma commune, Duby.

Hysterium Sambuci, Schum.

Melanconium truncatum, S.

Sicrosphaera Iledwigii, Lev.

Microsphaera Van Bruntiana. Gerard.

Microsphaera Alni. (DC.) Wint.

Microsphaera Grossulariae, Lev.

Ramularia sambucina, Pk.

Septoria sambucina, Pk.

Sphaeropsis macrospora, B. \& C.

$\{$ Dothiorella macrospora, Sact.

Sphaeropsis Sambuci, Pk.

Sphaerulina sambucina, Pk.

Tubercularia nigricans. (Bull.) DC.

Valsa pulviniceps. Pk.

Sambucus glauca, Nutt.

Hendersonia diplodioices, Ell. \& Ev.

Ophiobolus byssicola, Hark.

Phoma Asteriscus, B.

Phoma sambucina, Cke.

Rosellinia (Coniochaeta) rhyncospora, Hark.

\section{Sambucus nigra, L.}

Cercospora depazeoides, (Desm.) Sacc.

(Macroplodia sambucina, Cke.

Sphaeropsis sambucina, Sacc.

Sambucus racemosa, L.

Cercospora lateritia, El1. \& Halsted.

Dinemasporium Pezizula, B.

Excipula strigosa, Fr.

Exidia recisa, $F r$.

\{Hendersonia Sambuci, Pk.

\{Hendersonia pubens, Cke.

Irpex tabacinus, B. \& C.

Lophiostoma congregatum, Hark.
Nectria cinnabarina, (Tode) Fr.

Rosellinia ambigua, Sacc.

Septoria sambucina, Pk.

Sphaeria distincta, S.

Sambucus, sp. indet.

Cenangium acutum, (Schum.) Fr.

?Ceratosphaeria microdoma, Ell. \& Ev.

S Sphaeria corrata, Tode.

Chaetomium comatum, Fr.

Clasterosporium strepsiceras, (Ces.) Sacc.

Corticium sulfureum, Fr., var. ochroideum,

Hark. \& Moore.

Cystotricha stenospora, B. \& C.

Sphaeria spiculosa, A. \& S.

$\{$ Diaporthe spiculosa, Nits.

Sphaeria epidermidis, Fr.

Didymosphaeria epidermidis, Fckl.

\{ Peziza hispidula, Schrad.

$\{$ Dinemasporium hispidulum, M. A. Curtis.

Diplodia sambucina, Sacc.

f Valsa stellulata, Fr.

$\{$ Eutypella stellulata, Sacc。

\{ Sphaeria pulicaris, Fr.

\{Gibberella pulicaris, Sacc.

? Hendersonia caespitosa, B. \& C.

Stagonospora caespitosa, Sacc.

Sphaeria gelatinosa, Tode.

$\{$ Hypocrea gelatinosa, Fr.

Sphaeria diminuens, P.

Lophidium diminuens, Ces. \& DeNot.

Nectria cinnabarina, (Tode) Fr.

Nectria ochraceopallida, B. \& Br.

Peziza fusca, P.

Phoma exigua, Desm.

Phyllosticta Sambuci, Desm.

f Sphaeria Sambuci, Plow.

i Pleospora Sambuci, Sacc.

(Septoria maculans, B. \& C.

$\{$ Rhabdospora maculans, Sicc.

Sphaeria clandestina, Fr.

Sphaeria floccosa, Fr.

f Sphaeria myriocarpa, Fr.

\{ Psilosphaeria myriocarpa, Hark.

Sphaeria paetula, $\mathrm{Fr}$.

Sphaeria surculi, Fr.

Sphaeronema Sambuci, B. \& C.

$\{$ Dendrophoma Sambuci. Sacc.

Splateropsis mutica, B. \& Br.

?Sphaeropsis Pennsylvanica, B. \& C

Stereum purpureum, P.

$\{$ Sphaeria abnormis, Fr.

\{ Valsa abnormis, Fr.

\{Valsa sambucina, Pk.

Pse:udovalsa sambucina, Sacc.

Symphoricarpus mollis, Nutt.

Gloniopsis Miilleri. (Duby) Sacc.

Puccinia Symphoricarpi, Hark. 
Symphoricarpus oc̀cidentalis, Hook.

Aecidium abundans, $\mathrm{Pk}$.

Symphoricarpus oreophilus, Gray.

Aecidium abundans, $\mathrm{Pk}$.

Symphoricarpus racemosus, Michx.

Aecidium abundans, Pk.

Anthostomella perfidiosa, (DeNot.) Sacc.

Diplodia Symphoricarpi, Sacc.

Diplodia Thorniana, Gerard.

Microsphaera Symphoricarpi, Howe.

Phyllosticta Symphoricarpi, IVestd.

Septoria Symphoricarpi, Ell. \& Ev.

Sphaeria (Anthostoma?') gigaspora, Cke. \& Hark.

Thyridium cingulatum, (Mont.) Sacc.

Symphoricarpus vulgaris, Michx.

Aecidium abundans, $\mathrm{Pk}$.

Corticium incarnatum, (P.) Fr., forma consueta, Bresadola.

Microsphaera Symphoricarpi, Howe.

Symphoricarpus, sp. indet.

Microthyrinn microscopicum, Desm.

Triosteum perfoliatum, L.

Cladosporium Triostei, Pk.

Viburnum acerifolium, L.

Cercospora varia, $\mathrm{Pk}$.

(Microsphaera penicillata, I ev.

$\{$ Microsphaera Viburni. (S.) Howe.

(Microsphatera Alni, (DC.) Wint.

Viburnum dentatum, L.

Dothidea Viburni-dentati, S.

Iypoxylon notatum, B. \& C.

Sphaeropsis hyalina, B. \& C.

Phoma hyalina, Sacc.

(Macrophoma hyalina, Berl. \& Vogl.

$\{$ Massaria gigaspora, (Desm.) B.

\{ Massaria Corni, (Fr. \& Mont.) Sacc.

(Microsphaera sparsa, Howe.

$\{$ Microsphaera Viburni, (S.) Howe.

(Nicrosphiera Alni, (DC.) Wint.

S Sphaeria punicea, Schm.

Nectria punicea, Fr.

Sphaeria inclinata. S.

\{ Sphaeria subconcava, S.

\{ Nummularia subconcava, Ell. \& Ev.

Tympanis fasciculata, $\mathrm{S}$.

Valsa mesoleuca, B. \& C.

Valsa salicina, (P.) Fr.

Viburnum lantanoides, Michx.

$\{$ Peziza Viburni, S.

$\{$ Cenangium Viburni, $\mathrm{Fl}$.

$\{$ Dermatea minuta, $\mathrm{Pk}$.

Y Pezicula minuta, Pk.

Phyllosticta lantanoides, $\mathrm{Pk}$.

Sphaerographium lantanoides, $\mathrm{Pk}$.

Viburnum Lentago, L.

Cercospora varia, $\mathrm{Pk}$.

Coleosporium Viburni, Arthur.
$\{$ Cryptosporella Lentaginis, Rehm.

$\{$ Valsa Lentaginis, Ell. \& Ev.

Hendersonia Viburni, Ell.

\{ Massaria gigaspora, (Desm.) B.

\{Massaria Corni, (Fr. \& Mont.) Sacc.

Microsphaera Hedwigii, Lev.

Microsphaera penicillata, Lev.

Microsphaera sparsa, Howe.

Microsphaera Viburni, (S.) Howe.

Microsphaera Alni, (DC.) Wint.

Peziza Lentaginis, S.

Tympanis fasciculata, S.

Valsa ceratophora, Tul.

Viburnum nudum, L.

\{Sphaeronema hystricinum, Ell.

\{ Sphaerographium hystricinum, Sacc.

Viburnum Opulus, L.

Botryosphaeria Viburni, Cke.

Diplodia microspora, B. \& C.

$\{$ Hysterium variegatum, B. \& C.

\{Hypoderma variegatum, Duby.

Phoma mixtum, B. \& C.

Pistillaria elegans, B. \& C.

\{ Septoria interrupta, B. \& C.

Rhabdospora interrupta, Sacc.

Stictis glaucoma, B. \& C.

Stictis pupula, Fr.

Viburnum pauciflorum, Pylaie.

$\{$ Puccinia Linkii, Klotzsch.

$\{$ Puccinia Pringlei, Pk.

Viburnum plicatum, Thunb.

Cercospora tinea, Sacc.

Viburnum prunifolium, L.

S Sphaeria friabilis, $\mathrm{P}$.

Diatrype friabilis, M. A. Curtis.

\{ Sphaeria Viburni, S.

\{ Eutypa Viburni, Herb. Berk.

Hendersonia foliorum, Fckl, var. Viburni, Sacc.

\{ Massaria gigaspora, (Desm.) B.

\{ Massaria Corni, (Fr. \& Mont.) Sacc.

- Erysiphe Viburni, S.

Microsphaera Alni, (DC.) Wint.

Pestalozzia Jefferisii, Ell.

Polyporus conchatus, (P.) Fr.

Radulum molare, Fr.

Trametes sepium, B.

Tympanis turbinata, S.

S Sphaeria flabelliformis, S.

\{Xylaria flabelliforinis, Tul.

Viburnum pubescens, Pursh.

Microsphaera Alni, (DC.) Wint.

Viburnum Tinus, L.

Leptosphaeria Tini, Ell. \& Ev.

Viburnum, sp. indet.

Cenangium Viburni, Fr.

Corticium cinereum, $\mathrm{Fr}$.

$\{$ Sphaeria secreta, C. \& E.

\{ Cryptosphaeria secreta, Sacc. 
Dermatea carnea, C.. S E.

fDermatea purpurea, Ell.

\{ Dermatea viburnicola, Ell.

(Helmiuthosporium dubium, B. \& C.

? Helminthosporium Berkeleyı, Cke.

(Helminthosporium Beammontii, Sacc.

$\{$ Hydnum plumarium, B. S C.

Hydum conchiforme, Sacc.

$\{$ Dothidea frigoris, $\mathrm{S}$.

\{ Phyllachora frigoris, Sacc.

Sothidea subcuticularis, S.

? Phyllachora subcuticularis, Sacc.

Asteroma sp? Cke.

Sphateria elliptica, S.

\section{RUBIACEAE.}

\section{Cephalanthus occidentalis, L.}

Aecidium Cephalanthi, Seymour.

Ascochyta Cephalanthi, Ell. \& Ev.

$\{$ Peziza Cephalanthi, S.

$\{$ Cenangium Cephalanthi, $\mathrm{Fr}$.

Cercospora Cephalanthi, Ell. \& Kell.

Dendrophoma Cephalanthi, Pk.

Sphaeria Cephalanthi, S.

Diatrype Cephalanthi, B.

Diatrypella Cephalanthi, Sacc.

Epicoccum maculatum, Cke.

Lophiostoma prominens, $\mathrm{Pk}$.

Microsphaera semitosta, B. \& C.

Septoria Cephalanthi, Ell. \& Kell.

$\{$ Septoria verrucaeformis, B. \& C.

\{ $R$ habdospora verruciformis, Sacc.

Sphaerella maculiformis, (P.) Awd.

Valsa ceratophora, Tul.

Ciuchona, sp. indet.

Cercospora Cinchonae, Ell, \& Ev.

Diodia teres, Walt.

Aecidium Diodiae, Burrill.

Cercospura Diodeae, $\mathrm{Cke}$.

C Puccinia Spermacoces, S.

Caeoma Spermacoces, Lk.

Caeoma (Uredo) Spermacoces, S.

Uredo Spermacoces, Rav.

Uromyces Spermacoces, M. A. Curtis.

Galium Aparine, L.

Cercospora Galii, Ell. \& Holw.

Erysiphe Cichoracearum, DC.

Leptostroma clandestinum, S.

Mazzantia Galii, (Fr.) Mont.

Peronospora calotheca. D By.

S Puccinia Galii, S.

$\{$ Puccinia Galiorum, Lk.

Puccinia Galii, (P.) IVint.

Vermicularia scandentium, S.

Galium asprellum, Michx,

$\{$ Puccinia Galiorum, Lk.

\{ Puccinia Galii, (P.) Wint.

\section{Galium boreale, L.}

Peronospora calotheca, D By.

Peziza nigritella, Phil. \& Hark.

Phoma ellipticum, Pk.

$\{$ Puccinia Galiorum, Auct. Am. pp.

\{ Puccinia rubefaciens, Johans.

Galium Californicum, Hook \& Arn.

Puccinia Valantiae, P.

Galium concinnum, Torr. \& Gr.

Aecidium Galii, P.

$\{$ Puccinia Galiorum, Lk.

\{ Puccinia Galii, (P.) Wint.

Galium Nutuallii, Gray.

Puccinia Valantiae, P.

Galinm pilosum, Ait.

Cercospora Galii, Ell. \& Holw.

Puccinia Galii, S.

Septoria psilostega, Ell. \& Martin.

\section{Galium trifidum, L.}

Phacidium brunneolum, Pk.

Puccinia Galịorum, Lk.

Galium triflormm, Michx.

$\{$ Nectria Galii, Plow. \& Hark.

$\{$ Dialonectria Galii, Cke.

Peronospora calotheca, D By.

$\{$ Puccinia Galiorum, Lk.

\{ Puccinia Galii, (P.) Wint.

Galium, sp. indet.

$\{$ Hendersonia Galiorum, Cke. \& Hark.

\{ Camarosporium Galiorum, Sacc.

Dacryomyces epiphyllus, S.

Erysiphe communis, (Wallr.) Schlr.

\{ Septoria Galii, Ell

Septoria Galiorum, Ell.

\{ Xyloma herbartum, A. \& S.

P Phacidium repandum, $\mathrm{Fr}$.

Gardenia tilorida L.

Sphaerella Gardeniae, Cke.

Houstonia caerulea, L.

Houstonia purpurea, L.

Caeoma (Aecidium) houstoniatum, S.

Mitchella repens, L.

Meliolia Mitchellae, Cke.

Psychotria rufescens, Spr.

Pestalozzia maura, Ell. \& Ev.

Spermacoce glabra, Michx.

Puccinia lateritia, B. \& C.

$\{$ ?Uromyces Spermacoces, M. A. Curtis.

\{ Puccinia Spermacoces, B. \& C.

\section{VALERIANACEAE.}

Valeriana capitata, Pall.

$\{$ Excipula conglutinata, Ell. \& Ev.

\{ Excipulina conglutinata, Sacc.

Valeriana edulis, Nutt.

Aecidium Valerianearum, Duby. 


\section{DIPSACEAE.}

Dipsacus sylvestris, Mill.

Cercospora elongata, Pk.

Phoma oleracea, Sacc, var. Dipsaci, Sace.

Dipsacus, sp. indet.

Sphaerella asterinoides, Ell. \& Ev.

COMPOSITAE。

\section{Tubuliflorae.}

\section{Achillea Millefolium, L.}

$\{$ Dichomera Compositarum, Che. \& Hark.

Camarosporium Compositarum, Sacc.

Oidium erysiphoides, $\mathrm{Fr}$.

Actinella acaulis, Nutt.

Pleospora herbarum, (P.) Rabh.

CPuccinia variolans, Hark.

Puccinia Tanaceti.

var. Actinellae, Webber.

Actinomeris squarrosa, Nutt.

Cercospora anomala, Ell. \& Halsted.

$\{$ Erysiphe Cichoracearum, DC.

Erysiphe lamprocarpa, Lev. p. p.

Ambrosia artemisiaefolia, L.

$\{$ Cystopus cubicus, (Strauss) Lev。

\{Cystopus Tragopogonis, (P.) Schrt.

Entyloma Compositarum, Farl.

$\{$ Erysiphe Cichoracearum, DC.

Erysiphe lamprocarpa, Lev. p. p.

Oidium Ambrosiae, Thm.

$\{$ Peronospora Halstedii, Farl.

\{Plasmopara Halstedii, Berl.\& De Toni.

$\{$ Dothidea Ambrosiae, B. \& C.

$\{$ Pliyllachora Ambrosiae, Sacc.

Puccinia Xanthii, S., var. Ambrosiae, B. \& Rav.

Ambrosia psilostachya, DC.

Cystopus Tragopogonis, (P.) Schrt.

Entyloma Compositarum, Farl.

Erysiphe Cichoracearum, DC.

Plasmopara Halstedii, (Farl.) Berl. \& De Toni.

Puccinia Xanthii, S., var. Ambrosiae, B. \& Rav.

Ambrosia trifida, L.

Aecidium Compositarum, Mart.

Botrytis tephroidea, Sacc. \& Ell.

Cercospora ferruginea, Fekl.

Cercospora racemosa, Ell. \& Martin.

Cystopus Tragopogonis, (P.) Schrt.

Diaporthe Arctii, (Lasch) Nits.

Diatrypella herbacea, Ell. \& Fv.

Didymella prominens, E1l. \& Ev.

Entyloma Compositarum, Farl.
$\{$ Protomyces polysporus, Pk.

\{ Entyloma polysporum, Farl.

S Erysiphe Cichoracearum, DC.

\{rysiphe Ambrosiae, S.

Erysiphe lamprocarpa, Lev. p. p.

$\{$ Sphaeria fulgida, C. \& P.

Ophiobolus fulgidus, Sacc.

$\{$ Peronospora Halstedii, Farl.

\{ Plasmopara Halstedii, Berl. \& De Toni.

Peziza atrata, P.

Pyrenopeziźa atrata, Fckl.

Mollisia atrata, Karst.

Phlyctaena arcuata, B.

?Protomyces macrosporus, Ung.

Puccinia Xanthii, S., var. Anbrosiae, B. \& Rav.

Septoria bacilligera, Wint.

Vermicularia Dematium, (P.) Fr.

Ambrosia, sp. indet.

Leptostroma vulgare, $\mathrm{Fr}$.

Sphaeria Glomus, B. \& C.

$\{$ Ophiobolus? Glomus, Sacc.

Raphidospora Glomus, Cke.

$\{$ Sphaeria Anethi, P.

Phoma Anethi, Sacc.

Puccinia bullata, S.

Sphaeria nigrella, Fr.

\{ Didymella? nigrella, Sacc.

Antennaria âlpina, Gaertn.

Septoria cercosperma, Rostrup.

Sphaerélla confinis, Karst.

Antennaria plantaginifolia, Hook.

Phyllosticta Antennariae, Ell. \& Ev.

Aplopappus Bloomeri, Gray.

Lamproderma robustum, ElI. \& Ev.

Aplopappus ericoides, Hook. \& Arn.

Tremella moriformis, B.

Aplopappus integrifolius, T. C. Porter.

Pleospora herbarum, (P.) Rabh.

Aplopappus spinulosus, DC.

Puccinia variolans, Hark.

Arctium Lappa, L.

?) Sphaeria eriophora, Cke.

Leptosphaeria eriophora, Sacc.

Phyllosticta Lappae, Sacc.

Septoria Lapparum, Sacc.

Vermicularia Arctii, S.

Verticillium lateritium, (Ehrh.) Rabh.

Arnica alpina, Olin.

Pleospora herbarum, (P.) Rabh.

Arnica cordifolia, Hook.

Puccinia Arnicalis, Pk.

Arnica foliosa, Nutt.

Puccinia nuda, EIl. \& Ev. 
Artemisia Abrotanum, L.

Sphaeria Artemisiae, S.

Dothidea Artemisiae. B.

Nummularia Artemisiae, Sacc.

Artemisia Absinthium, L.

f Helminthosporium Absinthii, Pk.

\{ Cercospora Absinthii, Sacc.

Nidularia fascicularis, S.

Artemisia biennis, Willd.

Crstopus cubicus, (Strauss) Lcr.

Peronospora leptosperma, DBy.

Artemisia borealis, Pall.

S Sphaeria herbarum, P.

$\{$ Pleuspora herbarum, Rabh.

Pleospora vulgaris, Niessl.

Septoria cercosperma, Rostrup.

Artemisia Californica, Less.

f Sphaeria tuberculiformis, Ell.

$\{$ Plowrightia tuberculiformis, Sacc.

S Puccinia Tanaceti, DC.

Puccinia Artemisiarum, Schm. \& Kze.

Sphaeria (Montagnella) tumefaciens, Ell. \& Ilark.

Artemisia cana, Pursh.

Leptosphaeria Artemisiae, (Fckl.) Awd.

Puccinia Tanaceti, DC.

Artemisia Canalensis, Michx.

Puccinia Tanaceti, DC.

Artemisia discolor, Dongl.

Aecidium Compositarum, Mart.

Erysiphe Cichoracearum, DC.

Artemisia dracunculoides, Pursh.

Aecidium Compositurum, Mart.

Erysiphe Cichoracearum, DC.

Erysiphe Linkii, Lev.

Puccinia Tanaceti, DC.

Artemisia frigida, Willd.

Puccinia Tanaceti, DC.

Artemisia Ludoviciana, Nutt.

Aecidium Compositarum, Mart.

Erysiphe Cichoracearum, DC.

Frysiphe Linkii, Lev.

Peronospora Halstedii, Farl.

Peronospora leptosperma, DBy.

$\{$ Puccinia Tanaceti, DC.

\{ Puccinia Artemisiarum, Schm. \& Kze.

) Chaetomella (?) perforata, Ell. \& Ev.

i Venturia occidentalis, Ell. \& Ev.

Septoria Artemisiae, Pass.

Artemisia pycnocephala, DC.

Diplodia herbarım, (Cda.) Lev.

Gibberella Saubinetii, (Mont.) Sacc.

Puccinia Artemisiarum, Schun. \& Kze.

Artemisia serrata, Nutt.

Erysiphe Linkii, Lev.

Peronospora leptosperma, DBy.
Artemisia scopulor um, Gray.

(Spharopsis Raui, Pk.

$\{$ Phoma Raui, Sacc.

(Macrophoma Rani, Ber]. \& Vogl.

Artemisia tridentata, Nutt.

Puccinia Tanaceti, DC.

Sporidesmium macrosporoides, EH. \& Gall.

Artemisia vulgaris, L

S Sphaeria orthoceras, Fr.

Diaporthe orthoceras, Nits.

\{ Sphaeropsis opaca, C. \& E.

Haplosporella opaca, Sacc.

Phoma herbarum, Westd.

Septoria fusca, Pk.

Artemisia, sp. indet.

Aecidium Compositarum, Mart, var. Artemisiae, C. \& E.

Aiternaria hispidula, Ell.

\{Dichomera Compositarum, Cke. \& Hark.

Camarosporium Compositarum, Sacc.

Cucurbitaria solitaris, Ell.

Cucurbitaria umbilicata, E11.

Fusarium roseum, Lk.

$\{$ Cucurbitaria occidentalis, Ell.

Melanomma occidentalis, Sacc.

S Sphacria sulcata, Ell.

\{ Melanomma sulcatum, Berl. \& Vogl.

\{ Peziza Artemisiae, Lasch.

\{ Pyrenopeziza Artemisiae, Sacc.

Sphaeria ovalis, Ell.

\{Rosellinia ovalis, Sacc.

\{ Sporidesmium herbarum, Cke.

Sirodesmium herbarum, Sacc.

Sphaeria aculeata, S., var. artemisiaecola, S.

Stictis decipiens, (Karst.) Cke.

Tubercularia herbarum, Fr.

Aster acuminatus, Michx.

(Puccinia Asteris, Duby, var. purpurascens, C. \& P.

Puccinia purpurascens, C. \& P.

Aster adscendens, Lindl.

Erysiphe Cichoracearum, DC.

Aster azureus, Lindl.

Puccinia Astcris, Duby.

Aster canescens, Pursh.

Erysiphe Cichoracearum, DC.

Aster commutatus, Gray

Erysiphe Cichoracearum, DC.

Erysiphe communis, (Wallr.) Schl.

Puccina Asteris, Duby.

- Aster conspicuus, Lindl.

Coleosporium Sonchi-arvensis, (P.) Lev.

Erysiphe Cichoracearum, DC.

Puccinia Asteris, Duby.

Aster cordifolius, L.

Caeoma (Accidium) asteratum, S.

1 Uredo Solidaginis, S.

Coleosporium Sonchi-arvensis, (P.) Lev. 
Erysiphe Cichoracearum, DC.

Puccinia Asteris, Duby.

Septoria astericola, E1l. \& Ev.

Septoria atropurpurea, Pk.

Aster corymbosus, Ait.

Coleosporium Sonchi-arvensis, (P.) Lev.

Erysiphe Cichoracearum, DC.

Puccinia Asteris Duby.

Aster diffusus, Ait.

Erysiphe Cichoracearum, DC.

Aster Drummondii, Lindl.

Coleosporium Sonchi-arvensis, (P.) Lev.

Aster ericoides, L.

Coleosporium Sonchi, Lev.

Erysiphe Cichoracearum, DC.

Aster foliaceus, Lindl, var. Eatoni, Gray.

Erysiphe Cichoracearum, DC.

Aster junceus, Ait.

Erysiphe Cichoracearum, DC.

Aster laevis, L.

Aecidium Compositarum, Mart.

Coleosporium Sonchi, Lev.

$\{$ Erysiphe Cichoracearum, DC.

Erysiphe lamprocarpa, Lev. p. p.

Aster Lindleyanus, Torr. \& Gr.

Aecidium Compositarum, Mart.

Aster longifolius, Lam.

Erysiphe Cichoracearum, DC.

Aster macrophyllus, L.

$\{$ Coleosporium Sonchi, Lev.

$\{$ Coleosporium Sonchi-arvensis, (P.) Lev.

Erysiphe Cichoracearum, DC.

Puccinia Asteris, Duby.

Puccinia Gerardii, Pk.

Puccinia Asteris, Duby, var. purpurascens C. \& P.

Puccinia purpurascens, C. \& P.

Septoria atropurpurea, $\mathrm{Pk}$.

Aster multiflorus, Ait.

Erysiphe Cichoracearum, DC.

Aster Novae-Angiix, L.

(Peronospora entospora, (Roze \& Cornu) B. \& Br.

Peronospora simplex, Pk.

Plasmopara entospora, Schrt.

Puccinia Asteris, Duby.

CRamularia macrospora, Fres., var. Senecio-

nis, Trelease.

Ramularia macrospora, Fres., var. Asteris, Sacc.

Septoria atropurpurea, $\mathrm{Pk}$.

Aster oblongifolius, Nutt.

Y Accidium Asterum, S.

Aecidium Verbesina, S.

Accidium Solidaginis, S.

Cieoma (A ecidium) asteratum, Lk.

Uredo Asterum, Spreng.

Aecidium Compositarum, Mart., forma Asteris, Wint.
$\{$ Erysiphe Cichoracearum, DC.

\{Erysiphe Asterum, S.

Aster paniculatus, Lam.

Caeoma (Aecidium) asteratum, S.

$\{$ Puccinia Asteris, Duby.

\{ Puccinia Gerardii, Pk.

Aster ptarmicoides, Torr. \& Gr.

Phoma Iowana, Sacc.

Aster puniceus, L.

Entyloma Compositarum, Farl.

$\{$ Peziza assimilis, C. \& P.

\{Orbilia assimilis, Sacc.

Phoma Iowana, Sacc.

Ramularia filaris, Fres.

Septoria Punicei, Pk.

Aster sagittifolius, Willd.

SAecidium Asterum, S.

Aecidium Compositarum, Marț., forma Asteris, Wint.

f Coleosporium Sonchi, Lev.

\{ Coleosporium Sonchi-arvensis, (P.) Lev.

Erysiphe Cichoracearum, DC.

Puccinia Asteris, Duby.

Aster sericeus, Vent.

Aecidium Compositarum, Mart.

Aster Shortii, Hook.

Puccinia Asteris, Duby.

Aster Tradescanti, L.

Puccinia Asteris, Duby.

$\{$ Xyloma Asteris, S.

Rhytisma Asteris, S.

Aster umbellatus, Mill.

Coleosporium Sonchi-arvensis, (P.) Lev.

Erysiphe Cichorcearum, DC.

Erysiphe lamprocarpa, Lev. p. p.

Aster vimineus, Lam., var, foliolosus, Gray.

f Erysiphe Cichoracearum, DC.

IErysiphe lamprocarpa, Lev. p. p.

Puccinia Asteris, Duby.

Aster, sp. indet.

Diaporthe mucronulata, Sacc.

S Sphaeria orthoceras, Fr.

Diaporthe orthoceras, Nits.

Dothidea Haydeni, B. \& C.

Ophiodothis Haydeni, Sacc.

Rhopographus Haydeni. Cke.

(Dothidea Heliopsidis, S.

$\{$ Montagnella Heliopsidis, Sacc.

(Euryachora Heliopsidis, Cke.

\{ Hysterium expallens, S.

\{Hypoderma expallens, Cke.

f Sphaeria comatella, C. \& E.

\{ Leptosphaeria comatella, Sacc.

\{ Sphaeria Ogilviensis, B. \& Br.

\{Leptosphaeria Ogilviensis, Ces. \& De Not.

Sphaeria anguillida, Cke.

Ophiobolus anguillides, Sacc.

Raphidospora anguillida, Cke 
$\{$ Peziza astericola, C. \& E.

$\{$ Mollisia astericola, Sacc.

\{ Phoma macularis, B. \& C.

\{ Phoma maculifera, Sacc.

$\{$ Hypocenia herbarum, Cke. S Itark.

Stagonospora herbarum, Sacc.

Baccharis halimifolia, L.

Asterina melioloides, B. \& C.

Meliola Baccharidis, B، \& Rav.

Dimerosporium Baccharidis. Sacc.

Dimerosporium melioloides, Martiri.

Baccharis pilularis, DC.

Coleosporium Baccharidis, Cke. \& Hark.

Puccinia evadens, Hark.

Sphaeria bicuspidata, Cke. \& Hark.

$\{$ Leptosphaeria bicuspidata, Berl. \& Vogl。

Heptameria bicuspidata, Cke. \& Hark.

Sphaeria (Melanomma) seminis, Cke. \& Hark.

Baccharis viminea, DC.

Coleosporium Baccharidis, Cke. \& Hark.

Puccinia evadens, Hark.

Baccharis, sp. indet.

Corticium incarnatum, Fr.

Dothidea Baccharidis, Cke.

Sphaeria (Diaporthe) Baccharidis, Cke.

Stereum tabacinum, (Sow,) Fr.

Bahia, sp. indet.

Aecidium Bahiae, B. \& C.

Balsamorrhiza deltoidea, Nutt.

Balsamorrhiza macrophylla, Nutt.

Balsamorrhiza sagittata, Nutt.

(Puccinia flosculosorum, (A. \& S.) Roeh1.

T Trichobasis Balsamorrhizate, $\mathrm{Pk}$.

Puccinia Balsamorrhizae, Pk.

Uredo Balsamorrhizae, De Toni.

Bidens bipinnata, L.

Septoria Bidentis, Sacc.

Bidens cernua, L.

Peronospora Halstedii, Farl。

Bidens chrysanthemoides, Miclix.

Cercospora umbrata, Ell. \& Holw.

Peronospora Halstedii, Fari.

Sphaerotheca Castagnei, Lev.

Bidens connata, Muhl.

Erysiphe fuscata, B. \& C.

$\{$ Peronospora Halstedii, Farl.

\{ Plasmopara Halstedii, Ber\}. \& De Toni.

Sphaerotheca Castagnei, Ley.

Bidens frondosa, L.

Aecidium Compositarum, Mart.

Cercospora umbrata, Ell. \& Holw.

Erysiphe fuscata, B. \& C.

$\{$ Peronospora Halstedii, Farl.

\{ Plasmopara Halstedii, Berl. \& De Toni.

Sphaerotheca Castagnei, Lev.
Bidens, sp. indet.

$\{$ Sphaeria Ogilviensis, B. \& Br.

\{eptosphaeria Ogilviensis, Ces. \& De Not。

f Sphaeria acuminata, Sow.

T Ophiobolus acuminatus, Duby.

Sphaeria anguillida, Cke.

Ophiobolus anguillides, Sacc.

(Raphidospora anguillida, Cke.

Peziza fuscorubra, Rehm.

Peziza heterocarpa, Ell.

Ramularia concomitans, Ell. \& Ifolw.

Sporocybe byssoides, (P.) Fr.

Bigelovia Douglasii, Gray.

Erysiphe Cichoracearum, DC.

Aecidium Bigeloviæ, Pk.

Bigelovia graveolens, Gray., var. albicaulis, Gray.

Erysiphe Cichoracearum. DC.

Erysiphe sepulta, Ell. \& Ev.

Bigelovia, sp. indet.

Puccinia Bigeloviae, Ell. \& Ev.

Borrichia frutescens, DC.

Uromyces triannulatus, B. \& C.

Brickellia, sp. indet.

Puccinia Brickelliae, $\mathrm{Pk}$.

Cacalia atriplicifolia, L.

Aecidium Compositarum, Mart.

Septoria Cacaliae Ell. \& Kell.

Cacalia tuberosa, Nutt.

Septoria Cacaliae, Ell. \& Kell.

Callistephus Chinensis, Nees.

Coleosporium Sonchi-arvensis, (P.) Lev。

Carduus, sp. indet.

Sphaeria pellita, Fr.

Carphephorus tomentosus, Torr. \& Gr。

Fusisporium aurantiacum, Lk.

Chrysanthemum Leucanthemum, L.

Phoma herbarum, Westd.

Septoria herbarum, B. \& C.

Chrysopsis villosa, Nutt.

Erysiphe Cichoracearum, 1)C.

Cnicus altissimus, Willd.

$\{$ Erysiphe Cichoracearum, DC.

Erysiphe lamprocarpa, Lev. p. p.

Sphaeria acuminata, Sow.

Ophiobolus acuminatus, Duby.

Raphidospora acuminata, Rabh.

Septoria Cirsii, Niessl.

Septoria Commonsii, Ell. \& Ev.

Cnicus altissimus, Willd., var discolor, Gray.

$\{$ Puccinia flosculosorum, (A. \& S.) Roehı.

\{ Puccinia Compositarum, Schl.

$\{$ Chaetomella (?) perforata, Ell. \& Ev.

\{Venturia occidentalis, Ell. \& Ev. 
Cnicus arvenis, Hofim.

\{ Cystopus cubicus, (Strauss) Lev。

Cystopus spinulosus, DBy.

Phyllosticta Cirsii, Desm.

$\{$ Puccinia Compositarum, Schl.

\{ Puccinia Cirsii, Lasch.

('Trichobasis suaveolens, (P.) Lev.

$\{$ Puccinia obtegens, (Lk.) Tul.

Puccinia suaveolens, Rostrup.

Septoria Cirsii, Niessl.

Cnicus lanceolatus, Hoffm.

Cystopus cubicus, (Strauss) Lev.

CUredo flosculosorum, A. \& S.

Uredo Cichoracearum, D C.

Puccinia Hieracii, (Schum.) Mart.

$\{$ Puccinia flosculosorum, Roehl.

Puccinia variabilis, Grev.

Puccinia Compositarum, Schl.

(Puccinia Cirsii, Lasch.

Cnicus muticus, Pursh.

\{ Sphaeria mesoedema, B. \& C.

Heptameria mesoedema, Sacc.

Cnicus ochrocentrus, Gray.

Schizonella subtrifida, Ell. \& Ev.

Cnicus undulatus, Gray.

Cercospora obesa, Ell. \& Ev.

Erysiphe Cichoracearum,DC.

Puccinia flosculosorum, (A. \& S.) Roehl.

Cnicus, sp. indet.

$\{$ Pleospora baccata, Ell.

Clathrospora permunda, (Cke.) Berl.

$\{$ Sphaeria modesta, Desm.

$\{$ Leptosphaeria modesta, Karst.

Macrosporium commune, Rabh.

Coreopsis aurea, Ait.

Sphaerotheca Castagnei, Lev.

Coreopsis discoidea, Torr. \& Gr.

Peronospora Halstedii, Farl.

Dahlia variabilis, Desf.

Botryosporium pulchrum, Cda.

Erysiphe communis, (Wallr.) Schl.

Macrosporium erumpens, Cke.

Sphaerella Dahlize, C. \& E.

Vermicularia compacta, C. \& E.

Dysodia chrysanthemoides, Lag.

Erysiphe Cichoracearum, DC.

Elephantopus Carolinianus, Willd.

Cercospora Elephantopodis, Ell. \& Ev.

U Uredo Elephantopodis, S.

Caeoma Elephantopodis, Lk.

Coleosporium Sonchi, Lev.

Coleosporium Sonchi-arvensis, (P.) Lev,

Coleosporium Elephantopodis, Thm.

Elephantopus tomentosus, L.

Uredo Elephantopodis, S.

Erechthites hieracifolia, Raf.

Erysiphe communis, (Wallr.) Schl.
Sphaerotheca Castagnei, Lev.

Erigeron annuus, P.

(Caeoma (Aecidium) erigeronatum, S.

Aecidium Compositartm, Mart., forma

Erigerontis, Wint.

Cercospora cana, Sacc.

Cercospora griseëlla, $\mathrm{Pk}$.

$\{$ Fusisporium phyllogenum, C. \& P.

Fusarium phyllogenum, Sacc.

Leptothyrium punctiforme, B. \& C.

$\{$ Septoria Erigeronis, Pk.

\{ Septoria erigerontea, Sacc.

Erigeron armeriaefolius, Turcz.

Erysiphe Cichoracearum, DC.

Erigeron bellidifolius, Muhl.

Caeoma (Aecidium) erigeronatum, S.

$\{$ Aecidium Compositarum, Mart., forma Erigerontis, Wint.

Erigeron Canadensis, L.

Caeoma (Aecidium) erigeronatum, S.

Aecidium Compositarum, Mart., forma Erigerontis, Wint.

Fusidium canum, Pass.

Cercospora cana, Sacc.

Cercosporella cana, Sacc.

Ramularia cana, Halsted.

Diaporthe apiculosa, Ell.

Diaporthe incrustans, Nits. ?

Erysiphe Cichoracearum, DC.

$\{$ Peziza nigrescens, Cke.

$\{$ Helotium nigrescens, Rehm.

Leptosphaeria agnita, (Desm.) Ces. \& De Not., var. Erigerontis, Berl.

$\{$ Sphaeria Ogilviensis B. \& Br.

$\{$ Leptosphaeria Ogilviensis, Ces. \& De Not.

\{ Sphaeria bucera, C. \& E.

Metasphaeria bucera, Sacc.

\{ Sphaeria leiostega, Ell.

$\{$ Metasphaeria leiostega, Sacc.

(Peronospora entospora, (Rose \& Cornu) B. \& Br.

$\{$ Peronospora Basidiophora, Cornu.

Peronospora simplex, Pk.

Plasmopara entospora, Schrt.

$\{$ Septoria Erigeronis, Pk.

$\{$ Septoria erigerontea, Sacc.

Erigeron compositus, Pursh.

Pleospora herbarum, (P.) Rabh.

Pleospora platyspora, Sacc.

Sphaerella eriophila, Niessl.

Erigeron corymbosus, Nutt.

Erigeron divaricatus, Michx.

Erigeron glabellus, Nutt.

Erigeron micranthus, Nutt.

Erysiphe Cichoracearum, DC.

Erigeron Philadelphicus, L.

Caeoma (Aecidium) erigeronatum, S.

Entyloma Compositarum, Farl. 
Erigeron quercifolius, Lam.

Accidium Compositarum, Mart, forma

Erigerontis, Wint.

Erigeroul salsuginosus, Gray.

$\{$ Pleospora permunda, (Cke.) Sacc.

Clathrospora permunda, Berl.

Sphaerella subcongregata, Ell. \& Ev.

Erigeron strigosus, Muhl.

(Caeoma (Aecidium) erigeronatum, S.

Aecidium Compostarum, Mart., forma Erigerontis, Wint.

Erysiphe Cichoracearum, DC.

Septoria Erigeronis, Pk.

Septoria erigerontea, Sarc.

Erigeron uniflorus, L.

Laestadia circumtegens, Rostrup.

Torisla abbreviata, Cda.

Erigeron, sp. indet.

Asterina nigerrima, Ell.

Chaetomium olivaceum, C. \& E.

Dothidea Haydeni, B. \& C.

$\{$ Ophiodothis Haydeni, Sacc.

Rhopographus Haydeni, Cke.

Sphaeria anguillida, Cke.

$\{$ Ophiobolus anguillides, Sacc.

Peziza carneorubra, Ell.

$\{$ Trichopeziza carneorubra, Sacc.

$\{$ Peziza erigeronata, Sacc.

Mollisia erigeronata, Sacc.

$\{$ Peziza exigua, Cke.

$\{$ Pezizella exigua, Sacc.

Septoria Erigerontis, B. \& C.

Eriophyllum staechadifolium, Lag.

Heptameria mesoedema, (B. \& C.) Sacc.

Eupatorium ageratoides, L. f.

Cercospora ageratoides, Ell. \& Ev.

Cercospora Eupatorii, Pk.

Srysiphe communis, (Wallr.) Schl.

$\{$ Erysiphe Martii, Lev.

Hymenula olivacea, Pk.

Hypocrea phyllogena, Mont.

$\{$ Hypocrella phyllogena, Speg.

$\{$ Peronospora Halstedii, Farl.

Plasmopara Halstedii, Berl. \& De'Toni.

Seziza Solenia, Pk.

$\{$ Solenopezia Solenia, Sacc.

Cacoma (Aeciclium) tenue, S.

$\{$ Puccinia tenuis, Burrill.

Septoria Eupatorii, Rob.

Eupatorium album, L.

Cercospora ageratoides, Ell. \& Ev.

Cercospora Eupatorii, Pk.

Eupatorium altissimum, L.

Caeoma (Aecidium) tenue, S.

Eupatorium coelestinum, L.

Y Puccinia Centaureae, Berk.

Puccinia Conoclinii, Seymour.

Eupatorium coronopifolium, Willd.

S Sphaeria mesoedema, B. \& C.

Ileptameria mesoedcma, Sacc.
SDothiden Eupatorii, B. \& C.

\{ Phyllachora Eupatorii, Sacc.

$\{$ Sphaeria umbrinella, B. \& C.

$\{$ Rosellinia umbrinella, Sacc.

Eupatorium perfoliatum, L.

Aecidium Compositarum, Mart.

Caeoma (Aecidium) tenue, S.

Cercospora perfoliata, Ell. \& Ev.

Eupatorium purpureum, L.

f Sphaeria mortuosa, Ell.

$\{$ Anthostoma mortuosum, Sacc.

Caeoma (Aecidium) Compositarum, Lk., var. Eupatoriae, S.

Aecidium Compositarum, Mart., var. Eupatoriae, (S.) B.

Erysiphe Cichoracearum, DC.

\{Erysiphe lamprocarpa, Lev. p.p.

$\{$ Hysterium commune, Fr.

\{Hypoderma commune, Duby.

Leptostroma vulgare, $\mathrm{Fr}$.

\{ Peronospora Halstedii, Farl.

Plasmopara Halstedii, Berl. \& De Toni.

$\{$ Peziza Eupatorii, S.

\{richopeziza Eupatorii, Sacc.

Eupatorium purpureum, L., var. maculatum, Darl.

\{ Peziza longipila, Pk.

$\{$ Dasyscypha longipila, Sacc.

$\{$ Xyloma confluens, S.

$\{$ Rhytisma confluens, S.

Eupatorium serotinum, Michx.

Septoria Eupatorii, Rob.

Eupatorium, sp. indet.

Actinothyrium caulincolum, S.

Peziza atrata, $\mathrm{P}$.

Phacidium caulincolum, $\mathrm{S}$.

Sporidesmium irregulare, Cke.

Gaillardia aristata, Pursh.

Erysiphe Cichoracearum, DC.

Gaillardia pulchella, Fung.

Entyloma Compositarum, Farl.

Gnaphalium decurrens, Ives.

$\{$ Patellaria gnaphaliana, C. \& E.

Patinella gnaphaliana. Sacc.

Gnaphalium polycephalum, Michx. Caeoma (Aecidium) gnaphaliatum, S.

Puccinia investita, S.

Gnaphalium, sp. indet.

\{Cercospora gnaphaliacen, Cke.

Cercospora Gnaphalii, Hark.

Grindelia obusta, Nutt.

Sorosporium Californicum, Hark.

Grindelia squarrosa, Dunal.

Erysiphe Cichoracearum, DC.

Puccinia Grindeliae, Pk.

Ramularia Grindeliae, Ell. \& Kell.

Gutierrezia Euthamiae, Torr. \& Gr.

Erysiphe Cichoracearum, DC. 
Erysiphe communis, (Wallr.) Schl.

Pleospora herbarum, (P.) Rabh.

Helenium autumnale, $\mathrm{L}$.

Erysiphe Cichoracearum, DC.

Puccinia Helenii, S.

Septoria Helenii, Ell. \& Ev.

Helianthella Californica, Gray.

$\{$ Trichobasis Helianthellac, $\mathrm{Pk}$.

\{ Uredo Helianthellae, De Toni.

Helianthella Parryi, Gray.

Erysiphe Cichoracearum, DC.

Helianthus angustifolius, L.

Montagnella Heliopsidis, (S.) Sacc.

Puccinia Tanaceti, DC.

\section{Helianthus annuus, L.}

Alternaria tenuis, Nees.

Cercospora pachypus, Ell. \& Ev.

Entyloma Compositarum, Farl.

$\{$ Erysiphe Cichoracearum, DC.

\{rysiphe lamprocarpa, Lev. p.p.

Macrosporium inquinans, C. \& E.

- $\{$ Dothidea effusa, S.

\{ Phyllachora effusa, Sacc.

Plasmopara Halstedii, (Farl.) Berl. \& De Toni.

Polyactis simplex, (P.) Lk.

(Puccinia Tanaceti, DC.

$\{$ Puccinia Helianthi, S.

Puccinia Helianthorum, S.

Septoria Helianthi, Ell. \& Kell.

Vermicularia subeffigurata, S., var. Heli-

anthi, S.

Helianthus Californicus, DC.

$\{$ Puccinia Tanaceti, DC.

\{ Puccinia Helianthi, S.

Helianthus Californicus, DC., var. Utahensis, Gray.

Erysiphe Cichoracearum, DC.

Helianthus decapetalus, L.

Erysiphe Cichoracearum, DC.

$\{$ Puccinia Tanaceti, DC.

\{ Puccinia Helianthi, S.

Helianthus decapetalus, L., var. multiflortis, (?) Gray.

$\{$ Dothidea Heliopsidis, (S.) Fr.

\{Montagnella Heliopsidis, Sacc.

Helianthus divaricatus, L.

Caeoma (Aecidium) tracheliifoliatum, S.

Dothidea Heliopsidis, (S.) Fr.

$\{$ Puccinia Tanaceti, DC.

\{ Puccinia Helianthi, S.

Septoria paupera, Ell.

Helianthus doronicoides, Lam.

Aecidium Compositarım, Mart.

Caeoma (Aecidium) helianthatım, S.

Cercospora Helianthi, Ell. \& Ev.

$\{$ Erysiphe Cichoracearum, DC.

$\{$ Erysiphe lamprocarpa, Lev. p.p.
$\{$ Peronospora Halstedii, Farl.

\{ Plasmopara Halstedii, Berl. \& De Toni.

Septoria Helianthi, Ell. \& Kell.

Helianthus giganteus, L.

Caeoma (Aecidium) helianthatım, S.

$\{$ Caeoma (Uredo) Helianthi, S.

\{ Puccinia Tanaceti, DC.

Helianthus gracilentus, Gray.

Phyllachora effusa, (S.) Sacc.

Helianthus grosse-serratus, Martens.

Erysiphe Cichoracearum, DC.

$\{$ Peronospora Halstedii, Farl.

\{ Plasmopora Halstedii, Berl. \& De Toni.

$\{$ Puccinia Tanaceti, DC.

\{ Puccinia Helianthi, S.

Helianthus heterophyllus, Nutt.

\{ Puccinia Helianthi, S.

\{ Puccinia Helianthorum, S.

Helianthus laetiflorus, $\mathrm{P}$.

Puccinia Helianthi, S.

Helianthus Maximiliani, Schrad.

Cercospora Helianthi, Ell. \& Ev.

Puccinia Tánaceti, DC.

Helianthus mollis, Lam.

$\{$ Aecidium Helianthi-mollis, S.

\{ Caeoma (Aecidium) helianthatum, S.

Puccinia Tanaceti, DC.

Septoria Inulae, Sacc. \& Speg?

Helianthus occidentalis, Riddell.

$\{$ Peronospora Halstedii, Far\}.

\{ Plasmopara Halstedii, Berl. \& De Toni.

Puccinia Tanaceti, DC.

Helianthus petiolaris, Nutt.

Cercospora pachypus, Ell. \& Kell.

Puccinia Helianthi, S.

Helianthus rigidus, Desf.

- Caeoma (Aecidium) helianthatım, S.

Cercospora Helianthi, Ell. \& Ev.

Erysiphe Cichoracearum, DC.

$\{$ Puccinia Tanaceti, DC.

\{ Puccinia Helianthi, S.

Helianthus strumosus, L.

Aecidium Compositarum, Mart.

$\{$ Erysiphe Cichoracearum, DC.

\{ Erysiphe lamprocarpa, Lev. p.p.

Sphaeria Heliopsidis, S.

Dothidea Heliopsidis, Fr.

Montagnella Heliopsidis, Sacc.

(Euryachora Heliopsidis, Cke.

$\{$ Peronospora Halstedii, Farl.

\{ Plasmopara Halstedii, Berl. \& De Toni.

$\{$ Puccinia Tanaceti, DC.

$\{$ Puccinia Helianthi, S.

Septoria Heliantli, Ell. \& Kell.

Helianthus tracheliifolius, Willd.

Caeoma (Aecidium) tracheliifoliatum, S.

Plasmopara llalstedii (Farl.) Berl. \& De Toni. 


\section{Helianthus tuberosiu, L:}

Erysiphe Cichoracearum, DC.

$\{$ Peronospora Halstedii, Farl.

$\{$ Plasmopara Halstedii, Berl. \& De Toni.

Puccinia Tanaceti, DC.

Puccinia Helianthi, S.

Caeoma (Uredo) Helianthi, S.

Puccinia Helianthorum, S.

Trichobasis Helianthi, Rav. Fung. Am.

Helianthus, sp. indet.

Coleosporium Sonchi-arvensis, (P.) Lev..

Cyphella pezizoides, Zopf.

Macrosporium commune, Rabh.

S Septoria helianthicola, Cke. \& Hark.

$\left\{\begin{array}{l}\text { Rhabdospora helianthicola, Sacc. } \\ \text { R }\end{array}\right.$

(Sphaeria consessa, C. \& E.

$\{$ Leptosphaeria consessa, Sacc.

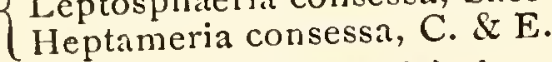

Sphaeria (Leptosphaeria) dumetorum,

(Niessl) C. \& E.

$\{$ Sporidesmium Fumago, Cke.

$\{$ Sirodesmium Fumago, Sacc.

Vermicularia subglabra, Cke. \& Hark.

Heliopsis laevis, $\mathrm{P}$.

Aecidium Compositarum, Mart., forma

Heliopsidis, Wint.

Sphaeria Heliopsidis, S.

Dothidea Heliopsidis, S.

Montagnella Heliopsidis, Sacc.

Euryachora Heliopsidis, Cke.

Heliopsis, sp. indet.

Puccinia Heliopsidis, S.

Inula Helenium, L.

$\{$ Sphaeria rostrospora, Gerard.

$\{$ Anthostomella rostrospora, Sacc.

Erysiphe Cichoracearum, DC.

Iva axillaris, Pursh.

Aecidium intermixtum, $\mathrm{Pk}$.

Puccinia intermixta, $\mathrm{Pk}$.

Iva ciliata, Willd.

Cystopus Tragopogonis, (P.) Schrt.

Septoria xantliifolia, Ell. \& Kell.

Iva frutescens, L.

Dothidea tetraspora, B. \& Br.

Phyllosticta ivaecola, Ell. \& Ev.

Iva xanthiifolia, Nutt.

Erysiphe Cichoracearum, DC.

\{ Plasmopara sp., Swingle.

\{eronospora Kellermanni, Ell. \& Halsted.

Sphaeria (Physalospora) Arthuriana, Sacc.

$\{$ Laestadia Arthuriana, Cke.

Puccinia intermixta, Pk.

Septoria xanthiifolia, Ell. \& Kell.

Kuhnia eupatorioides, L.

Puccinia Kuhniae, S.
Kuhnia, sp. indet.

Uredo flosculosorum, A. \& 9.

Lepachys pinnata, Torr. \& Gr.

Entyloma Compositarum, Farl.

Septoria infuscata, Wint.

Liatris scariosa, Willd.

Aecidium Compositarum, Mart. var. Liatri, Webber.

Madia Nuttallii, Gray.

Coleosporium Madiae, Cke.

Madia sativa, Molina.

Peronospora Halstedii, Farl.

Matricaria, sp. indet.

Cystopus cubicus, (Strauss) Lev.

Melanthera hastata, Miclix.

$\{$ Uromyces Melantherae, Cke.

\{Uromyces Martinii, Farl.

Mikania scânđenś, Willd.

Fusarium Mikaniae, B. \& C.

Mikania, sp. indet.

Aecidium Compositarum, Mart.

Onopordon acanthium, L.

Rhabdospora pleosporoides, Sacc.

Parthenium integrifolium, L.

Cystopus cubicus, (Strauss) Lev.

Perityle Californica, Benth., var. nuda,

Gray.

Cystopus cubicus, (Strauss) Lev.

Fetasites palmata, Gray.

Puccinia Nardosmii, Ell. \& Ev.

Ramularia variegata, Ell. \& Holw.

Pluchea borealis, Gray.

Puccinia splendens, Vize.

Rudbeckia hirta, L.

Septoria Rudbeckiae, Ell. \& Halsted.

Rudbeckia laciniata, L.

Entyloma Compositarum, Farl.

$\{$ Peronospora Halstedii, Farl.

$\{$ Plasmopara Halstedii, Berl. \& De Toni.

Ramularia Rudbeckiae, Pk.

Septoria Rudbeckiae, Ell. \& Halsted.

Uromyces Rudbeckiae, Arth. \& Holw.

\{ Uromyces Solidaginis, Auct. Am. p.p.

Rudbeckia occidentalis, Nutt.

Erysiphe Cichoracearum, DC.

Rudbeckia triloba, L.

Cercospora tabacina, Ell. \& Ev.

Peronospora Halstedii, Farl.

Septoria Rudbeckiae, Ell. \& Halsted.

Senecio aureus, L.

Aecidium Compositarum, Mart.

Aecidium Senecionis, Desm.

\{ Cystopus cubicus, (Strauss) Lev.

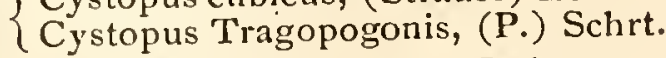

Entyloma Compositarum, Farl. 
Senecio lugens, Richards.

Cystopus cubicus, (Strauss) Lev.

Senecio Serra, Hook.

Cystopus Tragopogonis, (P.) Schrt.

Senecio triangularis, Hook.

Puccinia subcircinata, Ell. \& Ev.

Senecio vulgaris, L.

Coleosporium Senecionis, (P.) Fr.

Silphium integrifolium, Michx.

Aecidium Compositarum, Mart.

Ascochyta Treleasei, Berl. \& Vogl.

Cercospora Silphii, Ell. \& Ev.

Coleosporium Sonchi-arvensis, (P.) Lev.

Entyloma Compositarum, Farl.

$\{$ Peronospora Halstedii, Farl.

\{ Plasmopara Halstedii, Berl. \& De Toni.

Puccinia Silphii, S.

Silphium laciniatum, L.

Aecidium Compositarum, Mart.

Coleosporium Sonchi-arvensis, (P.) Lev.

Peronospora Halstedii, Farl.

Puccinia Silphii, S.

Silphium perfoliatum, L.

Aecidium Compositarum, Mart.

$\{$ Peronospora Halstedii, Farl.

\{ Plasmopara Halstedii, Berl. \& De Toni.

Puccinia Silphii, S.

Septoria Silphii, Ell. \& Ev.

Sphaeria Silphii, S.

Silphium terebinthinaceum, Jacq.

Aecidium Compositarum, Mart.

Chaetophoma maculans. Wint.

Uredo Terebinthinaceae, S.

$\{$ Caeoma Terebinthinaceae, S.

Coleosporium Sonchi-arvensis, (P.) Lev.

$\{$ Peronospora Halstedii, Farl.

$\{$ Plasmopara Halstedii, Berl. \& De Toni.

Puccinia Silphii, S.

Silphium trifoliatum, L.

$\{$ Peronospora Halstedii, Farl.

$\{$ Plasmopara Halstedii, Berl. \& De Toni.

Puccinia Silphii, S.

Silphium trifoliatum, L., var. latifolium, Gray.

Ellisiella mutica, Wint.

Silphium, sp. indet.

? Erysiplıe depressa, Lk.

? Erysiphe Linkii, Lev.

$\{$ Rhytisma Silphii, S.

Leptostroma Silphii, Cke.

$\{$ Dothidea Silphii, S.

\{Phyllachora Silphii, Sacc.

Vermicularia Silphii, S.

Solidago arguta, Ait.

Coleosporium Solidaginis, (S.) Thm.

Puccinia orbicula, Pk. \& Clinton.

Septoria solidaginicola, $\mathrm{Pk}$.
Solidago caesia, L.

Aecidium Asterum, S.

Rhytisma Solidaginis, S. Spec. Dub.

Solidago Canadensis, L.

(Aecidium Solidaginis, S.

$\{$ Aecidium Compositarım, Mart., forma Solidaginis, Wint.

$\{$ Coleosporium Sonchi-arvensis, (P.) Lev.

\{ Coleosporium Solidaginis, (S.) Thm.

(Erysiphe Cichoracearum, DC.

$\{$ Erysiphe lamprocarpa, Lev. p.p.

Oidium erysiphoides, $\mathrm{Fr}$.

$\{$ Peronospora Halstedii, Farl.

\{ Plasmopara Halstedii, Berl. \& De Toni.

Phyllachora Solidaginum, Sacc.

Ramularia Virgaureæ, Thm.

Septoria fumosa, Pk.

Septoria Solidaginum, Thm.

Spharella solidaginea, Ell. \& Kell.

Sphaeria Solidaginum, S.

Solidago lanceolata, L.

Rhytisma Solidaginis, S. Spec. Dub.

Solidago latifolia, L.

(Aecidium Asterum, S.

Aecidium Verbesinae, S.

Aecidium Solidaginis, S.

$\{$ Caeoma (Aecidium) asteratum, Lk.

Uredo Asterum, Spreng. p.p.

Aecidium Compositarum, Mart., forma Solidagir is, Wint.

Cercospora reticulata, (Pk:) Ell. \& Ev.

Coleosporium Sonchi-arvensis, (P.) Lev.

Solidago leptocephala, Torr. \& Gr.

Rhytisma Solidaginis, S. Spec. Dub.

Solidago Lindheimeriana, Scheele.

Ramularia Virgaureæ, Thm.

Solidago Missouriensis, Nutt.

Coleosporium Sonchi-arvensis, (P.) Lev.

Erysiphe Cichoracearum, DC.

Solidago nana, Nutt.

Erysiphe Cichoracearum, DC.

Solidago nemoralis, Ait.

Coleosporium Sonchi-arvensis, (P.) Lev.

Puccinia Solidaginis, Pk.

Solidago occidentalis, Nutt.

Didymaria spissa, Hark.

Erysiphe Cichoracearum, DC.

Solidago odora, Ait.

Septoria Solidaginis, Cke.

Solidago puberula, Nutt.

Coleosporium Solidaginis, Thm.

$\{$ Darluca Filum, (Biv.) Cast.

\{ Septoria Solidaginis, Thm.

Solidago pumila, Torr. \& Gr.

Puccinia Solidaginis, $\mathrm{Pk}$.

Solidago Riddellii, Frank.

Aecidium Compositarum, Mart.

\{ Peronospora Halstedii, Farl.

\{ Plasmopara Halstedii, Berl. \& De Toni. 
Solidago rigida, L.

Aecidium Asterum, S.

Aecidium Compositarum, Mart.

Erysiphe Cichoracearum, DC.

(Peronospora entospora, (Rose \& Cornu)

$\{$ B. \& Br.

Plasmopara entospora, Schrt.

S Stachylidium roseum, S.

Sporotrichum Schweinitzii, Sacc.

Solidago rugosa, Mill.

Aecidium Asterum, S.

$\{$ Cercosporella reticulata, $\mathrm{Pk}$.

$\{$ Cercospora reticulata, Ell. \& Ev.

Coleosporium Sonchi-arvensis, (P.) Lev.

$\{$ Puccinia Virgaureae, (DC.) Lib.

$\{$ Puccinia minutula, Pk.

Ramularia serotina, Ell. \& Ev.

Rhytisma Solidaginis, S., Spec. Dub.

Solidago rupestris, Raf.

Coleosporium Solidaginis, Thm.

Solidago sempervirens, $\mathrm{L}$.

Rhytisma bifrons, S., Spec. Dub.

Solidago serotina, Ait.

CUredo Solidaginis, S.

Caeoma Solidaginis, S.

Coleosporium Sonchi-arvensis, (P.) Lev.

Coleosporium Solidaginis, Thm.

Erysiphe Cichoracearum, DC.

Ramularia serotina, Ell. \& Ev.

Septoria Virgaureæ, Desm.

Solidago ulmifolia, Muhl.

Coleosporium Sonchi-arvensis, (P.) Lev.

Solidago Virgaurea, L., var. alpina, Bigel.

Septoria brevis, $\mathrm{Pk}$.

Solidago, sp. indet.

Diaporthe mucronulata, Sacc.

$\{$ Sphaeria orthoceras, Fr.

Diaporthe orthoceras, Nits.

Dothidea Heliopsidis, S. ?

Hymenula vulgaris, Fr.

Leptosphaeria Doliolum, (P.) De Not.

Sphaeria aulica, C. \& E.

$\{$ Metasphaeria aulica, Sacc.

Endophlaea aulica, C. \& E.

(Sphaeria Solidaginis, S. (in litt.)

$\{$ Dothidea Solidaginis, $\mathrm{Fr}$.

?Phyllachora Solidaginis, Sacc.

?Sphaeria Solidaginum, S.

Ophiobolus Solidaginis, Sacc.

Raphidospora Solidaginis, Cke.

Peziza atrata, $P$.

$\{$ Peziza atrocinerea, Cke.

$\{$ Mollisia atrocinerea, Phil.

$\{$ Peziza subatra, C. \& P.

\{Pyrenopeziza subatra, Sacc.

Phlyctaena arcuata, B.
$\{$ Sphaeria erratica, C. \& E.

Physalospora erratica, Sacc.

$\{$ Cryptosporium Solidaginis, C. \& E.

Rhabdospora Solidaginis, sacc.

Rhabdospora subgrisea, Pk.

Septoria gallarum, Ell. \& Ev.

Septoria intermedia, Ell. \& Ev.

Sphaeria linearis, Nees.

Uromyces Solidaginis, Niess].

Tagetes, sp. indet.

Sphaeria tageticola, S.

Tanacetum vulgaze, L.

$\{$ Sphaeria comatella, C. \& E.

\{Leptosphaeria comatella, Sacc.

Pistillaria coccinea, (Cda.) Fr.

Tetradymia canescens, DC.

Puccinia variolans, Hark.

Trilisia odsratissima, Cass.

Phoma minutissima, Cke.

Tussilago Farfara, L.

Ramularia brunnea, $\mathrm{Pk}$.

Verbesina enceliodes, Benth. \& Hook.

Erysiphe Cichoracearum, DC.

Verbesina occidentalis, Walt.

Puccinia Verbesinae, S.

Verbesina, sp. indet.

\{ Aecidium Asterum, S.

Aecidium Verbesinae, S.

Vernonia Baldwinii, Torr.

Cercospora oculata, Ell. \& Kell.

Cercospora Vernoniae, Ell. \& Kell.

Coleosporium Sonchi-arvensis, (P.) Lev.

Erysiphe Cichoracearum, DC.

$\{$ Peronospora Halstedii, Farl.

\{ Plasmopara Halstedii, Berl. \& De Toni.

Puccinia flosculosorum, (A. \& S.) Roehl.

Sphaerella decidua, Ell. \& Kell.

Vernonia fasciculata, Michx.

Cercospora Vernoniae, Ell. \& Kell.

Coleosporium Sonchi-arvensis, (P.) Lev.

$\{$ Erysiphe Cichoracearum, DC.

\{rysiphe lamprocarpa, Lev. p.p.

Puccinia flosculosorum, (A. \& S.) Roehl.

Puccinia Heliopsidis, S.

S Puccinia Vernoniae, S.

$\left\{\begin{array}{l}\text { Puccinia Tanaceti, DC., var. Vernoniae, } \\ \text { Burrill. }\end{array}\right.$

Vernonia Noveboracensis, Willd.

Aecidium Compositarum, Mart.

Ascochyta Treleasei, Berl. \& Vogl.

Cercospora Noveboracensis, Ell. \& Ev.

CUredo Solidaginis, S.

Caeoma Solidaginis, S.

Coleosporium Sonchi-arvensis, (P.) Lev.

Coleosporium Compositarum. Lev.

(Coleosporium Vernoniae, B. \& C. 
Sphaeria tenebrosa, B. \& Br.

$\{$ Didymosphaeria tenebrosa, Sacc.

Didymosphacrella tenebrosa, Cke.

Erysiphe Cichoracearum, DC.

Peronospora Halstedii, Farl.

Puccinia bullata, S.

Puccinia Tanaceti, DC.

Sphaerotheca Castagnei, Lev.

Vernonia Noveboracensis, Willd., var. latifolia, Gray.

Puccinia flosculosorurn, (A. \& S.) Roehl.

Viguiera, sp. indet.

Puccinia Viguierae, Pk.

Wyethia angustifolia, Nutt.

Trichobasis Wyethiae, Pk.

Uredo Wyethiae, De Toni.

Wyethia mollis, Gray.

Puccinia Wyethiae, (Pk.) Hark.

Septoria Wyethiae, Hark.

Xanthium Canadense, Mill.

Aecidium Compositarum, Mart.

Sothidea Xanthii, DC.

$\{$ Asteroma Xanthii, M. A. Curtis.

Phyllachora Xanthii, Sacc.

Erysiphe Cichoracearum, DC.

Erysiphe lamprocarpa, Lev. p.p.

Erysiphe Montagnei, Lev.

Erysiphe horridula, Lev.

Erysiphe spadicea, B. \& C.

Puccinia Xanthii, S.

Rhabdospora Xanthii, Pk.

Septoria Xanthii, Desm.

Sphaerella xanthicola, Cke. \& Hark.

Zinnia multiflora, L.

Cercospora Zinniae, Ell. \& Martin.

Sphaeria Zinniae, $\mathrm{S}$.

Ziunia, sp. indet.

Cryptosporium aurantiacum, Lk.

\section{Liguliflorae.}

Crepis glauca, Torr. \& Gr.

Puccinia Hieracii, (Schum.) Mart.

Ramularia Crepidis, Ell. \&.Ev.

Hieracium alpinum, L.

Septoria cercosperma, Rostrup.

Hieracium Canadense, Michx.

Erysiphe Cichoracearum, DC.

$\{$ Puccinia Hieracii, (Schum.) Mart.

\{Puccinia flosculosorum, (A. \& S.) Roehl.

Hieracium dovrense, Fr.

Leptosphaeria agnita. (Desm.) De Not.

Hieracium paniculatum, L.

Caeoma (Aecidium) hieraciatum, S.

Hieracium vulgatum, Fr.

Caeoma (Aecidium) hieraciatum, S.
Leptosphaeria agnita, (Desm.) De Not.

Puccinia Hieracii, (Schum.) Mart.

Hieracium, sp. indet.

Puccinia maculosa, S.

Sphaerotheca Castagnei, Lev.

Krigia amplexicaulis, Nutt.

Aecidium Compositarum, Mart.

Puccinia maculosa, S.

Krigia Dandelion, Nutt.

(Aecidium Compositarum, Mart.

Aecidium Dandelionis, S.

Caeoma Compositarum, Lk., var. Prenanthis, S.

Uredo Compositarum, Spreng. p.p.

Krigia Virginica, willd.

?Puccinia Asteris, Duby.

Lactuca acuminata, Gray.

Septoria unicolor, Wint.

Lactuca Canadensis, L.

Aecidium Compositarum, Mart.

Entyloma Compositarum, Farl.

Macrosporium inquinans, C. \& E.

$\{$ Bremia Lactucae, Regel.

$\{$ Peronospora gangliformis, (B.) D By.

Pleospora lactucicola, Ell. \& Ev.

Septoria lactucicola, E11. \& Martin.

Sphaerella Lactucae, Ell. \& Kell.

Vermicularia venturioidea, C. \& E.

Lactuca floridana, Gaertn.

Puccinia Prenanthis, (P.) Fckl.

Septoria lactucicola, Ell. \& Martin.

Lactuca leucophaea, Gray.

\{ Bremia Lactucae, Regel.

\{ Peronospora gangliformis, (B.) D By.

Lactuca Ludoviciana, DC.

Peronospora gangliformis, (B.) D By.

Lactuca pulchella, DC.

Aecidium hemisphaericum, Pk.

Erysiphe Cichoracearum, DC.

Peronospora gangliformis, (B.) D By.

Puccinia Prenanthis, (P.) Fckl.

Sphaeria (Dothidea) Lactucarum, S.

Tuberculina vinosa, Sacc.?

Lactuca sativa, L.

$\{$ Bremia Lactucae, Regel.

Peronospora gangliformis, (B.) D By.

Septoria consimilis, Ell. \& Martin.

Septoria Lactucæ, Pass.

Lactuca, sp. indet.

Dactylium roseum, (Lk.) B.

$\{$ Sphaeria subexserta, C. \& F.

$\{$ Didymella subexerta, Sacc.

Diplodia herbarum, Lev., var. Lactucae, C. \& E.

Dothidea crustosa, C. \& E. 
Macrosporium atrichum, C. \& E.

Macrosporium caudatum, C. \& E.

Macrosporium cladosporioides, Desm.

Myrothecium Verrucaria, (A. \& S.) Ditm.

Ovularia Carletoni, Ell. \& Kell.

$\{$ Sphaeria herbarum, $P$.

\{ Pleospora herbarum, Rabh.

Sphaerella depressa, Pk.

\{rastadia depressa, Berl. \& Vogl.

Lygodesmia juncea, Don.

Aecidium Compositarum, Mart., var. Lygodesmiae, Webber.

Darluca Filum, Cast.

Puccinia variolans, Hark.?, var. caulicola, Ell. \& Ev.

\{'Tubercularia persicina, Ditm.

\{ Tuberculina persicina, Sacc.

Lygodesmia spinosa, Nutt.

Puccinia Harknessi, Vize.

Malacothrix, sp. indet.

Synchytrium innominatum, Farl.

Prenanthes alba, L.

$\{$ Bremia Lactucae, Regel.

\{ Peronospora gangliformis, (B.) D By.

(Puccinia Hieracii, (Schum.) Mart.

$\{$ Puccinia variabilis, Grev.

Puccinia Compositarum, Schl.

Septoria Nabali, B. \& C.

Prenanthes altissima, L.

Cladosporium effusum, B. \& C.

$\{$ Bremia Lactucae, Regel.

$\{$ Peronospora gangliformis, (B.) D By.

Prenanthes aspera, Michx.

Cercospora Prenanthis, Ell. \& Kell.

Prenanthes racemosa, Michx.

Septoria Nabali, B. \& C.

Prenanthes, sp. indet.

Aecidium Compositarum, Mart.

(Puccinia Prenanthis, (P.) Fckl.

$\{$ Puccinia Chondrillae, Cda.

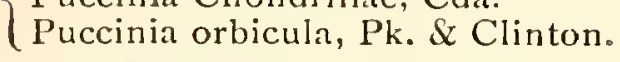

Septoria Nabali, B. \& C.

Septoria Prenanthis, Ell. \& Ev.

\{ Septoria simulans, B. \& C.

\{Phlyctaena simulans, Sacc.

Sphaerotheca Castagnei, Lev.

Rafinesquia Californica, Nutt.

Cercospora Rafinesquiae, Hark.

Sonchus asper, L.

\{ Bremia Lactucae, Regel.

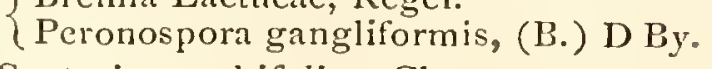

Septoria sonchifoliae, Cke.

Sonchus, sp. indet.

Sphaeria Lactucarum, S.

Stephanomeria minor, Nutt.

Puccinia cladophila, Pk.
Stephanomeria runcinata, Nutt.

Discula runcinata, Ell. \& Ev.

Puccinia cladophila, Pk.

Taraxacum officinale, Weber.

Aecidium Taraxaci, Schm. \& Kze.?

Erysiphe Montagnei, Lev.

Uredo flosculosorum, A. \& S.

Uredo Cichoracearum, DC.

Puccinia Hieracii, (Schum.) Mart.

Puccinia flosculosorum, Roehl.

Puccinia variabilis, Grev.

Puccinia Compositarum, Schl.

Trichobasis Compositarum, Lev.

Puccinia Cirsii, Lasch.

Ramularia lineola, Pk.

Ramularia Taraxaci, Karst.

Sphaerella Compositarum, Awd.

Sphaerotheca Castagnei, Lev.

Tragopogon porrifolius, L.

$\{$ Cystopus cubicus, (Strauss) Lev.

$\{$ Cystopus Tragopogonis, (P.) Schrt.

Troximon aurantiacum, Hook.?

?Ovularia compacta, Ell. \& Ev.

Puccinia Compositarum, Schl.

Puccinia rugosa, Billings.

Truximon cuspidatum, Pursh.

Puccinia flosculosorum, (A. \& S.) Roehl.

Puccinia Troximontis, Pk.

Troximon glaucum, Nutt.

Aecidium Compositarum, Mart.

Puccinia flosculosorum, (A. \& S.) Roehl.

Troximon gracilens, Gray.

Puccinia Troximontis, $\mathrm{Pk}$.

Troximon heterophyllum, Greene.

Puccinia 'Troximontis, $\mathrm{Pk}$.

Compositæ, sp. indet.

Leptosphaeria pyrenopezizoides, Sacc. \& Speg.

?Polyactis quadrifida, Lk.

Thecaphora pilulaeformis, B. \& C.

Uromyces compactus, Pk.

\section{LOBELIACEAE.}

Lobelia cardinalis, L.

Caeoma (Urcdo) Lobeliae-cardinalis, S.

Cercospora effusa, (B. \& C.) Ell. \& Er.

Lobelia inflata, L.

Cercospora effusa, (B. \& C.) Ell. \& Ev.

Entyloma Lobeline. Farl.

Septoria Lobeliae, Pk.

Lobelia puberula, Michx.

Cladosporium effusum, B. \& C.

$\{$ Puccinia Lobeliae, Gerard.

\{ Puccinia mierosperma, B.\& C.

Lobelia spicata, Lam.

Septoria Lobeliae, Pk. 
Lobelia syphilitica, L.

$\{$ Cladosporium effusum, B. \& C.

$\{$ Cercospora effusa, Ell. \& Ev.

Cercospora Lobeliae, Kell. \& Swingle.

$\{$ Puccinia Lobeliae, Gerard.

\{ Puccinia microsperma, B. \& C.

Septoria Lobeliae, Pk.

Lobelia, sp. indet.

Cladosporium pelliculosum, B. \& C.

Phoma devastatrix, B. \& Br.?

\section{CAMPANULACEAE.}

\section{Campanula Americana, L.}

Septoria Campanulae, (Lev.) Sacc.

Sphaerella Campanulae, Ell. \& Kell.

Campanula rotundifolia, L.

Pleospora herbarum, (P.) Rabh.

Sphaerella pachyasca, Rostrup.

Campanula uniflora, L.

Pleospora herbarum, (P.) Rabh.

Puccinia Campanulae, Carm.

Septoria cercosperma, Rostrup.

Sphaerella pachyasca, Rostrup.

Specularia biflora, Gray.

Specularia leptocarpa, Gray.

Septoria Speculariae, B. \& C.

S Septoria specularina, Sacc.

Specularia perfoliata, A. DC.

(Septoria Speculariae, B. \& C.

$\{$ Septoria Speculariae, Sacc.

Septoria specularina, Sacc.

$\{$ Uredo Campanulae, $\mathrm{P}$.

\{ Caeoma (Uredo) Campanularum, Lk.

\section{ERICACEAE.}

Andromeda ferruginea, Walt.

Asterina lepidigena, Ell. \& Martin.

Linospora ferruginea, Ell. \& Martin.

Septoria pulchella, B. \& C.

Andromeda ligustrina, Muhl.

$\{$ Sphaeria vexata, C. \& E.

Cryptosphaeria vexata, Sacc.

\{ Spheria grumata, Cke.

\{ Didymosphaeria grumata, Rehm.

Cucurbitaria longitudinalis, $\mathrm{Pk}$.

Exobasidium Andromedae, Pk.

Hypocrea rufa, (P.) Fr.

Melogramma fuliginosum, Ell.

Microsphaera penicillata, (Wallr.) Lev.

Sphaeria entaxia, C. S E.

$\{$ Melogramma fuliginosum, Ell. p.p.

Physalospora entaxia, Sacc.

$\{$ Xyloma Andromedae-ligustrinae, S.

$\{$ Rhytisma decolorans, Fr.
$\{$ Sphaeria punctiformis, P.

$\{$ Sphaerella punctiformis, $\mathrm{Fr}$.

$\{$ Hysterium Vaccinii, Carm.

Sporomega cladophila, (Lev.) Duby.

Caeoma (Uredo) Vacciniorum, Lk.

Valsa ligustrina, Cke.

Andromeda nitida, Bartr.

Corticium calceum, (P.) Fr.

$\{$ Hysterium Andromedae, S.

\{ Sporomega Andromedae, Duby p.p.

Sporomega Andromedae, Duby p.p.

$\{$ Hysterium variegatum, B. \& C.

Hypoderma variegatum, Duby.

Sphaeria Andromedae, S.

Uredo Andromedae, Cke.

Androneda polifolia, L.

Rhytisma Andromedae, (P.) Fr.

Venturia Cassandrae, $\mathrm{Pk}$.

Andromeda, sp. indet.

$\{$ Dermatea crypta, Cke.

$\{$ Scleroderris crypta, Sacc.

\{ Asterina clavuligera, Cke.

\{Dimerosporium clavuligerum, Martin.

(Venturia maculosa, El1.

$\{$ Meliola maculosa, Ell.

(Dimerosporium Ellisii, Sacc.

Hymenochaete agglutinans, Ell.

(Sphaeria sublanosa, Cke.

$\{$ Metasphaeria sublanosa, Sacc.

Endophlaea sublanosa, Cke.

$\{$ Peziza fuscidula, Cke.

$\{$ Dasyscypha fuscidula, Sacc.

$\{$ Peziza marginata, Cke.

Trichopeziza marginata, Sacc.

$\{$ Peziza mauriata, C. \& E.

\{ Patinella mauriata, Sacc.

Phacidium nigrum, Cke.

$\int$ Sphaeria microtheca, C. \& E.

$\{$ Calosphaeria microtheca, Sacc.

Physalospora microtheca, Sacc.

$\{$ Sphaeria arctespora, C. \& E.

Rosellinia arctispora, Sacc.

Sphaeria xylariaespora, C. \& E.

$\{$ Sphaeria detonsa, Cke.

Rosellinia xylarispora, Sacc.

Sphaeria Andromedarum, S.

$\{$ Sphaeria Andromedae, Rav. Fung. Amer.

(Sphaeria kalmicola, Rav. Fung. Amer.

Stereum ochraceoflavum, (S.) Rav.

? $\{$ Sphaeria salicina, P.

\{Valsa salicina, Fr.

Arbutus Menziesii, Pursh.

Corticium calceum, (P.) Fr.

Cryptosporium punctiforme, Cke. \& Hark.

Cyphelia Ravenelii, B.

i Diatrype prominens, Howe.

\{ Diatrypella prominens, Sacc.

Diplodia maculata, Cke. \& Hark.

Discosia minima, B. \& C. 
Exobasidium Vaccinii, (Fckl.) Wor.

Grandinia ocellata, Fr.

Phacidium Arbuti, Cke. \& Hark.

Phacidium quadratum, Schm.

(Dothidea rugodisca, Cke. \& Hark.

Homostegia rugodisca, Sacc.

Phyllachora rugodisca, Cke. \& Hark.

Melasmia arbuticola, Vize.

Rhytisma Arbuti, Phil.

Sphaerella arbuticola, Pk.

Sphaerella maculiformis, Awd.

SMacroplodia asterina, Cke. \& Hark.

S Sphaeropsis asterina, Sacc.

Sphaeropsis maculiformis, Cke. \& Hark.

Stictis radiata, $\mathrm{P}$.

Arctostaphylos alpina, Spreng.

Asteroma alpinum, Sacc.

Melampsora sparsa, Wint.

Arctostaphylos glauca, Lindl.

f Sphaeria Arctostaphyli, Plow.

Botryosphaeria Arctostaphyli, Sacc.

\{ Nacroplodia Arctostaphyli, Vize.

\{ Sphaeropsis Arctostaphyli, Sacc.

Arctostaphylos pumila, Nutt.

Venturia Arctostaphyli, Cke. \& Hark.

Arctostaphylos pungens, H. B. K.

Exobasidium Arctostaphyli, Hark.

Glonium stellatum, Muln.

Hysterium vulvatum, $\mathrm{S}$.

Macroplodia Arctostaphyli, Vize.

$\{$ Peziza labrosa, Phil. \& Hark.

\{ Trichopeziza labrosa, Sacc.

Arctostaphylos Uva-ursi, Spreng.

Asterina conglobata, B. \& C.

Exobasidum Vaccinii, (Fckl.) Wor.

Arctostaphylos, sp. indet.

Sphaeria sustenta, Plow.

$\{$ Anthostoma sustentum, Sacc.

Xylosphaeria sustenta, Cke.

Cryptosporium falcatum, Cke. \& Hark.

\{Sphaeria spiculosa, A. \& S.

Diaporthe spiculosa, Nits.

Exobasidium Vaccinii, (Fckl.) Wor.

Harknessia Arctostaphyli, Cke. \& Hark.

Sporidesmium Fumago, Cke., var. umbrinum, Cke. \& Hark.

Sirodesmium Fumago, Sacc., var. umbrinum, Sacc.

Sporidesmium induratum, Cke.

Bryanthus taxifolius, Gray.

Antennatula arctica, Rostrup.

Cassandra calyculata, Don.

Ascochyta Cassandrae, Pk.

Cenangium Cassandrae, $\mathrm{Pk}$.

Cenangium pezizoides, $\mathrm{Pk}$.

Discella arida, Pk.

\{ Exobasidium Vaccinii, (Fck1.) Wor.

$\{$ Exobasidium Cassandrae, $\mathrm{Pk}$.
Godronia Cassandrae, Pk.

Hysterium maculare, Fr.

Lophodermium orbiculare, (Ehrb.) Sacc.?

Synchytrium Vaccinii, Thomas.

Uredo Cassandrae, Pk. \& Clinton.

Caeoma (Uredo) Vacciniorum, Lk.

Venturia Cassandrae, $\mathrm{Pk}$.

Venturia pulchella, C. \& P.

Cassiope 1ycopodioides, Don.

$\{$ Hysterium gracile, Ehrb.

Lophodermium gracile, Sacc.

S Hysterium orbiculare. Ehrb.

Lophodermium orbiculare, Sacc.

f Sphaeria punctiformis, $\mathrm{P}$.

S Sphaerella punctiformis, Fr.

Cassiope tetragona, Don.

f Triblidium arcticum, Ehrb.

Cenangium arcticum, Fr.

Didymella hyperborea, (Karst.) Sacc.

Exobasidium Vaccinii, (Fckl.) Wor.

Leptosphaeria Andromedae, (Awd.) Sacc.

Metasphaeria Cassiopes, Rostrup.

Pleospora hyperborea, Fckl.

Sphaerella inconspicua, Schrt.

Sphaeria Hederae, Fr.

Venturia Myrtilli, Cke.?

Chimaphila maculata, Pursh.

Septoria Chimaphilae, E11. \& Ev.

Chimaphila umbellata, Nutt.

(Sphaeria Pyrolae, Ehrb.

$\{$ Depazea Pyrolae. Rabh.

Phoma Pyrolae, Rostrup.

Clethra alnifolia, L.

Sphaeronema clethrincolum, E11.

Cenangium urceolatum, E11.

Cenangiella ureceolata, Sacc.

$\{$ Nectria depauperata, Cke.

Dialonectria depauperata, Cke.

Phyllosticta clethricola, Ell. \& Martin.

$\{$ Stilbospora fenestrata, Ell. \& Ev.

\{ Steganosporium fenestratum, Sacc.

Synchytrium Vaccinii, Thomas.

$\{$ Valsa clethraecola, C. \& E.

$\{$ Valsaria clethricola, Sacc.

Valsa obtecta, C. \& E.

Clethra, sp. indet.

\{ Cenangium Urceolus, (A.\& S.) Fr.

\{ Godronia Urceolus, Sacc.

f Sphaeria viscosa, C. \& E.

TPhysalospora viscosa, Sacc.

$\{$ Sphaeronema rufum, Fr.

Sphaeronemella rufa, Sacc.

Sphaeropsis clethricola, C. \& E.

Epigaea repens, L.

\{ Erysiphe Vaccinii, S.

Microsphaera Vaccinii, C. \& P.

Phyllosticta Epigaeae, Pk. 
Gaultheria procumbens, L.

Asterina Gaultheriae, II. A. Curtis.

Cercospora Gaultheriae, Ell. \& Ev.

Discosia maculicola, Gerard.

Phyllosticta Gaultheriae, Ell. \& Ev.

Sphaerella Gautheriae, C. \& P.

Venturia Gaultheriae, Ell. \& Ev.

Gaultheria Shallon, Pursh.

Pestalozzia gibbosa, Hark.

Phyllosticta Gaultheriae, Ell. \& Ev.

Sphaerella Gaultheriae, C. \& P.

Gaylussacia dumosa, Torr. \& Gr.

$\{$ Peziza venturioides, E11. \& Er.

$\{$ Trichopeziza venturioides, Sacc.

Gaylussacia frondosa, Torr. \& Gr.

\{Hysterium abietinum, S. Syn. Car. p.p.

\{ Hysterium Vaccinii, S.

Xyloma Andromedae-ligustrinae, S., var. Vaccinii, S.

Rhytisma Vaccinii, Fr.

Gaylussacia resinosa, Torr. \& Gr.

Exobasidium Vaccinii, (Fckl.) Wor.

Melampsora Vaccinii, (A. \& S.) Wint.

Microsphaera Vaccinii, (S.) C. \& P.

Peziza venturioides, Ell. \& Ev.

Ramularia effusa, $\mathrm{Pk}$.

Kalmia angustifolia, L.

Peziza Kalmiae, Pk.

Dermatea Kalmiae, Cke.

Pezicula Kalmiae, Sacc.

Trichopeziza. Kalmiae, Sacc.

f Discella Kalmiae, Pk.

\{Discula Kalmiae. Sacc.

f Dothidea Kalmiae, Pk.

Dothidella Kalmiae, Sacc.

Leptosphaeria Kalmiae, Pk.

f Hysterium exaridum, C. \& P.

\{Lohodermium exaridum, C. \& P.

$\{$ Sphaeria (Depazea) kalmicola, S.

\{ Sphaerella colorata, Pk.

Synchytrium Vaccinii, Thomas.

Kalmia glauca, Ait.

$\{$ Sphaerella haematodes, B. \& C.

\{ Laestadia haematodes, Cke.

Venturia Kalmiae, Pk.

Kalmia latifolia, L.

Antennaria semiorata, B. \& Br.

f Stilbum atrocephalum, Ell.

Arthrobotryum atrocephalum, Sacc.

$\{$ Phacidium exasperans, S.

Asterina exasperans, B. \& C.

Cercospora sparsa, Cke.

Ceuthospora foliicola, (Cke.), var. Kalmiae, Sacc.

Cytospora foliicola, Lib., var. Kalmiae, Sacc.

Dacryomyces deliquescens, (Bull.) Duby.

Hysterium Kalmiae, S.
Lophodermium exaridum, C. \& P.

S Sphaeria longispora, Ell.

\{Ophioceras longisporum, Sacc.

Pestalozzia halmicola, Ell. \& Ev.

Sphaeria Kalmiarum, S.

Sphaeria (Depazea) kalmicola, S.

Depazea kalmicola, Rav.

Septoria kalmicola, B. \& C.

Phyllosticta kalmicola, Cke.

\{ Sphaeria Andromedarum, S.

S Sphaeria kalmicola, Rav. Fung. Amer.

S Valsa Peckii, Howe.

$\{$ Valsaria Peckii, Sacc.

Pseudovalsa Peckii, Cke.

Sphaeria atriella, C. \& E.

$\{$ Zignoella atrella, Sacc.

Psilosphaeria atrella, Cke.

Kalmia, sp. indet.

Arthrosporium compositum, Ell.

Cylindrocolla lactea, Sacc. \& Ell.

Helminthosporium subopacum, C. \& E.

$\{$ Peziza subiculata, S.

$\{$ Tapesia subiculata, Sacc.

$\{$ Sphaeria strigosa, A. \& S.

\{Lasiosphaeria strigosa, Sacc.

Sphaeria hysterioides, S.

$\{$ Lophiostoma hysterioides, Sacc.

Lophiosphaera hysterioides, Che.

$\{$ Dothidea chalybea, S.

\{ Phyllachora chalybaea, Sacc.

$\{$ Dothidea denigrans, S.

P Phyllachora denigrans, Sacc.

Sordaria lutea, E11. \& Ev.

Sphaeria atrovirens, A. \& S.

Xyloma sphaerioides, S. Syn. Car.

Ledum latifolium, Ait.

Aschochyta Ledi, Rostrup.

\{ Uredo Ledi, A. \& S.

\{ Chrysomyxa Ledi, D By.

Colpoma lacteum, $\mathrm{Pk}$.

Gloeosporium Ledi, Schrt.

Hypoderma commune, (Fr.) Duby.

$\{$ Hysterium sphaerioides, A. \& S.

\{Lophodermium sphaerioides, Duby.

Puccinia Ledi, B. \& C.

Uredo ledicola, $\mathrm{Pk}$.

Ledum palustre, L.

Chrysomyxa Ledi, (A. \& S.) D By.

Ledum, sp. indet.

Lophodermium maculare, (Fr.) De Not.

Leucothoe acuminata, Don.

Asterina diplodioides, B. \& C.

$\{$ Hysterium variegatum, B. \& C.

$\{$ Hypoderma variegatum, Duby.

Phyllosticta Leucothoes, E11. \& Martin.

Sphaeria atrovirens. A. \& S.

Xyloma sphaerioides, S. Syn. Car. 
Leucothoe axillaris, Don.

fHyterium Andromedac, S.

i Sporomega Andromedae. Duby p.p.

I Splaterella Leucothoes, Che.

Laestadia Leucothoes. Sacc.

Siloma hysterioides. P.

Hysterium foliicolum, $\mathrm{Fr}$.

Lophodermium lysterioides, Sacc.

Phoma Andromedae, S.

Sphaeria Andromedae, S.

Sphaeria Andromedarum, S.

Leucothoe racemosa, Gray.

Dermatea lobata, Ell.

Ramularia Andromedae, Ell. \& Martin.

Rhytisma decolorans, Fr.

( Hysterium Vaccinii, Carm.

\{ Sporomega cladophila, (Lev.) Duby.

Valsa delicatula, C. \& E.

Venturia pezizoides, Sacc. \& Ell.

Loiseleuria procumbens, Desv.

Phoma herbarum, Westd.

Sphaerella inconspicua, Schrt.

Monotropa uniflora, L.

Vermicularia Dematium, (P.) Fr.

Monotropa, sp. indet.

fHysterium planturum, S.

Hypoderma plantarum, Cke.

Oxydendrum arboreum, DC.

Racodium ramosum, S.

Oedemium ramorum, Fr.

(Dematium ramosum, S.

\{ Peziza gelatinosa, Bull.

Exidia recisa, Fr.

f Sistotrema olivaceum, S.

\{ Hydnum olivaceum, Fr.

Hysterium flexuosum, $\mathrm{S}$.

$\{$ Hysterium variegatum, B. \& C.

\{Hyoderma variegatum, Duby.

\{ Hysterium Vaccinii, Carm.

(Sporomega cladophila, (Lev.) Duby.

Stictis radiata, $P$.

Peziza Andromedae, S.

Cenangium Andromedae, Fr.

(Tympanis Andromedae, Fr.

Pirola elliptica, Nutt.

f Caeoma (Aecidium) pirolatum, S.

(Chrysomyxa pirolata, Wint.

\{ Uredo Pirolae. (Gmel.) Mart.

(Melampsora Pirolae, Schrt.

Pirola minor, L.

Chrysomyxa Pirolae, (DC.) Rostrup.

Pirola rotundifolia, L.

Caeoma (Aecidium) pirolatum, S.

Uredo pirolata, Körn.

Chrysomyxa pirolata, Wint.

Chrysomyxa Pirolae, (DC.) Rostrup.
CUredo Pirolae, (Gmel.) Mart.

Caeoma Pirolac, Sch1.

Trichobasis Pirolae, B.

Thecopsora? Pirolae, Karst.

Melampsora Pirolae, Schrt.

Phyllosticta Pirolae, Ell. \& Ev.

$\{$ Ovularia Pirolae, Trelease.

\{ Ramularia Pirolae, Ell. \& Ev.

Zygodesmus Pirolae, Ell. \& Halsted.

Pirola rotundifolia, L., var. pumila, Hook.

Cladosporium herbarum, (P.) Lk.

Heterosporium variabile, Cke.

Phoma Pirolae, (Ehrb.) Rostrup.

Pyrenophora comata, (Niessl) Sacc.

Sphaerella Pirolae, Rostrup.

Pirola secunda, L.

Chrysomyxa pirolata, (S.) Wint.

(Uredo Pirolae, (Gmel.) Mart.

$\{$ Trichobasis Pirolae, $\mathrm{B}$.

Melampsora Pirolae, Schrt.

Septoria Pirolae, Ell. \& Martin.

Rhododendron arboreum, Smith.

Sphaeria Kalmiarum, S.

Rhododendron Catawbiense, Michx.

Hendersonia concentrica, Ell. \& Ev.

Pestalozzia Guepini, Desm.

Rhododendron Lapponicum, Wahl.

Dimerosporium oreophilum, Speg.

Rhododendron maximum; L.

Agaricus Rhododendri, $\mathrm{Pk}$.

Cenangium palmatum, $\mathrm{S}$.

Cephalotrichum rigescens, Lk.

Coryneum Rhododendri, S.

Coryneum triseptatum, $\mathrm{Pk}$.

Pestalozzia Mariae, Clinton.

Dochmolopha Clintonii, Cke.

Cryptostictis Mariae, Sacc.

f Sphaeria denigrata, S.

\{ Eutypa denigrata, Cke.

Hendersonia concentrica, Ell. \& Ev.

(Hysterium Rhododendri, S.

Coccomyces Rhododendri, Cke.

(Lophodermium Rhododendri, Pk.

$\{$ Sphaeria inconstans, S.

OOhleria inconstans, Che.

Phacidium Rhododendri, S.

S Polyporus Rhododendri, S.

Poria Rhododendri, Cke.

Phyllosticta maxima, El]. \& Ev.

Sphaerella oblivia, Cke.

Rhododendron nudiflorum, Torr.

$\{$ Uredo minima, S.

\{ Caeoma (Uredo) Azaleae, S.

Nelampsora Vacciniorum, (Lk.) Schrt. 
Cysterium abietinum, S. Syn. Car. p. p. Hysterium Azaleae, S.

Sporomega Andromedae, Duby p. p.

Colpoma Azaleae, Cke.

Dacryomyces Azaleae, S.

Exobasidium Azaleae, Pk.

?Microsphaera Platani, Howe

Ramularia angustata, $\mathrm{Pk}$.

Sphaeria Azaleae, S.

\{ Periconia Azaleae, Pk.

$\{$ Sporocybe Azaleae, Sacc.

Rhododendron occidentale, Gray.

Diatrype asterostoma, B. \& C.

Exobasidium decolorans, Hark.

Sphaeria (Leptosphaeria) Californica, Cke. \& Hark.

Rhododendron Rhodora, Don.

Exobasidium Vaccinii, (Fckl.) Wor.

Caeoma (Uredo) Vacciniorum, Lk.

Rhododendron viscosum, Torr.

Caeoma (Uredo) Azaleae, S.

Exobasidium discoideum, Ell.

Exobasidium Vaccinii, (Fckl.) Wor.

Hypocrea consimilis, Ell.

S Sphaeria viscosa, C. \& E.

$\{$ IIelogramma fuliginosum, Ell. p. p.

(Physalospor: viscosa, Sacc.

f Sphaeria xylariaespora, C. \& E.

\{ Rosellinia xylarispora, Sacc.

f Sphaeronema hystricinum, Ell.

\{ Sphaerographium hystricinum, Sacc.

Synchytrium Vaccinii, Thomas.

Valsa decidua, C. \& E.

Rhododendron, sp. indet.

S Sphaeria aperta, S.

Amphisphaeria aperta, Cke.

f Sphaeronema Rhododendri, S.

A Aposphaeria Rhododendri, Sacc.

Botrytis laxa, S.

Coryneum depressum, Kze. \& Schm.

CHydnum sudans, A. \& S.

Dacrina hydnoideum, S. Am. bor.

Thelebolus sudans, Fr.

Dacryobolus sudans, Fr.

S Dermatea crypta, Cke.

Scleroderris crypta, Sacc.

Diatrype asterostoma, B. \& C., var. minor,

Cke. \& Hark.

Hysterium abbreviatum, S.

Hysterium teres, S.

$\{$ Irpex fuscescens, S.

Irpex cinnamomeus, Fr.

Patellaria Rhododendri, S.

Phacidium corticale, $\mathrm{S}$.

Physarum cæspitosum, S.

Schizoxylon Persoonii, S.

Septoria Rhododendri, Cke.

? Sphaeria strigosa, A. \& S.
Sphaeria truncata, Fr.

Sphaeronema cladoniscus, Fr.

\{ Sphaeronema echinatum, B. \& C.

Sphaerographium echinatum, Sacc.

Sphaeria (Thyridium) ambleia, C. \& E.

Tremella Stictis, A. \& S.

$\{$ Sphaeria rhizina, S.

$\{$ Valsa rhizina, Cke.

Valsa subclypeata, C. \& P.

Vaccinium arboreum, Marshall.

Corticium simulans, B. \& Rav.

$\{$ Peziza albocitrina, Cke.

Dasyscypha albocitrina, Sacc.

Podosporium praelongum, B. \& C.

Rhytisma Vaccinii, (S.) Fr.

Septoria albopunctata, Cke.

Sphaerella Vaccinii, Cke.

Vaccinium Canadense, Kalm.

\{ Calyptospora Goeppertiana, Kiihn.

\{ Melampsora Goeppertiana, Wint.

Synchytrium Vaccinii, Thomas.

Vaccinium corymbosum, L.

Calyptospora Goeppertiana, Kuihn.

\{ Cenangium asterinosporum, Ell. \& Ev.

Dothiora asterinospora, Sacc.

\{ Peziza Kalmiae, Pk.

Dermatea Kalmiae, Cke.

f Valsa corymbosa. C. \& E.

Diaporthe corymbosa, Sacc.

S Sphaeria concolor, S.

Diatrype concolor, Cke.

Hypocrea citrinella, Ell.

Marasmius cucullatus, Ell.

Sphaeria nidulans, S.

Chaetosphaeria nidulans, Rehm.

Meliola nidulans, Cke.

Microsphaera Friesii, var. Vaccinii, C. \& P.

Microsphaera Vaccinii. C. \& P.

(Peziza mollisiaeoides, S.

$\{$ Helminthosporium humile, Sacc.

Tapesia mollisioides, Sacc.

Ramularia Vaccinii. Pk.

Sphaerella Gallae, Ell. \& Ev.

Sphaerella Vaccinii, Cke.

Sphaeria vaccinicola, S.

Sphaeria vexata, C. \& E.

$\{$ Hysterium Vaccinii, Carm.

\{ Sporomega cladophila, (Lev.) Duby.

$\{$ Hysterium degenerans, Fr.

\{ Sporomega degenerans, Cda.

Caeorna (Uredo) Vacciniorum, Lk.

$\{$ Thecopsora Vacciniorum, Karst.

Melampsora Vacciniorum, Schroet.

Valsa delicatula, C. \& E.

$\{$ Sphaeria quadrifida, $\mathrm{S}$.

\{ Valsa quadrifida, Cke.

SValsa Peckii, Howe.

$\{$ Valsaria Peckii, Sacc.

(Pseudovalsa Peckii, Cke. 
Vaccinium macrocarpon, Ait.

Exobasidium Vaccinii, (Fcki.) Wor.

Pestalozzia Guepini. Desm.

Synchytrium Vaccinii, Thomas.

S Sphaeria cincinnata, Fr.

$\left\{\begin{array}{l}\text { Venturia cincinnata, Rostrup. } \\ \text { Ventura }\end{array}\right.$

Venturia compacta, Pk.

Vaccinium ovalifolium, Smith.

Calyptospora Goeppertiana. Kühn.

Vaccinium ovatum, Pursl.

Ceuthospora minima, Cke. \& Hark.

$\{$ Phacidium album, Phil. \& Hark.

i Coccomyces albus, Sacc.

Vaccinium Oxycoccus, L.

Ramularia multiplex, $\mathrm{Pk}$.

Venturia cincinnata, ( $\mathrm{Fr}$.) Rostrup.

Vaccinium Pennsylvanicum, Lam.

Lophodermium maculare, (Fr.) De Not.

f Calyptospora Goeppertiana, Kïhn.

Melampsora Goeppertiana, Wint.

(Erysiphe Vaccinii, S.

Microsphaera Friesii, rar. Vaccinii, C. \& P.

ULicrosphaera Vaccinii, C. S P.

Nonilia Peckiana, Sacc. \& Vogl.

Ramularia Vaccinii, Pk.

Septoria difformis, C. \& P.

Vaccininm stamineum, L.

S Xvloma punctatum, S. Syn. Car. p. p.

Rhytisma Vaccinii, (S.) Fr.

Vaccinium nliginosum, L.

Antennatula arctica, Rostrup.

Dothidella Vaccinii, Rostrup.

Exobasidium Vaccinii, (Fckl.) Wor.

Hysterium degenerans, Fr.

Lophodermium maculare, (Fr.) De Not.

Sphaeropsis cymbaespora, B. \& C.

\{ Phoma cymbispora, Sacc.

Phoma leptidea, (Fr.) Sacc.

$\{$ Podosphaera myrtillina, Kze.

Podosphaera Oxyacanthae, (DC.) D By.

Sphaerella myrtillina, Pass.

Venturia Myrtilli, Cke.

Vaccinium vacillans, Solander.

Nicrosphaera Vaccinii, (S.) C. \& P.

Vaccinium virgatum, Ait.

Rhytisma Vaccinii, (S.) Fr.

Vaccinium Vitis-Idaea, L.

Exobasidium Vaccinii, (Fckl.) Wor.

Leptostroma punctiforme, Wallr.

Lophodermium melaleucum, (Fr.) De Not.

Calyptospora Goeppertiana, Kühn.

Nelampsora Goeppertiana, Wint.

Phacidium Vaccinii, Fr.

Phyllachora latitans, (Fr.) Sacc.

Vaccinium, sp. indet.

Corticium cinereum, Fr. f Asterina clavuligera, Cke.

Dimerosporium clavuligerum, Martin.

Helminthosporium arctisporum, C. \& E.

Hymenochaete agglutinans, Ell.

$\{$ Corticium epichlorum, B. \& C.

$\{$ Hymenochaete epichlora, Cke.

Sphaeria leiostega, Ell.

$\{$ Mietasphaeria leiostega, Sacc.

Endophlaea leiostega, Cke.

Oidium compactum, C. \& E.

Peziza fusca, P.

$\{$ Peziza subiculata, S.

$\{$ Tapesia subiculata, Sacc.

$\{$ Peziza virginella, Cke.

$\{$ Dasyscypha virginella, Sacc.

Sphaeria punctulata, C. \& E.

Sphaeria picacea, C. \& E.

Anthostomella? picacea, Sacc.

Xylosphaeria picacea, C. \& E.

Anthostoma picaceum, C. \& E.

\{ Stictis linearis, C. \& E.

$\{$ Xylogramma linearis, Sacc.

Sphaeria paecilostoma, B. \& Br.

$\{$ Zignoella paecilostoma, Sacc.

Conisphaeria paecilostoma, Cke.

Sphaeria subvestita, Ell. \& Ev.

$\left\{\begin{array}{l}\text { Zignoella subrestita, Berl. \& Vogl. } \\ \text { Con }\end{array}\right.$

Conisphaeria subvestita, Cke.

\section{DIAPENSIACEAE.}

\section{Diapensia Lapponica, L.}

Sphaerella pachyasca, Rostrup.

Stictis phacidioides, Fr.

\{rochila phacidioides, Karst.

Galax, sp. indet.

Glenospora melioloides, M. A. Curtis.

\section{PLUMBAGINACEAE.}

Armeria Sibirica, Turcz.

Leptostroma herbarım, (Fr.) Lk.

Pleospora platyspora, Sacc.

Armeria vulgaris, Willd.

Aecidium Statices, Desm.

Uromyces Limonii, (DC.) Lev.

Statice Limonium, L.

$\{$ Aecidium Statices, Desm.

Aecidium Limonii, Pk.

Sphaeria Wrightii, B. \& C.

$\{$ Physalospora Wrightii, Sacc.

Laestadia Wrightii, Cke.

(Uromyces Limonii, (DC.) Lev.

Uromyces Statices, B. \& C.

Uredo Statices, De Toni.

Statice, sp. indet.

? Puccinia sclerotioidea, Cke. 
PRIMULACEAE.

Androsace occidentalis, Pursh.

$\{$ Peronospora candida, Fck\}.

$\{$ Peronospora Androsaces, Niessl.

Dodecatheon Meadia, L.

Phyllosticta Dodecathei, Trelease.

Puccinia melanconioides, Ell. \& Hark.

Glanx maritima, L.

Aecidium Glaucis, Dozy \& Molkenb.

Lysimachia quadrifolia, L.

S Aecidium Lysimachiae, S.

Uredo Lysimachiae, Spreng.

Caeoma (Aecidium) lysimachiatum, S.

, Caeoma (Aecidium) lysimachiatum, Lk.

? Aecidium Lysimachiae, (Schl.) Wallr.

Synchytrium aureum, Schrt.

Lysimachia stricta, Ait.

(Aecidium Lysimachiae, S.

$\{$ Uredo Lysimachiae, Spreng.

$\{$ Caeoma (Aecidium) Iysimachiatum, S.

? Caeoma (Aecidium) lysimachiatum, Lk.

? Aecidium Lysimachiae, (Schl.) Wallr.

Cercospora Lysimachiae, Ell. \& Halsted.

Lysimachia thyrsiflora, L.

Aecidium Lysimachiae, S. ?

Primula suffrutescens, Gray.

Uromyces Nevadensis, Hark.

Samolus Valerandi, L.

Pleospora herbarum: (P.) Rabh.

Steironema ciliatum, Raf.

?Aecidium Lysimachiae, S.

Aecidium Lysimachiae, (Schl.) Walir.

Puccinia Dayi, Clinton.

Septoria conspicua, Ell. \& Martin.

Septoria Lysimachiae, Westd.

Steironema lanceolatum, Gray.

Aecidium Lysimachiae, (Schl.) Wallr.?

Trientalis Americana, Pursh.

Septoria increscens, Pk.

Trientalis Europaea, L.

Sorosporium Trientalis, (B. \& Br.) Wor.

\section{MYRSINACEAE.}

Jacquinia armillaris, L.

Asterina paupercula, Ell. \& Ev.

\section{SAPOTACEAE.}

Bumelia lanuginosa, $\mathrm{P}$.

$\{$ Dothidea Bumeliae, S.

$\{$ Polystigma? Bumeliae, Sacc.

Bumelia, sp. indet.

Phoma circumscripta, Cke.

$\{$ Sphaeropsis maculans, B. \& C.

$\{$ Phoma maculans, Sacc.

Sphaerella Bumeliae, Cke.

\section{EBENACEAE.}

Diospyros Kaki, L. f.

Cercospora Kaki, Ell. \& Ev.

Diospyros Virginiana, L.

Syloma Diospyri, S.

$\{$ Dothidea Diospyri, Fr.

Asteroma Diospyri, M. A. Curtis.

Sphaeria Persimmons, S.

Dothidea parsimons, Fr.

Nelogramma Persimmons, M. A. Cnrtis.

Botryosphaeria Persimon, Sacc.

$\{$ Helminthosporium Diospyri, Thm.

\{ Cercospora Diospyri, Thm.

1 Cercospora fuliginosa, Ell. \& Kell.

C Cercospora atra, Ell. \& Ev.

Dendrina Diospyri, B. \& C.

Gloeosporium Diospyri, Ell. \& Ev.

Myxormia hypospila, Cke.

- Podosphaera Oxyacanthae, (DC.) D By.

Xyloma orbiculatum, S.

Jothidea orbiculata, Fr.

Phyllachora orbiculata, Sacc.

Sphaeria Diospyri, S.

$\{$ Diatrype Diospyri, M. A. Curtis.

Valsaria Diospyri, De Not.

Diospyros, sp. indet.

\{Agaricus meliigena, B. \& Cke.

$\{$ Mycena meliigena, Sacc. Syll.

Pestalozzia Guepini, Desm.

Trametes rigida, B. \& Mont.

Polyporus rigidus, Rav.

Polystictus rigidus, Cke.

Polystictus rigens, Sacc. \& Cub.

\section{STYRACACEAE.}

Halesia tetraptera, L.

Polyporus Halesiae, B. \& C.

Symplocos tinctoria, L' Her.

Exobasidium Symploci, Ell. \& Martin.

$\{$ Corticium epichlorum, B. \& C.

$\{$ Hymenochate epichlora, Cke.

Sacidium Symploci, Cke.

Septoria stigma, B. \& C.

Septoria Symploci, Ell. \& Martin.

\section{OLEACEAE.}

\section{Chionanthus Virginica, L.}

S Sphaeria piriospora, Ell.

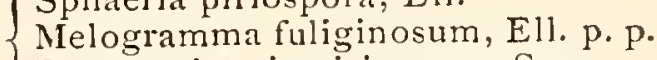

Botryosphaeria piriospora, Sacc.

$\{$ Nectria aurigera. B. \& Rav.

$\{$ Calonectria polythalama, Sacc.

Diplodia Chionanthi, C. \& E.

$\{$ Sphaeropsis diatrypea, C. \& E.

$\{$ Phoma diatrypea, Sacc.

Septoria Chionanthi, Cke.

Forestiera acuminata, Poir.

Microsphiera Alni, (DC.) Wint. 
Fraxinus Americana, L.

IYRENOMYCTES.

f Valsa fraxinicola, C. \& P.

Eutrpella fraxinicola. Sacc.

\{ Depazea fraxinicola, M. A. Curtis.

Laestadia fraxinicola, Sacc.

Leptosphacria Fraxini, Ell. \& Ev.

S Sphatria effigurata, S.

Sphaerella effigurata, Cke.

Sphaerella fraxinea, $\mathrm{Pk}$.

isphaeria fraxicola, S.

Sphaerelh fraxinicola, Cke.

Sphaeria maculiformis, P.

\{ Sphaerel\}a maculiformis, Awd.

Sphaeria samarae, $\mathrm{S}$.

Talsa fraxinina, Pk.

Valsa grisea, $\mathrm{Pk}$.

\section{SPHAEROPSIDEAE.}

Cytospora minuta, Thm.

Sphaeropsis hyalina, B. \& C.

Phoma hyalina, Sacc.

(Iacrophoma hyalina, Berl. \& Vog\}.

Plyyllosticta Fraxini, Ell. \& Martin.

Septoria Fraxini, Westd.

Septoria submaculata, Wint.

Sphaeria Spina, S.

Sphaeronema Spina, B. \& Rav.

Sphaeronema Flaxini, Pk.

Ceratostoma Spina, Sacc.

Sphaerographium Fraxini, Sacc.

Sphaeropsis biformis, Pk.

Sphaeropsis hyalina, B. \& C.

Sphaeropsis phomatella, $\mathrm{Pk}$.

\section{MELANCONIEAE.}

Gloeosporium aridum, Ell. \& Holw.

Gloeosporium decipiens, Ell. \& Ev.

\{ Gloeosporium punctiforme, Ell. \& Ev.

\{Gloeosporium Everhartii, Ell.

Gloeosporium irregulare, $\mathrm{Pk}$.

MISCELLANEA.

Aecidium Fraxini, S.

(Exosporium Tiliae, Lk.

$\{$ Coryneum clavaesporium, $\mathrm{Pk}$.

Exosporium clarisporum, Sacc.

Helotium sulfurellum, EH. \& Ev.

Phacidium fraxineum, S.

(Polyporus fraxineus, (Bull.) Fr.

Fomes fraxineus, Cke.

$\{$ Polyporus fraxinophilus, $\mathrm{Pk}$.

Fomes fraxinophilus, Sacc. Syll.

Polyporus salicinus, Fr.

$\{$ Epochnium quadratum, Cke.

Stemphylium quadratum, Sacc.

Fraxinus Oregana, Nutt.

\{ Septogloeum Fraxini, Hark.

\{ Gloeosporium Fraxini, Ell. \& Ev.

Phoma samararum, Desm.
Fraxinus pubescens, Lam.

Gloeosporium fraxineum, $\mathrm{Pk}$.

Piggotia Fraxini, B. \& C.

Sphaeria candida, $\mathrm{S}$.

$\{$ Enslinia candida, $\mathrm{Fr}$.

Poronia candida, M. A. Curtis.

Fraxinus sambucifolia, Lam.

Aecidium Fraxini, S.

$\{$ Sphaeria pruinosa, $\mathrm{Fr}$.

$\{$ Dendrophoma pruinosa, Sacc.

f Diatrype cercidicola, B. \& C.

$\{$ Hypoxylon suborbiculare, $\mathrm{Pk}$.

Michenera Atrocreas, B. \& C.

Peziza subvernalis, $\mathrm{Pk}$.

Fraxinus viridis, Michx. f.

Aecidium Fraxini, S.

Caeoma fraxinatum, Lk.

Caeoma (Roestelia) fraxinites, S.

Uredo Fraxini, Spreng.

Roestelia Fraxini, M. A. Curtis.

Cercospora Fraxini, Ell. \& Kell.

Cercosporella Fraxini, Ell. \& Kell.

Cylindrosporium Fraxini, Ell. \& Er.

Cercospora Texensis, Ell. \& Gall.

Cylindrosporium minor, Ell. \& Kell.

Cylindrosporium viridis, Ell. \& Ev.

Gloeosporium decipiens, Ell. \& Er.

$\{$ Phyllactinia guttata. Lev.

P Phyllactinia suffulta, (Reb.) Sacc.

Phyllosticta Fraxini, Ell. \& Martin.

Phyllosticta viridis, Ell. \& Kell.

Piggotia Fraxini, B. \& C.

Fraxinus, sp. indet.

PYRENOMYCETES.

Acrospermum Ravenelii, B. \& C.

(Diatrype rumpens, Cke.

$\{$ Anthostoma rumpens, Sacc.

Nummularia rumpens, Cke.

Sphaeria euomphala, B. \& C.

$\{$ Botryosphaeria euomphala, Sacc.

Byssosphaeria euomphala, Cke.

Nectria polythalama, B.

Nectria aurigera, B. \& Rav.

Calonectria polythalama, Sacc,

Calonectria aurigera, Sacc.

Chatomium elatum, Kze.

$\{$ Valsa Crataegi, Curr.

\{ Diaporthe Crataegi, Fckl.

\{ Sphaeria spiculosa, A. \& S.

\{Diaporthe spiculosa, Nits.

(Valsa angulata, Fr.

$\{$ Diatrypella angulata, Ces. \& De Not.

(Diatrypella nigroannulata, (Grev.) Nits.

Eutypa echinata, Ell. \& Ev.

$\{$ Vaisa stellulata, Fr.

Eutypella stellulata, Sacc.

\{ Sphaeria polycocca, B. \& Rav.

Fracchiaea lieterogenea, Sacc. 
Sphaeria petiolorum, S.

Sphaeria amoena, Auct. Amer.

Gnomoniella amoena, var. petiolorum, Sacc.

Hypoxylon argillaceum, (P.) Fr.

f Hypoxylon colliculosum, Rav. Fung. Am.

$\{$ ?Hypoxylon insidens, (S.) Ell. \& Ev.

Hypoxylon concentricum. (Bolt.) Grev.

S Sphaerla enteromela, S.

Hypoxylon enteromelum, B.

Hypoxylon fuscopurpureum, (S.) M. A.

Curtis.

Hypoxylon perforitium, (S.) Fr.

Hypoxylon rubricosum, (Fr.) Mont.

Hysterium samarae, Fr.

$\{$ Hysterium Fraxini. P.

Hysterographium Fraxini, De Not.

Massaria vomitoria, B. \& C.

Sphaeria albicans, S. Syn. Car.

Sphaeria mastoidea, Fr.

Melomastia Friesii, Nits.

Trematosphaeria mastoidea, Wint.

Lonisphaeria Friesii, Cke.

Pleospora pustulans, Ell. \& Ev.

S Sphaeria Aquila, Fr.

I Rosellinia Aquila, De Not.

$\{$ Sphaeria myriocarpa, Fr.

\{ Rosellinia myriocalpa, Cke.

Sphaeria foveolaris, S. Am. bor.

(Sphaeria obducens, Cke.

$\{$ 'Teichospora obducens, Fck].

Strickeria obducens, Cke.

Sphaeria corniculata, Ehrh.

$\{$ Diatrype corniculata, Rav. Fung. Car.

Valsa corniculata, M. A. Curtis.

Melogramma insidens, B. nec S.

$\{$ Melogramma grandinea, B. sec. Cke.

Valsaria grandinea, Berl. \& Vogl.

Valsaria purpurea, Pk.

\section{SPHAEROPSIDEAE.}

Cytospora Micheneri, B. \& C.

$\{$ Hendersonia sphaerosperma, B. \& C.

$\{$ Dichomera sphaerosperma, Cke.

Phoma infossa, Ell. \& Ev.

Phyllosticta osteospora, Sacc.

Phyllosticta variegata, Ell. \& Ev.

Septoria Besseyi, Pk.

Sphaeronemella carnea, Ell. \& Ev.

Sphaeropsis Pennsylvanica, B. \& C.

\section{IYPHOMYCETES.}

Cercospora fraxinites, E11. \& Ev.

Cladosporium simplex, S.

\{Helicoma Berkeleyi, M. A. Curtis.

\{Helicosporium Berkeleyi, Sacc.

Sporidesmium compactum, B. \& C.

$\{$ Tubercularia nigricans, S.

\{ Tubercularia dubia, Lk.
DISCOMYCETES.

$\int$ Peziza Fraxini, S.

Tympanis Fraxini, Fr.

Cenangium Fraxini, Tul.

\{ Peziza puberula, B. \& C.

Dasyscypha puberula, Sacc.

Vibrissea turbinata, Phil.

Helotium vibrisseoides, Pk.

Gorgoniceps turbinata, Sacc.

Gorgoniceps vibrisseoides, Sacc.

Peziza atrata, P.

Stictis radiata, $P$.

HYMENOMYCETES.

$\{$ Agaricus haerens, Pk.

Crepidotus haerens, Pk.

Coprinus fuscescens, (Schaeff.) Fr.

Corticium hepaticum, B. \& C.

$\{$ Corticium Oakesii, B. \& C.

Aleurodiscus Oakesii, Cke.

$\{$ Tremella albida, Huds.

\{ Exidia albida, Brefeld.

Hydnum coralloides, Scop.

Hydnum strigosum, Swartz.

Lentinus Dunalii, (DC.) Fr.

Lentinus vulpinus, Fr.

Marasmius minutulus, Pk.

Panus dealbatus, B.

\{ Auricularia mesenterica, (Dicks.) P.

Phlebia mesenterica, Fr.

Phlebia pileata, $\mathrm{Pk}$.

(Polyporus conchatus, (P.) Fr.

Fomes conchatus, Cke.

Mucronoporus conchatus, Ell. \& Ev.

Polyporus corticola, Fr., var. b.

Polyporus immitis, $\mathrm{Pk}$.

$\{$ Polyporus molluscus, (P.) Fr.

Poria mollusca, Cke.

Sphaeria pocula, S.

$\{$ Enslinia pocula, Fr.

Polyporus pocula, B. \& C.

( Polyporus subspadiceus, Fr.

\{ Poria subspadicea, Cke.

Schizophyllum comnune, Fr.

Peziza amorpha, $\mathrm{P}$.

Thelephora amorpha, Fr.

Corticium amorphum, Fr.

Aleurodiscus amorphus, Rabh.

Tremella colorata, Pk.

MISCELLANEA.

Didymium eximium, Pk.

STrichia angulata, $\mathrm{S}$.

$\{$ Perichacna angulata, Fr.

Semaspora magna, Grev.

Stilbospora magna, Berk.

Melanconium magnum, Berk. 
Ligustrum vulgare, L.

Sphaeria Ligustri, S.

Stictis Ligustri, S.

Olea Europaea, L.

Antennaria elacophila, Mont.

Olea, sp. indet.

Scptoria serpentaria, Ell. \& Martin.

Osmanthus Americanus, Benth. \& Hook.

Asterina discoidea. Ell. \& Martin.

Asterina oleina, Cke.

Asterina purpurea, Ell. \& Martin.

Capnodium elongatum, B. \& Desm.

Dialonectria erubescens, (Rob.) Cle.

Dothidea coccodes, (Lev.) B. \& C.

Fumago salicina, (Mont.) Tul.

Helotium castaneum, Sacc. \& Ell.

Neliola amphitricha, Fr.

Sphaerella oleina,Cke.

Phyllosticta oleina Cke.

Phyllosticta sinuosa, E11. \& Martin.

Syringa vulgaris, L.

\{ Sphaeria Syringae, S. Am. Bor.

Botryosphaeria Syringae, Cke.

Hysterium Syringre, S.

Triblidium dealbatum, Gerard.

Triblidium Syringae, Cke.

Pseudographis dealbata, Sacc.

Iysterographium Syringae, Sacc.

Erysiphe Syringae, S.

Microsphaera Friesii, Lev.

Erysiphe penicillata, Rav. Fung. Car.p.p.

Microsphaera Friesii, Lev., var. Syringae, C. \& P.

Microsphaera Alni, (DC.) Wint.

Phyllosticta Halstedii, Ell. \& Ev.

$\{$ Polyporus scutellatus, S.

Fomes scutellatus, Cke.

Sphaeropsis Syringae, Pk. \& Clinton.

Syringa, sp. indet.

S Sphaeria albomaculans, $S$.

Amphisphaeria albomaculans, Cke.

Cenangium fatiscens, $\mathrm{S}$.

Dacryomyces Syringae, (Schum.) Fr.

Dacryomyces syringicola, B. \& C.

Lichenopsis sphaeroboloidea, S.

Myrothecium roridum. Tode.

Phoma Syringae, B. \& C.

Phyllosticta Syringne, Westd.

Sporotrichum nitens, (P.) Lk.

Sphaeria decorticans, Fr.

Y Valsa decorticans, $\mathrm{Fr}$.

Sphatia leucopis, Fr.

(Valsa lencopis, Fr.

Valsa salicina, (P.) Fr.

APOCYNACEAE.

Amsonia angustifolia, Michx.

Torula microsora, Thm.
Coleosporium apocynaceum, Cke.

Amsonia Tabernaemontana, Walt.

Aecidium Apocyni, S.

Amsonia, sp. indet.

\{ Trichobasis Amsoniae, Cke.

Uredo Amsoniae, De Toni.

Apocynum androsaemifolium, L.

Cercospora Apocyni, Ell. \& Kell.

Cylindrosporium Apocyni, Ell. \& Ev.

Apocynum cannabinum, $\mathrm{L}$.

$\{$ Aecidium Apocyni, S.

\{ Caeoma (Aecidium) apocynatum, Lk.

$\{$ Cercospora Apocyni, Ell. \& Kell.

\{ Cercosporella A pocyni, Ell. \& Kell.

$\{$ Septogloeum Apocyni, Pk.

\{Glocosporium Apocyni, Ell. \& Ev.

Nectria Apocyni, Pk.

$\{$ Phyllosticta Apocyni. Trelease.

\{Phyllosticta Apocyni, Ell. \& Martin.

Septoria littorea, Sacc.

Apocynum, sp. indet.

Erysiphe Montagnei, Lev.

? Pestalozziella Andersoni, Ell. \& Gall.

Nerium Oleander, L.

Capnodium Nerii, Rabh.

Capnodium elongatum, B. \& Desm., var. Nerii, Clie.

Chaetophoma foeda, Sacc.

Dicranidion fragile, Hark.

Diplodia Nerii, Speg.

Haplosporella Nerii, Sacc.

Macrosporium Nerii, Cke.

Pestalozzia stellata. B. \& C., var. Nerii, Cke.

Phyllosticta Nerii, Westd.

Septoria oleandrina, Sacc.

Theclospora lateralis, Hark.

Trachelospermum difforme, Gray.

Cercospora repens, Ell. \& Ev.

Vinca minor, L.

$\{$ Diplodia Vincae, Sacc.

Sphaeropsis Vincae, Sacc. \& Wint.

Strumella Vincae, Cke. \& Hark.

\section{ASCLEPIADACEAE.}

Acerates longifolia, Elliott.

Aecidium Jamesianum, $\mathrm{Pk}$.

Acerates viridiflora, Elliott.

Aecidium Jamesianum, Pk.

Asclepiodora viridis, Gray.

$\{$ Cercospora Asclepiodorae, Ell. \& Kell.

\{ Cercospora fraxinea, E1l. \& Ev.

Asclepias amplexicaulis, Michx.

Macrosporium asclepiadeum, Cke.

Asclepias arenaria, Torr.

Accidium Jamesianum, $\mathrm{Pk}$.

$\{$ Cercospora clavata, (Gerard) Ckc.

$\{$ Cercospora Asclepiadis, Ell. 
Asclepias Cornuti, Decaisne.

Aecidium Jamesianum, Pk.

Alternaria tenuis, Nees.

$\{$ Cercospora Asclepiadis, Ell.

$\{$ Cercospora venturioides, $\mathrm{Pk}$.

$\{$ Asteroma capnoides, Ell.

Dimerosporium capnoides, Martin.

Gloeosporium fusarioides, Ell. \& Kell.

Hysterium librincola, S.

Sphaeria cinerascens, S.

$\{$ Sphaerella cinerascens, Cke.

Laestadia cinerascens, Sacc.

$\{$ Dothidea Asclepiadis, S.

TPhyllachora Asclepiadis, Sacc.

$\{$ Dothidea cinerascens, S.

$\{$ Phyllachora cinerascens, Sacc.

Phyllactinia guttata, Lev.

Phyllosticta Cornuti, Ell. \& Kell.

Sphaeria Asclepiadis, S.

Sphaeria lilacina, S.

Sphaeropsis sphaerospora, $\mathrm{Pk}$.

Trichobasis Howei, Pk.

$\{$ Uromyces Asclepiadis, Cke.

Uromyces Howei, Pk.

Asclepias incarnata, L.

Helminthosporium clavatum, Gerard.

Virgasporium clavatum, Cke.

Cercospora clavata, Cke.

Cercospora Asclepiadis, Ell.

I Cercospora venturioides, $\mathrm{Pk}$.

$\{$ Dothidea ornans, S.

$\{$ Phyllachora ornans, Sacc.

Septoria asclepiadicola, Ell. \& Ev.

Uromyces Howei, Pk.

Asclepias Jamesii, Torr.

Aecidium Jamesianum, Pk.

Cercospora Ascelepiadis, Ell.

Cercospora Asclepiodorae, Ell. \& Kell.

Asclepias obtusifolia, Michx.

Cercospora clavata, (Gerard) Cke.

Asclepias ovalifolia, Decaisne.

Aecidium Janesianum, $\mathrm{Pk}$.

Asclepias rizbrá, L.

Septoria asclepiadicola, Ell. \& Ev.

Asclepias speciosa, Torr.

Aecidium Jamesianum, $\mathrm{Pk}$.

Cercospora clavata, (Gerard) Cke.

Asclepias tuberosa, L.

Aecidium Jamesianum, Pk.

Diaporthe Asclepiadis, Ell. \& Ev.

Phyllosticta tuberosa, Ell. \& Martin.

Uromyces Howei, Pk.

Asclepias verticillata, L.

Aecidium Brandegei, $\mathrm{Pk}$.

Nectria Apocyni, Pk.

Asclepias, sp. indet.

Cladosporium molle, Cke.
Diplodia asclepiadea, C. \& E.

Fusarium roseum, Lk.

?Pestalozziella Andersoni, Ell. \& Gall.

Gonolobus, sp. indet.

Meliola furcata, Lev.

\{ Didymium obrusseum, B. \& C.

\{Physarum obrusseum, Rostf.

Puccinia Gonolobi, Rav.

\section{LOGANIACEAE.}

\section{Gelsemium sempervirens, Ait.}

Asterina stomatophora, Ell. \& Martin.

Cladosporium maculans, $\mathrm{S}$.

Sphaeria Jasmini, S.

Gelsemium, sp. indet.

Nectria mbicarpa, Cke.

$\{$ Sphaeria gelsemiata, Cke.

\{ Physalospora gelsemiata, Sacc.

S Sphaeria Aquila, Fr.

\{ Rosellinia Aquila, De Not.

\section{GENTIANACEAE.}

Frasera speciosa, Dougl.

Capnodium puccinioides, Ell. \& Ev.

Frasera, sp. indet. ? "Columbo.")

$\{$ Leptosphaeria Harknessiana, Ell. \& Er.

\{. Heptameria Harknessiana, Cke.

Gentiana affinis, Griseb.

Gentiana Andrewsii, Griseb.

Gentiana puberula, Michx.

Gentiana quinqueflora, Lam., var. occidentalis, Gray.

Puccinia Gentianae, (Strauss) Lk.

Gentiana alba, Muhl.

Phyllosticta gentianicola, (DC.) Fr.

Gentiana crinita, Fröl.

Cercospora gentianicola, Ell. \& Ev.

Gentiana linearis, Fröl.

Cercospora Gentianae, Pk.

Gentiana ochroleuca, Fröl.

$\{$ Dothidea Gentianae, S.

$\{$ Phyllachora Gentianae, Sacc.

Halenia deflexa, Griseb.

Puccinia Haleniae, Arth. \& Holw.

Limnanthemum lacunosum, Griseb.

Doassansia decipiens, Wint.

Menyanthes trifoliata, L.

(Protomyces Menyanthis, D By.

$\{$ Physoderma Menyanthis, D By.

Cladochytrium Menyanthis, D By.

Pleurogyne rotata, Griseb.

Sphaerella pachyasca, Rostrup.

Sabbatia angularis, Pursh.

Cercospora Sabbatiae, Ell. \& Ev. 


\section{POLEMONIACEAE.}

Gilia ciliata, Benth.

Gilia divaricata, Torr.

Gilia lougitlora, Don.

Puccinia Giliae, Ilark.

Gilia gracilis, Hook.

Aecidium Giliae, Pk.

$\{$ Puccinia plumbaria, Pk.

\{ Puccinia Wilcoxiana, Thm.

Sphaerotheca Castagnei, Lev.

Gilia linearis, Gray.

luccinia plumbaria, $\mathrm{Pk}$.

Sphaerotheca Castagnei, Lcv.

Gilia Nuttallii, Gray.

Aecidium Giliae, Pk.

Phlox caespitosa, Nutt.

Sphaerella pachyasca, Rostrup.

Phlox caespitosa, Nutt., var. condensata, Gray.

Puccinia Giliae, Hark.

Phlox divaricata, L.

Aecidium Polemonii, $\mathrm{Pk}$.

Accidium Vilcoxianum, Thm.

Cercospora omphakodes, Ell. \& Holw.

Puccinia plumbaria, $\mathrm{Pk}$.

\{ Septoria Phlogis, Sacc. \& Speg.?

\{ Septoria divaricata, Ell. \& Ev.

Vermicularia phlogina, Fairman.

Phlox Douglasii, Hook.

Aecidium Wilcoxianum, Thm.

Puccinia plumbaria, Pk., var. phlogina, Ell.

Sphaerella pachyasca, Rostrup.

Phlox Drummondii, Hook.

Erysiphe lamprocarpa, Lev. p. p.

Oidium Drummondii, Thm.

Phlox longifolia, Nutt.

Puccinia plumbaria, Pk.

Sphaerella phlogina, Ell. \& Ev.

Phlox paniculata, L.

(Erysiphe Cichoraccarum, DC.

$\{$ Erysiphe Plulogis, S.

Erysiphe lamprocarpa, Lev. p. p.

\{ Dothidea Phlogis, S.

Phyllachora Phlogis, Sacc.

S Sphaeria Dematium, P.

$\{$ Vermicularia Dematium, Fr.

Phlox pilosa, L.

Aecidium Polemonii, Pk.

Phlox, sp. indet.

\{ Erysiphe communis, (Wallr.) Sch?.

Erysiphe Martii, Lcv.

\{ Pleospora ciliata, Ell.

\{ Pyrenophora ciliata, Sacc.
Polemonium humile, Willd.

Phyllachora? Polemonii, Hark.

Pleospora herbarum, (P.) Rabh.

Polemonium reptans, L.

Accidium Polemonii, Pk.

Septoria Polemonii, Thm.

Septoria polemoniicola, Ell. \& Martin.

\section{HYDROPHYLLACEAE.}

Ellisia Nyctelea, L.

Aecidium Hydrophylli, Pk.

Eriodictyon glutinosum, Benth.

Torula glutinosa, Cke. \& Hark.

Hydrophyllum appendiculatum, Michx.

Hydrophyllum Cünadense, L.

Aecidium Hydrophylli, Pk.

Hydrophyllum Virginicum, L.

Aecidium Hydrophylli, Pk.

$\{$ Erysiphe Cichoracearum, DC.

Erysiphe lamprocarpa, Lev. p.p.

Puccinia Hydrophylli, Pk. \& Clinton.

Sphaerotheca Castagnei, Lev.

Phacelia circinata, Jacq. f.

$\Lambda$ ecidium Phaceliae, Pk.

Erysiphe Cichoracearum, DC.

Phacelia hispida, Gray.

Phacelia ramosissima, Dougl.

Aecidium Phaceliae, $\mathrm{Pk}$.

Phacelia Menziesii, Torr.

Erysiphe Cichoracearum, DC.

Phacelia sp. indet.

fDichomera Phaceliae, Cke. \& Hark.

\{ Camarosporium Phaceline, Sacc.

Diaporthe Phaceline, Cke. \& Hark.

\section{BORRAGINACEAE.}

\section{Cynoglossum Virginicum, L.}

$\{\Lambda$ ecidium asperifolii, $P$.

\{ Caeoma (Aecidium) urticatum, S. p.p.

Peronospora Cynoglossi, Burrill.

Cynoglossum, sp. indet.

Diplodia microscopica, Cke. \& Hark.

Echinospermum floribundum, Lehm.

Peronospora Myosotidis, D By.

Echinospermum Redowskii, Lehm.

Erysiphe Cichoracearum, DC.

Oidium erysiphoides, Fr.

Peronospora Myosotidis, D By.

Echinospermum Redowskii, Lehm., var. cupulatum, Gray.

Peronospora Cynoglossi, Burrill, var. Echinospermi, Swingle. 
Echinospermum Virginicum, Lehm.

$\{$ Erysiphe Cichoracearum, DC.

Erysiphe lamprocarpa, Lev. p. p.

Heliotropium Curassavicum, L.

Aecidium biforme, $\mathrm{Pk}$.

Aecidium Heliotropii, Tracy \& Gall.

Cercospora Heliotropii, Ell. \& Ev.

Kranitzkia, sp. indet.

Synchytrium Myosotidis, Kiihn.

Lithospermum arvense, $\mathrm{L}$.

(Erysiphe Cichoracearum, DC.

$\{$ Erysiphe lamprocarpa, Lev. p. p.

Erysiphe horridula, Lev. p. p.

Mertensia maritima, Don.

Septoria Stenhammariae, Roṣtrup.

Sphaerella pachyasca, Rostrup.

Mertensia Sibirica, Don.

$\{$ Erysiphe Cichoracearum, DC.

Erysiphe lamprocarpa, Lev. p. p.

Puccinia Mertensiae, Pk.

Mertensia, sp. indet.

Sphaeria Mertensiae, Ell.

$\{$ Leptosphaeria Mertensiae, Sacc.

Heptameria Mertensiae; Cke.

Myosotis verna, Nutt.

Aecidium Myosotidis, Burrill.

Peronospora Myosotidis, D By.

Onosmodium Carolinianum, DC.

Aecidium asperifolii, P. ?

Sphaeria onosmodina, Pk. \& Clinton.

$\{$ Sphaerella onosmodina, $\mathrm{Pk}$.

Didymella onosmodina, Sacc.

\section{CONVOLVULACEAE.}

\section{Convolvulus arvensis, $\mathrm{L}$.}

Puccinia Convolvuli, (P.) Cast.

Convolvulus luteolus, Gray.

Dothiden (Plowrightia) Calystegiae, Cke. \& Hark.

Puccinia Convolvuli, (P.) Cast.

Convolvulus sepium, L.

$\{$ Aecidium Calystegiae, Cast.

\{Aecidium dubium, Clinton.

Dothidea (Plowrightia) Calystegiae, Cke \& Hark.

Mazzantia sepium, Sacc. \& Penz.

Puccinia Convolvuli, (P.) Cast.

Septoria Calystegiae, Westd.

$\{$ Septoria Convolvuli, Desm.

Septoria flagellaris, Ell. \& Ev.

Convolvulus villosus, Gray.

Puccinia Convolvuli, (P.) Cast.

Convolvulus, sp. indet.

Phyllosticta Batatas, (Thm.) Cke.
\{ Sphaerella brachytheca, Ckc. \& Hark.

\{ Sphaerella Harknessii, Sacc.

Cuscuta Gronovii, Willd.

Protomyces Martindalei, Pk.

Evolvulus argenteus, Pursh.

Puccinia Lithospermi, Ell. \& Kell.

Evolvulus, sp. indet.

Aecidium Cressae, DC.

Ipomoea Batatas, Lam.

Ascophora nucuum, Cda.

Botrytis nivosa, Cke.

Cladosporium tuberum, Cke.

Coniosporium epiphyllum, Sacc.

Cystopus cubicus, Auct. p. p.

$\{$ Cystopus Convolvulacearum, Zalewski.

Cystopus Ipomoeae-panduranae, (S.) Farl.

Fusarium tuberum, Cke.

Mucor Mucedo, L.

Mystrosporium polytrichum, Cke.

Ozonium auricomum, Lk.

$\{$ Depazea Batatas, Thm.

Phyllosticta Batatas, Cke.

Phyllosticta bataticola, Ell. \& Martin.

$\{$ Sclerotium Batatas, S. ined.

\{ Rhizoctonia Batatas, Fr.

Typhula gyrans, (Batsch) Fr.

Ipomoea coccinea, L.

Vermicularia Ipomoearum, S.

Ipomoea commutata, Roem. \& Sch.

Cystopus cubicus, Auct. p. p.

Aecidium Ipomoene, B.

Cystopus Tragopogonis, Auct. p. p.

Cystopus Ipomoeae-panduranae, (S.) Farl.

Puccinia crassipes, B. \& C.

Ipomoea hederacea, Jacq.

Coleosporium Ipomoeae, (S.) Burrill.

$\{$ Cystopus Convolvulacearum, Zalewski.

\{ Cystopus Ipomoeae-panduranae, (S.) Farl.

Ipomoea Jalapa, Pursh.

(Cystopus cubicus, Auct. p. p.

$\{$ Cystopus Tragopogonis, Auct. p. p.

Cystopus Ipomoeae-panduranae, (S.) Farl.

Ipomoea lacunosa, $\mathrm{L}$.

Cercospora Ipomoeae, Wint.

Coleosporium Ipomoeae, (S.) Burrill.

Cystopus Ipomoeae-panduranae, (S.) Farl.

Ipomoea leptophylla, Torr.

(Cystopus cubicus, Auct. p.p.

$\{$ Cystopus Convolvulacearum, Zalewski.

Cystopus Ipomoeae-panduranae, (S.) Farl.

Ipomoea Nil, Roth.

Cystopus Ipomoeae-panduranae, (S.) Farl.

Ipomoea pandurata, Meyer.

Cercospora Ipomoeae, Wint.

(Uredo Ipomoeae, S.

$\{$ Caeoma Ipomoeae, Lk

Coleosporium Ipomoeae, Burrill. 
Accidium Ipomocae-panduranae, S.

Uredo Convolvuli, Spreng.

Caeoma (Aecidium) convolvulatum, Lk.

Aecidium rutilum, Bon.

? Accidium Convolvuli, M. A. Curtis.

Cystopus cubicus, Auct. p. p.

Aecidium I pomocae, $B$.

Cistopus Convolvulacearum, Zalewski.

Cistopus Tragopogonis, Auct. p. p.

Cystopus I pomoeae-pandurame, Farl.

Phyllosticta Ipomoeae, Ell. \& Kell.

Ipomoea purpurea, Lam.

Cercospora viridula, Ell. \& Ev.

Sphaeria capsularum, S.

Vermicularia Ipomoearum, S.

Ipomoea triloba, L.

Uredo Ipomoeae, S.

Caeoma I pomoene, Lk.

Colcosporium Ipomoeae, Burrill.

Xyloma sphaerioides, S. Syn. Car.

Ipomoea, sp. indet.

f Puccinia crassipes, B. \& C. ?

\{ Puccinia Ipomoeae, Cke.

\section{SOLANACEAE.}

\section{Capsicum annuum? L.}

Gloeosporium piperatum, Ell. \& Ev.

Capsicum, sp. indet.

Labrella Capsici, Fr.

Camaesaracha nana, Gray.

Aecidium Solani, Mont.

Puccinia Solani, Ckc.

Datura Stramonium, L.

Cercospora Daturae, $\mathrm{Pk}$.

SMacrosporium Solani, Ckc.

\{ Macrosporium Cookci, Sacc.

Macrosporium Solani, Ell. \& Martin.

Phlyctaena arcuata, B.

Datura Tatula, L.

Sphacria Daturae, S.

Datura, sp. indet.

Septoria phlyctacnoides, B. \& C.

\{ Tremella Stictis, P.

A Agyrium rufum, Fr.

Lycium Andersonii, Gray.

Puccinia tumidipes, $\mathrm{Pk}$.

Lycium Californicum, Nutt.

Puccinia globosipes, $\mathrm{Pk}$.

Lycium vulgare, Dunal.

Cercospora Lycii, Ell. \& llalsted.

Phyllosticta Lycii, Ell. \& Kcll

Puccinia Lycii, Kalchb.

Lycium, sp. indet.

Diplodina ramulorum, Ell. \& EV.

Tuhercularia subpedicellata, S.
Lycopersicum esculentum, Mill.

Cladosporium fulvum, Cke.

Cladosporium herbarum, (P.) Lk.

Dendryphium cladosporioides, Ell. \& Ev.

(Nectria perpusilla, Rav. Fung. Car.

$\{$ Nectria Peponum, B. \& C.

Dialonectria Peponum, Cke.

Fusarium Lycopersici, Sacc.

Fusarium Solani, (Mont.) Sacc.

Gloeosporium phomoides, Sacc.

Helminthosporium rhopaloides, Fres.

$\{$ Macrosporium Solani, Cke.

\{Macrosporium Cookei, Sacc.

Macrosporium Tomato, Cke.

Oidium lactis, Fres.

$\{$ Sphaeria rubella, $P$.

Ophiobolus porphyrogonus, (Tode) Sacc.

Phyllosticta Lycopersici, Pk.

Phytophthora infestans, (Mont.) D By.

f Sporidesmium herbarum, Cke.

\{ Sirodesmium herbarum, Sacc.

Stachyobotrys atrogrisea, Ell. \& Ev.

Nicotiana Bigelovii, Watson.

Peronospora sordida, B. \& Br.?

Nicotiana glauca, Graham.

Peronospora Hyoscyami, D By.

Physalis la aceolata, Michx.

Aecidium Solani, Mont.

Cercospora diffusa, Ell. \& Ev.

Cercospora Physalidis, Ell.

Cercospora Physalidis, Ell., var. concentrica, C. \& E.

Entyloma Physalidis, (Kalchb. \& Cke.) Wint.

Physalis pubescens, L.

Cercospora Physalidis, Ell.

Entyloma Physalidis, (Kalchb. \& Cke.) Wint.

Leptosphaeria Physalidis, Ell. \& Ev.

Physalis Virginiana, Mill.

Aecidium Solani, Mont.

(Protomyces Physalidis, Kalch. \& Cke.

$\{$ Entyloma Besseyi, Farl.

Entyloma Physalidis, Wint.

Physalis viscosa, L.

\{ Aecidium Solani, Mont.

\{ Aecidium Physalidis, Burrill.

$\{$ Entyloma Besseyi, Farl.

Entyloma Physalidis, (Kalchb. \& Ckc.) Wint.

Puccinia Physalidis, $\mathrm{Pk}$.

Solanum Dulcamara, L.

$\{$ Ramularia Dulcamarae, Pk.

Cercospora Dulcamarae, Ell. \& Ev.

Diplodia Dulcamarae, Fckl.

Solanum Melongena, L.

$\{$ Polyactis fascicularis, Cda.

Botrytis fascicularis, Sacc.

Mystrosporium polytrichum, Cke.

Phlyctaena arcuata, B. 
Solanum nigrum, L.

Entyloma Besseyi, Farl.

Macrophoma subconica, Ell. \& Ev.

Solanum triflorum, Nutt.

Entyloma Physalidis, (Kalchb. \& Ckc.) Wint.

Solanum tuberosum, L.

$\{$ Nectria Brassicae, Ell. \& Sacc.

\{ Dialonectria Brassicae, Ell. \& Ev.

Fusarium diplosporum, C. \& E.

$\{$ Fusisporium roseolum, H.O.Stephens.

Fusarium roscolum, Sacc.

$\{$ Fusisporium Solani, Mart.

Fusarium Solani, Sacc.

Sphaeria comatella, C. \& E.

$\{$ Leptosphaeria comatella, Sacc.

Heptameria comatella, C. \& E.

$\{$ Sphaeria Doliolum, P.

Leptosphaeria Doliolum, De Not.

Macrosporium Solani, Ell. \& Martin.

Sphaeria rubella, $P$.

Raphidospora rubella, Fckl.

Ophiobolus porphyrogonus, (Tode) Sacc.

Phoma eupyrena, Sacc.

$\{$ Sphaeria nebulosa, $P$.

Y Phoma nebulosa, Mont.

Botrytis infestans, Mont.

$\{$ Peronospora infestans, Casp.

Phytophthora infestans, D By.

Torula herbarum, Lk.

Verticillium lateritium, (B.) Cke.

Volutella ciliata, (Lk.) Fr.

Solanum umbelliferum, Esch.

Phoma Solani, Cke. \& Hark.

Solanum, sp. indet.

$\{$ Botrytis diffusa, var. b, S., Syn. Car.

Botrytis ramulosa, Lk.

Phyllosticta Solani, Ell. \& Martin.

Sphaeria rubicunda, S.

Sphaeria Solani, P.

\section{SCROPHULARIACEAE.}

Bartsia alpina, L.

Astcroma Bartsiae, Rostrup.

Mollisia atrata, (P.) Karst.

Septoria cercosperma, Rostrup.

Castilleia coccinea, Spreng.

Aecidium Pentastemonis, S.

Castilleia sessiliflora, Pursh.

Aecidium Pentastemonis, S.

Chelone glabra, L.

Aecidium Chelonis, Gerard.

Erysiphe Cichoracearum, DC.

Erysiphe Chelones, S.

Erysiphe lamprocarpa, Lev. p. p.

Erysiphe communis, Auct. Amer. p. p.

Microthyrium microscopicum, Desm.
Periconia albiceps, $\mathrm{Pk}$.

Septoria Wilsoni, Clinton.

Collinsia bartsiaefolia, Benth.

Entyloma Collinsiae, Hark.

Collinsia parviflora, Dougl.

Aecidium Collinsiae, Ell. \& Ev.

Gerardia grandiflora, Benth.

Sphaerotheca Castagnei, Lev.

Gerardia quercifolia, Pursh.

Aecidium Gerarcliae, Pk.

Cercospora clavata, (Gerard) Ckc.

Erysiphe Gerardiae, S.

$\{$ Sphaeria comatella, C. \& E.

\{Leptosphaeria comatella, Sacc.

Vermicularia Gerardiae, S.

Gratiola ramosa, Walt.

Septoria Gratiolae, Ell. \& Martin.

Herpestis, sp. indet.

Vermicularia rectispora, Cke.

Linaria Canadensis, Dumont.

Peronospora Linariae, Fckl.

Linaria vulgaris, Mill.

Entyloma Linariae, Schrt.

$\{$ Peziza corneola, C. \& P.

Heterosphaeria Linariae, (Rabh.) Rehm.

Mimulus alatus, Solander.

Cercospora Mimuli, Ell. \& Ev.

Mimulus glutinosus, Wendl.

\{ Amphisphaeria dothideaspora, Cke. \& Hark.

\{ Melanomma dothideaspora, Cke. \& Hark.

Sphaeria anisometra, Cke. \& Hark.

$\{$ Metasphaeria anisometra, Sacc.

Endophlaea anisometra, Cke. \& Hark.

Stictis radiata, P., var. pumila, Cke. \& Hark.

Mimulus Iuteus, L.

Erysiphe Cichoracearum, DC.

Ramularia Mimuli, Ell. \& Kell.

Mimulus ringens, $\mathrm{L}$.

Aecidium Pentastemonis, S.

Aecidium Pentastemonis, S., forma Mimuli, Wint.

Ramularia Mimuli, Ell. \& Kell.

Rhabdospora Kellermani, Ell. \& Martin.

Septoria Mimuli, Ell. \& Kell.

$\{$ Septoria Mimuli, Wint.

Septoria purpureocincta, Wint.

Mimulus, sp. indet.

Diatrype prominens, Cke. \& Hark.

Pedicularis Canadensis, L.

Puccinia Clintonii. Pk.

Pedicularis euphrasioides, Stephan.

Helminthosporium nanum, Nees.

Phoma Sceptri, Karst.

Sphaerella trichuphila, Karst.

Pedicularis flammea, L.

Phoma Sceptri, Karst. 
Pleospora vulgaris, Nicssl.

Sphaerella trichophila, Karst.

Pedicularis hirsuta, L.

Phoma herbarum, IVestd.

Phoma irregularis, Rostrup.

Phoma Sceptri, Karst.

Pleospora herbarum, (P.) Rabh.

Pleospora vulgaris, Niessl.

Pyrenophora chrysospora, (Niessl.) Sacc.

Sphaerella pedicularis, Karst.

Sphaerella trichophila, Karst.

Pedicularis Langsdorffi, Fisch., var. Ianata, Gray.

Sphaerella Pedicularis, Karst.

Pedicularis Lapponica, L.

Phoma herbarum, Westd.

Phoma Sceptri, Karst.

Pyrenophora comata, (Niessl.) Sacc.

Pentstemon barbatus, Nutt., var. Torreyi, Gray.

Aecidium Pentastemonis, S.

Pentstemon breviflorus, Lindl.

Triblidium turgidulum, Phil. \& Hark.

Pentstemon caeruleus, Nutt.

Aecidium Pentastemonis, S.

Pentstemon centranthifolius, Benth.

Septoria Pentstemonis, Ell. \& Ev.

Pentstemon Cobaea, Nutt.

Aecidium Pentastemonis, S.

Cercospora Pentstemonis, Ell. \& Kell.

Pentstemon corymbosus, Benth.

Septoria Pentstemonis, Ell. \& Ev.

Pentstemon glaber, Pursh.

Aecidium Pentastemonis, S.

Pentstemon gracilis, Nutt.

$\{$ Aecidium Pentastemonis, S.

\{ Caeoma (Aecidium) pentstemoniatum, S.

Pentstemon grandiflorus, Nutt.

Aecidium Pentastemonis, S.

Cercospora Pentstemonis, Ell. \& Kell.

Pentstemon laevigatus, Solander, var. Digitalis, Gray.

Septoria Pentstemonis, Ell. \& Ev.

Pentstemon linarioides, Gray.

Puccinia Pentstemonis, Pk.

Pentstemon pubescens, Solander.

(Aecidium Pentastemonis, S.

$\{$ Caeoma (Aecidium) pentstemoniatum, S.

Uredo Chelones, Spreng.

Scrophularia Californica, Cham.

Coniothyrium herbarum, C. \& E.

Coniothyrium Scrophulariae, (Fckl.) Sacc.

Diaporthe immutabilis, Cke. \& Hark.

Leptosphaeria consessa, (C. \& E.) Sacc.

Peronospora sordida, B. \& Br.
Septonema toruloideum, C. \& E.

Trichaegum atrum, Preuss.

Scrophularia nodosa, L., var. Marilandica, Gray.

Cylindrosporium Scrophulariae, Sacc. \& Ell.

(Lophiostoma Scrophulariae, Pk.

$\{$ Lophiotrema Scrophulariae, Sacc.

Lophiosphaera Scrophulariae, Cke.

Peronospora sordida, B. \& Br.

Rhabdospora Kellormani, Ell. \& Martin.

$\{$ Septoria Scrophulariae, Westd.

\{ Septoria Scrophulariae, Pk.

Sphaerella decidua, Ell. \& Kell.

Seymeria macrophylla, Nutt.

Fusarium parasiticum, Ell. \& Kell.

Puccinia Seymeriae, Burrill.

Synthyris, sp. indet.

Puccinia acrophila, Pk.

Verbascum Blattaria, L.

Septoria verbascicola, B. \& C.

Verbascum Thapsus, L.

Cercospora verbascicola, Ell. \& Ev.

\{ Sphaeria comatella, C. \& E.

Leptosphaeria comatella, Sacc.

\{ Sphaeria Thapsi, S.

Lophiostoma Thapsi, Sacc.

Phacidium capsulare, S.

$\{$ Sphacria verbascicola. S.

$\{$ Phoma verbascicola, Cke.

Phyllosticta verbascicola, Ell. \& Kell.

Ramularia variabilis, Fckl.

$\{$ Hysterium Verbasci, S.

\{ Schizothyrium Verbasci, Sacc.

Verbascum, sp. indet.

Patellaria Verbasci, S.

$\{$ Peziza fuscobarbata, S.

\{ Lachnella fuscobarbata, Sacc.

Peziza nigrescens, Cke.

Sphaeria nigrita, S.

Veronica alpina, L.

Leptosphaeria striata, Wint.

Peronospora grisea, Ung.

Phoma Veronicae, Roum.

Puccinia Porteri, Pk.

Puceinia Veronicarum, DC.

Septoria Veronicae, Rob.

Veronica arvensis, L.

Peronospora grisea, Ung.

Veronica peregrina, L.

Entyloma Linariae, Schrt.

Peronospora grisea, Ung.

Ramularia Veronicae, Fckl.

Veronica fruticosa, L.

Septoria semilunaris, Johans.

Septoria Veronicae, Rob. 
Veronica serpyllifolia, L.

Erysiphe Labiatarum, S. Am. bor.

Veronica Virginica, L.

Puccinia Veronicarum, D. C.

Septoria Veronicae, Rob.

Sphaerotheca Castagnei, Lev.

\section{BIGNONIACEAE.}

\section{Bignonia capreolata, L.}

$\{$ Polyactis grisea, S.

\{ Botrytis grisea, Sacc.

Capnodium elongatum, B. \& Desm.

Cladosporium Bignoniae, S.

Dimerosporium tropicale, Speg.

(Dothidea Bignoniae, $\mathrm{Fr}$.

Dothidea capreolatae, S.

Dothidea ribesia, Auct. Am. p. p.

$\{$ Meliola furcata, Rav. Fung. Am. 330 .

\{Meliola bidentata, Cke.

Oedemium atrum, Lk.

Sematium crucigerae, $\mathrm{S}$.

$\{$ Helminthosporium rigidum, $\mathrm{Fr}$.

Podosporium rigidum, S.

Sphaeria sulcata, Fr.

\section{Catalpa bignonioides, Walt.}

Cercospora Catalpae, Wint.

Cladosporium herbarum, (P.) Lk.

f Sporidesmium capsularum, Thm.

\{ Clasterosporium capsularum, Sacc.

Macrosporium Catalpae, Ell. \& Martin.

Microsphaeria elevata, Burrill.

$\{$ Phyllactinia guttata, Lev.

\{ Phyllactinia suffulta, (Reb.) Sacc.

Phyllosticta Bignoniae, Westd.

Phyllosticta Catalpae, Ell. \& Martin.

$\{$ Boletus distortus, S.

$\{$ Polyporus distortus, Fr.

Catalpa speciosa, Warder.

Capnodium axillatum, Cke.

Cercospora Catalpae, Wint.

Chaetophoma Catalpae, Cke.

Microsphaera elevata, Burrill.

Phyllactinia guttata, Lev.

\{ Sporidesmium compositum, B. \& Rav.

\{ Sirodesmium compositum, Sacc.

Catalpa, sp. indet.

Exidia saccharina, Fr.

Helminthosporium crustuosum, S.

$\{$ Sphaeria Catalpae, S.

\{Hypoxylon Catalpae, Sacc.

Dothidea Catalpae, B. \& C.

$\{$ Sphaeria subrugosa, S.

Lophiostoma subrugosum, Sacc.

Sphaeropsis Baculum, Gerard.

$\{$ Phoma Baculum, Sacc.

Macrophoma Baculum, Berl. \& Vogl.
Mucor truncorum, Lk.

Nummularia Clypeus, (S.) Cke.

Peziza leguminum, S.

Helotium leguminum, Cke.

Pezizella leguminum, Sacc.

\{ Sphaeria (Depazea) catalpicola, S.

Sphaerella catalpicola, Cke.

Sphaeronema Catalpae, S.

Torula olivascens, S.

S Sphaeria sphinctrina, Fr.

Valsa sphinctrina, Fr.

Vermicularia angustata, $\mathrm{S}$.

Vermicularia petiolorum, S.

Chilopsis saligna, Don.

Phyllosticta erysiphoides, Sacc.

Tecoma radicans, Juss.

(Sphaeria Van Vleckii, S.

$\{$ Melogramma Van Vleckii, B.

Botryosphaeria Van Vleckii, Sacc.

Cercospora duplicata, Ell. \& Ev.

Cercospora pallida, Ell. \& Ev.

Cercospora sordida, Sacc.

Cladosporium Bignoniae, S.

$\{$ Diplodia Tecomae, Cke.

$\{$ Diplodiella Tecomae, Sacc.

Erysiphe Cichoracearum, DC.

Hendersonia Peckii, Clinton.

Leptostroma hysterioides, Fr.

(Peziza leguminum, S.

$\{$ Helotium leguminum, Cke.

Pezizella leguminum, Sacc.

Sphaeria Bignoniae, S.

Valsa Bignoniae, M. A. Curtis.

Valsaria Bignoniae, S. in Herb. sec. Cke.

Pseudovalsa Bignoniae, Cke.

Sacidium Bignoniae, S.

Stictis Stigma, C. \& E.

$\{$ Sphaeria Sacculus, S.

$\{$ Torsellia Sacculus, Fr.

Sphaeria coronata, Hoffm.

$\{$ Valsa coronata, Fr.

Tecoma stans, Juss.

$\{$ Puccinia appendiculata, Wint.

$\{$ Puccinia ornata, Hark.

\section{PEDALIACEAE.}

Sesamum Indicum, L.

Diplodia herbarum, Lev.

Pistillaria micans, (P.) Fr.

\section{ACANTHACEAE.}

Dianthera Americana, L.

Cercospora Diantherae, Ell. \& Kell.

Dianthera humilis, Engelm. \& Gray.

Dimerosporium Langloisii, Ell. \& Martin. 
Finellia ciliosa, I'ursh.

Cercospora consociata, IVint.

Puccinia lateripes, B. \& Rav.

Ruellia strepens, L.

Puccinia lateripes, B. \& Rav.

Vermicularia ovata, $S$.

Ruellia, sp. indet.

Uromyces Texensis, B. \& C.

\section{VERBENACEAE.}

\section{Callicarpa Americana, L.}

S Melogramma Callicarpae, Cke.

\{ Botryosphaeria Callicarpae, Cke.

Cercospora Callicarpae, Cke.

Coniothyrium Callicarpae, Cke.

Diatrype Callicarpae, B. \& Rav.

\{ Meliola amphitricha, Rav. Fung. Amer. 84.

\{ Meliola Cookeana, Sacc. \& Speg.

Clerodendron, sp. indet.

Septoria phlyctaenoides, B. \& C.

Lippia nodiflora, Michx.

Cercospora Lippiae, Ell. \& Ev.

Phryma Leptostachya, L.

Aecidium Phrymae, Halsted.

Gymnosporium Harknessioides, Ell. \& Holw.

Coniosporium Harknessioides, Sacc.

Species spuria, sporae Piloboli.

Septoria Leptostachya, Ell. \& Kell.

Verbena angustifolia, Michx.

Aecidium verbenicolum, Ell. \& Kell.

Erysiphe Cichoracearum, DC.

Septoria Verbenae, Rob.

Verbena bracteosa, Michx.

$\{$ Aecidium Verbenae, Speg.

\{ Aecidium Verbenicolum, Ell. \& Kell.

Erysiphe Cichoracearum, DC.

Verbena hastata, L.

Aecidium verbenicolum, Ell. \& Kell.

Erysiphe Cichoracearum, DC.

Phyllosticta verbenicola, Martin.

\{ Septoria Verbenae, Rob.

\{ Septoria Verbenae, Gerard.

Verbena stricta, Vent.

$\{$ Aecidium Verbenae, Speg.

\{ Aecidium verbenicolum, Ell. \& Kell.

\{rysiphe Cichoracearum, DC.

Erysiphe lamprocarpa, Lev. p. p.

Verbena urticaefolia. L.

$\{$ Aecidium Verbenae, Speg.

Aecidium verbenicolum, Ell. \& Kell.

Crysiphe Cichoracearum, DC.

Erysiphe Verbenae, S.

Erysiphe lamprocarpa, Lev. p. p.

Erysiphe communis, Auct. Amer. p. p.

Septoria Verbenae, Rob.
Verbena Xutha, Lehm.

Cercospora verbenicola, Ell. \& Ev.

Verbena, sp. indet.

Oidium erysiphoides, Fr.

Vitex agnus-castus, L.

Cercospora Viticis, Ell. \& Ev.

Pyrenochacte minor, Ell. \& Er.

\section{LABIATAE.}

A udibertia humilis, Benth.

f Ophiobolus claviger, Hark.

\{ Raphidospora claviger, Cke.

A udibertia incana, Benth.

Puccinia nigrescens, $\mathrm{Pk}$.

A udibertia stachyoides, Benth.

$\{$ Patellaria nigrocyanea, Phil. \& Hark.

Durella nigrocyanea, Sacc.

Puccinia nigrescens, $\mathrm{Pk}$.

Blephilia hirsuta, Benth.

Puccinia Menthae, P.

Brunella vulgaris, $\mathrm{L}$.

Diaporthe Desmazierii, Niessl.

Ramularia Brunellae, Ell. \& Ev.

Septoria Brunellae, Ell. \& Holw.

Sphaerotheca Castagnei, Lev.

Calamintha Clinopodium, Benth.

Phacidium simulatum, B. \& C.

Phyllosticta Calaminthae, Ell. \& Ev.

f Trichobasis Labiatarum, Lev.

\{ Puccinia Menthae, P.

Collinsonia Canadensis, L.

$\{$ Spondylocladium tenellum, $\mathrm{Pk}$.

$\{$ Gonatobotryum tenellum, Pk.

Helminthosporium interseminatum, B. \& Rav.

Collinsonia, sp. indet.

Excipula majuscula, S.

\{ Dothidea elliptica, S.

Phyllachora elliptica, Sacc.

Cunila Mariana, L.

Puccinia Menthae, P.

Galeopsis Tetrahit, L.

Septoria Galeopsidis, Westd.

Hedeoma hispida, Pursh.

Peronospora Hedeomae, Kell. \& Swingle.

$\{$ Puccinia Menthae, $\mathrm{P}$.

\{ Puccinia Menthae, P., var. Americana, Pk.

$\{$ Septoria hedeomina, Pk.

\{ Rhabdospora hedeomina, Sacc.

Hyptis radiata, Willd.

Asterina spuria, B. \& C.

Uredo Hyptidis, M. A. Curtis.

Isanthus caeruleus, Michx.

Cercospora Isanthi, Ell. \& Kell.

Lamium, sp. indet.

Oidium erysiphoides, Fr. 
Leonotls nepetaefolia, R. Br.

Cercospora Leonotidis, Cke.

Leonurus Cardiaca, L.

Phyllosticta decidua, Ell. \& Kell.

Leonurus Marrubiastrum, L.

Septoria Lamii, Pass.

Lophanthus nepetoides, Benth.

Peronospora Lophanthi, Farl.

f Dothidea Hyssopi, S.

Phyllachora Hyssopi, Sacc.

$\{$ Stictis Hyssopi, S.

$\{$ Propolis Hyssopi, Cke.

(Puccinia Glechomatis, DC.

$\{$ Puccinia verrucosa, (Schultz) Lk.

Puccinia Hyssopi, S.

Septoria Lophanthi, Wint.

Lophanthus scrophulariaefolius, Benth.

Peronospora Lophanthi, Farlow.

$\{$ Puccinia verrucosa, (Schultz) Lk.

Puccinia Hyssopi, S.

Lophanthus, sp. indet.

Coniosporium Harknessioides, (E11. \& Holw.) Sacc.

Species spuria, sporae Piloboli

Sphaeria Lophanthi, B. \& C.

$\{$ Leptosphaeria Lophanthi, Sacc.

Heptameria Lophanthi, Cke.

Lycopus Europaeus, L.

Aecidium Lycopi, Gerard.

Puccinia Menthae, P.

Lycopus rubellus, Moench.

Cercospora Lycopi, Ell. \& Ev.

Lycopus Virginicus, L.

Aecidiutí Lycopi, Gerard.

Marrubium vulgare, L.

Sphaeria (Plenspora) Labiatarum, Cke. \&

Hark.

Synchytrium, sp.

Mentha Canadensis, L.

$\{$ Puccinia Menthae, P.

\{ Puccinia Menthae, P., var. Amcricana, Pk.

Ramularia menthicola, Sacc.

Mentha piperita, L.

Phyllosticta decidua, Ell. \& Kell.

Puccinia Menthae, $P$.

Mentha, sp. indet.

$\{$ Erysiphe Galeopsidis, DC.

Erysiphe lamprocarpa, Lev. p. p.

Monarda Bradburiana, Beck.

Puccinia Menthae, P.

Monarda citriodora, Cerv.

Puccinia Menthae, P., var. Americana, Pk.

Monarda fistulosa, L.

Secidium Menthae, DC.

$\{$ Puccinia Menthae, P.

Puccinia Menthae, P., var. Americana, Pk.

Synchytrium Holwayi, Farl.
Monarda punctata, L.

Puccinia Menthae, $P$

Nepeta Cataria, L.

\{ Sphaeria Catariae, C. \& E.

\{Didymella Catariae, Sacc.

$\{$ Diplodia herbicola, B. \& C.

\{ Diplodina herbicola, Sacc.

Phyllosticta decidua, Ell. \& Kell.

Septoria Nepetae, Ell. \& Ev.

Physostegia Virginica, Benth.

Puccinia Physostegiae, Pk. \& Clinton

Septoria Physostegiae, Ell. \& Ev.

Pycnanthemum incanum, Michx.

S Puccinia Menthae. P.

U Uredo Labiatarum, DC.

Aecidium Menthae, DC.

Puccinia Pycnanthemi, S. ?

Uredo Clinopodii, S.

Caeoma Labiatarum, Lk.

Trichobasis Labiatarum. Lev.

Trichobasis Labiatarum, Lev., var. Pycnan themi, Rav. Fung. Amer.

( Puccinia Menthae, P., var. Americana, Pk.

Pycnanthemum lanceolatum, Pursh.

Sphaeria Brachystemonis, S.

$\{$ Dothidea Brachystemonis, Fr.

Phyllachora Brachystemonis, Sacc.

Puccinia Menthae, P.

Pycnanthemum linifolium, Pursh.

Pycnanthemum muticum, P., var. pilosum, Gray.

Puccinia Menthae, $\mathrm{P}$.

Pycnanthemum, sp. indet.

Darluca Filum, (Biv.) Cast.

Sporidesmium Asteriscus, B. \& C.

Salvia azurea, Lam.

Puccinia Menthae, $P$.

Salvia azurea, Lam. var. grandiflora, Benth.

Puccinia nigrescens, $\mathrm{Pk}$.

Salvia ballotaeflora, Benth.

f Puccinia vertisepta, Tracy \& Galloway.

1 Diorchidium Tracyi, De Toni.

Salvia lanceolata, Willd.

Peronospora Swinglei, Ell. \& Kell.

$\{$ Puccinia nigrescens. Pk.

$\{$ Puccinia caulicola, Tracy \& Gall.

Salvia officinalis, $\mathrm{L}$.

Coniothyrium salviicolum, Ell. \& Ev.

Crucibulum vulgare, Tul.

Salvia, sp. indet.

\{ Sphaeria salviaecola, C. \& E.

$\{$ Diaporthe salviaecola, Sacc.

Salvia, sp. indet.

Ovularia ovata, (Fckl.) Sacc.

Peziza virginea, Batsch.

$\{$ Polyporus Salviae, B. \& C.

$\{$ Poria Salviae, Cke.

Volutella flexuosa, C. \& E. 
Scutellaria lateriflora, L.

\{ Erysiphe Galcopsiclis, DC.

Erysiphe communis, Auct. Amer. P. p.

Scutellaria parvula, Michx.

Erysiphe Galeopsidis, DC.

Scutellaria tuberosa, Benth.

Septoria Scutellariae, Thm.

Scutellaria versicolor, Nutt.

Cercospora Scutellariae, Ell. \& Ev.

Scutellaria, sp. indet.

Splsaeria rubella, P.

Ophiobolus porphyrogonus, (Tode) Siacc.

Sphacele calycina, Benth.

Hendersonia varians, Cke. \& Hark.

Stachys aspera, Michx.

Stachys bullata, Benth.

Septoria Stachydis, Rob.

Stachys palustris, L.

\{ Erysiphe Galeopsidis, DC.

Erysiphe Cichoracearum, Auct. Amer. p. p.

Teucrium Canadense, L.

Botrytis hypophylla, Ell. \& Kell.

Caeoma (Uredo) Teucrii, S.

Cercospora racemosa, Ell. \& Martin.

Cercospora Teucrii, Ell. \& Kell.

$\{$ Erysiphe Galeopsidis, DC.

\{ Erysiphe lamprocarpa, Lev. p. p.

(Gymnosporium Harknessioides, Ell. \&

$\{$ Holw.

Species spuria, sporae Piloboli.

Thymus Serpyllum, L.

Sphaerella pachyasca, Rostrup.

Trichostema dichotomum, L.

Septoria Trichostematis, Pk.

\section{PLANTAGINACEAE.}

Plantago decipiens, Barneoud.

$\{$ Pleospora herbarum, (P.) Rabh.

\{Pleospora Clarkeiana, El\}. \& Ev.

Plantago eriopoda, Torr.

Aecidium Plantaginis, Ces.

Plantago lanceolata, L.

Aecidium Plantaginis, Ces.

Cercospora Plantaginis, Sacc.

Peronospora alta, Fckl.

Ramularia Plantaginis, E1l. \& Martin.

Septoria inconspicua, B. \& C.

$\{$ Sphaeria plantaginicola, S.

Sphaerella plantaginicola, Cke.

Synchytrium plantagineum, Sacc. \& Speg.

Plantago major, L.

Aecidium Plantaginis, Ces.

Asterina Plantaginis, Ell.

Cercospora Plantaginis, Sacc.
Erysiphe Cichoracearum, DC.

$\{$ Peronospora alta, Fckl.

\{ Peronospora effusa, var. Plantaginis, Farl.

Ramularia Plantaginis, Ell. \& Martin.

$\{$ Septoria continua, B. \& C.

Rhabdospora continua, Sacc.

Septoria plantaginea, Pass., var. b. Sacc.?

Plantago maritima, L.

$\{$ Pleospora herbarum, (P.) Rabh.

\{leospora Clarkeiana, Ell. \& Ev.

Septoria semilunaris, Johans.

Sphaerella pachyasca, Rostrup.

Plantago Patagonica, Jacq., var. aristata, Gray.

Septoria inconspicua, B. \& C.

Plantago Virginica, L.

Aecidium Plantaginis, Ces. 


\section{APETALAE.}

\section{NYCTAGINACEAE.}

Abronia Crux-MaItae, Kellogg.

Peronospora Oxybaphi, Ell. \& Kell.

Abronia latifolia, Esch.

Heterosporium Abroniae, Hark.

Abronia umbellata, Lam.

Peronosporn Oxybaphi, Ell. \& Kell.

Oxybaphus nyctagineus, Sweet.

Ascochyta Oxybaphi, Trelease.

Cercospori Oxvbaphi, Ell. \& Halsted.

Peronospora Oxybaphi, Ell. \& Kell.

\section{ILLECEBRACEAE.}

\section{Gymnocarpus. sp. indet.}

Cercospora inquinans, Cke.

\section{AMARANTACEAE.}

\section{Acnida cannabina, L.}

Cystopus Bliti, (Biv.) Lev.

Acnida tuberculata, Moq.

Cystopus Bliti, (Biv.) Lev.

Alternanthera Achyrantha, R. Br.

Cercospora Alternantherae, Ell. \& Langlois.

Amarantus albus, L.

Cystopus Bliti, (Biv.) Lev.

Fusarium roseum, Lk. .

Amarantus blitoides, Watson.

Cystopus Bliti, (Biv.) Lev.

Amarantus chlorostachys, Willd., var. hybridus, Watson.

Cystopus Bliti, (Biv.) Lev.

Amarantus panizulatus, L.

(Caeoma (Uredo) Amaranthi, S.

Cystopus Bliti, (Biv.) Lev.

Cystopus Amaranthi, B.

Amarantus retroflexus, $\mathrm{L}$.

Cercospora brachiata. Ell. \& Ev.

Caeoma (Uredo) Amaranthi, S.

$\{$ Cystopus Bliti, (Biv.) Lev.

Cystopus Amaranthi, B.

Phyllosticta Amaranti. Ell. \& Kell.

Amarautus, sp. indet.
Sphaeria eriophora, Cke.
$\{$ Leptosphaeria eriophora, Sacc.
Heptameria eriophora, Cke.
S Sphaeria galbana, Fr
P Perisporium Galba, Fr.

\section{CHENOPODIACEAE.}

Atriplex Magdalenae, Brandegee.

Camarosporium Patagonicum, Speg.

Atriplex patulum, L., var. hastatum, Gray.

Peronospora effusa, (Grev.) Rabh.

Atriplex, sp. indet.

Ascochyta Atriplicis, Lasch, var. effusa, Ell. \& Kell.

Beta vulgaris, L.

$\{$ Cercospora beticola, Sacc.

Cercospora longissima, C. \& E.

(Uredo Betae, P.

$\{$ Trichobasis Betae, Lev.

Uromyces Betae, Kühn.

Blitum capitatum, L.

Septoria Atriplicis, (Lasch) Fck].

Chenopodium album, L.

Aecidium Ellisii, Tracy \& Gall.

$\{$ Cercospora Chenopodii, Fres.

Cercospora dubia, (Riess) Wint.

$\{$ Sphaeria picea, P. .

Diaporthe picea, Sacc.

$\{$ Diplodia hyalospora, C. \& E.

$\{$ Diplodina Ellisii, Sacc.

$\{$ Leptosphaeria eutypoides, Pk.

$\{$ Heptameria eutypoides, Cke.

$\{$ Sphaeria acuminata, Sow.

\{Ophiobolus acuminatus, Duby.

Peronospora effusa, (Grev.) Rahh.

Peronospora effusa, (Grev.) Rabh., var. minor, D By.

$\{$ Sphaeria longissima, $P$.

\{ Phoma longissima, Westd.

Pleospora calvescens, (Fr.) Tul.

Ascochyta Atriplicis, Lasch.

Septoria Atriplicis, Fckl.

Phyllosticta Chenopodii, N. A. F.

Phleospora Chenopodii. Ell. \& Kell.

Stagonospora Chenopodii, Pk.

Leptoria Westendorpii, Wint.

Chenopodium ambrosioides, L.

Phyllosticta ambrosioides, Thin.

Chenopodium ambrosioides, L., var. anthelminticum, Gray.

Chaetophoma anthelmintica, Cke.

$\{$ Fusisporium pallidoroseum, Cke.

$\{$ Fusarium pallidoroseum, Sacc.

Sphaeria anthelmintica, Cke.

$\{$ Leptosphaeria anthelrnintica. Sacc.

Heptameria anthelinintica, Cke. 
Sporocybe byssoides, (P.) Fr. Chenopodium hybridum, L.

Cercospora Clsenopodii, Fres.

Peronospora effusa, (Grev.) Rabh.

Chenopodium, sp. indet.

S Sphacria cuspina, C. \& E.

(Diaporthe euspina, Sacc.

Sphateria eriophora, Cke.

$\{$ Leptosphaeria eriophora, Sacc.

lleptameria eriophora, Cke.

Macrosporium commune, Rabh.

S Sphaeria galbana, Fr.

Perisporium Galba, Fr.

$\{$ Dothidea Chenopodii, S.

Phyllachora Chenopodii, Sacc.

\{ Dothidear ramosa, S.

P Phyllachora ramosa, Sacc.

? Puccinia bullata, S.

Sphaeria fumosa, S.

Sphaeria rubicunda, $\mathrm{S}$.

Torula herbarum, Lk.

Salicornia herbacea, L.

Plcospora Salsolae, Fckl.

Protomyces conglomeratus, Pk.

Sarcobatus vermiculatus, Torr.

$\{$ Aecidium Sarcobati, Pk.

Peridermium gracile, Hark.

Hendersonia heterophragmia, Ell. \& Ev.

Spinacia oleracea, Mill.

$\{$ Cladosporium nodulosum, Cke.

$\{$ Cladosporium sudnodosum, Cke.

Colletotriclaum Spinaciae, Ell. \& Halsted.

Entyloma Ellisii, Halsted.

Macrosporium Cheiranthi, Fr.

Pcronospora effusa, (Grev.) Rabh.

\section{PHYTOLACCACEAE.}

Phytolacca decandra, L.

PYRENOMYCETES.

Sphaeria aculeata, S.

Sphaeria Berkeleii, $\Lambda$ uct. Amcr.

Sphaeria spiculosa, var. Phytolaccae, C. \& E.

Diaporthe spiculosa, Ell. N. A. F.

Diaporthe aculeata, Sacc.

Spluaeria adelphica, Cke.

Didymosphaeria adelphica, Sacc.

Didymosphaerella adelpluica, Cke.

Sphaeria Himantia, P.

Dothidea Himantia, Fr.

SHymenula Phytolaccae, B. \& C.

Hymenella Phytolaccae, B. \& C.

Sphaeria clavigera, C. \& E.

Leptosphaeria clavigera, Sacc.

Heptameria clavigera, C. \& E.
(Dothidea crustacea, S.

Asteroma crustaceum, M. A. Curtis.

Phyllachora crustacea, Sacc. .

Dothidea elegans, S.

Asteroma elegans, M. A. Curtis.

Phyllachora elegans, Sacc.

(Dothidea inelegans, S.

Asteroma inelegans, M. A. Curtis.

Phyllachora inelegans, Sacc.

$\{$ Dothidea Phytolaccae, S.

Phyllachora Phytolaccae, Sacc.

Sphaeria (Didymosphaeria) Cupula, Ell.

Splaneria lilacina, $\mathrm{S}$.

SPHAEROPSIDEAE

Dinemasporium patellum, C. \& E.

Excipula erumpens, B., var. subhispida, C. \& E.

Phlyctaena orthospora, B. \& C.

\{ Septoria phlyctaenoides, B. \& C.

Phlyctaena septorioides, Sacc.

Phlyctaena vagabunda, Desm.

Phoma Phytolaccae, B. \& C.

Phyllosticta Phytolaccae, Cke.

Vermicularia Dematium, (P.) Fr.

\section{HYPHOM YCETES.}

Cercospora flagellaris, Ell. \& Martin.

(Helminthosporium interseminatum, B. \& Rav.

(Dendryphium nodulosum, Sacc.

$\{$ Fusisporium pallidoroseum, Cke.

$\{$ Fusarium pallidoroseum, Sacc.

Fusarium roseum, Lk.

Macrospoirium abruptum, C. \& E.

Macrosporium caudatum, C. S E.

Mncrosporium cladosporioides, Desm.

$\{$ Torula herbarum, Lk.

\{ Monilia herbarum, P.

MISCELLANEA.

SOphiotheca umbrina, B. \& C.

$\{$ Perichaena variabilis, Rostf.

\{ Peziza pulviscula, Cke.

$\{$ Pezizella pulviscula, Sacc.

\section{POLYGONACEAE.}

Chorizanthe pungens, Benth.

Uromyces Chorizanthis, Ell. \& Hark.

Eriogonum latifolium, Smith.

Phoma herbarum, Westd.

Eriogonum multiceps, Nees.

Uromyces Eriogoni, E11. \& Hark.

Eriogonum racemosum, Nutt.

Uromyces Arizonicus, Tracy \& Gall. 
Erigonum stellatum, Benth.

Uromyces intricatus, Ckc.

Uromyces Chorizanthis, Ell. \& Hark.

Urom yces Eriogoni, Ell. \& Hark.

\{ Uromyces Arizonicus, Tracy \& Gall.

Uromyces Eriogoni, Ell. \& Hark., var. foliicola, Ell. \& Ev.

Eriogonum tomentosum, Michx.

? Cercospora ferruginea, Fckl.

Cercospora rubella, Cke.

Pestalozzia? anomala, Hark.

Eriogonum umbellatum, Torr.

Uromyces Eriogoni, Ell. \& Hark.

Eriogonum virgatum, Benth.

Pestalozzia? anomala. Hark.

Uromyces Eriogoni, Ell. \& Hark.

Koenigia Islandica, L.

Sphaerella Polygonorum, (Crié) Sacc.

Ustilago Königiae, Rostrup.

Oxyria digyna, Hill.

Leptosphaeria Oxyriae, Rostrup.

Mollisia cincrea, (Batsch) Karst.

Puccinia Oxyriae, Fckl.

Pyrenophora comata, (Niessl) Sacc.

Septoria cercosperma, Rostrup.

Sphaerella Polygonorum, (Crié) Sacc.

Ustilago vinosa, (B.) Tul.

Polygonella articulata, Meisn.

Sphaeria curvicolla, $\mathrm{Pk}$.

$\{$ Gnomoniella curvicolla, Sacc.

Gnomonia curvicolla, Cke.

Polygonum acre, HBK.

Cercospora Polygonorum, Cke.

Cladosporium effusum, B. \& C.

Cladosporium pelliculosum, B. \& C.

Dialonectria (Nectriella) consors, E11. \& Ev.

Puccinia Polygoni-amphibii, P.

$\{$ Uredo Polygonorum, DC.

Trichobasis Polygonorum. Rav. Fung. Am.

Polygonum amphibium, L.

Puccinia Polygoni-amphibii, P.

Puccinia Polygoni, Reb.

Uredo Polygonoruin, DC.

Puccinia Polygoni-Pensilvanici, S.

Puccinia Polygonorum, Schl.

Puccinia concentrica, S.

Puccinia Amphibii, Fckl.

('Trichobasis Polygonorum, Rav. Fung. Am.

Ramularia rufomaculans, $\mathrm{Pk}$.

$\{$ Caeoma utriculosum, Nees.

\{Ustilago utriculosa, Tul.

Polygonum aviculare, $\mathrm{L}$.

Cercospora avicularis, Wint.

$\{$ Pcronospora Rumicis, Cda.

Peronospora Polygoni, Thm.

(Puccinia vaginalium, Lk.

$\left\{\begin{array}{l}\text { Uromyces Polygnni, (P.) Fckl. } \\ \text { Uromyces Aviculariae, Schrt. }\end{array}\right.$
Ustilago utriculosa, (Nees) Tul.

Polygonum cilinode, Michx.

$\{$ Ustilago anomala, J. Kunze.

$\{$ Ustilago pallida, Schrt.

Polygonum Convolvulus, L.

Cercospora polygonacea, Ell. \& Ev.

Peronospora Polygoni, Thm.

Polygonum dumetorum, L., var. scandens, Gray.

$\{$ Peronospora Rumicis, Cda.

Peronospora Polygoni, Thm

Ramularia rufomaculans $\mathrm{Pk}$.

Ustilago anomala, J. Kunze.

Polygonuin erectum, L.

Cercospora avicularis, Wint.

Uromyces Polygoni, (P.) Fckl.

Polygonum Hartwrightii, Gray.

$\{$ Puccinia Polygoni-amphibii, P.

\{ Puccinia Polygonorum, Schl.

Polygonum Hydropiper, L.

Botrytis hypophylla, Ell. \& Kell.

(Cercospora Polygonorum, Cke.

Helminthosporium Hydropiperis, Thm.

Cercospora Hydropiperis, Speg.

Septoria Polygonorum, Desm.

(Ustilago Candollei, Tul.

Sphacelotheca Hydropiperis, (Schum.)

Ustilago Austro-Americana, Speg.

Ustilago utriculosa, (Nees) Tul.

Polygonum lapathifolium, L., var. incarnatum, Watson.

$\{$ Ustilago Bistortarum, Auct. Amer.

Ustilago Austro-Americana, Speg.

Ustilago utriculosa, (Nees) Tul.

Polygonum Muhlenbergii, Watson.

Pleospora compressa, Hark.

(Puccinia Polygoni-amphibii, P.

$\{$ Puccinia Polygonorum, DC.

Puccinia Amphibii, Fckl.

Ramularia rufomaculans, $\mathrm{Pk}$.

Polygonum orientale, L.

Septoria Polygonorum, Desm.

Polygonum Pennsylvanicum. L.

$\{$ Patellaria discolor, Mont. \& Fr.

\{ Mollisia discolor, Phil.

Puccinia Polygoni, Reb.

Puccinia Polygoni-Pensilvanici, S.

Puccinia Polygonorum, Schl.

Puccinia Amphibii, Fckl.

Septoria Polygonorum, Desm.

Tilletia bullata, Auct. Amer.

? Ustilago Bistortarum, Auct. Amer.

Ustilago Austro-Americana, Speg.

Ustilago utriculosa, (Nees) Tul. 
Polygonum Persicaria, L

Peziza Polygoni, Lasch.

Niptera Polygoni, Rehm.

Peziza lactuosa, Cke.

Mollisia Polygoni, Gill.

Septoria Polygonorum, Desm.

Polygonum polymorphum, Ledeb.

$\{$ l'leospora compressa, Hark.

$\{$ Clathrospora permunda, (Cke.) Berl.

Kellermania Polygoni. Ell. \& Ev.

Polygonum ramosissimum, Michx.

Uromyces Polygoni, (P.) Fckl.

Polyơonum sagittatum, L.

Ustilngo Candollei, Tul.

Ustilago Hydropiperis, (Schum.) Schrt.

(Splı:ıcelotheca Hydropiperis, D By.

Sphaeria Polygoni-sagittati, S.

Polygonum Virginianum, L.

\{ Septoria complanata, B. \& C.

Phlyctaena complanata, Sacc.

(Puccinia Polygoni-amphibii, P.

Puccinia Polygoni, Reb.

Puccinia Polygonorum, Schl.

Puccinia Amphibii, Fckl.

Ustilago Austro-Americana, Speg.

Vermicularia Polygoni-Virginici, S.

Polygonum viviparum, L.

Pleospora platyspora, Sacc.

Puccinia Bistortae, (Strauss) DC.

Ramularia Bistortae, Fckl.

Rhytisma Bistortae, (DC.) Rostrup.

Septoria Polygonorum, Desm.

Sphacelotheca Hydropiperis, (Schum.) D By.

Sphaerella Polygonorum, (Crié) Sacc.

Ustilago Bistortarum, (DC.) Schrt.

Polygon um, sp. indet.

\{ Polyactis fascicularis, Cda.

Botrytis fascicularis, Sacc.

Sphaeria mortuosa, Ell.

Anthostoma mortuosum, Sacc.

Sphaeria eccentrica, C. \& P.

$\{$ Gnomoniella eccentrica, Sacc.

Gnomonia eccentrica, Cke.

$\{$ Sphaeropsis pulchrispora, $\mathrm{Pk}$. \& Clinton.

\{ Phoma callospora, Pk. \& Clinton.

Stigmatea Polygonorum, Fr.

Rheum Rhaponticum, L.

l'hyllosticta Rhei, Ell. \& Ev.

Septoria Rhapontici, Thin.

Vermicularia Polygoni-Virginici. S.

Rumex Acetosella, L

Cercospora Acetosellae, Ell.

Diaporthe discrepans, Sacc.

Sphaerella Tassiana, De Not.

Sphaeria (Didymella) lophospora, (Sacc. d Speg.) Ell.

Ustilago utriculosa, (Nees) Tul, var. Rumicis, $\mathrm{B}$
Rumex Brittanicus, L.

Puccinia ornata, Arth. \& Holw.

$\{$ Ramularia macrospora, Fres.

\{Ramularia decipiens, Ell. \& Ev.

Ramularia occidentalis, Ell. \& Kell.

Ramularia pratensis, Sacc.

Ustilago Parlatorei, Fisch. Waldh.

Rumex crispus, L.

Cercospora Acetosellae, Ell., var. maculosa, $\mathrm{Pk}$.

CRamularia macrospora, Fres.

Peronospora obliqua, Cke.

Ramularia obovata, Fckl.

Ovularia obliqua, Oud.

Ramularia decipiens, E11. \& Ev.

Rumex obtusifolius, L.

Cercospora Rumicis, Ell. \& Langlois.

Cylindrosporium pulchrum, Speg.

Ramularia macrospora, Fres.

\{ Ovularia obliqua, (Cke.) Oud.

Rumex verticillatus, L.

Excipula rumicicola, S.

Ramularia decipiens, Ell. \& Ev.

Rumex, sp. indet.

$\{$ Aecidium Rumicis, P.

\{ Caeoma (Aecidium) rubellatum, Lk.

Didymella lophospora, Sacc. \& Speg., var.

Acetosellae, Ell.

Phlyctaena arcuata, B.

\section{ARISTOLOCHIACEAE.}

Aristolochia Serpentaria, L.

Cercospora serpentaria, Ell. \& Ev.

Aristolochia tomentosa. Sims.

Sphaeropsis Squieriae, Clinton.

Asarum arifolium, Michx.

Sphaerella (Laestadia) asarifolia, Cke.

Asarum Canadense, L.

Synchytrium Asari. Arth. \& Holw.

Asarum Virginicum, L.

(Depazea concentrica, B. \& C.

$\{$ Sphaeria concentrica, Sacc.

Sphaerella concentrica, Cke.

Asarum, sp. indet.

$\{$ Puccinia asarina, Kze.

Puccinia Asari, Lk.

\section{PIPERACEAE.}

saururus cernuus, L.

Ccrcospora Saururi, Ell. \& Ev.

\section{LAURACEAE.}

\section{Lindera Benzoin, Blume.}

(Diatrype microplaca, B. \& C.

$\left\{\begin{array}{l}\text { Anthostoma microplacum, Sacc. } \\ \text { Nitom }\end{array}\right.$

Nummularia microplaca, Cke. 
\& Anthostomella ostiolata, Ell. \& Ev.

\{ Anthostoma ostiolatum, Cke.

Asterina ramularis, Ell.

Sphaeria conferta, S. Syn. Car.

\{ Sphaeria confertula, S. Am. bor.

Byssosphaeria conferta, Cke.

\{ Cenangium concinnum, B. \& C.

Scleroderis concinna, Sacc.

Thelephora coerulea, Schrad.

Thelephora Indigo, S.

Corticium coeruleum, Fr.

$\{$ Valsa sociata, C. \& E.

\{ Diaporthe sociata, Sacc.

Diatrype Hystrix, (Tode) Fr.

Didymella Linderae, Ell. \& Ev.?

Dothidea Linderae, Gerard.

Epicoccum neglectum, Desm.

Eutypella stellulata, (Fr.) Sacc.

Exidia glandulosa, (Bull.) Fr.

Helminthosporium Petersii, B. \& C.

Hendersonia pauciseptata, B. \& C.

f Sphaeria callostroma, S.

\{ Hypoxylon callostroma, B.

Hypoxylon fuscum, (P.) Fr.

\{ Graphium Linderae, Ell. \& Ev.

Isariopsis Linderae, Sacc.

Nectria ochroleuca, (S.) M. A. Curtis.

$\{$ Peziza Daedalea, $\mathrm{S}$.

\{ Tapesia Daedalea, Sacc.

$\{$ Dothidea lauricola, S.

\{ Phyllachora lauricola, Sacc.

Phyllosticta Linderae, Ell. \& Ev.

Sphaeria rivulosa, S.

Sphaeropsis Linderae, Pk.

$\{$ Coremium concentricum, S.

\{ Sporocybe concentrica, Sicc.

Valsa Linderae, Pk.

Zythia rufa, (Fr.) S.

Litsea geniculata, Benth. \& Hook.

\{ Helminthosporium pistillare, Cke.

\{ Cercospora Berkeleyi, Cke.

Persea Carolinensis, Nees.

\section{PYRENOMYCETES.}

(Diatrype hypophloea, B. \& Rav.

$\{$ Anthostoma hypophloeum, Sacc.

Nummularia hypophloea, Cke.

Sothidea Lauri-Borboniae, S.

$\{$ Phyllachora Lauri-Borboniae, Sacc.

Asterina? Lauri-Borboniae, Cke.

Asterina tenella, Cke.

Diatrype tenuissima, Cke.

\{ Hysterium lineolatum, Cke.

\{Gloniopsis lineolata, Sacc.

\{Hypoxylon Malleolus, B. \& Rav.

\{ Hypoxylon concentricum, Rav. Fung. Am.

Hypoxylon Sassafras, (S.) B.
Sphaeria Pezizula, B. \& C.

Lasiosphaeria Pezizula, Sacc.

Lophium mytilinum, (P.) Fr.

f Hysterium maculare, $\mathrm{Fr}$.

\{Lophodermium maculare, De Not.

Meliola amphitricha, Fr.

Melogramma mutilum, var., Rav. Fung. Am.

$\{$ Diatrype microplaca, B. \& C.

(Nummularia microplaca, Cke.

Phyllachora demersa, (Cda.) Sacc.

f Dothidea Scutula, B. \& C.

$\{$ Dothidella Scutula, Sacc.

Phyllachora Scutula, Cke.

(Hypoxylon subiculosum, B.

\{ Rosellinia subiculata, (S.) Sacc.

Sphaerella exutans, Cke.

Valsa tetraploa, B. \& C.

SPHAEROPSIDEAE.

Ceuthospora Cookei, Thm.

$\{$ Discosia nitidissima, B. \& C.

Discosia nitida, Lev., var.

f Excipula recurvispora, B. \& C

$\{$ Excipulina recurvispora, Sacc.

\{ Phyllosticta micropuncta, Cke.

\{ Phyllosticta Perseae, Ell. \& Martin.

\section{HYPHOMYCETES.}

$\{$ Cercospora purpurea, Cke.

\{ Cercospora Perseae, Ell. \& Martin.

Circinotrichum maculiforme, Nees.

Helicotrichum obscurum, (Cda.) Sacc.

Helminthosporium velutinum, Lk.

Menispora cylindrica, Cke.

Sporidesmium velutinum, Cke.

\section{HYMENOMYCETES.}

Corticium effuscatum, C. \& E.

Hydnum mucidum, Gmel.

Kneiffia setigera, $\mathrm{Fr}$.

Polyporus cinereus, S.

Polyporus crocatus, Fr.

$\{$ Polyporus fuscocarneus, $\mathbf{P}$.

Poria fuscocarnea, Cke.

\{ Polyporus mutabilis, B. \& C.

\{Polystictus mutabilis, Cke.

$\{$ Polyporus rufus, (Schrad.) Fr.

Poria rufa, Sacc. Syll.

$\{$ Xerotus Nigrita, Lev.

$\{$ Anthracophyllum Nigrita, Kalchb.

MISCELLANEA.

Acinula candicans, Fr.

Cenangium Magnoliae, B. \& C.

Pestalozzia laurina, Mont. 
Pestalozzia maura, Ell. \& Er.

Peziza scutellata, L.

Tremella Myricae, B. S Cke.

Trichocoma paradoxum, Rav. Fung. Car.

$\{$ 'Trichoscytale paradoxa, B. p. p.

Trichocuma lacvispora, Massee.

Perséa Carolinienisis, Nees, var. palus-

tris, Chapm.

Asterina carnea, Ell. S Martin.

Asterina delitescens, Ell. $\mathbb{S}$ Martin.

$\{$ Helminthosporium fumosum, Ell. \& Martin.

Brachysporium fumosum, Sacc.

Helotium maculosum, Eli. \& Martin.

Isariopsis clavata, Ell. \& Martin.

Neliola amphitricha, Fr.

$\{$ Peziza gelatinosa, Ell. \& Martin.

\{rbilia gelatinosa, Sacc.

Sassafras officinale, Nees.

PYRENOMYCETES.

\{ Sphaeria biglobosa, C. \& E.

$\{$ Diaportlie biglobosa, Sacc.

Sphaeria pentagona, S. Syn. Car.

Spliaeria goniostoma, S.

Valsa goniostoma, MI. A. Curtis.

Eutypella pentagona, Sacc. p. p.

Eutypella goniostoma, Sacc.

$\{$ Hysterium graphicum, Fr.

\{Glonium graphicum. Duby.

Gnomonia Sassafras, Ell. \& Ev.

S Sphaeria Sassafras, S.

\{ Hypoxylon Sassafras, B.

Hysterium acuminatum, Fr.

Lophium Sassafras, S.

$\{$ Sphaeria verrucosa, S.

$\{$ Nectria verrucosa, Sacc.

$\{$ Diatrype microplaca, B. \& C.

\{Nummularia microplaca, Cke.

Nummularia punctulata, (B. \& Rav.) Sacc.

\{ Dothidea Sassafras, S.

\{ Phyllachora Sassafras, Sacc.

S Sphaeria eriostega, C. \& E.

\{ Physalospora eriostega, Sacc.

Sphaerclla Sassafras, Ell. \& Er.

Sphacria albofarcta, S.

Sphacria tingens, $\mathrm{S}$.

Splaneria obducens, Fr.

$\{$ Teichospora obducens, Fekl.

Strickeria obducens, Cke.

Sphaeria acclinis, S.

$\{$ Valsa acclinis, M. A. Curtis.

Valsa inclinis. Sacc.

Sphaeria indistincta, S.

\{Valsa indistincta, Cke.

Valsa laurina, C. \& E.

Valsa nigrofacta, C. \& E.

$\{$ Valsaria nigrificata, Sacc.

Pseudovalsa nigrificata, Cke.
SPHAEROPSIDEAE.

Sphaeria sphaerocephala, S.

\{ytospora sphaerocephala, M. A. Curtis.

\{ Depazea frondicola, Fr.

S Sphaeria frondicola, Fr.

$\{$ Diplodia decorticata, C. \& E.

$\{$ Diplodiella decorticata, Sacc.

Sphaeropsis Sassafras, C. \& E.

Sphaeropsis seriatus, $\mathrm{Pk}$.

MELANCONIEAE.

Phyllosticta Sassafras, Cke.

$\left\{\begin{array}{l}\text { Phyllosticta affinis, Ell. \& Kell. } \\ \text { Gloeosporium affine, Ell. \& Ev. }\end{array}\right.$

Melanconium foliicolum, Pk.

Melanconium Sassafras, S.

Pcstalozzia funerca, Desm.

Pestalozzia Sassafias, Ell. \& Ev.

\section{HYPHOMYCETES.}

Acremonium fuscum, Schm.

Oidium subramosum, Lk.

Dematium Penicillus, S.

Actinocladium Penicillus, Fr.

Coremium concentricum, S.

Sporocybe concentrica, Sacc.

\section{DISCONYCETES.}

\{ Cenangium concinnum, B. \& C.

$\{$ Scleroderris concinna, Sacc.

\{ Sphaeria Patella, (Tode) P.

\{Heterosphaeria Patella, Grev.

Rhytisma Sassafras, S.

Xyloma concentricum, S. Syn. Car.

\section{HYMENOMYCETES.}

Merulius Corium, (P.) Fr.

$\{$ Boletus ferruginosus, Schrad.

Polyporus ferruginosus, Fr.

Polyporus Lindheimeri, B. \& C.

Solyporus Sassafras, S.

L Poria Sassafras, Cke.

Trametes sepium, B.

Umbellularia Californica, Nutt.

Acrospermum corrugatum, Ell.

Sphaeria Oreodaphnes, Cke. \& I Iark.

$\left\{\begin{array}{l}\text { Anthostomella Oreodaphnes, Berl. \& Vogl. } \\ \text { Anthostoma Orod }\end{array}\right.$

Anthostoma Oreodaphnes, Cke. \& Hark.

Asterina anomala, Cke. $\&$ Hark.

Sphaeria moriformis, Tode.

Bertia moriformis, De Not.

Psilosphaeria moriformis, Cke.

Blennoria Umbellulariae, Cke. \& Ilark.

Capnodium Tuba, Cke. \& Hark.

Ccuthospora foliicola, (Lib.) Cke.

Chalara brachyspora, Sacc. 
Cladosporium Fumago, Lk.

Corticium comedens, (Nees) Fr.

Corticium laeve, $\mathrm{P}$.

$\{$ Nectria Umbellularine, Plow. \& Hark.

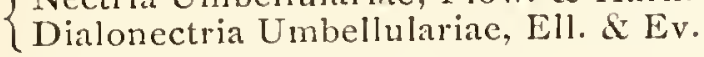

Diatrypella decipiens, Ell. \& Ev.

\{ Diplodia laurina. Cke. \& Hark.

\{ Diplodia Harknessii, Sacc.

Diplodia melaena, Lev.

Fusarium personatum, Cke.

Helotium aureum, $P$.

Helotium pallescens, (P.) Fr.

Iydnum Stevensoni, B. S Br.

Hypoxylon rubiginosum, (P.) Fr.

Hypoxylon serpens, (P.) Fr.

Irpex paradoxus, (Schrad.) Fr.

Massaria pulchra, Hark.

Midotis plicata, Phil. \& Hark.

Nectria cinnabarina, (Tode) Fr.

Nummularia Bulliardi, Tul.

Pestalozzia coryneoidea, Hark.

Polyporus igniarius, (L.) Fr.

Radulum molare, Fr.

Sphaerella Umbellulariae, Cke. \& Hark.

Sphaeria (Leptosphaeria) odora, Cke. $\mathcal{E}$

Hark.

\{ Epochnium glaucum, Cke. \& Hark.

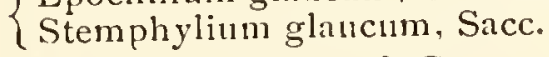

Valsa Americana, B. \& C.

Zygodesmus pannosus, B. \& C.

Lauraceae, sp. indet.

Cenangium Magnoliae, B. \& C.

$\{$ Sphaeria corniculata, Elirh.

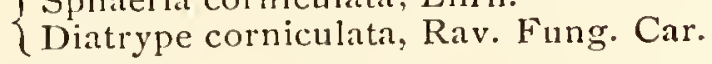

Thelephora episphaeria, Fr.

\section{THYMELAEACEAE.}

Dirca palustris, L.

Aecidium hydnoideum, B. \& C.

Fusarium lateritium, Nees.

Daphne Mezereum, L.

Dothidea Mezerei, Fr.

Fusarium inseptatum, S.

Sphaeria Daphnidis, S.

\section{ELAEAGNACEAE.}

Elaeagnus angustifolia.

Septoria argyrea, Sacc.

Elaeagnus longifolius, P.

Phyllosticta argyrea, Speg.

Shepherdia argentea, Nutt.

Sphaerotheca Castagnei, Lev.

Shepherdia Canadensis, Nutt.

Aecidium Allenii, Clinton.

Pleospora Shepherdiae, Pk.
LORANTHACEAE.

A reeuthobium pusillum, $\mathrm{Pk}$.

Sphaeria Arceuthobii, Pk.

$\{$ Wallrothiella Arceuthobii, Sacc.

Psilosphaeria Arceuthobii, Cke.

Phoradendron flavescens, Nutt.

Diplodia Phoradendri, Cke.

Diplodia Visci, (DC.) $F_{r}$.

\{ Sphaeria atrovirens, A. \& S.

Sphaeropsis atrovirens, Lev.

\{ Melogramma Phoradendri, B. \& C.

\{Valsaria Phoradendri, Berl. \& Vogl.

Verticillium rosellum, B. \& C.

\section{SANTALACEAE.}

Comandra pallida, A. DC.

Caeoma Comandrae, Pk.

Cronartium asclepiadeum, (Willd.) Fr., var.

$\{$ Thesii, B.

Cronartium Comandrae, $\mathrm{Pk}$.

\{ Puccinia Thesii, (Desv.) Chaill.

$\{$ Puccinia Comandrae, $\mathrm{Pk}$.

Comandra umbellata, Nutt.

\{ Aecidium pustulatum, M. A. Curtis.

\{ Aecidium Hamiltoniae, Thm.

Cronartium asclepiadeum, (Willd.) Fr., var.

$\left\{\begin{array}{c}\text { Thesii, } \mathrm{B} . \\ \text { Cronartium Comandrae, } \mathrm{Pk} .\end{array}\right.$

Tuberculina persicina, (Ditm.) Sacc.

\section{EUPHORBIACEAE.}

Acalypha Virginica, L.

Cercospora Acalyphae, Pk.

Buxus sempervirens, L.

Fusarium lateritium, Nees.

(Sphaeria Buxi, DC.

Diplodia Buxi, Fr.

Sphaeropsis Candollei, B. \& Br.

Phoma Candollei, Sacc.

Macrophoma Candollei, Berl. \& Vogl.

$\{$ Fusidium Buxi, Sclim.

$\{$ Fusisporium Buxi, Fr.

Penicillium roseum, Lk.

Sphaeria (Depazea) buxicola, Fr.

Volutella Buxi, (Cda.) B.

Croton Californicus, Miill.

Phyllachora Crotonis, (Cke.) Sacc.

Croton capitatus, Michx.

$\{$ Melampsora Crotonis, (Cke.) Burrill.

$\{$ Pucciniastrum Crotonis, De Toni.

Croton glandulosus, L.

Cercospora crotonifolia, Cke.

Melampsora Crotonis, (Cke.) Burrill.

Croton monanthogynus, Michx.

$\{$ Aecidium Crotonopsidis, Burrill.

$\{$ Aecidium splendens, Wint. 
Srichobasis Crotonis, Cke.

Melampsora Crotonis, Burrill.

Pucciniastrum Crotonis, De Toni.

Croton procumbens, Jacq.

\{ Trichobasis Crotonis, Cke.

Pucciniastrum Crotonis, De Toni.

Croton Texensis, Miil.

Melampsora Crotonis, (Cke.) Burrill.

Crotonopsis linearis, Michx.

\{Melampsorn Crotonis. (Cke.) Burrill.

\{ lucciniastrum Crotonis, De Toni.

Euplrorbia hicolor, Engelm. \& Gray.

Darluca Filum, (Biv.) Cast.

SUromvces Enphorbiae, C. \& P.

\{ Uromyces myristica, B. \& C.

Euphorbia commutata, Engelm.

Aecidium Cyparissiae, DC.

Euphorbia corollata, L.

Accidium Pammelii, Trelease.

Cercospora Euphorbiae, Kell. \& Swingle.

Microsphaera diffusa, C. \& P.

Microsphaera Euphorbiae, B. \& C.

Euphorbia dentata, Michx.

Aecidium Euphorbiae, S.

Uromyces Euphorbiae, C. \& P.

Euphorbia dictyosperma, Fisch. \& Mey.

Uromyces tuberculatus, Fckl. ?

Euphorbia g.yptosperma, Engelm.

Peronospora Euphorbiae, Fckl.

Uromyces Euphorbiae, C. \& P.

Euphorbia heterophylla, L.

Aecidium Euphorbiae, S.

Uromyces Euphorbiac, C. \& P.

Euphorbia humistrata, Engelm.

Uromyces Euphorbiae, C. \& P.

Euphorbia Ipecacuanhae, L.

Fusicladium fasciculatum, C. \& E.

Euphorbia maculata, L.

Aecidium Euphorbiae, S.

\{ Peronospora Euphorbiae, Fckl.

\{ Peronospora Cyparissiae, Farl. nec D By.

\}Uredo Euphorbiae, S. Syn. Car.

Uromyces Euphorbiae, C. \& P.

Uromyces Euphorbiae, C. \& P., var. minor, Arthur.

Euphorbia marginata, Pursh.

Aecidium Euphorbiae, S.

Aecidium Panmelii, Treleasc.

Botrytis cinerea, $P$.

Cladosporium solutum, Lk.

Helminthosporium herbarum, S.

Miciosphaera Euphorbiac, B. \& C.?

Splacria euphorbicola, S.

\{Tuhercularia persicina, Ditm.

Tuberculina persicina, Sacc.

Urnmyces Euphorbiac, C. \& P.
Uromyces Euphorbiae, C. \& P., var. minor, Arthur.

Euphorbia montana, Engelm.

Aecidium Euphorbine, S.

Uromyces scutellatus, (Schrank) Lev.

Euphorbia potaloidea, Engelm.

Fusicladium fasciculatum, C. \& E.

Uromyces Euphorbiae, C. \& P.

Euphorbia polygonifolia, L.

Accidium Euphorbiae, S.

Euphorbia Preslii, Guss.

Aecidium Euphorbine. S.

$\{$ Caeoma (Aecidium) Euphorbiae-hypericifoliae, S. Am. bor.

Erysiphe Euphorbiae, Pk.

Fusicladium fasciculatum, C. \& E.

Microsphaera Euphorbiae, B. \& C.

Peronospora Euphorbiae, Fckl.

Uredo Euphorbiae, S. Syn. Car.

Uredo scutellata, S. Syn. Car.

Cacoma punctuosum, S. Am. bor.

Uredo punctuosum, Curt. Cat.

Uromyces apiculosus, Curt. Cat. p. p.

Uromyces Euphorbiae, C. \& P.

Uromyces myristica, B. \& C.

Eupliorbia serpens, HBK.

Peronospora Euphorbiae. Fckl.

Uromyces Euphorbiae, C. \& P.

Euphorbia serpyllifolia, P.

Aecidium Euphorbiae, S.

Peronospora Euphorbiae, Fckl.

Uromyces Euphorbiae, C. \& P.

Euphorbia, sp. indet.

Botryosporium prorumpens, S

Cladosporium Chaetomium, Cke.

Mallotus Japonicus, Miill.

Cercospora Malloti, Ell. \& Ev.

Manilnot Carthagenensis, Miill.

Ascochyta Carthagenensis, Sacc.

Dothiorella botrya, Sacc.

Sphaeropsis Janiphae, Thm.

Phoma Janiphae, Sacc.

Macrophoma Janiphae, Berl. \& Vogl.

Macrosporium fasciculatum, C. \& E.

Ricinus communis, L.

$\{$ Cercospora ricinella, Sacc. \& Berl.

$\{$ Cercospora albidomaculans, Wint.

Cladosporium solutum, Lk.

Epicoccum nigrum, Lk.

Sphaeropsis Ricini, Cke.

$\{$ Phoma Ricini, Sacc.

Macrophoma Ricini, Berl. \& Vogl.

Macrosporium compactum, Cke.

Stillingia sebifera, Michx.

Cercospora Stillingiae, Fll. \& Ev.

Phyllosticta Stillingiae, Ell. \& Ev. 
URTICACEAE.

Boehmeria cylindrica, Willd.

Aecidium Urticae, Schum.

Cercospora Boehmeriae, Pk.

Septoria tenuissima, Wint.

Broussonetia papyrifera, Vent.

Ozonium auricomum, Lk.

S Sphaeria Papyriferae, S.

$\{$ Valsa Papyriferae, Cke.

Cannabis sativa, L.

(Septoria cannabina, Westd.

$\{$ Septoria Cannabis, (Lasch.) Sacc.

Septoria cannabina, $\mathrm{Pk}$.

Sphaeria Cannabis, S.

Celtis occidentalis, $\mathrm{L}$.

Cercospora Spegazzinii, Sacc.

Didymosporium Celtis, S.

Dinemasporium radiatum, Ell. $\&$ Ev.

Fusarium lateritium, Nees.

Gyroceras Celtidis, (Biv.) Mont. \& Ces.

Hendersonia Celtidis, Ell. \& Ev.

Hendersonia celtifolia, Cke.

$\{$ Hysterium hiascens, B. \& C.

Blitridium hiascens, Sacc.

Phleospora Celtidis. Ell. \& Martin.

Phyllosticta Celtidis, Ell. \& Kell.

Ramularia Celtidis, Ell. \& Kell.

Septoria Celti-gallae, Gerard.

Sphaerella maculiformis, (P.) Awd.

Sphaerotheca phytoptophila, Kell. \& Swingle.

Uncinula parvula, C. \& P.

(Uncinula polychaeta, B. \& C. in Grev.

$\{$ Pleochaeta Curtisii, Sacc. \& Speg., p. p.

Uncinula confusa, Massee.

Uncinula polychaeta, B. \& C. in Rav. Fung. Car.

Erysiphe polychaeta, B. \& C. in Grev.

(Pléochaeta Curtisii, Sacc. \& Speg., p. p.

Valsa Celtidis, Cke.

$\{$ Valsaria Celtidis, Sacc.

Pseudovalsa Celtidis, Cke.

Celtis, sp. indet.

Acrospermum foliicolum, B.

Sphaeria Celtidis, B. \& C.

$\{$ Didymella Celtidis, Sacc.

Endophlaea Celtidis, Cke.

Hymenochaete purpurea, Cke. \& Morg.

Hypoxylon notatum, B. \& C.

Lichenopsis sphaeroboloidea, S.

Macrosporium antennaeforme, B.\& C.

? Peziza succina, Phil.

Sphaeropsis Gallae, (S.) B. \& C.

Tricothecium roseum, $\mathrm{Fr}$.

$\{$ Diatrype obesa, B. \& C.

Valsaria quadrata, (S.) Sacc.

Ficus Carica, L.

$\{$ Ceratostoma hystricinum, Cke.

\{ Ceratostomella hystricina, Sacc.
Fusarium roseum, Lk.

Hysterinm (Gloniella) sycnophilum, Cke.

Melogramma (Botryosphaeria) Ficus, Cke.

Uredo Fici, Cast.

Ficus laurifolia, Lam.

Zygosporium oschenides, Mont.

Ficus, sp. indet.

Cytospora ficicola, B. \& C.

Fusarium lateritium, Nees.

Fusarium microspermum, B. \& C.

\{ Gibbera ficina, Cke. \& Hark.

Gibberella ficina, Sacc.

$\{$ Hendersonia fissurata, Cke.

\{ Hendersonia findens, Sacc.

Macrosporium caudatum, C. \& E.

Macrosporium Cheiranthi, Fr.

Nectria Cucurbitula, (Tode) Fr.?

Peziza vinosa, A. \& S.

Pilidium (?) Ficus, Cke.

Sporocybe byssoides, (P.) Fr.

Trichothecium roseum, Lk.

Tubercularia granulata, P.

Humulus Lupulus, L.

Cylindrosporium Humuli, Ell, \& Ev.

Erysiphe Cichoracearum, DC.

Pestalozzia compacta, B. \&. C.

Phyllosticta Humuli, Sacc. \& Speg., var. major, Ell. \& Ev.

Septoria lupulina, Ell. \& Kell.

Sphaerella erysiphina, (B. \& Br.) Ckc.

Sphaeria Penicillus, Schm.

Sphaerotheca Castagnei, Lev.

$\{$ Sphaeria humulina, $\mathrm{Pk}$.

$\{$ Zignoella humulina, $\mathrm{Pk}$.

Laportea Canadensis, Gandich.

Gloeosporium Laporteae, Pk.

Peronospora Urticae, (Lib.) D By.

Septoria Pileae, Thm.

Septoria Urticae, Rob.

\{ Peziza urticina, $\mathrm{Pk}$.

T'Trichopeziza urticina, Sacc.

Maclura aurantiaca, Nutt.

Corticium Sambuci, P.

Diplodia radicina, C. \& E.

Dothidea ribesia, (P.) Fr.

(Dothidea tetraspora, B. \& Br.

$\{$ Dothidea crystallophora, B. \& C.

Parodiella tetraspora, Kell. \& Carleton.

$\{$ Valsa tetraploa, B. \& C.

Eutypella tetraploa, Sacc.

Glonium parvulum, (Gerard) Cke.

Ovularia Maclurae, Ell. \& Langlois.

Phyllosticta Maclurae, Ell. \& Ev.

Boletus ferruginosus, Schrad.

Polyporus ferruginosus, Fr.

Poria ferruginosa, Karst.

Mucronoporus ferruginosis, Ell. \& Ev. 
Sphacropsis Machurac, Cke.

Sporidesmium Maclurac, Tlum.

Clasterosporium Maclurae, Pound.

SUredo Citri, Cke.

Uredo citrina, Dc Tonj.

Valsa Americana, B. \& C.

Valsa Maclurae, C. S E.

Morus alba, I.

1 Agracus adiposus, Fr.

( Pholiota adiposa, Sacc. Syll.

Cenangium fatisccns, S.

Cercospora moricola, Cke.

Coprinus micaceus, (Bull.) Fr.

$\{$ Splareria collecta, S.

\{ Diatrype collecta, Cke.

Dothidea moriformis, (Ach.) Fr.

$\{$ Didymosporium pezizoideum, S.

Epiclinjum pezizoideum, Fr.

Fracchiaea Americana, Berl.

Gonytrichum caesium, Nees.

Sphaeria olivaceohirta, S.

Massaria olivaceohirta, Cke.

Melanconjum conglomeratum, Lk.

Nectria dematiosa, (S.) M. A. Curtis.

$\{$ Sphaeria rerrucosa, $\mathrm{S}$.

\{ Nectria verrucosa, Sacc.

Ostracoderma spadiceum, S.

Ozonium auricomum, Lk.

Thelephora cinerascens, S.

Hymenochaete cinerascens, Lev.

Corticium cinerascens, B.

Peniophora cinerascens, Sacc.

Peniophora Schweiuitzii, Massce.

\{ Peziza conchella, S.

Pezizella conchella, Sacc.

$\{$ Peziza cruenta, S.

\{ Pezizella cruenta, Sacc.

\{ Septoria Mori, Lev.

Phleospora Mori, Sacc.

Phleospora moricola, (Pass.) Sacc.

Spermodermium rufum, S.

$\{$ Sphaeria Mori-albae, S.

Sphaerella Mori-albae, Cke.

Morus alba, L., var. latifolia, Bureau.

Diplodia Mori, B.

Dothidea Sambuci, (P.) Fr.

Fusarium lateritium, Nees.

Nectria coccinea, (P.) Fr.

S Sphaeria ochroleuca, S.

Nectria ochroleuca, M. A. Curtis.

Sphinctrina microscopica, B. \& C.

Valsa morigena, B. \& C. in Herb. B.

Valsa pusio, B. \& C.

Morus rubra, L.

Cercospora moricola, Cke.

$\{$ Cercospora pulvinulata, Sacc. \& Wint.

Cercospora Missouriensis, Wint.

Diplodia Mori, B.
Sphacria Mori-rubrae, S.

\{ Eutypa Mori-rubrae, Cke.

Massaria epilenca, B. \& C.

S Sphaeria dematiosa, S.

\{ Nectria dematiosa, M. A. Curtis.

(Peziza vinosa, $A$. \& S.

Calloria vinosa, $\mathrm{Fr}$.

Orbilia vinosa. Karst.

$\{$ Septoria Mori, Lev.

Plileospora Mori, Sacc.

Phoma longipes, B. \& C.

Uncinula geniculata, Gerard.

Morus, sp. indet.

\section{PYRENOM YCETES.}

(Botryosphacria Berengeriana, De Not.

Dothidea moricola, C. \& E.

Melogramma fuliginosum, Ell. p. p.

Bagnisiella moricola, Sacc.

Diatrype asterostoma, B. \& C.

Diatrype capnostoma, B. \& Rav.

Valsa stellulata, Rav. Fung. Am.

Eutypa ludibunda, Sacc.

Eutypella stellulata, (Fr.) Sacc., var., Ravenelii, Rehm.

\{ Sphaeria subcongregata, B. \& C.

\{Fracchiaea subconnata, (B. \& C.) Cke.

Hypoxylon Botrys, Nits.

\{ Hysterium Mori, S.

Hysterographium Mori. Rehm.

$\{$ Lophiostoma fenestralis, C. \& E.

\{Lophidium fenestrale, Sacc.

Nectria cinnabarina, (Tode) Fr:

Nectria Russellii, B. \& C.

Sphaeria botryosa, Tode.

Sphaerostilbe cinnabarina, Tul.

$\{$ Triblidium minor, Cke.

Triblidiella minor, Sacc.

Hysterium rufulum, Spreng.

$\{$ Triblidium rufulum, Cke.

'Triblidiella rufula, Sacc.

C Valsaria insitiva, Ces. \& De Not.

Diatrype Aethiops, C. \& E.

Valsaria Aethiops, Sacc.

Melogramma Aethiops, Cke.

\section{FUNGI IMPERFECTI}

Alternaria tenuis, Nees.

Diplodia atramentaria, C. \& E.

Diplodia moricola, C. \& E.

(Dinemasporium hispidulum, (Schrad.)

M. A. Curtis.

Excipula hispidula, C. \& E.

Fumago vagans, $P$.

Helminthosporium virgultorum, S.

Hendersonia epileuca, C. \& E.

Melanconium Dothidea, S.

Microcera coccophila, Desm. 
Sphacropsis valsoidea, C. \& E. Sporidesmium polymorphum, Cda.

Tubercularia mutabilis, Nees.

\section{DISCOMYCETES.}

Dermatea Cucurbitaria, Cke. Patellaria Cucurbitaria, Rehm.

Triblidium Cucurbitaria, Rehm.

Dermatella Cucurbitaria, Sacc.

Blitridium Cucurbitaria, Sacc.

\{ Patellaria indigotica, C. \& P.

Lecanidion infligoticum, Sacc.

Patellaria subsidua, C. \& E.

Blitridium subsiduum, Sacc.

$\{$ Stictis mollis, P.

Stictis Pupula, Fr.

\section{HYMENOMYCETES.}

Agaricus (Collybia) velutipes, Wm. Curtis,

$\{$ Stereum moricolum, B.

Peniophora moricola, Massee.

Polyporus adustus, (Willd.) Fr.

Polyporus Medulla-panis, (B.) Fr.

Parietaria debilis, Forst.

Erysiphe Cichoracearum, DC.

Parietaria Pennsylvanica, Muhl.

Peronospora Illinoensis, Farl.

Pilea pumila, Gray.

Erysiphe Cichoracearum, DC.

Septoria Pilene, Thm.

Ulmus alata, Michx.

$\{$ Phyllactinia guttata, Lev.

(Phyllactinia suffulta, (Reb.) Sacc.

Uncinula intermedia, B. \& C.

Uncinula macrospora, $\mathrm{Pk}$.

\section{Ulmus Americana, L.}

Acrospermum foliicolum, B.

Botrytis cinereoglauca, Ell. \& Kell.

Sphaeria phaeostroma, Dur. \& Mont.

$\{$ Chaetosphaeria phaeostroma, Fckl.

Byssosphaeria phaeostroma, Cke.

Coryneum tumoricola, $\mathrm{Pk}$.

$\{$ Valsa scoparia, (S.) M. A. Custis.

$\{$ Eutypa scoparia, Wint.

SXloma ulmeum, S.

$\{$ Sphaeria ulmea, Fr.

Gnomonia ulmea, Thm.

Hydnum parasitans, B. \& C.

Hypoxylon Caries, (S.) Sacc.

Hypoxylon effusum, Nits.

Melasmia ulmicola, B. \& C.

Microsphaera Alni, (DC.) Wint.

$\{$ Nectria (Calonectria) chlorinella, Cke.

$\{$ Dialonectria chlorinella, Cke.

Ozonicum auricomum, Lk.

Septoria Ulmi, Fr.

Phleospora Ulıni, Wallr.

$\{$ Dothidea Ulmi, (Duv.) Fr.

\{ Phyllachora Ulmi, Fckl.
\{ Peziza fibriseda, B. \& C.

\{ Pseudohelotium fibrisedum, Sacc.

Phoma cincta, B. \& C.

Phyllactinia suffulta, (Reb.) Sacc.

Boletus conchifer, S.

Boletus virgineus, $\mathrm{S}$.

Polyporus conchifer, Fr.

Polyporus virgineus, $\mathrm{Fr}$.

Polystictus virgineus, Cke.

Polystictus conchifer, Sacc. Syll.

$\{$ Polyporus tenerrimus, B. \& Rav.

Poria tenerrima, Cke.

Radulum molare, Fr.

Sacidium Ulmi-gallae, Kell. \& Swingle.

Uncinula macrospora, $\mathrm{Pk}$.

$\{$ Diatrype quadrata, (S.) B.

\{ Valsaria quadrata, Sacc.

Ulmus fulva, Michx.

Ceratophorum ulmicolum, Ell. \& Kell.

Corticium maculiforme, Fr.

Didymosporium effusum, S.

Eutypa scoparia, (S.) Wint.

Excipula ulmicola, S.

\{ Sphaeria ulmea, (S.) Fr.

\{ Gnomonia ulmea, Thm.

Irpex coriaceus, B. \& Rav.

Hypocrea bicolor, Ell. \& Ev.

Phyllosticta ulmicola, Sacc.

Septoria Ulmi, Fr.

$\{$ Sphaeria apertiuscula, $\mathrm{S}$.

\{ Sphaerella apertiuscula, Cke.

Stereum subpileatum, B. \& C.

Tubercularia vulgaris, Tode.

Uncinula macrospora, $\mathrm{Pk}$.

Ulmus, sp. indet.

\section{PYRENOMYCETES.}

Diatrype radiata, Ell.

Diatrype Stigma, (Hoffm.) Fr.

f Valsa innumerabilis, $\mathrm{Pk}$.

$\{$ Eutypella innumerabilis, Sacc.

Hypoxylon multiforme, Fr.

Hysteriurn pulicare, $\mathrm{P}$.

$\{$ Sphaeria ramulicola, $\mathrm{Pk}$.

Leptosphaeria ramulicola, Sacc.

$\{$ Lophiostoma Jerdoni, B. \& Br.

Lophiotrema praemorsum, (Lasch) Sacc.

(Nectria Canadensis, E11. \& Ev.

$\{$ Calonectria Canadensis, Berl. \& Vogl.

Tubercularia Canadensis, Berl. \& Vogl.

Nectria coccinea, (P.) $\mathrm{Fr}_{\mathrm{r}}$.

Nectria rhizogena, Cke.

Nectria Russellii, B. \& C.

$\{$ Sphaeria tubaeformis, Tode.

\{ Gnomoniella tubiformis, Sacc.

$\{$ Sphaeria phellogena, B. \& C.

$\{$ Teichospora phellogena, Sacc.

? Tympanis Fraxini, (S.) Fr.

Sphaeria Fraxini, Fr. 
Uncinula Bivonac, Ler.

Sphaeria ciliata, P.

Valsa ciliata, Fr.

Valsa coaperta, Cke.

\{ Sphaeria fibrosa, P.

\{ Valsa fibrosa, $\mathrm{Fr}$.

\{ Sphacria modesta, S.

Yalsa modesta, Clic.

f Sphaeria stcllulata, Fr.

Y Valsa stcllulata, Fr.

Spluaeria melastroma, Fr.

Valsa melastroma, Fr.

Valsaria melastroma, Sacc.

HYMENOMYCETES.

Agaricus (Mycena) corticola, Schum.

Agaricus (Pleurotus) euosmus, B.

Agaricus (Pleurotus) mastrucatus, $\mathrm{Fr}$.

$\{$ Agaricus sapidus, Kalchb.

$\{$ Pleurotus sapidus, Sacc. Syll.

f Agaricus subareolatus, Pk.

$\{$ Pleurotus subareolatus, Sacc. Syll.

$\{$ Agaricus ulmarius, Bull.

Pleurotus ulmarius, Quel.

Coprinus atratus, B. \& Br.

Coprinus fuscescens, (Schaeff.) Fr.

Corticium filamentosum, B. \& C.

$\{$ Corticium Oakesii, B. \& C.

$\{$ Aleurodiscus Oakesii, Cke.

$\{$ Corticium olivascens, B. \& C.

Coniophora olivascens, Massee.

Grandinia mucida, Fr.

Guepinia elegans, B. \& C.

Hirneola Auricula-Judae, (L.) B.

Hydnum Caput-medusae, Bull.

$\{$ Hydnum laeticolor, B. \& C.

Irpex laeticolor, Morg.

Hydnum udum, Fr.

\{Hymenochaete cinerascens: (S.) Lev.

Peniophora cinerascens, Sacc.

Lentinus vulpinus, $\mathrm{Fr}$.

Marasmius spodoleucus, B. \& Br.

Panus dealbatus, B.

Polyporus fragrans, $\mathrm{Pk}$.

Polyporus squamosus, (Huds.) Fr.

$\{$ Polyporus ulmarius, S. $\Lambda \mathrm{m}$. bor.

Polyporus scruposus, Fr.

$\{$ Polyporus ulmarius. (Sow.) Fr.

Fomes ulmarius, Cke.

Stereum disciforme, (DC.) Fr.

Stereum neglectum, Pk.

MISCELLANEA.

?Cercospora sphacriacformis, Ckc.

Cytospora carbonacca, Fr.

Helotium fibuliforme, (Bolt.) Fr.
Martindalea spironema, Sacc. \& Ell.

Pestalozzia insidens, Zabriskie.

$\{$ Peziza leonina, S.

$\{$ Trichopeziza leonina, Sacc.

Peziza rubella, P.

Rhytisma Ulmi, Fr.

Sporidesmium cellulosum, Fr.

Sporotrichum alutaceum, S.

f Hendersonia Lophiostoma, C. \& E.

\{ Stagonospora Lophiostoma, Sacc.

Urtica dioica, L.

Aecidium Urticae, Schum.

Urtica gracilis, Ait.

$\{$ Aecidium Urticae, Schum.

\{ Caeoma (Aecidium) urticatum, Lk.

Peronospora Urticae, (Lib.) D By.

Ramularia Urticae, Ces.

Sphaerella superflua, (Fckl.) Fll. \& Ev.

Urtica halosericea, Nutt.

Ramularia Urticae, Ces.

Sphaeropsis lanceolata, C. \& E.

Urtica, sp indet.

$\{$ Discella olivacea, Cke. \& Hark.

$\{$ Discula olivacea, Sacc.

Fumago vagans, $P$.

Helminthosporium Urticae, Pk.

Peziza fusarioides, B.

$\{$ Rhytisma elevatum, S.

\{ Phoma elevatum, Cke.

Phoma nebulosa, (P.) Mont.

Rhytisma Urticae, Fr.

Sphaeria Doliolum, P.

$\{$ Leptosphaeria Doliolum, De Not.

Heptameria Doliolum, Cke.

(Raphidospora Urticae, Rabh.

Sphaeria Urticae, Auct. Aner.?

Ophiobolus Urticae, Sacc.

\section{PLATANACEAE.}

\section{Platanus occidentalis, L.}

PYRENOMYCETES.

$\{$ Diatrype prominens, Howc.

\{Diatrypella prominens, Sacc.

Sphaeria Platani, S.

Y Valsa Platani, Cke.

Eutypella Platani, Sacc.

Sphaeria tinctor, B.

$\{$ Diatrype ? tinctor, Sacc.

IIypoxylon tinctor, Cke.

Sphaeria platanicola, Howe.

$\{$ Leptosphaeria platanicola, Sacc.

Heptameria platanicola, Cke.

Massaria atroinquinans, B. \& C.

Sphaeria platanoides, S. Syn. Car.

$\{$ Sphaeria stilbostoma, Fr.

Melanconis stilbostoma, Tul. 
$\{$ Microsphaera Platani, Howe.

\{ Microsphaera Alni, (DC.) Wint.

$\{$ Sphaeria macula, S. Syn. Car.

$\{$ Nummularia macula, Cke.

$\{$ Melanconis bicornis, Cke.

\{ Pseudovalsa bicornis, Sacc.

$\{$ Valsa hapalocystis, (B. \& Br.) Cke.

(Pscudovalsa hapalocystis, Sacc.

Sphaerclla maculiformis, (P.) Awd.

Sphaerella Platani, EH. \& Martin.

$\{$ Septoria platanifolia, Cke.

Sphaerella platanifolia, Cke.

Sphaeria ceratosperma, S. Syn. Car.

Sphaeria fulvopruinata, B.

Valsa fulvopruinata, Cke.

Diaporthe fulvopruinata, Sacc.

Valsaria fulvopruinata, Sacc.

\section{FUNGI IMPERFECTI.}

Cercospora platanicola, Ell. \& Ev.

Cladosporium epiphyllum, Nees.

Coryneum corticale, S.

$\{$ Didymosporium minutissimum, S.

Melanconium minutissimum, S.

$\{$ Discella Platani, Pk.

\{Discula Platani, Sacc.

Gloeosporium nervisequum, (Fckl.) Sacc.

Helminthosporium corticale, S.

$\{$ Ifendersonia Desmazieri, Mont.

\{Hendersonia Platani, Pk.

C Macrosporium echinellum, B. \& C.

Macrosporium Cheiranthi, Fr., var. cchinellum, B. \& C.

Macrosporium commune, Rabh., var.

echinellum, B. \& C.

Pestalozzia stellata, B. \& C.

Pestalozzia stictica, B. \& C.

Sphaeropsis Platani, $\mathrm{Pk}$.

Sporidesmium atrum, Lk.

Stigmella Platani, Fckl.

Stigmella visianica, Sacc. ?

Stilbospora quadriseptata, $\mathbf{S}$.

\section{MISCELLANEA.}

Achlya racemosa, Hildebrand, var.

$\{$ Thelephora albomarginata, S.

Peniophora albomarginata, Massee.

Phacidium Platani, S.

Stereum purpureum, $\mathrm{P}$.

Tremella mesenterica, Retz.

Trichia inconspicua, Rostf.

Platanus orientalis, L.

Glocosporium nervisequum, (Fckl.) Sacc.

Platanus racemosa, Nutt.

Diatrypella prominens, (Howe) Sacc.

Dictyosporium circinatum, Cke. \& Hark.
Glocosporium nervisequum, (Fckl.) Sacc.

Lasiosphaeria biformis, (P.) Sacc.

Sphaerella latebrosa, Cke.

f Stigmella Platani, Fckl.

Stigmina Platani, Sacc.

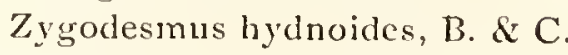

Zygodesmus pannosus, B. \& C.

Platanus, sp. indet.

\section{PYRENOMYCETES.}

Sphaeria ranella, B. \& Rav.

$\{$ Rebentischia ranella, Sacc.

Coniochaeta Rattus, (S.) Cke.

$\{$ Sphaeria comiculata, Ehrl.

\{ Diatrype corniculata, Rav. Fung. Car.

Diatrype Hystrix, (Tode.) Fr.

$\{$ Valsa aleurina, B. \& C. in Herb. B.

Eutypella aleurina, Ber\}. \& Vogl.

$\{$ Valsa tumidula, C. \& P.

\{ Eutypella tumidula, Sacc.

Glonium Ravenelii, Cke. \& Pliil.

$\{$ Sphaeria sublobata, S.

Hypocrea sublobata, Sacc.

$\{$ Hypoxylon coccineum, Bull.

Sphaeria fragiformis, P.

$\{$ Sphaeria illita, S.

\{Hypoxylon illitum, Sacc.

$\{$ Hypoxylon nummularium, Bull.

\{ Nummularia Bulliardi, Tul.

$\{$ Sphaeria rubiginosa, P.

Hypoxylon rubiginosun, Fr.

Hysterium angustatum, P.

$\{$ Hysterium pulicare, P., var. angustatum,

Sphaeria albocrustata, S.

$\{$ Sphaerella albocrustata, Cke.

Laestadia albocrustata, Sacc.

$\{$ Sphaeria canescens, P.

\{Lasiosphaeria canescens, Karst.

\{ Sphacria leucospila, B. \& C.

\{Linospora leucospila, Sacc.

$\{$ Sphaeria circumscissa, P.

Massaria circumscissa, M. A. Curtis.

$\{$ Sphaeria semitecta, B. \& C.

Massaria semitecta, Sacc.

$\{$ Sphaeria dematiosa, S.

Nectria dematiosa, M. A. Curtis.

$\{$ Sphaeria convergens, Tode.

I Pseudovalsa convergens, Sacc.

\{ Sphaeria Aquila, Fr.

\{ Rosellinia Aquila, De Not.

Sphaeria millegrana, S.

Psilosphacria millegrana, Rav. Fung. Am.

Rosellinia millegrana, Sacc.

Sphaeria Pupula, Fr.

Sphaerostilbe gracilipes, Tul.

Valsa salicina, (P.) Fr.

$\{$ Sphaeria stellulata, $\mathrm{Fr}$.

\{Valsa stellulata, Fr. 
FUNGI IMPERFECTI.

Botrytis rosen, Lk.

Campsotrichum simplex, Cke.

f Sporidesmium sticticum, B. \& C.

\{ Clasterosporium sticticum, Sacc.

\{ Stilbum didymum, Cke.

\{Didymobotryum Cookci, Sacc.

Diplodia Aceris, Fckl.

Diplodia fulvella, Cke.

Discosia Artocreas, (Tode) $\mathrm{Fr}_{\mathrm{r}}$.

llarpographium fasciculatum, Sacc.

Ilelicotriclum obscurum, (Cda.) Sacc.

(Sphaeropsis petiolata, Cke.

Phoma petiolata, Sacc.

Nacrophoma petiolata, Berl. \& Vogl.

\{Oedemium sparsum, B. \& Rav.

\{ Ocdcmium subsparsum, Sacc.

$\{$ Coccularia stictica, B.

Phoma stictina, Sacc.

Phyllosticta Platani, Sacc. \& Speg.

Sporidesmium epicoccoides, B. \& C.

Sporidesmium stygium, B. \& C.

Triposporium Ravenelii, Cke.

\section{HYMENOMYCETES.}

Exidia repanda, Fr.

Exidia spiculata, S.

Hydnum Erinaceus, Bull.

Boletus juglandinus, $\mathrm{S}$.

$\{$ Polyporus juglandinus, S.

Poria juglandina, Cke.

\section{JUGLANDACEAE.}

Carya alba, Nutt.

\section{PYRENOMYCETES.}

\footnotetext{
Sphaeria caryophaga, S. Sphaeria Curtisii, B.

Iypoxylon nucitena, B. \& C.

Nclanomma? nucitena, Sacc.

Amphisphaerıa caryophaga, Cke.

S Sphaeria juglandicola, S.

Sphaeria juglandina, Curr.

Diplodia juglandicola, Curr.

Valsa juglandicola, Cke.

Botryodiplodia juglandicola, Sacc.

\{ Hypocrea chlorina, Cke.

\{ Broomella chlorina, Sacc.

Cryptospora Caryae, $\mathrm{Pk}$.

Eutypa ludibunda, Sacc.

S Sphacria tubaeformis, Tode.

Gnomoniclla tubiformis, Sacc.

\{ Hysterium Cookeianum. Gerard.

\{ GIoniopsis Cookeana, Sacc.

Hysterium magnosporium, Gerard.

Microsphaer Alni, (DC.) Wint.
}

Nectria Missouriensis, Ell. \& Ev.

$\{$ Dothidea juglandicola, S.

(Phyllachora juglandicola, Sacc.

Sphacrella convexula, (S.) Thm.

Sphaeria pericarpii, S.

Xylaria Hypoxylon, (L.) Grev.

FUNGI IMPERFECTI.

Botrytis cinerclla, Sacc. \& Wint.

Discosia rugulosa, B. \& C.

Fusicladium effusum, Wint.

Hendersonia Davisii, Ell. \& Ev.

Illosporium caesium, S.

Melanconium intermedium, $\mathrm{Pk}$.

Melanconium pallidum, $\mathrm{Pk}$.

Microstroma leucosporum, (Mont.) Niessl.

Phyllosticta Caryae, Pk.

Ramularia albomaculata, $\mathrm{Pk}$.

Sphaeropsis Caryae, C. \& E.

Sphaeropsis pericarpii, $\mathrm{Pk}$.

DISCOMYCETES.

Peziza floccosa, S.

Rhytisma Juglandis, S.

\section{HYMENOMYCETES.}

$\{$ Agaricus chrysophlebius, B. \& Rav.

\{Pluteus chrysophlebius, Sace. Syll.

\{ Agaricus niger, S.

\{ Pleurotus niger, Sacc. Syll.

Clavaria spathulata, $\mathrm{Pk}$.

Corticium cinereum, $\mathrm{P}$.

Corticium cinereum, P., var. fumigatum, Pk.

$\{$ Polyporus Caryae, S.

Poria Caryae, Cke.

Polyporus conglobatus, B.

Stereum purpureum, $P$.

Carya amara, Nutt.

$\{$ Clasterosporium uncinatum, Clinton.

\{ Cerncophorum uncinatum, Sacc.

Fusicladium effusum, Wint.

Hysterium nucicolum, S.

Sphaerella convexula, (S.) Thm.

Carya aquatica, Nutt.

Pestalozzia sphaerelloides, Ell. \& Langlois.

Microstroma leucosporum, (Mont.) Niessl.

Carya olivaeformis, Nutt.

Dendrodochium simile, Ell. \& Ev.

Fusicladium caryigenum, Ell. \& Langlois.

Fusicladium effusum, Wint.

Carya porcina, Nutt.

Carya sulcata, Nutt.

$\{$ Dermosporium atrum, S.

Strumella atra, Sacc. 
Carya tomentosa, Nutt.

\{ Sphaeria caryophagga, S.

\{ Amphisphaeria caryophaga, Cke.

(Corticium fumigatum, Thm.

$\{$ Peniophora cinerea, (P.) Cke., var.

( fumigata, Sacc. Syll.

$\{$ Dematium cinnabarinum, $P$.

$\{$ Dacrina cinnabarina, $\mathrm{S}$.

$\{$ Sphaeria exarata, S.

Hypoxylon exaratum, M. A. Curtis.

Melanconium oblongum, $B$.

Microstroma leucosporum, (Mont.) Niessl.

Oidium pulvinatum, Cke.

Phlebia coccineofulva, $S$.

Rhytisma Juglandis, $\mathrm{S}$.

$\{$ Sphaeria convexula, S.

\{ Sphaerella convexula, Thm.

Stercum purpureum, P.

Stereum sanguinolentum, (A. \& S.) Fr.

$\{$ Dermosporium atrum, S.

$\{$ Strumella atra, Sacc.

$\{$ Sphaeria juglandicola, Schw.

\{Valsa juglandicola, Cke.

Carya, sp. indet.

\section{PYRENOMYCETES.}

Acrospermum viridulum, B. \& C.

Sphaeria concentrica, Bolt.

Hypoxylon concentricum, Grev.

(Daldinia concentrica, Ces. \& De Not.

$\{$ Valsa apocrypta, C. \& E.

$\{$ Diaporthe apocrypta, Sacc.

$\{$ Valsa Woolworthii, Pk.

$\{$ Diaporthe Woolworthii, Sacc.

Eutypa maura, (Fr.) Sacc.

$\{$ Valsa constellata, B. \& C. in Herb. B.

$\{$ Eutypella constellata, Berl. \& Vogl.

Fenestella amorpha, Ell. \& Ev.

Gihbera moricarpa, Cke.

Hypocrea gelatinosa, (Tode.) Fr.

Hysterium ellipticum, Fr.

f Hysterium Juglandis, S.

\{Hysterium pulicare, P., var. Juglandis, S.

Massaria (Massariella) seriata, Cke.

(Sphaeria leiostega, Ell.

$\{$ Metasphaeria leiostega, Sacc.

Endophlaea leiostega, Cke.

Nectria coccinea, (P.) Fr.

Nectria polythalama, B.

Rosellinia Aquila, ( $F_{r}$.) De Not.

(Sphaeria dendroides, S.

$\{$ Sphaerulina myriadea, (DC.) Sacc.

Sphaerella dendroides, Cke.

$\{$ Sphaeria punctiformis, P.

S Sphaerella punctiformis, Fr.

Sphaeria (Thyridium) ambleia, C. \& E.

Sphaeria drupivora, S.
Sphacrostilbe cinnabarina, Tul.

Sphaerostilbe gracilipes, Tul.

Triblidium insculptum, Cke.

Valsa caryigena, B. \& C.

Valsa chlorodisca, C. \& E.

Valsa conscripta, C. \& E.

Valsa decorticans, Fr.

Valsa deusta, Ell. \& Ev.

Valsa stellulata, Fr.

FUNGI IMPERFECTI.

$\{$ Excipula microspora, C. \& E.

Annerosporium microsporum, Sacc.

Cylindrosporium caryigenum, Ell. \& Ev.

Dactylaria mucronulata, Ell. \& Langlois.

Stilbum leucocephalum, B. \& C.

$\{$ Graphium leucocephalum, Tul.

Graphium Curtisii, Sacc.

\{ Stilbum rigidum, $P$.

\{ Graphium rigidum, Sacc.

Helminthosporium Arbuscula, B. \& C.

Hendersonia eximia, B. \& C.

$\{$ Hendersonia nobilis, B. \& C.

Hendersonia navicula, Cke.

$\{$ Rhytisma seriale, S.

Leptostroma seriale, Cke.

Lichenopsis sphaeroboloidea, S.

Melanconium gracile, Ell. \& Ev.

Melanconium intermedium, Pk.

Melanconium magnum, B.

\{ Phyllosticta Caryae, Ell. \& Ev.

$\{$ Phyllosticta caryigena, Ell. \& Ev.

Pilacre Petersii, B. \& C.

Septoria Caryae, Ell. \& Ev.

\section{HYMENOMYCETES.}

Agaricus (Pleurotus) atrocacruleus, Fr.

$\{$ Michenera Artocreas, B. \& C.

A Artocreas Micheneri, B. \& C.

Thelephora albidocarnea, S.

$\{$ Corticium albidocarneum, Rav.

Asterostroma albidocarneum, Massee.

Corticium Auberianum, Mont.

Corticium Leveillianum, B. \& C.

Cyphella Ravenelii, $\mathrm{B}$.

Gomphidius viscidus, Fr.

Grandinia tuberculata, B. \& C.

Hirneola Auricula-Judae, (L.) B.

Hydnum casearium, Morg.

Hydnum glabrescens, B. \& Rav.

Hydnum membranaceum, Bull.

Hydnum mucidium, Gmel.

Hymenochaete purpurea, Cke. \& Morg.

Irpex pallescens, Fr. 
Irpex Tulipiferae, S.

Panus farinaccus. (Schum.) Fr.

Polyporus abortivus, Pk., var. subglobosus, $\mathrm{Pk}$.

Polyporus Alabamae, B. \& C.

Favolus Canadensis, Klotzsch.

$\{$ Polyporus Boucheanus, (Klotzsch) Fr.

Favolus Europaeus, Auct. Am.?

Polyporus cuticularis, (Bull.) Fr.

Polyporus endocrocinus, B.

Polyporus hemileucus, B. \& C.

Polyporus igniarius, (L.) Fr., var.

Polyporus nidulans, Fr.

Polyporus niphodes, B. \& Br.

Polyporus pargamenus, Fr.

Polyporus plebeius, B.

Polyporus Salleanus, B. ?

Polyporus scutellatus, S.

(Polyporus spissus, S. in Fr. El.

$\{$ Poria spissa, Cke.

Mucronoporus spissus, Ell. \& Ev.

Polyporus vaporarius, (P.) Fr.

Polystictus purpureofuscus, Cke.

Radulum molare, Fr.

Radulum pallidum, B. \& C.

Stereum albobadium, (S.) Fr.

\section{MISCELLANEA.}

Myriangium Curtisii, B. \& Mont.

Juglans cinerea, L.

$\{$ Sphneria diffusa, S.

\{ Byssosphaeria diffusa, Cke.

f Sphaeria drupivora, S.

Ceratostoma drupivorum, Cke.

Corticium puberum, Fr.

Crepidotus haerens, Pk.

Depazea juglandina, $\mathrm{Fr}$.

\{ Valsa bicincta, C. \& P.

Diaporthe bicincta, Sacc.

Gloeosporium Juglandis, (Lib.) Mont.

Hysterium cinerascens, S.

Melanconium oblongum, B.

Microsphaera Alni, (DC.) Wint.

(Microstroma leucosporum, (Mont.) Niessl.

\{ Microstroma Juglandis, (Bereng.) Sacc., var. brachysporum, Sacc.

Ostropa rugulosa, S.

\{ Didymium subroseum, Pk.

\{ Physarum albicans, Pk.

Polyporus Radula, (P.) Fr.

Sphaeria amorphula, S.

Sphacria druparum, S.

Sphaeria umbonata, Fr.

Sphaeropsis Gallae, (S.) B. \& C.

Sphaeria lixivia, Fr.

\{ Valsa lixivia, $\mathrm{Fl}_{1}$.
Juglans nigra, L.

Cercospora Juglandis, Kell. \& Swingle.

Cladosporium pericarpinum, Cke.

Cytospora albiceps, Ell. \& Kell.

Diplodia Juglandis, Fr.

Sphaeria scoparia, S.

$\{$ Valsa scoparia, M. A. Curtis.

(Eutypa scoparia, Wint.

Gibbera pulicaris, Fr.

(Gloeosporium Juglandis, (Lib.) Mont.

\{ Marsonia Juglandis, Sacc.

Peziza auriformis, S.

Exidia auriformis, Fr.

Tremella auriformis, Spreng.

Hirneola auriformis, Fr.

S Sphaeria hispida, Tode.

\{Lasiosphaeria hispida, Fckl.

Lenzites vialis. $\mathrm{Pk}$.

Microsphaera Alni, (DC.) Wint.

$\{$ Microstroma leucosporum, (Mont.) Niess\}.

(Microstroma Juglandis, (Bereng.) Sacc.

(Boletus juglandinus, S.

$\{$ Polyporus juglandinus, $\mathrm{S}$.

Poria juglandina, Cke.

Sphaeria druparum, S.

Sphaeropsis pericarpii, $\mathrm{Pk}$.

Trichia difformis, $S$.

Triposporium Juglandis, Thm.

Sphaeria Juglandis, S. nec. Fr.

Septoria Juglandis, B. \& C.

$\{$ Rhabdospora Juglandis, Sacc.

Valsa Juglandis, S. Herb. sec. Ckc.

S Sphaeria sphinctrina, Fr.

\{ Valsa sphinctrina, Fr.

Valsa tetraploa, B. \& C.

Juglans regia, L.

Caryospora putaminum, (S.) De Not.

Diplodia Juglandis, Fr.

$\{$ Valsa juglandina, C. \& E.

$\{$ Eutypella juglandina, Sacc.

\{ Peziza fructigena, Bull.

\{ Helotium fructigenum, Karst.

Nelanconium magnum, B.

Microstroma leucosporum, (Mont.) Niessl.

$\{$ Peziza abdita, EH.

$\{$ Mollisia abdita, Sacc.

Sphaerella petiolicola, (Desm.) Awd.

Juglans, sp. indet.

Astcrophora effusa, S.

(Sphaeria Tubulina, A. \& S.

$\{$ Hypoxylon Tubulina, Fr.

Bolinia Tubulina, Sacc.

Cenangium Juglandis, B. \& C.

f Niclularia juglandicola, $\mathrm{S}$.

\{ Crucibulum juglandicolum, De Toni.

Dematium strigosum, (P.) Lk.

Dothidea (Ectostroma) petiolaris, S.

S Sphaeria spinosa, $P$.

\{ Eutypa spinosa, Tul. 
Hydnum Himantia, S.

Hydnum ischodes, B.

$\{$ Sphaeria ovina, $P$.

Lassiosphaeria ovina, Ces. \& De Not.

$\{$ Hysterium mytilinum, $\mathrm{P}$.

\{Lophium mytilinum, Fr.

Lycoperdon quercinum, $\mathrm{P}$.

Sphaeria gyrosa, S.

Peziza gyrosa, Spreng.

Melogramma gyrosum, Tul.

Endothia gyrosa, Tul.

Monilia aurea, var. aurantiaca, S. Syn. Car.

Nectria sanguinea, (Sibth.) Fr.

$\{$ Peziza Erinaceus, S.

\{ Lachnea Erinaceus, Sacc.

Phoma Pustula, (P.) Fr.

(? Sphaeria conferta, S. Am. bor.

$\{$ Dothidea acervulata, Fr.

Phyllachora acervulata, Sacc.

Physarum polyaedron, S.

Polyporus fumosogriseus, C. \& E.

Polyporus adustus, Fr., var. resupinatus, Cke.

$\{$ Polyporus cinereus, S.

Poria cinerea, Cke.

Peziza digitalis, S. Syn. Car.

Peziza pendula, S. Syn. Car.

Cyphella pendula, Fr.

Polyporus cupulaeformis, B. \& C.

Polyporus spongiosus, S. Am. bor.

$\{$ Agaricus alneus, L.

Schizophyllum commune, Fr.

Sphaeria putaminum, S., var.

Sphaeria Juglandis, $\mathrm{S}$.

Septoria Juglandis, B. \& C.

Rhabdospora Juglandis, Sacc.

(Valsa Juglandis, Cke.

\section{MYRICACEAE.}

\section{Myrica asplenifolia, Endl.}

Cronartium asclepiadeum, (Willd.) Fr.

Cucurbitaria Comptoniae, C. \& E.

Diaporthe Comptoniae, Ell. \& Ev.

$\{$ Diatrype Comptoniae, Ell. \& Ev.

\{Thyridaria Coinptoniae, Berl. \& Vogl.

Pcstalozzia flagellifera, Ell. \& Ev.

$\{$ Peziza rhabdocarpa, Ell.

\{ Erinella rhabdocarpa, Sacc.

$\{$ Sphaeria Comptoniac, S.

\{Valsa Comptoniae, Cke.

Valsa conscripta, C. \& E.

$\{$ Sphaeria rimicola, S.

\{ Valsa rimicola, Cke.

Myrica Californica, Cham.

Eutypa lata, (P.) Tul.

Lasiosphaeria biformis, (P.) Sacc.

Lophodermium xylomoides, (DC.) Chev.
Myrica cerifera, L.

$\{$ Melogramma graphideum, B. \& Rav.

\{ Botryosphaeria graphidea, Sacc.

Caeoma (Aecidium) myricatum, S.

Cercospora diffusa, Ell. \& Ev.

Cercospora dispersa, Ell. \& Ev.

Cercospora Penicillus, Ell. \& Ev.

Corticium calceum, Fr.

Dialonectria erubescens, (Rob.) Cke.

$\{$ Gibbera moricarpa, Cke.

Fracchiaea moricarpa, Sacc.

Gnomonia Myricae, C. \& E.

Helminthosporium inflatum, B. \& Rav.

Hymenochaete badioferruginea, (Mont.) Lev.

Hymenochaete rubiginosa, (Schrad.) Lev.

Hymenochaete scabriseta, Cike.

Hypocrea ochroleuca, B. \& Rav.

Hypoxylon marginatum, (S.) B., var. effusum, B.

Meliola manca, Ell. \& Martin.

$\{$ Peniophora flavidoalba, Cke.

$\{$ Corticium flavidoalbum, Rav. Fung. Am.

Pestalozzia Myricae, Ell. \& Martin.

$\{$ Peziza callochaetes, Ell. \& Ev.

$\{$ Dasyscypha callochaetes, Sacc.

Peziza pruinata, S.

$\{$ Solenia poriaeformis, (DC.) Fckl.

Tapesia pruinata, Sacc.

Phyllosticta Myricae, Cke.

$\{$ Polyporus Alabamae, B. \& Ckc.

$\{$ Poria Alabamae, B. \& Cke.

Sphaerella pardalota, C. \& E.

Stagonospora Myricae, Ell. \& Ev.

Sphaeria acanthostroma, Mont.

$\{$ Trichosphaeria acanthostroma, Sacc.

Byssosphaeria acanthostroma, Cke.

Xylaria corniformis, Fr.

Zygodesunus pannosus, B. \& C.

Myrica Gale, L.

Diaporthe Wibbei, Nits.

f Torula curvata, $\mathrm{Pk}$.

\{Hormiscium curvatum, Sacc.

Metasphaeria Myricae, $\mathrm{Pk}$.

$\{$ Ovularia monilioides, Ell. \& Martin.

$\{$ Ramularia monilioides, Ell. \& Ev.

$\{$ Peziza bicolor, Bull.

$\{$ Dasyscypha bicolor, Fckl.

$\{$ Peziza myricacea, $\mathrm{Pk}$.

$\{$ Trichopeziza myricacea, Sacc.

$\{$ Peziza sulphurella, $\mathrm{Pk}$.

$\{$ Dasyscypha sulphurella, Sacc.

Myrica, sp. indet.

Aspergillus Curtisii, B:

$\{$ Valsa phomaspora, C. \& E.

$\{$ Diaporthe phomospora, Sacc.

$\{$ Valsa tecta, Cke.

Diaporthe tecta. Sacc.

$\{$ Valsa Myricae, C. \& E.

\{ Eutypella Myricae, Sacc. 
? Farolus flaccidus, Fr.

Sphaeria subcongregata, B. \& C.

$\{$ Sphaeria subconnata, B. \& C.

Fracchiaea subconnati, Cke.

Glenospora Curtisii, B. \& Desm.

Grandinia granulosa, (P.) Fr.

$\{$ Hypoxylon polyspermum, Mont.

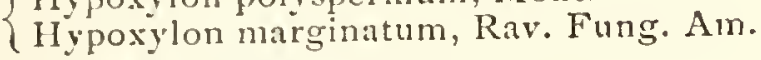

$\{$ Peziza alboviridis, Cke.

$\{$ Trichopeziza alboviridis, Sacc.

Polyporus barbaeformis, B. \& C.

Polyporus contiguus, (P.) Fr.

$\{$ Polyporus crocatus, Fr.

\{ Polystictus crocatus, Fr.

Polyporus Floridanus, B.

$\{$ Polyporus holoxanthus, B. \& Cke.

\{ Poria holoxantha, B. \& Cke.

Polyporus niphodes, B. \& Br.

Polyporus tenuis, S.

Stilbum glaucum, Cke.

Thelephora pedicellata, S.

Tremella Myricae, B \& Cke.

Valsa quernea, Curr.

\section{CUPULIFERAE.}

\section{Alnus incana, Willd.}

Anthostoma Ellisii, Sacc., var. exudans,

Pk.

r Valsa femoralis, $\mathrm{Pk}$.

$\{$ Cryptospora femoralis, Sacc.

Dermatea furfuracea, (Roth) Fr.

Diatrypella Tocciaeana, De Not.

Erysiphe aggregata, (Pk.) Farl.

Eutypella cerviculata, (Fr.) Sacc.

Hypoxylon fuscum, (P.) Fr.

Melanconis Alni, Tul.

(Microsphaera penicillata, (Wallr.) Lev.

Microsphaera penicillata, (Wallr.) Lev., var. Alni, C. \& P.

Microsphaera Alni, (DC.) Wint.

Pilacre orientalis, B. \& Br.

Pistillaria alnicola, $\mathrm{Pk}$.

Scolecosporium Fagi, Lib.

Septoria alnicola, Cke.

Sphaerella minutissima, $\mathrm{Pk}$.

- S Ascomyces Tosquinetii, Auct. Amer.

Taphrina alnitorqua, Auct. Amer.

Exonscus alnitorquas, Auct. Amer.

Exoascus amentorum, Sadebeck.

Taphrina Alni-incanae, (Kiihn) Magnus.

Thelephora rosella, $\mathrm{Pk}$.

Torula alnea, Pk.

Alnus rubra, Bongard.

Cryptosporium Neesii, Cda.

Gnomonia Alni, Plow.

Hydnum ciliolatum, B. \& C.
Melanconium sphaeroideum, Lk.

Naematelia nucleata, (S.) Fr.

Radulum orbiculare, Fr.

Asconyces Tosquinetii, Auct. Amer.

$\{$ Taphrina alnitorqua, Auct. Amer.

Taphrina Alni-incanae, (Kühn) Magnus.

Valsa femoralis, Pk.

Alnus rhombifolia, Nutt.

? Melampsora betulina, (P.) Tul.

Alnus serrulata, Willd.

Agaricus aurivellus, Batsch.

Capnodium elongatum, B. \& Desm.

$\{$ Ceratostoma capillare, Ell.

\{ Ceratostomella capillaris, Sacc.

\{ Cyphella fulva, B. \& Rav.

\{yphella Ravenelii, Sacc.

Cyphella subgelatinosa, B. \& Rav.

Diatrype megastoma, Ell. \& Ev.

$\{$ Diatrype aspera, Fr.

$\{$ Diatrypella aspera, Nits.

Diatrypella discoidea, C. \& P., var. Alni, Ckè.

Sphaeria verruciformis, Ehrla.

Diatrype verruciformis, $\mathrm{Fr}_{\mathrm{r}}$.

l Diatrypella verruciformis, Nits.

(Xyloma alneum, $\mathrm{P}$.

Dothidea alnea, Fr.

Discosia alnea, B.

\{rysiphella aggregata, $\mathrm{Pk}$.

Erysiphe aggregata, Farl.

(Valsa stellulata, Rav. Fung. Am.

$\{$ Eutypella stellulata, (Fr.) Sacc., var.

( cucurbitarioides, Rehm.

\{ Hysterium parvulum, Gerard.

$\{$ Gionium parvulum, Cke.

Helicosporium microscopicum, Ell.

Hymenochaete corrugata, (Fr.) Lev.

Hypocrea citrina, (P.) Fr.

Hypocrea rufa, (P.) Fr.

Hypoxylon fuscum, (P.) Fr.

\{Hypoxylon xanthocreas, B. \& C.

\{ Hypoxylon Peckianum. Sacc.

\{ Hypocrea viridirufa, B. \& Rav.

Hypoxylon viridirufum, Cke.

( Nelasmia alnea, Lev.

\{eptothyrium alneum, Sacc.

Microsphaera Alni, (DC.) Wiut.

(Peziza fascicularis, A. \& S.

$\{$ Dermatea fascicularis, Fr.

Cenangium populneum, (P.) Rehm.

Phyllactinia suffulta, (Reb.) Sacc.

Polyporus obliquus, Fr.

$\{$ Fomes obliquus, Cke.

Mucronoporus obliquus, Ell. \& Ev.

Polyporus vaporarius, (P.) $\mathrm{Fr}$.

$\{$ Valsa quaternata, (P.) Fr.

\{uarternaria Persoonii, Tul.

Sphaeropsis Alni, C. \& E.

Sphacrostilhe coccophila, Tul. 
Tremella enata, B. \& C.

$\{$ Plicatura Alni, Pk.

$\{$ Trogia Alni, Pk.

$\{$ Sphaeria Americana, B. \& C.

$\{$ Valsa Americana, B. \& C.

Valsa femolalis, $\mathrm{Pk}$.

Sphaeria haustellata, $\mathrm{Fr}$.

$\{$ Diatrype haustellata, Rav. Fung. Car.

Valsa haustellata, M. A. Curtis.

Valsa tetraploa, B. \& C.

Alnus viridis, DC.

Diaporthe marginalis, $\mathrm{Pk}$.

Dothidella Alni, Pk.

Sphaeria tubaeformis, Tode.

$\{$ Gnomonia tubiformis, Awd.

(Gnomoniella tubiformis, Sacc.

Leptothyrium alneum, (Lev.) Sacc.

Melanconium dimorphum, Pk.

Microsphaera divaricata, Lev.

Phoma Phillipsiana, Sacc. \& Roum

Polyporus contiguus, (P.) $\mathrm{Fr}$

Sphaerella alnicola, $\mathrm{Pk}$.

Alnus, sp. indet.

\section{PYRENOMYCETES.}

(Diatrype microspora, El].

$\{$ Anthostoma Ellisii, Sacc.

Fuckelia Ellisii, Cke.

Nectria diploa, B. \& C., var. diminuta, B.

Nectria diminuta, Sacc.

Dialonectria diminuta, Cke.

Dialonectria diploa, var. diminuta, Ell. \& Ev.

Calonectria diminuta, Berl. \& Vog].

$\{$ Valsa inconspicua, C. \& E.

Calospora ? inconspicua, Sacc.

Sphaeria suffusa, Fr.

$\{$ Valsa suffusa, $\mathrm{Fr}$.

Cryptospora suffusa, Tul.

$\{$ Valsa paucispora, $\mathrm{Pk}$.

\{ Cryptosporella paucispora, Berl. \& Vogl.

$\{$ Cucurbitaria alnea, Pk.

\{Otthia alnea, Sacc.

Daldinia concentrica, (Bolt.) Ces. \& De Not.

Diatrype Stigma, (Hoffin.) Fr.

Diatrypella Tocciaeana, De Not., var. subeffusa. E11. \& Ev.

Hypocrea gelatinosa, (Tode) Fr.

Stypoxylon Morsei, B. \& C.

$\{$ Hypoxylon Blakei, B. \& C.

Fuckelia Morsei, Cke.

Hypoxylon commutatum, Nits.

Hypoxylon commutatum, Nits., var.

Holwayanum, Sacc. \& Ell.

Hypoxylon multiforme, $\mathrm{Fr}$.

$\{$ Valsa thelebola. Fr.

Melanconis thelebola, Sacc.

\{ Erysiphe divaricata, S. Am. bor.

\{Microsphaera divaricata, Lev.
Nectria aglaeothele, B. \& C.

Nectria coccinea, (P.) Fr.

Nectria diploa, B. \& C.

$\{$ Nectria subcoccinea, Sacc. \& Ell.

\{ Nectria muscivora, Journ. Myc. II. I Io.

Valsa tubulosa, B. \& C.

Valsa tribulosa, B. \& C.

Pseudovalsa tubulosa, Sacc.

Calospora tribulosa. Sacc.

Scorias spongiosa, (S.) $\mathrm{Fr}$.

Sphaerella conglomerata, (Wallr.) Awd.

$\{$ Sphaeria Papilla, S.

\{Melanopsamma Papilla, Sacc.

$\{$ Sphaeria obducens, Fr.

\{ Teichospora obducens, Fckl.

Valsa Alni, Pk.

$\{$ Sphaeria centripeta, Fr.

$\{$ Valsa centripeta, Pk. 26th Rept.

$\{$ Diatrype moroides, C. \& P.

$\{$ Valsaria moroides, Sacc.

\section{FUNGI IMPERFECTI.}

Coniothyrium valsoideum, $\mathrm{Pk}$.

Cryptosporium Neesii, Cda.

$\{$ Valsa truncata, C. \& P.

\{ Cytospora truncata, Sacc.

Helminthosporium persistens, Cke.

$\{$ Sphaeropsis Amenti, Cke. \& Hark.

\{ Phoma Amenti, Sacc.

Polyactis pulvinata, B. \& C.

$\{$ Septoria maculans, B. \& C.

\{ Rhabdospora maculans, Sacc.

Rhinotrichum Curtisii, B.

Septonema dichaenoides, Pk. \& Clinton.

Sphaeronema pruinosum, Pk.

Sphaeropsis alnicola, $\mathrm{Pk}$.

Sphaeropsis Pennsylvanica, B. \& C.

Sporidesmium sicynum, Thm.

\{Dactylium sublutescens, $\mathrm{Pk}$.

\{'Trichothecium sublutescens, Sacc.

\section{DISCOMYCETES.}

Heliotium fastidiosum, $\mathrm{Pk}$.

Patellaria rhabarbarina, $B$.

Phacidium alneum, (Wormsk.) Fr.

\section{HYMENOMYCETES.}

Agaricus atrocaeruleus, Fr.

Corticium incarnatum, $\mathrm{Fr}$.

Corticium laeve, $\mathrm{P}$.

$\{$ Corticium scariosum, B. \& C.

$\{$ Corticium secedens, Sacc.

Grandinia granulosa, (P.) Fr.

Hydnum farinaceum, $P$.

Hymenochaete agglutinans, Ell. 
Irpex obliquus, (Schrad.) Fr.

Irpex paradoxus, (Schrad.) Fr.

Kneiffia setigera, Fr.

Marasmius praeacutus, Ell.

Serulius confluens, S.

$\{$ Merulius Corium, Fr.

Cantharellus confluens, S.

Panus operculatus, B. S C.

Polyporus aureonitens, Patouillard.

Polyporus pubescens, (Schm.) Fr.

Polyporus scutellatus, S.

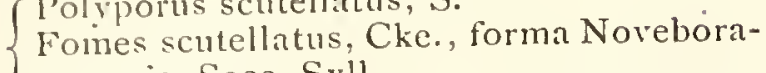
censis, Sacc. Syll.

Radulum molare, Fr.

Solenia ochracen, Hoffm.

Thelephora episphaeria, Fr.

Thelephora pedicellata, S.

Tremella intumescens, Sm.

$\left\{\begin{array}{l}\text { Merulius crispus, } P \text {. } \\ \text { Cantharellus crispus, Fr. } \\ \text { Trogia crispa, Fr. }\end{array}\right.$

Tympanis alnea, (P.) $\mathrm{Fr}$.

Betula carpinifolia, Sieb. \& Zucc.

Cenangium compressum, S.

Cenangium laminare, Fr.

Cenangium molliuscullum, S.

S Sphaeria transversa, S.

$\left\{\begin{array}{l}\text { Hypoxylon transversum, Sace. } \\ \text { Hetula }\end{array}\right.$

Betula glandulosa, Michx.

Dothidella betulina, (Fr.) Sacc.

Betula intermedia, Thom.

Dothidella betulina, (Fr.) Sacc.

Mollisia fusca, (P.) Karst.

\section{Betula lenta, L.}

Exidia picen, B. \& C.

Gelatinosporium betulinum, Pk.

$\{$ Sphaeronema seriatum, B. \& C.

Sphaerographium seriatum, Sacc.

Glocosporium Betularum, Ell. \& Martin.

$\{$ Valsa acrocystis, Pk.

\{Melanconiclla acrocystis, Berl. \& Vogl.

$\{$ Nicrosphaera penicillata, (Wallr.) Lev.

NIicrosphaera Alni, (DC.) Wint.

Septoria microsperma, $\mathrm{Pk}$.

Sphaeria scoriadea, Fr.

Betula lutea, Michx. f.

Asterosporium betulinum, $\mathrm{Pk}$.

$\{$ Diatrype betulina, $\mathrm{Pk}$.

$\{$ Diatrypella betulina, Sacc.

Gelatinosporium betulinum, $\mathrm{Pk}$.

Gelatinosporium fulvum, Pk.

Omphalia subgrisea, $\mathrm{Pk}$.

Septoria betulicola, Pk.

Polyporus pargamenus, Fr.

Polyporus elongatus, $B$.

Polystictus parganenus, Fr.

Polyporus pseudopargamenus, Thm.

(Polystictus pseudopargamenus, Sacc. Syll.
Diatrype ferruginea, Fr.

\{ Sillia ferruginea, Karst.

$\{$ Diatrype nigrospora, $\mathrm{Pk}$.

\{Valsaria nigrospora, Berl. \& Vogl.

Betula nana, L.

Didymosphaeria nana, Rostrup.

Dothidella betulina, (Fr.) Sacc.

Gnomonia campylostyla, Awd.

Taphrina bacteriosperma, Johanson.

Taphrina carnea, Johanson.

\section{Betula nigra, L.}

Botrytis minutula, S.

Coryneum compactum, B. \& Br.

$\{$ Hypoxylon concentricum, (Bolt.) Grev.

$\{$ Daldinia concentrica, Ces. \& De Not.

Eutypella angulosa, (Nits.) Sacc.

$\{$ Valsa niphoclina, Cke.

\{ Eutypella? niphoclina, Sacc.

Gloeosporium Betularum, Ell. \& Martin.

Hypoxylon fuscum, (P.) Fr.

Hysterium angustatum, $P$.

\{ Hysterium flexuosum, S.

Hysterographium flexuosum, Sacc.

Phyllactinia suffulta, (Reb.) Sacc.

S Sphaeria lanata, Fr.

Enchnoa lanata, Fr.

Betula occidentalis, Hook.

Phyllactinia suffulta, (Reb.) Sacc.

Betula papyracea, Ait.

Agaricus ostreatus, Jacq.

Diatrypella betulina, (Pk.) Sacc.

Diplodia valsoides, $\mathrm{Pk}$.

Eutypella cerviculata, (Fr.) Sacc.

Gnomonia Provancheriana, Thm.

Hypoxylon multiforme, Fr.

Hypoxylon transversum, (S.) Sacc.

Melanconium betulinum, Schm. \& Kze.

Melanconium bicolor, Nees.

Taphrina flava, Farl.

Trogia crispa, (P.) Fr.

Betula populifolia, Ait.

$\{$ Diplodia valsoides. Pk.

Botryodiplodia valsoides, Sacc.

Siphneria ciliatula, Fr.

$\{$ Valsa ciliatula, Fr.

Calosphaeria ciliatula, Karst.

i Cenangium betulinum, $\mathrm{Pk}$.

Scleroderris betulina, Sacc.

Cenangium seriatum, Fr.

Coryneum Kunzei, Cda.

Cryptospora Betulae, Tul.

$\{$ Valsa tomentella, $\mathrm{Pk}$.

\{ryptospora tomentella, Berl. \& Vogl.

$\{$ Diatrype discoiden, C. \& P.

\{ Diatrypella discoidea, C. \& P. 
CMelanconis lanciformis, (Fr.) Tul.

Melanconis elliptica, $\mathrm{Pl}$.

Diatrve elliptica, $\mathrm{Pk}$.

Pseudovalsa lanciformis, Ces. \& De Not., var. elliptica, Sacc.

Helminthosporium arbusculoides, $\mathrm{Pk}$.

Lenzites Cookei, B.

Melanconis (Melanconiella) Decorahensis, E11.

Melanconis stilbostoma, ( $\mathrm{Fr}_{\mathrm{r}}$.) Tul.

Melanconium betulinum, Schm. \& Kze.

Melanconium bicolor. Nees.

Polyporus salicinus, Fr.

Septoria Betulae, (Lib.) Westd.

Siphaeria ditricha, Fr.

Vermicularia ditricha, S.

Venturia ditricha, Karst.

Venturia maculans, $\mathrm{Pk}$.

Sphaeria flavovirens, Fr.

$\{$ Exoascus flavus, Farl.

\{ Taphrina flava, Farl.

Valsaria Niesslii, (Wint.) Sacc.

Valsella adherens, Fckl.

Betula pumila, L.

Diatrypella betulina, ( $\mathrm{Pk}$.$) Sacc.$

Melanconium betulinum, Schm. \& Kze.

Melanconis stilbostoma, ( $F r$. ) Tul.

Betula, sp. indet.

\section{PYRENOMYCETES}

\{ Sphaeria pannicola, B. \& C.

Chaetosphaeria pannicola, Sacc.

Cucurbitaria conglobata, (Fr.) Ces. \& De Not.

$\{$ Diatrype platasca, Pk.

$\{$ Diaporthe platasca, Sacc.

Diatrype asterostoma, B. \& C.

Diatrype disciformis, (Ioffm.) Fr.

$\{$ Sphaeria sordida, $\mathrm{P}$.

\{ Diatrype sordida, Cke.

Diatrype Stigma, (Hoftim.) Fr.

Sphaeria favacea, $\mathrm{Fr}$.

$\{$ Diatrype favacea, $\mathrm{Fr}$.

Diatrypella favacea, Ces. \& De Not.

f Sphaeria spinosa, $\mathrm{P}$.

(Eutypa spinosa, Tul.

Sphaeria Halseyana, S.

$\{$ Valsa Halseyana, Cke.

Eutypella Halseyana, Cke.

I Sphaeria setacea, P.

$\{$ Gnomonia setacea, Ces. \& De Not.

Sphaeria mirabilis, $\mathrm{Pk}$.

$\{$ Gnomoniella mirabilis, Sacc.

Gnomonia mirabilis, Cke.

Hypoxylon argillaceum, (P.) Fr.

Hypoxylon coccineum, Bull.

Hypoxylon Morsei, B. \& C.

Hypoxylon multiforme, Fr.
Hypoxylon perforatum, (S.) Fr.

Hysterium angustatum, P.

Ilysterium betulignum, S.

Hysterium pulicare, $P$.

Sphaeria monosperma, Pk.

$\{$ Julella monosperma, Sacc.

Delacourea monosperma, Cke.

$\{$ Sphaeria xestothele, B. \& C.

Lasiosphateria xestothele, Sacc.

Massaria Argus, (B. \& Br.) Fres.

Massaria siparia, (B. \& Br.) Ces. \& De Not.

Melanconis (Melanconiella) biansata, Ell. \&

Ev.

Melanconis Decorahensis, Ell., var. major, E11. \& Ev.

Melanconis lanciformis, (Fr.) Tul.

Mclanconis (Melanconiella) Meschuttii, E11. \& Ev.

Nectria Cucurbitula, (Tode) Fr.?

(Xyloma asteromorpha, S.

Dothidea asteromorpha, Fr.

Phyllachora asteromorpha, Sacc.

Sphaeria lichenalis, Pk.

$\{$ Pleospora lichenalis, Sacc.

Delacourea lichenalis, Cke.

$\{$ Sphaeria dissepta, Fr.

$\{$ Quaternaria dissepta, Tul.

\{ Sphaeria Desmazierii, B. \& Br.

$\{$ Rosellinia Desmazierii, Sacc.

Sphaerella sparsa, (Wallr.) Awd.

Sphaeria melasperma, Fr.

Sphaeria Verrucaria, Fr.?

Valsa angulosa, Nits., var elliptica, Pk.

Valsa Betulae, (Tul.) Ell. \& Ev.

Valsa pulchella, (P.) Fr.

(Sphaeria tuberculosa, S.

\{Xylosphaeria tuberculosa, Cke.

SPHAEROPSIDEAE.

Cytospora betulina, Ehrb.

Cytospora chrysosperma, (P.) Fr.

Cytospora melasperma, Fr.

$\{$ Sphaeria Artocreas, Tode.

$\{$ Discosia Artocreas, Fr.

Gelatinosporium magnum, E11.

Hendersonia glabra, Cke.

Prosthemium betulinum, Kze.

Sphaeria ? uberiformis, $\mathrm{Fr}$.

\{ Sphaeria oppilata, S. Am. bor.

\{ Stagonospora oppilata, $\left(\mathrm{Fr}^{\circ}\right.$ ) Sacc.?

MELANCONIEAE.

Coryneum disciforme, var. elllipticum, B. \& Br.

Cryptosporium Neesii, Cda. 
Didymosporium acuminatum, S.

Didymosporium eleratum, Lk.

Libertella betulina, Desm.

\{ Stilbospora microsperma, S. Syn. Car.

\{Melanconium sphaeroideum, Lk.

\{ Naemaspora crocea, P.

\{ MYxosporium croceum, Lk.

Myxosporium subviride, Ell. \& Ev.

\{ Naemaspora viridis, B. \& C.

\{ Mysosporium viride, Sacc.

f Corrncum irregulare, B. \& C.

\{ Steganosporium irregulare, Sacc.

Steganosporium muricatum, Bon.

\section{HYPHOMYCETES.}

Cladosporium lignicolum. Cda.

Nematogonium aurantiacum, Desm.

Sporotrichum cylindrosporum, Lk.

Zygodesmus granulosus, $\mathrm{Pk}$.

\section{DISCOMYCETES.}

?Cenangium Cerasi, (P.) Fr.

Cenangium seriatum, $\mathrm{Fr}$.

( Peziza sarcoides, (Jacq.) P.

$\{$ Bulgaria sarcoides, Fr.

Coryne sarcoides, Tul.

(Bulgaria bicolor, Pk.

$\{$ Peziza orbicularis, $\mathrm{Pk}$.

Discina orbicularis, Sacc.

Helotium aeruginosum, (Oeder) $\mathrm{Fr}$.

Seziza olivaceovirens, S.

$\{$ Patellaria olivaceovirens, $\mathrm{Fr}$.

Lemalis olivaceovirens, S.

$\{$ Peziza patula, $P$.

\{Dasyscypha patula, Sàcc.

Peziza populnea, $P$.

$\{$ Peziza fascicularis, A. \& S.

Cenanguim populneum, Rehm.

Phacidium coronatum, Fr.

Xylographa parallela, (Ach.) Fr.

HYMENOMYCETES.

Agaricus alnicola, Fr.

$\{$ Agaricus luteofolius, $\mathrm{Pk}$.

\{ Pholiota luteofolia, Sacc. Syll.

I Agaricus sapidus, Kalchb.

T Pleurotus sapidus, Sacc. Syll.

$\{\Lambda$ garicus semicaptus, B. \& C.

\{ Pleurotus semicaptus, Sacc. Syll.

Corticium martianum, B. \& C.

$\{$ Daedalea albida, S.

Daedalea discolor, Fr.

Daedalea confragosa, $P$.

Grandinia mucida, Fr.

Irpex pallescens, Fr.

$\{$ Thelephora setigera, Fr.

Kneifia setigera, Fr.

Lentinus lepideus, Fr.
Lenzites betulina, (L.) Fr.

Marasmius caespitosus, Pk.

Merulius tremellosus, Schrad.

Panus dorsalis, (Bosc.) Fr.

Phlcbia radiata, $\mathrm{Fr}$.

Polyporus annosus, S. Am. bor.

Polyporus aureonitens, Patouillard.

Boletus betulinus, Bull.

$\{$ Polyporus betulinus. Fr.

Polyporus albellus, $\mathrm{Pk}$.

S Polyporus biformis, $\mathrm{Fr}$.

$\{$ Polyporus Caroliniensis, B. \& C.

Polystictus biformis, $\mathrm{Fr}$.

Soletus alneus, S. Syn. Car.

$\{$ Polyporus cuticularis, S. Am. bor.

Polyporus conchatus, (P.) Fr.

Polyporus fomentarius, (L.) Fr.

$\{$ Polyporus igniarius, (L.) Fr.

(Fomes igniarius, Karst.

Polyporus marginatus, (P.) $\mathrm{Fr}$.

Polyporus nidulans, Fr.

Polyporus nigricans, Fr.

Fomes nigricans, Karst.

Mucronoporus nigricans, Ell. \& Ev.

Polyporus pubescens, (Schin.) Fr.

$\{$ Polyporus radiatus, (Sow.) Fr.

Polystictus radiatus, Cke.

Polyporus spissus, S.?

Polyporus subacidus, Pk., var. stalactiticus, Pk.

Poria subacida, Sacc. Syl]., var. stalactitica, Pk.

Polyporus ulmarius, (Sow.) Fr.

Polyporus vaporarius, (P.) Fr.

$\{$ Sistotrema spathulatum, S. Syn. Car.

Irpex spathulatus, S. Am. bor.

Polyporus vulgaris. Fr.

Boletus vitreus, S. Syn. Car.

Polyporus vitreus, S. Am. bor.

Poria vulgaris, Cke.

Solenia ochracea, Hoffm.

Stereum hirsutum, (Willd.) Fr.

Stereum purpureum, $P$.

Stereum rugosum, $P$.

MISCELLANEA.

Atractobolus lutescens, $\mathrm{S}$.

Ditiola radicata, (A. \& S.) Fr.

Mclampsora betulina. (P.) Tul.

Tremclla albida, Huds.

Carpinus Caroliniana, Walt.

PYRENOMYCETES.

Actidium? diatrypoides, Cke.

Sphaeria Betuli, P., var. tentaculata, S.

Sphaeria Carpini, Fr.

Valsa Carpini, Fr.

Diaporthe Carpini, Fckl. 
$\{$ Diatrype carpinigera, B. \& C.

Diaporthe carpinigera, Sacc.

S Valsaria actidia, B. \& Rav. in Heıb. B.

I Diatrype actidia, Cke.

Diatrype discostoma, Cke.

Sphaeria cerviculata, Fr.

$\{$ Valsa cerviculata, Fr.

Eutypella cerviculata, Sacc.

Sphaeria Leaiana, B.

$\{$ Valsa Leaiana, M. A. Curtis.

Eutypella Leaiana, Sacc.

Sphaeria callista, B. \& C.

$\{$ Cucurbitaria callista, B. \& C.

Fracchiaea callista, Sacc.

Sphaeria fimbriata, $P$.

$\{$ Gnomonia fimbriata, Fckl.

Gnomoniella fimbriata, Sacc.

Hypoxylon commutatum, Nits., var.

Holwayanum, Sacc. \& Ell.

Hypoxylon epiphaeum, B. \& C.

Hypoxylon fuscum, (P.) Fr.

Hypoxylon Morsei, B. \& C.

Sphaeria carpinea, Fr.

$\{$ Sphaerella carpinea, $\Lambda$ wd.

Laestadia carpinea, Sacc.

\{ Sphaeria canescens, P.

\{Lasiosphaeria canescens, Karst.

(Sphaeria subcorticalis, $\mathrm{Pk}$.

$\{$ Trichosphaeria subcorticalis, Sacc.

Lasiosphaeria subcorticalis, Cke.

$\{$ Melanconis chrysostoma, (Fr.) Tul.

\{Melanconiella chrysostoma, Sacc.

$\{$ Melogramma vagans, De Not.

\{ Melogramma Bulliardi, Tul.

Siatrype lateritia, Ell.

$\{$ Thyridaria lateritia, Sacc.

Melogramma lateritium, Cke.

Sypoxylon myriangioides, B. \& Rav.

$\{$ Thyridaria? myriangioides, Sacc.

(Melogramma myriangioides, Cke.

Microsphaera penicillata, (Wallr.) Lev.

Nectria coccinea, (P.) Fr.

$\{$ Phyllactinia guttata, Lev.

\{ Phyllactinia suffulta, (Reb.) Sacc.

$\{$ Diatrype Titan, B. \& Rav.

Pseudovalsa Titan, Sacc.

S Sphaeria acanthostroma, Miont.

Trichosphaeria acanthostroma, Sacc.

Valsa aurea, Fckl.

$\{$ Diaporthe Ellisii, Rehm.

\{Valsa Ellisii, Ell. \& Ev.

$\{$ Valsa stellulata, Fr.

$\{$ Diatrype haustellata, Pk. Rept.

\section{SPHAEROPSIDEAE.}

(Xyloma carpineum, S.

Sphaeria (Depazea) carpinicola, Fr.

Depazea carpinicola, M. A. Curtis.

Depazea carpinea, Sacc.
$\{$ Discella discoidea, C. \& P.

$\{$ Discula Peckiana, Sacc.

Plioma sordida, Sacc.

Sphaeropsis carpinea, Sacc. \& Br.

MELANCONIEAE.

Gloeosporium Carpini, (Lib.) Desm.

Glocosporium Robergei, Desm.

$\{$ Libertella betulina, Desm.

\{ Naemaspora aurea, $\mathrm{Fr}$.

Melanconium triangulare, Ell. \& Ev.

Stilbospora brevis, B. \& Rav.

Cheirospora botryospora, Fr.

Thyrsidium hedericolum, (De Not.) Dur. \&

Mont., var. Carpini, Sacc.

$\{$ Cheirospora Micheneri, B. \& C.

$\{$ Thyrsidium Micheneri, Sacc.

\section{HYPHOMYCETES.}

Fusisporium cinnabarinum, B. \& C.

$\{$ Helicoma Berkeleyi, M. A. Curtis.

\{Helicosporium Berkeleyi, Sacc.

Pilacre Petersii, B. \& C.

Septosporium velutinum, C. \& E.

$\{$ Periconia parasitica, $\mathrm{Pk}$.

Sporocybe parasitica, Sacc.

Streptothrix atra, B. \& C.

\section{DISCOMYCETES.}

Cenangium rubiginosum, Cke.

Dermatea carnea, C. \& E.

$\{$ Peziza flammea, A. \& S.

Lachnella flammea, Fr.

\Peziza carpinea, P.

Dermatea carpinea, Fr.

Patellaria carpinea, $\mathrm{B}$.

Pezicula carpinea, Tul.

Peziza nivea, (Hedw.) Fr.

$\{$ Peziza anomala, $P$.

Solenia a nomala, Fr.

\{ Tympanis Ravenelii, B. \& C.

$\{$ Cenangiella Ravenelii, Sacc.

\section{HYMENOMYCETES.}

Corticium lacteum, Fr.

Corticium Oakesii, B. \& C.

Corticium ochroleucum, Fr.

f Corticium chlorascens, Auct. Amer.

\{ Heterobasidium chlorascens, Massee.

Irpex deformis, $\mathrm{Fr}$.

Naematelia cerebriformis, Ell.

Thelephora hypnoides, S. Syn. Car.

Thelephora liydnoidea, S. Am. bor.

Radulum laetum, S. Am. bor.

Radulum molare, Fr. ? 
Radulum orbiculare, Fr.

Stereum complicatum, Fr.

Stereum pruinatum, Rav. Fung. Am.

\{Thelephora setiformis, B. S C.

Trametes serpens, Fr.

Xerotus viticola, B. \& C.

Castanea pumila, Michx.

$\left\{\begin{array}{l}\text { Splnaria Gnomon, Tode. } \\ \text { Gnomoniella vulgatris, (Ces. \& De Not.) } \\ \text { Sicc. }\end{array}\right.$ Sacc.

f Iclminthosporium caudatum, B. \& C.

Helminthosporium macrocarpon, Grev.

Nelanconiurs cinctum, B. \& C.

Pestalozzia concentrica, B. \& Rav.

Thelephora albomarginata, $\mathrm{S}$.

Castanea sativa, Mill., var. Americana,

Vatson.

\section{PYRENOMYCETES.}

Diatrype innata, B. \& C.

Valsa innata, B. \& C.

Cryptosporella innata, Sacc.

Diaporthe innata, Sacc.

Hysterium Castaneae, S.

Mclanconis modonia, Tul.

CMicrosphaera Friesii, Lev., var. Castaneae, C. \& P.

Microsphaera Hedwigii, Lev., var. Castaneae, Ell.

Microsphaera Alni, (DC.) Wint.

Erysiphe orbicularis, Ehrb.

Phyllactinia guttata, Lev.

Phyllactinia suffulta, (Reb.) Sacc.

S Sphaeria echinophila, S.

S Sphaerella echinophila, Awd.

\section{FUNGJ IMPERFECTI.}

SXyloma faginetum, Schrad.

Discosia Artocreas, (Tode) Fr.

Septoria ochrolcuca, B. \& C.

Cryptosporium epiphyllum, C. \& E.

Phyllosticta ochroleuca. Pk.

Gloeosporium ochroleucum, Ell. \& Ev,

IIclminthosporiurn macrocarpon, Grev.

Helminthosporium persistens, Cke.

Leptothyrium castanicolum, Ell. \& Ev.

Phoma castanea, $\mathrm{Pk}$.

Stilbum piliforme, $\mathrm{P}$.

S Tubercularia Castancac, P.

\{'Tubercularia minor, Lk.

\section{IIYMENOMYCETES.}

$\Lambda$ garicus Americanus, Pk.

(Lepiota Americana, Sacc. Syll.

A garicus (Itypholoma) sublateritius, Schaeff.

Dacdalea quercina, (L.) P

Fistulina lepatica, Fr.
Fistulina pallida, B. \& Rav.

IIydnum xanthum, ]3. \& C.

Polyporus hirsutus, (Schrad.) Fr.

(Polyporus pargamenus, Fr.

Polypor us elongatus, Berk.

Polystictus elongatus, $\mathrm{Fr}$.

Polystictus pargamenus, Fr.

Polyporus spumeus, Fr. ?

Polyporus sulfureus, (Bull.) Fr.

Polyporus versicolor, (L.) Fr.

MISCELLANEA.

Helotium papillare, (Bull.) Karst.

Arcyria globosa, S.

Craterium globosum, Fr.

Nassula globosa, Fr.

Lachnobolus globosus, Rostf.

Patellaria congregata, B. \& C.

Peziza alboviolascens, A. \& S.

(Peziza fructigena, Bull.

Helotium fructigenum, Karst.

Phialea fructigena, Gill.

Castanea, sp. indet.

\section{PYRENOMYCETES.}

S Sphaeria Eckfeldtii, Ell.

$\{$ Nelanomma Eckfeldtii, Sacc.

(Amphisphaeria Eckfeldtii, Cke.

Angelina rufescens, (S.) Duby.

Sphaeria Castaneae, S.

Melogramma Castaneae, M. A. Curtis.

Botryosphaeria Castaneae, Sacc.

Sphaeria assecla, S.

$\{$ Calosphaeria assecla, Sacc.

Valsa assccla, Cke.

Sphaeria scabriseta, S.

Eutypa scabriseta, B.

Valsa scabriseta, Cke.

Calosphaeria scabriseta, Sacc.

Sphaeria monstrosa, S.

Coniochaeta monstrosa, Cke.

$\{$ Valsa cinctula, C. \& P.

\{ryptosporal cinctuı, Sacc.

Sphaeria fibriseda, S.

$\{$ Nectria fibriseda, Sacc.

Dialonectria fibrjseda, Cke.

Diatrype Stigma, (Hoffm.) Fr.

(Sphaeria castanella, C. \& E.

Didymella castanella, Sacc.

Endophlaea castanella, C. \& E.

Sphaeria limaeformis, S.

\{ Eutypa spinosa, (P.) Tul.

(Valsa castanophila, B. \& C.?

$\{$ Valsa castanicola, B. \& C.

Fencstella castanicola, Sacc.

$\{$ Sphacria ferruginea, $P$.

\{ Sillia ferruginea, Karst. 
S Sphaeria enteromela, S.

\{Hypoxylon enteromelum, B.

f Hysterium Gerardi, C. \& P.

Hysterographium Gerardi, Sacc.

\{Hysterium variabile, C. \& P.

\{Hysterographinm variabile, Sacc.

$\{$ Sphaeria Pulvis-pyrius, P.

$\{$ Melanomma Pulvis-pyrius, Fckl.

f Dothidea Castaneate, S.

Y Phyllachora Castaneae, Sacc.

Sphaeria Corticium, S.

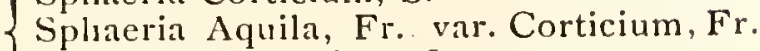

Rosellinia Corticium, Sacc.

Sphaterella sparsa, (Wallr.) Awd.

Sphaeria enteroxantha, S.

Sphaeria finscescens, S.

Sphateria inversa, Fr.

Valsa caryigena, B. \& C.

Valsa coronata, (Hoffm.) Fr.

Sphaeria oligostoma, S.

\{ Valsa oligostoma, Cke.

Valsa pullula, B. \& C. in Herb. B.

$\{$ Sphaeria pusilla, $P$.

\{ Valsa pusilla, Fr.

Sphaeria squalidula, C. \& P.

$\{$ Wall rothiella squalidula, Sacc.

Psilosphaeria squalidula, C. \& P.

FUNGI IMPERFECTI.

Coryneum epiphyllum. S.

$\{$ Sporidesmium epıphyllum, B. \& C.

Ceratophorum epiphyllum, Sacc.

Clasterosporium subulatum, C. \& E.

\{Ceratophorum subulatum, C. \& E.

$\{$ Coniothecium effusum, Cda.

\{ Sporidesmium Lepraria, B.

Coremium glancum, Lk.

Coryneum pustulatum, $\mathrm{Pk}$.

$\{$ Sphaeria Artocreas, Tode.

\{ Discosia Artocreas, Fr.

Fusarium coccineum, S.

Fusidium caesium, S.

$\{$ Dematium Castaneae, S.

\{Helminthosporium fasciculatum, S.

Helminthosporium obovatum, B. \& Br.

Oidium croceum, Lk.

Phoma brunneotincta, B. \& C.

$\{$ Stilbospora epiphylla, S.

$\{$ Septoria nigricans, Lk.

\{ Sphaeronema capillare, Ell. \& Hark.

S Sphaerographium capillare, Sacc.

Sphaeronema parabolicum, (Tode) Fr.

Sphaeronema ventricosum, (Ach.) Fr.

Spicaria fumosa, Ell. \& Ev.

Sporotrichum cohaerens, S.

Sporotrichum lateritium, Ehrb.

\{ Coryneum castanicolum, B. \& C.

$\{$ Steganosporium castanicolum, Sacc.
DISCOMYCETES

Agyrium nigricans, Fr.

Agyrium rufum, (P.) Fr.

Cenangium Castaneae, $\mathrm{S}$.

Cenangium fibrisedum, $\mathrm{S}$.

$\{$ Peziza chlora, S.

Chlorosplenium Schweinitzii, Fr.

Peziza punctiformis, Fr.

$\{$ Trichopeziza punctiformis, Fckl.

Cyphella punctiformis, Cke.

Dermatea purpurascens, Ell. \& Ev.

Haematomyces orbicularis, Pk.

$\{$ Peziza granuliformis, A. \& S.

Helotium papillare, (Bull.) Karst.

$\{$ Peziza Aranea, De Not.

\{ Trichopeziza Aranea, Sacc.

$\{$ Peziza cornuta, Ell.

Helotiella cornuta, Sacc.

Peziza echinophila, Bull.

Peziza rhaphidospora, Ell.

$\{$ Peziza translucida, B. \& C.

$\{$ Dasyscypha translucida, Sacc.

$\{$ Peziza turbinulata, S.

$\{$ Dasyscypha turbinulata, Sacc.

$\{$ Peziza vixvisibilis, S.

$\{$ Dasyscypha vixvisibilis, Sacc.

Phacidium coronatum, Fr.

\section{HYMENOMYCETES.}

Agaricus (Mycena) corticolus, Schum.

Corticium quercinum, (P.) Fr., var. scutellatum, Ell.

Favolus abnormis, S.

$\{$ Boletus radicatus, $S$.

$\{$ Fistulina radicata, Fr.

Hydnum fuscoatrum, Fr.

Tremella fragiformis. S. Syn. Car.

Dacryomyces fragiformis, S. Am. bor.

Hormomyces aurantiacus, Sacc.

Hypsilophora fragiformis, Cke.

Lenzites corrugata, (Klotzsch) B. \& C.

Merulius Corium, (P.) Fr.

$\{$ Boletus crispus, $\mathrm{P}$.

$\{$ Polyporus crispus, Fr.

Polyporus cupulaeformis, B. \& C.

Polyporus Pilotae, S.

\{ Polyporus dryadeus, S. Am. bor.

Polyporus scruposus, Fr.

$\{$ Polyporus tenuis, $\mathrm{S}$.

$\{$ Poria tenuis, Cke.

$\{$ Thelephora abietina, $\mathrm{P}$.

$\{$ Stereum abietirfum, Fr.

$\{$ Thelephora Chailletii, P.

S Stereum Chailletii, Fr.

Thelephora nuda, S. Am. bor.

Typhula ramealis, $\mathrm{S}$. 
MSCELI. NNA $\Lambda$.

Nucor echinophilus, S.

Sphacrobolus minutissimus, S.

\{helebolus? minutissimus, De Toni.

Castanopsis chrysophylla, A. DC.

(Taphrina cacrulescens, (Mont. \& Desm.)

$\{$ Tul.

Ascomyces Quercus, Cke.

Corylus Americana, Walt.

f Diatrypella Frostii, Pk.

\{ Diatrype Frostii, Cke.

Sphacria Tessera, Fr.

Diaporthe Tessera, Fckl.

Valsa mucronata, $\mathrm{Pk}$.

Valsa Tessera, Cke.

Diaporthe mucronata, Sacc.

f Splraeria tumulata, C. \& E.

\{ Diaporthe tumulata, Sacc.

\{ Gloensporium Coryli, (Desm.) Sacc.

\{ Phyllosticta corylina, Ell. \& Martin.

Gloeosporium profusum, Ell. \& Ev.

Gnomoniella Coryli, (Batsch) Sacc.

Hypoxylon fuscum, (P.) Fr.

(Microsphaera penicillata, (Wallr.) Lev.

$\{$ Microsphaera Hedwi rii, Lev.

Microsphaera Alni, (DC.) Wint.

(Sclerotium Erysiphe, P.

Erysiphe guttata, Lk.

Phyllactinia guttata, Lev.

Phyllactinia suffulta, (Reb.) Sacc.

Sphaeria Aquila, Fr., var. minor, C. \& E.

Sphaeronema Coryli, P.

Stereum ochraceoflavum, (S.) Rav.

Corylus rostrata, Ait.

Diaporthe sulfurea, Fckl.

Diaporthe Tessera, (Fr.) Fckl.

Dothidea corylina, Cke. \& Hark.

Glocosporium rostratum, Ell. \& Ev.

Sphaeria Coryli, Batsch.

$\{$ Gnomonia Coryli, Awd.

Gnomoniella Coryli, Sacc.

Gnomonia setacea, (P.) Ces. \& De Not.

Microsplaera penicillata, (Wallr.) Lev.

Septoria corylina, Pk.

Scptoria corylina, Pk., var. permaculata, $\mathrm{Pk}$.

Troposporium album, Hark.

Corylus, sp. indet.

Catinula turgida, (Fr.) Desm.

(Peziza furfuracea, Roth.

$\{$ Dermaten furfuracea, (Roth) Fr.

(Cenangium furfuraceum, Dc Not.

f Diatrype anomala, Pk.

C Cryptosporella anomala, Sacc.

S Spharia bullata, IIoffm.

(Diatrype bullata, Fr.

f Diatrypella Tocciacana, De Not.

Diatrype Tocciacana, Auct. Amer.

Diplodia glandicola. C. \& E.
$\{$ Fusisporium episphaericum, C. \& E.

$\{$ Fusarium episphaericum, Sacc.

Gloeosporium Coryli, (Desm.) Sacc.

Hydnum fragilissimum, B. S. C.

Botryosphaeria pustulata, Sacc.

Sphaeria pustulata, Cke.

Pliysalospora pustulata. Sacc.

Psilosphaeria moriformis, (Tode) Cke. "

Sphaeria ferruginea, $\mathrm{P}$.

\{ Sillia ferruginea, Karst.

(Sphacria conglobata, S. Am, bor.

$\{$ Cucurbitaria conglobata, (Fr.) Ces. \& De Not.?

Valsa corylina, Tul.

Fagus ferruginea, Ait.

\section{PYRENOMYCETES.}

Sphaeria atropunctata, S.

$\left\{\begin{array}{l}\text { Anthostoma atropunctatum, Sacc. } \\ \text { S }\end{array}\right.$

Anthostoma turgidum, (P.) Nits.

S Sphaeria vernicosa, $\mathrm{S}$.

$\{$ Daldinia vernicosa, Ces. \& De Not.

Hypoxylon vernicosum, M. A. Curtis.

S Sphaeria albopruinosa, S.

\{ Diatrype albopruinosa, C. \& E.

S Sphaeria disciformis, Hoffm.

\{ Diatrype disciformis, Fr.

i Sphaeria euphorea, Fr.

\{ Diatrype euphorea, Cke.

Diatrype verrucoides, $\mathrm{Pk}$.

Diatrype virescens, (S.) Rav. Fung. Car.

Dichaena caespitosa, $S$.

Dichaena faginea, (P.) Fr.

$\int$ Sphaeria Radula, $\mathrm{P}$.

$\{$ Valsa Radula, Cke.

(Eutypella grandis, (Nits.) Sacc.

Sphaeria errabunda, Rob.

$\{$ Sphaerella errabunda, Awd.

Gnomonia errabunda, $A$ wd.

Hypocrea polyporoides, B. \& C.

Hypoxylon argillaceum, (P.) Fr.

Hypoxylon coccineum, Bull.

Sphaeria fragiformis, $P$.

Isaria umbrina, P.

Institale acariforme, Fr.

Hypoxylon fragiforme, B.

$\{$ Sphaeria cohaerens, $P$.

Hypoxylon cohaerens, Fr.

Hypoxylon enteromelum, (S.) B.

Hypoxylon fuscum, (P.) Fr.

Hypoxylon rubiginosnm, (P.) Fr.

Hypoxylon stigmateum, Cke.

S Sphaeria turbinulata, S.

$\{$ Hypoxylon turbinulatum, B.

fHysterium tumidum, Fr.

Coccomyces tumida, De Not.

f Sphaeria crinita, P.

\{ Lasiosphaeria crinita, Sacc.

\{ Sphaerial viridicoma, C. \& P.

$\{$ Lasiosphaeria viridicoma, Sacc. 
Sphacria melogramma, S. Am. bor.

Sphaeria gyrosa, S.

$\{$ Peziza gyrosa, Spreng.

Nelogramma gyrosum, Tul.

Microsphaera erineophila, $\mathrm{Pk}$.

Microsphaera penicillata, (Wallr.) Lev.

Sphaeria quadrata, S.

Diatrype obesa, B. \& C.

Diatrype exasperans, Gerard.

Diatrype quadrata, B.

Nyrmaecium obesum, Rehm.

Valsaria quadrata, Sacc.

Melogramma obesum. Rav. Fung. Am.

Valsaria exasperans, B.

Myrmaecium quadratum, Rehm.

$\{$ Sphaeria coccinea, $\mathbf{P}$.

Nectria coccinea, Fr.

$\{$ Sphaeria Peziza, Tode.

$\{$ Nectria Peziza, Fr.

$\{$ Phyllactinia guttata, Lev.

\{ Phyllactinia suffulta, (Reb.) Sacc.

Sphaeria quaternata, $P$.

Valsa quaternata, $\mathrm{Fr}$.

Quaternaria Persoonii, Tul.

Sphaeria Bombarda, Batsch.

$\{$ Bombardia fasciculata, Fr.

Rosellinia fasciculata, Cke.

$\{$ Sphaeria mammaeformis. P.

\{Rosellinia mammaeformis, Ces. \& De Not.

Botrytis spongiosa, S.

$\{$ Dacryomyces giganteus, Spreng.

(Scorias spongiosa, Fr.

$\{$ Sphaeria maculiformis, $P$.

Sphaerella maculiformis, Awd.

Sphaeria radicalis, $\mathrm{S}$.

Valsa compta, 'Tul.

Valsa fagicola, Ell. \& Ev.

Valsa minutella, Pk.

Sphaeria carpophila, P.

$\{$ Xylaria carpophila, Fr.

\section{FUNGI IMPERFECTI.}

Bispora monilioides, Cda.

? Clasterosporium herculeum, Ell.

$\{$ Discosia faginea, Lib.

$\{$ Discosia Artocreas, (Tode) Fr.

Endobotrya elegans, B. \& C.

Fusarium lateritium. Nees.

Gloeosporium Fagi, (Rob.) Westd.

Melanconium ? grandis, C. \& E.

$\{$ Naemaspora crocea, $P$.

$\{$ Myxosporium croceum, Lk.

Phyllosticta fagicola, Ell. \& Morgan.

Phyllosticta faginea, $\mathrm{Pk}$.

Onygena faginea, $\mathrm{Fr}$.

Onygena decorticata, S. Syn. Car.

Pilacre faginea, B. \& Br.

Septonema radians, B. \& Rav.
Sporotrichum sulfuremm, Grev.

Stilbum rubicundum, Tode.

$\{$ Cheirospora botryospora, Fr.

Thyrsidium botryosporum, Mont.

Zygodesmus violaceofuscus, Sacc.

DISCONYCETES.

$\{$ Helotium albopunctum, Pk.

\{ Pezizella albop:nncta, Sacc.

Helotium macrosporum, $\mathrm{Pk}$.

\{ Patellaria pusilla, $\mathrm{Pk}$.

\{Lecanidion pusillum, Sacc.

\{ Peziza fuscescens, P.

Dasyscypha fuscescens, Rehm.

$\{$ Peziza pallidula, C. \& P.

\{ Geopyxis pallidula, Sacc.

HYMENOMYCETES.

Agaricus appendiculatus, Bull.

$\{$ Agaricus atrocoeruleus, Fr.

$\{$ Pleurotus atrocoeruleus, Sacc. Syll.

Agaricus (Pholiota) heteroclitus, Fr.

Agaricus (Mycena) Leaianus, B.

Agaricus (Pleurotus) lignatilis, $\mathrm{Fr}$.

$\{$ Agaricus limonellus, $\mathrm{Pk}$.

\{ Pholiota limonella, Sacc. Syll.

$\{$ Agaricus mucidus, Schrad.

\{Armillaria mucida, Sacc. Syll.

$\{$ Agaricus niger, S.

\{ Pleurotus niger, Sacc. Syll.

\{ Agaricus sapidus, Kalchb.

\{ Pleurotus sapidus, Sacc. Syll.

$\{$ Agaricus subcoerulea, $\mathrm{Pk}$.

\{ Mycena subcoerulea, Sacc. Syll.

Agaricus (Mycena) Tintinnabulum, Fr.

Corticium cinereum, $\mathrm{P}$.

Corticium subgiganteum, B.

Crepidotus croceitinctus, $\mathrm{Pk}$.

Exidia Auricula-Judae, (L.) Fr.

Exidia glandulosa, (Bull.) Fr.

Favolus Canadensis, Klotzsch.

Guepinia Spathularia. (S.) Fr.

Hydnum alutaceum, Fr.

Hydnum farinaceum, $P$.

Hydnum fuscoatrum, Fr.

Thelephora corrugata, Fr.

Corticium corrugatum, Fr.

Hymenochaete corrugata, Lev.

Hyinenochaete rubiginosa, (Schrad.) Lev.

Irpex ambiguus, Pk.

Lentinus ursinus, Fr.

Merulius himantioides, Fr.

Merulius rubellus, $\mathrm{Pk}$.

Panus conchatus, (Bull.) Fr.

Panus dorsalis, (Bosc) Fr. 
Phlebia pileata, Pk.

Phlebia radiata, Fr.

Polyporus fomentarius, (L.) Fr.

$\{$ liomes fomentarius, Karst.

Boletils graveolens, S.

Polyporus graveolens, $\mathrm{Fr}$.

Polyporus conglobatus. $B$.

Trametes graveolens, Ell.

Fomes graveolens, Cke.

fomes conglobatus, Sacc. Syll.

(Polyporus isidioides, B.

$\{$ Mucronoporus isidioides, Ell. \& Ev.

Polyporus lacteus, Fr.

Polyporus nigricans, Fr.

f Poria vitrea, $P$.

$\{$ Polyporus vitreus, Fr.

Solenia fasciculata, P.

$\{$ Thelephora lobata, Kze.

Stereum lobatum, Fr.

f Cantharellus crispus, (P.) Fr.

\{ Trogia crispa, Fr.

Fagus sylvatica, L.

Diaporthe galericulata, (Tul.) Sacc.

Diatrype virescens, (S.) Rav. Fung. Car.

$\{$ Patellaria rhabarbarina, B.

\{ Pezicula rhabarbarina, Tul.

Septonema radicans, B. \& Rav.

Fagus, sp. indet.

Helotium fagineum, (P.) Fr.

Libertella faginea, Desm.

Peniophora cinerea, (P.) Cke., var. fumigata, Sacc. Syll.

Ostrya Virginica, Willd.

Actidium? diatrypoides, Cke.

$\{$ Hypocrea Ravenelii, B.

\{Broomella Ravenelii, Sacc.

f Cenangium rubiginosum, Cke.

\{ Cenangium rubiginellum, Sacc.

$\{$ Sphaeria rostrata, Fr.

Ceratostomella rostrata, Sacc.

Corticium amorphum, (P.) Fr.

Corticium comedens, (Nees) Fr.

Corticium diminuens, B. $\&$ C.

Corticium Oakesii, B. \& C.

Daedalea aurea, Fr.

\{ Hypoxylon concentricum, (Bolt.) Grev.

\{ Daldinia concentrica, Ces. \& De Not.

\{ Valsaria actidia, B. \& Rav. in Herb B.

I Diatrype acticlia, Cke.

Sphaeria platystoma, S.

$\{$ Hypoxylon platystoma, Rav.

Diatrype platystoma, M. A. Curtis.

$\{$ Fusisporium udum, B.

P Pionnotes uda, Sacc.

\} Sphneria fimbriata, P.

\{ Gromoniclla fimbriata, Sacc.

Sphaeria Gnomon, Tode.

$\{$ Gnomonia vulgaris, Ces. \& De Not.

Gnomoniella vulgaris, Sacc.
CHypoxylon coccineum, Bull.

Institale acariforme, $\mathrm{Fr}$.

? Ptychogaster albus, Cda.

Hypoxylon fragiforme, (P.) $B$.

Hypoxylon Howeianum, Pk.

Phyllactinia guttata, Lev.

Polyporus obliquus, (P.) Fr.

Septoria Ostryae, Pk.

Stereum candidum, (S.) Fr.

Sphaeria culcitella, B. \& Rav.

$\{$ Trichosphaeria acanthostroma, (Mont.)

Sacc.

$\{$ Byssosphaeria corynephora, Cke.

\{ Trichosphaeria corynephora. Sacc.

Valsa hanstellata, (Fr.) M. A. Curtis.

Quercus agrifolia, Née.

Sphaeria decorticata, Cke. \& Hark.

$\{$ Amphisphaeria decorticata, Berl. \& Vogl.

Melanomma decorticata, Cke. \& Hark.

Ascomyces caerulescens, Mont. \& Desm.

Anlographum reticulatum, Phil. \& Hark.

Beltrania querna, Hark.

(Sphaeria Quercuum, S.

Mielogramma Quercuum, Fr.

$\{$ Melanops Quercuum, Rehm.

Melogramma fuliginosum, Ell. p. p.

Botryosphaeria Quercuum, Sacc.

Chaetophoma quercifolia, Cke.

Dichaena quercina, (P.) $\mathrm{Fr}$.

Erysiphe trina, Hark.

Gloeosporium quernum, Hark.

Helicosporium vegetum, Nees.

Helminthosporium persistens, Cke.

Helotium furfuraceum, Phil. \& Hark.

Hysterium pulicare, $P$.

Irpex obliquus, (Schrad.) Fr.

$\{$ Physalospora bina, Hark.

\{ Laestadia bina, Cke.

$\{$ Xyloma pezizoides, P.

\{ Phacidium coronatum, $\mathrm{Fr}$.

$\{$ Phillipsiella nigella, Phil. \& Hark.

$\{$ Microphyma nigellum, Sacc.

Phoma discosiaeformis, Cke. \& Hark.

Phyllosticta phomiformis, Sacc.

Pocillum Cesatii, (Mont.) De Not.

Polyscytalum sericeum, Sacc.

Sphaeropsis quercina, Cke. \& Hark.

\{ Oidium ventricosum, Hark.

Sphaerotheca lanestris, Hark.

Stereum ochraceoflavum, (S.) Rav.

Stereum rugosum, $P$.

$\{$ Rhytisma erythrosporum, B. \& C.

$\{$ Trabutia erythrospora, Cke.

Trabutia quercina, (Fr. \& Rud.) Sacc. \& Roum.

Zygodesmus pannosus, B. \& C. 
Quercus alba, L.

\section{PYRENOMYCETES.}

Acrospermum viridulum, B. \& C.

Systerium hiascens, B. \& C.

$\{$ Triblidium hiascens, Cke.

Blitridium hiascens, Sacc.

$\{$ Valsa farinosa, Ell.

Cryptosporella farinosa, Sacc.

Sphaeria Taleola, Fr.

Valsa Taleola, Fr.

Aglaospora Taleola, Tul.

Diaporthe Taleola, Sacc.

Diaporthe Raveneliana, Thm. \& Rehm.

f Diatrype Duriaei, Thm. M. U.

$\{$ Diatrype albopruinosa, (S.) C. \& E.

Dichaena quercina, (P.) Fr.

Didymosphaeria (Amphisphaeria) atrogrisea, C. \& P.

Eutypa velutina, (Wallr.) Sacc.

$\{$ Valsa phaeospora, Sacc.

\{Fenestella phaeospora, Sacc.

Sphaeria annulata, S.

Sphaeria marginata, S.

Hypoxylon annulatum, Mont.

Hypoxylon marginatum, B.

\{ Sphaeria durissima, S.

$\{$ Hypoxylon durissimum, Fr.

Hypoxylon perforatum, (S.) Fr.

$\{$ Hysterium stygium, Cke.

\{Hysterographium stygium, Sacc.

\{ Massaria bufonia, (B. \& Br.) Tul.

\{Massariella bufonia, Speg.

(Massaria sudans, $\mathrm{B}, \& \mathrm{C}$.

Massariella sudans, Sacc.

(Massariovalsa sudans, Sacc.

Melanconis chrysostoma, (Fr.) Tul.

$\{$ Microsphaera extensa, C. \& P.

$\{$ Microsphaera quercina. (S.) Burrill.

Sypoxylon punctulatum, B. \& Rav.

$\{$ Diatrype punctulata, B. \& Rav.

Nummularia punctulata, Sacc.

Sphaeria myriadea, DC.

Sphaeria dendroides, S.

$\{$ Sphaerella myriadea, Awd.

Sphaerulina myriadea, Sacc.

Sphaerella dendroides, Cke.

Valsa ceratophora, Tul.

Valsa lasiostoma, Ell. \& Ev.

Sphaeria leiphaemia, $\overrightarrow{\mathrm{F}}$.

$\{$ Valsa leiphaemia, Fr.

Diaporthe leiphaemia, Sacc.

Valsa leiphaemioides, B. \& C.

Valsa pulchelloidea, C. \& E.

Valsaria rubricosa, (Fr.) Sacc.

Sphaeria Orbicula, S.

$\{$ Venturia Orbicula, C. \& P.

FUNGI IMPERFECTI.

Cladosporium brevipes, $\mathrm{Pk}$.

Coryneum Kunzei, Cda.
Cylindrosporium microspilum, Sacc. \& Wint.

Cytospora pustulata, B. \& C.

Epidochium nigricans, Fr.

Gloeosporium Canadense, Ell. \& Ev.

Gloeosporium nervisequum, (Fckl.) Sacc.

Gloeosporium septorioides, Sacc.

Harknessia caudata, E11. \& Ev.

Helminthosporium Tiara, B. \& Rav.

Leptothyrium dryinum, Sacc.

Marsonia Martini, Sacc. \& Ell.

Melanconium bicolor, Nees.

$\{$ Pestalozzia monochaeta, Desm.

\{ Pestalozzia Peckii, Clinton.

Pestalozzia nervalis, E1l. \& Ev.

Pestalozzia pallida, Ell. \& Martin.

Pestalozzia Saccardoi, Speg.

Pestalozzia taphrinicola, Ell. \& Ev.

Phoma glandicola, Lev.

Phoma vixvisibilis, Thm.

Phyllosticta phomiformis, Sacc.

Phyllosticta Quercus, Sacc. \& Speg.

Phyllosticta tumoricola, $\mathrm{Pk}$.

$\{$ Sphaeropsis quercina, C. \& E.

$\{$ Dothiorella quercina, Sacc.

Sporidesmium fumosum, Ell. \& Ev.

Strumella coryneoidea, Sacc. \& Wint.

DISCOMYCETES.

Cenangium confusum, S.

Cenangium sphaeriaemorphum, S.

$\{$ Peziza triangularis, S.

$\{$ Cenangium triangulare, Fr.

Coccomyces triangularis, Sacc.

Dermatea lobata, Ell.

Dermatea tabacina, Cke.

$\{$ Peziza lenticularis, Bull.

$\{$ Helotium lenticulare, $\mathrm{Fr}$.

$\{$ Dermatea Cucurbitaria, Cke.

$\{$ Patellaria Cucurbitaria, Rehm.

CPeziza echinulata, Awd.

Peziza capitata, Pk.

'Trichopeziza ciliaris, (Schrad.) Rehm.

Trichopeziza capitata, Sacc.

Psilopezia flavida, B. \& C.

Schizoxylon moniliferum, Ell. \& Ev.

\{ Stictis niveopurpurea, Ell. \& Ev.

$\{$ Cryptodiscus niveopurpureus, Sacc.

(Taphrina caerulescens, (Mont. \& Desm.)

Tul.

(Taphrina Quercus, (Cke.) Sacc.

\section{HYMENOMYCETES.}

f Corticium Oakesii, B. \& C.

\{ Aleurodiscus Oakesii, Cke.

$\{$ Merulius incarnatus, S.

Cantharellus incarnatus, $\mathrm{S}$. 
Corticium caeruleum, (Schrad.) Fr.

Corticium citrinum, B. \& Rav.

Dacryomyces stillatus, Nees.

Daedalea quercina, (L.) P.

Daedalea unicolor, Fr.

Exidia recisa, $\mathrm{Fr}$.

Fistulina pallida, B. E Rav.

Hydnum adustum, S.

f Corticium corticola, B. \& Rav.

Hymenochate corticolor, B. \& Rav.

Stereum Curtisii, B.

$\{$ Thelephora Curtisii, B.

Hymenochaete Curtisii, Morg.

\{rpex fuscescens, S.

Irpex cinnamomeus, Fr.

Lenzites vialis, $\mathrm{Pk}$.

(Polyporus applanatus, $\mathrm{Fr}$,

Fonies applanatus, Karst.

Ganoderma applanatum, Pat.

Polyporus Beatiei, Banning.

(Boletus cinnabarinus, Jacq.

Polyporus cinnabarinus, Fr.

Sistotrema cinnabarinum, $\mathrm{S}$.

Hydnum cinnabarinum, Fr.

Trametes cinnabarina, Fr.

Polystictus cinnabarinus, Cke.

Polyporus pargamenus, Fr.

Polyporus elongatus, $\mathrm{B}$.

Polyporus laceratus, M. A. Curtis.

Polystictus elongatus, $F r$.

Polystictus pargamenus, Fr.

Polyporus pseudopargamenus, Thm.

Polystictus pseudopargamenus, Sacc. Syll.

$\{$ Polyporus versicolor, (L.) Fr.

$\{$ Polystictus versicolor, $\mathrm{Fr}$.

Stereum complicatum, Fr.

$\{$ Thelephora frustulata, P.

Stereum frustulosum, Fr.

Stereum versiforme, B. \& C.

\section{MISCELLANEA.}

\section{(Licea applanata, B. \\ Clathroptychium rugulosum, (Wallr.) \\ Rostf.}

Uredo Quercus, Brondeau.

Quercus aquatica, Walt.

Aulographum Liturae, Cke.

Aulographum maculare, B. \& Br.

Byssosphaeria acanthostroma, (Mont.) Cke.

Diatrype bispora, B. \& C.

$\{$ Discella quercina, Cke.

Discula quercina, Sacc.

$\{$ Hysterium chlorisum, B. \& C.

\{ Glonium chlorinum, Sacc.

Microsphaera Hedwigii, Auct. Amer.p.p.

Microsphaera penicillata, Auct. Amer. p. p.

Microsphaera extensa, C. \& P.

Microsphaera quercina, (S.) Burrill.

$\{$ Phyllactinia guttata, Lev.

\{Phyllactinia suffulta, (Reb.) Sacc.
Phyllosticta Ludoviciana, Ell. \& Martin.

Phyllosticta marginalis, Ell. \& Ev.

Boletus graveolens, S.

Polyporus graveolens, Fr.

Polyporus conglobatus, B.

Fomes graveolens, Cke.

Polyscytalum cylindroides, Sacc. \& Ell.

Sphaerella aquatica, Cke.

$\{$ Sphaeria punctiformis, $\mathrm{P}$.

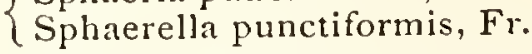

Taphrina caerulescens, (Mont. \& Desm.). Tul.

Quercus bicolor, Willd.

Hysterium hiascens, B. \& C.

$\{$ Microsphaera abbreviata, Pk.

$\{$ Microsphaera quercina, (S.) Burrill.

$\{$ Sphaerella spleniata, C. \& P.

\{ Sphaerella nigrita, Cke.

Quercus Catesbeyi, Michx.

Coniothyrium epiphyllum, Cke.

Graphium explicatum, B. \& C.

Microsphaera abbreviata, $\mathrm{Pk}$.

\{ Phoma Catesbeyi, Thm.

\{Chaetophoma Catesbeyi, Cke.

$\{$ Sphaeropsis nervisequa, Cke.

\{ Phoma nervisequa, Sacc.

Polyporus graveolens, (S.) Fr.

Sphaerella Catesbeyi, Cke.

Trichopeziza punctiformis, (Fr.) Fckl., var. nivea, Sacc.

Quercus chrysclepis, Liebm., vạr. vacciniifolia, Engelm.

$\{$ Hysterium foliicolum, Fr.

\{lophodermium hysterioides, (P.) Sacc.

Quercus cinerea, Michx.

f Torula insularis, Thm.

\{ Oospora insularis, Sacc. \& Vogl.

Phyllosticta vesicatoria, Thm.

(Taphrina caerulescens, (Mont \& Desm.) Tul.

Ascomyces Quercus, Cke.

Taphrina Quercus, Sacc.

Quercus coccinea, Wang.

\section{PYRENOMYCETES.}

(Diatrype dryophila, Curr., var. minor, Cke.

Anthostoma dryophilum, Sacc., var. minor, Rehm.

(Diatrype grandinea, B. \& Rav.

Anthostoma grandineum, Sacc.

Fuckelia grandinea, Cke.

Camarops grandinea, Cke.

Byssosphaeria luteobasis, (Ell.) Cke.

Diaporthe densissima, Ell.

Diatrype quadrata, (S.) B.

S Sphaeria (Didymosphaeria) Cupula, Ell.

\{ Didymosphaeria circinans, Hark. 
f Valsa lutescens, Ell.

$\{$ Eutypella lutescens, Sacc.

Sphaeria atropunctata, S.

$\{$ Hypoxylon atropunctatum, Frost's Cat.

Anthostoma atropunctatum, Sacc.

f Sphaeronema subcorticale, C. \& E.

\{ Hypsotheca subcorticalis, Ell. \& Ev.

$\{$ Nectria lasioderma, Ell.

$\{$ Lasionectria lasioderma, Cke.

$\{$ Physalospora quercifolia, Ell. \& Ev.

Laestadia quercifolia, Cke.

Microsphaera quercina, (S.) Burrill.

Phyllactinia suffulta, (Reb.) Sacc.

Sphaerella (Laestadia) polystigma. Ell. \& Ev.

Triblidium hiascens, (B. \& C.) Cke.

Valsa clausa, C. \& E.

Valsa multiplex, C. \& E.

Valsa pulchelloidea, C. \& E.

$\{$ Venturia Orbicula,(S.) C. \& P., var. sparsa, $\{$ Ell. \& Ev.

\section{FUNGI 1MPERFECTI.}

Cytospora chrysosperma, (P.) Fr.

Diplodia longispora, C. \& E.

Gloeosporium nervisequum, (Fckl.) Sacc.

Harknessia hyalina, Ell. \& Ev.

Leptothyrium dryinum, Sacc.

$\{$ Pestalozzia microspora, Ell. \& Ev.

\{Pestalozzia minor, Ell. \& Ev.

Pestalozzia taphrinicola, Ell. \& Ev.

Phoma parasitica, Ell. \& Ev.

\section{DISCOMYCETES.}

Bulgaria inquinans, (P.) $\mathrm{Fr}^{r}$.

$\{$ Dermatea tetraspora, Ell.

$\{$ Cenangium tetrasporum, Sacc.

\& Peziza quernea. S.

$\{$ Cenangium turgidum, Fr.

Dermatea tabacina, Cke.

$\{$ Patellaria cenangiicola, Ell. \& Ev.

$\{$ Patellea cenangicola, Sacc.

$\{$ Propolis glauca, Ell.

\{Propolidium glaucum, Sacc.

Schizoxylon moniliferum, Ell. \& Ev.

$\left\{\begin{array}{l}\text { Ascomyces caerulescens, Mont. \& Desm. } \\ \text { Taphrina caerulescens, Tul. } \\ \text { Ascomyces Quercus, Cke. } \\ \text { Exoascus caerulescens, Sadebeck. } \\ \text { Taphrina Quercus, Sacc. }\end{array}\right.$

\section{MISCELLANEA.}

Corticium rubrocanum, Thm.

Cronartium asclepiadeum, (Willd.) Fr., var. Quercuum, B. \& C.

$\{$ Thelephora pediccllata, S.

\{ Gausapia pedicellata, Fr.

Polyporus cupulacformis, B. \& C.
Quercus coccinea, Wang., var. tinctoria, Gray.

\section{PYRENOMYCETES.}

Sphaeria atropunctata, S.

Anthostoma atropunctatum, Sacc.

\{ Sphaeria cirrhosa, P.

\{ Ceratostomella cirrhosa, Sacc.

Diatrype platystoma, (S.) M. A. Curtis.

Diatrype roseola, Wint.

Eutypa velutina, (Vallr.) Sacc.

Hypoxylon annulatum, (S.) Mont.

f Hysterium Gloniopsis, Gerard.

\{ Gloniopsis Gerardiana, Sacc.

Hysterium pulicare, $\mathrm{P}$.

Sphaeria Pezizula, B. \& C.

$\{$ Lasiosphaeria Pezizula, Sacc.

Herpotricha Pezizula, Wint.

$\{$ Melanconis sigmoideum, C. \& E.

\{ Psendovalsa sigmoidea, Sacc.

\{ Erysiphe densissima, S.

Microsphaera densissima, C. \& P.

Microsphaera quercina, (S.) Burrill.

Nectria lecanodes, Ces.

Nummularia punctulata, (B. \& Rav.) Sacc.

? Ophiobolus hamasporus, Ell. \& Ev.

\{ Phyllactinia guttata, Lev.

$\{$ Phyllactinia suffulta, (Reb.) Sacc.

(Diatrype obesa, B. \& C.

Diatrype quadrata, (S.) B.

Valsaria quadrata, Sacc.

Melogramma obesum, Rav. Fung. Am.

$\{$ Sphaeria Orbicula, S.

$\{$ Venturia Orbicula, C. \& P.

Xylaria Hypoxylon, (L.) Grev.

FUNG I IMPERFECTI.

Asteroma tinctorium, Cke.

$\{$ Dichaena quercina, (P.) $\mathrm{Fr}$.

$\{$ Psilospora quercina, Wint.

Gloeosporium septorioides, Sacc.

Pestalozzia stellata, B. \& C.

Pestalozzia uncinata, Ell. \& Kell.

Polyscytalum sericeum, Sacc.

Septoria dryina, Cke.

Septoria querciti, Thm.

\{ Sphaeropsis quercina, C. \& E.

$\{$ Dothiorella quercina, sacc.

\section{DISCOMYCETES.}

$\{$ Ascomycetella quercina, $\mathrm{Pk}$.

$\{$ Cookella quercina, Sacc.

? Tympanis stictica, B. \& C.

? Cenangium sticticum, Sacc.

Peziza occidentalis, S.

Taphrina caerulescens, (Mont. \& Desm.) 'Tul.

Urnula Craterium, (S.) Fr. 
IYMEX゚OMYCETES.

Sichenera Artocreas, B. \& C.

Artocreas Michencri, B. \& C.

Aleurodiscus Micheneri, Massee.

Hymenochaete scabriseta, Ckc.

Irpex tabacinus, B. \& C.

Lenzites betulina, (L.) Fr.

Marasmius amadelphus, (Bull.) Fr.

Marasmins foetidus, (Sow.) Fr.

Panus stipticus, (Bull.) Fr.

Polyporus cupulaeformis, B. \& C.

Polyporus gilvus, (S.) Fr.

Polyporus pargamenus, Fr.

$\{$ Polyporus purpureus, Fr.

Poria purpurea, Cke.

Polyporus versicolor, (L.) Fr.

Radulum orbiculare, $\mathrm{Fr}$.

Radulum quercinum, (P.) Fr.

Stereum complicatum, Fr.

Stereum subpileatum, B. \& C.

Stereum versicolor, (Swartz) Fr.

Trametes cinnabarina, ( Jacq.) Fr.

\section{MISCELLANEA.}

Cronartium asclepiadeum, (Willd.) Fr., var.

Quercuum, B. \& C.

Lycoperdon leprosum, B. \& Rav.

Quercus densiflora, Hook. \& Arn.

Chalara setosa, Hark.

Laestadia caelata, Hark.

S Sphaeria conflicta, Cke.

\{Linospora conflicta, Sacc.

Quercus Douglasii, Hook. \& Arn.

Ascomyces Quercus, Cke.

Coryneum umbonatum, Nees.

Quercus falcata, Michx.

(Sphaeria atropunctata, S.

Diatrype atropunctata, M. A. Curtis.

Hypoxylon atropunctatum, Frost's Cat.

Anthostoma atropunctatum, Sacc.

f Cenangium concinnum, B. \& C.

Scleroderris concinna, Sacc.

Melogramma obesa, (B. \& C.) Rav. Fung. Am.

Valsaria quadrata, (S.) Sacc.

(Hypoxylon nummularium, Bull.

$\{$ Sphaeria nummularia, DC.

Nummularia Bulliardi, Tul.

Merulius incarnatus, S.

Microsphaera quercina, (S.) Burrill.

Phyllactina suffulta, (Reb.) Sacc.

Ramularia crypta, Cke.

Septoria dryina, Cke.

Quercus hypoleuca, Engelm.

Asteroma Pringlei, $\mathrm{Pk}$.
Quercus ilicifolia, Wang.

Corticium corrugatum, Fr.

SGloeosporium Quercus, Pk.

\{Marsonia Quercus, Pk.

Helminthosporium macrocarpon, Grev.

$\{$ Melanconis sigmoideum, C. \& E.

\{ Pseudovalsa sigmoidea, Sacc.

f Erysiphe quercina, $\mathrm{S}$.

\{Microsphaera quercina, Burrill.

Titaea Clarkei, Ell. \& Ev.

Quercus imbricaria, Michx.

Cenangium confusum, $S$.

Cenangium turgidum, Fr.

\{ Marsonia quercina, Wint.

Gloeosporium septorioides, Sacc.

$\{$ Microsphaera extensa, C. \& P.

$\{$ Microsphaera quercina, (S.) Burrill.

Stagonospora septorioides, Ell. \& Ev.

Stictis radiata, $P$.

Trematosphaeria mastoidea, (Fr.) Wint.

Quercus Kelloggii, Newberry.

Septogleum defolians, Hark.

Quercus laurifolia, Michx.

$\{$ Ascomycetella aurantiaca, Ell. \& Martin.

Leptophyma aurantiacum, Sacc.

Ascomycetella Floridana, Ell. \& Martin.

Asterina discoidea, Ell. \& Martin.

\{ Asterina erysiphioides, Ell. \& Martin.

' Asterina patelloides, Ell. \& Martin.

Asterina pustulata, Ell. \& Martin.

Asterina stomatophora, Ell. \& Martin.

Asterina subcyanea, Ell. \& Martin.

$\{$ Calonectria eruhescens, (Rob.) Sacc.

$\{$ Dialonectria erubescens, Cke.

Helminthosporium foliculatum, Cda., var.

Caroliniense, Sacc.

Helotium castaneum, Sacc. \& Ell.

Microsphaera densissima, (S.) C. \& P.

\{ Patellaria cyanea, Ell. \& Martin nec. Cke.

\{ Patellea cyanea, Sacc.

Polyporus gilvus, (S.) Fr.

Polyporus plebeius, B.

Saccardia Martinii, Ell. \& Sacc.

Septoria Serpentaria, Ell. \& Martin.

(Taphrina caerulescens, (Mont. \& Desm.)

$\{$ Tul.

Ascomyces Quercus, Cke.

Trabutia quercina, (Fr. \& Rud.) Sacc.

Venturia cupulata, Ell. \& Martin.

Quercus 1yrata, Walt.

\{ Sphaeria colliculosa, S.

Hypoxylon colliculosum, M. A. Curtis.

Quercus macrocarpa, Michx.

Microsphaera quercina, (S.) Burrill.

Clasterosporium uncinatum, Clinton.

$\{$ Sporidesmium helicosporum, Sacc.

Ceratophorum uncinatum, Sacc. 
Coryneum effiguratum, $\mathrm{S}$.

Helminthosporium macrocarpon, Grev.

\{ Hysterium pulicare, N. A. F.

$\{$ Hysterographium hiascens, Rehm.

Irpex flavus, Klotszch.

Microsphaera quercina, (S.) Burrill.

Phyllactinia suffulta, (Reb.) Sacc.

Phyllosticta Quercus, Sacc. \& Speg.

Polyporus Radula, (P.) Fr.

Rosellinia Gaudefroyi, Fabre.

$\{$ Acomyces extensus, Pk.

Taphrina extensa, Sacc.

Uredo Quercus, Brondeau.

Venturia Orbicula, (S.) C. \& P.

Volutella quercina, S.

Quercus nigra, L.

Angelina rufescens, (S.) Duby.

Bulgaria inquinans. (P.) $\mathrm{Fr}$.

Chaetophoma quercifolia, Cke.

Cronartium asclepiadeum, (Willd.) Fr., var.

Quercuum, B. \& C.

Diatrype dryophila, Curr., var. minor, Cke.

Diatrype subferruginea, B. \& Rav.

Dichaena strumosa, $\mathrm{Fr}$.

? Gloeosporium septorioides, Sacc., var. major, Ell. \& Ev.

Graphium explicatum, B. \& C.

Helminthosporium macrocarpon, Grev.

Hypoxylon glomiforme, B. \& C.

$\{$ Microsphaera penicillata, Auct. Amer. p. p.

Microsphaera quercina, (S.) Burrill.

Mucronoporus Everhartii, Ell. \& Gall.

Sphaeria Clypeus, S.

Hypoxylon Clypeus, M. A. Curtis.

Diatrype Clypeus, B.

Nummularia Bulliardi, Auct. Amer. p. p.

Nummularia Clypeus, Cke.

Pestalozzia hysteriiformis, B. \& C.

Phacidium dentatum, Schm. \& Kze.

Phoma dendritica, Thm.

$\{$ Boletus hispidus, Bull.

$\{$ Polyporus hispidus, Fr.

$\{$ Polyporus xantholoma, S.

I Poria xantholoma, Cke.

$\{$ Sphaeridium miniatum, Sacc.

\{ Cylindrocolla miniata, Sacc.

$\{$ Sphaeria Orbicula, S.

Venturia Orbicula, C. \& P.

Quercus palustris, Du Roi.

? Microcera coccophila, Desm.

Microsphaera extensa, C. \& P.

Pestalozzia maura, Ell. \& Ev.

Quercus Phellos, L.

$\{$ Sphaeria Pustula, P.

$\{$ Phoma Pustula, Fr.

(Hypospila Pustula, Karst.

$\{$ Sphaeria Phellos, S.

S Sphaerella Phellos, Cke.
Quercus prinoides, Willd.

Diatrype albopruinosa, (S.) C. \& E.

Marsonia Martini, Sacc. \& Ell.

Sphaerella spleniata, C. \& P.

Uredo Quercus, Brondeau.

Quercus Prinus, L.

$\{$ Valsa condensata, B. \& C.

Fenestella condensata. Sacc.

Sphaeria exilis, A. \& S.

Caelosphaeria exilis, Sacc.

Lasiosphaeria exilis, Cke.

Microsphaera quercina, (S.) Burrill.

$\{$ Peziza prinicola, Ell. \& Ev.

\{Mollisia prinicola, Sacc.

Sphaeropsis abundans, $\mathrm{Pk}$.

Stereum candidum, (S.) Fr.

Venturia Orbicula, (S.) C. \& P.

Quercus rubra, L.

Angelina rufescens, (S.) Duby.

$\{$ Ascomyces caerulescens, Mont. \& Desm.

\{Taphrina caerulescens, Tul.

Ascomyces rubrobrunneus, Pk.

$\{$ Botrytis macrospora, Lk.

Dactylium macrosporum, Fr.

Sphaeria luteobasis, Ell.

$\{$ Eutypa luteobasis, Sacc.

Byssosphaeria luteobasis, Cke,

Cytospora intermedia, Sacc.

Sphaeria spinosa, $P$.

Sphaeria penicillosa, S.

Sphaeria limaeformis, S.

Eutypa spinosa, Tul.

Eutypa limaeformis, B.

Gloeosporium quercinum, Westd.

$\{$ Hydnum Rhois, S.

$\{$ Hydnum flabelliforme, B.

Melanconium bicolor, Nees.

Erysiphe quercina, $\mathrm{S}$.

Microsphaera extensa, C. \& P.

Microsphaera abbreviata, $\mathrm{Pk}$.

Microsphaera quercina, Burrill.

Orcadella operculata, Wingate.

Orthotrichia microcephala, Wingate.

$\{$ Patellaria similis, Gerard.

$\{$ Durella similis, Sacc.

$\{$ Peziza pollinaria, Cke.

$\{$ Trichopeziza pollinaria, Sacc.

Phyllactinia suffulta, (Reb.) Sacc.

Phyllosticta Quercus-rubrae, Gerard.

Polyporus dryophilus, B.

Polyporus versicolor, (L.) Fr.

Septoria Querceti, Thm.

Tubercularia quercina, Opiz.

Sphaeria inflata, Ell.

$\{$ Zignoella inflata, Sacc.

Conisphaeria inflata, Cke.

Quercus stellata, Wang.

$\Lambda$ crospermum viridulum, B. \& C. 
Capnodium Caroliniense, B. \& Desm. Capnodium quercinum, (P.)B. \& Desm.

Cladosporium microspermum, B. \& C.

Eutypa relutina, (Wallr.) Sacc.

Excipula subcalva, Ell. \& Ev.

Cylindrium pallidum, Thın.

\{Fusidium pallidum, Sacc.

\{ Marsonia Martini, Sacc \& Ell.

\{ Gloeosporium Martini, Ell. \& Ev.

Helminthosporium macrocarpon, Grev. var. caudatum, B. \& C.

$\{$ Hendersonia variabilis, B. \& C.

Camarosporium variabile, Sacc.

f Stereum tabacinum, (Sow.) Fr.

Hymenochaete tabacina, Lev.

$\{$ Microsphaera penicillata, Auct. Amer. p. p.

YMicrosphaera quercina, (S.) Burrill.

$\{$ Phacidium dentatum, Schm. \& Kze.

\{ Coccomyces dentatus, Sacc.

Phoma innumerabilis, Thm.

Phoma vixvisibilis, Thm.

Phyllosticta phomiformis, Sacc.

$\{$ Boletus cinnabarinus, Jacq.

$\{$ Polyporus cinnabarinus, Fr.

Polyporus cupulaeformis, B. \& C.

Rhinotrichum breve, B. \& C.

$\{$ Sphaeria punctiformis, $P$.

Sphaerella punctiformis, Fr.

(Thelephora candida, S. Syn. Car.

$\{$ Thelephora candidissima, S. Am. bor.

Stereum candidum, Fr.

Stereum Curtisii, B.

$\{$ Valsa albofusca, C. \& E.

Cryptosporella albofusca, Sacc.

Valsa leiphaemioides, B. \& C.

Quercus virens, Ait.

Aulographum quercinum, Ell. \& Martin.

Cercospora polytricha, Cke.

Cronartium asclepiadeum, (Willd.) $\mathrm{Fr}^{\mathrm{r}}$, var. Quercuum, B. \& C.

$\{$ Hendersonia virens, Ell. \& Martin.

\{ Stagonospora virens, Ell. \& Martin.

Hymenochaete rubiginosa, (Schrad.) Lev.

IIypoxylon bicolor, Ell. \& Ev.

Spluaeria Janus, B. \& C.

$\{$ Leptosphaeria Janus, Sacc.

Heptameria Janus, Cke.

Patellaria cyanea, Ell. \& Martin nec C.ke.

Pestalozzia concentrica, B. \& Rav.

Pestalozzia maura, E11. \& Ev.

Phillipsiella atra, Cke.

Phoma glandicola, Lev.

Phyllosticta virens, Ell. \& Langlois.

Pocillum Americanum, Cke.

Polyscytalum cylindroides, Sacc. \& Ell.

Saccardia quercina, Cke.

Sphaerellopsis Qucrcuum, Cke.

Stictis quercifolia, C. \& E.
Trabutia quercina, (Fr. \& Rud.) Sacc. \& Roum.

Uredo Quercus, Brondeau.

Quercus Wislizeni, A.DC.

Laestadia auripuncta, Hark.

Quercus, sp. indet.

\section{PYRENOM YCETES.}

\section{Perisporiaceae.}

Asterina intricata, Ell. \& Martin.

Eurotium herbariorum, Lk., var. epixylon, Ell.

Meliola amphitricha, Fr.

Meliola manca, Ell. \& Martin.

\section{Sphaeriaceae.}

Sphaeria decastyla, Cke.

Acanthostigma decastylum, Sacc.

Venturia decastyla, Cke.

Sphaeria botulispora, Cke.

Amphisphaeria botulispora, Sacc.

Xylosphaeria botulispora, Cke.

? Fuckelia amplispora, Cke.

Anthostoma amplisporum, Berl. \& Vogl.

Sphaeria irregularis, Sow.

Sphaeria gastrina, Fr.

Diatrype gastrina, Rav. Fung. Car.

Melogramma gastrinum, Tul.

Valsa gastrina, M. A. Curtis.

Anthostoma gastrinum, Sacc.

Fuckelia gastrina, Cke.

S Sphaeria atrobarba, C. \& E.

$\{$ Chaetosphaeria atrobarba, Sacc.

Chaetosphaeria innumera, (B. \& Br.) Tul.

Cucurbitaria radicalis, Cke.

$\{$ Coelosphaeria radicalis, Sacc.

Nitschkia radicalis, Cke.

$\{$ Valsa trichispora, C. \& P.

$\{$ Cryptospora trichospora, Sacc.

Cucurbitaria confluens, Plow.

(Melogramma dichaenoides, B. \& C.

Diaporthe dichaenoides. Sacc.

Botryosphaeria dichaenoides, Cke.

$\{$ Eutypa subpyramidata, B. \& C.

$\{$ Diaporthe subpyramidata, Sacc.

$\{$ Valsa Woolworthii, Pk.

\{ Diaporthe Woolworthii, Sacc.

$\{$ Diatrype aspera, Fr.

Diatrypella aspera, Nits.

Diatrype bullata, (Hoffm.) Fr.

Diatrype corniculata, (Ehrh.) Rav. Fung. Car.

Diatrype disciformis, (Hoffm.) Fr.

Sphaeria ferruginea, $P$.

$\{$ Diatrype ferruginea, Fr.

Sillia ferruginea, Karst.

Diatrype platystoma, (S.) M. A. Curtis. 
$\int$ Sphaeria quercina, $\mathrm{P}$.

$\{$ Diatrype quercina, Fr.

Diatrypella quercina, Nits.

Diatrype quercina, (P.) Fr., var. lignicola, C. \& E.

\{ Sphaeria Stigma, Hoffm.

Diatrype Stigma, Fr.

Diatrype verrucaeformis, (Ehrh.) Fr.

Didymosphaeria denudata, Ell. \& Gall.

Sphaeria parietalis, B. \& C.

Enchnosphaeria parietalis, Sacc.

Byssosphaeria parietalis, Cke.

Eutypa lata, (P.) Tul.

$\{$ Sphaeria Mela, S.

\{Eutypa Mela, Cke.

Eutypa micropuncta, Cke.

$\{$ Hypoxylon rhypodes B. \& C.

¿Eutypa rhypodes, Sacc.

Eutypa velutina.(Wallr.) Sacc., var. quercina, Sacc.

Gnomonia clavulata, Ell.

Gnomonia setacea, (P.) Ces. \& De Not.

Hypoxylon argillaceum, (P.) Fr.

Hypoxylon cohaerens, (P.) Fr.

Hypoxylon coccineum, Bull.

Hypoxylon commutatum, Nits.

Hypoxylon fuscum, (P.) Fr.

Hypoxylon Howeianum, Pk.

$\{$ Sphaeria investiens, S.

\{Hypoxylon investiens, B.

Hypoxylon leucocreas, B. \& Rav.

Hypoxylon Malleolus, B. \& Rav.

Hypoxylon multiforme, Fr.

Hypoxylon notatum, B. \& C.

Hypoxylon pallidum, Ell. \& Ev.

Hypoxylon Petersii, B. \& C.

$\{$ Hypoxylon polyspermum, Mont.

\{Hypoxylon marginatum, Rav. Fung. Am.

$\{$ Hypoxylon confluens, Rav. Fung. Am.

Hypoxylon Ravenelii, Rehm.

$\{$ Sphaeria rubiginosa, $P$.

Hypoxylon rubiginosum, $\mathrm{Fr}$.

Hypoxylon serpens, (P.) Fr.

Hypoxylon stigmateum, Cke.

\{ Sphaeria uda, P.

\{ Hypoxylon udum, Fr.

S Sphaeria xanthostroma, S.

$\{$ Hypoxylon xanthostroma, Sacc.

Sphaeria pachyascus, C. \& E.

$\{$ Kalmusia pachyascus, Sacc.

Xylosphaeria pachyascus, Cke.

Sphaerella dryophila, Cke. \& Hark.

$\{$ Leptosphaeria dryophila, Sacc.

Sphaerulina dryophila, Cke. \& Hark.

$\{$ Massaria obesa, B. \& C.

$\{$ Valsaria quadrata, (S.) Sacc.

Melanconis longipes, Tul.

$\{$ Sphaeria Pulvis-pyrius, $P$.

$\{$ Melanomma Pulvis-pyrius, Fckl.
(Sphaeria Texensis, Cke.

$\{$ Melanopsanma Texensis, Sacc.

Conisphaeria Texensis, Cke.

Sphaeria gyrosa, S.

$\{$ Endothia gyrosa, Fr.

(Melogramma gyrosum, Tul.

Ohleria rugulosa, Fckl.

$\{$ Ohleria modesta, Fck\}.

\{Ohleria rugulosa, var. nigerrima, Ell.

$\{$ Sphaeria microtheca, C. \& E.

Physalospora microtheca, Sacc.

$\{$ Sphaeria Aquila, Fr.

\{ Rosellinia Aquila, De Not.

$\{$ Sphaeria Corticium, S.

\{ Rosellinia Corticium, Sacc.

(Sphaeria Bombarda, Batsch.

$\{$ Bombardia fusciculata, Fr.

Rosellinia fasciculata, Cke.

$\{$ Sphaeria mammiformis, $P$.

\{ Rosellinia mammiformis, Ces. \& De Not.

S Sphaeria millegrana, S.

Rosellinia millegrana, Sacc.

$\{$ Sphaeria pulveracea, Ehrh.

\{ Rosellinia pulveracea, Fckl.

Sphaeria subiculata, $\mathrm{S}$.

Hypoxylon subiculosum, B.

Rosellinia subiculata, Sacc.

Byssosphaeria subiculata, Cke.

$\{$ Sphaeria dryophila, S.

Sphaerella dryophila, Cke.

Sphaerella pandurata, Ell. \& Ev.

Sphaerella Ravenelii, Cke.

Sphaerella simulans, Cke.

\{ Sphaeria tigrinans, S.

Sphaerella tigrinans, Cke.

$\{$ Sphaeria bifrons, Schm. \& Kze.

$\{$ Hypospila bifrons, $\mathrm{Fr}$.

\{ Sphaeria botryosa, Tode.

$\{$ Cucurbitaria botryosa, Ces. \& De Not.

Sphaeria glandicola, S.

Sphaeria inversa, Fr.

\{ Sphaeria melanotes, B. \&. Br.

Anthostoma melanotes, Sacc.

Sphaeria Micheliana, Fr.

Sphaeria obscura, S.

$\{$ Sphaeria populina, P.

\{Cryptosphaeria populina, Sacc.

Sphaeria putaminum, S., var. b.

Sphaeria spissa, S.

Sphaeria stilbosporans, S.

Sphaeria tigrinans, S.

Sphaeria disseminata, B. \& C.

\{Trichospora disseminata, Sace.

$\{$ Sphaeria phellogena, B. \& C.

$\{$ Teichospora phellogena, Sacc.

Sphaeria acanthostroma, Mont.

$\{$ Trichosphaeria acanthostroma, Sacc.

(Byssosphaeria acanthostroma, Cke.

Ustulina vulgaris, Tul.

Sphaeria conspurcata, S.

\{ Valsa conspurcata, Cke.

Valsa coronata, (Hoffm.) Fr. 
Sphaeria deformis, Fr.

Y Valsa deformis, Fs.

f Sphaeria Frustrum-coni, S.

\{Valsa Frustrum-coni, M. A. Curtis.

f Sphaeria hanstellata, Fr.

\{ Valsa haustellata, M. A. Curtis.

Sphaeria Ootheca, B. \& C.

$\{$ Coronophora Ootheca, Sacc.

Valsa Ootheca, Cke.

Valsa subclyperta, C. \& P.

$\{$ Diatrype cincta, (Curr.) B. \& Br.

\{ Valsaria cincta, Sacc.

$\left\{\right.$ Vermicularia ditricha, $\left(\mathrm{Fr}^{\circ}\right)$ S.

YVenturia ditricha, Karst.

Sphaeria melanostigma, C. \& E.

$\{$ Wallrothiesla melanostigma, Sacc.

Psilosphaeria melanostigma, C. \& E.

Winteria crustosa, Ell. \& Ev.

Xylaria Hypoxylon, (L.) Grev.

Sphaeria cariosa, C. \& E.

$\{$ Zignoella cariosa, Sacc.

Psilosphaeria cariosa, C. \& E.

Sphaeria diaphana, C. S E.

$\{$ Zignoella diaphana, Sacc.

Psilosphaeria diaphana, Ell. \& Ev.

\section{Hypocreaceae.}

$\{$ Sphaeria contorta, S.

$\{$ Hypocrea contorta, B. \& C.

IIy pomyces aurantius, (P.) Tul.

Nectria coccinea, (P.) Fr.

$\{$ Nectria (Calonectria) fulvida, Ell. \& Ev.

\{ Dialonectria fulvida, Ell. \& Ev.

Ophionectria Everhartii, Ell. \& Gall.

\section{Dothideaceae.}

$\{$ Dothidea maculans, S.

\{ Phyllachora maculans, Sacc.

\section{Lophiostomaceae.}

\{Lophiostoma heterostomum, Ell. \& Ev.

\{Lophiosphaleria heterostoma, Berl. \& Vogl.

Sphateria abbreviata, S.

Lophiostoma abbreviatum, Sacc.

Lophiostoma hysterioides, Ell. \& Langlois, nec Sacc.

\section{Hysteriaceae.}

\footnotetext{
(Hysterium rufescens, S.

$\{$ Ascoholus conglomeratus, S.

Angelina rufescens, Duby.

(Hysterium quercinum, $\mathrm{P}$.

Cenangium quercinum, Fr.

Colpoma quercinurn, Wallr.

\{Hysterium hyalinum, C. \& P.

\{ Gloniella hyalina, Sacc.

\{ Hysterium ovatum, Cke.

\{Gloniella ovata, Sacc.

$\{$ Hysterium lineolatum, Cke.

\{loniopsis lineolata, Sacc.
}

\{ Hysterium stictoideum, C. \& E.

\{ Gloniopsis stictoidea, Sacc.

Glonium parvulum, (Gerard) Cke.

Glonium stellatum, Muhl.

Hypoderma ilicinum, De Not.

Hypoderma variegatum, Duby.

Hysterium complanatum, Duby.

Hysterium Gerardi, C. \& P.

$\{$ Hysterium lineare, B. nec. Fr.

\{ Hysterium lineariforme, Sacc.

IIysterium Mori, S.

Iysterium petiolare, (A. \& S.) Fr

Hysterium proteiforme, Duby.

Hysterium pulicare, P., var. lenticulare, Fr.

Hysterium punctiforme, Fr.

fHysterium tumidum, Fr.

\{ Coccomyces tumida, De Not.

Hysterium tumidum, Fr., var. trigonum, Fr.

Hysterium varium, Fr.

Hysterium versisporum, Gerard.

Hypoderma virgultorum, var. petiolare, Cke.

Hysterium virgultorum, var. petiolare, Cke.

\{ Hysterium flexuosum, S.

Hysterographium flexuosum, Sacc.

$\{$ Hysterium pulicare, N. A. F.

\{Hysterographium hiascens, Rehm.

$\{$ Hysterium subrugosum, C. \& E.

\{Hysterographium subrugosum, Sacc.

Cysterium vulvatum, $\mathrm{S}$.

Hysterium flexuosum, Rav. Fung. Car.

Hysterium flexuosum, Myc. Univ. I8r.

Hysterographium vulvatum, Rehm.

$\{$ Hysterium maculare, Fr.

Lophodermium maculare, De Not.

Lophodermium punctiforme, (Fr.) Fckl.

Ostropa hysterioides, S.

\section{SPHAEROPSIDEAE.}

Aposphaeria conica, Sacc.

Ascochyta Quercus, Sacc. \& Speg.

$\{$ Dothidea geographica, (DC.) Fr.

Asteroma geographicum, Desm.

Cytospora difformis, $S$.

Dinemasporium hispidulum, (Schrad.) Sacc.

Diplodina Gallae, Ell. \& Ev.

$\{$ Diplodia periglandis, Cke. \& Hark.

$\{$ Diplodina periglandis, Sacc.

\{ Sphaeria Artocreas, Tode.

$\{$ Discosia Artocreas, Fr.

Lichenopsis sphaeroboloidea, $\mathbf{S}$.

( Lichenopsis sphaeroboloidea, B. in Herb. p. p., nec. S.

Platysticta simulans, Cke. \& Massee.

Sphaeropsis dryina, B. \& C.

$\{$ Phoma dryina, Sacc.

Macrophoma dryina, Berl. \& Vogl. 
$\{$ Sphaeropsis quercina, $\mathrm{Pk}$.

\{ Phoma quercina, Sacc.

Septoria herbarmm, B. \& C.

Sphaeronema glomeratum, Mont.

Sphaeria Gallae, S.

Spharopsis Gallae, B. \& C.

(Diplodia Gallae, Auct. Amer.

Sphaeropsis insignis, B. \& C.

Sphaeropsis linearis, $\mathrm{Pk}$.

Staurochaeta membranacea, Cke.

\section{MELANCONIEAE.}

Coryneum pustulatum, $\mathrm{Pk}$.

Melanconium hysterioideum, C. \& E.

$\{$ Naemaspora crocea, $P$.

$\{$ Myxosporium croceum, Lk.

Pestalozzia Castagnei, Desm.

Stilbospora angustata, $P$.

Stilbospora multiseptata, S.

\section{HXPHOMYCETES.}

Arthrosporium compositum, Ell.

Asterophora effusa, S.

Bispora aterrima, B. \& Rav.

Botrytis atroviridis, C. $\&$ E.

Botrytis griseolilacina, Ell. \& Ev.

Botrytis prasina, B. \& C.

Chloridium glaucum, Ell. \& Ev.

C Corticium pactolinum, Cke. \& Hark.

$\{$ Chromasporium isabellinum, Ell. \& Sacc.

Chromasporium pactolinum, Cke.

Cladosporium epiphyllum, Nees.

Cladosporium lignicolum, Cda.

$\{$ Sporidesmium adscendens, B.

$\{$ Clasterosporium adscendens, Sacc.

\{ Sporidesmium atrum, Lk.

\{Clasterosporium atrum, Sacc.

$\{$ Sporidesmium strumarum, Cke.

$\{$ Clasterosporium strumarum, Cke.

Racodium ramosum, S.

Oedemium ramorum, Fr.

Dematium ramosum, S.

Glenospora Curtisii, B. \& Desm.

Glenospora ramorum, B. \& C.

Cylindrocolla quercina, C. \& E.

Fusarium glandicolum, Cke. \& Gerard.

Fusidium tenuissimum, S.

$\{$ Fusidium griseum, Lk.

\{ Fusisporium griseum, Fr.

Gonytrichum caesium, Nees.

Graphium subulatum, (Nees) Sacc.

Haplaria fusca, Cke.

Helicoma Berkeleyi, M. A. Curtis,

$\{$ Helicomyces aureus, Cda.

\{ Helicosporium aureum, Auct.

Helicosporium olivaceum, $\mathrm{Pk}$.
Helminthosporium apiculatum, Cda.

Ilelminthosporium corniculatum, S.

\{ Helminthosporium leptotrichum, C. \& E.

$\{$ Brachysporium leptotrichum, Sacc.

Helminthosporium rectum, B. \& C.

Helminthosporium septemseptatum, Pk.

Macrosporium subglobosum, Cke. \& Rav.

Menispora ciliata, Cda.

Oidium candidum, s;.

Oidium obductum, Ell. \& Langlois.

SOidium simile, B.

$\{$ Monilia aureofulva, C. \& E.

Oospora similis, Sacc.

Oospora cuboidea, Sacc. \& Ell.

Periconia calicioides, ( $\mathrm{Fr}$.) $\mathrm{B}$.

Podosporium glandicolum, $\mathrm{S}$.

$\{$ Aspergillus laneus, $S$.

$\{$ Rhinotrichum Curtisii, B.

Rhinotrichum ramosissimum, B. \& C.

Septonema multiplex, B. \& C.

Septonema spilomeum, B.

Sphaerosporium lignatile, $\mathrm{S}$.

Sporidesmium acinosum, B. \& C.

$\{$ Sporidesmium compositum, B. \& Rav.

S Sirodesmium compositum, Sacc.

Sporidesmium helicoides, Ell. \& Ev.

Sporidesminm inquinans, Ell. \& Ev.

Sporidesmium insulare, EIl. \& Ev.

$\{$ Coniothecium effusum, Cda.

$\{$ Sporidesmium Lepraria, B.

Sporidesmium mundulum, Cke.

Sporidesmium nigrum, B.

Sporidesmium Peziza, C. \& E.

Sporidesmium polymorphum, Cda.

Sporidesmium translucens, Cke.

Sporidesmium velutinum, Cke.

Sporotrichum gratum, S.

Sporotrichum intertextum, S.

Sporotrichum sulfureum, Grev.

\{ Epochnium glaucum, Cke. \& Hark.

Stemphylium glaucum, Sacc.

Streptothrix atra, B. \& C.

Torula abbreviata, Cda.

Torula binale, C. $\&$ E.

Torula dissita, B. \& C.

Torula sphaeriaeformis, C. \& E.

$\{$ Memnonium effusum, Cda.

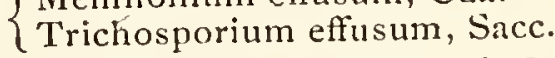

Verticillium puniceum, C. \& E.

$\{$ Botrytis atrofumosa, C. \& E.

$\left\{\begin{array}{l}\text { Virgaria atrofumosa, Sacc. } \\ \text { Virgaria fucopurpurea, }\end{array}\right.$

Virgaria fuscopurpurea, B. \& C.

Virgaria olivacea, Ell. \& Ev.

Virgaria uniseptata, B. \& C.

Zygodesmus effusus, B. \& C. 
$Z_{Y}$ godesmus fuscus, Cda.

$\mathrm{Z}$ ygodesmus rubiginosus, $\mathrm{Pk}$.

\section{DISCOMYETES.}

Agyrium nigricans, Fr.

$\{$ Bulgaria decolorans, B. \& C.

\{Ombrophila decolorans, Sacc.

Cenangium ferruginosum, $\mathrm{Fr}$.

Cenangium Abietis, (P.) Rehm.

f Dermatea fascicularis, (A. \& S.) Fr.

Cenangium populneum, (P.) Rehm.

(Peziza aeruginosa, (Oeder) $\mathrm{P}$.

Helotium aeruginosum, Fr.

Chlorosplenium aeruginosum, De Not.

\{ Peziza chlorascens, S.

Chlorosplenium repandum, Fr.

Peziza compressa, $\mathrm{P}$.

Durella compressa, Tul.

Durella macrospora, Fckl.

Peziza macrospora, Auct. Amer.

Peziza nigropunctula, Gerard.

Peziza Lecideola, Fr.

Patellaria Lecideola, Fr.

Durella Lecideola, Rehm.

$\{$ Haematomyces vinosus, C. \&. E.

Haematomyxa vinosa, Sacc.

Helotium citrinum, (Hedw.) Fr.

Helotium claroflavum, (Grev.) B.

Helotium fructigenum, (Bull.) Karst.

$\{$ Helotium versiforme, Fr.

Chlorosplenium versiforme, (P.) De Not.

$\{$ Patellaria atrata, (Wahl.) Fr.

\{ Lecanidion atratum, Rabh.

$\{$ Patellaria connivens, $\mathrm{Fr}$.

Durella connivens, Rehm.

Lecanidion fuscoatrum, Rehm.

Patellaria fuscoatra, El\}.

Durella fuscoatra, Rehm.

Patellaria nigrovirens, Ell. \& Sacc.

$\{$ Peziza Aurelia, P.

$\{$ Belonidium Aurelia, De Not.

$\{$ Peziza cinerea, Batsch.

\{ Mollisia cinerea, Karst.

$\{$ Peziza clandestina, Bull.

\{ Dasyscyphat clandestina, Fck\}.

Peziza coccinea, Jacq.

$\{$ Peziza coccinella, Sommf.

\{ Calloria coccinella, Fr.

$\{$ Peziza comata, S.

Trichopeziza comata, Sacc.

$\{$ Peziza crocitincta, B. \& C.

$\{$ Pezizella crocitincta, Sacc.

$\{$ Peziza culcitella. C. \& E.

\{'Tapesia culcitella, Sacc.

$\{$ Peziza epixantha, Cke.

Dasyscy pha epixantha, Sacc.

$\{$ Peziza fuscescens, P.

\{ Dasyscypha fuscescens, Rehm.
$\{$ Peziza introviridis, C. \& E.

Mollisia introviridis, Sacc.

$\{$ Peziza leucostigma, Fr.

Orbilia leucostigma, Fr.

$\{$ Peziza marginata, Cke.

Trichopeziza marginata, Sacc.

$\{$ Peziza nivea, (Hedw.) Fr.

\{Dasyscypha nivea, Sacc.

$\{$ Peziza obscura, Cke.

\{Trichopeziza obscura, Sacc.

$\{$ Peziza prolifica, E1l.

$\{$ Tapesia prolifica, Sacc.

Peziza pseudotuberosa, Rehm.

f Peziza radiocincta, Cke.

I Durella radiocincta, Sacc.

$\{$ Peziza rubella, P.

\{ Orbilia rubella, Karst.

Peziza rufula, S.

$\{$ Pezizella rufula, Sacc.

Orbilia rubella, (P.) Karst. var. rufula, Sacc.

$\{$ Peziza sanguinea, $\mathrm{P}$.

\{ Tapesia sanguinea, Fckl.

$\{$ Peziza succina, Phil.

\{ Dasyscypha succina, Sacc.

$\{$ Peziza uncinata, Phil.

\{Dasyscypha uncinata, Sacc.

Peziza variecolor, $\mathrm{Fr}$.

$\{$ Peziza vulgaris, Fr.

$\{$ Pezizella vulgaris, Sacc.

Peziza myceticola, B. \& C.

Peziza vulgaris, Fr., var. myceticola, B.

Pezizella vulgaris, Sacc., var. myceticola, Sacc.

Phacidium glandicolum, S.

Phacidium minutissimum, Awd.

Phacidium quercinum, $\mathrm{S}$.

\{ Rhytisma tostum, B. \& C.

Trabutia tosta, Cke.

Stictis dryophila, C. \& E.

$\{$ Stictis hysterina, Fr.

Mellitosporium hysterinum, Gill.?

$\{$ Stictis linearis, C. \& E.

\{Xylogramma linearis, Sacc.

Stictis quercina, Pk.

$\{$ Stictis versicolor, Fr.

\{ Propolis faginea, (Schrad.) Karst.

\section{HYMENOMYCETES.}

\section{Agaricini.}

$\{$ Agaricus applicatus, Batsch.

$\{$ Pleurotus applicatus, Sacc. Syll.

$\{$ Agaricus bombycinus, Schaeff.

$\{$ Volvaria bombycina, Sacc. Syll.

$\{$ Agaricus Californiensis, B. \& C.

Aycena Californiensis, Sacc. Syll.

A garicus (Naucoria) Erinaceus, Fr.

Agaricus (Omphalia) gracillimus, Weinm.

f Agaricus liratus, B. \& C.

T Galera lirata, Sacc. Syll. 
Agaricus (Armillaria) melleus, Vahl.

f Agaricus nidulans, P.

\{ Claudopus nidulans, $\mathrm{Pk}$.

Agaricus (Pleurotus) petaloides, Bull.

$\{$ Agaricus sapidus, Kalchb.

\{ Pleurotus sapidus, Sacc. Syll.

Agaricus (Pholiota) spectabilis, Fr.

Agaricus (Pholiota) squarrosus, Miill.

Agaricus (Mycena) strobilinus, P.

Agaricus (Hypholoma) sublateritius, Schaeff.

Agaricus (Pleurotus) tessulatus, Bull.

Cantharellus flabelliformis. B. \& Rav.

Coprinus fuscescens, (Schaeff.) Fr.

$\{$ Agaricus Chama, Bosc.

\{Lentinus Chama, Fr.

Lentinus Lecomtei, Fr.

Lentinus tigrinus, (Bull.) Fr.

Lenzites betulina, (L.) Fr.

Lenzites tricolor, (Bull.) Fr.

Marasmius brevipes, B. \& Rav.

Marasmius epiphyllus, Fr.

Marasmius insititius, Fr.

Marasmius rigidus, Mont.

Marasmius spodoleucus, B. \& Br.

Marasmius spongiosus; B. \& C.

Marasmius urens, (Bull.) Fr.

Marasmius viticolus, B. \& C.

Panus laevis, B. \& C.

Panus Robinsonii, B. \& Mont.

Pasus s trigosus, B.\& C.

Panus tomentosus, Bundy.

Panus torulosus, (P.) Fr.

Paxillus reniformis, B. \& Rav.

Schizophyllum commune, Fr.

Trogia crispa, (P.) Fr.

\section{Polyporei.}

Daedalea ambigua, $B$.

Daedalea aurea, Fr.

Daedalea Ravenelii, B.

Trametes sepium, B.

\{ Daedalea sepium, B.

$\{$ Boletus hepaticus, Huds.

\{Fistulina hepatica, Fr.

Fistulina spathulata, B. \& C.

Merulius ceracellus, B. \& C.

Merulius Corium, (P.) Fr.

Merulius porinoides, $\mathrm{Fr}$.

Merulius rufus, $P$.

(Boletus adustus, Willd.

$\{$ Polyporus adustus, Fr.

Myriadoporus adustus, Pk.

Polyporus amygdalinus, B. \& Rav.

Polyporus Anax, B.

$\{$ Polyporus argillaceus, Cke.

$\{$ Poria argillacea, Cke.
Polyporus Berkeleyi, Fr.

Solyporus biformis, $\mathrm{Fr}$.

$\{$ Polyporus Caroliniensis, B. \& C.

I Polystictus biformis, Fr.

$\{$ Polyporus Cremor, B. \& C.

\{ Poria Cremor, Cke.

Polyporus croceus, Fr.

Polyporus croceus, S. in litt.

Polyporus nitidus, Fr. El

Polyporus crociporus, B. \& C.

Poria crocipora, Cke.

$\{$ Boletus croceus, S. Syn. Car.

\{ Polyporus sulfureus, (Bull.) Fr.

$\{$ Polyporus cruentatus, Mont.

Poria cruentata, Cke.

f Fomes lucidus, Fr.

$\{$ Polyporus Curtisii, B.

Fomes Curtisii, Cke.

Polyporus dryadeus, Fr.

$\{$ Polyporus dryinus, B. \& Cke.

Poria dryina, B. \& Cke.

$\{$ Polyporus ectypus, B. \& C.

$\{$ Polystictus ectypus, Cke.

$\{$ Polyporus farinellus, Fr.

\{ Poria farinella, Cke.

$\{$ Polyporus Floridanus, B.

( Polystictus Floridanus, Fr.

$\{$ Boletus frondosus, S. Syn. Car.

Polyporus frondosus, Fr.

Polyporus giganteus, (P.) Fr.

Polyporus hirsutus, (Schrad.) Fr.

$\{$ Polyporus holoxanthus, B. \& Cke.

$\{$ Poria holoxantha, B. \& Cke.

Polyporus igniarius, (L.) Fr.

Polyporus intybaceus, $B$.

Polyporus labyrinthicus, Fr.

Polyporus licuoides, Mont.

Polyporus Lindbladii, B.

$\{$ Polystictus Lindbladii, Cke.

Poria Lindbladii, Cke.

Polyporus nidulans, Fr.

$\{$ Polyporus niger, B.

\{ Poria nigra, Cke.

(Boletus nigropurpureus, S.

$\{$ Polyporus nigropurpureus, S.

Poria nigropurpurea, Cke.

$\{$ Polyporus obducens, P.

\{ Poria obducens, Cke.

Polyporus quercinus, (Schrad.) Fr.

Polyporus radiatus, (Sow.) Fr.

f Polyporus Rhipidium, B.

\{ Favolus Rhipidium, Sacc. Syll.

(Boletus sanguineus, $\mathrm{L}$.

Polyporus sanguineus, Mey.

Odontia albominiata, B. \& C.

Polystictus sanguineus, Fr.

\{ Polyporus dryadeus, S. Am. bor.

$\{$ Polyporus scruposus, Fr.

Polyporus squamosus, (Huds.) Fr.

$\{$ Polyporus tabacinus, Mont.

$\{$ Mucronoporus tabacinus, Ell. \& Ev. 
f Poria tomentocinta, B. S Rav. sec. Cke.

Polyporus tomentocinctus, B. \& Rav. Herb.

Polyporus tomentosoquercinus, Johnson.

f Polyporus xanthus, Fr.

\{ Poria xantha, Cke.

Poria rufa, (Schrad.) Sacc. Syll., var. lilacina,

Ell. \& Ev.

(Porothelium lacerum, Fr.

$\{$ Boletus pezizoides, $\mathrm{S}$.

Porothelium pezizoides, S.

Porothelium subtile, (Schrad.) Fr.

Solenia anomala, (P.) Fckl.

Solenia ochracea, Hoffm.

Solenia villosa, Fr.

Trametes Kansensis, Cragin.

\section{Hydnei.}

Grandinia crustosa, (P.) Fr.

Hydnum Caput-medusae, Bull.

Hydnum cirrhatum, $P$.

Hydnum Erinaceus, Bull.

Hydnum fallax, Fr.

Hydnum farinaceum, $P$.

Hydnum laeticolor, B. \& C.

Hydnum ochraceum. $P$.

Hydnum pallidum, C. \& E.

Hydnum Ramaria, Fr.

Hydnum Stevensoni, B. \& Br.

Hydnum trichodontium, B. \& Rav.

Hydnum udum, Fr.

Irpex coriaceus, B. \& Rav.

Irpex crassus, B. \& C.

Irpex deformis, Fr.

Irpex lacteus, Fr.

Irpex mollis, B. \& C.

S Sistotrema niveum, S.

Irpex niveus, S.

Irpex sinuosus, Fr.

Kneiffia ambigua, Karst.

Kneiffia setigera, Fr.

Kneiffia tessulata, B. \& C.

$\{$ Phlebia hydnoidea, $\mathrm{S}$.

Odontia lateritia, B. \& C.

Phlebia anomala, B. \& Rav.

Phlebia orbicularis, B. \& C.

Phlebia rubiginosa, B. \& Rav.

Phlebia zonata, B. \& C.

Radulum Botrytis, Fr.

Radulum molare, Fr.

Radulum pallidum, B. \& C.

Sistotrema quercinum, $\mathrm{P}$.

\section{Thelephorei.}

\{ Corticium dryinum, B. \& C.

$\{$ Coniophora dryina, Massee.
(Thelephora laxa, Fr.

Corticium laxum, Fr.

Coniophora laxa, Sacc. Syll.

f Thelephora cinerascens, S.

\{ Corticium cinerascens, B.

Corticium diminuens, B. \& C.

Corticium laeve, $\mathrm{P}$.

Corticium lilacinofuscum, B. \& C.

Corticium puberum, Fr.

Corticium scutellare, B. \& C.

Cyphella trachychaeta, Ell. \& Ev.

Hymenochaete agglutinans, Ell.

Hymenochaete cervina, B. \& C.

Hymenochaete crocata, (Fr.) Lev.

Stereum umbrinum, B. \& C.

$\{$ Hymenochaete umbrina, B. \& C.

Stereum papyrinum, Rav. Fung. Am.

Peniophora intermedia. Massee.

$\{$ Stereum papyrinum, Mont.

\{ Peniophora papyrina, Cke.

Corticium quercinum, (P.) Fr.

$\{$ Peniophora quercina, Cke.

$\{$ Thelephora albobadia, S.

$\{$ Stereum albobadium, Fr.

Hymenochaete cervina, B. \& C.

Thelephora fasciata, S.

$\{$ Thelephora versicolor, var. fasciata, Fr.

Stereum fasciatum, Fr.

Stereum fimbriatum, Ell.

Stereum gausapatum, Fr.

Stereum Leveillianum, B. \& C.

Stereum purpureum, $\mathrm{P}$.

\{Thelephora spadicea, S. Syn. Car.

$\{$ Stereum spadiceum, Fr.

Thelephora granosa, B. \& C.

Tremellini.

Calocera glossoides, (P.) Fr.

Tremella Auricula-Judae, L.

Tremella Auricula, Mart.

Auricularia sambucina, Mart.

Exidia Auricula-Judae, Fr.

Hirneola Auricula-judae, B.

Exidia glandulosa, (Bull.) Fr.

Exidia glandulosa, (Bull.) Fr., forma levior,

Sacc.

Exidia truncata, Fr.

Naematelia encephala, (Willd.) Fr.

Naematelia nucleata, (S.) Fr.

Tremella enata, B. \& C.

$\{$ Tremella foliacea, $P$.

\{Ulocolla foliacen, Brefeld.

Tremella frondosa, $\mathrm{Fr}$.

Tremella marmorata, B. \& C.

Tremella mesenterica, Retz.

Tremella torta, Willd.

MISCELLANEA.

Capillaria grammica, $P$.

Ceratium hydnoides, (A. \& S.) Jacq. 
Endogone pisiformis, Lk.

Hymenula glandicola, Cke.

Lycoperdon quercinum, P.

Octaviana rosea, Hark.

Ozonium auricomum, Lk.

Pilacre Petersii, B. \& C.

Sparassis laminosa, Fr.

Sphaerobolus stellatus, Tode.

\section{SALICACEAE.}

\section{Populus alba, L.}

Cercospora populina, Ell. \& Ev.

Gloeosporium Castagnei, Desm. \& Mont.

$\{$ Gloeosporium Populi,(Lib.) Desm.\& Mont.

\{Marsonia Populi, Sacc.

Lenzites sepiaria, (Wulf.) $\mathrm{Fr}_{\mathrm{r}}$.

Populus augustifolia, James.

Melampsora populina, (Jacq.) Lev.

Uncinula Salicis, (DC.) Wint.

Populus balsamifera, L.

Melampsora populina, (Jacq.) Lev.

SOidium radiosum, Lib.

$\{$ Cladosporium ramulosum, Desm.

Fusicladium Tremulae, Frank.

$\{$ Polyporus biformis, Fr.

$\{$ Polystictus biformis, Fr.

Septoria populicola, Pk.

Septoria salicina, $\mathrm{Pk}$.

\{Uncinula adunca, Lev.

\{Uncinula heliciformis, Howe.

Populus balsamifera, L. var., candicans, Gray.

Eutypa subtecta, (Fr.) Fckl.

Gloeosporium brunneum, Ell. \& Ev.

Melampsora populina, (Jacq.) Lev.

Uncinula adunca, Lev.

Populus Fremontii, Watson.

Gloeosporium carpogenum, Cke. \& Hark.

Gloeosporıum Populi, (Lib.) Desm. \& Mont

Melampsora populina, (Jacq.) Lev.

$\{$ Asconyces aureus, Auct. Amer.

Taphrina rhizophora, Johans.

Populus grandidentata, Michx

Acalyptospora Populi, Pk.

Hypocrea Richardsonii, B. \& Mont.

$\{$ Lecythea ovata, Auct. Amer.

(Melampsora populina, (Jacq.) Lev.

f Torula populina, $\mathrm{Pk}$.

$\{$ Myxormia populina, Pk.

f Taphrina aurea, Auct. Aner.

\{ Taphrina rhizophora, Johans.

Uncinula Salicis, (DC.) Wint.

$\{$ Sphaeria nivea, Hoffin.

\{ Valsa nivea, Fr.
Populus heterophylla, L.

Uncinula adunca, Lev.

Uncinula luculenta, Howe.

Uncinula heliciformis, Howe.

Uncinula Salicis, (DC.) Wint.

$\{$ Dermea populnea, S.

$\{$ Cenangium Schweinitzii, Sacc.

Sphaeria exilis, A. \& S.

$\{$ Coelosphaeria exilis, Sacc.

Lasiosphaeria exilis. Cke.

Sphaeria variolosa, S.

\{Diatrype variolosa, Herb. B. sec. Cke.

Didymosporium minutissimum, $\mathrm{S}$.

$\{$ Sclerotium sphaeroides, $\mathrm{P}$.

Dothidea sphaeroides, Fr.

Uredo cylindrica, Strauss.

$\{$ Caeoma cylindricum, Schl.

Melampsora populina, (Jacq.) Lev.

(Melanconium hyalinum, Ell.

$\{$ Myxosporium Ellisii, Sacc.

Myxosporium hyalinum, Wint.

Monilia globosa, S.

$\{$ Sphaeria aperta, Schm.

\{ Valsa aperta. Cke.

Populus monilifera, Ait.

Capnodium avellanum, B. \& Desm.

Capnodium elongatum, B. \& Desm.

Cercospora populina, Ell. \& Ev.

\{ Sphaeria fusariospora, Ell. \& Ev.

\{Coelosphaeria fusariospora, Ell. \& Ev.

Cryptosporium coronatum, Fckl.

Cylindrosporium ? oculatum, Ell. \& Ev.

Gloeosporium stenosporum, Ell. \& Kell.

Sporocybe calicioides, Fr.

Periconia calicioides, B.

Hypsotheca calicioides, Ell. \& Ev.?

Caliciopsis Ellisii. Sacc.?

Lenzites sepiaria, (Wulf.) Fr.

Marsonia Populi, (Lib.) Sacc.

Sclerotium populinum, $\mathrm{P}$.

Uredo cylindrica, Strauss.

Caeoma cylindricum, Schl.

Melampsora populina, (Jacq.) Lev.

Lecythea populina, Lev.

Lecythea cylindrica, Auct. Amer.

Lecythea ovata. Auct. Amer.

Melampsora Medusae, Thm.

Peniophora occidentalis, Ell. \& Ev.

Phoma Populi, Fr.

Phyllosticta populina, Sacc.

Phyllosticta populina, Sacc., var. parva, Pk.

Septoria musiva, $\mathrm{Pk}$.

Sphaerella populifolia, Cke.

Sphaeronema Populi, B. \& C.

Teichospora pygmaen, Ell. \& Ev.

$\{$ Trametes Peckii, Kalchb.

\{ Trametes funalis, (Fr.) Ell. \& Ev.

Trametes Trogii, B.

Tremella subochracea, Pk. 
Uncinula Salicis, (DC.) Wint.

Zygodesmus obtusus, Ell. \& Ev.

Populus pyramidalis, Roz.

Agaricus ostreatus, Jacq.

Cenangium populinum, $\mathrm{S}$.

Saemaspora chrysosperma, P.

$\{$ Naemaspora populina, $P$.

Cytospora chrysosperina, (P.) Fr.

Hendersonia pauciseptata, B. \& C.

Hypoxylon Malleolus, B. \& Rav.

Myxosporium hyalinum, (Ell.) Wint.

$\{$ Phoma fibricola, B.

\{ A posphaeria fibricola, Sacc.

$\{$ Ascomyces aureus, Auct. Amer.

\{'Taphrina rhizophora, Johans.

Xylaria corniformis, Fr.

$\{$ Xyloma nervale, A. \& S.

\{ Rhytisina nèvale, Rehm.

Populus tremuloides, Michx.

Anthostoma flavoviride, Ell. \& Holw.

Cladosporium letiferum, $\mathrm{Pk}$.

Crytosphaeria millepunctata, Grev.

Cytospora chrysosperma, (P.) Fr.

Dermea populnea, S.

$\{$ Diatrype ferruginea, Fr.

\{ Sillia ferruginea, Karst.

Dimerosporium Populi, Ell. \& Ev.

Eutypa subtecta, (Fr.) Fckl.

Helicomyces roseus, Lk.

Hydnum velatum, B. \& C.

$\{$ Tubercularia pezizoidea, $\mathrm{S}$.

\{Hypocrea Richardsoni, B. \& Mont.

Hypoxylon Holwayii, Ell.

$\{$ Hysterium vulvatum, S.

\{Hysterographium vulvatum, Rehm.

Lentinus vulpinus, Fr.

Lophiotrema vestitum, Pk.

Melampsora populina, (Jacq.) Lev.

Lophodermium maculare, (Fr.) De Not.

Sidium radiosum, Lib.

Cladosporium ramulosum, Desm.

Fusicladium Tremulae, Frank.

$\{$ Patellaria fenestrata, C. \& P.

$\{$ Blitridium fenestratum, Sacc.

? Peziza Campanula, E11.

\{ Lachnella Campanula, Sacc.

Phlebia merismoides, Fr.

Phlebia spilomea, B. \& C.

Phoma Populi, Pk.

$\{$ Polyporus pargamenus, Fr.

Polyporus elongatus, B.

$\{$ Polyporus radiculosus, $\mathrm{Pk}$.

Poria radiculosa, Sacc. Syll.

Porothelium papillatum, $\mathrm{Pk}$.

Sphacrella inaculosa, Sacc.

$\left\{\begin{array}{l}\text { Taphrina aurea, Auct. Amer. } \\ \text { Ascomyces aureus, Auct. Amer. } \\ \text { Taphrina rhizophora, Johans. }\end{array}\right.$
$\{$ Uncinula heliciformis, Howe.

Uncinula Salicis, (DC.) Wint.

Valsa nivea, (Hoffm.) Fr.

Populus, sp. indet.

PYRENOMYCETES.

\{ Sphaeria mutila, S.

\{ Botryosphaeria mutila, Cke.

$\{$ Sphaeria acervata, Fr.

$\{$ Cucurbitaria acervata, Fr.

$\{$ Sphaeria sordida, P.

\{ Diatrype sordida, Cke.

Diatrypella hysterioides, Ell. \& Ev.

$\{$ Diatrypella angulata, (Fr.) Ces. \& De Not.

\{ Diatrypella nigroannulata, (Grev.) Nits.

Diatrypella Populi, Ell. \& Holw.

$\left\{\begin{array}{l}\text { Sphaeria eutypa, Fr. } \\ \text { Eutypa Acharii, Tul. } \\ \text { Cytospora Acharii, Sacc. }\end{array}\right.$

$\{$ Valsa Radula, Cke.

\{ Eutypella grandis, (Nits.) Sacc.

Hypoxylon serpens, (P.) Fr.

Sphaeria surrecta, Cke.

$\{$ Kalnuusia surrecta, Sacc.

Xylosphaeria surrecta, Cke.

$\{$ Melanconis apocrypta, Ell.

Melanconiella apocrypta, Sacc.

$\{$ Valsa quaternata, (P.) Fr.

Q Quaternaria Persoonii, Tul.

Sphaerella macularis, (Fr.) Awd.

Sphaerella orbicularis, $\mathrm{Pk}$.

$\{$ Sphaeria confluens, Tode.

(Hypoxylon udum, (P.) Fr.

Valsa a mbiens, (P.) Fr.

Valsa sordida, Nits.

FUNGI IMPERFECTI.

Cladosporium epiphyllum, Nees.

$\{$ Fumago vagans, $P$.

$\{$ Cladosporium Fumago, Lk.

Cytospora rubescens, Fr.

Helicoma Curtisii, B.

$\{$ Ilelicoma Mülleri, Cda.

$\{$ Helicosporium Mülleri, Sacc.

Melanconium populinum, $\mathrm{Pk}$.

Phoma urens, Ell. \& Ev.

$\{$ Dactylium sublutescens, Pk.

Trichothecium sublutescens, Sacc.

Vermicularia albomaculata, $\mathrm{S}$.

$Z$ ygodesmus atroruber, $\mathrm{Pk}$.

Zythia ovata, Pk.

HYMENOMYCETES.

$\{$ Agaricus atrocaeruleus, Fr.

\{Pleurotus atrocaeruleus, Sacc. Syll.

Agaricus Fibula, Bull., var. conicus, $\mathrm{Pk}$. 
$\{$ Agaricus fulvotomentosus, $\mathrm{Pk}$.

$\{$ Crepidotus fulvotomentosus, Sacc. Syll.

Agaricus salignus, Schrad.

$\{$ Agaricus haustellaris, Fr.

Crepidotus haustellaris, Sacc. Syll.

Crepidotus croceitinctus, $\mathrm{Pk}$.

Hydnum caryophylleum, B. \& C.

Irpex nodulosus, Pk.

Lenzites betulina, (L.) Fr.

Marasmius subvenosus, $\mathrm{Pk}$.

Phlebia zonata, B. \& C.

Polyporus spumeus, Fr.

Polyporus sulfurellus, Pk.

Polyporus versicolor, (L.) Fr.

Polystictus placentaeformis, B. sec. Cke.

\section{MISCELLANEA.}

Peziza populnea, $\mathrm{P}$.

Peziza fascicularis, A. \& S.

Dermatea fascicularis, Fr.

Cenangium populneum, Rehm.

Clathroptychium rugulosum, (Wallr.) Rostf.

Dermatea cinnamomea, C. \& P.

Phacidium coronatum, (Schum.) Fr.

Salix alba, L., var. vitellina, Koch.

Melampsora Vitellinae, Thm.

Salix amygdaloides, Anders.

$\{$ Melampsora salicina, Lev.

Melampsora Salicis-capreae, (P.) Wint.

Peniophora occidentalis, Ell. \& Ev.

Uncinula Salicis, (DC.) Wint.

Salix arctica, Pall.

Ceratostoma foliicolum, Fckl.

Cytospora capitata, Fckl.

f Rhizomorpha arctica, Fckl.

\{ Hypoxylon arcticum, Rostrup.

Melampsora salicina, Lev.

$\{$ Pleospora paucitricha, Fckl.

\{Pyrenophora pancitricha, Berl. \& Vogl.

Xylographa arctica, Fckl.

Salix arctica, Pall., var. Brownei, Anders.

Rhytisma salicinum, (P.) Fr.

Salix arctica, Pall., var. Groenlandica,

Anders.

Melampsora arctica, Rostrup.

Melanomma cinereum, (Karst.) Sacc.

Patellaria bacilligera, Karst.

Phoma salicina, Westd.

Pseudopeziza versicolor, (Wahl.) Rostrup.

Pyrenophora paucitricha, (Fckl.) Berl. \& Vogl.

Salix Babylonica, Tourn.

$\{$ Helicoma Curtisii, B.

\{ Helicosporium Curtisii, Sacc.

Lenzites Klotschii, B.
\{ Tympanis stictica, B. \& C.

Cenangium sticticum, Sacc.

Salix cordata, Muhl.

Melampsora salicina, Lev.

Melampsora Hartigii, Thm.

Melampsora Salicis-capreae, (P.) Wint.

Crysiphe adunca, Lk.

Erysiphe obtusata, Lk.

Uncinula adunca, Lev.

Uncinula luculenta, Howe,

Uncinula heliciformis, Howe.

Uncinula Salicis, (DC.) Wint.

Salix cordata, Mull., var. angustata, Anders.

(Hypoxylon Morsei, B. \& C.

$\{$ Hypoxylon Blakei, B. \& C.

Fuckelia Morsei, Cke.

Salix discolor, Muhl.

Corticium salicinum, Fr.

Exidia glandulosa, (Bull.) Fr.

Habrostictis ocellata, (P.) Fckl.

Lentinus suavissimus, Fr.

Lenzites sepiaria, (Wulf.) Fr.

$\{$ Melampsora Capraearum, Thm.

\{Melampsora Salicis-capreae, (P.) Wint.

Panus salicinus, $\mathrm{Pk}$.

Rhytisma salicinum, (P.) Fr.

Trimmatostroma Salicis, Corda.

Coniothecium toruloideum, M. A. Curtis, nec Corda.

Septonema connatum, B. \& C.

Trimmatostroma Americanum, Thm.

$\{$ Uncinula adunca, Lev.

\{Uncinula Salicis, (DC) Wint.

S Sphaeria salicina, $\mathrm{P}$.

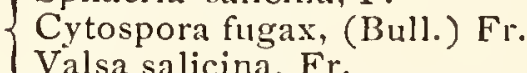

Salix flavescens, Nutt.

Melampsora Salicis-capreae, (P.) Wint.

Phlebia merismoides, $\mathrm{Fr}$.

Rhytisma salicinum, (P.) Fr.

Uncinula Salicis, (DC.) Wint.

Salix glauca, L.

Hypospila Groenlandica, Rostrup.

Leptosphaeria Coniothyrium, (Fckl.) Sacc.

Leptostroma punctiforme, Wallr.

Lophodermium hysterioides, (P.) Sacc.

Melampsora arctica, Rostrup.

Melampsora Salicis-capreae, (P.) Wint.

Pseudopeziza versicolor, (Wahl.) Rostrup.

Rhytisma salicinum, (P.) Fr.

Septoria salicina, $\mathrm{Pk}$.

Uncinula Salicis, (DC.) Wint.

Venturia chlorospora, (Ces.) Karst.

Salix herbacea, L.

Melampsora arctica, Rostrup.

Rhytisma salicinum, (P.) Fr.

Sphaerella salicicola, (Fr.) Fckl. 
Venturia chlorospora, (Ces.) Karst.

Salix In umilis, Marsh.

Melampsora Capraearum, Thm.

Rhytisma salicinum, (P.) Fr.

Salix lasiolepis, Benth.

Septogloeum maculans, Hark.

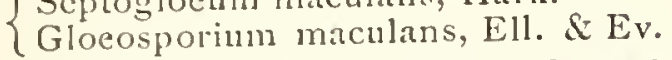

$\{$ Hysterium prominens, Phil. \& Hark.

$\{$ Hysterographium prominens, Berl. \& Vogl.

Salix lasiolepis, Benth., var. Bigelovii,

Bebb.

Melampsora Bigelovii, Thm.

Salix longifolia, Muhl.

Cercospora salicina Ell. \& Ev.

Gloeosporium Salicis, Westd.

SMelampsora salicina, Lev.

\{Melampsora Salicis-capreae, (P.) Wint.

Uncinula Salicis, (DC.) Wint.

Salix lucida, Muhl.

Thelephora salicina, Fr.

Thelephora cruenta, S. Syn. Car.

Corticium salicinum, Fr.

Corticium cruentum, (P.) Schrt.

Septoria Albaniensis, Thm.

Septoria salicina, $\mathrm{Pk}$.

Salix Myr'sinitis, L.

Asteroma Salicis, Rob. \& Desm.

Rhytisma salicinum, (P.) Fr.

Salix myrtilloides, L.

Melampsora Salicis-capreae, (P.) Wint.

Salix nigra, Marsh.

Cercospora salicina, Ell. \& Ev.

Darluca Filum, (Biv.) Cast.

Lenzites Klotschii, B.

CUredo Caprearum, DC.

Lecythea salicina, Lev.

Caeoma epiteum,

Uredo epiten,

Uredo Saliceti,

Lecythea epitea,

Auct. Amer.?

(Melampsora salicina, Lev.

Melampsora Capraearum, Thm.

Melampsora Hartigii, Thm.

Melampsora Salicis-capreae, Wint.

Melampsora farinosa, (P.) Schrt.

Melanconium salicinum, Ell. \& Ev.

Panus alliaceus. B. \& C.

Polyporus molluscus, (P.) Fr.

Prosthemiella hysterioides, Ell. \& Langlois.

(Triblidium clavaesporum, Pk.

$\{$ Triblidiella clavispora, Berl. \& Vogl.

(Patcllaria clavispora, Sacc.

Uncinula Salicis, (DC.) Wint.

Salix petiolaris, Smith.

Uncinula Salicis, (DC.) Wint.

Salix purpurea, L.

Rosellinia mastoidea, Sacc.

Tympanis saligna. Tode.
Salix rostrata, Rich.

Hypoxylon Blakei, B. \& C.

$\{$ Melampsora Salicis-capreae, Wint.

\{ Melampsora farinosa, (P.) Schrt.

Ramularia rosea, (Fckl.) Sacc.

Uncinula Salicis, (DC.) Wint.

Salix Russelliana, Smith.

$\{$ Septonema connatum. B. \& C.

\{ Trimmatostroma Salicis, Corda.

Salix sericea, Marsh.

$\{$ Gloeosporium salicinum, $\mathrm{Pk}$.

\{ Septogloeum salicinum, Sacc.

\{ Rhytisma maximun, Fr.

\{Cryptomyces maximus, Rehm.

Salix tristis, Ait.

Melampsora Caprearum, Thm.

Salix Uva-ursi, Pursh.

Melampsora Caprearum, Thm.

Rhytisma salicinum, (P.) Fr.

Salix, sp. indet.

PYRENOMYCETES.

$\{$ Sphaeria papillata, Schum.

\{ Amphisphaeria papillata, De Not.

Capnodium salicinum, Mont.

Sphaeria suffusa, Fr.

$\{$ Valsa suffusa, Fr.

Cryptospora sufiusa, Tul.

Cucurbitaria insecura, Ell.

$\{$ Valsa glyptica, B. \& Currey.

Diaporthe glyptica, Sacc.

Valsa mucronata, $\mathrm{Pk}$.

$\{$ Valsa Tessera, (Fr.) Cke.

Diaporthe mucronata, Sacc.

Sphaeria salicella, Fr.

$\{$ Diaporthe salicella, Sacc.

Endophlaea salicella, Cke.

\{ Sphaeria spiculosa. A. \& S.

$\{$ Diaporthe spiculosa, Nits.

Diatrype bullata, (Hoffm.) Fr.

Dimerosporium xylogenum, Ell. \& Ev.

Sphaeria oppansa, Fr.

\{ Eutypa oppansa, Cke.

Eutypa subtecta. (Fr.) Fckl.

Gibbera Saubinetii, Mont.

Hypocrea lenta, (Tode) B.

IIypoxylon Botrys, Nits.

Hypoxylon coccineum, Bull.

Hypoxylon investiens.(S.) B.

Hypoxylon Tinctor, (B.) Cke.

Hysterium fibritectum, S.

? Hysterium Mori, S.

Hysterographimm Mori, Rehm.

Hysterium rugulosum, S.

Leptosphaeria consimilis, Ell. \& Ev.

Lophiostoma erosum, Ell. \& Ev.

Lophrostoma Langloisii, Ell. \& Ev.

Lophiostoma turritum, C. \& P.

Nectria tremelloides, Ell. \& Ev. 
$\{$ Sphaeria myriocarpa, Fr.

$\{$ Rosellinia myriocarpa, Cke.

Sphaeria lineolans, $\mathrm{S}$.

Sphaerostilbe flammea, Tul.

Teichospora Helenae, Ell. \& Ev.

Valsa dissepta, Fr.

Valsa Orbicula, B. \& C.

Valsa pallida, Ell. \& Ev.

f Sphaeria Tessella, P.

\{ Valsa Tessella, Fr.

$\{$ Valsa translucens, De Not.

$\{$ Cytospora translucens, Sacc.

\section{SPHAEROPSIDEAE.}

Cytospora Salicis, (Cda.) Rabh.

Cytospora xanthosperma, Fr.

Diplodina Salicis, Westd.

Discella carbonacea, (Fr.) B. \& Br.

$\{$ Discella macrosperma, Pk.

\{Discula macrosperma, Sacc.

Discella microsperma, B. \& Br.

Dothiorella decorticata, Ell. \& Ev.

$\{$ Sphaeria hemisphaerica, A. \& S.

\{ Sphaeronema hemisphaericum, Fr.

HYPHOMYCETES.

Bactridium flavum, $\mathrm{K}_{z e}$.

Botrytis patula, Sacc. \& Berl.

Cladosporium Fumago, Lk.

Cladosporium polysporum, Lk.

Dematium cornutum, L.k.

Dematium viresens, $P$.

Dendrodochium densipes, Sacc. \& Ell., var. prolificum, Ell. \& Ev.

Helminthosporium simplex, Nees.

$\{$ Aspergillus laneus, S. Am. bor.

Rhinotrichum Curtisii, B.

Sphaerosporium lignatile, S.

Sporidesmium aurantiacum, B. \& C.

$\{$ Vermicularia clavuligera, S. Am. bor.

Sporidesmium concinaum, B.

Torula crustacea, S.

Volutella pulchra, B. \& C.

\section{DISCOMYCETES.}

$\{$ Peziza versiformis, $P$.

$\{$ Chlorosplenium versiforme, (P.) De Not.

Dermatea inclusa, Pk.

Helotium salicellum, Fr.

Patellaria difformis, (Fr.) S.

$\{$ Patellaria olivacea, (Batsch) B. \& Br.

\{ Patinella olivacea, Sacc.

$\{$ Peziza citrinella, S.

$\{$ Pezizella citrinella, Sacc.

? Peziza difformis,Fr.?

? Peziza nigrescens, Cke.

$\{$ Peziza salicina, P.

\{ Phialea salicina, Sacc.
$\{$ Stictis cylindrocarpa, $\mathrm{Pk}$.

Propolis cylindrocarpa, Sacc.

$\{$ Stictis mollis, P.

Stictis Pupula, Fr.

Sphaeria fuliginosa, $\mathrm{P}$.

$\{$ Cenangium fuliginosum, Fr.

Scleroderris fuliginosa, Karst.

\section{HYMENONIYCETES.}

? Agaricus disseminatus, $P$.

$\{$ Agaricus salignus, Schrad.

$\{$ Pleurotus salignus, Sacc. Syll.

Agaricus (Collybia) velutipes, Wm. Curtis.

\{ Corticium Oakesii, B. \& C.

\{ Aleurodiscus Oakesii, Cke.

Coprinus fuscescens, (Schaeff.) Fr.

Corticium evolvens, Fr.

Corticum lactescens, B.

Cyphella fasciculatá, B. \& C.

Dacryomyces cinnabarinus, $\mathrm{S}$.

Daedalea confragosa, $\mathrm{P}$.

Exidia impressa, (P.) Fr.

$\{$ Peziza gelatinosa, Bull.

Exidia recisa, Fr.

C Agaricus tenuissimus, $\leq$.

$\{$ Agaricus ringens, Fr., var. tenuissimus, $\mathrm{Fr}_{\mathrm{r}}$.

Lentinus tenuissimus. S.

Lenzites betulina, (L.) Fr.

Lenzites Cookei, B.

Marasmius saliguus, $\mathrm{Pk}$.

Marasmius salignus, Pk., var. major, Pk.

Omphalia tubaeformis, $\mathrm{Pk}$.

Panus foetens, Fr.

Polyporus pubescens, (Schum.) Fr.

(Boletus salicinus, Auct. p. p.

Polyporus salicinus, Fr.

Fomes salicinus, Karst.

Mucronoporus salicinus, Ell. \& Ev.

Polyporus salignus, Fr.

Stereum purpurellum, Fr.

Trametes odora, (Sommf.) Fr.

$\{$ Daedalea rubescens, A. \& S.

\{rametes rubescens, Fr.

Trametes suaveolens, Fr.

Tremella indecorata, Sommf.

Tremella intumescens, Sm.

\section{EMPETRACEAE.}

\section{Corema Conradii, Torr.}

Cucurbitaria Coremae, Ell. \& Ev.

Diaporthe Conradii, Ell.

Empetrum nigrum, L.

f Uredo Empetri, P.

\{ Chrysomyxa Empetri, Rostrup.

Metasphaeria Empetri, Sacc.

Septoria Empetri, Rostrup.

Sporomega Empetri, Rostrup. 





\section{A PROVISIONAL HOST-INDEX}

OF THE

Fungi of the United STATES

W. G. FARLOW AND A. B. SEYMOUR

PART III. 



\section{ENDOGENS.}

\section{ORCHIDACEAE.}

Corallorhiza multiflora, Nutt. $\{$ Leptosphaeria Corallorhizae. Pk. Heptameria Corallurhizae, Cke.

Cypripedium pubescens, Willd.

Puccinia Cypripedii, Arth. \& Holw.

Habenaria hyperborea, R. Br.

Cladosporium herbarum, (P.) Lk.

$\{$ Lachnum Groenlandicum, Rostrup.

$\{$ Dasyscypha Groenlandica, Sacc.

Orchidaceae, sp. indet.

Gloeosporium cinctum, B. \& C.

\section{SCITAMINEAE.}

\section{Canna Indica, L.}

Macrosporium bulbotrichum, Cke.

Musa, sp. indet.

Chaetophoma Musae, Cke.

Cladosporium pannosum, Cke.

Cyphella Bananae, Cke.

Fusarium semitectum, B. \& Rav.

Gloeosporium lagenarium, (Pass.) Sacc. \&

Roum., var. Musarum, Ell. \& Ev.

$\{$ Penicillium atrobrunneum, Cke.

$\{$ Haplographium atrobrunneum, Sacc.

\{ Hendersonia Musae, Cke.

\{ Stagonospora MIusae, Sacc.

Myxosporium Musae, B. \& C.

Phoma Musarum, Cke.

Sporidesmium Fumago, Cke.

Stachybotrys subsimplex, Cke.

Thalia dealbata, Roscoe.

Cercospora Thaliae, Ell. \& Langlois.

\section{BROMELIACEAE.}

\section{Tillandsia usneoides, L.}

\{ Psilonia cylindrospora, B. \& C:

$\{$ Volutella cylindrospora, Sacc.

\section{HAEMODORACEAE.}

Aletris a urea, Walt.

Aletris farinosa, L.

Puccinia Aletridis, B. \& C.

\section{IRIDACEAE.}

Belamcanda Chinensis, Adans.

Heterosporium gracile, Sacc.

Gladiolus, sp. indet.

Cyphella fumosa, Cke.

Diaporthe Gladioli, EIl. \& Ev.

Sphaeropsis elongata, B. \& C.

$\{$ Phoma elongata, Sacc.

Macrophoma elongata, Berl. \& Vogl.

Sphaeropsis Gladioli, Cke.

$\{$ Phoma Gladioli, Sacc.

Macrophoma Gladioli, Berl. \& Vogl.

Malcrosporium caudatum, C. \& E.

Macrosporium fasciculatum, C. \& E.

Polyactis cinerea, (P.) B.

$\{$ Sphaeria minimipuncta, Cke.

S Sphaerella minimipuncta, Cke.

Sphaeropsis lanceolata, C. \& E.

Iris Douglasiana, Herbert.

Iris longipetala, Herbert.

Caeoma (Uredo) Iridis, S.

Iris Missouriensis, Nutt.

Macrosporium Iridis, C. \& E.?

Iris sambucina, L.

Macrosporium Iridis, C. \& E.

Iris versicolor, L.

Aecidium Iridis, Gerard.

Cylindrosporium Iridis, E11. \& Halsted.

$\{$ Helminthosporium gracile, Wallr.

Heterosporium gracile, Sacc.

Sphaerella Iridis, Awd.

Uredo Iridis, DC.

Caeoma (Uredo) Iridis, S.

Puccinia Iridis, Duby?

Trichobasis iridicola, $\mathrm{Pk}$.

Uredo iridicola, De Toni. 


\section{Iris Virginica, I..}

Caeoma (Uredo) Iridis, S.

Cladosporium iridicolum, S.

Helminthosporium gracile, Wallr.

Sphaeria iridicola, S.

Vermicularia acuminata, S.

Iris, sp. indet.

Splacria Iridis, S.

Dothiclea Iridis, S. in IIcrb. B. sec Cke.

Darluca Filum. Cast.

Dothidea reticulata, (DC.) Fr.

Perisporinm Iridis, Fr.

Polyactis cinerea, (P.) B.

Vermicularia Dematium, (P.) Fr.

Sisyriuchium bellum, Watson.

Kellermania Sisyrinchii, Ell. \& Ev.

\section{ANARYLLIDACEAE.}

Agave Americana, L.

Melanconium Americanum, Pk. \& Clinton.

$\{$ Stachybotrys subsimplex, Cke?

$\{$ Stach ybotrys simplex, Hark.

Agave Shawii, Engelm.

\{ Dothidea Parryi, Farl. Mss.

\{ Endothia Parryi, Cke.

Agave, sp. indet.

Torula diversa, Cke.

4 maryllis, sp. indet.

Cercospora Amaryllidis, Ell. \& Ev。

Hypoxis erecta, L.

Urocystis Hypoxis, Thaxter.

Uromyces affinis, Wint.

Narcissus, sp. indet.

Aegerita candida, P.

\{ Hendersonia Curtisii, B.

\{ Stagonospora Curtisii, Sacc.

Pancratium coronarium, Leconte.

Cercospora Pancratii, Ell. \& Ev.

\section{DIOSCOREACEAE.}

\section{Dioscorea villosa, Torr.}

Cercospora Dioscoreae, Ell. \& Martin.

Dioscorea, sp, indet.

\{ Sphacria Dioscoreae, B. \& C.

i Didymella Dioscoreae, Sacc.

f Septoria Dioscoreae, Cke.

IPhyllosticta Dioscorene, Cke.

\section{LILIACEAE.}

\section{Allium Cepa, L.}

f Dactylium melanopus, S.

I Acrothecium melanopus, Sacc.

Cladosporium sparsum, $\mathrm{S}$.

S Fusisporium atrovirens, B.

\{Fusariellat atrovirens, Sacc. Syll.
Macrosporium Porri, Ell.

Macrosporium Sarcinula, I3., vas, parasıticum, Thm.

Mucor subtilissimus, B.

\{Peronospora Schleideni, Ung.

\{ Peronospora Schleideniana, D By.

Sothidea Cepae, S.

PPhyllachora Cepae, Sacc.

$\{$ Urocystis Cepulae, Frost.

UUrocystis Colchici, var. Cepulae, Cke.

Vermicularia circinans, 13 .

\{ Sphaeria Dematium, P.

\{ Vermicularia Dematium, Fr.

Allium mutabile, Michx.

Puccinia mutabilis, Ell. \& Gall.

Allium Nevadense, Watson.

Puccinia Allii, (DC.) Wint.

Allium Porrum, L.

Macrosporium Porri, Ell.

Allinm reticulatum, Fraser.

Aecidium Convallariae, Schum.

Allium Schoenoprasum, L.

Sphaeria allicina, Fr.

Allium stellatum, Fraser.

Aecidium alliicolum, Wint.

Allium tricoccum, Ait.

$\{$ Septoria Alliorum, Westd.

\{ Septoria viridetingens, M. A. Curtis.

Allium vineale, L.

Heterosporium Allii, Eli. \& Martin.

Heterosporium Ornithogali, (Klotzsch) Cke.

Allium, sp. indet.

Aspergillus niger, Van Tiegh.

$\{$ Dematium articulatum, P.

Cladosporium fasciculare, $\mathrm{Fr}$.

$\{$ Dothidea penicillata, S.

( Phyllachora penicillata, Sacc.

Rhinotrichum tenellum, B. \& C.

S Sphaeria exuberans, Fr.

Sphaeria Porri, var. exuberans, S.

Vermicularia Liliace urum, S.

Asparagus officinalis, L.

Cercospora caulicola, Wint.

Cladosporium fasciculare, $\mathrm{Fr}$.

Diplodia Asparagi, Pk.

Epicoccum purpurascens, Elırb.

Gibbera Saubinetii, Mont.

Leptosphaeria Asparagi, $\mathrm{Pk}$.

S Sphaeria comatella, C. \& E.

$\{$ Leptosphaeria comatella, Sacc.

(IIeptameria comatella, Cke.

Sphaeropsis lanceolata, C. \& E.

$\{$ Phoma lanceolata, Sacc.

Macrophoma lanceolata, Berl. \& Vogl.

Macrosporium caespitulum, Cke.

Macrosporium commune, Rabh. 
Phoma Asparagi, Sacc.

Phoma media, Ell. \& Ev.

Phoma microspora, B. \& C.

I Sphaeria herbarum, $P$.

\{ Pleospora herbarum, Rabh.

Puccinia Asparagi, DC.

Septoria phlyctaenoides, B. \& C.

Brodiaea capitata, Benth.

Puccina nodosa, Ell. \& Hark.

Brodiaea Howellii, Watson.

$\{$ Hendersonia cylindrocarpa, E11. \& Ev.

Stagonospora cylindrocarpa, Sacc.

Brodiaea laxa, Watson.

Uromyces Brodiaene, E11. \& Hark.

Calochortus Nuttallii, Torr. \& Gr.

Puccinia Calochorti, Pk.

Calochortus nudus, Watson.

Puccinia anachoreta, Hark.

Chlorogalum pomeridianum, Kunth.

$\{$ Sphaeria permunda, Cke.

\{ Pleospora permunda, Sacc.

Rhabdospora Chlorogali, Cke. \& Hark.

Clintonia borealis, Raf.

$\int \frac{\text { Puccinia mesomajalis, B. \& C. in Peck 25th }}{\text { Rept. }}$

Puccinia mesomegala, B. \& C. in Grev. III. 52.

Convallaria majalis, L.

Asteroma reticulatum, (DC.) Chev.

SDepazea cruenta, Fr.

$\{$ Phyllosticta cruenta, Kickx.

Dasylirion Wheeleri, Watson.

Torula quaternata, B. \& C.

Dasylirion, sp. indet.

fDothidea Dasylirii, Pk.

\{ Phyllachora Dasylirii. Sacc.

Dracaena Cooperi, Walt.

Cladosporium dracaenatum, Thm.

Dracaena, sp. indet.

Lophodermium Dracaenae, Phil. \& Hark.

Macrosporium abruptum, C. \& E.

Sphaeria anisometra, Cke. \& I Iark.

Metasphaeria anisometra, Sacc.

Endophlaea anisometra, Cke. \& Hark.

Pleospora phragmospora, (Dur. \& Mont.) Ces.

Erythronium Americanum, Smith.

Protomyces Erythronii, Pk.

Ustilago Erythronii. Clinton.

\{ Ustilago Ornithogali. (Schm. \& Kze.) Kühn, forma Erythronii, De Toni.

Fritillaria, sp. indet.

f Uromyces Liliacearum, Unger.

Uromyces Erythronii. (DC.) Pass.

Helonias bullata, L.

Sphaeria heloniaefolia. C. \& E.

Sphaerella heloniaefolia, C. \& E.

Hendersonia heloniaefolia. Cke.

Leptosphaeria heloniaefolia, Sacc.
Hemerocallis fulva, L.

Cyphella Capula, (Iolmsk.) Fr.

Heterosporium gracile, Sacc.

Hemerocallis, sp. indet.

$\{$ Xyloma Liliorum, S.

Excipula Liliorum, Fr.

(Dothidea Lineola, S.

Asteroma Lineola, M. A. Curtis.

Phyllaclrora Lineola, Sacc.

Vermicularia Liliacearum, S.

Syloma nitens, S.

Leptostroma sphaeroides, Fr., var. nitens, $\mathrm{Fr}$.

Lilium Canadense, L.

Aecidium Convallariae, Schum.

$\{$ Uromyces Erythronii, (DC.) Pass.

Uromyces Lilii, Clinton.

Lilinm candidum, L.

Aecidium Convallariae, Schum., var. Lilii, Farl.

Lilium pardalinum, Kellogg.

Peziza atrata, P., var. Liliacearum, Phil. \& Hark.

Lilin m Philadelphicum, L.

$\{$ Uromyces Liliacearum, Ung.

Uromyces Erythronii, (DC.) Pass.

Lilium superbum, L.

\{ Uromyces Erythronii, (DC.) Pass.

Uromyces Holwayi, Lagerheim.

Lilium tigrinnm, Ker.

Vermicularia Dematium, Fr.

$\{$ Vermicularia Liliacearum, S. ?

$\{$ Vermicularia Liliacearum, Westd.

Maiantlıenim Canadense, Desf.

Cercospora Maianthemi, Fckl.

Didymium Fairmani, Sacc.

Dothidea reticulata, (DC.) Fr.

Phoma Maianthemi, Pk.

Cercospora subsanguinea, Ell. \& Ev.

Nothoscordum striatum, Kunth.

Uromyces Ervthronii, (DC.) Pass.

Oakesia sessilifolia, Watson.

f Aecidium Uvulariae, S.

\{aeoma (Aecidium) uvulariatum, S.

Ornithogalum umbellatum, L.

Heterosporium Ornithogali, (Klotzsch) Cke.

Phormium tenax, Forst.

\{ Gloeosporinin phomiforme, Sacc. \& Ell.

$\{$ Gloeosporium punctiforme, Sacc. \& Ell.

Hymenula phormicola, Cke. \& Hark.

$\{$ Leptosphaeria phormicola, Cke. S Hark.

Heptameria phormicola, Che.

Stachybotrys scabra, Cke. \& Hark.

Polygonatum biflorum, Elliott.

Aecidium Convallariae, Schum.

Polygonatum giganteum, Dietrich.

Aecidium Convallariae, Schum. 
\{lysterium Polygonati, S.

Ulypoderma P'olygonati, Ckc.

Spluseria cruenta, Fr.

$\{$ Depazea cruenta. Fr.

I'hyllosticta crucuta, Kickx.

Sacidiun Polygonati, Ell. \& Martin.

Sphaeria tenuissima, S.

Urocystis Colchici (Schl.) Rabh.

Polygonatum, sp. indet.

Dothidea Asteroma, Fr.

Labrella nitida, S.

Sphaeria Polygonati, S.

Sphaerella Pölygonati, Ckc.

Laestadia Polygonati, Sace.

Periconia pycnospora, Fres.

f Dothidea Polygonati, S.

Phyllachora Poly gonati, Sacc.

Sphaeria subradians, Fr.

Vermicularia Polygonati, S.

Termicularia trichella, Fr.

Smilacina amplexicaulis, Nutt.

Phyllosticta cruenta, (Fr.) Kickx.

Smilacina racemosa, Desf.

$\{$ Aecidium Convallariae, Schum.

\{ Caeoma (Aecidium) convallariatum, Lk.

Diaporthe inquilina, ( $\mathrm{Fr}^{\circ}$ ) Nits.

f Depazea cruenta, Fr.

\{ Phyllosticta cruenta, Kickx.

Phyllosticta vagans, Pk.

Septoria Smilacinae, Ell. \& Martin.

Smilacina stellata, Desf.

Aecidium Convallariae, Schum.

Sphaeria smilacinina, Pk.

(Anthostomella smilacinina, Sacc.

Phyllosticta cruenta, (Fr.) Kickx.

Urocystis Colchici, (Schl.) Rabh.

Smilacina trifolia, Desf.

Peckia Clintonii, $\mathrm{Pk}$.

Smilax Bona-nox, L.

Diplodia smilacina, B.

Slysterium Smilacis, S.

Hypoderma Smilacis, Rehm.

Smilax glauca, Walt.

f Cercospora Smilacis, Auct. Amer.

\{ Cercospora smilacina, Sacc.

Diplodia smilacina, $B$.

Dothidea Dioscoreae, S.

? Phyllachora Dioscoreae, Sacc.

(Phuma Dioscoreae, Cke.

Helminthosporium Petersii, B. \& C.

Uredo Sinilacis, S.

Smilax herbacea, L.

Aecidium Smilacis, S.

Ceratium insociabile, Gerard.

Septoria smilacina, Dur. \& Mont.

Stagonospora Smilicis, (Fll. S Martin)

Sacc.
Vermicularia albomaculata, S.

Smilax hispida, Muhl.

Aecidium Smilacis, S.

Dendryphium subsessile, Ell. \& Ev.

Puccinia Smilacis, S.

Sphaeropsis brunneola, B. \& C.

Sphaeropsis Smilacis, Ell. \& Ev.

Smilax laurifolia, L.

Aecidium Smilacis, S.

Caeoma smilacinatum, Lk.

Uredo Smilacis, Spreng.

Aecidium verrucosum, Bon.

\{ Sphaeria sepelibilis, B. \& C.

Anthostomella sepelibilis, Sacc.

Leptostroma Smilacis, Cke.

CXloma Smilacis, S. Syn. Car.

Rhytisma Smilacis, Fr.

Dichaena smilacina, S. Am. bor.

Nicrothyrium Smilacis, Cke. an De Not?

Sphaerella smilacicola, (S.) Cke.

\{ Sphaeria sepulta, B. \& C.

\{Eutypa sepulta, Sacc.

Smilax Pseudo-China, L.

Coniothyrium Fuckelii, Sacc.

Smilax pumila, Walt.

Puccinia Smilacis, S.

Sinilax rotundifolia, $L$.

Aecidium macrosporum, Pk.

Aecidium Smilacis, S.

Caeoma smilacinatum, Lk.

Uredo Smilacis, Spreng.

(Aecidium verrucosum. Bon.

\{ Ascochyta Smilacis, Ell. \& Martin.

Steganosporium Smilacis, Sacc.

Colletosporium atrum, Lk., var. purpurascens, S.

Corticium incarnatum, $\mathrm{Fr}$.

Coryneum Smilacis, S.

$\{$ Dematium Smilacis, S.

Cladosporium Smilacis, Fr.

Diplodia smilacina, B.

Discosia maculicola, Gerard.

Dothidea abnormis, Howe.

Helminthosporium fragillissimum, B. \& C.

\{Hysterium Smilacis, S.

Hypoderma Smilacis, Rehm.

(Xyloma Smilacis, S. Syn. Car.?

Rhytisma Smilacis, Fr.?

Dichaena smilacina, S. Am. bor?

Microthyrium Smilacis, De Not.

Myiocopron Smilacis, Sacc.

Phlyctaena Smilacis, Cke.

\{ Sphaeropsis brunneola, B. \& C.

Y Phoma biunneola, Sacc.

Sphaeropsis smilacina, Pk.

$\{$ Phoma smilacina, Sacc.

(Macrophoma smilacina, Berl. \& Vogl. 
Uredo Smilacis, S.

Puccinia Smilacis, S.

(. Caeoma Smilacis, Lk.

\{ Sphaeria smilacicola, S.

\{ Diatrype smilacicola, M. A. Curtis.

S Sphaeria (Depazea) smilacicola, S.

Spharella smilacicola, Cke.

Smilax, sp. indet.

Acrospermum foliicolım, $\mathrm{B}$.

$\{$ Sphaeria eliminata, B. \& C.

Anthostomella eliminata, Sacc

Anthostomella Ludoviciana, Ell. \& Langlois.

Aspergillus pulvinatus, B. \& C.

Calosphaeria Smilacis, Karst. \& Hariot.

Capnodium elongatum, B. \& Desm.

Cercospora nubilosa, Ell. \& Ev.

Cercospora Smilacis, Thm.

Sphaeria aliquanti, C. \& E.

$\{$ Clypeosphaera? aliquanta, Sacc.

Heptameria aliquanta, Cke.

Cytospora Smilacis, Cke.

Sphaeria polysticta, B. \& C.

$\{$ Didymosphaeria polysticta, Sacc.

Didymosphaerella polysticta, Cke.

Dimerosporium nimbosum, Ell. \& Martin.

Diplodina ramulorum, E1l. \& Ev.

$\{$ Sphaerella smilacina, Ell. \& Ev.

\{Diplodina Smilacis, Ell. \& Ev.

Dothidea smilacicola, Cke. \& Gerard.

Fusarium marginatum, B. \& C.

$\{$ Graphium pubescens, C. \& E.

Didymobotryunı pubescens, Sacc.

Helminthosporium siliquosum, B. \& C.

Hypoxylon smilacicolum, Howe.

$\{$ Hysterium griseum, S.

\{ Sporomega griseum, Cke.

Hysterium virgultorum, (DC.) Rob. \& Desm.

Mystrosporium aterrimum, B. \& C.

Sphaeria filispora, C. \& E.

$\{$ Ophiobolus filisporus, Sacc.

Raphidospora filispora, C. \& E.

Pestalozzia clavata, C. \& E.

Pestalozzia funerea, Desm.

Pestalozzia Guepini, Desm.

$\int$ Sphaeria disrupta, B. \& C.

$\{$ Playsalospora disrupta, Sacc.

Phomatospora disrupta, Cke.

Ramularia subrufa, Ell. \& Holw.

Sphaeria erumpens, S.

$\{$ Sporidesmium obclavatum, Cke.

\{Clasterosporium obclavatum, Sacc.

Valsa tetraploa, li. \& C.

Tofieldia palustris, Huds.

Metasphaeria borealis, Rostrup.

Microsticta vagans, Desm.

Phoma Tofieldiae, Rostrup

Pleospora herbarum, (P.) Rabh.

Sphaerella Tassiana, De Not.

Trillium erythrocarpum, Michx.

$\{$ Vermicularia concentrica, Pk. \& Clinton.

$\{$ Vermicularia Peckii, Sacc.
Trillium recurvatum, Beck.

Aecidium Trillii, Burrill.

Septoria Trillii, Pk.

Uvularia grandiflora, Smith.

Aecidium Convallariae, Schum.

Phyllosticta Uvulariae, Galloway.

Uvularia perfoliata, L.

Aecidium Convallariae, Schum.

Aecidium Uvulariae, S.

Caeoma alliatum, Lk. p. p.

Uredo Uvulariae, Spreng.

Caeoma uvulariatum, s.

Uvularia, sp. indet.

Erysiphe communis, forma Liliacearum, $F_{1}$ Sphaeria Aegopodii, $P$.

Veratrum Càlifornicum, Durand.

Puccinia Veratri, Duby.

Uredo confluens, var, b., S. Syn. Car.

Veratrum viride, Ait.

Cylindrosporium veratrinum, Sacc. \& Wint.

Dothidea melanoplaca Desm.

Puccinia Veratri, Duby.

Veratrum Woodii, Robbins.

Puccinia atropuncta, Pk. \& Clinton.

Veratrum, sp. indet.

Peziza setigera, Phil.

Xerophyllum setifolium, Michx.

Asterina Xerophylli, Ell.

Hendersonia Xerophylli, Ell.

$\{$ Leptosphaeria hysterioides, Ell. \& Ev.

Heptameria hysterioides, Cke.

(Leptosphaeria Xerophylli, Ell.

$\{$ Metasphaeria Xerophylli, Sacc.

Sphaerulina Xerophylli, Cke.

Pestalozzia capitata, Ell.

Yucca aloifolia, L.

$\{$ Sphaeria nigroannulata, B. \& C.

\{Anthostomella nigroannulata, Sacc.

Cladosporium atriellum, Cke.

S Fusarium Yuccae, Cke.

$\{$ Nectria depauperata, Cke.

Dialonectria depauperata, Cke.

Diplodia circinans, B. \& C.

Diplodia circinans, B. \& Br., var. diffusa. B.

Dothidea Pringlei, $\mathrm{Pk}$.

(Discella anomala, Cke.

$\{$ Discula anomala, Sacc.

Kellermannia yuccaegena, EIl. \& Ev.

Pleospora Thümeniana, Sacc.

Sphaeria Yuccae, Fr.

Yucca angustifolia, Pursh.

Coniothyrium herbarum, C. \& E.

Cylindrosporium angustifolium, Ell. \& Kell.

Sphaeria yuccaegena, Cke.

Didymosphaeria yuccogena, Sacc.

Didymosphaerella yuccogena, Cke.

Kellermannia yuccaegena, Ell. \& Ev. 
Yucca brevifolia, Engelm.

Kellermannia yuccaegena, Ell. S Ev.

Yucca tilamentosa, L.

$\{$ Cercospora concentrica, C. \& E.

$\{$ Cercospora Yuccae, Cke.

(Phoma concentrica, Desm.

\{ Coniothyrium concentricum, Sacc.

f Phoma concentrica, Rav. Fung. Am.

Coniothyrium inops, Sacc.

Coniothyrium herbarum. C. \& E.

Diatrype acerrata, Ell. \& Ev.

Gloeosporinm yuccaegenum, Ell. \& Ev.

Kellermannia yuccaegena, Ell. \& Ev.

Leptospharia filamentosa, Ell. \& Ev.

Splsaeropsis filamentosa, Cke.

Phimma filamentosa, Sacc.

Jacrophoma filamentosa, Berl. \& Vogl.

Nacrosporium abruptum, C. \& E.

Macrosporium commune, Rabh.

Pestalozziella Yuccae, Karst.

Sphaeria argyrostigma, B.

$\{$ Sphaeria argyrostoma, Curt. Cat.

Pliomatosporit argyrostigma, Sacc.

$\{$ Dothidea scapincola, S.

\{Phyllachora scapincola, Sacc.

Sphatia scapincola, S.

Vermicularia subeffigurata, S., var. scapincola, S.

Yucca gloriosa, L.

Sphaeria Yuccae-gloriosae, S.

$\{$ Sphaeria Yuccae, Fr.

Septoria Yuccae, Sacc.

Yucca macrocarpa, Engelm.

(Dothidea Pringlei, Pk.

$\{$ Auerswaldia Pringlei, Sacc.

Phyllachora Pringlei, Cke.

Yucca, sp. indet.

Phoma Yuccae, Cke.

Phyllosticta yuccaegena, Ell. \& Ev.

Sphaeria uvispora, Cke.

Plhysalospora uvispora, Cke.

Sphaeropsis marginata, B. \& C.

Torula maculans, Cke.

Zygadenus elegans, Pursh.

Puccinia $Z$ ygadeni, Trelease.

Zygodenus Fremonti, Torr.

Zygadenus Nuttallii, Gray.

Zygadenus paniculatus, Watson.

Urom y'ces Zygadeni, Pk.

\section{PONTEDERIACEAE.}

Pontederia cordata, $L$.

Sphaerella Pontederiae, Pk.

$\left\{\begin{array}{l}\text { Uromyces Pontederiae, Cke. in Rav. Fung. } \\ \text { Am. } 793 \text { nec Gerard. } \\ \text { Uromyces Pontederia, Speg. }\end{array}\right.$

\section{COMMELINACEAE.}

Tradescantia Vir'ginica, L.

Cylindrosporium Tradescantiae, Ell. \& Kell.

Oidium erysiphoides, Fr.

\section{JUNCACEAE.}

Juncus arcticus, Willd.

Mollisia junciseda, Karst.

Juncus biglumis, L.

Leptosphaeria juncina, (Awd.) Sacc.

Juncus castaneus. Smith.

Mollisia junciseda, Karst.

Juncus effusus, L.

Sphaeria defodiens, Ell.

$\{$ Metasplaneria defodiens, Sacc.

Leptosphaeria defodiens, Ell.

Peziza luteodisca, Pk.

Phyllachora Junci, (Fr.) Fckl.

Puccinia Junci, Desm.

Puccinia Junci, S.

Uromyces Junci, Tul.

Tricliobasis Junci, Auct. Amer.

Ustilago Junci, (S.) B.

Juncus filiformis, $\mathrm{L}$.

Sphaeria striaeformis, var. Junci, A. \& S.

Sphaeria Junci, Fr.

$\{$ Dothidea Junci, Fr.

Phyllachora Junci, Fckl.

Juncus Greenii, Oakes \& Tuckerm.

\{ Sphaeria Michotii, Westd.

$\{$ Leptosphaeria Michotii, Sacc.

Juncus Lescurii, Bolander.

Hendersonia culmicola, Sacc.

Hendersonia scirpicola, Cke. \& Hark.

Leptothyrium juncinum, Cke. \& Hark.

Trullula Junci, Cke. \& Hark.

Juncus longistylis, Torr.

Uromyces Junci, (Desm.) Tul.

Juncus maritimus, L.

\{ Hendersonia trimera, Cke.

$\{$ Stagonospora trimera, Sacc.

Juncus Mertensianus, Bong.

Uromyces Junci, (Desm.) Tul.

Juncus temuis, willd.

(Caeoma (Uredo) Junci, S.

$\{$ Ustilago Junci, B.

Cintractia Junci, Trelease.

$\{$ Peziza stictoidea, C. \& E.

\{Mollisia stictoides, Sacc.

Uromyces Junci, (Desm.) Tul.

Juncus trifidus, L.

Mollisia junciseda, Karst.

Septoria Junci, Desm.

Troclsila juncicola, Rostrup.

Juncus triglumis, L.

Trochila juncicola, Ros,trup. 
Juncus, sp. indet.

Cimptoum cuspidatum, Cke. \& llark.

$\{$ Spháeria juucina, B. \& Rav.

\{Didymella juncina, Sacc.

$\{$ Discella tenuispora, Cke. \& Hark.

$\{$ Discula tenuispora, Sacc.

Fusarium pallens, B. \& C.

$\{$ Fusarium Curtisii, Cke.

Fusarium glumarum, Sacc.

Pleospora juncigena, Cke.

$\{$ Propolis sphaerelloides, Ell.

$\{$ Diplonaevia sphaerelloides, Sacc.

PSphaeria Graminis, P.

\{ Darluca Filum, (Biv.) Cast.

Sphaeronema consors, B. \& C.

Luzula arctica, Bl.

Pleospora Elynae, (Rabh.) Ces. \& De Not.

Trochila juncicola, Rostrup.

Luzula arcuata, Meyer.

$\{$ Hendersonia Luzulae, Westd.

\{ Stagonospora Luzulae, Sacc.

Leptosphaeria culmorum, Awd.

Trochila juncicola, Rostrup.

Luzula campestris, DC.

\{Hendersonia Luzulae, Westd.

\{ Stagonospora Luzulae, Sacc.

Leptosphaeria culmorum, Awd.

Puccinia obscura, Schrt.

Puccinia obscura, Schrt., var. vernalis, $\mathrm{Pk}$.

Sphaerella Tassiana, De Not.

Trochila juncicola, Rostrup.

Luzula confusa, Lindeb.

Pleospora Elynae, (Rabh.) Ces. \& De Not.

Sphaerella Luzulae, Cke.

Sphaerella Tassiana, De Not.

Trochila juncicola, Rostrup.

Luzula parviflora, Desv.

Mollisia luzulina, Karst.

Luzula spicata, Desv.

Leptosphaeria culmorum, Awd.

Leptostroma Luzulae, Lib.

Phoma Luzulae, Rostrup.

Pyrenophora comata, (Niessi) Sacc.

Septoria minuta, schrt.

Sphaerella Tassiana, De Not.

Stagonospora Luzulae, (IVestd.) Sacc.

Trochila juncicola, Rostrup.

PALMEAE.

Arenga saccharifera, Mart.

Chamaerops humilis, L.

Graphiola Phoenicis, Poit.

Cocos plumosa, Hook.

Septoria cocoina, Ell. \& Ev.
Phoenix dactylifera, L.

Graphiola Phoenicis, Poit.

Pritchardia, sp. indet.

Phoma Pritchardiae, Cke. \& Hark.

Sabal Adansonii, Guerns.

Sphaeropsis Sabal, Cke.

Sabal Palmetto, Roem. \& Schult.

Amerosporium sabalinum, Ell. $\&$. Ev.

Anthostomella melanosticta, E1l. \& Ev.

Asterina inquimans, EH. and Ev.

Chaetophoma Sabal, Cke.

Cladosporium Palmetto, Gerard.

Graphiola congesta, B. \& Rav.

Cyphella subcyanea, Ell. \& Ev.

Dermatea Sabalidis, Ell. \& Martin.

\{ Diatrypella deusta, Ell. \& Martin.

\{ Diatrype deusta, Cke.

Helminthosporium Palmetto, Gerard.

Helminthosporium spiculiferum, Ell. \& Ev.

Leptostroma micropunctum, Cke.

Linospora Palmetto, EH. \& Ev.

Ophiobolus versisporns, Ell. \& Martin.

Phyllosticta Palmetto, Ell. \& Ev.

$\{$ Rhytisma sparsum, Pk. \& Clinton.

\{ Cocconia sparsa, Sacc.

Sphaerella sabaligena, Ell. \& Ev.

Sphaeria sabalicola, Ell. \& Martin.

Venturia sabalicola, Ell. \& Ev.

Sabal serrulata, Roem. \& Schult.

Anthostomella minor, Ell. \& Ev.

Sphaeria (Anthostomella) leucobasis, Ell. \& Martin.

$\{$ Sphaeria sabalensioides, Ell. \& Martin.

Anthostomella sabalensioides, Sacc.

$\{$ Dermatea Sabalidis, Ell. \& Martin.

$\{$ Cenangium Sabalidis, Sacc.

$\{$ Diatrypella deusta, Ell. \& Martin.

Diatrype deusta, Cke.

$\{$ Didymosphaeria serruluta, Ell. \& Martin.

\{ Didymosphaerella serrulata, Cke.

Sphaeria Sabalensis, Cke.

$\{$ Dilophia Sabalensis, Sacc.

Metasphaeria Sabalensis, Cke.

Hypoxylon perforatum, (S.) Fr.

Hysterium lineolatum, Cke.

Sphaeria sabalicola, Ell. \& Mart.

$\{$ Leptosphaeria sabalicola, Sacc.

Heptameria sabalicola, Cke.

Melanconium Sabal, Cke.

$\{$ Meliola furcata, Ell., N. A. F. 1297 e.

Meliola palmicola, Wint.

| Ophiobolus versisporus, Ell. \& Martin.

\{Raphidospora versispora, Cke.

Sphaerella incisa, Ell. \& Martin.

Sphaerella serrulata, Ell. \& Ev. 
Sabal, sp. indet.

Sphaeria sabaligera, B. \& C.

Clypeosphaeria sabaligera, Sacc.

Leptosphacria sabaligera, Cke.

Coniosporium Arundinis, (Cda.) Sacc.

Melanconium Palmarum, Cke.

S Melola amphitricha, Rav. Fung. Am. SI.

\{Meliola furcata, Lev. sec. Speg.

Melogramma fuliginosum, Ell. p.p.

Sphaeria Palmetta, Cke.

\{ Metasphaeria Palmetta, Sacc.

Ravenelula Gainesvillensis, Speg.

Rhabdospora Sabalensis, Cke.

Splnaeria Palmarum, Duby.

Talsa sabalina, Cke.

Palmeae, sp. indet.

Sphaeria palmacea, Cke.

Authostomella palmacea, Sacc.

Didymosphaerella palmacea, Cke.

\{ Sphaeropsis maculata, Cke. \& Hark.

\{ Phoma maculata, Sacc.

\section{PANDANACEAE.}

Pandanus, sp. indet.

Physalnspora Pandani, Ell. \& Ev.

\section{TYPHACEAE.}

Sparganium eurycarpum, Engelm.

Uromyces Sparganii, C. \& P.

Typha angustifolia, L.

Cladosporium Typharum, Desm.

Melanconium Typhae, Pk.

Typha latifolia, L.

Cladusporium fasciculatum, Cda.

Cladosporium herbarum, (P.) Lk.

Cladosporium Typhae, S.

Cladosporium Typharum, Desm.

Dictyosporium opacum. Cke. \& Hark.

\{ Didymosphaeria Typhae, Pk.

\{Didymosphaerella Typhae, Cke.

Leptosphaeria luctuosa, Niessl.

Leptosphaeria Michotii, (Westd.) Sacc.

Leptosphaeria Typhae, Karst.

Leptosphaeria Typharum, Karst.

Peziza Typhae, Pk.

Phyllactinia suffulta, (Reb.) Sacc.

Pleospora Typhae, Pass.

Sphaerella Typhae, (Lasch) Awd.

Sphaeria Typhae, S.

Sphaeropsis typhina, Pk.

Tурha, sp. indet.

Chaetomium Typhae, S.

Sphaeria intercellularis, B. \& C.

Sphaerella intercellularis, Cke.

Didymella intercellularis, Sacc.
$\{$ Hysterium typhinum, Fr.

\{Lophodermium typhinum, Lamb.

SDarluca angusta, Cke.

Septoria angusta, Sacc.

\section{ARACE $A$ E}

Acorus Calamus, L.

Uromyces piriformis, Cke.

Arisaema Dracontium, Schott.

SAecidium Caladii, S.

$\{$ Caeoma (Aecidium) dracontiatum, S.

Uromyces Caladii, Farl.

A risaema triphyllum, Torr.

Caeoma (Aecidium) aroidatum, Lk.

Caeoma (Uredo) Ari-Virginici, S.

Caeoma (Aecidium) dracontiatum, S.

Puccinia Ari-triphylli, S.

Aecidium Ari, Auct. Amer.

Uromyces Peltandrae, Howe.

Uromyces Arisaemae, Cke.

Uromyces Ari, Farl.

Uromyces Caladii, Fart.

Call palustris, L.

Cercospora Callae, Pk. \& Clinton.

Colocasia esculenta, Schott.

Macrophoma subconica, Ell. \& Ev.

Orontium aquaticum, L.

\{ Polyactis streptothrix, C. \& E.

Botrytis streptothrix, Sacc.

Epicoccum Duriaeanum, Mont.

$\{$ Physalospora Orontii, Ell. \& Ev.

L Laestadia Orontii, Berl. \& Vogl.

Phyllosticta Orontii, Ell. \& Martin.

Ramularia Orontii, Ell. \& Martin.

Sphaerella Orontii, Ell. \& Ev.

Stilbum aciculosum, Ell. \& Ev.

Volutella diaphana, Ell.

Peltandra undulata, Raf.

Cercospora Callae, Pk. \& Clinton.

Gloeosporium paludosum, Ell. \& Gall.

\{ Sclerotium Caladii, S.

\{ Perisporium Caladii, S.

Pestalozzia aquatica, Ell. \& Ev.

Aecidium Caladii, S.

Ureclo Caladii, S.

Caeoma Caladii, Lk.

Caeona (Aecidium) aroidatum, Lk.

Uredo Calaidii, Spreng.

Caeoma (Uredo) Ari-Virginici, S.

Cáema (Aecidium) dracontiatum, S.

Puccinia A ri-triphylli, S.

Aecidium Ari, Auct. Amer.

Uromyces Peltandrae, Howe.

Uromyces Ari-Virginici, Howe.

Uromyces Pontederiae, Gerard.

Uromyces Arisaemae, Cke.

Uromyces Ari, Farl.

Uromyces Caladii, Farl.

Richardia Africana, Kunth.

Cercospora Callae, Pk. \& Clinton. 
Sphaeropsis Averyana, Gerard.

Symplocarpus foetidus, Salisb.

Cercospora Symplocarpi, Pk.

Septoria spiculosa, Ell. \& Holw.

\section{ALISMIACEAE.}

\section{Alisma Plantago, L.}

$\{$ Ascochyta Alismatis, (Oud.) Trail.

Ascochyta Alismatis, Ell. and Ev.

Cercospora Alismatis, Ell. \& Holw.

Doassansia Alismatis, (Nees) Cornu.

Septoria Alismatis, Oud.

Echinodorus rostratus, Engelm.

Doassansia Alismatis, (Nees) Cornu.?

Sagittaria variabilis, Engelm.

Cercospora Sagittariae, Ell. \& Kell.

CUredo Sagittariae, Westd.

Protomyces Sagittariae, Fckl.

Doassansia Sagittariae, Fisch.

Doassansia Alismatis, Auct. Amer. p.p.

\section{NAIADACEAE.}

Potamogeton fluitans, Roth.

Ramularia aquatilis, $\mathrm{Pk}$.

Potamogeton natans, L.

Doassansia occulta, (H. Hoffm.) Cornu.

Potamogeton perfoliatus, L., var. lanceolatus, Robbins.

Doassansia occulta, (H. Hoffim.) Cornu.

Potamogeton pusillus, L.

Doassansia occulta, (H. Hoffm.) Cornu.

Potamogeton Vaseyi, Robbins.

Sclerotium occultum, H. Hofim.

Doassansia Farlowii, Cornu.

Doassansia occulta, Cornu.

Triglochin palustre, L.

Asteroma Juncaginearum, Rabli.

\section{CYPERACEAE.}

\section{Carex adusta, Boott.}

Puccinia Caricis, (Scnum.) Reb.

Thecaphora aterrima. Tul.

Carex alpina, Swartz.

-Sphaerella pusilla, Awd.

Trochila diminuens, Karst.

Carex alpina, Swartz, var. holostoma, Bailey.

Sphaerella Tassiana, De Not.

Carex aquatilis, Wahl.

Pleospora pentamera, Karst.

Sphaerella Tassiana, De Not.

Sphaerella Wichuriana, Schrt.

Trochila fuscella. Karst.
Carex atrata, L.

Trochila diminuens, Karst.

Carex bicolor, All.

Sphaerella Tassiana, De Not.

Carex caespitosa, L.

Helotium Scutula, (P.) Karst., var, suspectum, (Nyl.) Karst.

Trochila diminuens, Karst.

Carex canescens, L.

Leptostroma caricinum, $\mathrm{Fr}^{\text {r }}$.

Trochila diminuens, Karst.

Ustilago urceolorum, Tul.

Carex canescens, L., var, alpicola, Wahl.

Trochila diminuens, Karst.

Carex capillaris, L.

Septoria punctoidea, Karst.

Carex capitata, L.

Trochila diminuens, Karst.

Carex cephaloidea, Dewey.

? Septoria caricinella, Sacc. \& Roum.

Carex crinita, Lam.

Goninsporium puccinioides, (DC.) Lk.

$\{$ Peziza crinella, Ell. \& Ev.

\{ Dasyscypha crinella, Sacc.

$\{$ Sphaeria Caricis, Fr.

\{ Phyllachora Caricis, Sacc.

$\{$ Puccinia Caricis, (Schum.) Reb.

\{ Puccinia Striola, L.k.

Ustilago urceolorum, Tul.

Carex Douglasii, Boott.

Puccinia Caricis, (Schum.) Reb.

Ustilago Caricis, (P.) Fckl.

Carex echinata, Murray, var. cephalantha, Bailey.

f Peziza multipuncta, $\mathrm{Pk}$.

\{Pyrenopeziza multipuncta, Sacc.

Ustilago urceolorum, Tul.

Carex festiva, Dewey.

Trochila diminuens, Karst.

Carex filifolia, Nutt.

Puccinia Caricis (Schum.) Reb.

Ustilago Caricis, (P.) Fckl.

Carex filiformis, L.

Puccinia caricina, DC.

Carex folliculata, L.

Leptosphaeria folliculata, Ell. \& Ev.

\{ Sphaeria Carectorum, B. \& C.

$\{$ Sphaerella Carectorum, Cke.

Metasphaeria Carectorum, Sacc.

$\{$ Sphaeria perigynicola, S.

$\{$ Sphaerella perigynicola, Cke.

Carex fusca, All.

$\{$ Caeoma Caricis, Lk.

\{ Ustilago Caricis, (P.) Fckl.

Carex glareosa, Wahl.

$\{$ Arthrinium curvatum, Kze.

$\{$ Camptoum curvatum, Lk. 
Cladosporium caricicolum, Cda.

Plcospora pentamera, Karst.

Ustilago Caricis, (P.) Fckl.

Carex glareosa, Wahl., var. caespltosa, Boeckeler.

Sphaerclla pusilla, Awd.

Carex intumescens, Rudge.

Puccinia Caricis, (Schum.) Reb.

Uromyces perigynius, Halsted.

Carex lagopina, Wahl.

Sphaerella Tassiana, De Not.

Trochila diminuens, Karst.

Carex livida, Willd.

Ustilago Caricis, (P.) Fckl.

Carex Magellanica, Lam.

Puccinia Striola, Lk.

Ustilago urceolorum, Tul.

Carex marcida, Boott.

Puccinia Caricis, (Schum.) Reb.

Carex microglochin, Wahl.

Leptosphaeria gigaspora, Niessl.

Trochila ignobilis, Karst.

Carex Nebraskensis, Dav.

Puccinia Caricis, (Schum.) Reb.

Carex misandra, R. Br.

Lophodermium caricinum, (Rob.) Duby.

Septoria punctoidea, Karst.

Sphaerella Tassiana, De Not.

Sphaerella Wichuriana, Schrt.

Carex nardina, Fr.

Pleospora discors, (Dur. \& Mont.) Ces. \& De Not.

Pleospora Elynae, (Rabh) Ces. \& De Not.

Septoria punctoidea, Karst.

Sphaerella Wichuriana, Schrt.

Ustilago Caricis, (P.) Fckl.

Carex obesa, All., var, minor, Boott.

Phoma Caricis, (Fr.) Sacc.

Pleospora Elynae, (Rabh.) Ces. \& De Not.

Pleospora heterospora, De Not.

Sphaerella pusilla, Awd.

Carex oligosperma, Michx.

Ustilago urceolorum, 'Tul.

Carex pedata, Wahl.

Septoria punctoidea, Karst.

Sphaerella saxatilis, Schrt.

Carex Pennsylvanica, Lam.

Phyllosticta Caricis, (Fckl.) Sacc.

Sphaeria Caricis, Fr.

Dothidea Caricis, Fr.

Phyllachora Caricis, Sacc.

Puccinia Caricis, (Schum.) Reb.

Geminella foliicola, Schrt.

Urocystis pusilla, C. \& P.

Geminella melanogramma, Magnus.

Schizonella melanogramma, (DC.) Schrt.
Sorosporium atrum, $\mathrm{Pk}$.

Uredo Caricis, P.

Caeoma Caricis, Lk.

Ustilago urceolorum, Tul.

Ustilago Caricis, Fckl.

Carex pilulifera, L.

Trochila ignobilis, Karst.

Carex pratensis, Drejer.

Sphaerella Wichuriana, Schrt.

Carex rariflora, Smith.

Cladosporium Graminum, Cda.

Leptostroma caricinum, Fr.

Sphaerella Wichuriana, Schrt.

Trochila ignobilis, Karst.

Carex rigida, Gooden.

Entyloma caricinum, Rostrup.

Leptostroma caricinum, Fr.

Metasphaeria macrotheca, Rostrup.

Sphaerella pusilla, Awd.

Sphaerella Wichuriana, Schrt.

Trochila ignobilis, Karst.

Ustilago Caricis, (P.) Fckl.

Carex riparia, W. Curtis.

$\{$ Clasterosporrum caricinum, S.

$\{$ Sporidesmium Closterisporium, Cda.

Carex rostrata, With.

Trochila ignobilis, Karst.

Carex rotundata, Wahl.

Sphaerella Wichuriana, Schrt.

Trochila diminuens, Karst.

Carex rufina, Drejer.

Trochila ignobilis, Karst.

Carex rupestris, All.

Cladosporium Graminum, Cda.

Pleospora pentamera, Karst.

Septoria punctoidea, Karst.

Sphaerella Tassiana, De Not.

Sphaerella Wichuriana, Schrt.

Trochila diminuens, Karst.

Ustilago Caricis, (P.) Fckl.

Carex salina, Wahl.

Lophodermium caricinum, (Rob.) Duby.

Trochila ignobilis, Karst.

Carex saxatilis, L.

Leptosphaeria epicarecta, (Cke.) Sacc.

Lophodermium caricinum, (Rob.) Duby.

Mollisia cymbispora, Rostrup.

Carex scirpoidea, Michx.

Cladosporium Graminum, Cda.

Pleospora Elynae, (Rabh.) Ces. \& De Not.

Sphaerella pusilla, Awd.

Trochila fuscella, Karst.

Ustilago Caricis, (P.) Fckl. 
Caréx scoparia, Schkuhr.

CPeziza cervinula, Cke.

Peziza multipuncta, Pk.

Pyrenopeziza multipuncta, Sacc.

Pyrenopeziza cervinula, Sacc.

Carex siccata, Dewey.

Carex stenophylla, Wahl.

Ustilago urceolorum, Tul.

$\{$ Ustilago Caricis, (P.) Fckl.

Carex straminea, Willd.

Puccinia Caricis, (Schum.) Reb.

Carex stricta, Lam.

Macrosporium transversum, Pk.

(Puccinia Caricis, (Schum.) Reb.

Puccinia caricina, DC.

Uredo caricina, DC.

Puccinia Striola, Lk.

(Puccinia Punctum, Lk.

$\{$ Uromyces Caricis, Pk.

$\{$ Puccinia Caricis-strictae, Dietel.

Ustilago urceolorum, Tul.

Carex stricta, Lam., var. angustata, Bailey.

Ascochyta teretiuscula, Sacc. \& Roum.

Phleospora Caricis, Ell. \& Ev.

Carex stylosa, C. A. Meyer.

Lophodermium caricinum, (Rob.) Duby.

Trochila ignobilis, Karst.

Carex trichocarpa, Muh1.

Puccinia caricina, DC.

Carex trichocarpa, Muhl., var. Deweyl, Bailey.

Ustilago subinclusa, Körn.

Carex umbellata, Schkuhr.

Ustilago urceolorum, Tul.

Carex utriculata, Boott.

$\{$ Helotium caricinellım, Pk.

\{ Niptera caricinella, Sacc.

Puccinia Caricis, (Schum.) Reb.

Ustilago urceolorum, Tul.

Carex varia, Muhl.

Septoria lineolata, Sacc. \& Speg.

$\{$ Sphaeria recutita, Fr.

Sphaerella recutita, Cke.

$\{$ Uredo Caricis, $P$.

Ustilago Caricis, Fckl.

Carex vulgaris, Fr.

Trochila fuscella, Karst.

Trochila ignobilis, Karst.

Ustilago Caricis, (P.) Fck].

Carex vulgaris, Fr., var. hyperborea,

Boott.

Leptosphaeria caricinella, Karst.

Lophodermium caricinum, (Rob.) Duby.

Metasphaeria macrotheca, Rostrup.

Mollisia cinerea, (Batsch) Karst.

Phoma Caricis, (Fr.) Sacc.

Septoria punctoidea, Karst.

Sphaerella Tassiana, De Not.
Sphaerella Wichuriana, Schrt.

Trochila fuscella, Karst.

Trochila ignobilis, Karst.

Ustilago Caricis, (P.) Fckl.

Carex, sp. indet.

Actidium caricinum, S.

Actinothyrium Graminis, Kze.

Amerosporium Caricum, (Lib.) Sacc.

Arthrinium caricicolum, Kze.

Chaetomium elatum, Kze.

Cladosporium nodulosum, Cda.

$\{$ Cryptosporium Caricis, Cda.

\{ Cryptomella Caricis, Sacc.

Cryptosporium nubilosum, Ell. \& Ev.

Cyphella caricina, $\mathrm{Pk}$.

Dinemasporium Graminum, Lev.

Epichloe typhina, (P.) Tul.

Epicoccum caricicolum, S.

\{ Sphaeria Michotii, Westd.

Leptosphaeria Michotii, Sacc.

Leptostroma caricinum, Fr.

Leptostroma inundatorum, S.

$\{$ Macrosporium caricinum, Fr.

$\{$ Cometella caricina, Fr.

$\{$ Sporocybe nigriceps, Pk.

$\left\{\begin{array}{l}\text { Periconia nigriceps, Sacc. } \\ \text { Pericenta }\end{array}\right.$

Periconia opaca, Cke.

Peziza melatephra, Lasch.

Peziza Yogoensis, Ell. \& Gall.

Septoria carnea, Ell. \& Ev.

Sphaeria Scirporum, S.

Cladium, sp. indet.

Meliola amphitricha, Fr.

Cyperus Baldwinii, Torr.

Sphaeria canaliculata, S.

$\{$ Dothidea canaliculata, B.

Phyllachora canaliculata, Sacc.

Cyperus Grayii, Torr.

$\{$ Ustilago axicola, B., var.

\{ Cintractia axicola, Cornu.

Cyperus ovularis, Torr., var. cylindricus, Torr.

Cyperus Schweinitzii, Torr.

(Puccinia Caricis, (Schum.) Reb.

$\{$ Puccinia caricina, DC.

Puccinia Striola, Lk.

Cyperus strigosus, L.

Puccinia angustata, $\mathrm{Pk}$.

Dulichium spathaceum, P.

\{ Puccinia Caricis, (Schum.) Reb.

$\{$ Puccinia Striola, Lk.

Eleocharis intermedia, Schult.

Puccinia Eleocharidis, Arth.

Eleocharis palustris, R. Br.

Epicoccum purpurascens, Ehrb.

Fusarium roseum, Lk.

Puccinia Eleocharidis, Arth. 
Eriophorum ang'ustifolinm, Roth.

Mollisia cymbispora, Rostrup.

Mollisia junciseda, Karst.

Sphaerella perexigua, Karst.

Sphacrella Wichuriana, Schrt.

Trochila ignobilis, Karst.

Eriophorum cyperinum, L.

Cheiromyces stellatus, B. \& C.

Puccinia angustata, Pk.

(Puccinia Caricis, (Schum.) Reb.

Puccinia Striola, Lk.

Puccinia Punctum: Lk.

Eriophorum polystachyon, L.

Puccinia angustata, Pk.

Eriophorum Scheuchzeri, Hoppe.

Mollisia crmbispora, Rostrup.

Eriophorum Virginicum, L.

$\mathrm{Puccinia}$ angustata, $\mathrm{Pk}$.

Fimbristylis autumpalis, Roem. \& Schult.

\{Hendersonia trimera, Cke.

Stagonospora trimera, Sacc.

\{ Ustilago axicola, B.?

\{Ustilago Fimbristylis, Thm.

Fuirena squarrosa, Michx., var.

Puccinia Fuirenae, Cke.

Kobresia caricina, Willd.

Sphaerella pusilla, Awd.

Trochila ignobilis, Karst.

Ustilago Caricis, (P.) Fckl.

Rhynchospora alba, Vahl.

f Ustilago Montagnei, Tul.

\{Ustilago Caricis, (P.) Fck\}.

Rhynchospora glomerata, Vahl.

(Ustilago Nontagnei, Tul., var. minor, Desm.

Ustilago Caricis, (P.) Fckl.

Rhynchospora macrostachya, Torr.

Amerosporium macrochaeta, Ell. \& Ev.

$\{$ Testicularia Cyperi, Klotzsch.

\{ Milleria herbatica, $\mathrm{Pk}$.

Rhynchospora miliacea, Gray.

IIeliola amphitricha, Fr.

Scirpus atrovirens, Muhl.

Puccinia angustata, $\mathrm{Pk}$.

Sphaeria scirpicola, DC.

Uromyces Scirpi, Burrill.

Scirpus caespitosus, L.

$\{$ Peziza scirpina, $\mathrm{Pk}$.

\{Mollisia scirpina. Sacc.

$\{$ Puccinia Scirpi, DC.

\{ Uredo scirpina, Westd.

Sphaerella Scirpi-lacustris, Awd.

Ustilago Caricis, (P.) Fckl.

Scirpus capillaris, L.

S Sphaeria gangraena, Fr.

Dothidea gangraena, Fr.

(Phyllachora gangraena. Fck!.
Scirpus fluviatilis Gray.

$\{$ Cryptosporium Scirpi, Pk.

$\{$ Septoria Peckii, Sacc.

Pestalozzia primaria, E11. \& Ev。

Uromyces Scirpi, Burrill.

Scirpus lacustris, L.

Camptoum curvatum, (Kze.) Lk.

$\{$ Hypoderma scirpinum, DC.

\{Hysterium scirpinum, Fr.

Naucoria scirpicoia, $\mathrm{Pk}$.

$\{$ Peziza luteodisca, Pl.

\{ Dasyscypha luteodisca, Sacc.

$\{$ Sphaeria herbarum, P.

Pleospora herbarum, Rabh.

Puccinia obtecta, $\mathrm{Pk}$.

Spilocaea Scirpi, Lk.

Uromyces Scirpi, Burrill.

Scirpus maritimus, L.

Epicoccum sphaerospermum, B. \& C.

Macrosporium Scirpi, Lasch.

Pestalozzia scirpina, Ell. \& Martin.

Septoria narvisiana, Sacc.

Scirpus Olneyi, Gray.

Pestalozzia scirpina, Ell. \& Martin.

Scirpus pungens, Vahl.

Gibbera Saubinetii, Mont.

Puccinia obtecta, Pk.

Scirpus sylvaticus, L.

Puccinia angustata, Pk.

Scirpus triquetrus, L.

Sphaeria Scirporım, S.

Uromyces Junci, var. Scirpi, Vize.

Scirpus, sp. indet.

Anthostoma mortuosum, (E11.) Sacc.

Cacoma (Uredo) rimosum, $\mathrm{Lk}$.

Camptoum cuspidatum, Cke. \& Hark.

?Dothidea Graminis, (P.) Fr.

Hendersonia scirpicola, Cke. \& Hark.

Peziza apala, B. \& Br.

Sporocybe nigriceps, $\mathrm{Pk}$.

Tetraploa scabra, Hark.

GRAMINEAE .

Agropyrum divergens, Nees.

Claviceps purpurea, (Fr.) Tul.

Puccinia Rubigo-vera, (DC.) Wint.

Agropyrum glaucum, Roem. \& Schult.

Claviceps purpurea, (Fr.) Tul.

Erysiphe Graminis, DC.

l’hyllachora Graminis, (P.) Fckl.

Puccinia coronata, Cda.

Puccinia Graminis, P.

Agropyrum repens, L.

Claviceps purpurea, ( $\mathrm{Fr}$.) Tul.

Oidium monilioides, Lk.

Puccinia Graminis, P.

Puccinia Rubigo-vera, (DC.) Wint. 
Pyrenophora relicina, Fckl.

Tilletia striaeformis, (Westd.) Wint.

(Urocystis Agropyli, (Preuss) Schrt.

$\{$ Urocystis occulta, (Wallr.) Rabh., var.

Tritici, Farl.

Agropyrum violaceum, Lange.

Claviceps purpurea, (Fr.) Till.

Hendersonia Agropyri, Rostrup.

Hendersonia crastophila, Sacc.

Phoma graminis, Westd.

Pleospora herbarum, (P.) Rabla.

Pleospora pentamera, Karst.

Puccinia graminis, P.

Sphaerella Tassiana, De Not.

Agrostis alba, L., var. vulgaris, Thurb.

Puccinia graminis, $\mathrm{P}$.

Tilletia striaeformis, (Westd.) Wint.

Agrostis canina, L.

Laestadia graminicola, Rostrup.

Lophodermium arundinaceum, (Schrad.) Chev.

Splraerella Tassiana, De Not.

Agrostis exarata, Trin.

Erysiphe graminis, DC.

Agrostis filiformis, Vill.

$\{$ Sphaeria Agrostidis, S.

$\{$ Phyllachora graminis, (P.) Fckl.

Agrostis scabra, Willd.

Claviceps (sclerotium), Sp.

Puccinia graminis, $\mathrm{P}$.

Agrostis stricta, Willd.

Sphaerella Tassiana, De Not.

Aira alpina, L.

$\{$ Leptopeziza Groenlandica, Rostrup.

\{ Durella Groenlandica, Sacc.

Mollisia cinerea, (Batsch) Karst.

$\{$ Mollisia graminis, Karst.

\{ Pyrenopeziza Karstenii, Sacc.

Phoma graminis, Westd,

Pleospora Elynae, (Rabh.) Ces. \& De Not.

Pleospora herbarum, (P.) Rabh.

Sphaerella ignobilis, Awd.

Sphaerella Tassiana: De Not.

Alopecurus alpinus, L.

Homostegia gangraena, (Fr.) Wint.

Leptosphaeria culmorum, Awd.

Sphaerella lineolata, (Rob.) De Not.

Sphaerella pusilla, Awd.

Sphaerella Wichuriana, Schrt.

Alopecurus geniculatus, L.

Fusicladium Alopecuri, E11. \& Ev.

Alopecurus geniculatus, L., var. aristulatus, Munro.

Scolecotrichum graminis, Fckl.

$\{$ Uromyces Dactylidis, Ottl.

U Uromyces Alopecuri, Seymour.
Ammophila arundinacea, Host.

CHysterium gramineum, M. \& N.

Hysterium culmigenum, var. gramineum, Fr.

Lophodermium arundinaceum, (Schrad.)

( Chev. var. gramineum, Duby.

$\{$ Pleospora infectoria, Fckl.

Sphaeria infectoria, $\mathrm{Pk}$.

Tilletia striaeformis, (Westd.) Wint.

Andropogon argyraeus, Schult.

Ustilago Brunkii, Ell. \& Gall.

Andropogon furcatus, Muhl.

Asteroma graminis, Westd. ?

Epichloe typhina, (P.) Tul.

$\{$ Peziza distincta, $\mathrm{Pk}$.

Trichopeziza distincta, Sacc.

(Caeoma (Uredo) Andropogi, S.

$\{$ Puccinia Andropogi, S.

Puccinia Ellisiana, Thm.

Sphaeria graminis, $\mathrm{P}$.

Sphaeria Andropogi, S.

Sphaeria andropogicola, S.

Dothidea graminis, Fr.

Sphaeria luteomaculata, S.

(Phyllachora graminis, Fckl.

Puccinia Phragmitis, (Schum.) Körn.

Sorosporium Ellisii, Wint., var, provincialis, Ell. \& Gall.

(Sorosporium Ellisii, Wint., var. occidenta$\{$ lis, Seymour.

Ustilago Andropogonis, Kell. \& Swingle.

Andropog on Hallii, Hackel.

Puccinia Andropogi, S.

Andropogon macrourus, Michx.

$\{$ Peziza dinemasporioides, E11. \& Ev.

\{ Gorgoniceps dinemasporioides, Sacc.

Andropogon muricatus, Retz.

Chaetostroma aterrimum, (Cke.) Sacc.

Didymella Andropogonis, Ell. \& Ev.

Didymosphaeria Andropogonis, Ell. \& Langlois.

Haplosporella tingens, Ell. \& Langlois.

Langloisula spinosa, Ell. \& Ev.

Ophiobolus Medusae, Ell. \& Ev., var. minor, Ell. \& Ev.

Andropogon scoparius, Michx.

Ellisiella caudata, Sacc.

$\{$ Peziza planodisca, Pk. \& Clinton,

$\{$ Pezizella planodisca, Sacc.

Phoma culmicola, S.

Phyllachora graminis, (P.) Fckl.

$\{$ Puccinia Andropogi, S.

(Puccinia Ellisiana, Thm.

Puccinia Baryi, (B. \& Br.) Wint.

Puccinia Phragmitis, (Schım.) Körn.

Sphaeria nervisequia, $\mathrm{S}$. 
Andropogon Virginicus, L.

Spliaeria mortuosa, Ell.

Anthostoma mortuosum, Sacc.

Xylosplaneria mortuosa, cke.

Aulogruplum culmigenum, Ell.

f Fusisporimm Andropogonis, Ckc.

$\{$ Fusalium Andropogonis, Cke.

Puccinia Ellisiana, Thm.

Caeoma Syntherismae, S. Am. bor.

L'redo Syntherismac, Rav. Fung. Car.

Ustilago Syutherismae, Pk.

Ustiligo Cesatii Fisch. Wald.

Sorosporium Ellisii, WVint.

Sorosporium Everbartii, Ell. \& Gall.

Andropogon, sp. indet.

Spphaeria phaeosticta, B.

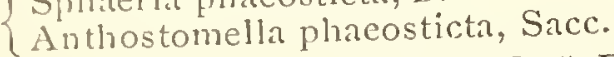

Chatomella Andropogonis, C. \& E.

Chaetomium olivaceum, C. \& E.

S Gymnosporium Arundinis, Cda.

$\{$ Coniosporium Arundinis, Sacc.

Corticium graminicolum, Ell. \& Ev.

Dinemasporium minimum, C. \& E.

Gonytrichum fulvum, Ell.

Helotium rhizogenum, Ell. \& Er.

\{ Hypocrea atramentosa, B. \& C.

Hy pocrella atramentosa, Sacc.

Sphaeria culmicola, Fr.

$\{$ Leptosphaeria culmicola, Karst.

(Splsaeria latebrosa, Ellis.

$\{$ Leptosphaeria latebrosa, Sacc.

Heptameria latebrosa, Cke.

? Lophiostoma implexum, Ell. \& Ev.

Patellaria aureococcinea, B. \& C.

S Patellaria clavata, Ell.

Lecanidion clavatum, Sacc.

f Patellaria tuberculosa, Ell.

$\{$ Lecanidion tuberculosum, Sacc.

Peziza aberrans, Pk.

Peziza Andropogonis, B. \& C.

Mollisia Andropogonis, Rehm.

Belonium Andropogonis, Sacc.

Belcnium aberrans, Sacc.

Peziza albotestacea, Desm.

\{ Peziza atriella, Cke.

Mollisia atriella, Sacc.

(Peziza Atropae, A. \& S.

$\{$ Peziza relicinat, $\mathrm{Fr}$.

Trichopeziza relicina, Fckl.

(P'eziza stenostoma, B. \& C.

Mollisia stenostoma, Sace.

(Pcriza vexata. De Not.

I'eziza subgibbosa, Ell.

Belonium subgibbosum, Sacc.

S Sphaeria phomatospora, B. \& Br.

\{ Phomatospora Berkcleyi, Sacc.

Puccinia graminis, P. ?

Stictis arundinacea, $P$.

Schmitzomia arundinacea, Karst.

Stictis Sesleriae, N. $\Lambda$. F. $45^{2}$
?Ustilago cylindrica, Pk.

Vermicularia velutina, B. \& Rav.

Arotagrostis latifolia, Griseb.

Laestadia graminicola, Rostrup.

Leptosphaeria Rousseliana, (Desm.) Ces. \&

De Not.

Mollisia graminis, Karst.

Sphaerella Tassiana, De Not.

Aristida basiramea. Engelm.

Uromyces Aristidae, Ell. \& Ev.

Aristida dichotoma, L.

Sphaeria Aristidae, S.

Dothidea Aristidae, Ell.

I Phyllachora Aristidae, Sacc.

Sorosporium Ellisii, Wint.

Aristida purpurea, Nutt.

Uromyces Aristidae, Ell. \& Ev.

Aristida Rusbyi, Scribner.

SUstilago Aristidæ, Pk.

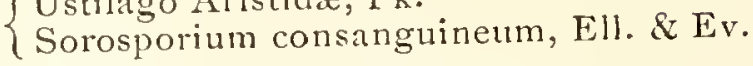

Aristida stricta, Michx.

Dothidea Aristidae, (S.) Ell.

Hendersonia effusa, B. \& C.

Arrhenatherum avenaceum, Benur.

Ustilago segetum, (Bull.) Ditm.

Arundinaria macrosperma, Michx.

$\{$ Dactylium crustaceum, S.

Acrothecium crustaceum, Sacc.

\{ Sphaeria apiospora, Dur. \& Mont.

\{ Apiospora Montagnei, Sacc.

Asteroma Robergei, Desm., var.

Arundinariae, Sacc.

Aulographum Arundinariae, Cke.

Botrytis pallida, B. \& C.

Cladosporium compactum, B. \& C.

Caryospora Langloisii, Ell. \& Ev.

Corticium pezizoideum, Ell. \& Ev.

Sporodum atropurpurem, B. \& C.

\{ Dematium atropurpureum, Sacc.

Dendryphium Arundinariae, Cke.

Diatrype consobrina, Mont.

Diatrype pustulans, Ell. \& Ev.

(Sphaeria combulliens, B. \& C.

\{ Didymella combulliens, Sacc.

(Sphaeria eumorpha, B. \& C.

$\{$ Sphaerella eumorpha, Cke.

(Didymella enmorpha, Sacc.

Dothidea graminis, (P.) Fr.

\{ Epicoccum sphaerospermum, B. \& C.

$\{$ Epicoccum simplex, B. \& C.

\{ Gymnosporium graminenm, Ell. \& Ev.

\{ Coniosporium gramineum, Sacc.

Gymnosporium inquinans, B.

Helicoma Berkeleyi, M. A. Curtis.

f Hypocrea tuberiformis, B. \& Rav.

\{Dussiella tuberiformis, Patouillard.

Hypoxylon culmorum, Cke. 
Hypoxylon perforatum, (S.) Fr.

Spharia arundinacea, Sow.

Sphaeria striaeformis, var. Arundinis, A. \& S.

Leptosphaeria arundinacea, Sacc.

lleptameria arundinacea, Cke.

$\{$ Hysterium arundinaceum, Schrad.

Lophodermium arundinaceum, Chev.

$\{$ Phoma erumpens, B. \& C.

\{ Macrophoma erumpens, Berl. \& Vogl.

$\{$ Stilbospora sphaerosperma, P.

\{ Melanconium sphaerospermum, Lk.

Meliola tenuis, B. \& C.

$\{$ Stigmatea Arundinariae, Cke.

Metaspheria ? Arundinariae, Sacc.

Myxotrichum simile, B. \& C.

(Peziza Arundinariae, B. \& C. p. p.

$\{$ Peziza cannea, B.

Dasyscypha Arundinariae, Sacc.

( Peziza Arundinariae, B. \& C. p.p.

Peziza eustegiaeformis, B. \& C.

Pyrenopeziza Arundinariae, Sacc.

Belonium? eustegiaeforme, Sacc.

$\{$ Peziza caulicola, Fr.

\{ Dasyscypha caulicola, Sacc.

Physalospora conica, Ell. \& Ev.

Puccinia Arundinariae, S.

Stigmatea sclerotoidea, Cke.

Ustilago hypodytes, (Schl.) Fr.

Arundo Donax, L.

Leptostroma Donacis, S.

Monilia sparsa, Lk.

Asprella Hystrix, Willd.

Phyllachora graminis, (P.) Fcki.

Puccinia apocrypta, Ell. \& Tracy.

Avena elatior, L.

Cintractia Avenne, Ell. \& Tracy.

Avena sativa, L.

Fusicladium destruens, $\mathrm{Pk}$.

Puccinia coronata, Cda.

Puccinia graminis, $\mathrm{P}$.

Puccinia Rubigo-vera, (D.C.) Wint.

(Caeoma segetum, (Bull.) Lk. p. p.

Uredo segetum, P. p p.

Ustilago segetum, Ditm. p.p.

Ustilago Carbo, Aud.p.p.

Ustilago Avenae, (P.) Jensen.

Ustilago Avenae, (P.) Jensen, var. levis,

Kell. \& Sivingle.

Avena, sp. indet.

Erysiphe graminis, DC.

Helminthosporium avenaceum, M. A. Curtis.

Bambusa, sp. indet.

Cladosporium graminum, Cda.

Diplodia Bambusae, Ell. \& Langlois.

? Graphiola Phoenicis: Poit.

Gymnosporium Bambusae, Thm.

Macrosporium graminum, Cke.

Torula graminis, Desm.
Beckmannia erucaeformis, Host.

Erysiphe Graminis, DC.

Boutelona hirsuta, Lag.

$\{$ Puccinia vexans, Farl.

Uredo Boutelouae, Arth.

Bouteloua oligostachya, Torr.

Epichloe typhina, (P.) Tul.

Puccinia graminis, $\mathrm{P}$.

Puccinia vexans, Farl.

Ustilago Boutelouae, Kell. \& Swingle.

Bonteloua racemosa, Lag.

Uromyces Brandegei, Pk.

$\{$ Puccinia vexans, Farl.

Uredo Boutelouae, Arth.

Brachyelytrum aristatum, Beauv.

Sphaeria typhina, $\mathrm{P}$.

Dothidea typhina, Fr.

Epichloe typhina, Tul.

$\{$ Sphaeria graminis, $P$.

Plhyllachora graminis, Fckl.

Briza maxima, L.

Puccinia graminis, P.

Bromus ciliatus, L.

$\{$ Pleospora infectoria, Fckl.

\{ Pleospora vulgaris, Niessl.

$\{$ Puccinia tomipara, Trelease.

\{ Rostrupia tomipara, Lagerheim.

Urocystis Agropyri, (Preuss) Schrt.

Ustilago bromivora, Fisch. Wald., var. macrospora, Farl.

Bromus secalinus, $\mathrm{L}$.

Phoma graminella, Sacc?

Buchloe dactyloides, Engelm.

Cercospora seminalis, Ell. \& Ev.

Fusarium graminum, Cda.

Tilletia buchloeana, Kell. \& Swingle.

Ustilago Buchloes, Ell. \& Tracy.

Calamagrostis Canadensis, Beauv.

Acrospermum graminum, Lib.

Claviceps (sclerotium), Sp.

Epichloe typhina, (P.) Tul.

Hadıotriclıum lineare, $\mathrm{Pk}$.

\{ Peziza agrostina, Pk.

\{Trichopeziza agrostina, Sacc.

Pilidium graminicolum, $\mathrm{Pk}$.

Pttccinia graminis, $P$.

(Puccinia linearis, $\mathrm{Pk}$.

$\{$ Puccinia striatula, $\mathrm{Pk}$.

(Puccinia Baryi, (B. \& Br.) Wint.

Calamagrostis Groenlandica, Kunth.

Claviceps purpurea, (Fr.) Tul.

Calamagrostis lanceolata, Roth.

Pleospora vagans, Niessl.

Calamagrostis, Lapponica, Trin.

Mollisia graminis, Karst.

Puccinia Rubigo-vera, (DC.) Wint. 
Calamagrostis longifolia, Hook.

Niptera Ellisii, N. A. F. 2329

$\{$ Peziza clavigera, Ell. \& Ev.

Mollisia clavigera, Sacc.

Puccinia graminis, P.

Calamagrostis phragmitoides, Hartm.

Hendersonia crastophila, Sacc.

Lophodermium arundinaceum, (Schrad.)

Chev.

Septoria nebulosa, Rostrup.

Sphaerella Tassiana, De Not.

Calamagrostis Pickeringii, Gray.

$\{$ Ustilago Salveii, B. \& Br.

Tilletia striaeformis, (Westd.) Wint.

Calamagrostis purpurascens. Br.

Lophodermitum arundinaceum, (Schrad.)

Chev.

Physalospora leptosperma, Rostrup.

Calamagrostis stricta, Trin.

Lophodermium arundinaceum, (Schrad.)

Chev.

Septoria nebulosa, Rostrup.

Cenchrus tribuloides, L.

$\{$ Ustilago Syntherismae, (S.) $\mathrm{Pk}$.

U Ustilago Cesatii, Fisch. Wald.

Chloris petraea, Thunb.

Cercospora caespitosa, Ell. \& Ev.

Chrysopogon nutans, Benth.

Caeoma (Uredo) Andropogi, S.

Colletotrichum lineola, Cda. var. pachyspora, Ell. \& Kell.

Ellisiella caudata, Sacc.

Everhartia hymenuloides, Sacc. \& Ell.

S Peziza hydrophila, Karst.

$\{$ Mollisia hydrophila, Karst.

Niptera hydrophila, Sacc.

Chrysopogon nutans, Benth. var. avenaceus, Gray.

$\{$ Patellaria leucochaete, Ell. \& Ev.

\{ Scutularia leucochaete, Sacc.

\{ Dothidea culmicola, S.

\{Phyllachora culmicola, Sacc.

Puccinia graminis, P.

Vermicularia punctans, S.

Cinna arundinacea, L.

Acrospermum compressum, Tode.

Puccinia coronata, Cda.

Ctenium Americanum, Spreng.

Puccinia Campulosi, Thm.

Cynodon Dactylon, P.

Dimerosporium erysiphoides, Ell. \& Ev.

Dactylis glomerata, L.

Epichloe typhina, (P.) Tul.

Phyllachora Graminis, (P.) Fckl.

Puccinia coronata, Cda.

Scolccotrichum graminis, Fckl.

Dactyloctenium Aegypticum, Willd.

Lștilago destruens, Schl.
Danthonia spicata, Beauv.

Ustilago segetum, (Bull.) Ditm.

Deschampsia atropurpurea, Scheele.

Lophodermium arundinaceum, (Schrad.)

Chev.

Mollisia graminis, Karst.

Deschampsia caespitosa, Beauv.

Tilletia cerebrina, Ell. \& Ev.

Deschampsia flexuosa, Trin.

Lophodermium arundinaceum, (Schrad.)

Chev.

Sphaerella Tassiana, De Not.

Distichlis maritima, Raf.

Phyllachora Tracyi, Ell. \& Ev.

Puccinia graminis, $\mathrm{P}$.

Uromyces Peckianus, Farl.

Dupontia Fischeri, R. Br.

Septoria arctica, B. \& C.

Dupontia psilosantha, Rupt.

Sphaerella Tassiana, De Not.

Eatonia obtusata, Gray.

Puccinia Rubigo-vera, (DC.) Wint.

Eatonia Pennsylvanica, Gray.

Puccinia Rubigo-vera, (DC.) Wint.

Eleusine Indica, Gaertn.

$\{$ Helminthosporium nodosum, B. \& C.

Helminthosporium nodulosum, Sacc.

Myrothecitim roridum, Tode.

Elymus arenarius, L.

Cladosporium graminum, Cda.

Lophodermium arundinaceum, (Schrad.; Chev.

Mollisia graminis, Karst.

Pleospora vagans, Niessl.

Elymus Canadensis, L.

Claviceps purpurea, (Fr.) Tul.

Phyllachora graminis, (P.) Fckl .

Puccinia graminis, $\mathrm{P}$.

Puccinia Rubigo-vera, (DC.) Wint.

Urocystis Agropyri, (Preuss) Schrt.

Urocystis occulta, (Wallr.) Rabh.

Ustilago hypodytes, (Schl.) Fr.

Elymus Canadensis, L., var. glaucifolius, Gray.

'Tilletia striaeformis, (Westd). Wint.

Elymus condensatus, Presl.

Claviceps purpurea, (Fr.) Tul.

Erysiphe graminis, DC.

Puccinia Rubigo-vera, (DC.) Wint.

Elymus mollis, Trin.

Lophodermium arundinaceum, (Schrad.) Chev.

$\{$ Puccinia triarticulata, B. \& C.

\{Rostrupia Elymi, (Westd.) Lagerheim.

Elymus striatus, Willd.

Acrospermum graminum, Lib. 
Elymus Virginicus, L.

Claviceps purpurea, (Fr.) Tul.

$\{$ Sphaeria graminis, $\mathrm{P}$.

\{Phyllachora graminis, Fckl.

Puccinia Rubigo-vera, (DC.) Wint.

Septoria Bromi, Sacc.

Uromyces graminicola, Burrill.

EIyınus, sp. indet.

$\{$ Pleospora planispora, Ell.

$\{$ Clathrospora planispora, Berl.

Epichloe typhina, (P.) Tul.

Eragrostis capillaris, Nees.

$\{$ Dothidea vorax, B. \& C.

Dothidea atramentaria, B. \& C.

Eragrostis major, Host.

Helminthosporium hadotrichoides, Ell. \& EV.

Ustilago spermophora, B. \& C.

Eragrostis pectinacea, Gray.

Puccinia emaculata, S.

Erianthus saccharoides, Michx.

Cladosporium Erianthi, Thm.

Erianthus alopecuroides, Ell.

(Sphaeria orthogramma, B. \& C.

$\{$ Leptosphaeria orthogramma, Sacc.

Heptameria orthogramma, Cke.

Erianthus, sp. indet.

Dothidea graminis, (P.) Fr.

Euchlaena luxurians, Dur. \& Asch.

Ustilago Zeae-Mays, (DC.) Wint.

Eulalia aponica, Trin.

Coccularia graminis, Cke.

Festuca microstachya, Nutt.

Tilletia fusca, E11. \& Ev.

Festuca ovina, L.

Cladosporium graminum, Cda.

Lophodermium arundinaceum, (Schrad.) Chev.

Pleospora pentamera, Karst.

Sphaerella Tassiana, De Not.

Uromyces Dactylidis, Otth.

Festuca rubra, L.

Lophodermium arundinaceum, (Schrad.) Chev.

Sphaerella pusilla, Awd.

Festuca tenella, Willd.

$\{$ Peziza denigrata, (J. Kunze) Ell.

\{Pyrenopeziza denigrata, Relim.

Septoria tenella, C. \& E.

Festuca, sp. indet.

Hendersolia trimera, Cke.

Glyceria arctica, Ilook.

Leptostroma marginatum, S.

Glyceria arundinacea, Kunth.

Ustilago longissima, (Sow.) Tul.

Glyceria fluitans, R. Br.

Claviceps purpurea, (Fr.) Tul.
Glyceria nervata, Trin.

Claviceps (sclerotium).

Epichloe typhina, (P.) Tul.

Erysiphe graminis, DC.

Glyceria vaginata, Lange.

Cladosporium graminum, Cda.

Glyceria Vahliana, Fr.

Lophodermium arundinaceum, (Schrad.) Chev.

Sphaerella Tassiana, De Not.

Glyceria vilfoidea, Fr.

Lophodermium arundinaceum, (Schrad.)

Chev.

Septoria Arundinis, (Mont.) Sacc.

Sphaerella Tassiana, De Not.

Gynerium argenteum, Nees.

$\{$ Psilonia aterrima, Cke.

\{ Chaetostroma? aterrima, Sacc.

Fusarium Gynerii, Cke. \& Hark.

$\{$ Gymnosporium Arundinis, Cda.

Ustilago Gynerii, Vize.

Helminthosporium parvulum, Cke.

Isaria repens, Cke.

Mystrosporium consors, Thm.

\{ Coniothyrium lineare, Thm.

Plioma linearis, Sacc.

Sphaeria apiospora, Dur. \& Mont.

Torula fusoidea, Cke. \& Hark.

Hierochloa alpina, Roem. \& Schultes.

Lophodermium arundinaceum, (Schrad.) Chev.

Pleospora macrospora, Schrt.

Sphaerella Tassiana, De Not.

Hilaria Jamesii, Benth.

Ustilago Hilariae, E11. \& Tracy.

Hordeum jubatum, L.

Claviceps purpurea, (Fr.) Tul.

Erysiphe graminis, DC.

Puccinia graminis, P.

\{ Uredo Rubigo, Auct.

Puccinia Rubigo-vera, (DC.) IVint.

Ustilago segetum, (Bull.) Ditm.

Hordeum pratense, Huds.

Puccinia graminis, $P$.

Puccinia Rubigo-vera, (DC.) Wint.

Hordeum vulgare, L.

Uredo segetum, (Bull.) P.p.p.

Ustilago segetum, Ditm.p.p.

Ustilago Carbo, Auct. p.p.

U Ustilago Hordei, (P.) Kell. \& Swingle.

(Ustilago segetum, (Bull.) Ditm. p.p.

$\{$ Ustilago Carbo, Auct. p.p.

(Ustilago nuda, (Jensen) Kell. \& Swingle.

Hordeum, sp. indet.

Fusarium incarnatum, (Rob.) Sacc.

Koeleria cristata, P.

Claviceps purpurea, (Fr.) Tul. 
Puccinia Rubigo-vera, (DC.) Wint.

Koelleria subspicata, Rchb.

Lophodermium arundinaceum, (Schrad.)

Cliev.

Leer'sia oryzoides, Swz.

\{ Dactylium graminum, S.

\{ Dactylaria graminum, Sacc.

Phyllachora graminis, (P.) Fckl.

Piricularia grisea, (Cke.) Sacc.

Sphaerella Leersiae, Pass.

Tilletia Corona, Scribner.

Leersia Virginica, Willd.

Phyllachora graminis, (P.) Fckl.

Piricularia grisea, (Cke.) Sacc.

Tilletia Coroni, Scribner.

\{ Uromyces digitatus, Halsted.

\{romyces Halstedii, De Toni.

Milium, sp. indet.

S Sphaeria rubella, P.

OOphiobolus porphyrogonus, (Tode) Sacc.

Muhlembergia glomerata, Trin.

Phyllachora graminis, (P.) Fckl.

$\{$ Trichothecium griseum, Cke.

\{ Piricularia grisea, Sacc.

Puccinia graminis, $\mathrm{P}$.

Puccinia Windsoriae, S.

Ustilago Montaniensis, Ell. \& Holw.

Muhlenbergia Mexicana. Trin.

Phyllachora graminis, (P.) Fckl.

Puccinia graminis, $\mathrm{P}$.

Puccinia Windsoriae, S.

? Sphaerella Nuhlenbergiae, Ell.

Muhlenbergia, sp. indet.

$\{$ Dothidea Muhlenbergiae, Ell.

$\{$ Phyllachora Muhlenbergiae, Sacc.

Nardus stricta, L.

Leptosphaeria Nardi, (Fr.) Ces. \& De Not.

Lophodermium arundinaceum, (Schrad.)

Chev.

Sphaerella pusilla, Awd.

Trochila exigua, Rostrup.

Oryza sativa, L.

Phoma glumarum, Ell. \& Tracy.

Oryzopsis melanocarpa, Muhl.

Septoria graminum, Desm.

Panicum agrostoides, Muhl.

(Dothidea vorax, B. \& C.

Epichloe Hypoxylon, Pk.

Ophiodothis vorax, Sacc.

Euryachora vorax, Cke.

Hypocrea Hypoxylon, (Pk.) Ell. \& Ev.

$\{$ Ustilago Syntherismae (S.) Peck.

Ustilago destruens, Auct. Amer.p.p.

Ustilago Panici-Miliacei, Auct. Amer.p.p.

Panicum brizanthum, Hocket.

Phyllachora stenostoma, Ell. \& Tracy.
Panicum capillare, L.

$\{$ Puccinia emaculata, S.

$\{$ Puccinia graminis, P., var. brevicarpa, $\mathrm{Pk}$.

Caeoma Syntherismae S. Am. bor.

Uredo Syntherismae, Rav. Fung. Car.

Ustilago Syntherismae, Pk.

Ustilago Cesatii, Fisch. Wald.

Ustilago destruens, Auct. Amer. p.p. nec. Schl.

Ustilago Panici-Miliacei, Auct. Amer. p.p.

Sorosporium Ellisii, Wint.

Sorosoporium Syntherismae, Farlow.

Panicum Crus-galli, L.

Puccinia flaccida, B. \& Br.

$\{$ Sorosporium lullatum, Schrt.

$\{$ Tolyposporium bullatum, Schrt.

Sphaerella Crus-galli, Ell. \& Kell.

Ustilago sphaerogena, Burrill.

Panicum Cizritisiti, Chapm.

Metasphaeria punctulata, Ell. \& Ev.

Sphaeria (Metasphaeria) stenotheca, Ell.

$\&$ Ev.

Venturia erysiphioides, Ell. \& Ev.

Panicum dichotomum, L.

Sphaeria Panici, S.

Sphaeria Punctum, S.

Dothidea graminis, (P.) Fr.

Phyllachora graminis, Fckl.

Piricularia grisea, (Cke.) Sacc.

Panicum divaricatum, L.

Phyllachora graminis, (P.) Fckl.

Panicum glabrum, Gaudin.

of Ustilago Rabenhorstiana, Kühn.

\{Ustilago destruens, Auct. Amer. p.p.

Panicum latifolium, L.

Phyllachora graminis, (P.) Fckl.

Panicum miliaceum, L.

SUstilago destruens, Auct. Amer. p.p.

$\{$ Ustilago Panici-miliacei, Auct. Amer.p.p.

Sorosporium Syntherismae, (S.)Farlow.

Panicum proliferum, Lam.

Vermicularia discoidea, Ell. \& Langlois.

Panicum sanguinale, L.

$\{$ Trichothecium griseum, Cke.

\{ Piricularia grisea, Sacc.

Septoria graminum, Desm.

Ustilago diplospora, Ell. \& Ev.

Ustilago Rabenhorstiana, Kühn.

(Caeoma (Uredo) Syntherismae, S.

? Ustilago Syntherismae, $\mathrm{Pk}$.

Ustilago Cesatii, Fisch. Wald.

Panicum virgatum, L

Puccinia emaculata, S.

Uromyces graminicola, Burrill.

(Ustilago Maclagani, B. ?

Ustilago rotundata, Arth.

Tilletia rotundata, Ell. \& Ev.

Panicum, sp. indet.

Conoplea hispidula, P. 
S Sphaeria culmifraga, Fr.

$\{$ Leptosphaeria culmifraga, Ces. \& De Not.

S Sphaerella Panicum, Cke.

Metasphaeria Panicorum, Sacc.

S Sphaeria rubella, P.

Ophiobolus porphyrogonus, ('Tode) Sacc.

(Dothidea Panici, S.

Asteroma Panici, M. A. Curtis.

Phyllachora Panici, Sacc.

Septoria graminum, Desm.

Vermicularia sanguinea, E1l. \& Halsted.

Volutella Ellisii, Langlois.

Paspalum ciliatum, H.B.K.

Sclerotium Paspali, S.

Spermoedia Paspali, Fr.

Splaacelia Páspali, Bornet.

Paspalum digitatum, Kunth.

Fusarium heterosporum, Nees.

Paspalum lasve, Michx.

Spermoedia Paspali, (S.) Fr.

Paspalum platycaule, Poir.

$\{$ Cerebella Paspali, Cke. \& Massee.

Langloisia tremelloides, Ell. \& Ev.

Paspalum setaceum, Michx.

Trichothecium griseum, Cke., var. leptospermum, Ell. \& Kell.

Paspalum setaceum, Michx., var. dasyphyllum, Vasey.

Phyllachora graminis, (P.) Fckl.

Paspalum, sp. indet.

?Leptostroma Spiraeae, Fr.

Phalaris arundinacea, $\mathrm{L}$.

Claviceps purpurea, ( $\mathrm{Fr}$.) Tul.

Puccinia coronata, Cda.

Puccinia striatula, $\mathrm{Pk}$.

Ustilago echinata, Schrt.

Phalaris Canariensis, L.

Cladosporium velutinum, Ell. \& Tracy.

Phippsia algida, R. Br.

Leptosphaeria algida, Rostrup.

Mollisia graminis, Karst.

Pleospora herbarum, (P.) Rabh.

Sphaerella Tassiana, De Not.

Phleum alpinum, L.

Scolecotrichum gramınis, Fckl.

Sphaerella pusilla, Awd.

Sphaerella Tassiana, De Not.

Phleum pratense, L.

Epichloe typhina, (P.) Tul.

$\left\{\begin{array}{c}\text { Lophodermium arundinaceum, (Schrad.) } \\ \text { Chev., var. gramineum, Duby. } \\ \text { Hysterium arundinaceum, Schrad., var. } \\ \text { gramineum, C. \& E. }\end{array}\right.$
Sphaeria (Physalospora) oxyspora, Ell. \&

Sacc. in N. A. F.

Physalospora? oxystoma, Sacc. \& Ell.

Laestadia oxyspora, Cke.

Puccinia graminis, $\mathrm{P}$.

SUstilago Salveii, B.\& Br.

$\{$ Tilletia DeBaryana, Fisch. Wald.

(Tilletia striaeformis, (Westd.) Wint.

Vermicularia culmigena, Desm.

Phragunites communis, Trin.

Claviceps microcephala, (Wallr.) Tul.

$\{$ Gymnosporium Arundinis, Cda.

\{ Coniosporium Arundinis, Sacc.

$\{$ Leptosphaeria clavicarpa, Ell. \& Ev.

$\{$ Heptameria clavicarpa, Cke.

Lophodermium arundinaceum, (Schrad.) Chev.

Napicladium arundinaceum, (Cda.) Sacc.

Peziza albotestacea, Desm.

Puccinia graminis, P.?

Puccinia arundinacea, Hedw.

$\{$ Puccinia Arundinariae, S.

Puccinia Phragmitis, (Schum.) Körn.

Systerium clavisporum, C. \& P.

$\{$ Dothidea clavispora, $\mathrm{Pk}$.

(Rhopographus clavisporus, Sacc.

Scolecotrichum maculicolum, Ell. \& Kell.

Poa abbreviata, R. Br.

Pleospora pentamera, Karst.

Poa alpina, L.

Lophodermium arundinaceum, (Schrad) Chev.

$\{$ Mollisia graminis, Karst.

\{ Pyrenopeziza Karstenii, Sacc.

Sphaerella Tassiana, De Not.

Uromyces Dactylidis, Otth.

Poa annua, L.

Septoria graminum, Desm.

Poa caesia, Smitl.

Sphaeria arctica, Fckl.

Leptosphaeria culmorum, Awd.

Lophodermium arundinaceum, (Schrad.) Chev.

Mollisia graminis, Karst.

Pleospora pentamera, Karst.

Septoria nebulosa, Rostrup.

Sphaerella Tassiana, De Not.

Poa compressa, L.

Claviceps (sclerotium).

Poa flexuosa, Muhl.

Leptosphaeria culmorum, Awd.

Lophodermium arundinaceum, (Schrad.) Chev.

Mollisia graminis, Karst.

Sphaerella Tassiana, De Not. 
Poa pratensis, L.

Epichloe typhina, (P.) Tul.

Erysiphe graminis, DC.

Lophodermium arundinaceum, (Schrad.)

Chev.

Mollisia graminis, Karst.

?Piricularia grisea, (Cke.) Sacc.

Puccinia graminis, $\mathrm{P}$.

Sphacrella Tassiana, De Not.

Poa temuifolia, Nutt.

Cladosporium graminum, Cḍa.

Erysiphe graminis, DC.

Poa, sp. indet.

Claviceps purpurea, (Fr.) Tul.

Dothidea vorax, B. \& C.

Dothidea atramentaria, B. \& C.

Ophiodothis vorax, Sacc., var. atramentaria, Sacc.

Oidium monilioides, Lk.

Vermicularia denudata, S.

Puccinellia maritima, Parl.

Lophodermium arundinaceum, (Schrad.)

$$
\text { Chev. }
$$

Saccharum offictnarum, L.

Sphaeropsis Sacchari, Cke

$\{$ Phoma Sacchari, Sacc.

Macrophoma Sacchari, Berl. \& Vogl.

Schedonnardus Texanus, Steud.

Puccinia Schedonnardi, Kell. \& Swingle.

\section{Secale cereale, L.}

Ceuthospora phaeocomes, (Reb.) S.

Cladosporium compactum, B. \& C.

(Sclerotium Clavus, DC.

$\{$ Cordyceps purpurea, Fr.

Claviceps purpurea, Tul.

Fusarium heterosporum, Nees.

Gibberella Saubinetii, (Mont.) Sacc.

Puccinia graminis, $\mathrm{P}$.

Uredo Rubigo-vera, DC.

Caeoma Rubigo, Lk.

Trichobasis Rubigo, Lev.

Uredo Rubigo, Auct.

Puccinia Rubigo-vera, (DC.) Wint.

Urocystis occulta, (Wallr.) Rabh.

Secale, sp. indet.

Marasmius graminum, (Lib.) B.

Pyrenophora sarcocystis, (B. \& C.) Rav.

Fung. Am.

Pyrenophora trichostoma, (Fr.) Sacc.

Setaria glauca, Beauv.

Piricularia grisea, (Cke.) Sacc.

Ustilago destruens, Auct. Amer.p.p.

$\{$ Ustilago neglecta, Niessl.

(Ustilago Panici-glauci, (Wallr.) Wint.

Setaria Italica, Kunth.

\{ Peronospora graminicola, Sacc.

Sclerospora graminicola, Schrt.

Piricularia grisea, (Cke.) Sacc.
Setaria viridis, Beauv.

$\{$ Peronospora graminicola, Sacc.

$\{$ Sclerospora graminicola, Schrt.

Septoria graminum, Desm.

Ustilago neglecta, Niessl.

Setaria, sp. indet.

Ustilago viridis, Ell. \& Ev.

Sorghum halapense, P.

Cercospora Sorghi, Ell. \& Ev.

Lophiostoma implexum, Ell. \& Ev.

Puccinia graminis, P., var. Sorghi, Cke.

Sorghum saccharatum, P,

Cladosporium graminum, Lk., var. Sorghi, Rav. Fung. Am.

Colletotrichum Lineola, Cda.

$\{$ Sphaeropsis Sacchari, Cke.

\{ Phoma Sacchari, Sacc.

Puccinia purpurea, Cke.

Ustilago Sorghi, (Lk.) Pass.

Sorghum vinlgare, $\mathrm{P}$.

Ustilago Reiliana, Kühn.

Ustilago Sorghi, (Lk.) Pass.

Sorghum, sp. indet.

$\{$ Excipula punctiformis, B.

Amerosporium punctiforme, Sacc.

Epicoccum sphaerospermum, B. \& C.

(Helminthosporium Sorghi, S.

$\{$ Helminthosporium Sorghi, Cke.

Helminthosporium Cookei, Sacc.

Sphaeria sorghophila, Pk.

$\{$ Leptosphaeria sorghophila, Sacc.

Heptameria sorghophila, Cke.

Macrosporium culnorum, Cke. S Hark.

Puccinia Sorghi, S.

\{ Sporidesmium culmigenum, Cke

$\{$ Sirodesmium culmigenım, Sacc.

Spartina cynosuroides, Willd.

Ascochyta Spartinae, Trelease.

Claviceps purpurea, (Fr.) Tul.

Puccinia arundinacea, Hedw.

$\{$ Puccinia Arundinariae, S.

(Puccinia Plragmitis, (Schum.) Körn.

Sphaerella Spartinae, Ell. \& Ev.

Uromyces acuminatus, Arth.

Spartina grabra, Mulu.

Uredo periclermiospora, Ell. \& Tracy.

Spartina goacilis, Trin.

PucciniaPhragmitis, (Schum.) Körn.

Uromyces acuminatus, Arth.

Spartina juncea, Willd.

?Microthyrium microscopicum, Desm.

Uromyces Spartinae, Farl.

Spartina polystachya, willd.

$\{$ Didymosphaeria pardalina, Ell. \& Ev.

\{ Didymosphaerella pardalina, Cke.

\{ Dimerosporium Spartinae, El\}. \& Ev.

\{Meliola Spartinae, Berl. \& Vogl.

Peziz? atriella, Cke. 
\{ Peziza heteromorpha, Ell. \& Ev.

$\{$ Belonidium heteromorphum, Sacc.

\{ Puccinia arundinacea, Hedw.

\{Puccinia Arundinariae, S.

Spartina stricta, Roth.

\{ Uromyces Junci, var. Spartinae, Farl.

\{ Uromyces Spartinae, Farl.

Spartina, sp. indet.

Sphaeria (Leptosphaeria) Californica, Cke. \& Hark.

$\{$ Leptosphaeria discors, Sacc. \& Ell.

Metasphaeria discors, Sacc.

Sphaeria incarcerata, B. \& C.

$\{$ Leptosphaeria incarcerata, Sacc.

Heptameria incarcerata, Cke.

\{Leptosphaeria marina, Ell. \& Ev.

\{ Heptaneria marina, Cke.

\{ Leptosphaeria Spartinae, Ell. \& Ev.

IIleptameria Spartinae, Cke.

(Leptosphaeria albopunctata, (Westd.) Sacc.

$\{$ Leptosphaeria sticta, Ell. \& Ev.

Heptameria sticta, Cke.

\{Ophiobolus Medusa, Ell. \& Ev.

\{ Raphidospora Medusae, Cke.

\{ Sclerotium Clavus, DC.

\{ Spermoedia Clarus, Fr.

Sporobolus asper, Kunth.

Puccinia graminis, $\mathrm{P}$.

Puccinia Sporoboli, Arth.

Sporobolus asperifolins, Thurb.

Phyllachora graminis, (P.) Fchl.

Tilletia asperifolia, Ell. \& Ev.

Sporobolus cryptandrus, Gray.

Puccinia Sporoboli, Arth.

Sporobolus cuspidatus, Torr.

Puccinia vexans, Farl.

Sporobolus depauperatus, Vasey.

Leptosphaeria Sporoboli, Ell. \& Gall.

Sporobolus gracillimus, Vasey.

\{ Tilletia montana, Ell. \& Ev.

\{ Tilletia asperifolia, Ell. \& Ev.

Sporobolus heterolepis, Gray.

Puccinia Sporoboli, Arth.

Sporobolus Indicus, R. Br.

$\{$ Dactylium Helminthosporii. Thm.

Trichothecium IIelminthosporii, Sacc.

Helminthosporium Ravenelii, M. A. Curtis.

Sporobolus vaginaeflor us, Vasey.

Puccinia Sporoboli, Arth.

Ustilago Vilfae, Wint.

Sporobolus, sp. indet.

Uromyces simulans, $\mathrm{Pk}$.

Stipa comata, Trin.

Puccinia Stipae, Arth.

$\{$ Ustilago hypodytes, (Schl.) Fr.

\{ Ustilago minima, Arth.
Stipa spartea, Trin.

Puccinia Stipae, Arth.

\{ Ustilago liypodytes, (Schl.), Fr.

U Ustilago minima, Arth.

Stipa viridula, Trin.

Puccinia Stipae, Arth.

Sorosporium granulosum, Ell. \& Tracy.

Triodia cuprea, Jacq:-

Puccinia emaculata, S.

Puccinia Windsoriae, S.

Tripsacum dactyloides, L.

Claviceps purpurea, (Fr.) Tul.

\{ Spermoedia Tripsaci, M. A. Curtis.

Sphacelia Tripsaci, Bornet.

Vermicularia culmigena, Desm.

Trisetum subspicatum, Beauv.

Cladosporium graminum, Cda.

Lophodermium arundinaceum, (Schrad.)

Chev.

Pleospora herbarum, (P.) Rabh.

Septoria graminum, Desm.

Septoria nebulosa, Rostrup.

Sphaerella Tassiana, De Not.

Triticum vulgare, L.

Claviceps purpurea, $\left(F^{r}\right.$.) Tul.

Erysiphe graminis, DC.

Gibbera pulicaris, Fr.

$\{$ Dothidea glumarum, B. \& C.

\{Gibberella Saubinetii, (Mont.) Sacc.

Hymenula glumarum, Cke. \& Hark.

Sphaeria trichostoma, Fr.

Pyrenophora trichostoma, Sacc.

Sphaeria sarococystis, B. \& C.

Pyrenophora sarcocystis, Rav. Fung. Arn.

Pleospora sarcoycstis, Sacc.

Puccinia coronata, Cda.

Uredo linearis, $P$.

$\{$ Caeoma lineare, Schl.

Puccinia graminis, P.

\{ Trichobasis Rubigo-vera, Lev.

\{ Puccinia Rubigo-vera, (DC.) Wint.

\{ Septoria Tritici, B. \& C.

\{ Septoria Curtisiana, Sacc.

Ustilago foetens, B. \& C.

$\{$ Tilletia laevis, Kühn.

Tilletia foetens, Trelease.

Uredo foetida, Bauer.

$\{$ Tilletia Caries, (DC.) Tul.

(Tilletia Tritici, (Bjerk.) Wint.

Trichaegum culmorum, Cke.

Uredo segetum, (Bull.) P. p.p.

Ustilago segetum, (Bull.) Ditm. p.p.

Ustilago Carbo, Auct. p.p.

Ustilago Tritici, (P.) Jensen.

Triticum, sp. indet.

\{ Chaetomium elatum, Kze.

\{Chaetomium comatum, (Tode) Fr. 
Splneria culmiti"aga, Fr.

$\{$ beptosphacria culmifraga, Ces. \& De Not.

(leptameria culmifraga, Cke.

Uniola gracilis, Michx.

Ustilago Uniolae, Ell. E Ev.

Uniola, sp. indet.

Dothidea rorax, B. \& C.

Dothidea pilulaeformis, B. E C.

Ophiodothis vorax, Sacc., var. pululaeformis Sacc.

Zea Mays, L.

\section{PYRENOMYCETES.}

Spluaria mortuosa, Ell.

Anthostoma mortuosum, Sacc.

Sectria Curtisii, B.

Calonectria Curtisii, Sacc.

Dialonectria Curtisii, Cke.

Chaetomium melioloides, C. \& P.

Dialonectria gibberelloides, Ell. \& Ev.

Diaporthe Kellermanniana, Wint.

Dinemasporimm bicristatum, Cke.

Splueria pulicaris, Fr.

Gibbera pulicaris, Fr.

Nectria pulicaris, 'Tul.

Gibberella pulicaris, Sacc.

Sphaeria Zeae, S.

Dothidea Zeae, S.

Sphaeria Maydis, B.

Diplodia Zeae, Lev.

Diplodia Maydis, Sacc.

Sphaeria Saubinetii, Mont.

Gibbera Saubinetii, Mont.

Gibberelila Saubinetii, Sacc.

(Sphaeria ceratispora, B. S C.

?

Leptosphaeria ceratispora, Sacc.

Conisphaeria ceratispora, Cke.

Heptameria ceratispora, Cke.

Spluaria interspersa, Cke.

$\{$ Leptosphaeria interspersa, Sacc.

Heptameria interspersa, Cke.

Sphaeria orthogramma, B. \& C.

Leptosphaeria orthogramma, Sacc.

lleptameria orthogramma, Che.

Lophiostoma Arundinis, (Fr.) Ces. \& De Not.

S Spharia ceratotheca, Cke.

Netasphaeria ceratotheca, Sacc.

Leptosphaeria hyalospora, Sacc.

Sphaeria hyalospora, N. A. F.

Metasphaeria hyalospora, Sacc.

Perisporium Zeae, B. \& C.

$\{$ Dothidea nigrescens, S.

$\{$ Phyllachora nigrescens, Sacc.

Physilospora zeicola, Ell. \& Ev.

Pleospora rubicunda, Niessl.

Sphaerella paulula, Cke.

Sphaeria fissa, P. ?

Sphaeria striaeformis, var. g., S.

FUNGI IMPEREECTI,

Aspergillus alutaceus, B. \& C.
Cercospora Sorghi. Ell. \& Ev.

Cladosporim herbarum, (P.) Lk.

Cladosporium herbarum, (P.) Lk., var. indutum, Thm.

Cladosporium tenuissimum, Cke.

$\{$ Colletotrichum Lineola, Cda.

\{ Psilonia apalospora, B. \& C.

? Dactylium dendroicles, (Bull.) Fr.

$\{$ Vermicularia graminum, Lib.

\{ Dinemasporium graminum, Lev.

Diplodia frumenti, Ell. \& Ev.

Epicoccum neglectum, Desm.

Epicoccum sphaerospermum, B. \& C.

$\{$ Fusisporium cerealis, Cke.

Fusarium cerealis, Sacc.

Fusarium graminum, Cda.

$\{$ Fusisporium rimosum, Pk.

\{ Fusarium rimosum, Sacc.

- Fusarium roseum, Lk.

Fusisporium aurantiacum, Lk.

Fusisporium graminum, Ces.

Fusisporium roseum, Lk.

Haplographium griseum, Ell. S Langlois.

Helicomyces mirabilis, $\mathrm{Pk}$.

Helicosporium vegetum, (Nees).

Helminthosporium foliculatum, Cda

Helminthosporium inconspicuum, C. E E.

f Hymenella haematococca, B. S C.

\{ Hymenula haematococca, Sacc.

lllosporium pallidum, Che.

Isariopsis subulata, Ell. \& Ev.

Macrosporium culmorum, Cke. S Hark.

Macrosporium Maydis, C. S. E.

Monilia Martinii, Ell. \& Sacc.

Mystrosporium erectum, Ell. \& Ev.

Penicillium armeniacum, B.

$\{$ Sporocybe digitata, Cke.

Periconia digitata, Sacc.

Rhinotrichum cucumerinum, B. \& C.

Septosporium praelongum, Sacc.

\{ Sporidesmium tessarthra, B. \& C.

\{ Spegazzinia tessarthra, Sacc.

Sporotrichum flavissimum, Lk?

Sporotrichum fuliginosum, $\mathrm{P}$.

Sporotrichum Himantiae, S.

Tetraploa Ellisii, Cke.

Trichosporium sphaericum, Sacc.

Tubercularia sarmentorum, Fr.

Vermicularia atramentaria, B. \& Br.

Zygodesmus chlorochaites, Ell.

MISCELLANEA.

$\{$ Agaricus demetriacus, B. \& Mont.

Entoloma demetriacum, Sacc. Syll.

Clavaria trichomorpha, S.

S Patellaria imperfecta, Ell.

Karschia imperfecta, Sacc. 
$\{$ Puccinia Sorghi, S.

\{ Puccinia Maydis, Berenger.

Uredo Zeae, S Syn. Car.

Caeoma Zeae, S. Am. bor.

Ustilago Maydis, (1)C.) Cda.

Ustilago scliweinitzii. 'Tul.

Ustilayo Zeae, (S.) Curt. Cat.

(Ustilago Zene-Mays, (DC.) Wint.

Zizania aquatica, L.

Claviceps (sclerotium).

$\{$ Entyloma crastophilum, Sacc.

\{Ustilago lineata, Cke.

Sphaeria Zizaniae, S.

Sphaerella Zizanjae, Ell. \& Ev.

Zizania, sp. indet.

S Arthrinium Sporophleum, Kze.

\{ Sporophleum gramineum, Lk.

S Sphaeria rimosa, S. Herb.

Sphaeria zizaniaccola, B. S C.

Leptosphaeria zizaniaecola. Sacc.

(Heptameria zizaniaecola, Cke.

Leptostroma Zizaniae, S.

S Sploneria Arundinis, Fr.

Lophiostoma Arundinis, Ces. \& De Not.

Puccinia Zizaniae, S.

Sphaeria sulcigena, S.

Gramineae, sp. indet.

PYRENOMYCETES.

Byssonectria rosella, Cke. \& Ilark.

Chaetomium elatum, Kze.

Chaetomium lanosum, $\mathrm{Pk}$.

S Sphaeria Nebraskae, B. \& C.

¿ Didymella Nebraskae, Sacc.

CDothidea vorax, B. \& C.

Hypocrea atramentaria, B. \& C.

Hypocrea atramentosa, B. \& C.

Dothidea atramentaria, B. \& C.

Dothidea pilulaeformis, B. \& C.

Epichloe Hypoxylon, Pk.

Hypocrella Hypoxylon, Sacc.

Hy pocrella atramentosa, Sacc.

Ophiodothis vorax, Sacc.

Ophiodothis vorax, var. atramentaria. Sacc.

Ophiodothis vorax, var. pilulaeformis, Sacc.

Euryachora vorax, Cke.

(Hypocrea Hypoxylon, Ell. \& Ev.

Ephelis borealis, Ell. \& Ev.

Erysiphe communis, (Wallr.) Schl.?

Helotium album, Schum.

Hypocrea subviridis, B. \& C.

Sphaeria Beaumontii, B. \& C.

$\{$ Leptosphaeria Beaumontii, Sacc.

Heptameria Beaumontii, Cke.

Leptosphaeria culmifraga, (Fr.) Ces. \& De Not.

$\{$ Hysterium culmigenum, Fr.

Lophode mium culmigenum, Karst.
Splatia stictispora, C. S E.

$\{$ Ophiobolus stictisporus, Sacc.

(Raphidospora stictispora, C. \& E.

Ophiobolus trichisports, Ell. \& Ev.

f Dothidea delicatula, S.

Plyyllachora delicatula, Sacc.

\{ Leptosphaeria straminis, Cke. \& IIark.

L Pleospora Iarknessii, Berl. \& Vogl.

Pleospora laxa, Ell. E Gall.

Pyrenophora relicina, Fckl.

Sphaerella Californica, Cke.

\{ Sphaeria coccineomaculata, S.

Sphaerella coccineomaculata, S.

Sphaerella perpusilla, (Desm.) Sacc.

Sphaerella philochorta, Cke.

Xylaria Clavulus, B. \& C.

Dylaria graminicola, Gerard.

FUNGI IMPERFECTI.

f Excipula leucotricha, Pk.

\{ Amerosporium leucotrichum, Sacc.

Botryosporium pulchrum, Cda.

Cladosporium graminum, Cda.

Coelosporium fruticulosum, Lk.

Cryptosporium atrum, Lk.

Dinemasporium cruciferum, Ell.

Excipula hispidula, (Schrad.) C. \& E.

Fusisporium foeni, B. S $\mathbb{S}^{\star}$ Br.

Gloeosporium graminicolum, Ell. \& Ev.

?Helminthosporium septemseptatum, Pk.

Illosporium helicoideum, Sacc. \& Ell.

Leptostroma cereale, S.

Macrosporium pinguedinis, I.

Myrothecium Verrucaria, (A. \& S.) Ditm.

Mystrosporium Rubigo, B. \& C.

Penicillium sparsum, Lk.

Vermicularia hispidula, (Lk.) S.

f Sphaeria relicina, Fr.

\{ Vermicularia relicinia, Fr.

Vermicularia straminis, Cke. \& Hark.

Verticillium pyramidale, Bon.

Volutella Ellisii, Langlois.

MISCELLANEA.

$\{$ Peziza culmicola, Desm.

\{ Phialea culmicola, Gill.

\{Peziza nigrocincta, B. \& C.

\{ Trichopeziza nigrocincta, Sacc.

$\{$ Polyporus incrustans, B. \& C.

Poria incrustans, Cke.

Thelephora caesia, P.

Thelephora cristata, (P.) Fr.

Thelephora sebacea, P.

Uromyces scaber, Ell. \& Ev. 


\section{GYMNOSPERMAE.}

CONIFERAE.

Abictineae.

Abies balsamea, Mill.

$\{$ Agaricus mitis, P.

\{ Pleurotus mitis, Quel.

Agyrium rufum, (P.) Fr.

Asterina nuda, Pk.

Cenangium balsameum, Ph.

Cenangium ferruginosum, $\mathrm{Fr}$.

f Cenangium pityum, B. \& C.

Scleroderris pitya, Sacc.

$\{$ Nectria Cucurbitula, Curr.

Chilonectria Cucurbitula, Sacc.

$\{$ Nectria balsamea, C. \& P.

$\{$ Clsilonectria Rosellinii, (Carest.) Sacc.

Clitocybe sulfurea, $\mathrm{Pk}$.

CPeziza amorpha, P.

Thelephora amorpha, $\mathrm{Fr}^{\mathrm{r}}$.

Corticium amorphum, $\mathrm{Fr}$.

Nodularia balsamicola, $\mathrm{Pk}$.

Aleurodiscus amorphus, Rabh.

Peziza balsamicola, Cke. nec. Pk.

f Corticium giganteum, Fr.

Peniophorat gigantea, Massee.

Corticium sulfureum, Fr.

Corticium thelephoroides, Ell. \& Ev.

Cryptosporium Noveboracense, B. \& C.

Dermatea phyllophila, $\mathrm{Pk}$.

$\{$ Diplodia lignicola, Pk.

$\{$ Diplodiella liguicola, Sacc.

Fusarium Berenice, Herb. Curt.

$\{$ Fusisporium Berenice, B. \& C.

Fusarium lierenice, Sacc.

Hirneola Auricula-Judae, (L.) B.

Hypoderma nervisequum, DC.

Lentinus strigosus, (S.) Fr.

Narasmius filipes, $\mathrm{Pk}$.

Meliola balsamicola, $\mathrm{Pk}$.

Merulius aureus, Fr.

Paxillus Curtisii, B.

Peridermium balsameum, Pk.

Aecidium elatinum, A. \& S.

Peridermium elatinum. Schm. \& Kze.

Pestalozzia ? campsosperma, $\mathrm{Pk}$.

$\{$ Peziza Agassizii, B. \& C.

\{ Dasyscypha Agassizii, Sacc.

$\{$ Peziza balsamicola, Pk.

Tapesia balsamicola, Sacc.
Phacidium abietinum, Schm.

$\{$ Didymium flavidum, $\mathrm{Pk}$.

Physarum flavidum, $\mathrm{Pk}$.

Polyporus abietinus, (Dicks.) Fr., var. irpiciformis, $\mathrm{Pk}$.

$\{$ Polyporus balsameus, $\mathrm{Pk}$.

\{ Polystictus balsameus, Che.

Polyporus violaceus, Fr.

$\{$ Polyporus volvatus, $\mathrm{Pk}$.

Fomes volvatus, Cke.

Sacidium Pini, (Cda.) Fr.

Steganosporium cenangioides, Ell. \&

Rothrock.

Sterenm balsameum, $\mathrm{Pk}$.

$\{$ Stictis hysterina, Fr.

XXlogramma hysterinum, Rehm.

C Peziza Pinastri, P.

Cenangium Pinastri, Fr.

Tympanis Pinastri, Tul.

Cenangiella Pinastri, Sacc.

Valsa Friesii, (Duby) Fckl.

Abies Fraseri, Lindl.

$\{$ Peziza crocea, S.

\{Phialea crocea, Sacc.

Trichosphaeria parasitica, R. Hartig.

Abies grandis, Lindl.

Macrophoma parca, Ell. \& Ev.

Abies, sp. indet.

\section{MYXOMYCETES.}

'Trichia erecta, Rex.

Trichia subfusca, Rex.

PYRENOMYCETES.

$\{$ Triblidium morbidum, Pk.

$\{$ Colpoma morbidum, Sacc.

Cucurbitaria macilenta, C. \& E.

$\{$ Wallrothiella macilenta, Sacc.

(Gibberidea macilenta, C. \& E.

Hypocrea lenta, (Tode) B.

Hypoderma conigenum, (P.) Sacc.

Lophiostoma excipuliforme, (Fr.) Ces. \&

De Not., var. Abietis, Ell. \& Ev.

Lophodermium Pinastri, (Schrad.) Chev.

Meliola fenestrata, C. \& E.

Sphaeria acuum, Cke. \& Hark.

$\{$ Metasphaeria acuum, Sacc.

Sphaerulina acuum, Cke. 
Sphaeria squamata, C. \& E.

$\{$ Metasphaeria squamata, Sacc.

Endophlaea squamata, C. \& E.

$\left\{\begin{array}{l}\text { Hysterium Pinastri, Curt. in Herb. Duby } \\ \text { Aporia obscura, Duby. } \\ \text { Schizothyrium obscurum, Sacc. }\end{array}\right.$

FUNGI IMPERFECTI.

Chalara acuaria, C. \& E.

Diplodia megalospora, B. \& C.

Eustilbum Rehmianum, Rabh.

Helicosporium ellipticum, Pk.

Helminthosporium obclavatum, Sacc.

Sphaeridium citrinum, Sacc.

? Sphaeropsis Pinastri, C. \& E.

Sporidesmium pallidum, B. \& C.

Streptothrix abietina, $\mathrm{Pk}$.

Tubercularia nigricans, (Bull.) DC.

\section{DISCOMICETES.}

Helotium lutescens, (Hedw.) Fr.

Helotium subtile, Fr.

Mitrula cucullata, Fr.

$\{$ Peziza acuum, A. S S.

$\{$ Dasyscypha acuum, Sacc.

Peziza badia, P.

Peziza calycina, Schum.

$\{$ Peziza fumosella, C. \& E.

$\{$ Phialea fumosella, Sacc.

Peziza hyalina, $P$.

Peziza lachnoderma, B.

$\{$ Peziza nigripes, $\mathrm{P}$.

\{ Helotium nigripes, Schum.?

$\{$ Peziza resinae, $\mathrm{Fr}$.

Tromera resinae, Körb.

$\{$ Peziza strobilina, $\mathrm{Fr}$.

\{ Phialea strobilina, Sacc.

$\{$ Peziza subtilissima, Cke.

\{Dasyscypha subtilissima, Sacc.

Stictis fimbriata, S.

\section{HYMENOMYCETES.}

$\{$ Agaricus Austini, Pk.

\{Omphalia Austini, Sacc. Syll.

$\{$ Agaricus Blakei, B. \& C.

\{ Pletrotus Blakei, Sacc. Syll.

Agaricus (Mycena) purpureofuscus, $\mathrm{Pk}$.

Clavaria abietina, $P$.

Corticium chlorinum, B. \& C.

Corticium cremoricolor, B. \& C.

Corticium subincarnatum, Pk.

Dacryomyces chrysocomus, (Bullø) Tul.

Hydnum Auriscalpium, L.

Hymenochate abnormis, $\mathrm{Pk}$.

Lenzites heteromorpha, Fr.

Lenzites sepiaria, (Wulf.) Fr., var. porosa, $\mathrm{Pk}$.
Merulius bellus. B. \& C.

$\{$ Merulius confluens, $S$.

\{Merulius Corium, Fr.

Merulius molluscus, $\mathrm{Fr}$.

Odontia fusca, C. $\mathcal{E}$ E.

Polyporus carneus, Nees.

Polyporus circinatirs, Fr.

Polyporus dualis, $\mathrm{Pk}$.

Polystictus circinatus, Cke.

Mucronoporus circinatus, EIl. \& Ev.

Mucronoporus dualis, Ell. \& Ev.

Polyporus fulvus, Fr.

Polyporus destructor, (Schrad.) Fr.

Polyporus leucospongia, Cke. \& Hark.

$\{$ Polyporus odorus, $\mathrm{Pk}$.

\{ Poria odora, Sacc. Syll.

$\{$ Polyporus Stephensii, B. \& Br.

\{ Trametes serpens, Fr.

$\int$ Polyporus sinuosus, Fr.

Trametes sinuosa, Cke. \& Quel.

Poria sinuosa, Cke.

Polyporus vulgaris, Fr.

Trametes odoratus, (Wulf.) Fr.

Tremella lutescens, P.

Ozonium auricomum, Lk.

Larix Americana, Michx

Agaricus (Mycena) strobilinus, $\mathrm{P}$.

Chilonectria Cucurbitula, (Curr.) Sacc.

Corticium amorphum, (P.) Fr.

Corticium giganteum, Fr.

(Thelephora ochracea, S. Syn. Car.

$\{$ Thelephora subzonata, Fr.

Corticium subzonatum, $\mathrm{Fr}$.

Irpex tabacinus, B. \& C.

Lentinus lepideus, Fr.

Lentinus maximus, Johnson.

Lentinus tigrinus, (Bull.) Fr.

Peziza Agassizii, B. \& C.

Polyporus abietinus, (Dicks.) Fr.

Polyporus abietinus, (Dicks.) Fr., var. resupinatus, Thm.

Polyporus pinicola, (Swartz) Fr.

Trametes Pini, (Brot.) Fr.

Tremella mesenterica, Retz.

Trichothecium roseum, Lk.

Larix, sp. indet.

Agaricus (Clitocybe) cerrusatus, Fr.

Agaricus (Pleurotus) serotinus, Schrad.

Thelephora pubera, S. Am. bor.

Corticium Auberianum, Mont.

S Sphaeria strobilina, $\mathrm{Fr}$.

Dichaena strobilina. Fr.

Lenzites abietina, (Bull.) Fr.

Nectria coccinea, (P.) Fr.

Phlyctaena phomatella, Sacc.?

$\{$ Polyporus annosus, Fr.

\{ Fomes annosus, Cke. 
S Polyporus armeniacus. B.

\{ Polyporus adiposus, B. S Br., var.

armeniacus. Cke.

Polvporus destructor, (Schrad.) Fr.

I Poria incarnata, $P$.

Polyporus incarnatus, Fr.

Polvporus violaceus, Fr.

Trametes Abietis, Karst.

Picea alba, Lk.

f Cenangium pityum, B. S C.

Scleroderis pitya, Sacc.

Lenzites sepiaria, (Wult.) Fr.

Phragmotrichum Chailletii, Kze.

Picea concolor, Gordon.

Aecidium elatinum, $A$. $\mathbb{S}$ S.

Picea Engelmanni, Engelm.

Peridermium abietinum, (A. \& S.) Thm.

Peridermium Engelmanni, Thm.

Picea excelsa, Lk.

Nectria coccinea, (P.) Fr.

(Aecidium conorum-Piceae, Reess.

Peridermium conorum, Thm.

Pestalozzia abietina, Vogl.

Pestalozzia Stevensonii, Pk.

Pestalozzia truncatula, Farl. in N. A. F. an Fckl.?

Pestalozzia strobilicola, Speg.

Pestalozzia conorum-Piceae, Vou Tubeuf.

Phoma lineolata, Desm.

Picea nigra, Lk.

Agaricus (Mycena) liemalis, Osbeck.

Corticium subaurantiacum. Pk.

Hypodermium sparsum, Lk.

Irpex fuscoviolaceus, (Schrad.) Fr.

Marasmius perforans, (Hoftin.) Fr.

Merulius Ravenelii, B.

Mucronella calva, (A. S S.) Fr.

Aecidium abietinum, Auct. Amer. pp.

Peridermium abietinum, Auct. Amer. pp.

Peridernium decolorans, Pk.

Peridermium abietinum, var. decolorans, Thm.

Polyporus marginellus, Pk.

Poria mutans, Pk., var. tenuis, $\mathrm{Pk}$.

Polyporus piceinus, $\mathrm{Pk}$.

Polyporus variformis, Pk.

Polyporus variiformis, Pk., var. interruptus, Pk.

Polyporus variiformis, Pk., var. nodulosus, Pk.

Polyporus variiformis, $\mathrm{Pk}$., var. resupinatus, Pk.

SPolyporus violaceus, Fr.

Poria violacea, Cke.

$\{$ Polyporus volvatus, Pk.

Fomes volvatus, Cke.

Schizophyllum commune, Fr.

Stereum abietinum, (P.) Fr.
Picea Sitchensis, Carr.

Peziza fuscosanguinea, (Relim) Ell. \& Ev.

Trametes Pini, (Brot.) Fr.

Pinus albicaulis, Engelm.

Comatricha Suksdorfii, Ell. \& Ev.

Hysterium formosum, Cke.

Pinus Austriaca, Höss.

Pestalozzia peregrina, Ell. \& Martin.

Pinus contorta, Dougl.

Sphaeria tumulata, Cke.

Amphisphaeria tumulata, Sacc.

Xylosphaeria tumulata, Cke.

Corticium carneum; B. \& Cke.

\{ Peniophora carnea, Cke.

\{ Dacryomyces deliquescens, (Bull.) Duby.

\{ Dacryomyces tortus, Fr.

S Hysterium formosum, Cke.

Hysterographium formosum, Sacc.

\{ Hysterium Pinastri, Schrad.

Lophodermium Pinastri, Chev.

f Sphaeria Pulvis-pyrius, P.

Melanomma Pulvis-pyrius, Fckl.

Peridermium Harknessii, Moore.

(Peziza Agassizii, B. E C., var. rufipes, Phil.

Dasyscypha Agassizii, Sacc., var. rufipes, Sacc.

f Peziza hyalina, P.

Pseudohelotium hyalinum, Fckl.

Polyporus labyrinthicus, Fr.

Polyporus obvolutus, B. \& Cke.

Polyporus volvatus, Pk., var. obvolutus, Pk.

Fomes volvatus, Cke., var. obvolutus, Sacc. Syll.

Sphaeria myriocarpa, Fr.

Psilosphaeria inyriocarpa, Hark.

Rosellinia myriocarpa, Cke.

Secotium nubigenum, Hark.

Pinus inops, Ait.

Coniosporium strobilinum, S.

Hypodermium sulcigenum, Lk.

SHysterium Nova-Caesariense, Ell.

$\{$ Mytilidion Nova-Caesariense, Rehm.

Hysterographium Nova-Caesariense, Roum.

Periconia nana, Ehrb.

Cacoma pineum. Lk. $\left\{\begin{array}{l}\text { Peridermium Pini, Lev., var.corticolum, } \\ \text { Auct. }\end{array}\right.$

Peziza conorum, Ell.

Phacidium lacerum, Fr.

Stictis fimbriata, S.

Pinus insignis, Dougl.

Cenangium ferruginosum, Fr.

S Phoma acuum, C. \& E.

$\{$ Coniothyrium acuum, Hark.

Dermatea Pini, Phil. \& Hark.

Sphaeria Vermicularia, Nees.

$\{$ Eriosphaeria Vermicularia, Sacc.

Lasiosphaeria Vernicularia, Cke. 
Lophium mytilinum, (P.) Fr.

Peridermium Harknessii, Moore.

Peridermium Pini, Lev. var. corticolum, Auct. Sphaerella acicola, Cke. \& Hark.

Tubercularia insignis, Cke. $\mathbb{S}$ Hark.

Vermicularia Tiliae, Lk.

$Z_{\text {y godesmus fuscus, Cda. }}$

Zygodesmus marginatus, Cke. \& Hark.

Pinus Lambertiana, Dougl.

$\{$ Polyporus argillaceus, Cke.

$\{$ Poria argillacea, Cke.

Pinus mitis, Michx.

Corticium laeve, $\mathrm{P}$.

Hysterographium flexuosum, (S.) Sacc.

Lentinus lepideus, Fr.

f Peridermium oblongisporium, Fckl, var.

\{ Peridermium orientale, Cke.

$\{$ Cryptosporium acicolum, Thm.

\{ Septoria acicola, Sacc.

Pinus Murrayana, Balf.

Didymosphaeria euryasca, E11. \& Gall.

Hymenochaete fimbriata, Ell. \& Ev.

Trametes Pini, (Brot.) Fr.

Pinus palustris, Mill.

Boletus hemichrysus, B. \& C.

S Sphaeria pilifera, Fr.

\{Ceratostoma piliferum, Fckl.

Corticium arachnoideum, B.

Hydnum pityophilum, B. \& C.

Lentinus lepideus, Fr.

\{ Hysterium Pinastri, Schrad.

Lophodermium Pinastri, Chev.

\{ Merulius fugax, Rav. Fung. Car.

$\{$ Merulius ambiguus, B.

Merulius lacrymans, (Wulf.) Schuın.

$\{$ Agaricus dorsalis, Bosc.

\{ Panus dorsalis, Fr.

Tubercularia carnea, Bosc.

Peridermium oblongisporium, Fckl., var. Ravenelii, Thm.

Peridermium orientale, Cke.

Polyporus abietinus, (Dicks.) Fr., var. resupinatus, Thm.

Polyporus carneus, Nees.

Polyporus palustris, B. \& C.

Stictis strobilina, Cke.

Propolis strobilina, Sacc.

Trametes Pini, (Brot.) Fr.

Pinus ponderosa, Dougl.

Cenangium ferruginosum, Fr.

Peridermium filamentosum, Pk.

Peridermium Harknessii, Moore.

(Peridermium Pini, Lev., var. corticolum, Auct.

(Peridermium cerebrum, $\mathrm{Pk}$.

Phacidium Pini, (A. \& S.) Schm.
Scoriomyces Andersoni, Ell. \& Gall.

f Hymenula aciculosa, Ell. \& Hark.

\{Volutella aciculosa, Sacc.

Pinus zesinosa, Ait.

Sphaeria pholidigena, Ell.

Anthostomella pholidigena, Ell.

Anthostoma pholidigena, Cke.

Lophodermium Pinastri, (Schrad.) Chev.

Pinus rigida, Mill.

Asterina Pinastri, Sacc. \& Ell.

$\{$ Nectria Cucurbitula, Curr.

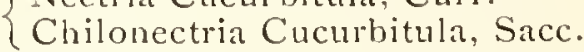

Cenangium acuum, C. \& P.

Dacryomycas corticioides, Ell. \& Ev., var. conigenus, Ell. \& Ev.

Hendersonia rostrata, Sacc. \& Ell.

Hymenula fumosa, C. \& E.

Hypoderma Desmazierii, Duby.

Lophium mytilinum, (P.) Fr.

Lophodermium Pinastri. (Schrad.) Chev.

Marasmius straminipes, $\mathrm{Pk}$.

Sphaeria squamata, C $\& \mathrm{E}$.

Mletasphaeria squamata, Sacc.

Leptosphaeria squamata, Ell.

Systerium Nova-Caesariense, Ell.

\{Mytilidion Nova-Caesariense, Rehm.

Parodiella rigida, Ell. \& Ev.

SAecidium Pini, P. p.p.

Peridermium Pini, var. acicolum, Wallr.

Peridermium oblongisporium, Fckl.

Cecidium Pini, P. p.p.

Caeoma pineum, Lk.

Peridermium Pini, Lev., var. corticola, Auct.

Peridermium cerebrum, $\mathrm{Pk}$.

Peridermium corticolum, Rav. Fung. Am.

\{ Peziza conorum, Ell.

\{ Propolis thodoleuca, (Sommf.) Phil.

Peziza lachnoderma, B.

Peziza Ellisiana, Rehm.

Dasycypha lachnoderma, Rehm.

Helotium Ellisianum, Wettstein.

Dasyscypha Ellisiana, Sacc.

$\{$ Dermatea phyllophila, J'k.

$\{$ Peziza lethalis, Ell.

(Peziza Pinastri, C. \& P.

$\{$ Cenangium acuum, C. \& P.

Mollisia Pinastri, Sacc.

Peziza pulverulersta, Lib.

Peziza solfatera, C. $\&$ E.

Dasyscypha pulverulenta, Sacc.

Dasyscypha solfatera, Sacc.

CPolyporus obvolutus. B. \& Cke.

Poly porus volvatus, Pk., var. obvolutus, $\mathrm{Pk}$.

Fomes volvatus, Cke., var. obvolutus, Sacc. Syll.

$\{$ Propolis leucaspis, Ell.

\{ropolis Leonis, (Tul.) Relim.

(Sphaeria abietina, var. trichota, C. \& E.

$\{$ Rosellinia abietina, Fckl., var. tricota, Sacc. 
Trametes Pini, (Brot.) Fr.

Venturia barbula, (B. \& Br.) Cke, var. foliicoln, Ell.

Sphaeria albocincta, C. N E.

$\{$ Zignoella albocincta, Sacc.

Conisphaeria albocincta, C. \& E.

Pinus Sabinfana, Dongl.

Aulographum acicolum, II ark.

Cenangium ferruginosum, Fr.

Peridermium Harknessii, Moore.

Trichosporium fuscescens, Cke. \& Hark.

\section{Pinus Strobus, L.}

Agaricus (Armillaria) melleus, Vahl.

Caliciopsis pinea, Pk.

Cenangium acuum, C. \& P.

Cenangium ferruginosum, $\mathrm{Fr}$.

Cenangium Abietis, (P.) Rehm.

Cenangium Pinastri, (P.) Fr.

Sectria Curcurbitula, Curr.

$\{$ Chilonectria Cucurbitula, Sacc.

Diplodia pinea, (Desm.) Kickx.

Excipula Strobi, Fr.

Haplosporella Pini, Pk.

Hirneola A uricula-Judæ, (L.) B.

Sypoderma nervisequum, DC.

$\{$ Rhytisma lineare, Pk.

Hypoderma lineare, $\mathrm{Pk}$.

Hypodermium effusum, S.

Lentinus lepideus, Fr.

SHysterium Pinastri, Schrad.

Lophodermium Pinastri, Chev.

Merulius Iacrymans, (Wulf.) Schum.

Phacidium abietinum, Schm.

Shacidium Pini, (A. \& S.) Schm.

Phacidium crustaceum, B. \& C. in Herb.

Curtis.

Coccomyces Pini, Karst.

Polyporus Vaillantii, (DC.) Fr.

Phoma strobilinum, P. \& C.

Sphaeronema strobilinum, Desm.

f Sphaeronema subtile, B. \& C.

Aposphaeria hysterella, Sacc.

Stictis hysterina, Fr.

\{ Xylogramma hysterinum, Rehm.

Sphaeria Spraguei, B. \& C.

$\{$ Mielogramma Spraguei, B. \& C.

Thiridium Spraguei. Sacc.

f Polyporus annosns, Fr.

Trametes radiciperda, R. Hartig.

Trametes Pini, (Brot.) Fr.

Sphaeria Colliculus, Wormsk.

\{ Vilsa Colliculus, Cke.

Pinus silvestris, $L$.

f Sphaeropsis Pinastri, C. \& E.

¿Sphaeropsis Ellisii, Sacc.

Pinus Taeda, L.

Diplodia megalospora, P. \& C.
Peridermium orientale, Cke.

Peridermium Pini, Lev var.

Peridermium corticolum, Rav. Fung. Am.

\{ Phacidium elegans, B. \& C.

\{Dothiora elegans, Sacc.

Pinus, sp. indet.

MYXOMYETES.

Sadhamia penetralis, C. \& E.

$\{$ Lamproderma Ellisiana, Cke.

Comatricha Ellisiana. Eli. \& Ev.

$\{$ Diderma albescens, Phil.

Chondrioderma albescens, Cke.

Cribraria dictydioides, Cke. $\mathbb{S}$ Balf.

Physarum pulcherrimum, B. \& Rav.

Stemonitis porphyra, B. \& C.

\section{PYRENONYCETES.}

Sphaeria Scopula, C. \& P.

$\{$ Lasiosphaeria Scopula, C. \& P.

Acanthostigma Scopula, Pk.

Capnodium australe, Mont.

Capnodium Pini, B. \& C.

Ceratostoma conicum, EH. \& Ev.

Ceratostoma fallax, Cke. \& Sacc.

$\{$ Sphaeria parallela, Fr.

Endoxyla parallela, Sacc.

Sphaeria luteobasis. Ell.

$\{$ Eutypa luteobasis, Sacc.

Byssosphaeria luteobasis, Cke.

Glonium stellatum, Mulsl.

Hypocrea olivacea, C. \& E.

$\{$ Hysterium conigenum, P.

$\{$ Hypoderma conigenum, Sacc.

Hypoderma nervisequum, DC.

$\{$ Nectria Tegillum, B. \& C.

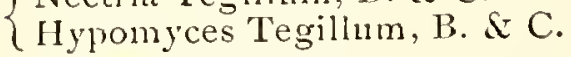

Sphaeria nudicollis, B. \& C.

$\{$ Hypoxylon nudicolle, B. \& C.

(Valsaria? nudicollis, Sacc.

Hysterium Gloniopsis, Gerard.

Hysterium macrosporium, Pk.

S Sphaeria canescens, P.

$\{$ Lasiosphaeria canescens, Karst.

Sphaeria hispida, Tode.

Lasiosphaeria hispida, Fckl.

Sphaeria solaris, C. \& E.

$\{$ Lasiosphaeria solaris, Sacc.

(Byssosphaeria solaris, Cke.

\{ Sphaeria strigosa, A. \& S.

$\{$ Lasiosphaeria strigosa, Sacc.

Lophium fusisporum, Cke.

Melanomma ramincolım, S. Herb.

Sphaeria graopsis, Ell.

Melanopsamma graopsis, Sacc.

Meliola fenestrata, C. \& E. 
Sphaeria Coulteri, Pk.

Lasiosphaeria acicola, Cke.

Amphisphaeria? acicola, Sacc.

Enchnosphaeria Coulteri, Sacc.

Neopeckia Coulteri, Sacc.

Byssosphaeria acicola, Cke.

Sphaeria hirtissima, $\mathrm{Pk}$.

$\{$ Rosellinia hirtissima, Sacc.

Coniochaeta hirtissima, $\mathrm{Pk}$.

$\left\{\begin{array}{l}\text { Hysterium Pinastri, M. A. Curtis in Herb. } \\ \text { Duby. }\end{array}\right.$

Aporia obscura, Duby.

Schizothyrium obscurum, Sacc.

(Sphaeria fissurarum, B. \& C.

$\{$ Trichosphaeria fissurarum, Sacc.

(Lasiosphaeria fissurarum, Cke.

Sphaeria pilosa, P.

$\{$ Triclıosphaeria pilosa, Fckl.

Lasiosphaeria pilosa, Cke.

Valsa Abietis, Fr.

Valsa Pini, (A. \& S.) Fr.

$\{$ Sphaeria tortuosa, Fr.

$\{$ Valsa tortuosa, Cke.

(Sphaeria diaphana, C. \& E.

$\{$ Zignoella diaphana, Sacc.

Psilosphaeria diapliana, Che.

Sphaeria soluta, C. \& E.

$\{$ Zignoella soluta, Sacc.

Conisphaeria soluta, C. S E.

\section{SPIIAEROPSIDEAE.}

Leptostroma confluens, Cke.

Leptostroma durissimum, Cke.

Leptostroma Pinastri, Desm.

\{ Sacidium Pini, (Cda.) Fr.

$\{$ Leptothyrium Pini, Sacc.

Phoma fibricola, B.

Sphaeropsis micromegala, B. \& C.

$\{$ Phoma micromegala, Sacc.

Macrophoma micromegala, Berl. \& Vogl.

Phoma strobiligena, Desm.

$\{$ Cryptosporium Pini, B. \& C.

$\{$ Rhabdospora Pini, Sacc.

\section{MELANCONIEAE.}

Stilbospora pinicola, B. \& C.

\section{IIY PIOMYCETES.}

Botrytis elegantula, Cke.

?Botrytis epigaea, Lk.

$\{$ Circinotrichum candidum, S.

\{ Botrytis? Schweinitzii, Sacc.

Chalara acuaria, C. \& E.

Chalara fusidioides, Cda.

Cylindrocolla cylindrophora, Ell. \& Ev.

Epicoccum scabrum, Cda.

$\{$ Fusisporium miniatum, B. \& C.

$\{$ Fusarium miniatum, Sacc.
Fusarium roseum, Lk.

$\{$ Helicomyces aureus, Cda.

IIelicosporium auresm, Auct.

Helicosporium fuscum, B. \& C.

Helicosporium olivaceum, Pk.

Helicosporium thysanophorum, Ell. \& Hark.

Illosporium coccinellum, Cke.

Illosporium persicinum, $\mathrm{Fr}$.

Isaria radiata, B. \& C.

Macrosporium leptotrichum, C. \& E.

Monilia antennata, $P$.

fOidium simile, B.

\{ Oospora similis, Sacc.

Riessia semiophora, Fres.

Rhinotrichum corticioides, Cke.

Rhinotrichum ramosissimum, B. \& C.

Septonema dendryphioides, B. \& C.

Septonema tabacinum, Ell. \& Hark.

Septonema toruloideum, C. \& E.

Sphaeridium citrinum, Sacc.

Sporidesmium antiquum, Cda.

$\{$ Sporidesmium translucens, Che.

\{ Sirodesmium translucens, Sace.

Sporotrichum aeruginosum, S.

Stigmella pityophila, Cke.

$\{$ Stilbum smaragdinum, A. S S.

\{ Graphium smaragdinum, Sacc.

Tortula sparsa, B. \& C.

Tubercularia microsperma, B. \& C.

Virgaria indivisa, Sacc.

Volutella citrina, E1l. \& Ev.

Zygodesmus marginatus, Che. S Hark.

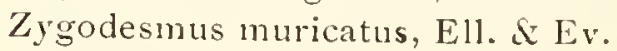

Zygodesmus olivascens, B \& C.

$Z$ ygodesmus pannosus, B. \& $C$.

Zygodesmus ramosissimus, B. \& C.

Zygodesmus sublilacinus, Ell. \& Holw.

$Z_{y}$ godesmus trachychaetes, E11. \& Ev.

DISCOMYCETES.

Agyrium brunneolum, B. \& C.

Agyrim rufum, (P.) Fr.

$\{$ Ascobolus carneus, P.

\{Ascophanus carneus, Boud.

$\{$ Ascomyces anomalus, Ell. S llark.

$\{$ Exoascus a nomalus, Sacc.

Cenangium pityum. Fr.

Helotium subtile, Fr.

Mitrula cucullata, Fr.

Peziza abdita, Ell.

Peziza bicolor, Bull.

Peziza calycina, Schum.

Peziza clamaeleontina, Pk.

$\{$ Peziza conigena, $\mathrm{P}$.

$\{$ Helotium conigenum, $F$ r. 
Peziza fascicularis, A. $\mathbb{S}$.

Dermatea fascicularis, $\mathrm{Fr}$.

Cenangium populneum, (P.) Rehm.

( Peziza Hexella. (Ach.) Fr.

Patinella Hexella, Sacc.

Peziza fumosella, C. $\mathbb{S}$ E.

Dasyscypha fuscosanguinea, Rehm. Peziza arida, Phil.

Peziza fuscosanguinea, E1l. \& Ev.

Dasyscypha arida, Sacc.

Peziza latebrosa. Ell.

Dasyscypha latebrosa, Sacc.

f Peziza ochracea, S.

\{ Dasyscypha ochracea, Sacc.

(Peziza Pineti, Batsch.

Peziza lurida, P.

Peziza pulchella, var. b., A. \& S.

Peziza resinae, Fr.

f Peziza rhaphidospora, Ell.

Erinella thaphidospora, Sacc.

Peziza scutellata, L.

Peziza strobilina, Fr.

( Peziza uncinata, Phil.

Dasyscypha uncinata, Sacc.

(Peziza subtilissima, Cke.

$\{$ Dasyscypha subtilissima, Sacc.

f Peziza vinosa, A. S S.

Orbilia vinosa, Karst.

f Stictis decidua, Ell. \& Ev.

Propolis decidua, Sacc.

Stictis hemisphaerica, Fr.

\section{HYMEYOMYCETES.}

\section{Agaricini.}

Agaricus Campanella, Batsch.

S Agaricus chrysophyllus, Fr.

Omphalia chrysophylla, Karst.

$\{$ Agaricus conigenoides, E1l.

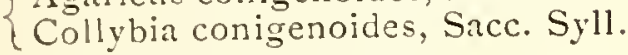

$\{$ Agaricus conigenus, P.

\{ Coliybia conigena, Sacc. Syll.

I Agricus flavescens, Pk.

Tricholoma flavescens, Sacc. Syll,

Agaricus (Mycena) intertextus, B. E C.

(Agaricus nidulans, $P$.

Claudopus nidulans, Pk.

f Agaricus niphetus, Ell.

$\{$ Pleurotus niphetıs, Sacc. Syll.

f Agaricus porrigens, $P$.

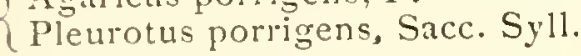

f Agaricus rubescentifolius, Pk.

Collvbia rubescentifolia, Pk.

Agaricus (Flammula) sapineus, Fr.

Agaricus (Tricholoma) scalpturatus, Fr.

Agaricus (Collybia) stipitarius, Fr.

$\{$ Agaricus striatulus, $P$.

$\{$ Pleurotus striatulus, Sacc. Syll.

Agaricus (Pleurotus) sulfureoides, Pk.
Cantharellus Ravenelii, B. S C.

Cortinarius limonius, Fr.

Cortinarius opimus, Fr.

Lentinus cochleatus, Fr.

Lentinus cochleatus, Fr., var. dentatus, S.

Lenzites rhabarbarina, B. S C.

Lenzites sepiaria, (Wulf.) Fr.

Lenzites striata, (Swartz) Fr.

Lenzites vialis, $\mathrm{Pk}$.

Narasmius candidus, (Bolt.) Fr.

Marasmius flammans, Cke.

Marasmius perforans, (Hoffm.) Fr.

Marasmius pityophilus, B. \& C.

Marasmius praeacutus, El].

Mycena crystallina, Pk.

Panus foetens, Fr.

Paxillus atrotomentosus, (Batsch), Fr.

Paxillus Curtisii, B.

Paxillus panuoides, Fr.

Paxillus rudis, B. \& C.

\section{Polyporei.}

f Daedalea rhabarbarina, B. \& Cke.

Daedalea Berkeleyi, Sacc.

Merulius aureus, Fr.

Merulius bellus, B. \& C.

Merulius Corium, (P.) Fr.

Merulius incrassatus, B. \& C.

Merulius lacrymans, (Wulf.) Schum., var. tenuissimus, B.

Merulius porinoides, $\mathrm{Fr}$.

f Merulius serpens, Rav. Fung. Car.

Merulius Raveneliı, B.

Merulius tremellosus, Schrad.

(Boletus abietinus, Dicks.

$\{$ Polyporus abietinus, Fr.

Polystictus abietinus, Cke.

Polyporus aneirinus, Sommf.

Trametes aneirina, Cke. \& Quel.

(Poria aneirina, Cke.

f Polyporus aurantiopallens, B. \& C.

Poria aurantiopallens, Cke.

Polyporus caesius, (Schrad.) Fr.

( Polyporus cinnabarinus, (Jacq.) Fr.

\{Hydum cinnabarinum, (S.) Fr.

Polyporus corticolus, Fr.

f Polyporus deglubens, B. S C.

Polystictus deglubens, Cke.

$\{$ Polyporus dependens, B. $\mathbb{E}$ C.

Polystictus dependens, Cke.

Polyporus epileucus, Fr.

(Polyporus farinellus, Fr.

$\{$ Polyporus tenellus, N. A. F.

Poria farinella, Cke.

$\{$ Polyporus tenellus, B. S Cke.

$\{$ Poria tenella, B. \& Cke. 
( Poria incarnata, P.

$\{$ Polyporus incarnatus, Fr.

Polyporus lacteus, Fr.

Polyporus Lindbladii, B., var.

Polyporus lucidus, (Leys.) Fr.

Poria mucida, $P$.

$\{$ Boletus mucidus, $P$.

Polyporus mucidus, Fr.

f Polyporus Radula, Rav. Fung. Am.

Poria onoema, B. sec Cke.

(Polyporus parvulus, S.

$\{$ Polystictus parvulus, Cke.

Polystictus pusio. Sacc. \& Cub.

Polyporus pineus, Pk.

S Polyporus pinicola, (Swartz) Fr.

$\{$ Fomes pinicola, Cke.

\{ Polyporus salmonicolor, B. S C.

Poria salmonicolor, Cke.

$\{$ Polyporus sanguineus, (L.) Mey.

\{ Polystictus sanguineus, Fr.

SPolyporus triqueter, (Secr.) Fr.

(Polystictus triqueter, Cke.

Poria vaporaria, P.

$\{$ Polyporus vaporarius, Fr.

( Polyporus velutinus, (P.) Fr.

$\{$ Polystictus velutinus, Cke.

Polyporus vesiculosus, B. \& C.

Polyporus subacidus, Pk., var. vesiculosus, Pk.

Poria vesiculosa, Cke.

Poria subacida, Sacc. Syll., var. vesiculosa, Pk.

\{ Polyporus vulgaris, Fr.

Poria vulgaris, Cke.

Polyporus xanthus, Fr.

\{ Poria xantha, Cke.

Poria favillacea, (B. \& C.) Cke.

Porothelium confusum, B. \& Br.

Porothelium Friesii, Mont.

Trametes odorata, (Wulf.) Fr.

Trametes sepium, B.

Trametes versatilis, $B$.

$\{$ Polystictus versatilis, Fr.

\section{Hydnei.}

Grandinia alutacea, B. \& Rav.

Grandinia granulosa, (P.) Fr.

Hydnum alutaceum, Fr.

Hydnum auriscalpium, L.

Hydnum ciliolatum, B. \& C.

Hydnum Ellisianum, Thm.

Hydnum farinaceum, P.

Hydnum nudum, B. \& C.

Hydnum plumosum, Duby.

Hydnum Stevensoni, B. \& Br.

Hydnum velatum, B. \& C.

Hydnum xanthum, B. \& C.

Irpex coriaceus, B. \& Rav.
Irpex fusco-violaceus, (Schrad.) Fr.

Kneiffia setigera, Fr.

Odontia fusca, C. \& E.

\section{Thelephorei,}

Asterostroma corticola, Massee.

Thelephora albidocarnea, S.

Corticium albidocarneurn, Rav.

Corticium clrysocieas, B. \& C.

Asterostroma albidocarneum, Massee.

Corticium Auberianum, Mont.

Corticium Berkeleyi, Cke.

\{Thelephora calcea, Fr.

\{ Corticium calceum, Fr.

Corticium echinosporum, Ell.

\{Hymenochaete Ellisii, B. S Cke.

Corticium (Coniophora) Ellisii, Cke.

(Thelephora gigantea, Fr.

Corticium giganteum, Fr.

Peniophora gigantea, Massee.

Corticium incarnatum, $\mathrm{Fr}$.

Corticum incarnatum, Fr., var. maculans, Ell.

\{ Corticium leucothrix, B. \& C.

\{ Coniophora leucothrix, Sacc. Syll.

Corticium ochroleucum, Fr., var. spumeum, B. \& Rav.

Corticium punctulatum, Cke.

Corticium saccharinum, B. \& C.

Corticium seriale, Fr.

f Corticium suffocatum, Pk.

Coniophora suffocata, Massee.

Corticium tessulatum, Cke.

Corticium vagum, B. \& C.

Stereum radiatum, $\mathrm{Pk}$.

\{ Thelephora sanguinolenta, A. \& S.

Stereum sanguinolentum, Fr.

$\{$ 'Thelephora arida, $\mathrm{Fr}$.

\{ Coniophora arida, Sacc. Syll.

Thelephora chalybaea, P.

$\{$ Thelephora concentrica, A. \& S.

$\{$ Thelephora ochracea, Fr.

Thelephora puteana, Schum.

Theiephora zygodesmoides, Ell.

Clavariei.

Clavaria pyxidata, P.

Tremellini.

Arrhytidia flava, B. \& C.

Calocera stricta, Fr.

$\{$ Calocera albipes, (Nont.) B

Calocera cornea, (Batsch) Fr.

Tremella Aurantia, S.

\{ Dacryomyces chrysosperma, B. \& C.

Dacryomyces corticioides, Ell. \& Ev.

Tremella abietina, $P$.

$\{$ Dacryomyces stillatus, Nees. 
Ditiola madicata, (.1. S. S.) Fr.

Exidia pedunculata, B. \& C.

Exidia recisa, $\mathrm{Fr}_{\mathrm{r}}$.

Sydnum gelatinosum, Scop.

$\{$ Hidnoglaeum gelatinosum, Curr.

Tiemellodon gelatinosum, Fr.

Tremella pinicola, $\mathrm{Pk}$.

MSCELLANEA.

Crucibulum vulgare, Tul.

Nidularia rubella, Ell. \& Ev.

Ozonium auricomum, Isk.

Peridermium pyriforme, $\mathrm{Pk}$.

f Xylostroma giganteum, Tode.

\{ Racodium Xylostroma, P.

Rlizoctonia bicolor, Ell.

Sphaerobolus stellatus, Tode.

Pseudotsuga Douglasii, Carr.

Cenangium leptospermum, B. \& C.

Haplaria Ellisii, Cke.

Hymenochaete rugispora, Ell. \& Ev.

Lasiosphaeria Pezizula, (B. \& C.) Sacc.

\{ Patellaria abietina, Cke.

Patinella abietina, Sacc.

Phoma strobiligena, Desm.

Polyportus hispidus, (Bull.) Fr.

Spiaerella conigena, E11. \& Er.

Sporidesmium translucens, Cke.

Trametes Pini, (Brot.) Fr.

Tsuga Canadensis, Carr.

ASCOMXETES.

\{ Sphaeria Scopula, C. \& P.

Acanthostigma Scopula, Pk.

Cenangium balsameum, Pk., var. abietinum, $\mathrm{Pk}$.

Cenangium Pinastri, (P.) Fr.

$\{$ Peziza chamaeleontina, $\mathrm{Pk}$.

Dasycypha chamaeleontina, Sacc.

$\{$ Peziza elatina, A. \& S.

\{ Chlorosplenium elatinum, Sacc.

(Bulgaria deligata, Pk.

$\{$ Peziza leucobasis, Pk.

Pyronema leucobasis, Sacc.

Seziza pitya, P.

$\{$ Sarea pitya, S.

( Pitya vulgaris, Fokl.

SRizina Helvetica, Fckl.

Peziza Warnei, Pk.

(Discina Warnei, Sacc.

Sphaerclla conigena, $\mathrm{Pk}$.

\{ Stictis Tsugae, Farl.

\{ropolidium Tsugae, Sacc.

f Splnaeria livida. P.

I'Thyridium lividum, Sacc.

Valsa Abictis, Fr.

\section{FUNGI IMPERFECTI.}

Botrytis ceratioides, $\mathrm{Pk}$.
Botrytis lilacina, S.

\{ Isaria monilioides, var. b., S. Syn. Car.

Cephalotrichum monilioides, Lk.

Cryptosporium Noveboracense, B. \& C.

Gelatinosporium abietinum, Pk.

Helicosporium ellipticum, Pk.

Helicosporium olivaceum, $\mathrm{Pk}$.

Phoma Libertiana, Speg. \& Roum.

$\{$ Fusarium capitatum, S.

$\{$ Pionnotes capitata, Fr.

\section{IIYMENOMYCETES.}

$\{$ Agaricus bellulus, Pk.

$\{$ Naucoria bellula, Sacc. Syll.

Agaricus Campanella, Batsch.

Agaricus (Mycena) epipterygius, Scop.

Agaricus (Armillaria) melleus, Vahl.

$\{$ Agaricus porrigens, $P$.

\{ Pleurotus porrigens, Sacc. Syll.

$\{$ Agaricus (Omphalia) rugosodiscus, $\mathrm{Pk}$.

MYycena rugosodisca, Sacc. Syll.

$\{$ Agaricus striatulus, P.

\{ Pleurotus striatulus, Sacc. Syll.

$\{$ Agaricus (Collybia) succosus, $\mathrm{Pk}$.

MIycena succosa, Sacc. Syll.

$\{$ Corticium suffocatum, Pk.

Coniophora suffocata, Massee.

Hydnum farinaceum, P.

Lenzites sepiaria, (Wulf.) Fr.

Marasmius filipes, Pk.

Merulius himantioides, $\mathrm{Fr}$.

Mucronella calva, (A. \& S.) Fr.

Pantıs stipticus, (Bull.) Fr.

Paxillus atrotomentosus, (Batsch) $\mathrm{Fr}$.

Paxillus pantroides, Fr.

Polyporus abietinus, (Dicks.) Fr.

$\{$ Polyporus applanatus, (P.) Wallr.

\{Fomes applanatus, Karst.

Fomes applana:us, (P.) Karst, var. abietinus, Thm.

$\{$ Polyporus benzointus, (Wahl.) Fr.

\{ Trametes benzoina, $\mathrm{Fr}$.

Polyporus borealis, (Wahl.) Fr.

Polyporus cinnabarinus, (Jacq.) Fr.

Polyporus crispellus, $\mathrm{Pk}$.

Polyporus epileucus, Fr.

Polyporus epileucus, Fr., var. candidus, Pk.

Polyporus giganteus, (P.) Fr.

Polyporus lucidus, (Leys.) Fr.

Polyporus Medulla-panis, (P.) Fr.

Polyporus Pilotae, S.

Polyporus Pini-Canadensis, S.

Polyporus hypococcinus, B.

(Fomes Pini-Canadensis, Cke.

Polyporus pinicolus, (Swartz) Fr.

Polyporus resinosus, (Schrad.) Fr.

Polyporus rhodellus, Fr. 
\{ Polyporus subacidus. Pk., var. tenuis, Pk.

\{ Poria subacida, Siacc. Syll, var. tenuis, Pk.

Polyporus undosus, Pk.

Polyporus Vaillantii, (DC.) Fr.

Polyporus versicolor, (L.) Fr.

Polyporus Weinmanni, Fr.

Poria tomentocincta, B. \& Rav. sec Cke.

Radulum Pini-Canadensis, S.

Solenia candida, P.

Solenia villosa, Fr., var. polyporoidea, Pk.

Stereum radiatum. $\mathrm{Pk}$.

Stereum sanguinolentum, (A. \& S.) Fr.

Tremella Aurantia, S.

MISCELLANEA.

Caeoma Abietis-Canadensis, Farl.

Chrysomyxa Abietis, (Wallr.) Unger.

Comatricha subcaespitosa, Pk.

Crucibulum rulgare, Tul.

Aecidium columnare, Auct. Amer.

Peridermiun columnare, Anct. Amer.

Peridermium Peckii, Thm.

Xylostroma giganteum, Tode.

Tsuga Pittoniana, Engelm.

f Anthostomella brachystoma, Ell. \& Ev.

\{Xylosphaeria brachystoma, Cke.

Lasiosphaeria stuppea. Ell. \& Ev.

\{ Patellaria signata, Ell. \& Ev.

$\{$ Blitrydium signatum, Sacc.

\section{Araucarieae.}

Araucaria imbricata, Pavon.

Pestalozzia funerea, Desm.

Sphaeria (Leptosphaeria) Californica, Cke. \& Hark.

Stictis Araucariae, Phil. \& Hark.

Cupressiueae

"Cedar," gen. indet.

$\{$ Agaricus niphetus, Ell.

\{ Pleurotus niphetus, Sacc. Syll.

Sphaeria aquatica, Ell. \& Ev.

$\{$ Amphisphaeria aquatica, Berl. \& Vogl.

(Didymosphaeria aquatica, Ell. \& Ev.

Amphisphaeria deformis, Ell. \& Langlois.

Daedalea quercina, (L.) P.

Dendrodochium densipes, Sacc. \& Ell.

Dendryphium Harknessii, Ell.

Glonium nitidum, Ell.

Ielicomyces aureus, Cda.

Hydnum Stephensoni, B. S Br.

Hypocrea armeniaca, B. S C.

Hypocrea minima, Sacc. \& Ell.

Marasmius candidus, (Bolt.) Fr.

Marasmius praeacutus, Ell.

$\{$ Polyporus circinatus, Fr.

$\{$ MIucronoporus circinatus, Ell. \& Ev.

Odontia fusca, C. \& E.

Ombrophila aurea, Ell.
Ombrophila subamea. Cke.

Peziza ancilis, Pers?

Peziza caucus, Reb.

$\{$ Phoma fibricola, B.

Aposphaeria fibricola, Sacc.

(Polyporus farinellus, Fr:

Polyporus tenellus. N. A.F.

Poria farinella, Cke.

Polyporus Schweinitzii, Flo.

Solenia villosa, Fr.

Xylographa parallela, (Ach.) Fr.

Chamaecyparis sphaeroidea, Spach.

$\{$ Agaricus Campanella, Batsch.

SOmphalia Campanella, Karst.

Agaricus (Armillaria) melleus, Vỉhl.

$\{$ Venturia cupressina, Rehm.

Asterina cupressina: Cke.

Coniothyrium subtile, Cda.

Diplodia thyoidea, C. \& E.

Gymnosporangium biseptatum, Ell.

Gymnosporangium biseptatum, Ell, vax. folicolum, Farl.

Podisoma Ellisii, B.

Hamaspora Ellisii, Körn.

Gymnosporangium Ellisi, Farl.

Phragmidium Ellisii, De Toni.

Hendersonia foliicola, (B.) Fckl.

Hendersonia thyoides, C. S E.

\{ Hydnum subtile, S. Am. bor.

\{Hydnum Ellisianum, Thm.

Hypsotheca thujina, Ell. \& Ev.

Hysteromyxa effugiens, Sacc. \& Ell.

$\{$ Nectria thujana, (Relım.) Sacc.

\{ Dialonectria thujana, Cke.

$\{$ Nectria truncata, Ell.

$\{$ Dialonectria truncata, Cke.

? Paxillus aurantiacus, Ell.

Pestalozzia unicornis, C. \& E.

Peziza Cupressi, Batsch.

$\{$ Propolis grisea, C. \& E.

$\{$ Nemacyclus griseus, Sacc.

Cryptomeria Japonica, Don.

Pestalozzia Cryptomeriae, Cke.

Cupressus macrocarpa, Hartw.

Diplodia cyparissa, Cke. S Hark.

Splueria anisometra, Cke. \& Hark.

Metasphaeria anisometra, Sacc.

Endophlaea anisometra, Cke. \& Hark.

Sphieropsis Cupressi, Cke. \& Hark.

\{ Macrophoma Cupressi, Berl. \& Vogl.

Pestalozzia funerea, Desm.

Pestalozzia heterospora, Desm.

Polyscytalum sericeum, Sacc,

Cupressus, sp. indet.

\{ Dothidea Sequoine. Cke. \& Hak.

Stigmatea Sequoire, Silcc.

Juniperus communis, L.

Coryneum juniperinum, Ell. 
Dermatea juniperina, Ell.

Guepinia elegans, B. \& C.

(Gimnosporangimm clavariactome, (Jacq.) DC.

l'odisoma Juniperi, Cke.

Grmnosporangium claripes, C. \& P.

Gymnosporangium fuscum, DC?

isphaerella juniperina, Ell.

L Laestadia juniperina, Sacc.

Lophodermium juniperinum, (Fr.) De Not.

I Coryneum unicolor, M. A. Curtis.

Pesta\}ozzia uniculor, B. \& C.

Juniperus communis, L., var. alpina,

Gaud.

Corsneum paraphysatum, Rostrup.

IIenriquesia cinerascens, (Duby) Sacc.

Lophoderminm juniperinum, (Fr.) De Not.

Juniperus occidentalis, Hook.

Grmnosporangium speciosum, $\mathrm{Pk}$.

Hysterum formosum, Cke.

Hysterographium formosum, Sacc.

Juniperus squamata, Don.

Discosia Artocreas, (Tode) Fr., var.

Juniperi, Rav. Fung. Am.

Peziza pitya, P.

\section{Jumiperus Virginiana, L.}

\section{PYREXOMYCETES.}

Ceratostoma juniperinum, Ell. \& Ev.

Sphaeria junipericola, S.

Sphaeropsis junipericola, Cke.

Cucurbitaria junjpericola, Cke?

S Sphaeria stipitata, S.

\{ Cucurbitaria stipitata, Cke?

\{ Valsa cenisia, De Not.

\{ Cytospora cenisia, Sacc.

$\{$ Sphaeria griseotingens, B. \& C.

\{ Diaporthe griseotingens, Sacc.

Diatrype fibritecta, C. \& E.

Sphaeria acervalis, var. Juniperi, Desm.

Gibherella acervalis, var. Juniperi-

Virginianae, Sacc.

f Iysterium tortile, S.

Y Mytilidion tortile, Sacc.

(Hysterium Petersii, B. \& C.

Colpoma juniperinum, C. \& P.

Lophodermium Petersii, Sacc.

Melanopsamma cupressina, Ell. \& Ev.

Sphatia thelebola, Fr.

$\{$ Valsa thelebola, Fr.

(Melanconis thelebola, Sacc.

Splracria thyoidea, C. \& E.

$\{$ Ielogramma fuliginosum, Ell. p. p.

(Physalospora thyoidea, Sacc.

Nicrothyrium Juniperi, (Desm.) Sacc.

Mytilidion Juniperi, Ell. \& Ev.

Splueria deerrata, C. \& E.

Rosellinia deerrata. Sacc.

Amphisphaeria deerrata, Cke.
Valsa Abietis, Fr.

Valsa coryneoides, B. \& C. in Herb. B.

Talsa juniperina, Cke.

FUNGI IMPERFECTI.

Cercospora Sequoiae, Ell. S Ev., var.

Juniperi, Ell. \& Ev.

f Sporidesmium larvatum, C. AE.

\{ Clasterisporium larratum, Sacc.

Diplodia Virginiana, Cke.

Discosia alnea, (P.) B.

Discosia Virginiana, Thm.

? Phoma longipes, B. \& C.

Sphaeropsis Juniperi, Pk.

Stilbum lichenoidenm, B. \& C.

Streptothrix abietina, $\mathrm{Pk}$.

Streptothrix atra, B. \& C.

Zygodesmus rudis, Ell.

\section{DISCOMYCETES.}

\{ Cenangium deformatum, Pk.

\{ Cenangiella deformata, Sacc.

Cenangium Sequoiae, Plow.

Patellaria dispersa, Gerard.

f Peziza cedrina, Cke. \& Gerard.

Lachnella cedrina, Sacc.

\{ Peziza Cupressi, Batsch.

\{ Peziza cupressina, Fr.

(Peziza incrustata, Ell.

\{Pseudohelotium incrustatum, Sacc.

UREDINEAE.

Aecidium Bermudianum, Farl.

Gymnosporangium clavipes, C. \& P.

Gymnosporangium Juniperi, var. clavipes, Cke.

Podisoma Gymnosporangium, var. clavipes, Cke.

Podisoma fuscum, Auct. Amer. p.p.

Gymnosporangium fuscum, var. globosum, Farl.

Gymnosporangium Sabinae, var. globosum, Trelease.

Gymnosporangium globosum, Farl.

Gymnosporangium Juniperi-Virginianae, S. Gymnosporangium macropus, Lk.

Gymmosporangium Virginianum, Spreng.

Podisoma Juniperi-Virginianae, Fr.

Podisoma macropus, Lk.

Podisoma Juniperi, Auct. Amer. p.p.

Grumosporangium Juniperi, Auct. Amer. p.p.

Gymnosporangium conicum, Auct. Amer. p.p.

Gymnosporangium juniperinum, Auct. IT.8 Amer. p.p.

Gymnosporangium clavipes, Farl. nec C. \& P.

Gumnosporangium Nidus-avis, Thaxter. HYMENOMYCETES.

$\{$ Stereum acerinum, var. nivosum, Cke.

$\{$ Corticium acerinum, var. nivosum, Thm. 
f Corticium brum neolum, B. \& C.

\{Coniophora brunneola, Sacc. Syll.

Corticium calceum, Fr.

Slymenochaete Ellisii, B. S Cke.

$\{$ Corticium (Coniophora) Ellisii, Cke.

Coniophora Ellisii, Sacc. Syll.

Crucibulum vulgare, Tul.

Cyphella cupulieformis, B. \& Rav.

Grandinia tabacina, C. $\&$ E.

Polyporus sericeohirsutus, Klotzsch.

$\{$ Hexagona sericea, $\mathrm{Fr}$.

Hexagona sericeohirsuta, M. A. Curtis.

Kneiftia candidissima, B. \& C.

Kneiffia setigera, Fr.

Peniophora tlavidoalba, Cke.

Polyporus carneus, Nees.

f Polvstictus barbatulus, Fr.

$\{$ Polyporus barbatulus, Rav.

Porothelium Friesii, Mont.

Trametes sepium, $B$.

Junipexus, sp. indet.

Agyrium rufum, (P.) Fr.

$\{$ Phoma fibricola, B.

Aposphaeria fibricola, Sacc.

Cantharellus Petersii, B. \& C.

$\{$ Corticium cervicolor, B. \& C.

Asterostroma cervicolor, Massee.

Corticium deglubens, B. \& C.

Dendryphium Ellisii, Cke.

Diplodia cupressina, Cke.

Epicoccum scabrum, Cda.

Grandinia papillata, B. S C.

Gymnosporangium fuscum, DC.?

Hydnum Caput-medusae, Bull.

Illosporium coccinellum, Cke.

Sphaeria inusta, Cke.

Kalmusia inusta, Sacc.

Iylosphaeria inusta, Cke.

Ombrophila aurea, Ell.

\{ Peziza flexella, (Ach.) Fr.

\{ Patinella flexella, Sacc.

$\{$ Dothidea sphaeroidea, Cke.

\{ Phyllachora sphaeroidea, Cke.

Polyporus abietinus, (Dicks.) Fr.

? $\{$ Polyporus leucolomeus, Lev.

Poria leucolomea, Cke.

Polyporus vulgaris, Fr., var. calceus, Fr.

Sphaeria pustulata, $P$.

Sporidesmium larvatum, C. \& E.

Clasterosporium larvatum, Sacc.

Sporidesmium rude, Ell.

Stereum hirsutum, (Willd.) Fr.

Streptothrix fusca, Cda.

Sphaeria albocincta, C. $\mathbb{E}$ E.

$\{$ Zignoella albocincta, Sacc.

Conisphaeria albocincta, C. S E.

Zygodesmus bicolor, C. \& E.
Libocedrus decur'rens, 'Tor'r.

(Cucurbitaria macilenta, C. \& E.

$\{$ Wallrothiella macilenta, Sacc.

Gibberidea macilenta, C. \& E.

Daedalea vorax, Hark.

Gymnosporangium biseptatum, Ell.

$\{$ Peziza alutipes, Phil.

\{ Phialea alutipes, Sacc.

Tiruja occidentalis, L.

\{ Sphaeria thujina, $\mathrm{Pk}$.

Amphisphaeria thujina, Sacc.

Coniosporium punctoideum, Karst.

(Peziza rubella, P.

Ombrophila rubella, Quel.

Dacryomyces conglobatus, $\mathrm{Pk}$.

Diplodia thujina, Pk. \& Clinton.

Hymenochaete tenuis, Pk.

Hysterium Thujarum, C. \& P.

Hysterium tortile, S.

Pestalozzia conigena, Lev.

CPeziza Cupressi, Batsch.

Peziza cupressi, Fr.

Pitya cupressina, Fckl.

Helotium thujinum, $\mathrm{Pk}$.

Pitya Cupressi, Sacc.

Pitya thujina, Sacc.

$\{$ Peziza enterochroma, $\mathrm{Pk}$.

Ombrophila enterochroma, Sacc.

Phoma magnifructa, Pk.

Sphaerella conigena, $\mathrm{Pk}$.

Sphaerella Pinsapo, Thm.

\{ Periconia corticalis, C. \& P.

(Sporocybe corticalis, Sacc.

Valsa Thujae, Pk.

Thuja, sp. indet.

$\{$ Phoma albistrata, $\mathrm{Pk}$.

Coniothyrium albistratum, Sacc.

Cylindrium elongatum, Bon.

Cytospora Thujae, Sacc. \& Ell.

Micropera tenella, Sacc. \& Ell.

Pestalozzia foedans, Sacc. \& Ell.

Pestalozzia funerea, Desm.

Valsa Thujae, Pk., var. foliicola, Ell. \& Ev.

\section{Taxaceae.}

\section{Taxus Canadensis, Willd.}

Agaricus (Mycena) Leaianus, B.

Agaricus terraeolens, Pk.

Sphaeria taxicola, $\mathrm{Pk}$.

$\{$ Leptosphaeria taxicola, Sacc.

Heptameria taxicola, Cke.

Nerulius subaurantiacus, Pk.

S Sphaeria Taxi, Sow.

Diplodia Taxi, DeNot. 


\section{Turodicue.}

Sequoia gigantea, Decaisne.

Cenangium Sequoine, Plow.

Scleroderris Sequoiate, Sacc.

Cercospora Sequoiae, Ell. a Er.

Clavaria cristata, P.

Dacryomyces stillatus, Nees.

Hydnum niveum, P.

Mytilidion Californicum, Ell. \& Hark.

P’eziza bulgarioides, Rabh.

\{ Peziza Sequoiae, Phil.

\{ Lachnea Sequoine, Sacc.

Sphaeria consociata. Ell. \& Ilark.

P'hysalospora consociata, Sacc.

(Laestadia consociata, Cke.

$\{$ Venturia Sequoine, Plow.

\{ Acanthostigma Sequoiae, Sacc.

f Sphaeria Sequoiae, Plow.

$\{$ Zignoella Sequoiae, Sacc.

Conisphaeria Sequoiae, Che.

Sequoia sempervirens, Endl.

(Sphaeria Wellingtoniae, Cke. \& Hark.

$\{$ Amphisphaeria Wellingtoniae, "Berl. \& Vogh.

Xylosphatria Wellingtoniae, Che. \& Hark.

Corticium caeruleum, (Schrad.) Fr.

Corticium lacteum, $\mathrm{Fr}$.

Corticium sanguineum, Fr.

Corticium umbrinum, A. \& S.

Crtospora Pinastri, Fr.

Hymenochaete tabacina, (Sow.) Lev.

Hy pocrea rufa, (P.) Fr.

Hysterium angustatum, $\mathrm{P}$.

Lasiosphaeria canescens, (P.) Karst.

Leptostroma Sequoiae, Cke. \& Ilark.

$\{$ Sphaeria confertissima, Plow.

\{. Melanopsamma confertissima, Sacc.

Merulius molluscus, Fr.

Morchella conica, P.

Mystrosporium turbinatum, Cke. \& Hark.

Nyxotrichum ochraceum, B. S Br.

Naematelia encephala, (Willd.) Fr.

\{ Peziza acum, A. S S.

\{ Dasyscypha acuum, Sacc.

\{ Peziza chloromela, Phil. \& Hark.

\{Chlorosplenium chloromelum, Sacc.

Peziza Cupressi, Batsch.

Peziza cupressina, Fr.

Pitya cupressina Fckl.

Pitya Cupressi, Sacc.

Peziza fusca, P.

\{ Peziza gemmea, Phil.

\{ Barlaea gemmea, Sacc.

! Peziza pitya, P.

(Pitỹa vulgaris, Fckl.

$\{$ Peziza sphaerophoroides, Phil. \& Ilark.

\{ Helotium spaerophoroides, Sacc.

Peziza ustorum, B. \& Br.

f Rosellinia mutans. (C. \& P.) Sacc.

( Büssosphneria mutans, Cke.
Solenia fasciculata, P.

Stictis rersicolor, Fr.

Tremella mesenterica, Retz.

Tremellodon gelatinosum, (Scop.) Fr.

Sequoia sp. indet.

$\{$ Polyactis rulgaris, Lk.

\{ Botrytis rulgaris, Fr.

Peziza carbonaria, A. \& S.

Sporidesmium induratum, Clie.

Taxodium distichum, Richard.

Aulographum caespitosum, Ell. \& Ev.

Cenangium fallax, B. S Rav.

$\{$ Peziza chlorascens, S.

\{Chlorosplenium repandum, $\mathrm{Fr}$.

\{Coniothecium effusum, Cda.

\{ Sporidesmium Lepraria, B.

Cytospora pallida, E1l. \& Ev.

Daedalea sepium, B.

\{Hysterium lineare. B. nec Fr.

Hysterium lineariforme, Sacc.

Melanopsamma cupressina, Ell. \& Ev.

(Peziza pomicolor, B. \& Rav.

$\{$ Pseudohelotium pomicolor, Sacc.

Trichopeziza poinicolor, Sacc.

$\{$ Peziza Taxodii, B.

$\{$ Blitrydium Taxodii, Sacc.

$\{$ Polyporus Stephensii, B. \& Br.

\{ Trametes serpens, Fr.

Sphaerella Taxodii, Cke.

Sphaeria (Metasphaeria) cavernosa, Ell. \& Ev.

Trametes sepium, B.

Coniferae, sp. indet.

$\{$ Hypoxylon Beaumontii, B. \& C.

\{Valsaria? Beaumontii, Sacc.

Omphalia umbellifera, (L.) var. abiegnus, $\mathrm{Pk}$.

Patellaria subvelata, Ell. \& Ev.

Peziza connivens, Fr.

Plebia vaga, $F$ r.

Phoma Pini, Cke. \& Hark.

Sphaeria (Winteria) coerulea, Ell. \& Ev.

\section{GNETACEAE.}

Ephedra, sp. indet.

SAecidium Pini, var. Ephedrae, B. \& C.

$\{$ Peridermium Pini, var minor, B. \& C.

Peridermium Ephedrae, Cke.

\section{CXCADACEAE.}

Cycas, sp. indet,

Chaetophoma Cycadis, Cke.

Macrosporium commune, Rabh. 


\section{CRYPTOGAMIA.}

\section{EQUISETACEAE.}

\section{Equisetum arvense, L.}

Gloeosporium Equiseti, Ell. \& Ev.

Leptosphaeria arvensis, Speg.

$\{$ Peziza Persooni, Moug.

Stamnaria Equiseti, (Hoffm.) Sacc.

Equisetum hiemale, L,

$\{$ Excipula Equiseti, Pk.

$\{$ Amerosporium Equiseti, Sacc.

f Peziza Persoonii, Moug.

$\left\{\begin{array}{l}\text { Stamnaria Equiseti. (Hoffm.) Sacc. } \\ \text { Stictis Saccardoi }\end{array}\right.$

Stictis Saccardoi, Rehm.

Equisetum laevigatum, Braun.

Gloeosporium Equiseti, Ell. \& Ev.

Leptosphaeria Equiseti, Karst.

Equisetum, sp. indet.

Epicoccum Equiseti, B.

Helotium cyathoideum, (Bull.) Karst.

Septoria Equiseti, Desm.

\section{FILICES.}

Aspidium marginale, Swz.

Fusidium Pteridis, Kalchb.

Aspidium, sp. indet.

Leptostroma Aspidiorum, S.

Cheilanthes Pringlei, Daven port.

Caeoma Cheilanthis, $\mathrm{Pk}$.

Cystopteris fragilis, Bernh.

Uredo Polypodii, (P.) DC.

Caeoma Filicum, Lk.

Uredo Filicum, Chev.

Uredo Aspidiotus, Pk.

Uredo Phegopteris, Auct. Amer.

Onoclea sensibilis, L.

$\{$ Agaricus pterigenus, $\mathrm{Fr}$.

Mycena pterigena, Sacc. Syll.

Septoria mirabilis, $\mathrm{Pk}$.

Uredo macrosperma, Cke.

Gloeosporium Phegopteridis, Pass.

(Melampsora Scolopendrii, (Fckl.) Farl.

?Uredo Filicum, (Lk.) Chev.

Vermicularia filicina, S.

Onoclea Struthiopteris, Hoffm.

? Leptostromella filicina, (B. \& C.) Sacc.
Osmunda cinnamomea, L.

$\{$ Cyphella candida. Pk.

$\{$ Cyphella Perkii, Sacc.

$\{$ Cryptosporium filicınum, B. \& C.

Leptostromella filicina, Sacc.

Solenia filicina, Pk.

Stictis filicina, Pk.

Typhula filicina. Pk.

Osmunda regalis, L.

Caeoma (Aecidium) osmundatum, S.

Hysterium Osmundae, S.

\{ Hypoderma Osmundie, Sacc.

Dothidea Osmundae, Clinton.

Dothidella Osmundae, Sacc.

Phyllachora Osmundae, Cke.

Ustilago Osmundae, $\mathrm{Pk}$.

Osmunda, sp. indet.

Leptostroma filicinum, $\mathrm{Fr}$.

Leptostroma litigiosum, Desm.

f Peziza frondicola, Ell. \& Ev.

$\{$ Pirottaea frondicola. Sacc.

\{ Peziza Osmundae, C. \& E.

\{Trichopeziza Osmundae, Sacc.

Peziza Pteridis, A. \& S.

$\{$ Peziza tenella, C. \& E.

$\{$ Mollisia tenella, Sacc.

Peziza virginea, Batsch.

Vermicularia filicina, S.

Pellaea gracilis, Hook. Uredo Polypodii, (P.) DC.

Phegopteris Dryopteris, Fée.

$\{$ Uredo Polypodii, (P.) DC.

$\{$ Uredo Aspidiotus, Pk.

Polypodium vulgare, L.

Uredo Filicum, (Lk.) Chev.

Pteris aquilina, L.

Agyrium herbarum, Fr.

Cladosporium grumosum, (P.) Lk.

$\{$ Sphaeria pteridicola, B. \& C.

\{ Didymella pteridicola, Sacc.

Gloeosporium necans, E1l. \& Ev.

Gloeosporium leptospermum, Pk.

Gloeosporium Pteridis, Hark.

$\{$ Hysterium Pteridis, S.

\{ Hypoderma Pteridis, Sacc.

Leptostroma filicinum, Fr.

Sphaeria epipteridis, Cke. \& Hark.

Metasphaeria epipteridea, Sacc. 
Ceziza Pteridis, A. S S.

Trichopeziza Pteridis, Rehm.

Trichopeziza pulverulenta, Thm. Myc. Univ.

Septostroma litigiosum, Desm.

$\{$ Dothidea Filicum, S.

Phyllachora Filicum, Sacc.

S Siphaeria Aabella, S.

Dothidea Habella, li. \& C.

Plivllachora Aabellat, Sacc.

f Dothidea leptostromoidea, Cke.

(Phyllachora leptostromoidea, Cke.

(1)othidea Pteridis, (Reb.) Fr.

(Phyllachora Pteridis, Fckl.

Spluaria filicina, Fr.

Dothidea filicina, Fr.

Rbopographus filicinus, Nits.

Sphaerella indistincta, Pk.

f Sploaeria aquilina, Fr.

S Spaerella aquilina, Awd.

Uredo Filicum, (Lk.) Cher.

Filices, sp. indet.

Cyphella filiciola, B. \& C.

Leptostromella filicina, (B. \& C.) Sacc.

f Dialonectria filicina, Cke. \& Hark.

[Nectria filicina, Berl. \& Vog\}.

Phoma aquilina, Sacc. \& Penz.

\section{LYCOPODIACEAE.}

Lycopodium alpinum, L.

Leptosphneria Crepini, (Westd.) DeNot.

Lycopodium annotinum, L.

Sphneria Crepini, Westd.

$\{$ Leptosphaeria Crepini, DeNot.

Heptameria Crepini, Cke.

Sphaeria lycopodina, Nont.

Leptosphaeria lycopodina, Sacc.

Heptameria lycopodina, Cke,

Sphaeria Marciensis, Pk.

$\{$ Leptosphaeria Marciensis, Sacc.

Heptameria Marciensis, Cke.

Lycopodium clavatum, L.

$\{$ Leptosphaeria lycopodiicola, Pk.

\{lleptameria lycopodicola, Cke.

Sphaerella Lycopodii, Pk.

Lycopodiam Selago, L.

\{ Sphaeria Marciensis, Pk.

\{Leptosphaeria Marciensis, Sacc.

Lycopodium, sp. indet.

I Sphaeria Lycopodii, B. \& C.

i. Metasphaeria Lycopodii, Sacc.

\section{MUSCI.}

Bartramia pomiformis, lledw.

Cladosporium epibryum, Cke. \& Massee.

Bartramia, sp. indet,

Dematium Miuscormm, Schleich.
Climacium Americanum, Brid.

$\{$ Pistillaria muscicola, (P.) Fr.

\{Typluta musicola, Fr.

Dicranum scoparium, Hedw.

Vermicularia thecicola, S.

Encalypta rhabdocarpa, Schwaegr.

Grimmia Donniana, Smith.

Grimmia ovata, Web. \& Mohr.

Cladosporium epibryum, Cke. \& Minssee.

Hypnum delicatulum, L.

Helotium bryogenum, Pk.

Pistillaria musicola, (P.) Fr.

Hypnum Nuttallii, Wils.

Leptosphaeria bryophila, Sacc.

Polytrichum formosum, Hedw.

\{ Hendersonia Rauii, Ell.

S Stagonospora Rauii, Sacc,

Polytrichum juniperinum, Willd.

Coniosporium Polytricli, Pk.

Macrosporium Polytrichi, Pk.

Septoria thecicola, B. \& $\mathrm{Br}$.

Ptychomitrinm Gardneri, Lesq.

Cladosporium epibryum, Cke. \& Massee.

Sphagnum, sp. indet.

\{ Agaricus paluster, Pk.

\{Mycena palustris, Sacc. Syll

Agaricus Sphagnorum, P.

Agaricus umbelliferus, L.

Botrytis rhinotrichoides, Sacc. \& El].

Botrytis Sphagnorum, Cke.

Lizonia Sphagni, Cke.

Ulota phyllantha, Brid.

Cladosporium epibryum, Cke. \& Massee.

Musci, sp. indet.

f Agaricus delicatulus, $\mathrm{Pk}$.

\{Nolanea delicatula, Sacc. Syll.

$\{$ Agaricus furfuraceus, P.

\{ Tubaria furfuracea. IV. Smith.

Agaricus Hypnorum, Batsch.

Agaricus (Tubaria) Muscorum, Hoffin.

\{ Agaricus sphaerosporus, B.

OOmplatia sphaerospora, Sacc. Syll.

\{ Agaricus tremulus, Schaeff.

\{ Pleurotus tremulus, Quel.

Badhamia lilacina, ( $F r$.$) Rostf.$

Botrytis Micheneri, B. \& C.

Cantharellus muscigenus, (Bull.) $\mathrm{Fr}$.

Cantharellus Muscorum, (Roth.) Fr.

1 Diderma gasterodes, Phil.

\{ Chondrioderma gasterodes, Cke.

Clavaria tenuis, $\mathrm{S}$.

Cyphella galeata, (Schum.) Fr.

Cyphella muscigena, (P.) Fr.

Helvella lacunosa, Afz.

Hygrophorus coccineus, Fr.

Oligonema brevifilum, Pk. 
$\{$ Perichaena flavidum, Pk.

Oligonema flavidum, Pk.

Peziza axillaris, Nees.

Peziza cerea, Sow.

Peziza subcarnea, C. S P.

Polyporus Macouni, Pk.

$\{$ Polyporus dichrous, Fr., var. Macouni, Sacc. Syll.

f Reticularia aftinis, B. S C.

\{ Trichosporium Curtisii, Massee.

Rhizoctonia Muscorum, Fr.

Thelephora cristata, (P.) Fr.

Thelephora scoparia, Pk.

\section{HEPATICAE.}

Jungermannia, sp. indet.

Sphaeria muscivora, B. \& Br.

$\{$ Nectria muscivora, Cke.

Calonectria muscivora, Sacc.

Hepaticae, sp. indet.

\{ Peziza subcarnea, C. \& P.

\{ Phialea subcarrea, Sacc.

\section{LICHENES.}

Cladonia cariosa, (Ach.) Spreng.

Sphaeropsis Cladoniae, Ell. \& Ev.

Lecidea, sp. indet.

Capillaria rhizomorphina, S.

Parmelia saxatilis, (L.) Fr.

$\{$ Dothidea Pigsotii, B. \& Br.

Homostegia Piggotii, Karst.

Parmelia, sp. indet.

\{ Diplodia Parmeliae, B. \& C.

Diplodina Parmeliae, Sacc.

$\{$ Nectria lecanodes, Wint.

$\{$ Nectria diplocarpa, Ell. \& Ev.

$\{$ Sphaeria Peltigerae, Mont.

\{ Rhaphidospora Peltigerae, Mont.

Peltigera aphthosa, (L.) Hoffm.

Illosporium carneum, Fr.

Peltigera, sp. indet.

$\{$ Agaricus peltigerinus, $\mathrm{Pk}$.

\{ Clitocybe peltigerina. Sacc. Syll.

Pertusaria comnunis, DC.

Illosporium coccineum, Fr.

Pertusaria, sp. indet.

Spinctrina turbinata, (P.) Fr.

Physcia obscura, (Ehrh.) Nyl.

Illosporium roseum, (Schreb.) Mart.

Sticta pulmonaria, (L.) Ach.

Celidium Stictarum, Tul.

Usnea barbata. (L.) Fr.

Fusarium barbatum, Ell. \& Ev.

Lichenes, sp. indet.

Dematium rupicolum, Lk.

Dendrodochium subeffusum, Ell. \& Ev.

Nectria rubefaciens, Ell. \& Ev.

Stigmatella aurantiaca, B. \& C.
FUNGI.

MYYOMYCE'TES.

Arcyria cinerea, (Bull.) Schum.

Stilbum echinatum, Ell. \& Ev.

Stibum tomentosum, Schrad.

Aroyria, sp. indet.

Verticillium Rexianum, Sacc.

Chondrioderma spumarioides, (Fr.) Rostf.

S Nectria Rexiana, Ell.

\{ Lasionectria Rexiana, Cke.

Hemiarcyria clavata, (P.) Rostf.

Stilbum tomentosum, Schrad.

Physarumn, sp. indet.

Verticillium botryoides, Sacc.

Stemonitis, sp. indet.

Botrytis thinotrichoides, Sacc. \& Ell.

Trichia varia, P.

Stilbun capillare, Ell. \& Ev.

\section{PYRENOMYCETES.}

Bertia moriformis, (Tode) De Not.

Barya parasitica, Fckl, var. caespitosa, $\mathrm{Pk}$.

Botryosphaeria Hibisci, (L.) Sacc.

Stigmatella aurantiaca, B. \& C.

Diatrype platystoma, (S.) M. A. Curtis.

\{ Nectria poliosa, EH. \& Ev.

\{Lasionectria poliosa, Ell. \& Ev.

Diatrype Stigma, (Hofim.) Fr.

(Sphaeria cerea, B. \& C.

\{ Calonectria cerea, Sacc.

Cenangitm episphaerium, S.

Thelephora episphaeria, $\mathrm{Fr}$.

$\{$ Corticium episphaerium, M. A. Curtis.

Hymenochaete episphaeria, Massee.

Sphaeria episphaeria, Tode.

$\{$ Nectria episphaeria, Fr.

Dialonectria episphaeria, Cke.

f Tremella fragiformis, S. Syn. Car.

$\{$ Hypsilcphora fragiformis, Cke.

Lophiostoma Floridanum, Ell. \& Ev.

Melanomma parasiticum, Ell. \& Ev.

?Nectria poliosa, Ell. \& Ev.

Ophionectria Everhartii, Ell. \& Gall.

Sothidea episphaeria, Pk.

$\{$ Phyllachora episphaeria, Sacc.

Teichospora mycogena, Ell. \& Ev.

Diatrype tremellophora, Ell.

Byssosplaneria barbicincta, Ell. \& Ev.

\{ Stictis parasitica, Ell. \& Ev.

$\{$ Propolis parasitica, Sacc.

Diatroype, sp. indet.

Cornularia Persicae, (S.) Sacc.

Cucurbitaria setosa, Ell. \& Ev.

f Fusisporium episphaericum, C. \& E.

\{ Fusariun episphaericum, Sacc. 
Itelminth sporium episphaericum, C. \& P.

Sporotriclium anceps. Sacc. \& Ell.

Dichaena quercina, (P.) Fr.

Titaca Clarkei. EH. \& Ev.

Dichaena strumosa, Fr.

\{Helotium strumosum, Ell. \& Ev.

Pseudohelotium strumosum, Sacc.

II ysotheca subcorticalis, (C. \& E.) Ell.

\& Ev.

Titaeil Clarkei, Ell. \& Ev.

Dimerosporium Collinsii, (S.) Thm.

$\{$ Fusisporium parasiticum, $\mathrm{Pk}$.

\{ Fusarium Peckii, Sacc.

Dothidea Kalmiae, Pk.

Peziza Kalmiae, $\mathrm{Pk}$.

Epichloe typhina, (P.) Tul.

$\{$ Capillaria Sphaeriae-typhinae, Cda.

\{ Botrytis Sphaeriae-typhinae, Sacc.

Erysipheae variae.

Cicinnobolus Cesatii, DBy.

Eutypa limaeformis, (S.) B.

Cenangium episplaterium, $\mathrm{S}$.

Eutypella lutescens, (Ell.) Sacc.

S Nectria lasioderma, Ell.

Lasionectria lasioderma, Cke.

Eutypella stellulata, (Fr.) Sacc.

(Nectria episphaeria, (Tode) Fr.

Dialonectria episphaeria, Cke.

Hypoxylon coccineum, Bull.

Botrytis geniculata, Cda.

Tubercularia fungicola, $\mathrm{Pk}$.

Hypoxylon Morsei, B. \& C.

Helotium episphaericum, $\mathrm{Pk}$.

Lophiotrema parasiticum, Pk.

Hypoxylon Sassafras, (S.) B.

Melanomma Commonsii, Ell. \& Ev.

Hypoxylon Tinctor, (B.) Cke.

(Sphaerostilbe gracilipes, Tul.

$\{$ Stilbum coryneoides, Ell. \& Ev.

Graphium coryneoides, Sacc.

Hypoxylon, sp. indet.

\{Amphisphaeria Hypoxylon, Ell. \& Ev.

Melitnomma Iypoxylon, Cke.

Botrytis atroviridis, C. \& E.

Botrytis geniculata, Cda.

Clasterosporium herculenm, Ell.

Nectria aglaeothcle, B. \& C.

f Nectria episphateria, (Tode.) Fr.

\{ Dialonectria episphaeria, Cke.

Peziza episphaeria, Mart.

Sphaeropsis parasitans, B. \& Rav.

Venturia parasitica, Ell. \& Ev.

Massaria sudans, B. \& C.

Valsa parasitica, C. \& E.

Massaria, sp. indet.

Calonectria Dearnessii, Ell. \& Ev.
Nummularia Bulliardi, Tul. ?

Sphaeria sphaerincola, S.

Plowrightia morbosa, (S.) Sacc.

Nectria coccinea, (P.) Fr.

Ilendersonula morbosa, Sacc.

Sphaeronema Persicae, (S.) Ell.

SPericonia sphaerophila, $\mathrm{Pk}$.

$\{$ Sporocybe spluaerospila, Sacc.

Sphaeria clȳpeata Nees.

Sphaeria lagenaria, P.

Sphaeria, sp. indet.

Spluaeria scabriseta, S.

Eutypa scabriseta, B.

Valsa scabriseta, Cke.

Calosphaeria scabriseta, Sacc.

Nectria diploa, B. \& C., var. diminuta, B.

Nectria diminuta, Sacc.

Dialonectria diminuta, Che.

Dialonectria diploa, var. diminuta, Ell. \& Ev.

Calonectria diminuta, Berl. \& Vogl.

Cladosporium episphaerium, S.

\{ Sphaeria viridiatra, S.

\{ Coniochaeta viridiatra, Cke.

Dematium episphaericum, S.

Dematium virescens, $P$.

$\{$ Mucor Fimbria, Nees.

\{ Hydrophora Fimbria, Fr.

Nectria sanguinea, (Sibth.). Fr.

$\{$ Dactylium sublutescens, Pk.

$\{$ Trichothecium sublutescens, Sacc.

Triblidiella rufula, (Spreng.) Sacc.

Peziza Ravenelii, B. \& C.

Ustulina vulgaris, Tul.

Isaria congesta, B, \& C.

$\{$ Nectria episphaeria, (Tode) Fr.

$\{$ Dialonectria episphaeria, Cke.

Valsa Abietis, Fr.

Sphaeria (Nectria) Purtoni, (Grev.) Curr.

Valsa didymospora, Ell.

Cenangium microspermum, Sacc. \& Ell.

FUNGI INIPERFECTI.

Cercospora Polygonorum, Cke.

Botrytis hypophylla, Ell. \& Kell.

Fusarium roseum, Lk.

Epicoccum purpurascens, Ehrb.

Helminthosporium Ravenelii,

II. A. Curtis.

f Dactylium Helminthosporii, Thm.

$\{$ Tricliothecium Helminthosporii, Sacc.

Trichothecium roseum, Fr.

Helmintzosporium, sp. indet.

Helicosporium brunneolum, B. \& C.

Micropera, sp. indet.

$\{$ Sphaeronema Microperae, Cke.

$\{$ Sphaerographium Microperae, Sacc. 
Rhinotrichum, sp. indet.

Helicosporinm griseum, B. \& C.

Sphaeropsis, sp. indet.

Cheiromyces stellatus, B. \& C.

Thyrsidium botryospormm, (Fr.) Mont.

Periconia parasitica, $\mathrm{Pk}$.

Tubercularia, sp. indet.

Tetracolium Tuberculariae, (Nees) Lk.

\section{DISCOMYCETES.}

Cenangium turgidum, $S$.

$\{$ Patellaria cenangiicola, Ell. \& Ev.

\{ Patellea cenangicola, Sacc.

Dermatea olivacea, Ell.

Oospora hyalinula, Sacc.

Geoglossum op liioglossoides, (L.) Sacc.

Hypomyces Geoglossi, Ell. \& Ev.

Papulaspora candicla, Sacc.

Verticillium agaricinum, (Lk.) Cda., var. clavisedum, Sacc.

Geoglossum hirsutum, P.

Hypomyces Geoglossi, Ell. \& Ev.

Helvella elastica, Bull.

f Mycogone cervina, Ditm.

\{ Sepedonium cervinum, Fr.

Patellaria nigrocinnabarina, S.

\{ Peziza Ravenelii, B. \& C.

\{ Cenangium Ravenelii, Sacc.

Peziza helnispherica, Wigg.

Asterophora Pezizne, Cda.

Peziza macropus, P.

SMycogone cervina, Ditm.

$\{$ Sepedonium cerrinum, Fr.

Peziza hinnulea, B. \& Br.

\{ Gymnosporium fulvum, B. \& C.

\{Chromosporium fulrum, Sacc.

Peziza, sp. indet.

Nucor albovirens, Fr.

Rhytisma acerinum, (P.) Fr.

Exosporium sociatum, Ell. \& Ev.

Spathularia, sp. indet.

Hypocrea alutacea, (P.) Ces. \& DeNot.

Taphrina coerulescens, (Mont. \& Desm.)

Tul.

Pestalozzia taphrinicola, Ell. \& Ev.

Phoma parasitica, Ell. \& Ev.

\section{AGARICINI.}

Agaricus abortivus, B. \& C.

$\{$ Sporodinia grandis, Lk.

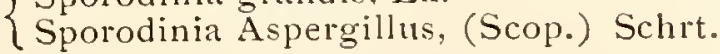

Agaricus dryophilus, Bull.

Tremella mycetophila, $\mathrm{Pk}$.
Agaricus melleus, Vahl.

Aspergillus repens, (Cda.) Sacc.

Cladosporium epimyces, Cke.

Sterigmatocystis butyracea, Bain.

Agaricus salignus, P.

Sphneria cladosporiosa, $\mathrm{S}$.

Agaricus sphaerosporus, B.

Stilbum arcticum, B.

Agaricus velutinus, P.

Cucor ramosus, Bull.

Aspergillus maximus, Ehrb.

Sporodinia grandis, Lk.

Syzygites megalocarpus, Ehrb.

Sporodinia dichotoma, Cda.

Nucor Syzygites, DBy.

[Sporodinia Aspergillus, (Scop.) Schrt.

Agaricus, sp. indet.

Aspergillus candidus, Lk.

Aspergillus virens, Lk.

Botrytis ramosa, S. Syn. Car.

Dactylium dendroides. (Bull.) Fr.

$\{$ Nectria perforata, Ell. \& Holw.

$\{$ Dialonectria perforata, Ell. \& Holw.

Diplocladium minus, Bor.

Sphaeria subulata, Tode.

Sphaeronema subulatum. Fr.

Zythia subulata, S.

Eleutheromyces subulatus, Fckl.

Heterosporium fungicolum, Ell. \& Ev.

Hypomyces ochraceus, (P.) Tul.

$\{$ Hypocrea tomentosa, B.

\{ Hypomyces tomentosus, B.

S Isaria agaricina, P.

\{ Isaria brachiata, (Batsch) Schum.

$\{$ Monilia candida, $\mathrm{Pk}$.

\{ Monilia mycophila, Sacc.

Mucor flavidus, P.

\{ Mucor fusiger, Lk.

$\{$ Spinellus fusiger, Van Tiegh.

Myrothecium fungicolum, Pk.

Myrothecium inundatum, Tode.

Sporotrichum agaricinum, Lk.

Stilbum byssinum, P.

Cantharellus aurantiacus, (Wulf.) Fr.

$\{$ Sphaeria aurantia, P.

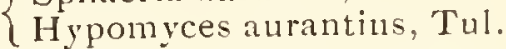

Cantharellus cibarius, $\mathrm{Fr}$.

Hypomyces transformans, $\mathrm{Pk}$.

Cantharellus, sp. indet.

Hypomyces Lactifluorum, (S.) Tul.

Gomphidius, sp. indet.

Hypomyces Lactifluorum, (S.) Tul.

Lactarius deliciosus, (L.) Fr.

Lactarius Indigo, (S.) Fr.

( Hypocrea lateritia, Fr.

$\left\{\begin{array}{l}\text { Hypomyces lateritius, Tul. } \\ \text { Hyctas. }\end{array}\right.$

Lactarius piperatus, (Scop.) Fr.

$\{$ Asterophora physaroides, Fr.

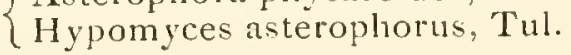


Sphaeria Lactifluorum, S.

Hypomyces Lactitluorum, "Tul.

(Hypocrea Lactilluorum, M. A. Curtis.

Lactarius subdulcis, (Bull.) Fr.

Verticillium Lactari, Pk.

Lactarius torminosus, (Schaefi.) Fr.

Hypocrea Hoccosa, Fr.

Iypomyces lateritius, (Fr.) Tul.

Lactarius, sp. indet.

Iyponyces Banningii. Pk.

f Hypociea tomentosa, B.

$\{1$ ypomyces tomentosus, B.

Lentinus ursinus, $\mathrm{Fr}^{\circ}$.

Coniosporium mycophilum, Ell. \& Langlois. Russula adusta, (P.) Fr.

Hypomrces asterophorus, Tul.

Syaricus lycoperdonoides, Bull.

Asterophora agaricoides, Fr.

Nyctalis Asterophora, Fr.

Russula alutacea, Fr.

Sphaeria viridis. A. S S.

Sphaeria luteovirens, Fr.

Hypocrea luteovirens, Fr.

Hypomyces luteovirens, Tul.

Russula foetens, (P.) Fr.

(Sphaeria hyalina, S.

$\{$ Hypocrea hyalina, Fl:

IIPpomyces hyalinus, $\Gamma u$.

Nictalis parasitica, Fr.

Russula nigricans, (Bull.) Fr.

Nyctalis Asterophora, Fr.

Russula, sp. indet.

Agaricus tuberosus, Bull.

\section{POLYPOREI.}

Boletus, sp. indet.

Aspergillus virens, Lk.

Botrytis ramosa, S. Syn. Car.

Botrytis spicata, Mich.

$\{$ Spliaeria aurantia, P.

\{ Hypomyces aurantius, 'Tul.

Hypomyces chlorinus, Tul: ?

Hypomyces luteovirens, (Fr.) Tul.

Isaria : saccharina, P.

IIucor capitato-ramosus, S.

SMcor paradoxus, B. \& C.

Thamnidium? parodoxum, Berl. \& De'Toni.

Sepedonium chrysospermum, (Bull.) Fr.

Sspergillus maximus, Lk.

Syzygites megalocarpus, Ehrb.

Sporodinia dichotoma, Cda.

Nucor Syzygites. DPy.

(Siporodinia Aspergillus, (Scop.) Schrt.

Daedalea unicclor, Fr.

Stilbum aurifilum. Gerard.

Daedalea, sp. indet.

Calocera fasciculatil, S.
Merulius tremellosus, Schrad.

\{ Sphaeronema oxysporum, B.

\{ Sphaeronemella oxyspora, Sacc.

Merulius, sp. indet.

Hyalopus parasitans, B. \& C.

Polyporus abietinus, (Dicks.) Fr.

Sphinctrina tigillaris, B. \& Br.

Polyporus acanthoides, (Bull.) Fr. Sepedonium flavidum, Sacc. \& Ell.

Polyporus applanatus, Fr.

Ceratostoma parasiticum, Ell. \& Ev.

Thelephora dendritica, B.

Polyporus Berkeleyi, Fr.

Aspergillus repens, (Cda.) Sacc.

Sepedonium flavidum, Sacc. \& Ell.

Polyporus caesius, (Schrad.) Fr.

Polyporus chioneus, $\mathrm{Fr}$.

Hypocrea pallida, Ell. \& Ev.

Polyporus Curtisii, B.

$\{$ Sphaeria Stereorum, S.

Hypocrea Stereorum, B. \& C.

Polyporus elegans, (Bull.) Fr.

$\{$ Pterula setosa, $\mathrm{Pk}$.

Lachnocladium setosum, Sacc. Syll.

Polyporus fomentarius, (L.) Fr.

Helotium mycetophilum, $\mathrm{Pk}$.

Polyporus frondosus, (Schrank.) Fr

Zy thia compressa, S.

Polyporus igniarius,, (L.) Fr.

Institale? maximum, S.

Peziza mycogena, Ell.

Polypor'us loricatus, P.

Hypomyces rosellus, (A. \& S.) Tul.

Polyporus mucidus, (P.) Fr.

Aspergillus maximus, Ehrb.

Nucor ramosus, Bull.

Mucor Syzygites, DBy.

Sporodinia Aspergillus, (Scop.) Schrt.

Polyporus pargamenus, Fr.

Coniosporium mycophilum, Ell. \& Langlois.

S Sphinctrina tigillaris, B. \& Br.

\{ Calicium tigillare, Sacc.

Polyporus piceinus, $\mathrm{Pk}$.

Solenia fasciculata, P.

Polyporus picipes, Fr.

Heterosporium fungicolum, Ell. \& Ev.

Polyporus Schweinitzii, Fr.

Rhinotrichum armeniacum, B. \& C.

Polypor us sulfureus, (Bull.) Fr.

? Sphaeria boleticola, S.

\{Hypomyces boleticolus, Sacc.

Sphaeria cladosporiosa, S.

$\{$ Lasiosphaeria cladosporiosa, Cke.

Coniochaeta cladosporiosa, Cke. 
Polyportus versicolor, (L.) Fr.

Sphaeria aurantia, P.

$\{$ Nectria aurantia, Fr.

Hypomyces aurantius, Tul.

Hypomyces polyporinus, Pk.

Polyporus, sp. indet.

Agaricus (Pleurotus) septicus, Fr.

$\{$ Dictydium magnum, Pk.

Sadhamia magna, Pk.

$\{$ Helminthosporium leptotrichum, C. \& E.

\{ Brachysporium leptotrichum, Sacc.

Cladosporium epiphyllum, Nees.

Diplocladium minus, Bon.

Hydnum nudum, B. \& C.

Hypocrea citrina, (P.) Fr.

Hy pocrea fungicola, (Karst.) Sacc.

Hypocrea rufa, (P.) Fr.

Sphaeria flavescens, Fr., var. epimyces, S.

$\{$ Hypomyces flavescens, Cke.

Botrytis agaricina, Lk.

Botrytis ramosa, S. Syn. Car.

Verticillium agaricinum, Cda.

Hypomyces ochraceus, (P.) Tul.

Hypomyces rosellus, (A. \& S.) Tul.

Hypsilophora fragiformis, Cke.

S Sphaeria lagenaria, P.

$\{$ Melanospora lagenaria, Fckl.

Nucor capitato-ramosus, S.

Mucor rufus, S. Am. Bor.

Mycogone rosea, Lk.

$\{$ Peziza incrustata, E11.

Pseudohelotium incrustatum, Sacc.

Peziza myceticola, B. \& C.

Peziza vulgaris, Fr., var. myceticola, B.

Pezizella vulgaris, Sacc., var. myceticola, Sacc.

?Phyllopta biparasitica, Fr.

$\{$ Polyporus favillaceus, B. \& C.

Poria favillacea, Cke.

Sphaeria mycophila, Fr.

$\{$ Rosellinia mycophila, Sacc.

(Amphisphaeria mycophila, Cke.

Sepedonium chrysospermum, (Bull.) Fr.

$\{$ Sphaeronema oxysporum, B.

Sphaeronemella oxyspora, Sacc.

Stemonitis maxima, S.

Stilbum pellucidum, Schrad.

$\{$ Graphium Berkeleyi, Mont.

\{ Stysanus Berkeleyi, Sacc.

Thelephora granosa, B. \& C.

Trametes, sp. indet.

Sepedonium flavidum, Sacc. \& Ell.

HYDNEI.

Hydnum Erinaceus, Bull.

Hypocrea parasitans, B. \& C.

Hydnum me mbranaceum, Bull.

Fusarium hydnicolum, Ell. \& Ev.
Hydnum, sp. indet.

Dematium parasiticun, $\mathrm{Pk}$.

S Sphaeria hydnicola, S.

\{ Hypoxylon hydnicolum, Sacc.

\{ Peziza cyphelloides, Ell. \& Ev.

Lachnellula cyphelloides, Sacc.

Virgaria hydnicola, $\mathrm{Pk}$.

\section{THELEPHOREI.}

Corticinm, sp. indet.

Chlorosplenium epinyces, Cke.

Melanomma spiniferum, Ell. \& Ev.

Zygodesmus bicolor, C. \& E.?

Peniophora, sp. indet.

Dendryphium pachysporum, Ell. \& Ev.

Stereum bicolor, (P.) Fr.

$\{$ Leptosphaeria stereicola, Ell.

$\{$ Heptameria stereicola, Cke.

Stereum fasciatum, (S.) Fr.

$\{$ Sphaeria Stereorum, S.

\{ Hypocrea Stereorum, B. \& C.

Stereum frustulosum, (P.) Fr.

Stictis stereicola, B. \& C.

Stereum spadiceum, Fr.

Penicillium Hypomycetis, Sacc.

Stilbum sebaceum, E11. \& Ev.

Stereum subpileatum, B. \& C.

Diplocladium melleum, (B. \& Br.) Sacc.

Stereum sp. indet.

Dialonectria sulfurea, E11. \& Calkins.

Hypomyces aurantilis, (P.) Tul.

$\{$ Sphaeria Porothelia, B. \& C.

$\{$ Melanomma Porothelia, Sacc.

Thelephora, sp. indet.

?Phyllopta biparasitica, Fr.

\section{CLAVARIEI.}

Clavaria gigantea, S.

\{ Tremella parasitica, S.

\{ Phyllopta parasitica, Fr.

Clavaria cristata, $\mathrm{P}$.

Chaetosphacria Clavariarum, (Desm.)

Massee.

Clavaria pistillaris, L.

?Hypomyces apiosporus, Cke.

Clavaria, sp. indet.

Dactylium dendroides, (Bull.) Fr.

$\{$ Helminthosporium Clavariarum, Desm.

$\{$ Scolecotrichum Clavariarum, Sacc.

\section{TREMELLINI.}

Exidia, sp. indet.

Hypocrea citrina, (P.) Fr.

Tremella, sp. indet.

Sphaeronema epiglaeum, B. \& C. 


\section{GASTEROMYCETES.}

'Cyathus vernicosus, (Bull.) DC. Fusarium miniatum, (B. \& C.) Sacc. Lycoperdon, sp. indet.

Cladosporium lycoperdinum, Cke. Scleroderma vulgare, Hornem.

Boletus parasiticus, Bull.

Fusarium Sclerodermatis, Pk.

\section{UREDINEAE.}

\section{Uredineae variae.}

Darluca Filum, (Bir.) Cast.

('Tubercularia persicina, Ditm.

\{ Tuberculina persicina, Sacc.

Aecidium Podophylli, S.

Macrosporium Podophylli, Ell. \& Ev.

Aecidium porosum, $\mathrm{Pk}$.

Tubercularia persicina, Ditm.

Sinchytrium Jonesii, Pk.

Tuberculina Jonesii, Sacc.

Tuberculina persicina, Sacc.

Gymnosporangium macropus, Lk.

Discosia Podisomatis, C. \& E.

Puccinia Seymeriae, Burrill.

Fusarium parasiticum, EH. \& Kell.

\section{MISCELLANEA.}

Achlya, sp.indet.

Olpidium Saprolegniae, Braun.

Elaphomyces granulatus, Fr.

Cordyceps ophioglossoides, (Ehrh.) Lk.

Mucor Mucedo, L.

$\{$ Botrytis Jonesii, B. \& Br.

\{ Chaetocladium Jonesii, Fres.

\section{Pachyma Cocos, S.}

Granularia eurotioides, Sacc. \& Ell.

Hypocrea solenostoma, B. S Rar.
Peronospora obovata, Bon.

Macrosporium parasiticum, Thm.

$R$ hizomorpha, sp. indet.

$\{$ Peziza Rhizomorpha, Ell. \& Ev.

$\{$ Plectania Rbizomorpha, Sacc.

Ustilago Avenae, (P.) Jensen.

Fusarium Ustilaginis, Kell. \& Swingle.

Macrosporium utile, Kell. \& Swingle.

Frungi, sp. indet.

\{ Agaricus cirrhatus, Schum.

$\{$ Collybia cirrhata, Sacc. Syll.

$\{$ Agaricus epimyces, Pk.

\{Panneolus epimyces, Sacc. Syll.

Cladosporium herbarum, (P.) Lk.

Sphaeria tubericola, S.

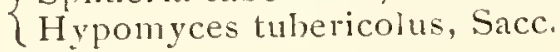

Isaria citrina, $P$.

Isaria intricata, $\mathrm{Fr}$.

Isaria monilioides, var. b., S. Syn. Car.

S Monilia candida, Pk.

\{ Monilia mycophila, Pk.

Myrothecium fungicolum, $\mathrm{Pk}$.

Myrothecium inundatum, Tode.

Nectria mycetophila, $\mathrm{Pk}$.

$\{$ Nectriella nuycetophila, Sacc.

Dialonectria mycetophila, Cke.

Penicillium bicolor, Fr.

Penicillium candidum, Lk.

Sepedonium brunneum, Pk.

Sepedonium roseum, (Lk.) Fr.

Zygodesmus bicolor, C. \& E.

\section{ALGAE.}

Callithamnion cruciatum, Agardh. Callithamnion Plumula, Lyngb. Callithamnion Pylaisaei, Mont. Chytridium Plumulae, Cohn.

Sphacelaria, sp. indet.

Chytridium Sphacellarum, Kny. 


\section{ANTMALS.}

Under the present heading we have included only the species of fungi which specially concern systematic mycologists and have thought it best to omit an enumeration of the pathogenic forms which are specially treated in works on hmman or animal pathology. By far the greater part of the latter belong to the Schizomycetes, an order not included in the present work for reasons already stated in the preface. The fungi like Trichoplyton tonsurans, Microsporon Furfur, etc., to which is attributed the eause of certain skin diseases and the Aspergilli which inhabit the external ear are fonnd in the United States as in other parts of the world but they are more conveniently treated in medieal than in botanical works like the present. There is a small number of fungi which inhabit vertebrate animals which for the convenience of the student, may be mentioned in this connection without any attempt to present a complete list. Sporendonema myophitum, Sacc. has been recorded on a living monse and on the same animal an unnamed Torula or Oidium has been noted by Leidy, Proc. Acad. Nat. Sei. Philad. 1873, 260; Onygena equina, (Willd.) P. has occasionally been found on dead hoofs of horses while on bones of animals occur Fusisporium ossicolum, B. \& C. (Fusa, rium ossicolum, Sacc.) ; Gymnoascella aurantiaca, Pk., referred by Saceardo first to Myxotrichum and later to Gymnoascus; Rhopalomyces elegans, Cda. and R. strangulatus Thaxter. On feathers of birds occurs Fusarium gallinacenm, Cle. \& Hark. On turtles and frogs Achlya racemosa Hildb. and Ach. polyandra Hildb. have been noted in this country as well as an unnamed Saprolegnia on the salamander, MFenobranchus lancenlatus. Saprolegnia ferax, (Gruith.) Kütz. has been reported on several kinds of fish, especially salmon; the donbtful fungus, Devoea infundibilis, Lockwood, ocenrs on Hippocampus heptagona and on dried codfish there is occasionally found Torula pulvinata, Farl. (Oidium pulvinatum, Farl. nec B. \& C. and O. Morrhuae Farl.). For the animals below fishes we have included all the species of fungi, excluding Schizom. ycetes, known to occur in the United States and we are indebted to Prof. Roland Thaxter for revising the fungi found on insects. Among the latter Isaria sphecophita Ditm. on Polistes sp. and Botrytis Bassiana Bals. on Hyphantria have been omitted, the determination in those cases being erroneons. 


\section{INSEC'TS.}

HEXAPODA.

H MIENOPTERA.

Formizariae, sp. indet.

Cordiceps myrmecophila, Ces.

Halictus?, sp indet.

Empusa sphaerosperma, (Fres.) Thaxter.

Iclnneumonidae, sp. indet.

Empusa sphaerosperma, (Fres.) Thaxter.

Tentlr redinidae, sp. inclet.

Empusa Tenthredinis, (Fres.) Thaxter.

Hymenoptera, sp. indet.

$\{$ Botrutis tenella, Sacc.

Botrytis Bassiana, Bals., var. tenella, Sacc.

\section{LEPIDOPTERA.}

Agrotis femnica, (Tausch).

Empusa virescens, Thaxter.

Agrotis, sp. indet.

Empusa Grylli, (Fres.) Nowakowski, forma

Aulicae, (Reich.)

Arctiidae, sp. indet.

Empusa Grylli, (Fres.) Nowakowski.

Bombyx Mori, L.

Botrytis Bassiana, Bals.

Colias philodice, Godt.

Empusa sphaerosperma, (Fres.) Thaxter.

Deltoidinae, sp. indet.

Empusa apiculata, Thaxter.

Eupithecia, sp. indet.

Empusa greometralis, Thaxter.

Hyphantria cunea, Drury.

Empusa apiculata, Thaxter.

Empusa Grylli, (Fres.) Nowakowski, torma Aulicae, (Reich.)

Lithophanae, sp. indet.

Mamestra, sp. indet.

Orgyia nova, Fitch.

Empusa Grylli, (Fres.) Nowikowski, forma Anlicae, (Reich.)

Petrophora, sp. indet.

Empusa apiculata, Thaxter.

Empusa geometralis, 'Thaxter.

Phlegethontius Carolina, lluebn.

Phlegethontius Celeus, Burm.

Empusa Grylli, (Fres.) Nowakowski, forma

Aulicae, (Reich.)

Pieris Rapae, L.

Empusa sphaerosperma, (Fres.) Thaxter.
Plusia Brassicae, Riley.

Botrytis Rileyi, Farl.

Splingidae, sp. indet.

J Isaria Sphingum, S.

Torrubia Sphingum, Tul.

lCordyceps Sphingum, Sacc.

Thera, sp. indet.

Empusa geometralis, Thaxter.

Tortrix, sp. indet.

Empusa apiculata, Thaxter.

Lepidoptera, sp. indet.

Aspergillus flavus, Lk.

Potrytis tenella Sacc.

Cordyceps isarioides, M. A. Curtis.

\{ Isaria farinosa, Fr.

$\{$ Silbum ramosum, Pk.

Isaria nigripes, S.

Isaria Sphingum S.

\section{DIPTERA.}

Chironomus, sp. indet.

Empusa conica, (Nowakowski) Thaxter.

Empusa montana, Thaxter.

Culex, sp. indet.

Empusa Culicis, A. Braun.

Culicidae, sp. indet.

Empusa sphaerosperma, (Fres.) Thaxter.

Drosophila nigricomis, Loew.

(Appendicularia entomophila, Pk.

$\{$ Appendiculina entomophila, Berl.

Stigmatomyces entomophilus, Thaxter.

Lucilia Caesar, L.

Empusa Americana, Thaxter.

Empusa Muscae, Cohn.

Mussa domestica, L.

Empusa Americana, Thaxter.

f Empusa Muscæ. Cohn.

\{ Entomophthora Muscae, Fres.

Musca vomitoria, L.

Empusa Americana, Thaxter.

Empusa Muscae, Cohn.

Musca,:sp. indet.

Empusa sphaerosperma, (Fres.) Thaxter.

Mycetophilidae, sp. indet.

Empusa dipterigena, Thaxter.

Empusa sphaerosperma, (Fres.) Thaxter.

Sapromyza Iongipennis, Meigen.

Enpusa echinospora, Thaxter. 
Syrphus sp. indet.

Empusa Muscre, Cohn.

Tipula, sp.indet.

Empusa Caroliniana, Thaxter.

Empusa conglomerata, (Sorokin) Thaxter?

Empusa dipterigena, Thaxter.

Tipulidae, sp. indet.

Empusa Grylli, (Fres.) Nowakowski?

Empusa sepulchrilis, Thaxter.

Empusa sphaerosperma, (Fres.) Thaxter.

Diptera, sp. indet.

Achlya prolifera, Nees.

Empusa apiculata, Thaxter.

Saprolegnia ferax, (Gruith.) Kiitz.

Diptera (gnats).

Empusa gracilis, Thaxter.

Empusa papillata, Thaxter.

Empusa variabilis, Thaxter.

\section{COLEOPTERA.}

Anchylonyca, sp. indet.

Cordyceps Ravenelii, B. \& C.

Bembidium, sp. indet.

Laboulbenia truncata Thaxter.

Bidessus lãustris, Say.

Zodiomyces vorticellarius, Thaxter.

Bledius assimilis, Faurel.

Cantharomyces Bledii, Thaxter.

Carabidas, sp. indet.

Laboulbenia paupercula, Thaxter.

Casnonia Pennsylvanica, Dejean.

Laboulbenia Casnonize, Thaxter.

Chauliognathus Pannsylvanicus, De G.

Empusa Lampyridarum, Thaxter.

Chilocorus bivulnerus, Mulsant.

Hesperomyces virescens, Thaxter.

Haltica bimarginata. Say.

Sporot richum larvicolum, $\mathrm{Pk}$.

Harpalus Pennsylvanicus, De G.

Laboulbenia archata, Thaxter.

Laboulbenia conferta, Thaxter.

Laboulbenia elegans, Thaxter.

Laboulbenia Harpali, Thaxter.

Lachnosterna fusca, Frohl.

Botrytis tenella, Sacc.

$\{$ Torrubia Melolon thae, Tul.

Cordyceps Melolonthae, Sacc.

Cordyceps Ravenelii, B. \& C.

$\{$ Torrubia elongata, Riley.

Torrubia Ravenelii, B \& C.

Lachuosterna quercina, Knoch.

$\{$ Cordyceps militaris, (L.) Lk.

\{ Torrubia militaris, Tul.

Lampyridae, sp. indet.

Empusa sphaerosperma, (Fres.) Thaxter.

Nebria pallipes, Say.

Laboulbenia Nebriae, Peyritsch.
Nictobates?, sp. indet.

f Cordyceps Carolinensis, B. \& Rav.

\{ Cordyceps acicularis, B. \& Rav.

Passalus cornutus, Fabr.

Achlya prolifera, Nees.

Arthromitus cristatus, Leidy.

f Clidophytum ramosissimum, Leidy.

\{ Cladophytum comatum, Leidy.

Corynocladus radiatus, Leidy.

Cryptodesma tenuis, Leidy.

Enterobryus attenuatus, Leidy.

Patrobus longicornis, Say.

Laboulbenia brachiatil, Thaxter.

Phytonomus punctatus. Fabr.

SEtomophthora Phytonomi, Arthur.

(Empusa sphaerosperma, (Fres.) 'Thaxter.

Platynus cincticollis, (Say).

Laboulbenia elongata, Thaxter.

Laboulbenia fumosa, Thaxter.

Laboulbenia Rougetii, Mont. \& Robin.

Peyritschiella curvata, Thaxter.

Peyritschiella minima, Thaxter.

Platynus extensicollis, Say.

Laboulbenia paupercula, Thaxter.

Laboulbenia scelophila, Thaxter.

Ptilodactyla serricollis, (Say).

Empusa apiculata, Thaxter, var. major, Thaxter.

Scarabeidae, sp. indet.

Cordyceps Miquelii, (Tul.) Sacc.

Sunius longiusculus, Mann.

Cantharomyces verticillatus, Thaxter.

\section{HEMIPTERA.}

Aphis Mali, Fabr.

Empusa Fresenii, Nowakowski.

Aphis, sp. indet.

Cladosporium Aphidis, Thm.

Empusa sphaerosperma, (Fres.) Thaxter.

4 plides, sp. inclet.

Empusa Aphidis, (Hoffm.) Thaxter.

Empusa lageniformis, Thaxter.

Empusa occidentalis, Thaxter.

Empusa Planchoniana, (Cornu?) Thaxter.

Cicada septendecem, L.

Massospora cicadina, Pk.

Cicada, sp. indet.

Vermicularia cicadina, Ell. \& Kell.

Coccus, sp. indet.

?Nectria aglaeothele, B. \& C.

Coccidae, sp. indet.

S Nectria coccicola, Ell. \& Ev.

Ophionectria coccicola, Berl. \& Vogl.

(Dialonectria coccicola, Ell. \& Ev. 
Saprolegnia ferax, (Gruith.) Kütz.

Sphaerostilbe coccophila, Tul.

Lecanium, sp. indet.

Sorrubia clavulata, S.

$\{$ Cordyceps pistillariaeformis, B. \& Br.

Cordrceps clavulata, Ell. \& Ev.

Schizonenra imbricator, (Fitch).

Scorias spongiosa (S.) Fr.

Typhlocyba Mali,

Empusa sphaerosperma, (Fres.) Thaxter.

Typhlocyba,.sp. indet.

Empusa apiculata, Thaxter.

\section{THYSANOPTERA.}

Thrips, sp. indet.

Empusa sphaerosperma, (Fres.) Thaxter.

\section{ORTHOPTERA.}

Acridii, sp. indet.

$\{$ Empusa Grylli, (Fres.) Nowakowski.

\{ Entomophthora Calopteni, Bessey.

Caloptenus differentialis, Thomas.

\{ Empusa Grylli, (Fres.) Nowakowski.

Entomophthora Calopteni, Bessey.

Ceuthophilus, sp. indet.

Empusa Grylli, (Fres.) Nowakowski.

Gryllidae, sp, indet.

Cordyceps albida, B. \& C. Herb.

Tomonotus, sp. indet.

Empusa Grylli, (Fres.) Nowakowski.

\section{NEUROPTERA.}

Phryganeidae, sp. indet.

Empusa rhizospora, Thaxter.

\section{MISCELLANEA.}

Hexapoda, sp. indet.

$\{$ Cordyceps entomorrhiza. (Dicks.) Fr.

\{ Torrubia entomorrhiza, Tul.

Cordyceps herculea, (S.) Sacc.

Cordyceps insignis, Cke. and Ray.

Cordyceps palustris, B. \& Br.

Cordyceps stylophora, B. \& Br.

$\{$ Torrubia superficialis, Pk.

Cordyceps superficialis, Sacc.

Isaria crassa, S.

Isaria furcata, S.

Isaria tenuipes. Pk.

\section{ARACHNIDA.}

Araneina, sp. indet.

Isaria arachnophila, Ditm.

Isaria aranearum, S.

\section{MYRIAPODA}

JuIus marginatus, Say.

Artluromitus cristatus, Leidy.

Cladophytum comatum, Leidy.

Enterobryus elegans, Leidy.

Julus pusillus, Say.

Enterobryus spiralis, Leidy.

Polydesmus granulatus, Say.

Eccrina moniliformis, Leidy.

Polydesmus Virginiensis, (Drury).

Arthromitus cristatus, Leidy.

Cladophytum comatum, Leidy.

Eccrina longa, Leidy.

Spirobolus marginatus, (Say.)

$\{$ Arthromitus nitidus, Leidy.

\{Arthromitus cristatus, Leidy.

Enterobryus elegans, Leidy.

\section{CRUSTACEANS.}

Crawfish.

Saprolegnia ferax, (Gruith.) Kütz.

\section{WORMS.}

Aorurus, sp. indet.

Enterobryus elegans, Leidy.

Ascaris infecta, Leidy.

Arthromitus cristatus, Leidy.

Cladophytum comatum, Leidy.

Enterobryus elegans, Leidy.

Ascaris sp indet.

Achlya prolifera, Nees.

Streptostomum agile, Leidy.

Arthromitus cristatus, Leidy.

Cladophytum comatum, Leidy.

Enterobryus elegans, Leidy.

Taenia Solium, Rud.

Mucor Taeniae, Fairman.

Thelastomum attenuatum, Leidy.

Arthromitus cristatus, Leidy.

Cladophytum comatum, Leidy .

Enterobryus elegans, Leidy. 


\section{ADDENDA.}

\section{RANUNCULACEAE.}

Aconitum Fischeri, Reich.

Aecidium Aconiti-Napelli, (DC.) Wint.

\{Uromyces Aconiti, Fckl.

\{ Uromyces Aconiti-Lycoctoni, (DC.) Wint.

Anemone Caroliniana, Walt.

Aecidium punctatum, P.

Anemone cylindrica, Gray.

Aecidium Ranunculacearum, DC.

Didymaria Ungeri, Cda.

Phleospora Anemones, Ell. \& Kell.

$\{$ Puccinia Anemones-Virginiunue, S.

$\{$ Puccinia compacta, DBy.

Anemone dichotoma, L.

Anemone Hepatica, L.

$\{$ Peronospora pygmaea. Ung.

\{ Plasmopara pygmaea, Schrt.

Anemone multifida, DC.

Aecidium Ranunculacearum, DC.

Peronospora pygmaea, Ung.

Puccinia fusca, (Relh.) Wint.

Anemone nemorosa, L.

\{ Peronospora pygmaea, Ung.

$\{$ Plasmopara pygmaea, Schrt.

Anemone parviflora, Michx.

Pleospora stenospora, Schrt.

Anemone Virginiana, L.

Phleospora Anemones, Ell. \& Kell.

Aquilegia Canadensis, L.

Cercospora Aquilegiae, Kell. \& Swingle.

Erysiphe communis, (Wallr.) Schl.

Aquilegia coernlea, James.

Aecidium Aquilegiae, $P$.

Aquilegia Jonesii, Parry.

Sphaerella Aquilegiae, Ell. \& Ev.

Clematis Douglasii, lJook.

Aecidium Clematidis, DC.

Clematis Jackmanii, Spec. cult.

Cylindrosporium Clematidis, Ell. \& Ev., var. Jack manii, E1l. \& Ev.

Clematis ligus icifolia, Nutt.

\{ Lophiostoma Montaniense, Ell.

$\{$ Ceriospora Montaniensis, Berl.

Cercospora Rubigo, Cke. \& Hark.

Cercospora squalidula, $\mathrm{Pk}$.

$\{$ Pleospora permunda, (Cke.) Sacc.

\{ Clathrospora permunda, Berl.

Erysiphe communis, (Wallr.) Schl.
Parodiclla fruticola, Ell. \& Ev.

Spharella applanata, EIl. \& Ev.

Clematis Virginiana, L.

\{Caeoma (Aecidium) Clematitatum, S.

$\{$ Aecidium clematitatum, $P k$.

Coptis trifolia, Salisb.

Septoria cercosperma, Rostrup.

Paeonia officinalis, DC.

Cladosporium Paeoniae, Pass.

Paeonia, sp. indet.

$\{$ Polyactis vulgaris, Lk.

$\{$ Botrytis vulgaris, Fr.

Erysiphe communis, (Wallr.) Schl.

Phyllosticta Commonsii, Ell. \& Ev.

Ranunculus abortivus, L.

Septocylindrium Ranunculi, Pk.

Peronospora Ficariae, Tul.

Ramularia Ranunculi, Pk.

Ranunculus acris, L.

Cladosporinm herbarum, (P.) Lk.

Ranunculus affinis, R. Br.

Leptosphaeria Ranunculi, Rostrup.

Ranunculus Cymbalaria, Pursh.

Aecidium Ranunculacenrum, DC.

Erysiphe communis, (Wallr.) Schl.

Ranunculns nivalis, L.

Septoria cercosperma, Rostrup.

Sphaerella pachyasca, Rostrup.

Stigmatea Ranunculi, Fr.

Ramunculus pygmaens, Wahl.

Stigmatea Ranunculi, Fr.

$R$ anunculus repens, L.

Erysiphe communis, (Wallr.) Schl.

Ramularia gibba, Fckl.

Ranunculus repens, L. var. hispidus, Torr.

$\{$ Peziza singularia, $P k$.

$\{$ Mollisia singularis, Sacc.

Ranunculus sceleratus, L.

Erysiphe communis, (Wallr.) Schl.

Ranunculus, sp. indet.

Ascochyta? infuscans, E11. \& Ev.

Thalictrum alpinum, L.

Aecidium Sommerfeltii, Johanson.

Leptosphaeria Thalictri, Wint.

Lizonia Thalictri, Rostrup.

Sphaerella pachyasca, Rostrup.

Thalictrum Cornuti, L.

Aecidium punctatum, $\mathrm{P}$. 
S Accilimm Thelictri, crres.

Aecidium Thalictri-tla.i, (1)(.) Wint.

Accidium Sommerfeltii, Johanson.

Prziza Thalictri, Pk.

Pyrenoperiza Thalictri, Sacc.

Thalictrum Fendleri, Engelm.

1 Aecidium Thalictri, Grew.

Tecidium Thalictri-flui, (DC.) Wint.

Aecidium punctatum, P.

Thalictrum purpurascens, L.

Accidium Sommerfeltii, Johanson.

Septoria Thalictri, Ell. \& Ev.

Symchytrium Anemones, (1)C.) Wor.?

\section{CALYCANTHACEAE.}

Calycanthus, sp. indet.

I Patellaria nigrocinnabarina, S.

Blitrydium nigrocinnabarinum, Sacc.

Tubercularia Calycanthi, S.

\section{MIGNOLIACEAE.}

\section{Liriodendron Tulipifera, L.}

llypoxylon rubiginosum, (P.) Fr.

(Polyporus corticola, Fr., var. Tulipiferae, Fr.

$\{$ Irpex Tulipiforae, S.

Poria Tulipiferae, Sacc. Syll.

(Leptosphueria stictoides, (B. \& C.) Sacc

Leptameria stictoides. Cke.

Monitio aurea, var. aurantiaca, S. Syn. Car.

f Patellaria nigrocinnabarina, S.

$\{$ Blitrydium nigrocinnabarinum. Sacc.

$\{$ Peziza Aurclia, P.

\{ Belonidium Aurelia, DeNot.

\{ Pczizu Erinucens. S

L Lachnea Erinaceus, Sacc.

\{ Peziza ainosa, A. d S.

OOrbilia vinosa, Karst.

f Polyporns cincrens, S.

\{ Poriat cinerea, Cke.

I Polyporus fuleus, Fr.

\{ Fomes fulvus, Karst.

(Polyporus nigromarginatus, S.

Polystictus hirsutus, (Schrad.) Fr.

(Polystictus nigromarginatus, Cke.

$\{$ Polyorus Nitgheriensis, Mont.

$\{$ Polystictus Nilgheriensis, Fr.

$\{$ Polyporus scarrosus, B. \& C.

\{ Polystictus scariosus. Cke.

Ramularia Liriodendri. Ell. SiEv.

$\{$ Sphacria Tulipiferae, $S$.

S Sphaerella Tulipiferae, Cke.

Sporidesunium sanguineum, Ell. \& Ex.

I Stictis histerima, Fr.

i Kylogrammal hysterinum, Rehm.

Marnoliz glanca, L.

\{ Corticium subsigantom, $B$.

\{ leniophora subgigantea, Massce.

F) iaporthe Amcricana, Speg.
Exidia glandulosa, (Bull.) Fr.

ITelminthosporium Arbuscula, B. \& C., var. trichellum, Sacc.

Nummularia hypophlaea, (B. \& Rav.) Cke.

S Peziza albopileata, Cke.

\{Dasyscypha albopileata, Sacc.

Seziza protrusa, B. \& $C$.

$\{$ Pyrenopeziza protrusa, Sacc.

Pseudopeziza protrusa, Sacc.

Saccardia Martini, Ell. \& Sacc.

Volutella conorum, Ell. \& Ev.

\section{Magnolia grandiflora, L.}

Asterinula Langloisii, Ell. \& Ev.

(Discosia alnea, (P.) B.

$\{$ Discosia nitida, Lev.

Discosia ocellata, B. \& C.

$\{$ Peziza hystricula, Ell. \& Ev.

\{ Dasyscypha hystricula, Sacc.

Magnolia, sp. indet.

PYRENOMYCETES.

Anthostomella Magnoliae, Ell. \& Ev.

Diatrype Stigma, (Hoffim.) Fr.

Cucurbitaria congesta, C. \& E.

$\{$ Dothidea Magnoliae, Cke.

\{Homostegia Magnoliae, Sacc.

Dothidea Scutula, B. \& C.

Hypocrea minima, Sacc. \& Ell.

Hypoxylon annulatum, (S.) Mont., var. b.

Hypoxylon tinctor, (B.) Clie.

Lasiosphaeria subvelutina, Ell. \& Ev.

?Lophodermium exaridum, C. \& P.

fIlysterium foliicolum. Fr.

Lophodermium hysterioides, (P.) Sacc.

\{Hysterium maculare, Fr.

Lophodermium maculare, De Not.

Nectria Russellii, B. \& C.

Sphaerella annulata, Cke.

Venturia parasitica, Ell. \& Ev.

Xylaria corniformis, Fr.

Xylaria filiformis, (A. S S.) Fr.

SPIIAEROPSIDEAE.

Diplodia Ravenelii, Cke.

Phoma atomispora, Cki.

fDiplodia tephrospora, M. A. Curtis.

S Splacropsis tephrospora, B. \& C.

MELANCONIEAE.

Melanconium ramulorum, Cda.

Naeinaspora rufa, B. \& C.

HYPHOMYETES.

Acrothecium obovatum, C. \& E.

$\{$ Polyactis curta, B. \& C.

\{ Botrytis curta, Sacc.

Dendrodochium rubellum, Sacc., var. macrosporum, Sacc.

Dendryphium quadriseptatum, Cke.

Glenospora Curtisii, B. \& Desm.

Haplaria chlorina, Ell. \& Ev. 
f IIelicoma velutinum, Ell.

\{Ielicosporium velutinum, Sacc.

$\{$ Illosporium acaroides, Sacc.

$\{$ Volutellaria acaroides, Sacc.

?Illosporium Pezizula, Ell. \& Sacc.

Menispora glanconigra, C. \& E.

Ovularia monilioides, Ell. S Martin.

Penicillium repens, C. \& E.

Polyscytalum sericeum, Sacc.

Speira minor, Sacc.

Stachylidium fuscum, C. \& E.

Verticillium candelabrum, Bon.

Virgaria globiger, Sacc. \& Ell.

Zygodesmus laevisporus, Cke.

Zygodesmus pannosus, B. S C.

\section{DISCOMYCETES.}

Chlorosplenium aeruginosum, (P.) De Not.

Dermatea Magnoliae, B. \& C.

$\{$ Peziza diaphanula, Cke.

\{ Pezizella diaphanula, Sacc.

\{ Peziza fumigata, Ell. \& Ev.

\{ Mollisia fumigata, Sacc.

\{ Peziza Glenospora, Ell. \& Ev.

\{Mollisia Glenospora, Sacc.

\{ Peziza gracilipes, Cke.

Sclerotinia gracilipes, Sacc.

f Peziza incondita, Ell.

$\{$ Sclerotinia incondita, Sacc.

\{ Peziza introspecta, Cke.

$\{$ Belonidium introspectum, Sacc.

\{ Peziza phlegmacea. El\}.

$\{$ Belonidium? phlegmaceum, Sacc.

\section{HYMENOMYCETES.}

\{ Agaricus conigenoides, Ell.

$\{$ Collybia conigenoides, Sacc. Syll.

Coniophora fulva, Massee.

Corticium glabrum, B. \& C.

Corticium laeve, $\mathrm{P}$.

Hymenochaete corticolor, B. \& Rav.

Irpex sinmosus, Fr.

Kneiffia setigera, Fr.

Polyporus adustus, (Willd.) Fr.

Poly porus cinnabarinus, (Jacq.) Fr.

Polyporus hemileucus, B. \& C.

Polyporus spumens, Fr.

Polyporus velutinus, (P.) Fr.

Poria nitida, $\mathrm{P}$.

Solenia sulfurea, Sacc. \& Ell.

\section{ANONACEAE.}

\section{Asimina triloba, Dunal.}

Corticium incarnatum, $\mathrm{Fr}$.

Phoma microsporella, Karst. \& Hariot.

\section{MENISPERMACEAE.}

Menispermum Canadense, L.

Cercospora Menispermi, Ell. \& IIolw.

\section{BERBERIDACEAE.}

Berberis repens, Lindl.

Aecidium Perberidis, Gmel.

Podophyllum peltatum, L.

$\{$ Accidium Podophylli, S.

$\{$ Puccinia aurea, Spreng.

Gloeosporium podophyllinum, Ell. \& Ev.

Macrosporium Podoplyyli, Ell. \& Ev.

NYMPIIAEACEAE.

Nelnmbinm, sp. indet.

Dothiorella Nelumbii, E1l. \& Anders.

Nupliar advena, Ait. f.

Phyllosticta fatiscens, $\mathrm{Pk}$.

Phyllosticta Orontii, Ell. \& Martin, var. advena, Ell. \& Ev.

Sphaerella paludosa, E11. \& Ev.

\section{SARRACENIACEAE.}

Sarracenia flava, L.

Seziza atrata, $P$.

$\{$ Pyrenopeziza itrata, Fckl.

Mollisia atrata, Karst.

Sarracenia purpurea, L.

f Leptosphatria scapophila, (Pk.) Sacc.

IIeptameria scapophila, Cke.

\section{PAPAVERACEAE.}

Argemone platyceras. Lk. \& Otto.

Altemaria lancipes, Ell. \& Ev.

Bocconia cordata, Willd.

$\{$ Leptosphaeria Bocconiae, (C. \& E.) Sacc.

\{Heptameria Bocconiae, Cke.

Eschscholtzia Californica, Cham.

\{ Peziza Eschscholtziae, Phil. \& Hark.

\{ Phialea Eschscholtziae, Sacc.

Papaver nudicanle, L.

Physalospora polaris, Rostrup.

Pleospora papaveracea, (De Not.) Sacc.

Sphaerella arthopyrenioides, Awd.

\section{FUMARIACEAE.}

Corydalis anrea, Willd, var. occidentalis, Gray.

(Aecidium Dicentrae, Trelease, forma Corydalis, Ell. \& Kell.

Aecidium Fumariacearum, Kell. \& Swingle.

Aecidium Corydalis, Webber. 


\section{CRUCIFERAE.}

Arabis alpina, L.

Metasphacria Arabidis, Johanson.

Septoria arabidicola, Rostrup.

Sirococcus cylindroides, Sacc.

Sphaerelia pachyasca, Rostrup.

Arabis Holboellii Hornemann.

SAecidium Holboellii, Homemann.

$\{$ Puccinia Holboellii, Rostrup.

Hendersonia Arabidis, Rostrup.

Pleospora platyspora, Sacc.

Sphaerella pachyasca, Rostrup.

Arabis Hookeri, Lange.

Septoria Arabidis, Sacc.

Arabis Lyallii, Watson.

S Sphieronema sphaeroidem, Ell.

\{Cornuliria sphaeroidea, Sacc.

Arabis 1jrata. L.

Sphaerella Cruciferarum, (Fr.) Sacc.

Arabis, sp. indet.

Puccinia arabicola, E.ll. \& Ev.

Barbarea vulgaris, R. Br.

Ramularia Barbareae, $\mathrm{Pk}$.

Brassica campestris, L.

Cystopus candidus, (P.) Lev.

Peronospora parasitica, (P.) Tul.

Brassica oleracea, L.

Coprinus Brassicae, Pk.

$\{$ Peziza brassicaccola, B.

$\{$ Pezizella brassicaecola, Sacc.

Plasmodiophora Brassicae, Wor.

Brassica, sp. indet.

S Sarea brassicaecola, S.

Helotium brassicaecolum, Sacc.

\{Leptosphaeria olericola, (B. \& C.) Sacc.

\{ Heptameria olericola, Cke.

Cardamine bellidifolia, $\mathrm{L}$.

Septoria cercosperma, Rostrup.

Sphaerella Cruciferarum, (Fr.) Sacc.

Cardamine hirsuta, L.

Peronospora parasitica, (P.) Fr.

Cardamine pratensis, L.

Phoma nebulosa, (P.) Mont.

Sphaerella Cruciferarum, (Fr.) Sacc.

Cardamine rhomb idea, DC.

Peronospora parasitica, (P.) $\mathrm{Fr}$.

Septoria Dentariae, Pk.

Cheiranthus pygmateus, Adams

Puccinia Cheiranthi, Ell. \& Ev.

Draba alpina, L., var. corgmbosa, Durand.

Septoria cercosperma, Rostrup

Sphaerella pachyasca, Rostrup.

Draba androsacea, Wahl.

IIymeunla macrospora, Sacc. \& Roum.
Phoma nebulosa, (P.) Mont.

Pleospora Drabae, Schrt.

Plcospora herbarum, (P.) Rabh.

Pyrenophora chrysospora, (Niessl) Sacc.

Septoria cercosperma, Rostrup.

Septoria pleosporoides, Sacc.

Draba"aurea, Vabl.

Aecidium Drabae, Tracy \& Gall.

Pleospora platyspora, Sacc.

Septoria cercosperma, Rostrup.

Draba Caroliniana, Walt.

Cystopus candidus, (P.) Lev.

Draba crassifolia, Graham.

Sphaerella Cruciferarum, (Fr.) Sacc.

Draba hirta, L.

Cladosporium herbarum, (P.) Lk.

Erysiphe communis, (Walhr.) Schl.

Laestadia circumtegens, Rostrup.

Pleospora Drabae, Schrt.

Puccinia Drabae, Rudolphi.

Pyrenophora chrysospora, (Niessl) Sacc.

Sphaerella pachyasca, Rostrup.

Draba incana, L.

Puccinia Drabae, Rudolphi.

Draba incana, L., var. arabisans,

Watson.

Pyrenophora hispida, (Niess1) Sacc.

Eutrema Edwardsii, R. Br.

Sphaerella Cruciferarum, (Fr.) Sacc.

Lepidium intermedium, Gray. Aecidium Lepidii, Tracy \& Gall. Cystopus candidus, (P.) Lev.

$L$ spidium montanum, Nutt.

Aecidium Lepidii, Tracy \& Gall.

Lepidium Virginicum, L.

(Leptosphaeria planiuscula, (Riess.) Ces. \& DeNot.

Leptosphaeria Virginica, (C. \& E.) Sacc.

Heptameria Virginica, Cke.

Lyrocarpa Coulteri, Hook. \& Harv.

Pleospora herbarum, (P.) Rabh.

Nasturtium palustre, DC.

Ramularia Armoraciae, Fckl.

Nasturtium sessiliflorum, Nutt.

Cystopus candidus, (P.) Lev.

Raphanus sativus, L.

Plasmodiophora Brassicae, Wor.

Sisymbrium canescens, Nutt.

Ascochyta Sisymbrii. Ell. \& Kell.

Peronospora parasitica, (P.) Fr.

Sisymbrium linifolium, Nutt.

Aecidium monoicum, Pk.

Cystopus candidus, (P.) Lev.

Puccinia aberians, Pk. 
Puccinia consimilis, Ell. \& Ev.

Sisymbrium officinale, Scop.

Cercospora Cruciferarum, Ell. \& Ev.

Cercospora Nasturtii, Pass.

Vesicaria alpina, Nutt.

Helminthosporium nanum, Nees.

Phoma punctiformis, Desm.

Pleospora herbarum, (P.) Rabh.

Pyrenophora comata, (Niessl) Sacc.

Septoria cercosperma, Rostrup.

Septoria Vesicariae, Rostrup.

Sphaerella Sibirica, Thm.

Vesicaria arctica, Richards.

Pleospora platyspora, Sacc.

Septoria cercosperma, Rostrup.

Sphaerella Cruciferarum, (Fr.) Sacc.

\section{CAPPARID.ICEAE.}

\section{Cap?aris Jamaicensis, Jacq.}

Asterina lepidigenoides, Ell. \& Ev.

Cl zome integrifolia, Torr. \& Gr.

Aecidium Cleomis, Ell. \& Anders.

Ieterosporium hybridum, Ell, \& Ev.

Cleome pungens, Willd.

Cercospora Clcomis, Ell. \& Halsted.

\section{VIOLACEAE.}

\section{Viola blanda, Willd.}

Cercospora granuliformis, Ell. \& Hark.

Septoria Violae, Westl., var. oligocarpa, $\mathrm{Pk}$.

Viola Canadensis, L.

Aecidium Violae, Schum.

Vicla hastata, Michx.

(Puccinia hastatu, Cke

$\left\{\begin{array}{l}\text { Puccinia Fergussoni, B. \& Br., var. } \\ \text { hastata, DeToni. }\end{array}\right.$

Viola Nuttallii, Pursh.

Puccinia Violae, (Schum.) DC.

Viola odorata, L.

Gloeosporium Violae, B. \& Br.

Zygodesmus albidus, Ell. \& Ilalsted.

Viola pubescens, Ait.

Cercospora murina, Ell. \& Kell.

Phyllosticta Violae, Desm.

\section{Viola tricolor, L.}

Peronospora Violae, DBy.

Phyllosticta Violae, Desm.

Viola, sp. indet.

Sphaerotheca Humuli, (DC.) Burrill.
POLYGALACEAE.

Polygala polygama, Walt.

Gloeosporium ramosum, Ell. \& Ev.

Polygala sp. indet.

\{ Gibbera Sanbinetii, Mont.

\{ Gibberella Silubinetii, Sacc.

\section{CARYOPIIYLLACEAE.}

Arenaria biflora, Wrtson.

$\{$ Leptopeziza Groenlandica, Rostrup.

\{ Durella Groenlandica, Sacc.

Pyrenophora comata, (Niessl) Sacc.

Sphaerella Stellarianearum, (Rabh.) Karst.

Sphatella Tassiana, DeNot.

Arenaria Groenlandica, Spreng.

Sphaerella Stellarianearum, (Rabh.) Karst.

Arenaria Michanxii, Ilook. f.

Pleospora herbarum, (P.) Rabh.

Arenaria peploidos, L.

Phoma herbarum, Westd.

Pleospora herbarum, (P.) Rabh.

Pyrenophora comata, (Niessl) Sacc.

Arenaria pungens, Nutt.

Puccinia fragilis, Tracy \& Gall.

Arenaria verna, L.

Pleospora herbarum, (1) Rabh.

Sphaerella Stellarianearum, (Rablr.) Karst.

Arenaria verna, L., var. hirta, Watson.

Hendersonia tenella, Schrt.

Sphaerella Tassiana, DeNot.

Cerastium alpinum, L.

Phoma herbarum, Westd.

Puccinia Arenariae, (Schum.) Wint.

Pyrenophora comata, (Niessl) Sacc.

Cerastium aroticum, Lange.

Pleospora herbarum, (P.) Rabh.

Cerasútum arovense, L.

Uredo Caryophyllacearum, DC.

$\{$ Melampsora Cerastii, (P.) IVint.

(Melampsorella Cerastii, Sclurt.

Phoma lierbarum, Westd.

Puccinia Arenariae, (Schum.) IVint.

Sphaerella Stellarianearum, (Rabh.) Karst.

Cerastium nutaus, Raf.

Peronospora Alsinearum, Casp.

Cerastium trigynum, Vill.

Sphaerella Stellarianearum, (Rabh.) Karst.

Cerastium vulgatum, L.

Isariopsis pusilla, Fres.

Isariopsis alborosella, Sacc.

Dianthus barbatus, L.

$\{$ Puccinia Lychnidearum, Lk.

(Puccinia Arenariae, (Schum.) Wint. 
Lychnis attinis. Vahl.

Clatosporium herbarum. P.) Lk.

Pleospora herbarum, (P.) Rahh.

Pyrenophoral comata, (Niessl) Sacc.

Sphaerella Sibirici, Thm.

Lychmis ap tala. L.

Sphaerella Sibirica, Thm.

Lychnis tritlora, R. Br.

Cladosporium herbarum, (P.) Lk.

Leptosphaeria Vahlii, Rostrup.

Pleospora herbarum, (P.) Rabh.

Pyrenophora comata, (Niessl) Sacc.

Sphaerella Sibirica, Thm.

Lyclnnissp. indet.

I Uitilago antherarum, F,.

\{Ustilago violacea, (P.) Fckl.

Sagina nivalis, Lindbl.

Septoria nivalis, Rostrup.

Silene acaulis, L.

Leptosphaeria Silenes-acaulis, De Not.

Pleospora herbarum, (P.) Rabh.

Sphaerella Sibirica, 'Tlım.

Splacella Tassiana, DeNot.

Silene antirrhina, L.

Aicochyta Silenes, E11. \& Ev.

Peronospora Arenariae, (B.) D By., var.

macrospora. Farl.

Ph:llosticta Silenes, Pk.

Spergrila arvensis, L.

Macrosporium parasiticum, Thm.

Peronospora obovata, Bon.

Puccinia Spergulae. DC.

Stellaria glauca, With.

Puccinia Arenariae, (Schum.) IVint.

Stellaria humifusa, Rottb.

Leptosphaeria Stellariae, Rostrup.

Septoria Stellariae, Rob.

Sphaerella Stellarianearum, (Rabh.) Karst.

Stellaria longipes, Goldie.

Leptosphaeria Stellariae, Rostrup.

Phoma herbarum, Westd.

Succinia Dianthi, DC.

Puccinia Lychnidearum. Lk.

(Puccinia Arenariae, (Schum.) Wint.

Trochila Stellariae, Rostrup.

Stellaria media, Smith.

Septoria Stellariae, Rob.

\section{PORTULACACEAE.}

Calandrinia Menziesii, Hook. Claytonia perfoliata, Donn.

Peronospora Claytoniae, Farl.

Lewisia rediviva, Pursh.

Uromyces Spragneae, Hark.
HYPERICACEAE.

Asayrum Clax-Andreae, L.

Fusicladium ascyrinum, Ell. \& Ev.

Hypericum anreum. Barton.

$\{$ Aecidium Hyferici-frondosi, S.

Uredo Ilyperici, Spreng.

Hypericum corymbosum, Muhl.

Aecidium Hyperici-frondosi, S.

Hypericum mutilum, L.

Gloeosporium cladosporioides, Ell. \& Halsted.

Septoria sphaerelloides, Ell. \& Kell.

Hypericum Sazothra, Michx.

SPeziza atrocinerea, Che.

\{Mollisia atrocinereal, Phil.

Hypericum, sp. indet.

?Aecidium Hypericorum. B. a C C.

\{ Physalaspora hypericina, (B. \& C.) Sacc.

$\{$ Phomatospora hypericina, Cke.

MALVACEAE.

Althaea rosea, L.

Colletotrichum Althaeae, Southworth.

Colletotrichum Malrarum, (Braun. \& Casp.) Southworth.

Puccinia Malvastri, Pk.

Septoria Fairmani, Ell. \& Ev.

Callirrlnoe involucrata, Gray.

Aecidium tuberculatum, Ell. \& Kell.

Vermicularia sparsipila, EH. \& Kell.

Gossypium herbacein, L.

Cercospora gossypina, Cle.

Colletotrichum Gossypii, Southworth.

Microsporium nigricantium. Atkinson.

Ozonium auricomum, Lk.

Phyllosticta gossypina, Ell. \& Martin.

Ramularia areola, Atkinson.

Gossypium, sp. indet.

Pyrenophora hyphasmatis, EH1. \& Ev.

Phoma corvina, Rav.

Hibis?us esculentus, L.

Ophiobolus consimilis, Ell. \& Ev.

Hibiscus grandirlorus, Michx.

Creoma (Aecidium) hibisciatum, S.

Hibiscus Maninot, L.

Vermicularia hibiscina, Ell. \& Ev.

Hibiscus Moschentos, L.

Phyllosticta Hibisci, Pk.

Hibiscus Syriacus, L.

Uromyces heterogenus, Cke?

Hibiscus Trionum, L.

Cercospora althaeina, Sacc.

Fusarium roseum, Lk. 
Hibiscus, sp. indet.

Phyllosticta hibiscina, Ell. \& Ev. Malva rotundifolia, L.

Phoma Lyndonvillensis, Fairman.

Puccinia Malvacearum, Mont.

Malva sylvestris, L.

Puccinia Malvacearum, Nont.

Sida spinosa, L.

Cercospora sidaecola, Ell. \& Ev.

Ozonium auricomum, Lk.

\section{TILIACEAE.}

Tilia Americana. L.

$\{$ Agariuns tiliofhilus, $P k$.

$\{$ Crepidotus tiliophilus, Pk.

Corticium bavile, $\mathrm{Pk}$.

f Valsa femoralis, Pk.

Cryptospora femoralis. Sacc.

Cyphella pezizoides, Zopf.

Disporthe farinosa. Pk.

$\{$ Rabenhorstia Tiliae. Fr.

IIercospora Tiliae, (P.) 'Tul.

Melanconium Tiliae, Pk.

Ozonium auricomum, Lk.

f Peziza Tiliae, $P_{k}$.

(Trichopeziza Tiliae, Sacc.

Radulum pendulum, Fr.

Sporotrichum sulfureum, Grev.

Tilia. sp. indet.

Aposphaeria aranea, Pk.

Chaetomium pusillum, Ell. \& Ev.

Chaetomium sphaerospermum, C. \& E.

\{ Coryne Ellisii, B.

\{ Dacryopsis Ellisiana, Missee.

Fusariun obtusisporum, Cke. \& Hark.

Hysterium Fraxini, P.

Myriococcum consimile, Ell. \& Ev.

Sacidium lignarium, $\mathrm{Pk}$.

\section{LINACEAE.}

T,innm perenne, L.

Linum rigidum, Pursl.

Velampsora Lini, (P.) Desm.

Li7nmm sulcatuini, Riddel.

Cercospora Lini, Ell. \& Ev.

\{Peronospora Lini. Schrt. ?

\{ Peronospora Lini, Ell. \& Kell.

\section{GERANIACEAE.}

Floerlea proserpinacoides, Will.

Entyloma Floerkeae, Holw.

Geranium Garolinianzm, L.

Cercospora Geranii, Kell. \& Swingle.
Cylindrosporium Gernnii, Ell. \& Ev.

f Peronospora Geranii, Pk.

\{ Plasmopora Geranii, Berl. \& De Toni.

Geranium incisum, Nutt.

Sphaerotheca Castagnei, Lev.

Geranium maculatum, L.

f Aecidium Geranii, DC.

\{ Ureclo hemisphaerica, Spreng.

\{ Peronospora Geranii, Pk.

$\{$ Plasmopara Geranii, Berl. \& De Toni.

Geranimm Richazdsoni, Fisch. \& Mey.

Erysiphe communis, (Wallr.) Schl.

Puccinia Geranii-sylvaticae, Karst.

Sphaerotheca Castagnei, Lev.

Geranium Roberíinmm, L.

f Peronospora Geranii, Pk.

\{ Plasmopara Geranii, Berl. \& De Toni.

Geranium, sp. indet.

Cercospora Brunkii, E11. \& Gall.

Impatiens fulva, Nutt.

SPeronospora obducens, Schrt.

Plasmopara obducens, Schrt.

(Peronospora Impatientis, Ell. \& Ev.

Impatiens pallida, Nutt.

\{Peronospcra obducens, Sehrt.

$\{$ Plasmopara obducens, Schrt.

Inzpatiens, sp. indet.

(Aecillium Impatientis, S.

Caeoma (Aecidium) impatientatum, S.

(Uredo Schweinitziana, Spreng.

Trichopeziza sulfurea, (P.) Fckl.

Oxalis corniculata, L., var. stricta, Sav. Aecidium Oxalidis, Thm. ?

Ustilago Oxalidis, Ell. \& Tracy.

Pelargonium zonzle, Willd.

fLeptosphueria subcacspitosa, Cke. of Hark.

Ileptimeria subcaespitosa, Cke.

\{ Physalostora Geranii, CKe. \& Hark.

\{ Phomatospora Geranii, Cke.

\section{RUTACEAE.}

Citrus Aurantium, L.

Aschersonia turbinata, $\mathrm{B}$.

Botryodiplodia diplocirpa, Ell. \& Ev.

Cladosporium herbarum, (P.) Lk.

Fusarium sarcochroum, (Desm.) Sacc.

Gloeosporium hysterioideum, Ell. \& Ev.

Pliyllosticta adusta, Ell. \& Martin.

Sphaerostilbe cinnabarina, Tul.

Citrusus, sp. indet.

Gloeosporium intermedium, Sacc.

Mucor clavatus, Lk.

Ptelea trifoliata, L.

Septoria Pteleae, Ell. \& Ev. 
Xanthoxylum, sp. indet.

Discula Xanthoxyli, Ell. \& Ev.

Materophona Xanthoxyli, EH. \& Ev.

\section{SIMARUBACEAE.}

\section{Ailantlnus glandulosus, L.}

Cllendersonia subfenestrata, B. \& C.

Hendersonia Sartwellii, Herb. Curt.

Camarosporium subfenestratum, sacc.

Crtosporina Ailanthi, Sacc.

Haplosporella Ailanthi, Ell. \& Ev.

Iypoderma virgultorum, DC.

Monilia aurantiaca, Pk. \& Sacc.

Phomin Ailanthi, Sacc.?

\{'Tubercularia vulgaris, Tode.

$\{$ Tubercularia Ailanthi, Cke.

S Valsa glandulosa, Cke.

Valsa clavulata. Cke.

Eutypella glandulosa, E1l. \& Ev.

Koerberlinia spinosa Zucc.

Diplodina Koerberliniae, Ell. \& Ev.

\section{MELIACEAE}

Melia Azedarach, L.

$\{$ A garicus meliigena, B. \& Clie.

$\{$ Mrcena meliigena, Sacc. Syll.

Cercospora leucosticta, Ell. \& Ev.

f Fusisporium azedarachinum, Thm.

Fusarium azedarachinum, Sacc.

Ozonium auricomum, Lk.

Phyllosticta Meliae, Ell. \& Ev.

Eutypella paradisaica, Speg.

\{ Spaerostilbe gracilifos, Tul.

$\{$ Stilbum corynoides, Ell. \& Ev.

Thiridaria eutypoides, Ell. \& Ev.

Valsa subscripta, (Fr.) M. A. Curtis.

\section{ILICINEAE.}

Ilex desidua, Walt.

f Tympanis Ravcnelii, B. \& $C$.

\{ Cenangiella Ravenelii, Sacc.

Phyllosticta concomitans, Ell. S Ev.

Ilex glabra, Gray.

$\{$ Asterina Ilicis, Ell.

\{Microphyma Llicis, Speg.

Valsa cercophora, Ell.

Ilex opaca, Ait.

$\{$ Peziza Aquifolinc, C. if $E$.

\{ Pezizella Aquifolii, Sacc.

\{ Polythorus ilicincola. B. \& $C$.

\{ Polystictus ilicincolus, Cke.

Valsa subcuticularis, C. \& E.

Ilex verticillata, Gray.

Phyllosticta Prini, Pk.
Ilex, sp. indet.

Coniothorium ilicinum, Ell. \& Anders.

Phoma ilicina. Ell. \& Anders.

$\{$ Physaluspora philoprina, (B. d C.) Sacc.

( Laestadia philoprina, Cke.

Nemopanthes Canadensis, DC.

f Tympanis Nemopanthis, Pl.

\{ Godronia Nemopanthis, Sacc.

CYRILLEAE.

Cyrilla racemiflora, L.

Sphaeria abrupta, B. \& C.

Botryosphaeria abrupta, B. \& C. in Herb. B. sec. Cke.

S Sphaeria flavidocompta, B. \& C.

\{ Chaetosphaeria flavidocompta, Sacc.

fDiatrype leioplaca, Fr.

\{Eutypa leioplaca. Cke.

Glenospora Curtisii, B. \& Desm.

fllysterium Cyrillae, B. \& C.

\{Glonium Cyrillate, Sacc.

Sphaeria rhodospila. B. \& C.

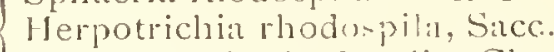

Bysosphateria rhodospila, Cke.

Hypocrea citrina, (P.) Fr.

Sphacria cyrillicola, B. \& C.

Melanomma cyrillicolum, Sacc.

Conisphaeria cyrillicola, Cke.

f Polyporus continguus. (P.) Fr.

\{ Poria contigua, Karst.

S Sphateria mammiformis, P.

$\{$ Rosellinia mammiformis, Ces, \& DeNot.

Ifypoxylon mammiforme, B.

Sphaeria assecla, S.

Stilbum cinereorubrum, B. \& C.

Torula aequalis, B. \& C.

\section{CELASTRACEAE.}

Celastrus scandens, L.

Nicrosphaera Alni, (DC.) Wint.

Euonymus Americanus, L.

Cercospora Euonymi, Ell.

Phyllosticta Enonymi, Sacc.

Euonymus atropurpuretz, Jacq.

Haplasporella Euonymi, Ell. \& Ev.

Phoma Weldiana, Fairman.

Solenia ochracea, Hoff̈m.

\section{RIIANINACEAE.}

Ceanothus ovatus, Desf.

Cercospora Ceanothi, Kell. \& Swingle.

Ceanothus thyrsifloris, Esch.

Cylindrosporium Ceanothi, Ell. \& Ev.

Ceanothas, sp. indet.

Gloniopsis connivens, (Clie. \& Hark.) Pazschke. 
Sphaeria vaccinicola, S.?

Rhirmnus Frangula, L

\{ Sphaeria punicea, Schm.

I Nectria punicea, $\mathrm{Fr}_{\mathrm{r}}$.

Rhamnus lanceolatus, Pursh.

Cercospora aeruginosa, Cke.

Cercospora Rhamni, Fckl.

\section{VITACEAE.}

Ampelopsis quinquefolia, Michx.

Gloeosporiun Ampelopsidis, Ell. \& Ev.

Laestadia Bidwellii, (Ell.) Viala \& Ravaz.

(Phoma uvicola, B. \& $C$.

$\{$ Sphaeropsis Ampelopsidis, C. \& E.?

Phyllosticta Labruscae, Thm.

Vermicularia compacta, C. \& E.?

Ampelopsis tricuspidata Sieb. \& Zucc.

Peronospora viticola, (B. \& C.) DBy.

Plyyllosticta Ampelopsidis, Ell. \& Martin.

Cissus stans, $\mathrm{P}$.

Diatrypella'Vitis, Ell. \& Ev.

Fusarium Volutella, Ell. \& Ev.

Laestadia Bidwellii, (Ell.) Viala \& Ravaz.

Vitis, species variae.

Naemaspora ampelicida, Engelm.

Phoma uvicola, B. \& C.

Phoma ustulatum, B. \& C.

Sphaeropsis uvarum, B.\& C.

Sphaeropsis Ampelopsidis, C. \& E.?

Depazea Labruscae, Engelm.

Septoria viticola, B. \& C.

Phoma uvicola, B. \& C., var. Labruscae,

Thm.

Phyllosticta viticola, Thm.

Phyllosticta Labruscae, Thm.

Ascochyta Ellisii, Thm.

Sacidium viticolum, Cke.

Phoma Ampelopsidis, Sacc.?

Phoma uvarum, Sacc.

Phyllosticta Ampelopsidis, Ell. \& Martin.

I Macrophoma Ampelopsidis, Berl. \& Vogl.?

Sphaeria Bidwellii, Ell.

Physalospora Bidwellii, Sacc.

Laestadia Bidwellii, Viala \& Ravaz.

Sphaerella Bidwellii, Ell.

$V i$ is aestivalis, Michx.

Cladosporium viticolım, Ces.

$\{$ Peronospora viticola, $(B . \& C) D B$.$y .$

\{ Plasmopara viticola, Berl. \& De Toni.

Pistillaria viticola, $\mathrm{Pk}$.

(Rhytisma monogramme, $B$. \& $C$.

$\{$ Rhytisma Vitis, $S$.

Fungi dubii.

Vitis Californica, Benth.

\{ Septosporium Fuckelii, Scribner nec Thm.

\{ Septosporium heterosporum, Ell. \& Gall.

Vitis cinerea, Engelm.

Uncinula Ampelopsidis, Pk.
Vitis Labrusca, L.

Acro spermum foliicolum, B.

\{ Greneria fuliginca, Scribner \& Viala.

\{Melanconium fuligineum, Cavara.

$\{$ Peronospora vilicola, (B.\& C.) DBy.

\{ Plasmopara viticola, Berl. \& De Toni.

$\{$ Pcziza ascoboloidea, $S$.

\{ Lachnella ascoboloidea, Fr.

$\{$ Peziza penicillata, S.

\{Trichopeziza penicillata, Sacc.

f Peziza vinosa, A. \& S.

Calloria villosa, Fr.

Orbilia vinosa, Karst.

$\{$ Peziza viticola, $P$. nec S.

\{Patellaria viticola, Sacc.

$\{$ Peziza Vitis, S.

$\{$ Trichopeziza Vitis, Sacc.

$\{$ Polyporus papyraceus, S.

$\{$ Poria papyracea, Cke.

$\{$ Polyporus viticolus, $S$.

\{ Poria viticola, Cke.

Vitis riparia, Michx.

$\{$ Phyllosticta viticola, Thm.

$\{$ Phyllosticta Labruscae, Thm.

Vitis vini era, L.

$\{$ Peziza vinosa, $A$. \& $S$.

$\{$ Orbilia vinosa, Karst.

$\{$ Peziza viticola, P. nec $S$.

\{ Patellaria viticola, Sacc.

$\{$ Peronospora viticola, (B.\& C.) DBy.

\{ Plasmopara viticola, Berl. \& De Toni.

\{ Thyridium antiquum, Ell. \& Ev.

$\{$ Pleospora antiqua, Ell. \& Ev.

\{ Uredo Vitis, Thm.

$\{$ Species excludenda.

Vitis vulpina, L.

$\{$ Patellaria atrofusca, $B . \&$ \& $C$.

$\{$ Lecanidion atrofuscum, Sacc.

$\{$ Peziza cillereofusca; $S$.

$\{$ Lachnella cinereofusca, Sacc.

Vitis, sp. indet.

Dendryphium acinorum, Ell. \& Ev.

$\{$ Gloeosporium fructigenum, B.

\{ Gloeosporium versicolor, B. \& C.

Gloeosporium Physalosporae, Cavara.

Hysterographium Ruborum, Cke., forma Vitis, Rehm.

Mucronoporus ferruginosus, (Schrad.)

E11. \& Ev.

f Peziza occulta, Rehm.

TOrbilia occulta, Sacc.

Peziza poriaeformis, DC.

Peziza pruinata, S.

Solenia poriaeformis, Fckl.

[Tapesia pruinata, Sacc.

Phyllactinia, sp.

$\{$ Polyporus barbaeformis, B. \& C.

$\{$ Poria barbaeformis, Cke.

Rosellinia Langloisii, Ell. \& Ev.

Sporotrichum Himantiae, S. 


\section{SAPINDACEAE.}

Acer dasycarpum, Ehrlh.

Ozonium auricomum, Lk.

Sphaerella maculiformis, Awd.

Acer glabrum, Torr.

Phyllosticta minutissima, Ell. \& Ev.

Tejchospora megastega, Ell. \& Ev.

Acer Pennsylvanicum, L.

f Didymella sphaerellula, (Pk.) Sacc.

\{ Endophlaea sphaerellula, C.ke.

Acer r'ubrum, L.

Diatrype Macounii, Ell. \& Ev.

Exosporium sociatum, Ell. \& Ev.

Gloeosporium nervisequum, (Fckl.) Sacc.

Gnomonia emarginata, Fckl.?

Gnomonia tenella, Ell. \& Ev.

Helmintlosporium subolivaceum, Ell. \& Ev.

(Massariella sudans, (B. 느 C.) Sacc.

\{Massariovalsa sudans, Sacc.

\{ Peziza miniopsis, Ell.

Erinella miniopsis, Sacc.

Ramularia lethalis, Ell. \& Ev.

Stach ybotrys elongata, Pk.

Valsa myinda, C. \& E.

Valsa quaternata, Fr.

Acer saccharinum, Wang.

$\{$ Agaricus saccharinophilus, $P k$.

Hypholoma saccharinophilum, Sacc. Syll.

Agaricus (Collybia) velutipes, Wm. Curtis.

Cylindrosporium saccharinum, Ell. \& Ev.

Trametes incana, $B$.

Dredalea ambigua, $\mathrm{B}$.

Trametes lactea, B.

Trametes ambigua, $\mathrm{Fr}$.

Lenzites glaberrima, B. \& C.

Daedalea glaberrima, B. \& C.

Diatrypella Frostii, Pk.

Gloeosporium saccharinum, Ell. \& Ev.

Helotium fraternum, $\mathrm{Pk}$.

Melanconium magnum, B.

Merulius Corium, (P.) Fr.

Merulius molluscus, Fr.

Naemaspora microsperma, Ell. \& Ev.

Naematelia nucleata, (S.) Fr.

Omphalia striipilea, (Fr.) Sacc. Syll., var. albogrisea, $\mathrm{Pk}$.

$\{$ Patellaria leptosperma, $P k$.

$\{$ Lecanidion leptospermum, Sacc.

$\mathrm{Phlebia}$ acerina, $\mathrm{Pk}$.

Polyporus applanatus, (P.) Wallr.

$\{$ Polyporus connatus, Fr.

$\{$ Fomes connatus, Cke.

Poria aurea, $\mathrm{Pk}$.

(Rhytisma punctatum, (P.) Fr.

\{ Rliytisma punctiforme, Mayr.

Septonema breviusculum, B. \& C.
Septoria saccharina, Ell. \& Ev.

Steganosporium cellulosum, Cda., var

major, Ell. \& Ev.

Trametes mollis, (Sommf.) Fr.

Trametes pallidofulva, (B.) Fr.

Valsa coronata (Hoffm.) Fr.

Acer spicatum, Lam.

S Sphaeria albidostoma, $P k$.

$\{$ IIerpotrichia leucostoma, Pk.

Polyporus aureonitens, Patouillard.

Pseudovalsa stylospora, Ell. \& Ev.

Acer, sp. indet.

$\{$ Agaricus albocrenulatus, $P k$.

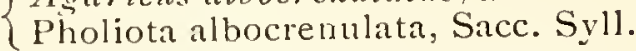

$\{$ Agaricus fulvotomentosus. $P k$.

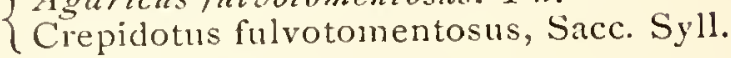

$\{$ Agaricus herbarum, $P k$.

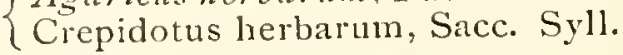

$\{$ Asaricus hirtosquamulosus, $P k$.

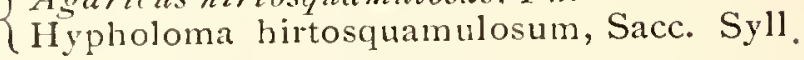

$\{$ Agaricus squarrosoides, $P k$.

$\{$ Pholiota squarrosoides, Sacc. Syll.

\{ Anthostoma saprophilum, Ell. \& Ev.

\{Xylosphaeria saprophila, Cke.

?Anthostomeila eructans, Ell. \& Ev.

Anthostomella ? picacea, (C. \& E.) Sacc.

Bulgaria purpurea, Fckl.

Bulgaria sarcoides, (Jacq.) Fr.

Byssosphaeria subiculata, (S.) Cke.

Camarosporium acerinum, Ell. \& Ev.

$\{$ Ceratostomella barbirostris, Sacc.

\{eratostoma barbirostris, (Duf.) Cke.

Clasterosporium caespitulosum, Ell. \& Ev.

Coniosporrum corticale, Ell. \& Ev.

Corticium ochroleucum, Fr.

Discella pilosula, Ell. \& Ev.

Helotium albovirens, Cke

Helotium epiphyllum, (P.) Fr.

Hirneola Auricula-Judae, (L.) B.

?Hypoxylon commutatum, Nits., var.

Holwayanum, Sacc. \& Ell.

$\{$ Hypoxylon serpens, Ell. N. A. F.

Hypoxylon insidens, (S.) Ell. \& Ev.

Hypoxylon multiforme, Fr., var. effusum,

C. \& E.

Hypoxylon perforatum, (S.) Fr.

Hypoxylon rubiginosum, (P.) Fr.

fIlysterium Prostii, Duby.

$\{$ Hysterium ellipticum, N.A. F.

Libertella acerina, Westd. ?

Lophiostoma rhopaloides, Sacc., var. pluriseptatum, Fairman.

Martindalea spironema, Sacc. \& Ell.

Massaria (Massariella) bispora, Cke.

$\{$ Amphisphaeria Papilla, (S.) Sacc.

\{ Melanopsamma Papilla, Sacc. 
Menispora glauconigra, C. \& E.

$\{$ Patellaria cylindrospora, Ell.

\{ Scutularia cylindrospora, Sacc.

Peniophora flavidoalba, Cke.

$\{$ Peziza acerina, C. \& E.

$\{$ Trichopeziza acerina, Sacc.

$\{$ Peziza aurata, B. \& Rav.

Ombrophila aurata, Phil.

$\{$ Peziza aureofulva, Cke.

Mollisia aureofulva, Sacc.

$\{$ Peziza compressa, P.

\{ Durella compressa, Tul.

$\{$ Peziza floriformis, Pk.

Pezizella floriformis, Sacc.

- Peziza paullopuncta, C. \& E.

\{ Mollisia paullopuncta, Sacc.

$\{$ Peziza simulata, Ell.

\{Phialea simulata, Sacc.

?Phlebia vaga, Fr.

SPolyporus contiguus, N.A.F.

Polyporus ferruginosus, (Schrad.) $\mathrm{Fr}$.

Mucronoporus ferruginosus, Ell. \& Ev.

$\{$ Polyporus pargamenus, Fr.

$\{$ Polyporus elongatus, B.

$\{$ Polyporus fimbriatellus, $\mathrm{Pk}$.

$\{$ Poria fimbriatella, Sacc. Syll.

$\{$ Polyporns semitinctus, $P k$.

Y Poria semitincta, Cke.

Porothelium confusum, B. \& Br.

Rhinotrichum ramosissimum, B. \& C.

Solenia ochracea, Hofim.

Sporidesmium compositum, B. \& Rav.

Sporonema pallidum, Ell. \& Ev.

Stilbum aeruginosum, Desm.

Stilbum atrocephalum, Ell.

$\{$ Teichospora vetusta, (Ell.) Sacc.

Strickeria vetusta, Cke.

Trametes mollis, (Sommf.) Fr.

Tubercularia nigricans, (Bull.) DC.

$\{$ Tympanis acerina, $P k$.

Scleroderris acerina, Sacc.

? $\{$ Tympanis bicolor, Ell.

$\{$ Cenangium bicolor, Sacc.

$\{$ Tympanis rhabdospora, B. \& C.

$\{$ Godronia rhabdospora, Sacc.

Zygodesmus limoniisporus, Ell. \& Ev.

Aesculus arguta, Buckley.

Aecidium Aesculi, Ell. \& Kell.

Macrosporium baccatum, E11. \& Kell.

Aesculus flava, Ait.

Phyllosticta sphaeropsoidea, Ell. \& Ev.

Aesculus Hippocastanum, L.

?Aecidium Aesculi, Ell. \& Kell.

$\{$ Agaricus sapidus, Kalchb.

\{ Pleurotus sapidus, Sacc. Syl1.

Diplodia Aesculi, Lev.

Haplosporella Hippocastani, Ell. \& Ev.

Phyllosticta sphaeropsoidea, Ell. \& Ev.
Schizothyrella Hippocastani, Ell. \& Ev.

Negundo aceroides, Moench.

Cercospora Negundinis, Ell. \& Ev.

Dendrodochium nigrescens, Ell. \& Ev.

Diplodia atrata, (Desm.) Sacc.

Gloeosporium apocryptum, Ell. \& Ev.

Helminthosporium subcuticulare, Ell. \& Ev.

Phyllosticta Negundinis, Sacc. \& Speg.

Rosellinia Kellermanni, Ell. \& Ev.

Sphaeronema canum, Ell. \& Ev.

Negundo Californicum, Torr. \& Gr.

Phleospora Aceris, (Lib.) Sacc.

Negundo, sp. indet.

Stictis phacidioides, Fr.

Staphylea trifolia, L.

Phyllosticta Staphyleae, Dearness.

$\{$ Metasphaeria staphylina (Pk.) Sacc.

Endophlaea staphylina, Pk.

Plowrightia staphylina, E11. \& Ev.

$\{$ Sphaeria Staphyleae, L.

$\{$ Sphaerella Staphyleae, Cke.

\section{ANACARDIACEAE.}

Rhus aromatica, Ait.

$\{$ Pileolaria effusa, $\mathrm{Pk}$.

\{Uromyces? effusus, De Toni

$R h u s$ copallina, L.

$\{$ Gonatobotrys m̈aculicola, Wint.

\{ Gonatrobotryum maculicolum, Sacc.

Sphaeronema Rhois, B. \& C. ?

\{ Cornularia Rhois, Karst.

$\{$ Taphrina purpurascens, Robinson.

$\{$ Exoascus purpurascens, Sacc.

(Sphaeria aculeans, $S$.

Valsa albovelata, B. \& C.

Valsa acnleans, $B$.

Diaporthe albovelata, Sacc.

Rhus Cotinus, L.

Hainesia rhoina, E11. \& Sacc.

Rhus diversiloba. Torr. \& Gr.

Valsonectria virens, Hark.

Rhus glabra, L.

(Anthostoma atrofuscum, (B. \& C.)

$\{$ Berl. \& Vogl.

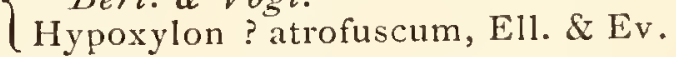

Rhus Toxicodendron, L.

Cercospora Bartholomei, Ell. \& Kell.

$\{$ Pileolaria brevipes, $B$. \& Rav.

$\{$ Pileolaria Terebinthi, Auct. Amer. nec Cast

Rhus Tuxicodendron, L., var. radicans, Torr.

Lophiostoma radicans, Ell. \& Ev.

$\{$ Lophiotrema vagabundum, Sacc., var. radicans, (E1l.) Berl.

\{ Dematium crucigerae, S.

$\{$ Podosporium rigidum, S. 
Rhus typhina, L.

Sphaerotheca pruinosa, C. \& P.

$R$ hus venenata, DC.

Sphaeria rufescens, S.

Sphacria aculeans, S.

Ílsa rufescens, M. A. Curtis.

Valsa albovelata, B. \& C.

Valsa aculeans, $B$.

Diatrype rhoina, $C$. \& $E$.

Valsa phoiphila, C. \& $E$.

Diaporthe albovelata, Sacc.

Calospora aculeans, Sacc.

Calospora rhoina, Sacc.

Cercospora infuscans, Ell. \& Ev.

\{ Dermatea pallidula, Cke.

$\{$ Scleroderris pallidula, Sacc.

\{ Pcziza thcioidea, C. \& E.

Lachnellula theioiden, Sacc.

Radulum orbiculare, Fr.

\section{LEGUMINOSAE.}

Papilionaceae.

Amorpha canescens, Nutt.

\{ Puccinia Amorphae, M. A. Curtis.

\{Uropyxis Amorphae, Schrt.

Amorpha fruticosa, L.

Puccinia Amorphae, M. A. Curtis.

$\{$ Uropyxis A morphae, Schrt.

Aecidium Amorphae, Cke.

Uredo Kansensis, Kell. \& Swingle.

Amphicarpaea monoica, Elliott.

SDothidea Glycineos, S.

\{ Phyllachora Glycineos, Sacc.

Apios tuberosa, Moench.

Uromyces Fabae, (P.) DBy.

Astragalus adsurgens, Pall.

Erysiphe communis, (Wallr.) Schl.

Microsphaera Ravenelii, B.

Uromyces Astragali, (Opiz.) Sacc.

Astragalus bisulcatus, Gray.

\{ Physalospora megastoma, (Pk.) Sacc.

$\{$ Laestadia megastoma, Cke.

Astragalus caryocarpus, Ker.

Colletotrichum carpophilum, Kell. \& Swingle.

Astragalus Chamaeleuce, Gray.

Napicladium Astragali, Ell. \& Ev.

Astragalus decumbens, Gray.

Erysiphe communis, (Wallr.) Schl.

Astragalus Drummondii, Dougl.

Volutella occidentalis, E11. \& Anders.

Astragalus flexuosus, Dougl.

Dendryphium nubilosum, Ell. \& Ev.

Volutella occidentalis, Ell. \& Anders.

Astragalus hypoglottis, L.

Erysiphe communis, (Wallr.) Schl.

Fusicladium brevipus, Ell. \& Ev.
Pnysalospora megastoma, (Pk.) Sacc.

Astragalus junceus, Gray.

Erysiphe communis; (Wallr.) Schl.

Astragalus lactiflorns, Hook.

Peronospora Trifoliorum, D By.

Astragalus mollissimus, Torr.

Uromyces Astragali, (Opiz.) Sacc.

Astragalus multiflorus, Gray.

Erysiphe communis, (Wallr.) Schl.

Astragalus pectinatus, Dongl.

Physalospora aurantia, Ell. \& Ev.

Astragalus scopulorm, Porter.

Sorosporium Astragali, $\mathrm{Pk}$.

Astragalus triplnyllus, Pursh.

Erysiphe communis, (Wallr.) Schl.

Baptisia leucoplraea, Nutt.

\{Accidium anfhigcnum, Ell. \& Kell.

\{Aecidium Kellermannii, De Toni.

Baptisia perfoliata, R. Rr.

$\{$ Sphaeropsis Baptisiae, Thm.

\{ Coniothyrium Baptisiae, Roum.

Baptisia tinctoria, R. Br.

\{Leptosphaeria comatella, (C.\&E.) Sacc.

\{ Ieptameria comatella, Cke.

Dalea laxiflora, Pursh.

Aecidium Daleae, Kell. \& Swingle.

Desmanthus brachylobus, Benth.

Erysiphe communis, (Wallr.) Schl.

Desmodium strictum, DC.

(Nctasphaeria dissiliens, (C.\& E.) Sacc.

\{Leptosphaeria dissiliens, Ell.

\{Uromyces Hedysari-paniculati, (S.) Farl.

\{ Coryneum Hedysari, Roum. Fung. Sel.

Desmodium, sp. indet.

$\{$ Leptosphaeria distributa, (C. \& E.) Sacc.

$\{$ Heptameria distributa, Cke.

$\{$ Peziza atrocinerea, Cke.

$\{$ Mollisia atrocinerea, Phil.

Phyllosticta Desmodii, Ell. \& Ev.

Dolichos melanophthalmus, DC.

Cercospora Dolichi, Ell. \& Ev.

Dolichos Sinensis, L.

Amerosporium oeconomicum, Ell. \& Tracy.

Cercospora Dolichi, Ell. \& Ev.

Dolichos, sp. indet.

Amerosporium Dolichi, Ell. \& Ev.

$\{$ Physalospora phlyctanoides, (B.\& C.) Sacc.

$\{$ Phomatospora phlyctanoides, Cke.

Genista, sp. indet.

?Eutypa ludibunda, Sacc.

Glycyrrhiza lepidota, Pursh.

Septoria Glycyrrhizae, Ell. \& Kell.

Uromyccs Trifolii, forma Glycyrrhizae, Ell. \& Kell.

Uromyces Glycyrrhizae, (Rabh.) Magnus. 
Hedysarum boreale, Nutt.

Uromyces Hedysari-paniculati, (S.) Farl.

Hosackia, sp. indet.

\{ Ditopella Hosackiae, (Ckc. \& Hark.) Sacc.

\{ Physalospora llosackiae, Cke.

Lathyrus latifolius, L.

Cercospora lathyrina, E11. \& Ev.

Septoria Lathyri, Ell. \& Ev.

Lathyrus maritimus, Bigel.

Phoma herbarum, IVestd.

Septoria emaculata, Pk. \& Clinton.

Lathyrus ochroleucus, Hook.

Dicoccum lathyrinum, E11. \& Gall.

Lathyrus paluster, L.

Phleospora reticulata, E11. \& Ev.

Lathyrus polymorphus, Nutt.

Erysiphe communis, (Wallr.) Schl.

Uromyces Fabae, (P.) D By.

Laingrus sativus, L.

Pleospora hyalospora, Ell. \& Ev.

Lathyrus venosus, Muhl.

\{ Didymosphaeria Vizcana, (Cke.) Sacc.

\{ Didymosphaerella Vizeana, Cke.

Lespedeza capitata, Michx.

Cercospora latens, E11. \& Ev.

Microsphaera diffusa, C. \& P.

Lupinus argenteus, Pursh.

Uromyces Genistae-tinctoriae, (P.) Wint.

Uromyces Lupini, B. \& C.

Lupinus argenteus, Pursh., var. argo-

phyllus, Watson.

Erysiphe communis, (Wallr.) Schl.

Lupinus Douglasii, Agardh.

$\{$ Uromyces Lupini, Ell. N. A. F.

Uromyces Genistae-tinctoriae, (P.) Wint.

Lupinus parviflorus, Nutt.

Erysiphe communis, (WVallr.) Schl.

Lupinus perennis, L.

\{ Erysiphe communis, (Wallr.) Schl.

\{Erysiphe Martii, Lev.

Lupinus Plattensis, Watson.

Phoma Lupini, Ell. \& Ev.

Uredo Lupini, B. \& C.

Uromyces Lupini, B. \& C.

Uromyces Astragali. (Opiz.) Sacc., var. Lupini, De Toni.

Lupinus rivularis, Dougl.

Uromyces Lupini, B. \& C.

Lupinus villosus, Willd.

Cercospora longispora. $\mathrm{Pk}$.

Medicago denticulata, Willd.

Cercospora Medicaginis, Ell. \& Ev.

Medicago sativa, L.

$\{$ Phacidium Medicaginis, Lib.

\{Pseudopeziza Medicaginis, Sacc.

Rhizoctonia Medicaginis, 1$)$ C.
Melilotus alba, Lam.

Cercospora Davisii, E11. \& Ev.

Melilotus, sp. indet.

$\{$ Leptosphaeria viridella, (Pk.) Sacc.

\{I Ieptameria viridella, Cke.

Oxytropis Lamberti, Pursh.

Erysiphe communis, (Wallr.) Schl.

Phleospora Oxytropidis, Ell. \& Gall.

$\{$ Trichobasis Oxytropidis, $P k$.

U Uredo Oxytropidis, De Toni.

Oxytropis monticola, Gray.

Pleospora herbarum, (P.) Rabh.

Oxytropis Uralensis, DC.

Ascochyta Oxytropidis, Schrt.

Oxytropis Uralensis, DC. var. pumila, Ledeb.

Pleospora herbarum, (P.) Rabh.

Parkinsonia aculeata, L.

Phyllosticta Parkinsoniae, E1l. \& Ev.

Petalostemon candidus, Michx.

Aecidium Petalostemonis, Kell. \& Carleton.

\{ Puccinia Petalostemonis, Farl.

\{ Uropyxis Petalostemonis, De Toni.

Petalostemon multiflorus, Nutt.

Aecidium Petalostemonis, Kell. \& Carleton.

Puccinia Petalostemonis, Farl.

Petalostemon violaceus, Michx.

$\{$ Puccinia Petalostemonis, Farl.

Uropyxis Petalostemonis, De Toni.

Phaseolus lielvolus, L

Erysiphe communis, (Wallr.) Schl.

Phaseolus Iunatus, L.

Phytophthora Plaseoli, Thaxter

Phaseolus pauciflorus, Benth.

Uromyces appendiculatus, (P.) Lev.

Pliaseolus perennis, IValt.

Erysiphe communis, (Wallr.) Schl.

Phaseolus vu?garis, L.

$\{$ Glocosporium Lindemuthianum, Sacc.

$\{$ \& Magmus.

Colletotrichum Lindemuthianum, Scribner.

Pliaseolus, sp. indet.

Corticium pezizoideun, Ell. \& Ev.

Isariopsis griseola, Sacc.

Macrosporium fasciculatum, C. \& E.

Pisum sativum, L.

Ascochyta Pisi, Lib.

Phytomyxa Leguminosarum, (Frank.)

Schrt.

$\{$ Pleospora herbarum, (I.) Rabl.

\{ Pleospora Pisi, (Sozu.) Fckl.

Pleospora hyalospora, E11. \& Ev.

Septoria Pisi, Westd.

Psoralea argopliglla, Pursh.

Aecidium Psoraleae, $\mathrm{Pk}$.

Parodiella perisporioides, (B. \& C.) Speg 
Psoralea macrostachys, DC.

$\{$ Calloria myriospora, Plil. \& Hurk.

Orbilia myriospora, Sacc.

Psoralea tenuiflora, Pursh.

$\{$ Uromyces Fabae, (P.) DBy.

\{ Uromyces Orobi, Fckl.

Robinia Pseudacacia, L.

\{ Valsa ludibunda, Sacc.

\{ Eutypa ludibunda, Sacc.

Gloeosporium revolutum, Ell. \& Ev.

Herpotrichia rhodomphala, (B.) Sacc.

$\{$ Polyporus rimosus, $B$.

$\{$ Fomes rimosus, Cke.

Rosellinia pulveracea, (Ehrh.) Fckl.

\{ Corticium muscigenum, B. \& Br.

\{ Stereum muscigenum, Cke.

Robinia viscosa, Vent.

Cytospora orthospora, B. $\&$ C.

Robinia, sp. indet.

(Boletus triqueter, S. Syn. Car.

$\{$ Polyporus cuticularis, S. Am. bor.

Polyporus conchatus, (P.) Fr.

(Metasphacria anisometra (Cke. \&

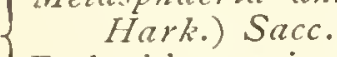

(Endophlaea anisometra, Cke.

Rosellina Aquila, ( $F_{1}$.) De Not.

Teichospora pezizoides, Sacc. \& Speg.

Sesbania macrocarpa, Mubi.

Physalospora Sesbaniae, Ell. \& Ev.

Sphaerella Sesbaniae, E1l. \& Ev.

Sesbania, sp. indet.

Botryosphaeria minor, Ell. \& Ev.

Sophora secundiflora, Lag.

Phyllosticta Sophorae, Ell. \& Ev.

Thermopsis montana, Nutt.

Erysiphe communis, (Wallr.) Schl.

Thermopsis rhombifolia, Riclard.

Phoma Thermopsidis, Ell. \& Gall.

Trifolium dasyphyllum, Torr. \& Gr.

Uromyces minor, Schrt.

Trifolium hybridum, L.

Uromyces 'Trifolii, (lledw. .) Lev.

Trifolium involucratum, Will.

Erysiphe communis, (Wallr.) Schl.

Uromyces Trifolii, (Hedw. f.) Lev.

Trifolium longipes, Nutt.

Erysiphe communis, (Wallr.) Schl.

Trifolium Parryi, Gray.

Uromyces Trifolii, (Hedw. f.) Lev.

Trifolium pratense, L.

$\{$ Ophiobolus collapsus, Ell. \& Sacc.

\{ Raphidospora collapsa, Cke.

\{ Phacidium Trifoliz, (Bernh.) Boud.

\{Pseudopeziza 'Trifolii, Fckl.

Verticillium dichotomum, Ell. \& Ev.

Volutella flexuosa, C. \& E.

Trifolium repens, L.

\{ Schinzia Leguminosarum, Frank.

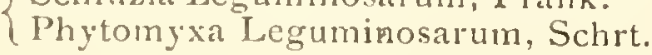

Stagonospora Trifolii, Ell. \& Ev.

Trifolium, sp. indet.

Lophiostoma caulium, (Fr.) Ces. \& De Not.

Streptothrix glauca, Ell. \& Ev.

Vicia Americana, Muhl.

?Peronospora Trifoliorum, DBy.

Vicia Americana, Mull.. var. linearis,

Watson.

Erysiphe communis, (Wallr.) Schl.

Microsphaera Ravenelii, B.

Vicia sativa,:L.

$\{$ Pleospora hyalospora, E11. \& Ev.

\{ Pleospora Americana, Ell. \& Ev.

\section{Caesalpinicae.}

Cassia Chamaecrista, L.

Septoria cassiaecola, Kell. \& Swingle.

Cercis Canadensis, L.

(Peziza cinereofusca, $S$.

$\{$ Cyphella cinereofusca, Sacc.

Lachnella cinereofusca, Sacc.

Cercis, sp. indet.

\{ Patellaria Lecideola, Fr.

\{ Durella Lecideola, Rehm.

Gleditschia triacanthos, L.

$\{$ Calloria rubrococcinea, Relmm. \& Wint.

Orbilia rubrococcinea, Sacc.

$\{$ Leptostroma hypophyllum, B. \& Rav.

\{Melasmia Gleditschiae, E1l. \& Ev.

Myriangium Curtisii, B. \& Mont.

Nectria sphaerospora, Ell. \& Ev.

Gleditschia, sp. indet.

$\{$ Peziza cinereofusca, S.

\{ Lachnella cinereofusca, Sacc.

Gymnocladus Camadensis, Lam.

Amerosporium subclausum, Ell. \& Kell.

\{ Cercospora Gymmocladi, Ell. \& Kell.

$\{$ Cercospora superflua, Ell. \& Holw.

\section{Mimoseae.}

Acacia, sp. indet.

Coniothyrium puncturn, Cke. \& Hark.

Diplodia Phyllactiniae, Cke. \& Hark.

\{Physarum nodulosum, Cke. \& Balf.

\{ Badhamia nodulosa, Massee.

Leguninosae, sp. indet.

$\{$ Triphragmium deglubens, $B$. \& $C$.

\{ Pliragmidium ? deglubens. De Toni.

ROSACEAE.

Amygdaleae.

Prunus Americana, Marshall.

$\{$ Septoria corasina, $P k$.

\{ Cylindrosporium Padi, Karst. 
\{Podosphaera Oxyacanthae, (DC.) D By. \{ Podosphaera tridactyla, (Wallr.) DBy.

Valsa leucostoma, (P.) Fr.

Prunus Amygdalus, Baill.

(Puccinia Pruni-spinosae, P.

$\{$ Puccinia Pruni, DC.

Uredo Castagnei, Rav. Fung. Car.

Prunus Armeniaca, L.

Septoria cerasina, Pk.

$\left\{\begin{array}{l}\text { Septoria Pruni, Ell. } \\ \text { Cying }\end{array}\right.$

Cylindrosporium Padi, Karst.

Prunus Cerasus, L.

$\{$ Sphaeronema Persicae, (S.) Ell.

$\{$ Cornularia Persicae, Sacc.

Cylindrosporium Padi, Karst.

Prunus Chicasa, Michx.

Taphrina Pruni, (Fckl.) Tul.

Prunus domestia, L.

$\{$ Septoria cerasina, $P k$.

$\{$ Cylindrosporium Padi, Karst.

Podosphaera Oxyacanthae, (DC.) DBy.

$\{$ Puccinia Pruni-spinosae, $P$.

$\{$ Puccinia Pruni, DC.

Prunus Lauro-Cerasus, L.

Cercospora cladosporioides, Sacc.

Piunus maritima, Wang.

Puccinia Pruni-spinosae, $P$.

Prunus Padus, L.

Cylindrosporium Padi, Karst.

Prunus Pennsylvanica, L. f.

Irpex paradoxus, (Schrad.) Fr.

Odontia Pruni, Lasch.

Poria latemarginata, (Dur. \& Mont.) Sacc.

Taphrina deformans, (B.) Tul.

Prunus Persica, Benth. \& Hook.

Cladosporium carpophilum, Thm.

$\{$ Cercuspora Persica, Sacc.

$\{$ Cercosporella Persica, Sacc.

$\{$ Septoria cerasina, $\mathrm{Pk}$.

Cylindrosporium Padi, Karst.

\{ Polyporus cervinus, (S.) Fr.

\{ Polystictus cervinus, Cke.

Polyporus versicolor, (L.) Fr.

$\{$ Oidium leucoconium, Desm.

Sphaerotheca pannosa, (Wallr.) Lev.

Prunus serotina, Ehrh.

$\{$ Septoria cerasina, Pk.

Cylindrosporium Padi, Karst.

Gloeosporium ovalisporum, Ell. \& Ev.

Lophiostoma Pruni, Ell. \& Ev.

$\{$ Puccinia Pruni-spinosae, $P$.

\{ Puccinia Pruni, DC.

\{ Taphrina Pruni, Tul.

\{ Taphrina Farlowii, Sadebeck.

Prunus subcordata, Benth.

?Plowrightia morbosa, (S.) Sacc.
Prunus Virginiana, L.

Dendryphium muricatum, Ell. \& Ev.

Lachnella flammea, ( $\Lambda$. \& S.) Fr.

Peniophora occidentalis, Ell. \& Ev.

$\{$ Phyllosticta destruens, Desm.

\{ Phoma Virginiana, Ell. \& Halsted.

$\{$ Puccinia Pruni-spinosae, f'.

$\{$ Puccinia Pruni, DC.

Septoria cerasina, Pk.

Teichospora Helenae, E11. \& Ev.

Valsa leucostoma, (P.) Fr.

Prunus (Plum).

$\{$ Monilia fructigena, $P$.

\{ Oidium fructigenum, Kze. \& Schm.

Exoascus deformans, (B.) Fckl.

Hypoxylon commutatum, (Nits.)

Massaria conspurcata, (Wallr.) Sacc

Pestalozzia adusta, Ell. \& Ev.

Prunus (Cherry).

$\{$ Monilia fructigena, $\mathrm{P}$.

\{ Oidium fructigenum, Kze. \& Schm.

\{ Sphaeria pustulata, (Sacc.) Cke.

\{ Physalospora pustulata, Sacc.

Tubercularia vulgaris, Tode.

Valsaria quadrata, (S.) Sacc.

Prunus, sp. indet.

Cucurbitaria setosa, Ell. \& Ev.

$\{$ Sphaeria excavata, $S$.

$\{$ Nummularia excavata, Ell. \& Ev.

Septoria gummigena, Ell. \& Ev.

Winteria tuberculifera, Ell. \& Ev.

Rosaceae proprieae.

Agrimonia Eupatoria, L.

Uredo Agrimoniae-Eupatoriae, ( $D C$. )

Wint.

Uredo Agrimoniae, Schrt.

Thecopsora Agrimoniae, Dietel.

Alchemilla alpina, L.

Sphaerella melanoplaca, (Desm.) Awd.

Alchemilla vulgaris, L.

Coleroa Alchemillae, (Grev.) Wint.

Phoma herbarum, Westd.

\{ Uromyces intrusus, (Grev.) Lev.

$\{$ Trachyspora Alchemillae, (P.) Fckl.

Dryas octopetala, L., var. integrifolia,

Cham. \& Schl.

Laestadia rhytismoides, Sacc.

Massarina Dryadis, Rostrup.

Melasmia Dryadis, Rostrup.

$\{$ Pleospora Dryadis, Fckl.

\{ Didymosphaeria Dryadis, Rostrup.

Sphaerella ootheca, Sacc.

Fragaria vesca, L.

Ascochyta colorata, $\mathrm{Pk}$. 
Fragarla Virginiana, Ehrh.

Ascochyta Fragariae, Sacc.

Synchytrium aureum, Schrt.

Fragaria, sp. indet.

\{ Pezizu Earliuna, Ell. \& Ev.

\{ Mollisia Earliana, Sacc.

Heteromeles arbutitolia, Roem.

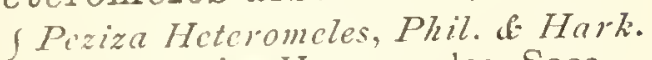

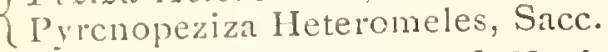

Neiliia opulifolia, Benth. \& Hook.

$\{$ Lophiotrema Spiraene, (Pk.) Sacc.

Lephiosphaera Spiraeae, Cke.

\{ Pezizae Opulifoliae, S.

$\{$ Trichopeziza Opulifoliae, Sacc.

Potentilla Anserina, L.

Trochila Potentillae, Rostrup.

Potentilla ar"gentea, L.

$\{$ Peziza Dchnii, Rabh.

\{Nollisia Dehnii, Karst.

Potentilla Canadensis, L.

$\{$ Taphrina Potentillae, Johanson.

\{ Exoascus Potentillae, Sacc.

Potentilla dissecta, Pursh.

Phragmidinm Potentillae, (P.) Wint.

Potentilla emarginata, Pursh.

Leptostroma Potentillae, (Fr.) Karst.

Pleospora vulgaris, Niessl.

Trochila Potentillae, Rostrup.

Potentilla gracilis, Dougl.

Phragmidium Potentillae, (P.) Wint.

Potentilla maculata, Pourret.

Nicrothyrium arcticum, Oud.

Physalospora Potentillae, Rostrup.

Pleospora herbarum, (P.) Rabl.

Pleospora vulgaris, Niessl.

Septoria potentillica, Thm.

Sphaerella punctiformis, (P.) Fr.

Potentilla nivea L.

Septoria semilunaris, Johanson.

'Trochila Potentillae, Rostrup.

Potentilla Norvegica, L.

\{ Peziza Dchnii, Rabh.

\{Mollisia Dehnii, Karst.

Phragmidium Fragariae, (DC.) Wint.

Potentilla Pennsylvanica L.

Phragmidium Potentillae, (P.) Wint.

Potentilla pulchella, R. Br.

Pleospora herbarum, (P.) Rabh.

Potentilla tridentata, Solander.

Phyllosticta potentillica, Sacc.

Trochila Potentillae, Rostrup.

Rosa Arkansana, Porter.

Cercospora rosicola, Pass.

Phragmidium speciosum, Fr.
Coleosporium miniatum, Bon.

Phragmidium mucronatum, Auct. Amer.

Phragmidium subcorticium, (Schrank) ilint.

f Oidium leucoconium, Desm

\{ Sphaerothecat pannosa, (Wallr.) Lev.

Rosa Californica, Cham. \& Schl.

Cercospora rosicola, Pass.

Rosa lucida, Ehrh.

Sphaeronemella Rosae, Ell. \& Ev.

Rosa rubiginosa, L.

S Sphaeria rhodina, B. \& C.

\{ Physalospora rhodina, Cke.

Sphaerella spinicola, Ell. \& Ev.

Rosa, sp. indet.

S Sthaeria Rauii, Ell. \& Eu.

$\{$ Didymella Rauii, Ell. \& Ev.

Endophlaea Rauii, Cke.

Lophiotrema anctum, Sacc.

\{ Mctasthaeria brachytheca, (B. \& C.) Sacc

Endophlaea brachy theca, Cke.

SMetasphaeria leiostegra, (Ell.) Sacc.

$\{$ Endophlaea leiostega, Cke.

Pestalozzia discosioides, Ell. \& Ev.

\{ Peziza Rosac, P.

Tapesia Rosae, Fckl.

$\{$ Pcziza umbilicata, B.\& C.

$\{$ Trichopeziza umbilicata, Sacc.

Phragmidium mucronatum, var. Amer-

$\{$ icanum, Pk.

I Phragmidium Rosae-alpinae, (DC.) Wint.

$\{$ ? Sphaeria subclypeata, B. \& C.

$\{$ Valsa subclypeata, C. \& P .

Sporotrichum elneochroum, Fr.

Rubus Canadensis, L.

Phoma lethalis, Ell. \& Martin.

Rubus Chamaemorts, L.

Leptostroma virgultorum, Sacc., var. rubinum, Karst.

Rubus delici sus, James.

$\{$ Lecythca speciosa. Pk.

\{ Uredo speciosa, De Toni.

Rubus Nutkanus, Nocino.

$\{$ Peziza bicolor, Bull.

$\{$ Dasyscypha bicolor, Fckl.

$\{$ Peziza scabrozillosa, Phil.

Dasyscypha scabrovillosa, Sacc.

Plimgmidium Rubi-Idaei, (P.) Wint.

Rubus occidentalis, L.

Asterina rubicola, Ell. \& Ev.

Chrysomyxa albida, Kühn.

Vermicularia compacta, C. \& E.

Rubus odoratus, L.

$\{$ Peziza lacerata, C. \& P.

Pyrenopeziza lacerata, Sacc.

$\{$ Peziza subochracea, C. \& P.

$\{$ Trichopeziza subochracea, Sacc. 
Phyllosticta bicolor, Pk.

Septoria Rubi, IVestd,

Rubus strigosus, Michx.

Botrytis patula, Sacc. \& Berl.

$\{$ Clypcosphacriu Hcndersoniae, (Ell.) Sacc.

$\{$ Leptosphaeria Hendersoniae, Cke.

$\{$ Gloeosporium Venetum, Speg.

$\{$ Gloeosporium necator, Ell. \& Ev.

Peronospora Rubi, Rabh.

Phyllosticta Ruborum, Sacc.

Valsa sepincola, Fckl.

Rubus trivialis, Michx.

Septoria Rubi, Westd.

Rubus ursinus, Cham. \& Schl.

Septoria Rubi, Westd.

Fubus villosus, Ait.

Gloeosporium Rubi, Ell. \& Ev.

$\{$ Patellaria rhabarbarina, $B$.

$\{$ Pezicula rhabarbarina, Tul.

Phoma lethalis, Ell. \& Martin.

Tubercularia carpogena, $\mathrm{Pk}$.

$R u b u s$, sp. indet.

$\{$ Conangium Rubi, (Fr.) Duby.

\{ Pyrenopeziza Rubi, Rehm.

SMetasphaeria anisometra, (Cke. \& Hark.) Sacc.

$\left\{\begin{array}{c}\text { Endophlae anisometra, Cke. \& Hark. } \\ \text { Ecziza. }\end{array}\right.$

$\{$ Peziza rufo-olivacea, $A$. \& $S$.

$\{$ Lachnella rufo-olivacea, Sacc.

$\{$ Peziza vinosa, A. \& $S$.

\{ Orbilia vinosa, Karst.

Sibbaldia procumbens, L.

Sphaerella innumerella, Karst.

Spiraea hypericifolli, Sieb. \& Zucc.

Diplodia spiraeicola, Ell. \& Ev.

Spiraea salicifolia, L.

Cylindrosporium Filipendulae, Thm.

Spiraea, sp. indet.

Phyllosticta filipendulina, Sacc. \& Speg.

\section{Pomeae.}

Amelanchier alnifolia, Nutt.

Entomosporium maculatum, Lev.

Erysiphe communis, (Wallr.) Schl.

Amelanchier Canadensis Torr. \& Gr.

$\{$ Metasphatia Peckii, (Spes.r.)..Sacc.

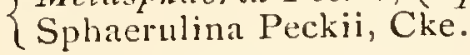

Nummularia discreta, (S.) Tul.

Gymnosporangium Nidus-avis, Thaxter. (Aecidium.)

Grataegus Crus-galli, L.

Corticium nudum, $\mathrm{Fr}$.

Podosphaera Oxyacanthae, (DC.) DBy.

Gymnosporangium globosum, Farl.

(Aecidium.)

Crataeguis Oxycantha, L.

Podosphaera Oxyacanthae, (DC.) D By.
Crataegus parviflora, Ait.

Entomosporium Thumenii, (Cke.) Sacc.

Roestelia aurantiaca, $\mathrm{Pk}$.

Crataegus tomentosa, $\mathrm{L}$.

Daedalea confragosa, $P$.

Crataegus, sp. indet.

Hypoxylon albocinctum, Ell. \& Ev.

Morthiera Thumenii, Cke., var. sphaerocysta, Pk.

$\{$ Patellaria nigrocinnabarina, $S$.

$\{$ Blitrydium nigrocinnabarinum, Sacc.

Septoria Crataegi, Kickx.

Cydonia vulgaris, $\mathrm{P}$.

Morthiera Mespili, (DC.) Fckl., var.

Cydoniae, C. \& E.

Nectria mammoidea, Phil. \& Plow.

f Podosphaera Oxyacanthac. (DC.) DBy.

\{ Podosphaera tridactyla, (Wallr.) DBy.

Gymnosporangium globosum, Farl. (Aecidium.)

Gymnosporangium Nidus-avis, Thaxter. (Aecidium.)

Sphaeropsis Cydoniae, C. \& E.

Pirus Americana, DC.

$\{$ Ccnangium Aucupariae, (P.) Fr.

$\{$ Tympanis Aucupariae, Wallr.

Coronophora gregaria, (Lib.) Fckl.

Diplodia maura, C. \& E., var. Americana, Ell.

Nummularia repanda, (Fr.) Nits.

$\{$ Aecidium cornutum, Gmel.

\{Roestelia cormuta, Fr.

Gymnosporangium globosum, Farl. (Aecidium.)

Valsa leucostoma, (P.) Fr.

Pirus arbutifolia, L.

Roestelia aurantiaca, $\mathrm{Pk}$.

Roestelia cornuta, (Gmel.) Fr.

Roestelia transformans, Ell., var. fructigena, Thm.

Pirus communis, L.

$\{$ Dacryomyces syringicola, B. \& C.

Dacryomyces destructor, B. \& Rav.

Dendrodochinm subeffusum, EH. \& Gall.

Entomosporium maculatum, Lev.

Entomosporium maculatum, Lev., var. carpogenum, Ell. \& Ev.

Setasphaeria subcutanea, (C. \& E.) Sacc.

$\{$ Endophlaea subcutanea, Cke.

Leptosphaeria subcutanea, Ell.

S Monilia fructigena, $\mathrm{P}$.

\{ Oidium fructigenum, Kze. \& Sckm.

Ozonium auricomum, Lk.

Gymnosporangium Nidus-avis, Thaxter. (Aecidium.)

Thelephora pedicellata, S. 
Pirus coronaria, L.

Cercospora pirina, Ell. \& Ev.

Oidium pirinum, Ell. \& Ev.

S Septoria Piri, Cisst.

\{ Septoria piricolir, Desm.

Pirus Malus, L.

Agaricus adiposus, Fr.

$\{$ Asuricus fulrinatus, $P$.

\{Pleurotus pulvinatus, Sacc. Syll.

Ceratostomella Mali. Ell. \& Ev.

Cercospora Malı: Ell. \& Ev.

Clitopilus conissans, Pk.

Didynella Mali, Ell. \& Ev.

Entomosporium maculatum, Lev.

\{ Gloeosporim fructigenum, B.

\{ Gloeosporium versicolor, B. \& C.

IIendersonia foliorum, Fckl.

Hendersonia Mali, Thm.

Hypoxylon Morsei, B. \& C.

$\{$ Monilia fructigena, P.

\{ Oidium fructigenum, Kze. \& Schm.

Nectria mammoidea, Phil. \& Plow.

Ozonium auricomum, Lk.

\{Peziza inquinans, Cke.

\{Patinella inquinans, Sacc.

$\{$ Peziza regalis, C. \& E.

Pezizella regalis, Sacc.

Phoma piricola, Ell. \& Ev.

Phyllosticta pirina, Sacc.

\{ Podosphaera Kunzei, Lev.

Podosphaera Oxycanthae, (DC.) DBy.

S Sphaeria collapsa, S.

S Sphaerella collapsa, Cke.

Sphaerella sentina, (Fr.) Sacc.

$\{$ Sphacria subbullaus, $S$.

S Sphaerella subbullans, Cke.

Sphaeropsis Malorum, B.

Sphaeropsis Malorum, B., var. foliicola,

Eli. \& Ev.

Sporotrichum cinereum, $\mathrm{Pk}$.

Polyporus igniarius, (L.) Fr.

\section{SAXIFRAGACEAE.}

\section{Dentzia, sp. indet.}

Ply yllosticta Deutziae, Ell. \& Ev.

Heuchera cylindrica, Dougl.

Puccinia Saxifragae, Schl.

Heuchera parvifolia, Nutt.

Phyllactinia suffulta, (Reb.) Sacc.

Sphaerotheca Castagnei, Lev.

Hydrangea arborescens, L.

$\{$ Uredo Hydrangeae, $B$. \& $C$.

\{ Melampsora Hydrangeae, Farl.

$\{$ Peziza fracta, B. \& $C$.

TPyenopeziza fracta, Sacc.
Hydrangea arborescens, L., var. cor-

data, Torr. \& Gr.

$\{$ Peziza Hydrangeae, S.

P Pirottaea ? Hydrangeae, Sacc.

Hydrangea, sp. indet.

Phyllosticta Hydrangeae, E11. \& Ev.

\{ Sphaerobolus sparsus, $S$.

Stictis sparsa, Sacc.

Parnassia Caroliniana, Miclix

$\{$ Didymosphaeria Parnassiae, (Pk.) Sacc.

\{ Didymosphaerella Parnassiae, Cke.

Philadelphus inodorus, L.

$\{$ Peziza Philadelphi, S.

$\{$ Pezicula ? Philadelphi, Sacc.

Philadelphus Lewisii, Pursh.

?Cucurbitaria Kelseyi, Ell. \& Ev.

Ribes cereun, Dougl.

Sphaerotheca Mors-uvae, (S.) B. \& C.

Ribes floridum, L'Her.

Asteroma ribicolum, Ell. \& Ev.

Microsphaera Grossulariae, Lev.

Sphaerotheca Castagnei, Lev.

Ribes lacustre, Poir.

Diaporthe Strumella, (Fr.) Fckl.

Ribes nigrum, L.

Microsphaera Grossulariae, Lev.

Ribes oxyazanthoiles, L.

Peronospora ribicola, Sclurt.

Ribes rotundifolium, Michx.

Cucurbitaria Ribis, Niessl.

?Homostegia Kelseyi, Ell, \& Ev.

Microsphaera Grossulariae, Lev.

Sphaerotheca Mors-uvae, (S.) B. \& C.

Ribes rubrum, L.

Gloeosporium Ribis, (Lib.) Desm. S Mont. Ribes, sp. indet.

Diplodia Dearnessii, Ell. \& Ev.

$\left\{\right.$ Corticium incarnatum, $\mathrm{Fr}_{\mathrm{l}}$.

\{ Thelephora ribesia. S. Am. bor.

Saxifraga Aizoon, Jacq.

Exobasidium Varmingii, Rostrup.

Sphaerella minor, Karst.

Saxifraga caespitosa, L.

Pyrenophora chrysospora, (Niessl) Sacc.

Saxifraga cernua, L.

Sphaerella pachyasca, Rostrup.

Saxiliaga mivalis, L.

Phoma Saxifragarum, Westd.

Pyrenophora chrysospora, (Niess1) Sacc.

Saxifraga oppositifolia, L.

Sphaerella minor, Karst.

Saxifraga Pennsylvanica, L.

Septoria albicans, Ell. \& Ev.

Saxifraga punctata, L.

$\{$ Puccinia Adoxae, DC.

\{ Puccinia pallidomaculata, Ell. \& Ev. 
$\{$ Puccinia Chrysosplenii, Grev.

$\{$ Puccinia spreta, N. A. F. 2233.

Saxifraga tricuspizata, Retz.

Cladosporium herbarum, (P.) Lk.

Puccinia Saxifragae, Schl.

Saxifraga Virginiensis, Michx.

f Puccinia Saxifragae, Schl.

\{ Puccinia striata, Cke.

\section{CRASSULACEAE.}

Sodum F:hodiola, DC.

Cladosporium herbarum, (P.) Lk.

\section{HAMAMELACEAE.}

\section{Hamamelis Virginiana, L.}

Cercospora Hamamelidis, Ell. \& Ev.

Dermatella Hamanelidis, Ell. \& Ev.

SPolyporus scutellatus, $\mathrm{S}$.

$\{$ Fomes scutellatus, Cke., forma Nove-

boracensis, Sacc. Syll.

$\{$ Patellaria Hamamelidis, Pk.

$\{$ Lecanidion Hamamelidis, Sacc.

Liquidambar Styraciflua, L.

Cercospora tuberculans, Ell. \& Ev.

$\{$ Clasterosporium subulatum, C. \& E.

\{ Ceratophorum subulatum, C. \& E.

Corticium Liquidambaris, B. in Herb.

Corticium prasinum, B. \& C.

Dendrodochium verticillatum, Cke. S Massee.

(Gnomonia petiolomm, (S.) Cke.

$\{$ Gnomoniella amoena, val. petiolorum, Sacc.

I Peziza cimmabarina, S.

L Lachnella cinnabarina, Fr.

$\{$ Peziza saccharifera, $B$.

$\{$ Psendohelotium sacchariferum, Sacc.

(Peziza vulgaris, Fr., var. sanguinella, $B$.

$\{$ Pezizelia vulgaris, Sacc., var. sanguinella, Sacc.

\{ Soleniu poriaeformis, (DC.) Fckl.

Tapesia pruinata, Sacc.

f Polyporus biformis, Fr.

Polystictus biformis, Fr.

\section{MYRTACEAE.}

Eucalyptus globulus, Labill.

f Calloria Eucalypti, Phil. \& Hark.

$\{$ Orbilia Eucalypti, Sacc.

$\{$ Didymosphaeria circinans. Hark.

$\{$ Didymosphaerella circinans, Cke.

\{ Peziza emergens, Phil. ㄴ IIark.

$\{$ Pyrenopeziza emergens, Sacc.

$\{$ Peziza subcornea, Phil. a Hark.

$\{$ Mollisia subcornea, Sacc.
Eucalyptus, sp. indet.

$\{$ Agaricus sacchariferus, $B$. \& $B r$.

Mycena saccharifera, Sacc. Syll.

\{ Dermatea Euculyti, Cke. de Hark.

$\{$ Scleroderris Eucalypti, Sacc.

SMetasphaeria anisontra, (Cke. \&

$\{$ Hark.) Sacc.

Endophlaea anisometra, Cke. \& Hark.

(Sphaeria plagarum, Clie. of IIark.

$\{$ Metasphaeria plagarum, Berl. \& Vogl.

Endophlaea plagarum, Cke. S Ilark.

\{ Peziza carneorosea, Cke. d Hark.

$\{$ Pezizella carneorosea, Sacc.

\{ Peziza rufo-olivacea, A. A $S$.

\{Lachnellá rufo-olivacea, Sacc.

\{ Propolis versicolor, Fr.

\{ Propolis faginea, (Sclirad.) Karst.

\section{LYTHRACEAE.}

\section{Lagerstroemia Indica, L.}

Phoma Liggerstroemiae, Speg., var. foliicola, E11. \& Ev.

Phyllosticta Lagerstroemiae, Ell. \& Ev.

Botryodiplodia varians, Ell. \& Langlois.

Nesaea verticillata, HBK.

$\{$ Peziza cyathoidea, Bull.

$\{$ Phialea cyathoidea, Gill.

\{ Peziza subatra, C. \& P.

$\{$ Pyrenopeziza subatra, Sacc.

\section{ONAGRACEAE.}

Epilobium alpinum, L.

Puccinia Epilobii, DC.

Epilobium coloratum, Muhl.

$\{$ Melampsora Epilobii, (P.) Fckl.

$\{$ Pucciniastrum Epilobii, Otth.

Epilobium hirsutum, L.

$\{$ Uredo pustulata, $P$.

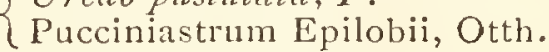

Epilobium latifolium, L.

Asterella Chamaenerii, Rostrup.

Gnomonia fenestrans, (Duby) Sacc.

Marsonia Chamaenerii, Rostrup.

Pleospora herbarum, (P.) Rabh.

Ramularia Chamaenerii, Rostrup.

Sphaerella pachyasca, Rostrup.

Epilobium spicatum, Lam.

Cercospora Epilobii, Schneid.

Laestadia Epilobii, (Wallr.) Sacc.

Marsonia Chamaenerii, Rostrup.

$\{$ Melampsora Epilobii, (P.) Fckl.

$\{$ Pucciniastrum Epilobii, Otth.

Sphaerella microspila, (B. et $\mathrm{Br}$.) Cke.

Epilobium tetragonum, L.

SLecythea macrosora, $P$.

\{ Uredo macrosora, De Toni. 
Gaura biennis, L.

Cercospora Gaurae, Kcll. \& Swingle.

Sphaerella granulata, Ell. \& Ev.

Gaura coccinea, Nutt.

$\{$ Trichobasis gun rima, $P$.

U Uredo gaurina, De Toni.

Gaura parviflor:a, Dougl.

Peronospora Arthuri, Farl.

Gayophytum ramosissimum, Torr. \& Gr.

Ustilago Gayophyti, Hark.

Ludwigia polycarpa, Short \& Peter.

Septoria Ludwigiae, Cke.

Ludwigia, sp. indet.

SPhysalospora Ludgrvigiae, (Cke.) Sacc.

\{ Pliomatospora Ludwigiae, Cke.

Oenothera albicaulis, Nutt.

Erysiphe communis, (Wallr.) Schl.

Oenothera bienmis, L.

$\{$ Accidium Oenotherae, $\mathrm{Pk}$.

\{ Aecidium Peckii, De Toni.

$\{$ Peziza Osnotherae, C. \& E.

$\{$ Pezizella Oenotherae, Sacc.

Puccinia Epilobii, DC.

Oenothera cardiophylla, Torr.

Puccinia Oenotherne, Vize.

Oenothera serrulata, Nutt.

$\{$ Aecidium Oenotherae, Pk.

\{ Aecidium Peckii, De 'Toni.

Oenothera sinuata, L.

Peronospora Arthuri, Farl.

$\{$ Physalospora Oenotherae, (B.\& C.) Sacc.

Phomatospora Oenotherae, Cke.

Oenothera speciosa, Nutt.

Peronospora Arthuri, Farl.

\section{LOASACEAE.}

Mentzelia laevicaulis, Torr. \& Gr.

Septoria Mentzeliae, Ell. \& Kell.

\section{PASSIFLORACEAE.}

\section{Passiflora incarnata, L.}

Cercospora fuscovirens, Sacc.

\section{CUCURBITACEAE.}

Citrullus vulgaris, Schrad.

(Gloeosporium lagenarium, (Pass.) Sacc. \& Roum.

Gloeosforimm Lindemuthianum, N. A. F., I 73 b nec Sacc. \& Magnus.

Gloeosporium lagenarium, (Pass.) Sacc.

\& Roum., var. foliicolum, Ell. \& Ev.

Septoria Citrulli, Ell. \& Ev.

Cucumis Melo, L.

Gloeosporium lagenarium, (Pass.) Sacc. \& Roum.
Oospora Cucumeris, Pk.

Cucumis sativus, L.

Cladosporium cucumerinum, Ell. \& Arth.

Gloeosporium lagenarium, (Pass.) Sacc.

\& Roum., var. foliicolum, Ell. \& Ev.

$\{$ Peronospora Cubensis, B. \& C.

\{ Plasmopara Cubensis, Humphrey.

Pythium DeBaryanum, Hesse.

Cucurbita foetidissima, HBK.

Macrosporium Sarcinula, B.

Cucurbita Melopepo, L.

Gloeosporium lagenarium, (Pass.) Sacc. \& Roum.

Vermicularia Wallothii, Sacc.

Cucurbita Pepo, L.

Coniosporium Fairmani, Sacc.

Diplodia Cucurbitacene, Ell. \& Langlois.

Peronospora Cubensis, B. \& C.

Rhopalonyces Cucurbitarum, B. \& Rav.

Torula herbarum, Lk.

Cucurbita (Squasls).

Coniosporium Fairmani, Sacc.

Epicoccum purpurascens, Ehrb.

Fusarium lateritium, Nees, var. aurantiacum, Cda.

Gloeosporium lagenarium, (Pass.) Sacc. \& Roum.

Gloeosporium orbiculare, B.

f Peronospora Cubensis, B. \& C.

$\{$ Plasmopara Cubensis, Humplirey.

$\{$ Peziza Cucurbitue, Gerard.

\{ Pezizella Cucurbitae, Sacc.

Rhopalomyces elegans, Cda.

Echinocystis lobata, Torr. \& Gr.

\{ Peronospora australis, Speg.

\{ Plasmopara australis, Swiugle.

Sicyos angulatus, L.

SPeronospora australis, Speg.

Peronospora sicyicola, Trelease.

Plasmopara australis, Swingle.

\section{CACTACEAE.}

Mamillaria vivipara, Hawortl.

Phoma Mamillariae, Ell. \& Ev.

Opuntia Missouriensis, DC.

Phoma Mamillariae, Ell. \& Ev.

Opuntia, sp. indet.

Gloeosporium lunatum, Ell. \& Ev.

Sphaerella Opuntiae, Ell. \& Ev.

FICOIDEAE.

Mollugo verticillata, L.

Phyllosticta Molluginis, Ell. \& Ialsted. 


\section{UMBELLIFERAE.}

Angelica gemuflexa, Nutt.

(Puccinia Oreoselini, (Strauss) Korn.

Puccinia Angeticae, Ell. at Eu.

Puccinia Pimpinellae, Farl. p.p.

Puccinia Ellisii, De Toni.

Angelica lucida, L

$\{$ Sphacria Anselicac-Iucidue, S.

Sphaerella Angelicae-lucidae, Cke.

Archangelica a tropurpurea, Hofim.

Fusicladium Angelicae, Ell. S Ev.

Septoria Dearnessii, Ell. \& Ev.

Sphaerella Angelicae, Ell. \& Ev.

Arcliangelica officinalis, Hoffin.

Asteroma Robergei, Desm.

$\{$ Calloria minutissima, Rostrup.

\{ Orbilia minutissima, Sacc.

Heterosphaeria Patelia, (Tode) Grev.

Laestadia Archangelicae, Rostrup.

Peziza cyathoidea, Bull.

$\{$ Helotium cyathoideum, Karst.

Phialea cyathoidea, Gill.

Phoma complanata, (Tode) Desm.

Pyrenophora phaeocomoides, Sacc.

Septoria cercosperma, Rostrup.

Cicuta maculata, L.

Cylindrosporium Cicutae, Ell. \& Ev.

$\{$ Peziza cyathoidea, Bull.

\{ Phialea cyathoidea, Gill.

Cymopterus bipinnatus, Watson.

Pleospora herbarum, (P.) Rabh.

Puccinia Jonesii, Pk.

Daucus Carsta, L.

$\{$ Leptosphaerict comatella, (C. \& E.) Sacc.

Heptameria comatella, C. \& E.

Macrosporium Carotae, Ell. \& Langlois.

Musenium tenuifolium, Nutt.

Puccinia Jonesii, Pk.

Osmorrhiza longistylis, DC.

Cercospora Osmorthizae, Ell. \& Ev.

Pastinaca sativa, L.

\{ Helotium pastinacinum, Cke.

Pezizella Pastinacae, (S.) Sacc.

Petroselinum, sp. indet.

Pleospora diaportheoides, Ell. \& Ev.

Sium cicutaefolium, Gmel.

Cercospora Sii, Ell. \& Ev.

Septoria Sii, Rob.

Thaspium barbinode, Nutt.

Ascochyta Thaspii, Ell. \& Ev.

Thaspium aureum, Nutt., var. tritoliatum, Coult. \& Rose.

Cercospora Thaspii, Ell. \& Ev.

Zizia cordata, DC.

Cylindrosporium Ziziae, Ell. \& Ev.
Umbelliferae, sp. indet.

\{ Sphaeria aculeata, S.

\{ Diaporthe aculeata, Sacc.

?Metasphaeria Thwaitesii, (B. \& Br.) Sacc.

$\left\{\right.$ Peziza extricata, $B$. a $^{\circ} C$.

Lachnella extricata, Sacc.

ARALIACEAE.

Aralia racemosa, L.

Ramularia repens, Ell. \& Ev.

Aralia spinosa, L.

Hypoderma virgultorum, DC.

Aralia trifolia, Decaisre \& Planchon.

Puccinia Araliae, Ell. \& Ev.

Aralia, sp. indet.

$\{$ Peziza sctigere, Phil.

\{ Trichopeziza setigera, Sacc.

Hedera Helix, L.

\{Metasphaeria hederifolia, (Cke.) Sacc.

S Sphaerulina hederifolia, Cke.

\{ Physalospora Eunotia, (B. \& C.) Succ.

\{ Phomatospora Eunotia, Cke.

$\{$ Mctasphacria helicicola, (Desm.) Sacc.

\{ Sphaerulina helicicola, Cke.

CORNACEAE.

\section{Cornus florida, L.}

$\{$ Leptosphacria oluzispora, (B. \& C.) Sacc.

Heptameria olivispora, Cke.

$\{$ Peziza exidiella, B. \& $C$.

$\{$ Pezizella exidiella, Sacc.

$\{$ Peziza miltophthalma, $B$. \& $C$.

Mollisia miltoph thalma, Sacc.

$\{$ Peziza roseoalba, S.

\{ Trichopeziza roseoalba, Sacc.

$\{$ Solenia poriaeformis, (DC.) FCkl.

Tapesia pruinata, (S.) Sacc.

Sphaeria Comi, S.

$\{$ Sphaerella cormifolia, Cke.

Sphaerella Corni, Cke.

Cornus sэricea, L.

Ascochyta cornicola, Sacc.

Cornus stolonifera, Michx.

(Microsphara Alni, (DC.) Hint.

$\{$ Microsphaera Corni, Mayr.

( Podosphaera Corni, Mayr.

Phyllactinia suffulta, (Reb.) Sacc.

Ramularia stolonifera, E11. \& Ev.

Cornus Suecica, L.

Hypoderma commune, (Fr.) Duby.

Leptostroma herbarum, ( $F_{1}$, $)$ Lk.

$\{$ Patellaria applanata, $B$. $C$ C

$\{$ Patinella applanata, Sacc.

$\{$ Patellaria rhabarobarina, $B$.

\{ Pezicula rhabarbarina, Tul.

Cornus, sp. indet.

$\{$ Dermatea farocinerea, Phil.

$\{$ Cenangiella flarocinerea, Sacc. 
I Didrmella Corni, (Sow.) Sacc.

(Diplodia papillosa. Ell. S Ev.

( Rhlisma maximum. Fr.

(Cryptomyces maximus, Rehim.

sporidesminum toruloides, Ell. S Er.

Garrya elliptica. Dougl.

I Jezizella lautilla, Phil. "Hakk.

Dasyscypha tautilla, Sacc.

Phillificilla furfurea, Phil. of Mlark.

llarknessiella purpurea, Sacc.

Nyssa multiflora, Nang.

( Peziza nlsadegena, Ell.

$\{$ Ciboria nyssogena, Sacc.

f Plicosfori thurictonta, (C. GE. S Sacc.

\{ Delacourea thuridonta, Che.

Nyssa, sp. indet.

Corticium Nyssae, B. S C.

(Didymella sesna, (C. dE. E. Sacc.

\{ Endophlaea segna, Cke.

l Iydnum pulcherrimum, B. \& C.

SPetellaria lignyota, Fr.

Karschia lignyota, Sacc.

Sporidesmium adscendens, $B$.

Steleum purpureum, $P$.

'Torula bigemina, C. $\mathbb{E}$ E.

Uredo Nyssae, Ell. \& Tracy.

\section{CAPRIFOLIACEAE.}

\section{Adoxa Moschatellina, L.}

Puccinia Adoxae, DC.

Lonicera involucrata, Banks. (Metasphacria anisometra, (Cke. \& Hark.) Sacc.

(Endophlaea anisometra, Cke.

Lonicera oblongifolia, Muhl.

Anthostomella limitata, Sacc.

Lonicera sempervirens, L.
S Sflacria Lonicerae, Sow.
Melomastia Friesii, Nits.
Conisphaeria Friesii, Cke.

Lonicera, sp. indet.

Leptothyrium Periclymeni, (Desm.) Sacc.

Leptothyrium Periclymeni, (Desm.) Sacc., var. Americanum, Ell. \& Ev.

Suetasphaeria aulica, (C. d E.) Sacc.

Endophlaea aulica. Cke.

Leptosphaeria aulica, Ell.

\{ Peziza atrata, $P$.

\{ Pyrenopeziza atrata, Fckl.

Sambucus Canadensis, L.

Diaporthe megalospora, Ell. \& Ev.

Nectria Sambuci, E11. \& Ev.

Valsa ambiens, (P.) Fr.

Sambucus nigra, L., var. laciniata,

Aecidium Sambuci, S.
Sambucus racemosa, L.

S Sphacria distincta, S.

\{ Massaria distincta, Cke.

Microsphaera Alni, (DC.) Wint.

Sambucus, sp. indet.

Graphium squarrosum, Ell. \& Langlois.

Haplosporella seriata, Ell. \& Ev.

$\{$ Pleospora Sambuci, (Plow.) Sacc.

\{ Delacourea Sambuci, Cke.

Rosellinia ligniaria, (Grev.) Nits, var. microspora, Ell. \& Ev.

Symphoricarpus occidentalis, Hook.

Nicrospliaera Symphoricarpi, Howe.

Plowrightia Symphoricarpi, Ell. \& Ev.

Rosellinia parasitica, Ell. \& Ev.

Septoria Symphoricarpi, Ell. \& Ev.

Teichospora umbonata, Ell. \& Ev.

Symphoricarpas vulgaris, Michx.

Cercospora Symphoricarpi, Ell. \& Ev.

Triostenm perfoliatum, L.

Cylindrosporium Triostei, Kell. S Swingle.

Viburnum dentatum, L.

Dinemasporium hispidulum, (Schrad.)

M. A. Curtis.

Plasmopara Viburni, $\mathrm{Pk}$.

Viburnum Lentago, L.

Pestalozzia Veneta, Sacc.

\{ Peziza Lentaginis, S.

Dasyscypha Lentaginis, Sacc.

Ramularia Viburni, Ell. \& Ev.

Viburnum Tinus, L.

Hendersonia Tini, Ell. \& Langlois.

Viburnum, sp. indet.

\{ Dermatea purpurea, Ell.

\{ Cenangim Ellisii, Sacc.

\section{RUBIACEAE.}

Cephalanthus occidentalis, $\mathrm{L}$.

Coniothyrium Cephalanthi, Ell. \& Ev.

Galinm Aparine, L.

Septoria Aparine, Ell. \& Kell.

Galinm boreale, L.

Hainesia borealis, Ell. \& Ev.

$\{$ Peziza Migritella, Phil. \& Hark.

\{Pyrenopeziza nigritella, Sacc.

Galinm circaezans, Michx.

Cercospora Galii, EH. \& Holw.

Septoria psilostega, Ell. \& Martin.

Galinn stellatum, Kellogg.

Mazzantia Galii, (Fr.) Mont.

Galinm, sp. indet.

Melasmia ? Galii, Ell. \& Ev.

Houstonia caerulea, L.

Cercospora Houstonine, Ell. \& Ev. 


\section{COMPOSITAE.}

\section{Tubuliftome.}

Antenuaria plantaginifolia, Hook.

Cy'stopus Tragopogonis, (P.) Schrt.

Arnica cordifolia, llook.

Ramularia arnicalis, Ell. \& Ev.

Arctium Lappa, L.

Helotium fumosum. Ell. \& Ev.

Sphaeropsis Lappae, Ell. \& Ev.

Artemisia cana, Pursh.

Clasterosporium dothideoides, Ell. \& Ev

Melanomma Tetonensis, Ell. \& Ev.

\section{Aster ericoides, L.}

(Dothidea Haydeni, b. \& C.

Ophiodothis Haydeni, Saçc.

(Rhopographus Haydeni, Cke.

Aster Tradescanti, L.
Xyloma Asteris, S.
Rhytisma Asteris, S.
Rhytisma astericolum, Sacc.
Fungus dubius.

Bidens cernua, L.

Sphaerotheca Castagnei, Lev.

Bidens chrysanthemoides, Michx.

$\{$ Peronospora Halstedii, Farl.

\{ Plasmopara Halstedii, Berl. \& De Toni.

Bigelovia, sp. indet.

Dothidea Bigeloviae, Ell. \& Ev.

?Dothidea Montaniensis, Ell. \& Ev.

?Macrosporium puccinioides, Ell. \& Anders.

Boltonia asteroides, L'Her.

Septoria Erigerontis, B. \& C., var.

Boltoniae, Webber.

Chrysopsis villosa, Nutt.

Aecidium Chrysopsidis, Ell. \& Anders.

Erechtites hieracifolia, Raf.

Septoria Erechtitis, Ell. \& Ev.

Erigeron annuls, P.

Ramularia macrospora, Fres, var.

Erigeron Canadensis, L.

Sphaerotheca Castagnei, Lev.

Erigerou Philadelphicus, L.

Septoria Erigerontis, B. \& C.

Erigeron, sp. indet.

Macrosporium atrichum, C. \& E.

$\{$ Peziza erigeronata, Cke.

\{Mollisia erigeronata, Sacc.

Gutierrezia Euthamize, Torr. \& Gr.

Aecidium Chrysopsidis, Ell. \& Anders.

Puccinia Bigeloviae, Ell. \& Ev.

Helenium autumnale, L.

Septoria nubilosa, Ell. \& Ev.

Helenium Hoopesii, Gray.

Septoria Helenii, Ell. \& Ev.
Helianthus tuberosus, L.

Sphaeria consessa, C. \& E.

$\{$ Leptosphaeria consessa, Sacc.

Heptameria consessa, Cke.

Iva xanthiifolia, Nutt.

Cuccinia Compositarum, N. A. F. 2252 nec Schl.

Puccinia xantliifolia, E11. \& Ev.

Liatris punctata, Hook.

Aecidium Compositarum, Mart, var. Liatridis, Vebber ?

Aecidium Liatridis, Ell. \& Anders.

Liatris scariosa, Willd.

Aecidium Compositarun, Mart.,

var. Liatridis, Webber.

Mikania scandens, Willd.

Cercospora Mikaniae, Ell. \& Ev.

Petasites palmata, Gray.

Phyllosticta Petasitidis, Ell. \& Ev.

Stagonospora Petasitidis, Ell. \& Ev.

Rudbeckia laciniata, L.

Phoma Rudbeckiae, Fairman.

Senecio aureus, L.

Cercospora Senecionis, Ell. \& Ev.

Senecio triangularis, Hook.

(Puccinia conglomerata, (Strauss) Sclum.

$\left\{\begin{array}{l}\text { Kze. } \\ \text { Priacina }\end{array}\right.$

Puccinia subcircinata Ell. \& Ev.

Solidago Canadensis, L.

Septoria Canadensis, Ell. \& Davis.

Septoria solidaginicola, Pk.

Solidago latifolia, L.

Septoria dolichospora, Ell. \& Ev.

Solidago nemoralis, Ait.

Ramularia Virgaureae, Thm.

Solidago rigida, L.

Ramularia Virgaureae, Thm.

Solidago, sp. indet.

I Metasphaeria aulica, (C. \& E,) Sacc.

Leptosphaeria aulica, Ell.

Mollisia atrata, (P.) Karst., var. megalospora, Ell. \& Ev.

Xanthium,Canadense, Mill.

Oidium erysiphoides, Fr.

\section{Liguliftorae.}

Crepis acuminata, Nutt.

Aecidium crepidicolum, Ell. \& Ev.

Lactuca Cauadeusis, L.

\{ Sphaeria subexserta, C. \& E.

$\{$ Didymella subexserta, Sacc.

Stephanomeria minor, Nutt.

$\{$ Puccinia Harknessii, Vize.

$\{$ Puccinia cladophilu, Pk.

Troximou glaucum, Nutt.

Puccinia Hieracii, Mart. 
ERICACEAE.

Andromeda ligustrina, Muhl.

S Exobasidinm Andromedne, Pk.

Exobasidium Vaccinii, Wor, vitr.

Andromedae, Ell.

Andromeda nitida, Bartr.

I Sphacriul-ludromedue, s.

Sphaerella Andromedeae, Cke.

Andromeda politolia, L.

Sphaerelka polifolia, Ell. \& Er.

Andromeda, sp. indet.

$\{$ Calosphacria microtheca, (C. d-E.) Sacc.

\{ralsa microtheca, Cke.

Cenangium urceolatum, Ell.

S.Metaphaeria sublanosa, (Cke.) Sacc.

Leptosphaeria sublanosa, Ell.

Talsa delicatula, C. E E.

Cassiope tetragona, Don.

SLeplosphaeric Andromedac, (Awd.) Sacc.

Sphaerulina Andromedae, Cke.

Clethra alnitolia, L.

Sphaeropsis clethricola, C. \& E.

Gaultheria procumbens. L.

Synchytrium Vaccinii, Thomas.

Gajlussacia resinosa, Torr. \& Gr.

Synchytrium Vaccinii, Thomas.

Kalmia latifolia, L.

Cercospora Kalmiae, Ell. \& Ev.

Kalmia, sp. indet.

Phacidium sphaeroideum, C. \& E.

S Sphacria kalmicola, Sz.

Sphaerella kalmicola, Cke.

Leucothoe axillaris, Don.

S Sphacria Andromedae, S.

Sphaerella Andromedae, Cke.

Lencothoe racemosa. Gray.

Dialonectria depauperata, Cke.

Rhododendron viscosum, Torr.

(Erobasidium Vaccinii, (Fckl.) Hor.

$\{$ Exobasidium discoideum, Ell.

(Exobasidium Vaccinii, var. discoideum, Ell.

Rhododendron, sp. indet.

Torula opaca, Cke.

Vaccinium corymbosum, L.

Ramularia Andromedae, Ell. \& Martin.

Zignoella paecilostoma, (B. \& Br.), Sacc.

Vaccinium, sp. indet.

Cryptosphaeria vexata, (C. \& E.) Sacc.

Hendersonia delicatuliı, C. \& E.

Speira punctulata, C. \& E.
Steironoma ciliatum, Raf.

Aecidium Lysimachiae, (Schl.) Wallr.

Leptosphaeria dumetorum, Niessl.

Leptosphaeria Steironematis, Ell. \& Ev.

Peziza Dearnessii, E11. \& Er.

Sphaerella ciliata, Ell. \& Ev.

\section{EBENACEAE.}

Diospyros Virginiana, L.

Ozonium auricomum, Lk.

OLEACEAE.

Chionanthus Virginica, L.

Dermatea Chionanthi, Ell. \& Ev.

Fraxinus Americana, L.

Corticium basale, $\mathrm{Pk}$.

Ozonium auricomum, Lk.

Phyllosticta Coryli, Westd.

Phyllosticta variegata, Ell. \& Ev.

Fraxinus viridis, Michx. f.

Chilonectria crinigera, Ell.\& Ev.

Hypoxylon fuscum, (P.) Fr.

?Nectria sphaerospora, Ell. \& Ev.

Fraxinus, sp. indet.

Calonectria Dearnessii, Ell. \& Er.

Sphaeria Fraxini, (S.) Fr.

\{Cenangiun Fraxini, Tul.

Cucurbitaria Fraxini, Ell. \& Ev.

Dermatella Fraxini, Ell. \& Ev.

Diaporthe congener, Ell. \& Ev.

?Didymosphaeria accedens, Sacc.

f Dimerosporium pulchrum, Sacc.

\{ Sarcinella heterospora, Sacc.

Epidochium olivaceum, Ell. \& Ev.

Helminthosporium macrocarpum, Grev.

Nectria chlorinella, Cke.

Sphaeronema sphaeropsoideum, Ell. \& Ev.

Thyridaria Fraxini, Ell. \& Er.

Syringa, sp. indet.

Phoma herbarum, Westd.

APOCINACEAE.

Apocynum, sp. indet.

Laestadia Apocyni, Ell. \& Ev.

?Pestalozziella Andersoni, Ell. \& Ev. 
Asclepias Cornuti, Dexaisne,

Didymella cornuta, Ell. \& Ev.

Cercospora clavata, (Gerard) $\mathrm{Pk}$.

Fusarium roseum, Lk.

Asclepias, sp. indet.

?Pestaloziella Andersoni, Ell. \& Er.

\section{GENTIANACEAE.}

Gentiana Saponaria, L.

Sphaerella Gentianae, Niessl?

Gentiana, sp. indet.

Macrosporium compactum, Cke.?

Menjanthes trifoliata, L.

Septoria Menyanthis, (Lib.) Desm.

Gilia longiflora, Don.

Puccinia Giliae, Hark.

CONVOLVULACEAE.

Ipomoea Batatas, Lam.

Acrocystis Batatas, Ell. \& Halsted.

Cercitocystis fimbriata, Ell. \& Halsted.

Monilochaetes infuscans, Ell. \& Halsted.

Phoma Batatae, Ell. \& Halsted.

Rhizopus nigricans, Ehrb.

\section{SOI.ANACEAE.}

Capsicum annum, L.

Colletotrichum nigrum, Ell. \& Halsted.

Lycopersicum esculentum, Mill. Cladosporium lycoperdinum, Cke.?

Sporotrichum solubile, S.

Pirysalis, sp. indet.

Podosporium rigidum, S.

Solanum Dulcamara, L.

Vermicularia solanoica, Fairman.

Solanum tuberosum, L.

Helminthosporium interseminatum, B. \& Rav.

Periola tomentosa, $\mathrm{Fr}$.

Rhopalomyces elegans, Cda.

Torula convoluta, Harz.

SCROPHULARIACEAE.

Castilleia, sp. indet.

Aecidium micropunctum, Ell. \& Ev.

Pentstemon glaber, Pursh.

Pentstemon grandiflorus, Nutt.

Cercospora Pentstemonis, Ell. \& Kell.

Pentstemon virgatus, Gray.

Aecidium Palmeri, Anderson.
BIGNONIACEAE.

Bignunia capreolata, L.

Asterina Bignoniae, Ell. \& Ev.

\section{I.ABIATAE.}

Brunella vulgaris, L.

Leptosphaeria Brunellae, Ell. \& Ev.

Rhabdospora Brunellae, Ell. \& Ev.

Leonurus Cardiaca, L.

Coniosporium culmigenum, (B.) Sacc., var. minor, $\mathrm{Pk}$.

Helotium fumosum, Ell. \& Ev.

Lycopus Virginicus, L.

?Phyllosticta Lycopodis, Ell. \& EV.

Monarda, sp. indet.

Sphaerella Monardae, Ell. \& Ev.

Nepeta Cataria, L.

$\{$ Didymella Catariae, (C. \& E.) Sacc.

\{ Plysalospora Catariae, Ell.

Salvia officinalis, L.

Diaporthe salviaecola, (C. \& E.) Sacc.

Scutellaria lateriflora, L.

Septoria Scutellariae, Thm.

Scutellaria parvula, Michx.

Cercospora Scutellariae, Ell. \& Ev.

\section{PLANTAGINACEAE.}

Plantago Rugelii, Decaisne.

Plantago Virginica, L.

Peronospora alta, Fckl.

AMARANTTACEAE.

Acnida cannabina. L.

Cercospora Acnidae, Ell. \& Ev.

Acnida tuberculata, Moq.

\{ Cystopus Bliti, (Biv.) Lev.

Cystopus Amaranti, (S.) B.

Amarantus retroflexus, $\mathrm{L}$.

Fusarium roseum, Lk.

Phoma longissima, (P.) Westd.

\section{CHENOPODIACEAE.}

Atriplex patulum, L., var. hastatum, Gray.

Phoma longissima, (P.) Westd.

Chenopodium, sp. indet.

Phoma picea, (P.) Sacc., ral. Chenopodii, Karst. \& Hariot.

Enrotia lanata, Noq.

Aecidium Eurotiae, Ell. \& Ev. 
Sarcobatus vermiculatus, Torr.

Teichospora mammoides, Ell. \& Ev.

Spinacea oleracea, Mill.

Cladosporium macrocarpum, Preuss.

\section{POLYGONACEAE.}

Fagopyrum esculentum, Moench.

Polyatis rulgaris, Lk.

Ranularia rufomaculans, Pk.

Polygonum dumetorum, L., var. scandens, Gray.

Puccinia mammillata, Schrt.

Polygonum Pennsylvanicum, L.

Cercospora Hydropiperis, (Thm.) Speg.

Polygonum, sp. indet.

Peziza atrocinerea, Cke.

Rumex crispus, L.

Ramularia pratensis. Sacc.

\section{LAURACEAE.}

Lindera Benzoin, Blume.

Diplodia Linderae, Ell. \& Ev.

Sassafras officinale, Nees.

Diatrype Stigma, (Hoffm.) Fr.

Valsa subclypeata, C. \& P.

\section{ELAEAGNACEAE.}

Elaeagnus argentea, Pursh.

Septoria argyrea, Sacc.

Shepherdia argentea, Nutt.

Clasterosporium dothideoides, Ell. \& Ev.

Curreya Shepherdiae, Ell. \& Ev.

Fomes Ellisianus, Anders.

\section{SAN'TALACEAE.}

Comandra umbellata, Nutt.

Cercospora Comandrae, Ell. \& Dearness.

\section{EUPHORBIACEAE.}

\section{Buxus sempervirens, L.}

Verticillium Buxi, (Lk.) Awd. \& Fleisch.

Euphorbia marginata, Pursl.

Asteroma Euphorbiae, Fckl.

\section{URTICACEAE.}

Celtis occidentalis, L.

Fusarium Celtidis, Ell. \&'Pracy.

Gloeosporium Celtidis, Ell. \& Ev.

Ficus Carica, L.

Ozonium auriconum, Lk.
Ficus elastica, L.

Leptostromella elastica, Ell. \& Ev.

Ficus, sp. indet.

$\{$ Polyactis vulgaris, Lk.

$\{$ Botrytis vulgaris, Fr.

Maclura anrantiaca, Nutt.

Coelosphaeria corticata, Ell. \& Ev.

Leptosphaeria Maclurae, Ell. \& Ev.

Sphaerella Maclurae, Ell. \& Ev.

Morus alba, L.

$\{$ Thelephora cinerascens, S.

Stereum cinerascens, Massee.

Ulmus Americana, L.

Eutypella longirostris, $\mathrm{Pk}$.

Pyrenophora Zabriskieana, Ell. \& Ev.

Thyronectria chrysogramma, Ell. \& Ev.

Ulmus fulva, Nichx.

Cryptosporella hypodermia, (Fr.) Sacc.

Didymosporium effusum, S., var. distinctum, $\mathrm{Pk}$.

UImus, sp. indet.

Anthostoma gastrinum, (Fr.) Sacc.

Aposphaeria fibricola, (B.) Sacc.

Calonectria Dearnessii, Ell. \& Ev.

Cornularia ulnicola, Ell. \& Ev.

Diatrype Daldiniana, De Not.

Diatrype Hochelagae, Ell. \& Ev.

Hymenochaete corticolor, B. \& Rav.

Massaria Ulmi, Fckl.

Myriococcum consimile, Ell. \& Ev.

Pestalozzia insidens, Zabriskie.

Pestalozzia rostrata, Zabriskie.

Sphaeropsis ulmicola, Ell. \& Ev.

Typhula subfasciculata, Ell. \& Ev.

Urtica, sp. indet.

Cylindrocolla Urticae, (P.) Bon.

PLATANACEAE.

Platanus occidentalis, L.

Discosia Artocreas, (Tode) Fr.

Metasphaeria rubida, Ell. \& Ev.

Nectria athroa, Ell. \& Ev.

$\{$ Massaria Platani, Ces.

Massaria atroinquinans, B. \& C.

\section{JUGLANDACEAE.}

Carya alba, Nutt.

Gloeosporium Caryae, Ell. \& Dearness.

Carya tomentosa, Nutt.

Cercospora Halstedii, Ell. \& Ev.

Carya, sp. indet.

Thelephora retiformis, B. \& C.

Phyllosticta caryigena, Ell. \& Ev., var. subfusca, Ell \& Ev. 
SHypoxylon nucitenu. B. \& C.

Amphisphaeria nucitena, Cke.

Melanomma nuclearium, (De Not.) Berl.

Diatrype Stigma, (Hoffm.) Fr.

Pseudovalsa Fairmani, Ell. \& Ev.

Valsaria insitiva, Ces. S De Not.

Juglans cinerea, L.

$\{$ Gloeosporium Fuglandis, (Lib.) Mtont.

$\{$ Marsonia Juglandis, Sacc.

Phoma albovestita, Friman.

Juglans, sp. indet.

Polyporus sulfureus, (Bull.) Fr.

MYRICACEAE.

Myrica asplenifolia, Endl.

$\left\{\begin{array}{c}\text { Thyridaria Comptonice, (Ell. \& Ev.) } \\ \text { Berl. \& Vogl. } \\ \text { Diatrypella Comptoniae, Ell. }\end{array}\right.$

CUPULIFERAE.

\section{Alnus incana, Willd.}

Anthostoma ? microsporum, Karst.

Alnus serrulata, Willd.

Tremella enata, B. \& C.

$\{$ Dacryomyces enatus, Massee.

Alnus, sp. indet.

Calosphaeria alnicola, Ell. \& Ev.

Diaporthe nivosa, Ell. \& Holw.

Nectria pithoides, Ell. \& Ev.

Betula lutea, Michx. f.

Ditiola conformis, Karst.

Irpex rimosus, $\mathrm{Pk}$.

$\{$ Peziza cerina, $P$.

\{ Lachnella cerina, Phil.

Betula, sp. indet.

$\{$ Tremella sarcoides, (Jacq.) With.

\{ Bulgaria sareoides, Fr.

Dacryomyces fragiformis, S.

$\{$ Diatrype Stigma, (Hoffim.) Fr., var.

$\{$ Diatrype Dearnessii, Ell. \& Ev.

Lentinus strigosus, (S.) $\mathrm{Fr}$.

Melanconium gracile, Ell. \& Ev.

Thyrsidium botryosporum, (Fr.) Mont.

Valsa angulosa, Nits.

Valsa oxyspora, $\mathrm{Pk}$.

Carpinus Caroliniana, Walt.

Calosphaeria microsperma, Ell. \& Ev.

Clasterosporium cornigerum, Ell. \& Ev.

Coryneum umbonatum, Nees.

Cylindrosporium Dearnessii, Ell. \& Ev.

Dacryomyces fragiformis, $\mathrm{S}$.

Diaporthe leucosarca, Ell. \& Ev.

Diatrypella informis, Ell. \& Ev.

Fusicladium effusum, Wint., var. carpineum, Ell. \& Ev.
Sphaeria melogramma, P.

Melogramma campulosporum, Fr.

Melogramma vagans, De Not.

Sphaeria fuscospora, $\mathrm{S}$.

Melogramma Bulliardi, Tul.

Iypocrea Ravenelii, B.

Hypoxylon myriangioides, $B$. \& $R$ \&v.

Diatrype lateritia, Ell.

Thyrialaria lateritia, Sacc.

Thyriaria? myriangioides, Sacc.

Nelogramma ? fuscosporum, Sacc.

Broomella Ravenelii. Sacc.

Melogramma myriungioides, CRe.

Melogramma lateritia, Cke.

Stereum purpureum, Pers.

(Pseudovillsa Titan, (B. \& Rav.) Sacc.

T Titania Berkeleyi, Berl.

Castanea sativa, Mill, var. Japonzca.

Laestadia orientalis, Ell. \& Ev.

Pestalozzia affinis, Ell. \& Ev.

Castanea sativa, Mill., var. Americana.

Watson.

Hymenochaete insularis, B.

Castanea, sp. indet.

Ceratostoma carpophilum, Ell.

Iypoxylon effusum, Nits.

(Mclanomma Eckfeldtii, (Ell.) Sacc.

$\{$ Didymella Eckfeldtii, Berl.

Zignoella Eckfeldtii, Ell.

Septonema spilomeum, B.

Fagus ferruginea, Ait.

Barya parasitica, Fckl., var. caespitosa, Pk.

Gloeosporium Fagi, (Rob.) Westd., var.

Americana, Ell. \& Ev.

Haematomyces faginea, $\mathrm{Pk}$.

Polyporus marginatus, (P.) Fr.

Ostrya Virginica, Willd.

$\{$ Melogramma vagans, De Not.

$\{$ Hypocrea Ravenelii, $B$.

Quercus alba, L.

Daldinia vernicosa, (S.) Ces. \& De Not.

Hydnum pallidum, C. \& E.

Stereum sulfuratum, B. \& Rav.

Quercus coccinea, Wang.

Dichaena strumosa, Fr.

Gloeosporium septorioides, Sacc.

Marsonia Martini, Sacc. \& E11.

Pestalozzia hysteriiformis, B. \& C.

Pestalozzia taphrinicola, EH. \& Ev.

Quercus coccinea. Wang., var. tinctoria, Gray.

Gnomonia clavulata, Ell.

Quercus falcata, Miclix.

Thelephora Perdix, Hartig.

Quercus ilicifolia, Wang.

Dichaena strumosa, $\mathrm{Fr}$.

Quercus macrocarpa, Michx.

Gloeosporium septorioides, Sacc. 
IIirneola Auricula-Judae, (L.) B.

Marsonia Martini, Sacc. \& Ell.

sphaerella nigrita, Che.

Quercus rubra, L.

?Gloeosporium septorioides, Sacc.

Phoma allantella, Pk.

Valsaria cincta. (Curr.) Sacc.

Quercus virens, Ait.

Rosellinia glandiformis, Ell. \& Ev.

Quercus, sp. indet.

\section{PIREYOMYCETES.}

Byssosphaeria Aquila. (Fr.) Che.

Ceratostoma cirrhosum, (P.) Fckl.

Chatosphaeria atrobarba, (C. \& E.) Sacc.

Cryptovalsa sparsa, Ell. \& Ev.

Diaporthe crinigera, Ell. \& Er.

Didymosphaeria Cupula, (Ell.) Sacc.

Eutypa Acharii, Tul.

Helotium subtile, Fr. ?

Hypocrea melaleuca, E11. \& Ev.

Hysterium Cookeanum, Gerard.

\{Kalmusia pachyasca, (C. dE. E.) Sacc.

\{. Melanomma pachyascum, Ber\}.

fLophiostoma hysterioides, Ell. \&

Langlois, nec Sacc.

Lophiotrema hysterioides, Berl.

Lophiostoma scelestum, C. \& E.

Massaria inquinans, (Tode) $\mathrm{Fr}$.

Melanomma fuscidulum, Sacc.

Sphaerella maculiformis, (P.) Awd.

Valsa albofusca, C. \& E.

(Wallrothiella melanostigma, (C.\&E.)

$\{$ Sacc.

(Melanopsamma melanostigma, Ell.

FUNGI IMPERFECTI.

Botrytis atroviridis, C. \& E.

Clasterosporinm Hirudo, Sacc.

Cylindrium elongatum, Bon.

Diplodia glandicola, C. E E.

Helminthosporium inflatum, B. \& Rav.

Nenispora glaucorigra, C. S E.

Pilidium quercinum, Cke. in Ellis Cat.

Speira minor, Sacc.

Stilbum rulgare, Tode.

DISCOMICETES.

Agyrium rufum, (P.) Fr.

Bulgaria purpurea, Fckl.

Bulgaria sarcoides, (Jacq.) Fr.

f Peziza chlora, s.

Chlorosplenium Schweinitzi, Fr.

f Peziza Fairmani. El\}. \& Ev.

? Pseudohelotiun Fairmani, Sacc.
Peziza fusca, P.

Peziza fuscidula, Clie.

URED1 YEAE.

Crinula paradoxa, B. \& C.

Cronartium asclepiadeum, (Willd.) Fr., var. Quercuum, B. \& C.

Pistillaria paradoxa, Cke.

Cronartium paradoxum. FarI.

\section{HYMENONYCETES.}

Chromosporium vitellinum, Sacc. \& Ell.

Corticium auriforme, B. \& C.

Corticium prasinum, B. \& C.

Grandinia granulosa (P.) Fr.

Hydnum Caput-Medusae, Bull.

Hymenochaete spreta, $\mathrm{Pk}$.

Lentinus strigosus, (S.) Fr.

Nucronoporus Andersoni, Ell. \& Ev.

Pholiota aeruginosa, $\mathrm{Pk}$.

Polyporus caesius, Fr.?

$\{$ Polyporus contiguus, (P.) Fr.

$\{$ Nucronoporus contiguus, Ell. \& Ev.

\{ Polytorus igniarius, (L.) Fr.

$\{$ Nucronoporus igniarius, Ell. \& Er.

Polyporus marginatus, (P.) Fr.

Polyporus Medulla-panis, (P.) Fr.

\{ Poria Medulla-panis, Cke.

Polyporus obtusus, B.

Stereum acerinum, (P.) Fr.

Stereum coffeatum, B. \& C.

\{ Tremella enata, B. d $C$.

$\{$ Dacryomyces enatus, Nassee.

Tremella stipitata, Pk.

\section{SALICACEAE.}

\section{Populus alba, L.}

$\{$ Gloeosporium Castagnei, Desm, \& Mont. \{ Narsonia Castagnei, Sacc.

Populus balsamifera, L.

Septoria musiva, $\mathrm{Pk}$.

Populus monilifera, Ait.

Hypocrea contorta, (S.) B. \& C.

Teichospora Kansensis, Ell. \& Ev.

Valsa floriformis, Ell. \& Ev.

Populus tremuloides, Michx.

Corticium mutatum, Pk.

Lentinus pholiotoides, Ell. \& Anders.

Nectria sulfurata, Ell. \& Ev.

Septonema griseofulyum, Ell. \& Ev.

Septoria musiva, Pk.

Sporidesmium tabacinum, Ell. \& Ev.

Populus, sp. indet.

Coniosporium subgranulosum, Ell. \& Ev.

S Kalmusia surrecta, (Cke.) Sacc.

\{Nelanomma surrectum, Berl. 
Poplar (Populus ?).

Alternaria tenuis, Nees.

$\{$ Amphisphaeria suliculosa, EH. \& Ev.

\{ Herpotrichia diffusa, (S.) Ell. \& Ev.

Corticium rhodellum, $\mathrm{Pk}$.

Salix alba, L.

Corticium Berkeleyi, Cke.

Saiz Babylonica, Tourn.

Comatricha longa, $\mathrm{Pk}$.

Salix chloropliylla, And.

Diatrypella Demetrionis, Ell. \& Ev.

Salix cordata, Muhl.

$\{$ Melampsora Salicis-Capreae, (P.) Wint.

\{ Melampsora farinosa, (P.) Schrt.

Salix humilis, Marsh.

Uncinula adunca, Lev.

Salix longifolia, Muhl.

$\{$ Uncinula adunca, Lev.

\{ Uncinula Salicis, (DC.) Wint.

Salix Incida, Muhl.

Marsonia apicalis, Ell. \& Ev.

Salix nigza, Marsh.

$\{$ Lophiostoma Langloisii, Ell. \& Ev.

\{ Melanomma Langloisii, Berl.

$\{$ Uncinula adunca, Lev.

\{Uncinula Salicis, (DC.) Wint.

Valsa nivea, (Hoffin.) Fr.

Salix, sp. indet

Anthostoma Ontariense, Ell. \& Ev.

Coniothecium toruloides, Cda.

Daedalea pallidofulva, B.

Didymella Canadensis, Ell. \& Ev.

Marsonia nigricans, Ell. \& Ev.

Melanconis salicina, Ell. \& Ev.

Teichospora Helenae, Ell. \& Ev.

Teichospora megastega, Ell. \& Ev.

Teichospora papillosa, Ell. \& Ev.

Trametes Peckii, Kalchb.

Valsa boreella, Karst.

Valsa canodisca, Ell. \& Holw.

Valsaria salicina, Ell. \& Ev.

Volutella occidentalis, Ell. \& Anders.,

var. minor, Ell. \& Anders.

\section{SCITAMINEAE.}

Musa, sp. indet.

Acrostalagmus cinnabarinus, Cda.

$\{$ Myxosporium Musae, B. \& $C$.

$\{$ Gloeosporium Musae, Ell. \& Ev.

\section{DIOSCOREACEAE.}

Dioscorea villosa, Torr.

Ramularia Dioscoreae, Ell. \& Ev.

\section{LILIACEAE.}

Allizm, sp. indet.

Chaetocladium Brefeldii, Van Tieghem \& Le MIon.

Pleospora Allii, (Rabh.) Ces. \& De Not.

Dasylirion, sp. indet.

Coniothyrium hysterioideum, Karst.

Lilium, sp. indet.

Polyactis cana, $B$.

Polygonatum giganteum, Dietrich.

Leptostroma Polygonati, Lasch.

Smilax hispida, Muhl.

Ascochyta Smilacis, Ell. \& Nartin.

Cercospora smilacina, Sacc.

Smilax, sp. indet.

Anthostomella Ludoviciana, Ell. \& Langlois.

Aspergillus pulvinatus, B. \& C.

Cyphella subcyanea, EH. \& Ev.

Helicosporium diplosporum, Ell. \& Ev.

Veratrum viride, Ait.

Vermicularia veratrina, Ell. \& Ev.

PALMEAE.

Chamaerops humilis, L.

Graphiola ? compressa, E. Fischer.

ARACEAE.

Peltandra undulata, Raf.

Cercospora pachyspora, Ell. \& Ev.

\section{LEMNACEAE.}

Spirodela polyrhiza, Schleid.

Cornuella Lemnae, Setchell.

\section{ALISMACEAE.}

\section{Alisma Plantago, L.}

Cercospora pachyspora, Ell. \& Ev.

Sagittaria graminea, Michx.

Doassansia Sagittarue, (Westd.) Fisch.

Sagittaria variabilis. Engelm.

Burrillia pustulata, Setchell.

Doassansia deformans, Setchell.

Doassansia opaca, Setchell.

Doassansia obscura, Setchell.

\section{NAIADACEAE.}

Potamogeton, sp. indet.

$\left\{\begin{array}{c}\text { Doassunsia Farlowiz, Cornu. } \\ \text { Doussansia occulta, (Hoffm.) Cormu., } \\ \text { var. Farlowii, Setcliell. }\end{array}\right.$

Doassansia Martianoffiana, (Thm.) Schrt. 


\section{CIPERACEAE.}

Carex conoidea, Sclik.

Ramularia Canadensis, Ell. E Er. Carex stricta, Lam.

Puccinia dioicre, Magnus.

Carex. sp. indet.

Phleospora Caricis, Ell. \& Ev., var. emaculata, Ell. \& Ev.

Septoria carnea, Ell. \& Ev.
Cyperus oylindricus, Chapm.

Stagonosporal Cyperi, Ell. \& Tracy.

GRAMINEAE.

Andropogon, sp. indet.

Stictis Sesleriae, Lib,

Ustilago Nealii, Ell. \& Anders.

Paspalzm platycaule, Poir.

Coryneum Paspali, Ell. \& Ev.

\section{CORRIGENDA.}

[*** Abbicviations: 1 , right hand column, l, left hand column; t denotes lines from top of page, b from bottom.]

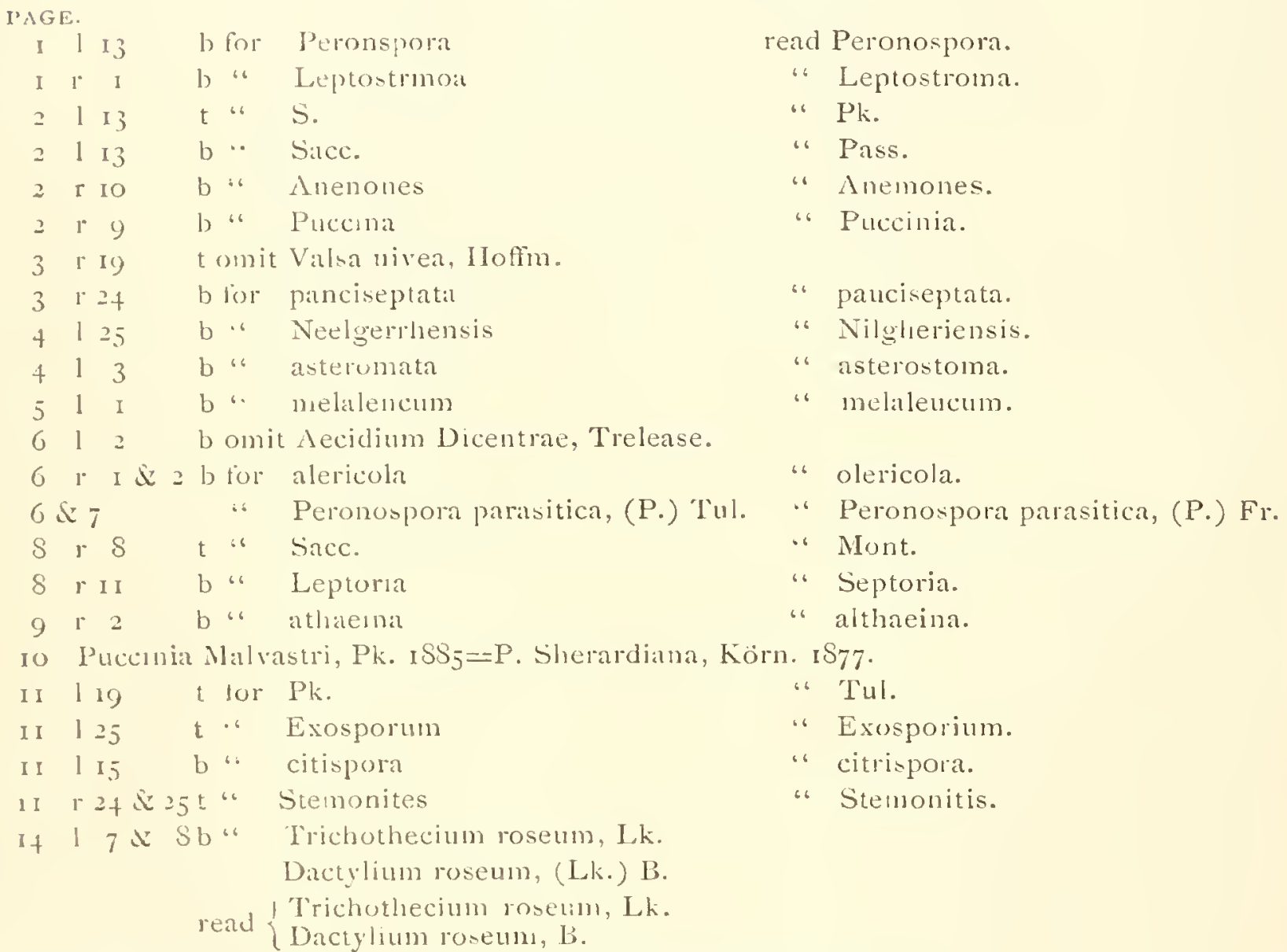

5 Puccinia dighatal, Ell. E Hark, r83f=P. Mesnieriana, Thm. IS77.

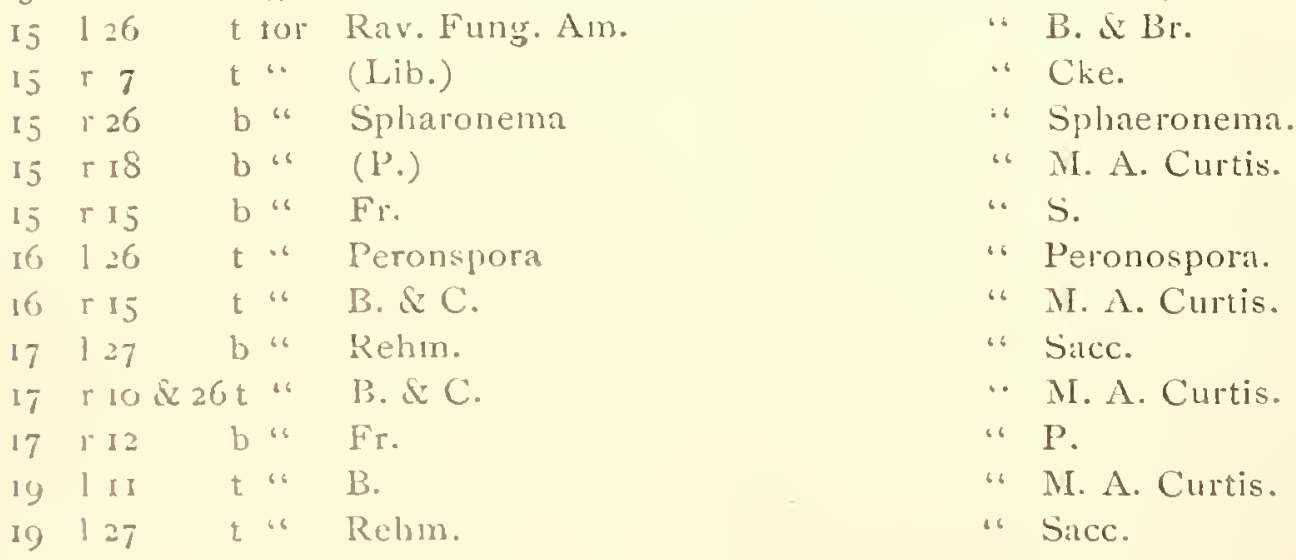


" M. A. Curtis.

-6 spodoleucus.

2 I $\quad$ r I 7 b $\cdots$ spondoleucus

22123 bomit Patellaria congregata, B. \& C.

2419 b for Apiosphaeria

28117 t " lanatus

$2 S$ r 5 t omit Cercospora latens, Ell. \& Ev.

29 r 22 b for Cke.

301 I6 b" (A. \& S.) Wint.

30 r I9 b " Sacc.

34 rit t " Rehm.

$3+\quad 2_{2} \quad t$ " Clie.

37 I 2 t " deliciosa

$40 \mathrm{r} I \mathrm{t}$. $\mathrm{Fr}$.

$44 \quad 124 \quad t$ " Fr.

4417 b " M. A. Curtis

$471 I_{5}$ b " Mucur

471 I4 bomit Mucospurtus, B. \& C.

50 r I9 $t$ for Rosellina

$50 \quad \mathrm{r} 27 \quad \mathrm{t}$ “ Cke.

5 I 120 t " Cke.

5 I $122 \quad t$ " Eutypella

5 I 13 b " strictum

5618 t " Hydum

59 r 25 b " Cichorcearum

62 r 8 b " obusta

66 I I3 b “ C. \& E.

77 r 3 b for Nemaspora

$\mathrm{S}_{4}$ r 3 b " fruticosa

9 I $\quad 25$

9915

I0O 1 I9

IOO $r$ I

IO3 $\mathrm{I} 3$

I 2015

I 2 I 1 I I

123 r IO

I23 $\mathrm{IO}$

I 2811

I $29 \quad 1$ I6

I $30 \quad 122$

I3I 1 I

$132 \quad 125$

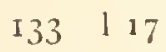

I33 17

13719

I39 17

139 I IS

142 r 32

$149 \quad 114$

15212

$169 \quad \mathrm{r} 22$

I 70 I I 2

ISO 1 I 4

ISI 129

ISI I $S$

t exclude from brace Septoria Polygonorum, Desm. b for Ozonicum

$t$ " halosericea

b "Tnl.

b " mucidium

b" Phyllactina

t " Acomyces

b " Trichospora

b " Sace.

$t$ " tomentocinta

$t$ " augustifolia

$t$ "Crytosphaeria

b tक Klotscbii

b " Klotschii

b " concinaum

$t$ " Corticum

$t$ " Puccina

t * Spharella

$t$ "aliquanti

t. Call

b " Aud.

$t$ "6 Koehleria

t ، cupressi

b " Plebia

b " Lithophanae

b " Carabidas

b " B. \& C.
"Apospharia.

" linatus.

" M. A. Curtis.

" (Hedw. f.) Lev.

“ Ell \& Ev.

"Since.

"M. A. Curtis.

" speciosa.

“(Fr.) M. A. Curtis.

“P.

"Fr.

"Mucor.

“ Rosellinia.

" M. A. Curtis.

"M. A. Curtis.

“Eutypa.

" striatum.

"6 Hydnum.

6 Cichoracearum.

"robusta,

"Cke.

“ Naemaspora.

"fruticulosa.

read Ozonium.

" holosericea.

"Tul.

" mucidum.

“Phyllactinia.

"Ascomyces.

“Teichospora.

"Sacc.

" tomentocincta.

" angustıfolia.

"Cryptosphaeria.

“ Klotzschii.

"Klotzschii.

" concinnum.

"Corticium.

“Puccinia.

“ Sphaerella.

" aliquanta.

" Calla.

"Auct.

" Koeleria.

" cupressina.

‘ Phlebia.

" Lithophane.

" Carabidae.

" Cke. 
On issuing the thind and final part of the Index, we wish to return our thanks to Mr. Sereno Wratson for aid in determining the names of host-plants, to Dr. George Vasey for the revision of the mames of the grasses; and to Prof. Roland Thaxter for a revision of the list of fungi on insects. We also would acknowledge with thanks information with regard to symonymy and corrections kindly furnished by Mr. J. B. Eilis, Dr. O. Pazschke, Prof. C. H. Peck, and Mr. W. T. Swingle.

The general plan of the Index was sufficiently set forth in the preface of Part $I$, and it only remains to state the plan pursued in the Addenda. Where species are intronnced either as new to this Index, or as occmring on hosts not recorded in the borly of the Index, they are printed in "ommon type. Where new varieties of species previonsly given are introduced, the varietal name only is given in common tyle, the slecific name being in italies. Where the néw names are synonymons with names given previously in the Index, they are placert in braces with one or more of the nunes previonsly given, the latter being printed in italics. Some of the species before reported are now known either to have been incorrectly determined or not to occur on the hosts originally given, and these facts have been bronght out as fill as possible by the synonymy or by the use of the words "species excludenda." As lefore, the arrangement of synonyms in braces is chronological and, in using the Intex, it should not be forgotten that unpublished names are not given except in a few eases where necessary to avoid confusion of synonymy.

Cambrigge, Fune 13, isqr.

W. G. FARLOW. 


\section{N D E X.}

\begin{tabular}{|c|c|c|c|c|c|c|c|}
\hline Abies & $15 S$ & Anemone & $\mathrm{I}, \mathrm{I} \mathrm{S}_{3}$ & Audibertia & 86 & Calandrinia & 9, I $S S$ \\
\hline Abronia & 89 & Anemonella & I & Avena & I 49 & Calla & $1+2$ \\
\hline Abutilon & 9 & Angelica & 48,203 & & & Callicarpa & \\
\hline Acacia & 31,199 & Animals & 179 & Baccharis & 60 & Callirhoe & Io, ISS \\
\hline Acalypha & 95 & Anonaceae & $5, \mathbf{I S}_{5}$ & Bahia & 60 & Callistephus & 60 \\
\hline Acanthaceae & 85 & Antennaria, & 57,205 & Balsamorrhiza & 60 & Callithamnion & I 78 \\
\hline Acer & 18,192 & Aorurus & I $S_{2}$ & Bambusa & I 49 & Calochortus & 137 \\
\hline Acerates & 78 & Aphides & I $\mathrm{S}$ I & Baptisia & 25,194 & ptenus & I $8_{2}$ \\
\hline chillea & 57 & Aphis & I $S_{I}$ & Bubarea & 6, I 86 & Caltha & 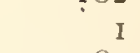 \\
\hline Achlya & I 78 & Apios & 25,194 & Bartsia & $8_{3}$ & Calycanthaceae & $3, I S_{7}$ \\
\hline Acnida & 89,207 & Apium & 48 & Bartramia & 172 & Calycanthus & $3, \mathrm{I} 8_{4}$ \\
\hline Aconitum & 183 & Aplopappus & 57 & Beckmannia & I 49 & esaracha & $8 z$ \\
\hline Acorus & 142 & Apocynaceae & 78,206 & Belamcanda & I 35 & $\mathrm{Ca}$ & II \\
\hline Acridii & I $\$ 2$ & num & 78,206 & Bembic & ISI & liaceae & 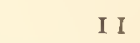 \\
\hline Actaea & I & Aquilegia & $\mathrm{I}, \mathrm{I} \mathrm{S}_{3}$ & Berberidaceae & $5, I S 5$ & $\mathrm{Ca}$ & 9 \\
\hline ctinella & 57 & Arabis & $6, \mathrm{I} 86$ & Berberis & 5,185 & aceae & 59 \\
\hline omeris & 57 & Araceae & $\mathrm{I}+2,2 \mathrm{II}$ & Berche & 15 & & 135 \\
\hline Adenostoma & 34 & Arachis & 25 & Bertia & 173 & abis & 97 \\
\hline doxa & 53,204 & Arachnida & 182 & $\mathrm{Be}$ & S9 & rellus & 175 \\
\hline fidium & I78 & Aralia & 49,203 & la & IOS, 209 & idaceae & IS7 \\
\hline lus & 22,193 & Araliaceae & 49,203 & $\mathrm{Bic}$ & 60,205 & $\mathrm{Ca}$ & $\mathrm{I} S_{7}^{\prime}$ \\
\hline cini & 175 & ina & $\mathrm{IS}_{2}$ & $\mathrm{Bi}$ & $\mathrm{I} \mathrm{S}_{1}$ & $\mathrm{C}$ & 53,204 \\
\hline cus & 175 & & 167 & & 60,205 & & \\
\hline $\mathrm{lg}$ & 136 & $\Lambda r^{\prime}$ & 69 & & $S_{5}, 207$ & & $8 z, 207$ \\
\hline onia & 34,197 & obium & 95 & niaceae & 85,207 & & I $S_{\text {I }}$ \\
\hline yrum & 146 & gelica & $4 S, 203$ & $\mathrm{Bl}$ & ISi & ine & 7,186 \\
\hline Agr & I 47 & stis & 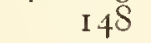 & 1.a & 86 & $\mathrm{C}$ & 60 \\
\hline & I So & & I & & 89 & & 212 \\
\hline Ailanthus & 12,190 & Arc & 57,205 & Bo & 6,185 & pephorus & 60 \\
\hline Aira & I 47 & taphylos & 70 & & 97 & $\mathrm{C}$ & I IO, 209 \\
\hline milla & 34,197 & & 173 & valia & 46 & $\mathrm{Ca}$ & $102,20 S$ \\
\hline is & 135 & & 8. IS7 & & 176 & $\mathrm{Ca}$ & ae $S$, is $S_{7}$ \\
\hline Algae & 178 & & $14 \mathrm{I}$ & & 205 & & $I S I$ \\
\hline & 3,211 & one & 6,185 & & I So & ndra & 70 \\
\hline aceae & $3,2 I I$ & & 142 & $\mathrm{Bc}$ & So & & 30,196 \\
\hline & $2 \mathrm{II}$ & & I 48 & & 60 & & 70,206 \\
\hline Al & 106,209 & ochia & 92 & haeria & 173 & & I I 2,209 \\
\hline ecurus & I 47 & ochiacea & 92 & & I 49 & psis & I I 4 \\
\hline anthera & Sq & Ar & 74 & lytrum & 149 & eia & $S_{3}, 207$ \\
\hline 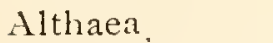 & $9, \mathrm{I} S S$ & Arr & 57,205 & & 6, IS6́ & $\mathrm{C}:$ & 85 \\
\hline itace & S9.207 & 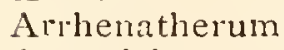 & $n \quad I+S$ & rellia & 60 & phyllum & - \\
\hline tus & 89,207 & Art & 58,205 & & I 49 & lothus & I5, 190 \\
\hline lidacere & I36 & aria & I $4 S$ & & 137 & & 167 \\
\hline lis & I36 & & I 49 & iaceae & I 35 & ceae & If, 190 \\
\hline sia & 57 & & 92 & & I 49 & 11 & I 4,190 \\
\hline chier & $3^{8,}$ I99 & & I $\delta_{2}$ & netia & 97 & & 97,208 \\
\hline hila & 147 & iadaceae & $7 S, 206$ & & 86,207 & ngium & 175 \\
\hline & 24,194 & & 78,206 & & 70 & & 150 \\
\hline S, & I5, I9I & dora & 78 & & 149 & hus & 56,204 \\
\hline nicarpaea & 24,194 & & ISS & lia & 7.5 & stium & $S, I_{7}$ \\
\hline & 78 & & 5,185 & Buxus & 95,208 & & $30,19^{6}$ \\
\hline aceae & 32,193 & & 136 & & & & 174 \\
\hline 1 lis & 206 & $\mathrm{um}$ & I $7 \mathrm{I}$ & & 00 & us & I $\delta_{2}$ \\
\hline onyca & $18 \mathrm{I}$ & & 149 & & $4 S, 202$ & & $4^{S}$ \\
\hline meda, & 69,206 & & 58,205 & & 48 & $11 \mathrm{um}$ & $4^{S}$ \\
\hline dropogon & 147,212 & lus & I94 & & I 49 & paris & 167 \\
\hline ndrosace & & Atriplex & $\mathrm{Sg}, 207$ & Calamintha & 86 & Chamaerops & $2 \mathrm{II}$ \\
\hline
\end{tabular}




\begin{tabular}{|c|c|c|c|c|c|c|c|}
\hline Iognathus & 131 & Corylus & 114 & Dimerosporium & 174 & Eupithecia & I So \\
\hline Cheilanthes & 171 & Crassula & 43 & Diodia & 56 & Eurotia & 207 \\
\hline Cheiranthus & 7,156 & Crassulaceae & 43,201 & Dioscorea 13 & $136,2 \mathrm{II}$ & Eutrema & IS6 \\
\hline Chelone & & Crataegus & 39,199 & Dioscoreaceae 13 & 136,211 & Eutypa & 174 \\
\hline Chenopodiaceat & e So, 207 & $\begin{array}{l}\text { Crawfish } \\
\text { Crepis }\end{array}$ & $1 S 2$ & Diospyros & 75,206 & Eutypella & $\begin{aligned} \\
\text { I } 74 \\
\text { SI }\end{aligned}$ \\
\hline Chenopodium S & 59,207 & $\begin{array}{l}\text { Clepis } \\
\text { Crotalaria }\end{array}$ & $\begin{array}{r}67,205 \\
25\end{array}$ & $\begin{array}{l}\text { Dipsaceae } \\
\text { Dipsacus }\end{array}$ & $\begin{array}{l}57 \\
57\end{array}$ & $\begin{array}{l}\text { Evolvu } \\
\text { Exidia }\end{array}$ & 177 \\
\hline Chilocorus & isi & roton & 95 & Diptera & ISo, 1 SI & Eysenhardtia & 36 \\
\hline Chilnpsis & $s_{5}$ & rotonopsis & 96 & & 95 & & \\
\hline hila & 70 & ruciferae & $6,7,186$ & Discomycetes & 175 & Fagopyrum & 208 \\
\hline Chicnanthus & 75,206 & Crustaceans & I S2 & Distichlis & 150 & & I I 4,209 \\
\hline Chironomus & 1 So & Cryptomeria & 167 & Dodecatheon & 75 & Festuca & I 51 \\
\hline hloris & 150 & ryptotaenia & $4^{S}$ & Dolichos & 26,194 & Fendlera & 4 \\
\hline galum & 137 & Cter & 150 & sidea & 174 & & \\
\hline Chondrioderma & 173 & & 47,202 & Draba & $7, \mathrm{IS6}$ & deae & 48,202 \\
\hline Chorizanthe & $9^{\circ}$ & $\mathrm{Cuc}$ & $47,2 \mathrm{O} 2$ & Dracaena & 137 & Ficus & 97208 \\
\hline Chrysinthemum & 60 & Cucurbitaceae & 47,202 & phila & I So & & 71,173 \\
\hline pogon & 150 & Culex & I So & Dryas & 34,197 & Fimbristylis & 146 \\
\hline Chrysopsis & 60,205 & Culicidae & 180 & hium & I 45 & & I 9 \\
\hline Cic & I $S \mathbf{I}$ & Cur & S6 & & 150 & culum & $4 S$ \\
\hline & $4^{S}, 203$ & $\mathrm{Cu}$ & 45 & Dysodiat & $6 I$ & Forestiera & \\
\hline ifuga & I & $\mathrm{C}_{11}$ & 167 & & & & IS \\
\hline Cin & $5^{6}$ & ferae & 106,209 & Eatonia & 150 & Fo & 1 \\
\hline & 150 & Cus & SI & & 75,206 & $\mathrm{Fra}$ & 34,197 \\
\hline Cir & 46 & s & I $7 \mathrm{~S}$ & tus & $4^{S}$ & & \\
\hline & 191 & aceae & 170 & & 47,202 & & 76,206 \\
\hline $\mathrm{Cis}$ & 7 & $v_{c}$ & 170 & is & I 43 & itia & II \\
\hline Cit & 47,202 & Cy & 39,199 & num & So & aria & 137 \\
\hline & $\mathrm{I} 2, \mathrm{I} \mathrm{S}_{9}$ & terus & 203 & ceae & 95,208 & & \\
\hline $\mathrm{Cla}$ & 145 & $\mathrm{Cy}$ & 150 & & $95,20 S$ & & 146 \\
\hline & 173 & ossum & So & yces & $17 \mathrm{~S}$ & ariaceae & $6,1 S_{5}$ \\
\hline $\mathrm{Cl}$ & 46 & ceae & 143,212 & is & 145 & $F_{u}$ & 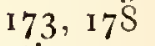 \\
\hline $\mathrm{Cla}$ & 177 & $\mathrm{Cy}$ & 145,212 & opus & $6 \mathrm{I}$ & i Imperfec & $\operatorname{cti} \quad 174$ \\
\hline & 177 & edium & I 35 & & 150 & Fusarium & 174 \\
\hline & 9, IS8 & 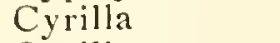 & 14,190 & & So & & \\
\hline $\mathrm{Cle}$ & $2, \mathrm{I} \mathrm{S}_{3}$ & ae & 14,190 & & 9 & & \\
\hline & $1 S_{7}$ & $\mathrm{Cyt}$ & 25 & & I 50 & & 26 \\
\hline endron & 56 & Cystopteris & 171 & ae & 133 & & 4 \\
\hline $\mathrm{Cl}$ & 70,206 & & & & 133 & 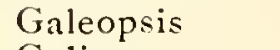 & \\
\hline um & 172 & & I 50 & & $17^{2}$ & & $56,2 O_{4}$ \\
\hline $\mathrm{Cli}$ & 137 & ctenium & I 50 & & 170 & jia & \\
\hline $\mathrm{Cn}$ & 60 & lea & 176 & loe & 174 & & $5 \mathrm{I}, 2 \mathrm{O}_{4}$ \\
\hline $\mathrm{Co}$ & ISI & & $6 I$ & & 70 & $C$ & 177 \\
\hline Co & I SI & & 25, I94 & & 46,201 & a & 71,206 \\
\hline $\mathrm{Co}$ & 141 & Lonia & I 50 & & I $7 \mathrm{I}$ & & $46,2 O_{2}$ \\
\hline tera & $I S_{1}$ & & 95 & & 171 & 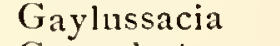 & 71,206 \\
\hline & I Sn & rio & 137,211 & & I 5 I & $\mathrm{m}$ & $46,2 \mathrm{O}_{2}$ \\
\hline & $S_{3}$ & & 22 & & $6 \mathrm{I}, 205$ & 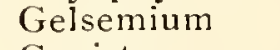 & \\
\hline nia & S6 & & $S_{2}$ & & I 5 I & & 26,194 \\
\hline Col & $1+2$ & 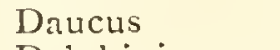 & $4^{S}, 203$ & & 69,206 & & 79,207 \\
\hline & 25 & $\mathrm{~m}$ & 2 & & $6 \mathrm{I}, 205$ & & 79,207 \\
\hline dia & $95,20 \mathrm{~S}$ & nae & ISo & & So & & 175 \\
\hline cene & 140 & mecon & 6 & & 90 & ae & 11,18 \\
\hline $57, \mathrm{C}$ & $6 S, 205$ & & 7 & & 146 & & I I , I $S_{9}^{\prime}$ \\
\hline & 158, i 70 & & I 75 & & 62 & 1. & \\
\hline Co & 137 & & 150 & & II & & \\
\hline 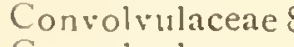 & 81,207 & & 25, I94 & & $4^{S}$ & & So \\
\hline lvilus & $S_{I}$ & dium & 25,194 & & 174 & a & 35 \\
\hline & $2, \mathrm{I} S_{3}$ & & $4 I, 200$ & & 26 & olus & \\
\hline $\mathrm{Co}$ & 135 & era & 85 & 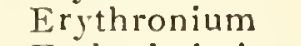 & 137 & & \\
\hline & I33 & & 8, I 87 & & $6, \mathrm{I} \mathrm{S}_{5}$ & a & I, 196 \\
\hline & $6 \mathrm{I}$ & sia & 74 & ptus, & 44,201 & & \\
\hline & 24 & siaceae & 74 & 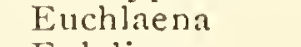 & $15 \mathrm{I}$ & & 26,194 \\
\hline & 24 & se & I 73 & & $15 \mathrm{I}$ & $\mathrm{dm}$ & $\sigma^{\top}$ \\
\hline Co & 50,203 & & 6 & & 14,190 & & 170 \\
\hline & 50,203 & & 174 & & 62 & ius & 175 \\
\hline Cor & 177 & Dicranuin & 172 & rbia & 96,208 & & \\
\hline & $6,1 S_{5}$ & Diervilla & 53 & Euphorbiaceae & $95,20 S$ & Gordonia & \\
\hline
\end{tabular}


Gossypium 10, 1SS

Gramineae 146,157 . 2 I 2

Gratiola

Grimmia

Grindelia

$\mathrm{S}_{3}$

Gryllidae

172

Gryllidae.

62

Gutiemezia 62,205

Gymnocarpus $\quad \mathrm{Sg}$

Gymnocladus 31,196

Gymosporangium 178

Gynerium $15 \mathrm{r}$

\section{Habenaria}

Hamodoraceae 135

Ilalenia

Halesia

Halictus

Halorageae

Haltica

Hamainelaceae 43 , 201

Hamamelis $\quad 43,201$

Harpalus

$43, \frac{201}{1 S I}$

Hedeoma

Heder:

lledysarum

50,203

Helenium

26,195

Helian thella

63,205

Helianthus

Heliopsis

63,205

64

Heliotropium SI

Helminthosporium 174

Helonias .

I37

Helvella

175

Hemerocallis

137

Hemiarcyra

Hemiptera

173

Hepaticae

Heracleum

Herpestis

Heteromeles

Heuchera

Hexapoda

Hibiscus

Hieracium

Hierochloa

Hilaria

Hordeum

Hosackiá

Houstonia

Hudsonia

Humulus

Hydnei

Hydnum

$1 \mathrm{SI}$

173

$4 \mathrm{~S}$

$\mathrm{S}_{3}$

35 , IgS

41,200

${ }_{1} S_{2}$

I0, I SS

67

15 I

I 5 I

$15 \mathrm{I}$

26, 195

56,204

\section{7}

97

177

Hydrangea

177

Hydrocotyle

42,200

Hydrophyllaceae So

Hydrophyllum So

Hymenoptera i 7 , ISo

Hypericaceae 9, ISS

Hypericum 9, ISS

Hyphantria

I So

Hypnum

Hypoxis

172

Hypoxylon

136

Hyptis

174
56

Ichnellmonidae 180

Ilex

I3, I90
Ilicineae

Hllecebraceae

Impatiens

Indigotera

Insects

Inula

Ionidium

lpomoea

Iridaceae

Iris

lsanthus

Isomeris

Isopyrum

Iva

13190
89
12.189
26
180
64
7
S1, 207
135
135
56
7
2
64.205

Jacquinia

Juglandaceae

Juglans

Julus

Juncaceae

Juncus

Jungermannia

Juniperus

Jussiáea

Kalmia

Kerria

Kobresia

Koeleria

Koenigia

Koerberlinia

Kranitzia

Krigia

Kuhnia

\section{Labiatae}

Lachnosterna

Lactarius

Lactuca

Lagenaria

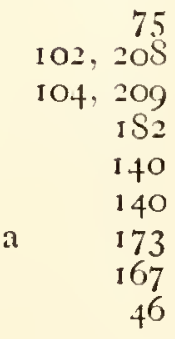

7.5

Og

$\mathrm{S}_{2}$

140

173

46

$\begin{array}{lr}\text { Lagenaria } & 47 \\ \text { Lagerstroemia } & 45,201\end{array}$

Lamium

Lampyridae

Laportea

Larix

Lathyrus

71,206

35

I 46

1
91

I 90

SI

67

64

Lauraceae

Lavatera

Lecanium

S6, 207

$1 \mathrm{SI}$

175

67,205

Lecidea

Ledum

Leersia

Leguminosae

S6

I $\mathrm{I}$

97

I 59

$26,19.5$

$92,95,20 \dot{S}$

182

173

71

152

Lemnaceae

$24,32,194,196$

Lentinus

Leonotis

Leonurus

Lepachys

Lepidium

Lepidoptera

Lespedeza

Leucothoe

Lewisia

Liatris

Libocedrus

Lichenes

Ligusticum

176

7,156

I So

27,195

71,206

I SS

64,205

169

I 73

$4 \mathrm{~S}$
Ligustrum

Liliaceae I 6,211

Lilium 137, 2 I I

Limnanthemum $\quad 79$

Linaceae II, IS9

Linaria

Lindera

Linnaca

Linum

Lippia

Liquidambar

Liriodendron

Lithophanae

Lithospermum

Litsea

Loasaceae

Lobelia

Lobeliaceae

Loganiaceae

Loiseleuria

Lonicera

Lophan thus

Loranthaceae

Lucilia

Ludwigia

Lupinus

Luzula

Lychnis

Lycium

Lycoperdon

Lycopersicum Sa,

L2, 207

Lycopodiaceae

Lycopodium

Lycopus

Lygodesmia

Lyrocarpa

Lysimachia

Lythraceae

Lythrum

Maclura

Madia

Magnolia

Magnoliaceae

Maian themum

Malacothrix

Mallotus

Malva

Malvaceae

Malvastrum

Malvaviscus

Mamestra

Mamillaria

Manihot

Narrubium

Massaria

Matricaria

S7, 202

Matthiola

64 Medicago

Megarrhiza

Melanthera

Melia

Meliaceae

Melilotus
Menispermum 5

Mentha

Mentzelia

47,202

Menyanthes 


\begin{tabular}{|c|c|c|c|c|c|c|c|}
\hline Orontium & 142 & phragmites & I 53 & Pteleil & $\mathrm{I} 2, \mathrm{I} \mathrm{S}_{9}$ & Schedonnardus & \\
\hline Orthoptera & IS: & Phryganeidae & $I S 2$ & Pteris & I 7 I & Schizoneura & I S 2 \\
\hline Oryzil & 152 & Pliryma & S6 & Ptilodactyla & ISI & Scleranthus & \\
\hline Oryzopsis & 152 & Physalis & $S_{2}, 207$ & Ptychomitrium & 172 & Scirpus & 146 \\
\hline Osinantluss & $7 S$ & Plivsarum & 173 & Puccinellia & I 54 & Scitamineae & I 35,2 I I \\
\hline O-morrliza & $4 S, 203$ & Physcia & 173 & Puccinia & I $7 \mathrm{~S}$ & Sclerederma & I $7 \mathrm{~S}$ \\
\hline Osmunda & 171 & Pliysostegia & 87 & Pycnanthemum & $S_{7}$ & Scrophularia & 8 \\
\hline O-trya & I 16,209 & Phytolacca & 90 & Pyrenomycetes & I 73 & Scrophulariacea & re \\
\hline Oxali: & I 2, IS9 & Phytolaccaceae & 90 & & & & $\mathrm{~S}_{3}$ \\
\hline Oxybaphus & S9 & Phytonomus & I 8 I & Quercus & II 6,200 & Scutellaria & 207 \\
\hline Oxidendrum & 72 & Picea & $\begin{array}{l}160 \\
\text { I So }\end{array}$ & Rafinesquia & $6 S$ & $\begin{array}{l}\text { Secale } \\
\text { Sedum }\end{array}$ & $\begin{array}{r}154 \\
201\end{array}$ \\
\hline $\begin{array}{l}\text { Oxyria } \\
\text { Oritropis }\end{array}$ & $\begin{array}{r}91 \\
\text { 2S. I9: }\end{array}$ & $\begin{array}{l}\text { Pieris } \\
\text { Pilea }\end{array}$ & $\begin{array}{r}\text { I So } \\
99\end{array}$ & Ranunculaceae & $\mathrm{I}, \mathrm{IS} S_{3}$ & Senecio & 64,205 \\
\hline Oxytropis & & Pimpinelia & 49 & Ranunculus & $2, \mathrm{IS}_{3}$ & Sequoia & 170 \\
\hline achyma & I $T S$ & Pinus & 160 & Raphanus & $7, \mathrm{IS6}$ & Sesamum & $\mathrm{S}_{5}$ \\
\hline Pachistima & I5 & Piperaceae & 92 & Reseda & 7 & Sesbania & 29,196 \\
\hline Paleonia & $2, \mathrm{I} S_{3}$ & irola & 72 & Resedaceae & 7 & Setaria & I 54 \\
\hline Palmeae It & It $2,21 \mathrm{I}$ & Pirus. & $39 \cdot 199$ & Rhamnaceae & I 5, I 90 & Seymeria & $\mathrm{S}_{4}$ \\
\hline Pancratium & I36 & Pisum. & $2 S, 195$ & Rhamnus & I 5, I $9 \mathrm{I}$ & herdia & $95,20 S$ \\
\hline Pandinaceae & I 42 & istacia & 22 & & 92 & $\mathrm{Si}$ & 199 \\
\hline inus & I +2 & Pittosporaceae & 7 & trichum & 175 & $\mathrm{Si}$ & 47,202 \\
\hline$P a t$ & 152 & $n$ & 7 & morpha & I $7 S$ & $\mathrm{Si}$ & $\mathrm{IO}, \mathrm{I}_{9}$ \\
\hline & $6, \mathrm{IS}_{5}$ & inaceae & SS, 207 & Rhododendron & 72,206 & Sidalcea & I I \\
\hline aceae & 6. $\mathrm{IS}_{5}$ & go & SS, 207 & Rhus & 22,193 & & $S$, ISS \\
\hline Pai & 99 & ceae & $100,20 S$ & ichosia & 28 & um & 65 \\
\hline nia & I 95 & & $100,20 S$ & chospora & 146 & ibeae & I 2,190 \\
\hline Par & 173 & $\mathrm{Pla}$ & I $S_{1}$ & R! & 175 & 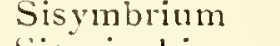 & $7, \mathrm{IS} 6$ \\
\hline Par & $t^{2}, 200$ & ne & 79 & & 42,200 & rinchium & 136 \\
\hline hieae & 9 & gintia & I 74 & ardia & I 42 & & 49,203 \\
\hline Parthenitum & 64 & $\mathrm{Pl}$ & 64 & & 96 & kia & \\
\hline Pals & 153,2 I 2 & baginaceae & 74 & nia & $2 S, 196$ & & 130 \\
\hline P.15 & ISI & Plusia & I So & Ro & 36, I $9 S$ & & I $3 S, 2$ II \\
\hline dora & 47,202 & $\mathrm{P}$ & I 53 & $\mathrm{R}$ & 32,196 & $\mathrm{~s}$ & 52.207 \\
\hline ceae & 47,202 & tophyllum & $6, \mathrm{IS}_{5}$ & ceae & 56,204 & & 82,207 \\
\hline $\mathrm{P}_{3}$ & 49.203 & iceae & So, 207 & & 36,198 & & 65,205 \\
\hline & 17.5 & & So & eckia & 64,205 & & $6 S$ \\
\hline$P$ & I S I & ius & I $S=$ & & 86 & & 29,196 \\
\hline eae & $\mathrm{S}_{5}$ & & S, IS7 & & 92,208 & & I 54 \\
\hline aris & $S_{3}$ & alaceae & $S, \boldsymbol{s} S_{7}$ & & 176 & iium & 142 \\
\hline nium & 12,189 & ceae & 90,208 & Rutaceae & 12,189 & & I 54 \\
\hline Pell & I $7 \mathrm{I}$ & uin I & I 37,2 I I & & & ria & 175 \\
\hline $\mathrm{Pe}$ & $1+2,2 \mathrm{II}$ & 11 a & $9 \mathrm{I}$ & & I 4 I & & \\
\hline & I 73 & & $91,20 S$ & $\mathrm{~S}$ & 79 & & I SS \\
\hline ora & 177 & 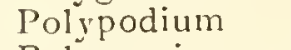 & I 7 I & arum & I54 & oce & \\
\hline & 43 & $p$ & 176 & & I $S S$ & aria & I 75 \\
\hline mon & $S_{4}, 207$ & $\mathrm{~s}$ & I 76 & 1 & 143,2 I I & & \\
\hline Per & 64 & Pol & 49 & & I 29,2 Iо & & 174 \\
\hline sppora & I $7 \mathrm{~S}$ & & 172 & nia & 90 & psis & 175 \\
\hline $\mathrm{Pe}$ & 93 & 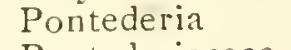 & I 40 & & $\mathrm{I} 3 \mathrm{I}, 2 \mathrm{I} \mathrm{I}$ & & 172 \\
\hline Pertu: & 173 & deriaceae & I 40 & & $S_{7}, 207$ & idae & I So \\
\hline mon & $2 S, 195$ & & 210 & & 54,204 & & 90,208 \\
\hline & 64,205 & & 29,2 IO & & 75 & & $3 S, 199$ \\
\hline hora & I So & & & aria & 6 & & I 82 \\
\hline linum & 203 & aceae & 9, I SS & & 49 & & 2 I I \\
\hline danum & 49 & yeton & I 43, 2 I I & & $95.20 S$ & olus & I 55 \\
\hline & 175 & & 35, I $9 S$ & ceae & IS, L92 & & \\
\hline & So & & 36 & & 22 & & \\
\hline Phalaris & 153 & renanthes & $6 S$ & & S & ylea & 22,193 \\
\hline Pha-eolu & $2 S .195$ & & 75 & & 75 & & \\
\hline & 171 & & 75,206 & & ISOO & S & 75,206 \\
\hline Philadelphus & 42,200 & ardia & It I & Sarcobatus & 90,208 & & 9. ISS \\
\hline Phippsia & I.53 & inaca & 47 & Sarracenia & $6, \mathrm{IS}_{5}$ & onitis & 173 \\
\hline Phlegethontius & I $\mathrm{S}$ & & 3 & niaceae & e $6 . \mathrm{IS}_{5}$ & & \\
\hline & 153 & & $3=196$ & Sassafras & $94,20 S$ & meria & 68,205 \\
\hline & So & ya & I 66 & & $9^{2}$ & aceae & I I \\
\hline Phoenix & $1 \dashv 1$ & sidium & 45 & a & 43,200 & $n m$ & I 7 \\
\hline dron & 95 & & 19.5 & & $4 \mathrm{I}, 200$ & & I 73 \\
\hline (1) & 137 & Psychotria & $5^{6}$ & carabeidae & I $S_{\text {I }}$ & Stillingia & \\
\hline
\end{tabular}




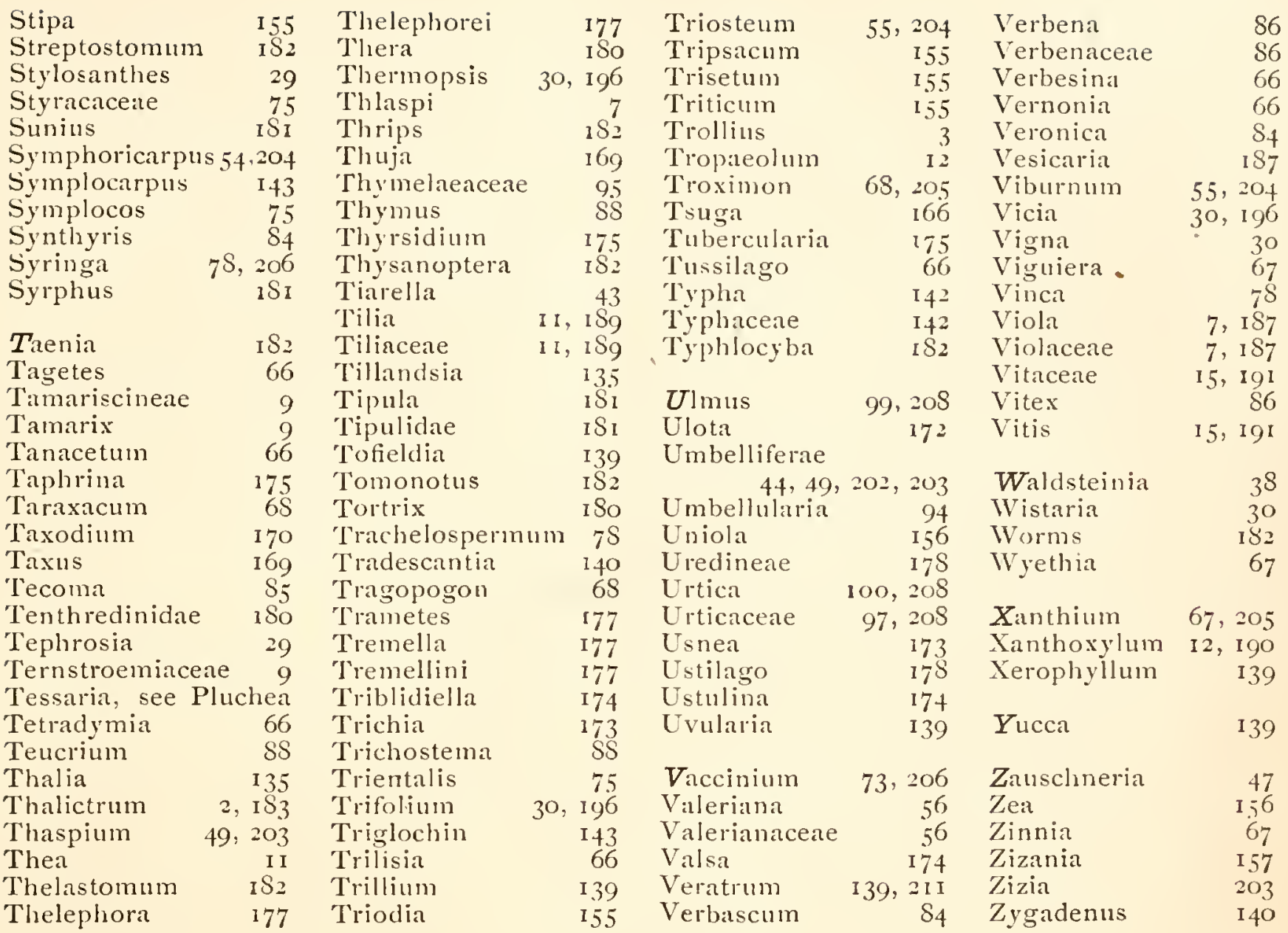









\section{Lilurary of harbard antwersity.}

\section{Bibliographical CONTRibutions.}

EDITED BY JUSTIN WINSOR, LIJRARIAN.

No. 25.

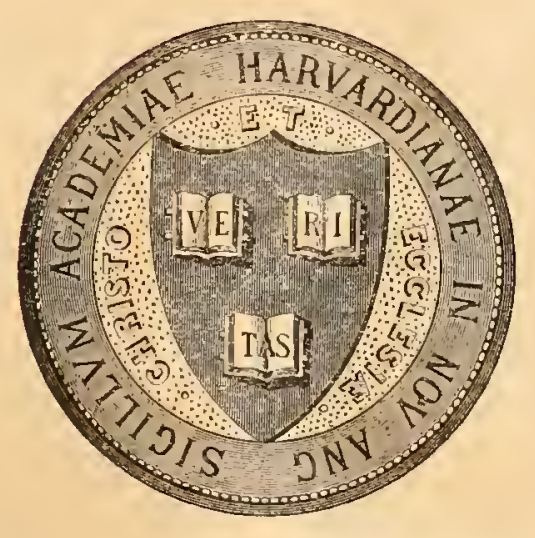

A LIST OF WORKS ON NORTH AMERICAN FUNGI. By W. G. FARLOW AND WILLIAM TRELEASE.

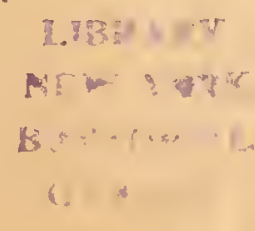

CAMBRIDGE, MASS. :

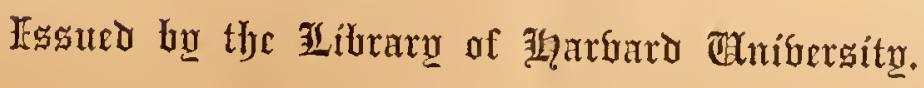
I 887 


\section{Already issued or in preparation:}

A Star prefixed indicates they are not yet ready.

\section{VOL. I.}

I. EDward S. IIOLden. Index-Catalogue of Books and Memoirs on the Transits of Mercury.

2. Justin Wrinsor. Shakespeare's Poems : a Bibliography of the Earlier Editions.

3. Charles Eliot Norton. Principal books relating to the Life and Works of Michelangelo, with Notes.

4. Justix Winsor. Pietas et Gratulatio. An Inquiry into the authorship of the several pieces.

5. List of Aplaratus in different Laboratories of the United States, available for Scientific Researches involving Accuratc Measurements.

6. The Collection of Books and Autograpus, bequenthed to Harvard College Library, by the Honor. able Charles Sumner.

7. Williair C. Lane. The Dante Collections in the Harvard College and Boston Public Libraries.

S. CALENDAR of the Artlur Lee Manuscripts in Harvard College Library.

Q. George Lincoln Goodale. The Floras of different countries.

10. JUSTIN WInsor. Halliwelliana : a Bibliography of the Publications of James Orchard Halliwell-Phillipps.
11. Samuer H. Scudder, The Entomological Libraries of the United States.

12. A LIST OF the PUBlications of Harvard University and its Officers, IS7O-rSSo.

13. Samuel H. Scudder. A Bibliograplyy of Fossil Insects.

I4. William H. Tillingmast. Notes on the Historical Hydrography of the Handkerchief Shoal in the Bahamas.

15. J. D. Whitney. List of American Authors in Geology and Palæontology.

16. Ricinard Bliss. Classified Index to the Maps in Petermann's Geographische Mittheilungen. IS55ISSI.

I7. Richakd Buiss. Classified Index to the Maps in the Royal Geographical Society's Publications. 1\$30ISS3.

is. Justin WInsor. The Bibliography of Ptolemy's Geography.

19. Justin Winsok. The Kohl Collection of Early Maps.

20. William C. Lane. Index to Recent Reference Lists, ISS4-ISS5.

\section{VOL. II.}

21. A List of tife Publications of Harvard University and its Officers, ISSO-1SS5.

*22. Justin Winsor. Calender of the Sparks Manuscripts in Harvard College Library.

23. Williain H. Tillinginast. List of the Publications of IIarvard University and its Officers, ISS5-1SS6.
24. William C. Lane. Index to Recent Reference I.ists, ISS5-iSS6.

25. W. G. Farlow and William Trelease. I.ist of Works on North American Fungi. 


\title{
LIST OF WORKS ON NORTH AMERICAN FUNGI,
}

\section{WITH THE EXCEPTION OF SCHIZOMYCETES, PUBLISHED BEFORE 1887.}

\author{
BY W. G. FARLOW AND WILLIAM TRELEASE.
}

The following list has been prepared from a card-catalogue of authors used in indexing the species of fungi found in the United States and the northern parts of North America. The list does not include works on Schizomycetes, as that group is now studied principally by specialists, and ineludes forms the greater part of whieh belong rather to the department of medicine than to botany proper, and the literature of the subjeet is already well indexed elsewhere. The present list aims to inelude mainly works of greater or less value to working botanists, and it would be diffieult and, perhaps, unneeessary to add the very large number of papers of a popular and indefinite elaraeter relating to fungi not specifically named whieh are scattered through the various agrieultural, hortieultural, and other journals. Neither has it been thought best to enumerate the general and, to botanists, well-known treatises which inelude fungi as well as other orders of plants, unless special referenee is made to North American speeies, or unless they eontain figures of our species not easily found elsewhere. As it is, the pres. ent list, whieh is the first of the kind yet published, will show that the general belief of botanists not speeially studying the subject that very little has been written on North American myeology is by no means correct. The means for obtaining good summaries of the current literature have, within the last few jears, been muel improved; and, in order to complete the record of the past as far as possible, botanists are requested to send correetions of and additions to the present ist, whieh may be issued in a supplement. Thanks are due to a number of authors who have kindly consented to revise the list of their works for publieation.

Where the pages of reprints differ from the original they are put in parenthesis. The dates after authors' names indicate the date of birth, and death is indicated by a cross $(t)$.

Cambidide, February 23, 1887.

W. G. FARLOW.

\section{Agricultural Reports.}

For papers on fungi in the reports of the Department of Agriculture up to the year 1876 consult the following:-

A general index of the Agricultural Reports of the Patent Office for twenty-five years from 18371861, and of the Department of Agriculture for fifteen years from 1862-1876. $8^{\circ}$. pp. 225 . Washington. 1879.

Subsequent papers under authors' names.

3. 2. American Naturalist, The. Monthly, illustr. Vol. I.-XX. March, 1867-Jan. 1887. To be continued.

Devoted to all branches of natural history. The first nine volumes published at Salem, Mass., X.-XI. at Boston, XII.XX. at Philadelphia. For X.-XI. there was an associate editor for botany, G. L. Goodale, and since 1881, XV., C. E. Bessey. Articles on fungi given here under author's' names. Reviews of mycologieal works by the editors.

Andrews, Charles Lowell.

3. Contributions to the Mycology of Massachusetts. Proc. Boston Soc. Nat. Iist. V. pp. 321-323. April, 1856.

Remarks on the study of fungi and their economical importance, followed by a list of 36 species collected by the author.

\section{Arctic Manual. See Manual.}

Arthar, Joseph Charles. Lowville, N. Y. 11 Jan. 1850. See Bessey, C. E. See Botanical Gazetre.

$+5 .-$ The interpretation of Schweinitzian and other early descriptions. Am. Nat. XVII. 77, 78. Jan. 1883.

+6 . Descriptions of Iowa Uromyces. Bull. Minnesota Acad. Nat. Sci. II. 13-37. Minneapolis. Printed 31 May, 1883.

Descriptions of 12 species, one of them new, $U$. acuminatus, with index of synonyms and host-plants.
* 7. American Aecidia on Ranunculi. Bot. Gaz. IX. 177. Oct. 1884.

Note on aecidia on Ranunculus abortivus and Anemone nemorosa.

+ 8. - Preliminary list of Iowa Uredineae. Bull. Iowa Agr. College, Dept. of Botany. 151171. Nov. 1884. Issued Jan. 1885.

Enumerates 134 species, and describes 8 new speeies with index of host-plants. See no. 65. Names of new species in Jour. MIyc. I. 47.

+ 9. - Memorandum of Iowa Ustilagineae. Bull. Iowa Agr. College, Dept. of Botany. 172174. Nov. 1884. Issued Jan. 1885.

Enumerates 25 species, of which 2 are new. This paper and the preceding were subsequently issued together as a separate paper with original paging.

ro. Hollyhock disease and the cotton plant. Science, V. 2. Jan. 2, 1885. Also Jour. Myc. I. 27. Feb. 1885.

Reports experiments by Plowright to show that Puccinia Mralvaceamem will not grow on the cotton plant.

- Ir. - The Aecidium of Adoxa. Bot. Gaz. X. 369. Sept. 1885. Also Jour. Myc. I. 141. Nov. 1885.

States that Aecidium albescens is an annual.

\% r2. - Report of the Botanist to the New York Agricultural Experiment Station. $3 d$ Annual Rept. N. Y. Agr. Exp. Station for 1884. pp. 353-385. Fig. 6. $8^{\circ}$. Albany.

Notices of pear blight, F'usicladium pyrinum, $F$. dendriticum, Morthiera Mespili, Podosphaera tridactyla, Sphaeropsis Cydoniae, Exoascus deformans, peach yellows, gummosis, Gloeosporium phomoides, Ustilago segelum, Clematis disease, ete. For Clematis disease see also Country Gentleman, 25 .June, 1885. This report was distributed about 1 Sept. 1885. See Rev. in Bot. Centralblatt, XXIV. 335. 
Arthur, Joseph Charles.

13. A new larval Entomophthora. Bot. Gaz.

XI. 14-17. PI. 2. Ja1. 1886.

Describes and figures Ent. Phytonomi on $P$. punctatus. See also Jour. Myc. 1I. 35, and tth Kept. N. I. Agr. Exp. Station. See vext title.

$\therefore$ r4. Report of the Botanist to the New York Agricultural Fiperiment Station. 4th Amn. Rept. N. Y. Agr. Exp. Station for 1885. 241-265. Fig. 10. Ist elition, Rochester, issued 30 Jan. 1886 ; 2d edition, Albany, Dec. 1886.

Contains acconnts of Morthiera Mespili $\mathbf{r}$. Cydoniae Septoria Lactucae, Peronospora gangliformis, Oidium fructigenum, Entomophthora Phytonomi, pear blight, the rotting of tomatoes, ete. The second erlition has separate paging, but is accompanied by a table referring to original paging.

Atkinson, Isaac Edmondson. Baltimore, Md. 23 Jan. 1846.

15. The botanical relations of Trichophyton tonsurans. N. Y. Med. Jour. XXVIII. 561-575 (1-17). Fig. 3. New York, Dec. 1878.

Account of germination of spores and formation of cyst-like eells.

Austin, Coe Finch. Closter, N. J., 20 Jume, 1831. tCloster, N. J., 18 March, 1880.

is 6 . Agaricus with the odor of chlorine. Torr.

Bull. VI. 278, 279, and 298. Dec. 1878.

Charneters of Agaricus chlorinosmus, Peck, with notes on its orlor.

Bagnis, Carlo. 1854. † Aisone, 6 Aug. 1879. 17. Le Puccinie. 4\% pp. 83. Pl. 1-11. Rome. 1876. Ext. Att. Reale Acad. Lincei. 2 ser. Vol. III. Part 2. 6\$1-721.

The author reduces the number of species of Puccinia to 61, and gives the syuony'ns of formus recognized by him, including a number of American species.

Bailey, Jacob Whitman. Ward, Mass., 29 April, 1811. + IYest Point, N. Y., 26 Feb. 1857.

8. Curious microscopic fungus, Craterium pyriforme. Am. Jour. Sci. Arts, XIII. 195. 1842. Reports finding this species on rocks at West Point.

Banning, Mary Elizabeth. Talbot Co., Md. 1832.

I9. Notes on the fungi of Maryland. Field and Forest, III. 42-47, 59-63. Sept.-Oct. 1877.

1 20. - Notes on Fungi. Bot. Gaz. V. 5-10, 23. Jan.-Fcb. 1880.

General remarks on the occurrence and habit of a number of species.

\% 2r. - New species of fungi found in Mary-

land. Bot. Gaz. VI. 165, 166. Jan. 1881.

Characters of $\downarrow \Lambda$ garies.

22. - Maryland Fungi. Bot. Gaz. VI. 200-

202, 210-213. April-May, 1881.

Notes on a number of IIymenomycetes and Gasteromycetes.

Barnes, Charles Reid. Madison, Jefferson Co., Indiana, 7 Sept. 1858. See Botanical Gazette. \{-23. Oospores in Capsella. Bot. Gaz. IX. 194. Dec. $188 t$.

Finds oospores of Cystopus candidus in Capsella.

Bary, (IIeinrich) Anton DE. Frankfort-on-theMain, 26 Jan. 1831.

24. Recherches sur le développement de quelques champignons parasitcs. Mémoire pour servir de réponse à une question proposéc par l'Académie des Scicnces en 1861 et pour servir de supplément aux travaux sur la question des générations dites spontanées. Ann. Sci. Nat. Bot. 4 ser. XX. j$148(1-144)$. Pl. 1-13. 1863.

Detailed account of observations on the stmeture and de. relopment of Cystopus, Peronospors, and different Uredineae followed by an "Essai l'un swnopsis des Péronosporées," with full deseriptions of all known species, including $P$. viticola from the United States. This monograph appeared originally in the Anuales, and is not a French trauslation from the German as is sometimes stated in this country.

25. Ueber den sogenannten Brenner (Pech) der Reben. Ann. der Oenologie, IV. Heft $2(1-3)$. 1873.

Reprint only seen. A communeation to the editor giving an account of Sphaceloma ampelinum, witl a note on the Nuemaspora ampelicida of Engelmam in America.

Other papers of interest, although not treating strietly of North American fungi, are: "Die gegenwärtig lierrschende Kartoffelkranklıeit, ihre Ursache und ihre Verhiitung," $8^{\circ}$, pp. 75 , pl. 1, Leipzig, 1861; and "Researelies into tlie vature of the potato-fungus, Phytophthora infestans," $8^{\circ}$, pp. 33 , fig. 8, London, 1876; and Jour. Royat Agr. Soc. England, XiI. part 1.

Berkeley, Miles Joseph. Biggin, Parish Oundle, Northampton, England, 1 April, 1803. See Cooke, M. C., Curtis, M. A., Klootzsch, I. J., Lea, T. G., de Sernes, J., Wrain, J.

26. - Descriptions of exotic fungi in the collection of Sir WV. J. Hooker, from nemoirs and notes of J. F. Klotzsch, with additions and corrections. Ann. Nat. Hist. III. 375-401. 1839.

Review of species in Klotzscl's papers in Limnaea, VIT. 193, and VIII. 478. Notes on 42 speeies from North America.

27. - Supplement to descriptions of exotic fungi in Annals of Natural History, vol. III. 322 and 375. Ann. Nat. Hist. VII. 451-454. 1841.

Principally notes on species collected by Richardson in Britisl America, with deseriptions of 3 new species.

28. - Descriptions of fungi collected by R. B. Hinds, Esq., principally in the islands of the Pacific. London Jour. Bot. I. 447-457 (1-11). Pl. 14, 15. 1842 .

Enumerates 7 species from the Columbia River, Sitka, and California, and deseribes Polyporus Columbiensis.

29. - Notices of fungi in the herbarium of the British Museum. Ann. Mag. Nat. Hist. X. 369-385. Pl. 9-12. Jan. 1843.

Contains a deseription of Polypomıs Floridanus from Florida, and also speeies from Jamaien.

30. - On Agaricus crinitus, I., and some allied species. Trans. Linn. Soc. XX. 109-112. P1. 9. 18 Feb. 1845.

Contains a description and figure of Lentinus tener.

31. - Decades of Fungi. London Jour. Bot. and Hooker's Jour. Bot.

The following parts relate to North American species: Nos. 74-100 in Jondon Jour. Bot. IV. 299-313 (35-49), pl. 11, 12, fig. 1-5, 1845, collected, with few exeeptions, by T. G. Lea in Ohio; pl. 11 represents Cyclomyces Greeneii from Massaehuisetts; - nos. 111-140, l. c. VI. 312-326 (1-15), 1847, all from Olio, colleeted by Lea, are mostly Hymenomycetes with a new genus Psilopezia; - nos. 201-240, Ilooker's Jour. Bot. I. 97-10t (1-8), 231-239 (105-110), 1849, have the title "North and South Carolina Fungi; by the Rer. M. J. Berkeley and Rev. M. A. Curtis"; - no. 485, 1. c. VI. 227 (2), 1854, deseribes Dothidea vorax from Sonth Carolina. The species given in the Decades are most of them to be found ilso in Lea's Catalogue and Berkeley's Notices of North American Fungi, $q$. $v$., and a few of the figures are in Berkeley's Introduction to Cryptogamic Botany. See also Curtis, M. A.

32. - On the white rust of cabbages. Jour. Royal Ilort. Soc. III. 265-271. Aug. 1848.

Gives a figure of conidia of Uredo (Cystopus) Amaranthi, Schweinitz.

33. - Indian Bread or Tuckahoe. Gardeners' Chronicle, p. 829.16 Dec. 1848.

Includes a note of Prof. Ellet on the chemical properties of tuckahoe.

34. - Enumeration of some fungi from St. Domingo. Ann. Mag. Nat. Hist. 2 ser. IX. 192203. Pl. 8. March, 1852.

Fungi collected by A. Sallé, ineluding 67 species, of which 20 are new, and 1 new genus. Species may be expected to occur in Florida. 
Berkeley, Miles Joseph.

35. On two new genera of fungi. Trans. Linn. Soc. XXI. 149-154. Pl. 19, fig. 4. June, 1852.

Has description of Corynites Ravenelii, with figure.

36. Ravenelia. Gard. Chron. 26 Feb. 1853. p. 132.

In an editorial without title there is a note and figure of R. glandulaeformis.

37. - On some entomogenous Sphaeriae. Jour. Linn. Soc. I. 157-159. Pl. 1. 4 Nov. 1856.

Includes descriptions and figures of 5 new species of Cordyceps from the United States, collected by $\mathbf{H}$. W. Ravenel.

38. - Introduction to Cryptogamic Botany. 8. pp. 604, with 127 illustrations. London. 1857.

Incidental references to Ameriean species of fungi, with figures of some of them. In a few instances the figures are the earliest published records of the species.

39. - On some new fungi. Trans. Linn. Soc. XXII. 129-131. Pl. 25. $5 \mathrm{May}, 1857$.

Description and figure of Mitremyces Ravenelii.

40. An account of the plants collected by Dr. Walker in Greenland and Arctic America during the expedition of Sir Francis McClintock, R.N., in the yacht Fox. By .T. D. Hooker, M.D. Jour. Linn. Soc. V. 79-89. 1861. Read 21 June, 1860.

On p. 86 , a list of 8 species, all but one Agaricini, repeated in Manual Arctic Expedition, part 2, p. 526.

41. On some new fungi from Mexico. Jour. Linn. Soc. 1X. 423-425. Pl. 12. 1867. Read 15 Nov. 1866.

Describes 6 new species sent by Curtis, collected by Botteri near Orizaba, and a new genus Wynnea, of which a figure is given. The species are attributed to Berkeley and Curtis. +42 . Notices of North American Fungi. Grevillea, I. 33-39, Sept. 1872, nos. 101-125 ; 4955 , Oct. 1872, nos. 126-149; 65-71, Nov. 1872, nos. $150-175 ; 97-102$, Jan. 1873, nos. 176-200; 145-150, April, 1873, nos. 201-225; 161-166, May, 1873 , nos. $226-250 ; 177-180$, June, 1873 , nos. $251-$ 275 ; - II. 3-7, July, 1873, nos. 276-300; 17-20, Aug. 1873, nos. 301-319; 33-35, Sept. 1873, nos. $320-327 ; 49-53$, Oct. 1873 , nos. $328-350 ; 65-69$, Nov. 1873 , nos. 351-375; 81-81, Dec. 1873, nos. $376-401$; 97-101, Jan. 1874 , nos. 451-475; 153157, April, 1874, nos. 476-500; 177-181, June, 1874, nos. 402-414; - III. 1-17, Sept. 1874, nos. $415-450$ and $501-525 ; 49-64$, Dec. 1874 , nos. 526$600 ; 97-112$, March, 1875, nos. 601-681; 145-160, June, 1875, nos. 682-750; - IV. 1-16, Sept. 1875, nos. $751-813$; 45-52, Dec. 1875 , nos. 814-844; 93-108, March, 1876, nos. 845-902; 141-162, June, 1876 , nos. $903-1005$.

This series is a continuation of the "Centuries of North American Fungi," by Berkeley and Curtis (see below), in which 100 species are described. The present series begins with 101, and the numbers represent new species described by Berkeley and Curtis, a small number of whieh had been previously published in Berkeley's "Decades of Fungi" and "Contributions to North American Mycology," by Berkeley and Curtis. Besides the numbered species, a large number of others are enumerated without numbers. See CUnTIs, MI. A., and TUCKERMAN, E.

43. - Enumeration of the fungi collected during the Arctic expedition, 1875-76. Jour. Linn. Soc. XVII. 13-17. 1880. Read 7 March, 1878.

Enumeration of 24 species, 7 described as new.

44. - Iypsilophora destructor. Gard. Chron. XI. new ser. 299. Fig. 41. 8 March, 1879.

Description and figure of IIypsilophora (Dacrymyces) destructor on pear stems, and reference to 2 other species.
45. - Truffles in California. Gard. Chron. XXI. new ser. 833. 28 June, 1884.

Short note on the occurrence of truffles in California. and Broome, C. E.

46. Enumeration of the fungi of Ceylon. Part 2. Jour. Linn. Soc. XIV. 29-140. Pl. 2-10. 3 April, $1873(1875)$.

Includes descriptions of Artocreas Micheneri, p. 73, and Patcllaria upplanata, p. 108, from the United States, and notes on several species described in Cuban Fungi.

— and Curtis, M. A.

+47. Contributions to the Mycology of North America. Am. Jour. Sci. Arts, 2 ser. VIII. 401403, Nov. 1849; IX. 171-175, March, 1850; X. 185-188, Sept. 1850 .

Continuerl from Am.Jour. Sci. Arts, VI. 353. See Cortis, M. A. Enumeration of 100 species, numbered 31-130, of which 26 are new; and one new genus Arrhytidia. Most of the species are repeated in Notices of North American Fungi, for which see above, no. 42 .

48. —— Descriptions of new species of fungi collected by the U.S. Exploring Expedition under C. Wilkes, U.S.N., Commander. Am. Jour. Sci. Arts, XI. 2 ser. 93-95. Jan. 1851.

Describes 9 species, all from the Pacific Islands. American species of this expedition were publisbed elsewhere. See CuRtis and Berkeley.

49. - - Exotic fungi from the Schweinitzian herbarium, principally from Surinam. Jour. Acad. Nat. Sci. Philadelphia. II. new ser. 277-294. Pl. 25. April, 1853.

Includes 7 species from the United States.

50. - Centuries of North American fungi. Ann. Mag. Nat. Hist. 2 ser. XII. 417-435 (1-19), Dec. 1853; 3 ser. IV. 284-296 (1-13), Oct. 1859.

Descriptions of 100 new species of Hymenomycetes and Polyporei, with notices of a few others. About half of them are repeated in Notices of North American Fungi. No. 42.

5r. — - A commentary on the Synopsis Fungorum in Americâ Boreali mediâ degentium, by L. D. de Schweinitz. Jour. Acad. Nat. Sci. Philadelphia. New ser. III. 20气ั-224. 1856.

Critical notes on 326 species, principally $\Pi$ ymenomycetes, from the Schweinitz herbarium, including deseriptions of 2 new species, Polyporus crociporus, $\mathrm{B}$. and $\mathrm{C}$., and $P$. cervino. nitens, Schw.

52. - Characters of new fungi, collected in the North Pacific Exploring Expedition by Charles Wright. Am. Acad. Arts and Sci. Boston. IV. 111-130. 14 Dec. 1858.

Includes 8 new species from California and N. W. coast.

53. —— Fungi Cubenses. Jour. Linn. Soc. X. 280-341, 341-392. 1869.

Part 1, inclnding IIymenomycetes, was read 2 May, 1867. Part 2, including Gasteromycetes, Physomycetes, Coniomycetes, Hyphomycetes, and Ascomycetes, was read 16 Jan. 1868. This work gives frequent references to localities in the United States where Cuban specics oecur.

\section{and Desmazières, J. B. H. J.}

54. On some moulds referred by authors to Fumago and on certain allied or analogous forms. Jour. Royal Hort. Soc. IV. 243-260 (3-19). Fig. 11. 1849.

Describes 5 Capnodia and Glenospora Curtisii, and figures also Scorias spongiosa.

and Montagne, C.

55. Sixième centurie de plantes cellulaires nouvelles, tant indigènes qu'exotiques. Décade 7 . Ann. Sci. Nat. Bot. 3 ser. XI. 235-246. 1849.

Includes descriptions of Trametes rigida and Exidia protracta from the United States. 
Berlese, Angusto Napoleone, and Voglino, P. Padua, 21 Oct. 1864. See Saccardo, P. A.

56. Sopra un nuovo genere di funglii Sferopsidei. Att. Soc. Venet. Trent. X. 176-205 (2-32). Pl. 2, 3. Padua. 1887.

A ccount of n new genus, Aracrophoma, with descriptions of 32 species from the United States; figures of 3 species, formerly included in Thoma and Sphneropsis. The reprint is dated 1856, but the volume of the Atti, 1887.

Bertoloni, Antonio. Sarzana, 11 Feb. 1775. + Bologna, 17 April, 1869.

57. Viscellanea Botanica, XVII. 22. Pl. 2.

Fig. 2. Bologna. 1857.

Gives a description and figure of Thelephora lobata from Alabama.

Bessey, Charles Edwin. See American NatuRalst. Milton, Wayne Co., O., 21 May, 1845.

58. On injurious fungi. The Blights, Erysiphei. 7th Biennial Rept. Iowa Agr. College. 8. $^{\circ}$ pp. 185-204 (1-20). Pl. 1, 2. Dec. 1877.

A description of the species known in the United States, with synopsis and figures of 10 species.

59. - Simblum rubescens, Gerard, in Iowa. Torr. Bull. VIII. 126, Nov. 1881. Am. Nat. XVI. 42, Jan. 1882.

Species found in Iowa.

1. 6o. A sketch of the progress of botany in the United States in 1880. Am. Nat. XV. 947955. Dec. 1881.

Includes an enumeration of United States fungi described in 1580 .

6r. The diseases of plants. Trans. Iowa State Hort. Soc. XVI. 85-98. 1882.

Gires, pp. 93-98, an account of sereral diseases caused by fungi.

62. - Some observations on the action of frost upon leaf-cells. Proc. Am. Ass. Adv. Sci. XXXI. 464, 465. Aug. 1882.

Finds that leaf-cells attacked by the mycelium of Aecidium Fraxini have less power of resisting cold than other cells.

\& 63. - A new species of insect-destroying fungus. Am. Nat. XVII. 1280-1281, 1286. Dec. 1883.

Description of Entomophthora Calopteni.

+ 64. - An enormous puff-ball. Am. Nat. XVIII. 530. May, 1884.

Reports the finding by Prof. R. E. Call in New York State of an immense specimen of $L y$ coperdon giganteum.

+65 . Bulletin of the Iowa Agricultural College, issued by the Dept. of Botany, Nov. 1884. 8. pp. 109-174. Cedar Rapids, Iowa.

Contains the following papers on fungi by C. E. Bessey : The Wheat Smut, pp. 115-126, fig. 3,4; Smut of Indian Corn, pp. 127-129, tig. 5, 6; Ergot, pp. 130-132, fig. 7; Treliminary lists ol Cryptogams, pp. 133-150, including between 200-300 fungi on pp. 134, 135-148. For other papers in this bulletin, see AlituUR, J. C. Sient to press Nov. 1884, but not issued until about Jan. 1855, as we are informed by the anthor.

f66. - The abundance of ash rust. Am.

Nat. XIX. 886-887. Sept. 1885.

Notes the abundance of Aecidium Fraxini in Nebraska.

67. - Injurious fungi in their relation to the discases of plants. Am. Pomolog. Soc. Session 1885. pp. 35-43.

A general account of the properties of injurious fungi and the diseases cansed $b y^{\circ}$ them, followed by a list of 41 species which occur in the United States.

68. - The rust of the ash tree. Am. Nat. XX. 806. Sept. 1886.

Tinports a marked absence of Aecidium Fraxini in Ne. brasks and Iowa in 1886.
Billings, John Shaw. Switzerland Co., Indiana, 12 April, 1838.

68. The study of minute fungi. Am. Nat. V. 323-329. Fig. 75. Aug. 1871.

A popular account of Iralsa stellulata, Fr., with figure, and directions for studying fungi.

$68^{\mathrm{b}}$. - The genus IIysterium and some of its allies. Am. Nat. V. 626-631. Yl. 11. Oct. 1871.

General account of the structure of the genus, followerl by notes on the spores of 54 species, with figures of the spores of 21 speeies. The specimens examined were principally from the herbaria of Scliweinitz, Ravenel, and Curtis.

\section{and Curtis, E.}

69. Report of results of examinations of fuids of diseased cattle, with reference to cryptogamic growths. Rept. Commissioner Agr. on Diseases of Cattle in U. S. $4^{\circ}$. pp. 174-190, with plate. Washington. 1871.

Account of cultures made with fluids of animals infected with contagious pleuro-pneumonia and 'Texas fever, with plate of forms found, some of them bacteria.

and Frost, C. C.

70. United States geological exploration of the fortieth parallel, Clarence King, geologist in charge. Botany by Sereno Watson aided by Prof. Daniel C. Eaton and others. $4^{\circ}$. pp. liii., 525. Pl. 1-40. Washington. 1871.

On pp. 413, 414 is a list of 14 fungi, including 3 new Pucciniae. The Aecidia and Pucciniae were detcrmined by J. S. Billings; the other fungi by C. C. Frost.

Bonorden, Hermann Friedrich. Herford, 28 Aug. 1801.

7 . Zur kenntniss einiger der wichtigsten gattungen der Coniomyceten and Cryptomyceten $4^{\circ}$. pp. 63. Pl. 1-3. Halle. 1860. Extr. Abhandl. Nat. Ges. Halle, V. 167-229. 1860.

Mentions 7 species of Uredineae from North America.

72. Die zweite deutsche Nordpolarfalirt in den jahren 1869 und 1870 unter führung des Kapitän Karl Koldewey, herausgegeben von dem Verein für die deutsche Nordpolarfahrt in Bremen. $8^{\circ}$. Leipzig. 1874.

The fungi are given in vol. II. 88-96. The fleshy fungi by Bonorden, pp. 88-90, include 4 species and some moleternined forms. The endophytic fungi by L. Fuckel include 13 species, all but one new, pp. 90-96, with pl. 1 . Names of species given in Arctic Mranual, part 2, p. 585.

Bosc, Louis (Augustin Guillanme). Paris, 20 Jan. 1759. +Paris, $10 \mathrm{July,} 1828$.

73. Mémoire sur quelques espèces de champignons des parties méridionales de l'Amérique Septentrionale Gesell. Natur. Freunde, Mag. $4^{\circ}$. V. 83-89 (1-7). Pl. 4-6. Berlin. 1811.

Separate copies of this paper are very rare. Fifteen species are described, all but one with figures: Boletus alveolarins ; B. hydnatinus; Agaricus pinarius: Ag. (Panus) dorsalis; Ag. (Lentinus) chuma; Clathmes coluninatus (copied in Corda, Anleitung, and Nees, System); Mlorchella caroliniana (Guromitra); Phallus duplicatus (copied in Corra and Nees); Saty'us rubicundus (copied in Corda?); Lycoperdon' (Cauloglossum) transversarium (in Nees); $L$. heterogeneum (Mitremyces lutescens); L. cyathiforme, Uperhiza (IIyperrhiza carolinensis); Tubercularia carnca (Peridermium); Tremella (Leotia) stipitata (copied in Nees).

- 74. Botanical Balletin. Edited by J. M. Coulter. $8^{\circ}$. Crawfordsville, Ind. Vol. I. Nov. 1875-Nov. 1876.

Published monthly in parts of 4 pages. In Nor. 1876 the name was changed to the following.

75. Botanical Gazette. Continuation of Bot. Bulletin. See above. Vol. II.-XI. Nov. 1876 -Dec. 1886. To be continued.

Tol. IT. ended Oct. 1877, and vol. IIT. began Jan. 187s. II. and III. have 8 pages in each number; IV.-VII. 12; 
VIII.-X., 16; XI., 24 pages; some numbers more. From I. $X$. there is an index with every second volume, and a gencral index to rols. I. $-\mathrm{X}$. appeared in 1886 . Later volumes are illustrated. The editors of yols. II.-VI. were J. MI. and MI. S. Coulter. Since then, J. M. Coulter, C. R. Barnes, and J. C. Arthur. Articles on fungi from this journal appear here under authors' names.

Brendel, Frederick. Erlangen, Germany, 20 Jan. 1820.

+ 76. Flora Peoriana. Die vegetation im clima von Mittel-Illinois. Természetrajzi Füzetek. V. Parte 2-4. pp. 299-405. 1882. A Musaeo Nationali Hungarico edito. Budapest. 1882.

Separate clition with pp. $10 \%$, large $8^{\circ}$. On p. 94 (392) is an enumeration of 39 species of fungi without notes.

Britton, Elizabeth G. See Bulletin Torrex Cluis.

Britton, Nathaniel Lord. New Dorp, Staten Island, N. Y., 15 Jan. 1859.

77. Geological survey of New Jersey. A preliminary catalogue of the flora of New Jersey. $8^{\circ}$. pp. 233. New Brunswick, N. J. 1881.

This work was distributed among botanists, bound and interleared, with the request that the copies be returned with corrections and comments to be embodied in the final publication. A list of about 950 fungi, determined by J. B. Ellis, is given on pp. 167-203.

Brongniart, Adolphe Théodore. Paris, 14 Jan. 1801. tParis, 18 Feb. 1876.

78. Essai d'une classification naturelle des cliampignons ou tableau méthodique des genres rapportés jusqu'à présent à cette famille. $8^{\circ}$. pp. 99 . Pl. 1-8. Paris. 1825.

This is a reprint of the article Mycologie in Dictionnaire des Science Naturelles, XXXIII. 492-588. 1824. The plates are taken from Turpin, Végetaux acotylédons. On p. 70 (559) is a reference to Calostoma cinnabarinum, Desv. = Mitre. myces cinnabarinus, Schw.

Broome, Christopher Edmund. Berkhampstead, Hertford, $24 \mathrm{July,} 1812 .+$ Bath, England, 15 Nor. 1886. See Berlecer, M. J.

Brown, A. E.

+79. Diseased Vaccinium. Torr. Bull. VI. 164. July, 1877.

Note on dcformity of fruit due to Exobasidium Vaccinii.

Brown, Robert. See Suiтu, W. G.

+8o. Bulletin of the Torrex Botanical Club, Vol. I.-XIII. Jan. 1870-Dec. 1886. To be continued. Montlily. 8. - New York. Illustr.

There is an index of I.-V. at the end of V. Vol. VI. extends from Jan. 1875 to Dec. 1879, with index. Since then the voluraes are annnal and have an index. Editors, 18701881, W. H. Leggett; 1882, W. H. Leggett and W. R. Gerard; 1883-1885, W. I. Gerard; 1886, E. G. Britton and F. J. II. Merrill. Deroted to all branches of botany. The articles on fungi here given under authors' names.

Bundy, W. F.

8r. A partial list of the fungi of Wisconsin, with descriptions of new species. Geology of Wisconsin, Survey of 1873-79. 4.. I. 396-401. 1883.

Enumerates more than 300 speeies of 76 genera with notes on loealities. Two new species described. The types of this paper have been lost, and there are no means of verifying species named.

Burnett, Swan Moses. New Market, Tenn., 16 Marclı, 1847.

82. Otomyces purpureus (Wreden) in the human ear. Archives of Otology, X.319-326. Dec. 1881. Also trans. in Zeitschr. für Ohrenheilkunde, XI. 89-95. 1882.

Account of the fungus, including a note by W. G. Farlow.

Barrill, Thomas Jonathan. Pittsfield, Mass., 25 April, 1839.

83. Aggressive parasitism of fungi. Trans. Illinois State Hort. Soc. 1873, VII. 217-22I. Chicago. 1874.
84. Lettuce mould and leaf-blights. Trans. Illinois State Hort. Soc. 1875, IX. 139144. Fig. 5. Chicago. 1876.

An account of injury done by Peronospora gangliformis in Illinois, and of lilac blight, pea blight, Verbena mould, and rose mildew, with figures of the fungi which eause them.

+ 85. - Upon parasitic fungi. Bull. Illinois State Lab. Nat. Hist. I. part 1, 52-59. Pl. 1-4. Bloomington. Dec. 1876 .

Gencral account of fungus discases, with specific account of those caused by Peronosporeae and Perisporiaceae, with figtres of several species.

86. Injurious fungi.

Trans. Illinois State Hort. Soc. X. 213-220. 1876.

An account of the harm done by scveral species, Peronospora riticola, Phragmidium, bunt in wheat, disease of Austrian pine, etc.

87:- Cherry leaf-blight. Trans. Illinois State Hort. Soc. XI. 134-136. 1877.

A popular account of Podosphaera Kunzei and the ravages caused by it.

+88. - First annual report upon useful and noxious plants. Trans. Dept. Agr. Illinois, XVII. 560-568. Springfield, Ill. 1880. Reprint, $8^{\circ}$, pp. 9. Springfield. 1880.

Contains a chapter on "Fungi in living plants," giving general aeconnt of growth and classification of fungi, with the effects thcy produce on other plants.

+89. - The Bacteria. 8. pp. 65. Fig. 2. Pl. 1. Springfield, Ill. 1882.

Taken from 11th Rept. Illinois Industrial Univ. pp. 93157. Besides Bacteria, this paper contains Saccharomycetes, pp. 62-65.

90. Notes on parasitic fungi. Agr. Review, 86-92. New York. Nov. 1882.

Gives an account of apple-scab, Fusicladium dendriticum, orange rust of blackberry, Caeoma luminatum, and blackberry cane rust.

9I. - An orchard seourge. Trans. Miss. Valley Ilort. Soc. I. 202-207. Indianapolis. 1883. Account of ravages duc to Fusicladium dendriticum.

92. - The grape rots. Trans. Illinois State Ilort. Soc. XVII. 42-45. 1883.

Notes the occurrence of the true Sphaceloma ampelinum in Illinois, with account of some other fungi. See no. 96.

93. - Broom-corn rust. Prairie Farmer, LVI. 532. Chicago. Aug. 1884.

Notice, with 2 figures, of an unnamed Chaetostroma on Sorghum.

Marasitic fungi of Illinois. Part 1. Uredineae. Bull. Illinois State Laboratory, II. 141-255. Peoria. 1885.

A full account of the Eredineae of Illinois, with synopsis of genera, descriptions and synonymy of species, with critical notes and index of species and losts. Also given in 12th liept. Illinois Industrial Univ. pp. 93-152, without hostindex, and some notes.

95. - The Uredineae of Illinois. A list of the species. Proc. Am. Soc. Aficros. VIII. 93-102 (1-10). 1885 .

A list of species, with introduction taken substantially from Parasitic fungi of Illinois. See title above.

96. — Grape Rots. Am. Pomolog. Soc., Session 1885. pp. 47-49.

Notes the occurrenec of the true Sphaceloma ampelinum in Illinois, with an account of its action compared with Phoma uvicola. See no. 92.

97. - Orange rust of blackberry leaves. Prairie Farmer, LVII. 762. Chicago. 4 Nov. 1885.

Account, with figure, of the spermogonia of Caeoma nitens. Substance of article and tigure given in paper by SEYMOUR, A. B., q. . 
Bnxrill, Thomas Jonathan.

98. Vegetable pathology. Trans. Ilinois State Jlort. Soc. IIX. 218-226. 1885.

Guneral renuks on disease in plints.

—_ and Seymour, A. B.

99. Nuw species of Uredineae. Bot. Gaz. IX. 187-191. 1)ec. 1884 .

Describes 13 species from Illinois. On p. 191, 4 other species descrihed by Sermovis, $\Lambda$. 13 .

Busbberg Cataloguo. See Ergelmany, Georice.

Calkins, William Wirt. Farm Ridge, La Salle Co., Ill., $29 \mathrm{May}, 1842$.

Ioo. Notes oll Florida fungi. Tour. Myc. II. 6, 7, Jan. 1886; 23, Feb. 1886 ; 42, April, 1886; 53. 5t, May, 1886 ; 70, June, 1886; 80, 81, July, 1856; 89-91; Aug. 1886; 10t-106, Sept. 1886; 126-128, Nov. 1886.

Part 3 of this series is called "The leaf fungi of Florida"; part 4, "Cryptogamic 13otany of a Florida log"; parts 7-9 give a list of species determined by J. B. Ellis.

tror. Polyporlus officinalis, Fr. Jour.

Myc. II. 107. Sept. 1886.

Notes the occurrence of this species in Michigan.

Cist, Jacob.

102. Notice of the Melolontha or May-bug. Am. Jour. Sci. Arts, VIII. 269-271. Pl. 4. Aug. 182!. Gives a note and figure of an unnamed species of Cordyceps on Melolontha.

Clayton, John. Fulham, England, 1685. †15 Dec. 1773. See Gronorius; J. F.

Cooke, Mordecai Cubitt. Horning, Norfolk, England, 12.July, 1825. See Eowaros, A. M. See Ellis, J. B. See Grevillea. See Ravenet and lienu, exsiceati in supplement. See Stevensox, W. C.

t ro3. Decades of Maine Fungi. Proc. Soc. Nat. Irist., Portland, I. 179-185. Fig. 9. 1862.

Descriptions of 21 species of Uredineae, 2 of them new.

1. 104. - Handbook of British Fungi, with full descriptions of all the speeies, and illustrations of the genera. $8^{\circ}$. 2 vol. pp. 981. Pl.7, col. and fig. 408. London. 1871.

After each species it is stated whether it occurs also in the United States. There appeared in Grevillea, beginning in 1583, a revised second edition of this work. Up to date, 176 pares, descriptions of Agarici, hare been published in the different numbers of that journal, and also issued separntely.

105. - Notes on Podisoma. Jour. Quekett Micros. Club, II. 255-268 (1-14). I'. 18, 19.

Real $2+$ Nor. 1871.

ITas figures of Gym. clavipes and Gym. macropus from the

Uniter States; also notes on some of our species.

†- го6. - Carpology of Peziza. Grev. III. 30-31, pl. 27-30, Sept. 1874; 73, 74, pl. 31-3t, I)ec. $1874 ; 127,128$, pl. $38-41$, March, $1875 ; 186$, pl. 43, 44, June, 1875; - IV. 41, pl. 50, 51, Sept. $1875 ; 132$, pl. 65, March, $1876 ; 168$, pl. 69, June, 1876.

Figures of asci, spores, and, in some cases, paraphyses of Pezizae, including American species.

1. ro7. - Revision of Geoglossum. Grev. III. 133, 194. March, 1875.

Revision of the species of Cooke's IIandbook; refers to two American species.

f- ro8. - Synopsis of the Discomycetous fungi of the United States. I'art 1. Bull. Soc. Nat. Sci. Buffalo, II. 285-300, March, 1875.-T' Iart 2. 1. c. III. 21-32; 33-37, Scpt. and Nov. 1875 .

Part 1 gires a list of 357 species, with localities and characters of the new or little-known forms. J'art 2 has remarks on some of the speries in part 1, with a list of 212 additional вpecies and descriptions of new species.
+ Iog. On Corticium amorphum, Fr. Grev. III. 136-138. Marcl, 1875.

Synonymy of this species with reference to Aleurodiscus amorphus, Rabl., including Nodularia balsamicola, Peck. See next title.

firo. On Corticium Oakesii, B. and C. Grev. III. 172. June, 1875.

Continuation of paper on C. amorphum. See abore.

fIII. - Phragmidium. Grev. III. 171. Pl. 45. June, 1875.

Figures $P$. specios $21 n$ from the United States.

f Ir2. - British Fungi. Grevillea.

The following references to North Ameriean fungi: Grev. III. 179, 150, June, 1875, note on Puecinia fiolarn, $P$. Asteris, $P$. Gercardi, description and figure of $I$. leastala, pl. 49 , fig. $10 \mathrm{~b}$; p. 182 , l. c. descriptiou of genus I'irgatsporium and note on $\boldsymbol{V}$. clacrtum; p. 185 , 1 . e. note on Ppeiza Agassizii; IV. 69, Dec. 1875, note on species of Iirgasporium and Claslerisporium.

I13. - Pezizae Amerieanae. IIedwigia, XIV. 81-S5. June, 1875.

Describes 21 new species from Maine, New Tork, New Jersey, and 1 fiom Mlexico. Some previonsly described in Torr. Bull. VI. 31, 32.

+ II4. - Podisoma on Juniperus phoenicia. Grev. III. 189. 190. June, 1875.

Includes a note on the germination of Gymnosporangium clavipes.

+ II5. - New Scotch Peziza. Grev. IV. 91,92 . Dee. 1875 .

A foot-note gives a description of Peziza scubalonta, Gerard, from New York.

+ Ir6. - On Peziza brunnea. Grev. IV. 125, 126. March, 1876.

Note on $P$. confusa, Cooke, of the United States.

+ II7. — Triblidium. Grev. IV. 182. Pl.67.

June, 1876.

Includes figures of 5 Triblidia and Ostreichnion Americanum from the United States.

+ Ir8. - Observations on Peziza calycina. Grev. IV. 169-172. Pl. 66. June, 1876.

A criticism of Rehm's Note on Peziza calycina. See Rem. t Irg. - The IIyphomycetous Fungi of the United States. Bull. Soc. Nat. Sci. Buffalo, III. 189-192, Feb. 1877 ; 193-202, July, 1877.

Enumeration of 304 species, with synonymy in some cases. I20. - Caution to mushroom eaters. Field and Forest, II. 146 . Feb. 1877.

Calls attention to the rescmblance between Agaricus campestris and A. fastibilis.

121. - On black moulds. Jour. Quekett Micros. Club, IV. 246-277. (1-28.) P1. 24-27. Read 23 Feb. 1877.

Has colored figures of 8 species of IIyphomycetes of the United States.

I22. On Valsa vitis, Schw. Grev. V. 125. March, 1877.

Separates the European form of this name under the name of $\boldsymbol{T}$. vitigera.

f 123. - The Valsei of the United States. Proc. Acad. Nat. Sci. Philadelphia. pp. 110-127. Apr. May, 1877.

Deseribes 93 species of Talsa and 5 of Melanconis, with enumeration of 40 doubtful species, principally from the Schweinitz herbarium. See Stevenson, W. C.

t. I24. - The Myxomycetes of the United States. Ann. Lyceum Nat. IJist. New York, XI. 378-109. June, 1877. Read 16 April, 1877.

An arrangement of species taken from Rostafinski, with synonymy and, in some cases, spore measurements; 154 species cnumerated, 3 described as new. 
Cooke, Mordecai Cubitt.

+125 . Spore formation in Urocystis. Grev. V. 158. June, 1877.

Summary of papers by Fischer von Waldheim and Farlow, q. v.

+ 126. - North American Fungi. Grev. V.

150-154. June, 1877.

Notices of 54 species from Maine, New York, and Sonth Carolina, 13 described as new. See nos. 129 and 152.

127. - Some allied species of Aecidiacei. Bull. Soc. Bot. France, XXIV. 314, 315. Transl. Quelques espèces voisines d'Aecidiacées, l. c. 316, 317. 22 Oct. 1877.

Includes notes on Peridermium cerebmum, Peck, and Roestelia hyalina, Cooke.

+ 128. - Praecursor ad monographiam IIendersoniae. Nuov. Giorn. Bot. Ital. X. 17-27. Jan. 1878.

Mentions 32 species of the United States, and gives detaila of the spores in some species.

129. - North American Fungi. Hedwigia, XVII. 37-40. March, 1878.

Description of 21 new species in Ravenel's Fungi Americani Exsiccati. See no. 126, 135, and 152.

130. - On fungoid diseases of the Vine. Jour. Roy. Hort. Soc. V. new ser. 68-76, 90-94, 115-123, March, July, Dec. 1878.

In a notice of Farlow's American Grape Vine Mildew there are given critieal notes on Oidium Tuckeri, Uncinula spiralis, U. Americana, $U$. subfusca, and Sphaerotheca Morsuvae. Also descriptions of Septoria viticola, B. and C., $S$. ampelina, B. and C., S. falx, B. and C. Last part has notes on Capnodium elongatum, with review of Cornu's papers on Anthraenose, Cladosporium viticalum, ete., with notes on Phoma uvaecola, B. and C., Graphium clavisporum and Cladosporia. See no. 133.

131. - The fungi of Texas. Jour. Linn. Soc. XVII. 141-144. 1880. Read 4 April, 1878.

Descriptions of 25 new species collected hy II. W. Ravenel. See next title.

+ 132. The fungi of Texas. Ann. Acad. Sci., New York, I. 177-187. May, 1878.

A list of 149 species collected by $H$. W. Ravenel, including descriptions of 25 , same as in last paper.

+ r33. - Vine diseases. Grev. VI. 147-150. Juwe, 1878.

A review of Pirotta's Funghi parassiti dei Vitigni and Thuemen's Pilze des Weinstockes, q. V., with notices of American viticolous fungi. See no. 130.

+ 134. - Enumeration of Polyporus. Trans. Bot. Soc. Edinburgh, XIII. 131-159. 1878. Read $2 \mathrm{July}, 1878$.

Alphabetical list of known species of Polypomes, with their distribution. See no. 163.

+ 135. - Ravenel's Ameriean fungi. Grev. VI. 129-146, June, 1878; VII. 32-35, Sept. 1878; 43-54, Dec. 1878.

Enumeration of species in Ravenel's Fungi Americani Exsiccati, see supplement, with descriptions of 143 nêw species and figures of some of the species on pl. 101. This plate is in vol. VI., the deseription of plate in VII. 96.

Two new genera, Phillipsiella and Saccardia, figured on pl. 119. Some of the species named are described in ITedvigia, XVII. 37-10, March, 1878.

+ 136. - On Chaetophoma.

Grev. VII. 24-

26. Pl. 118, 119. Sept. 1878.

Describes and figures 6 species from Carolina. Descriptions repeated without figures in IIed wigia, XVII. 134.

† 137 - Some Extra-European Fungi. Grev. VII. 13-15. Sept. 1878.

Includes an enumeration of 34 species collected by W. $R$. Gerard in New York, 5 deseribed as new.

+ 138. - Californian Fungi. Grev. VII. 1-4, Sept. $1878 ; 101,102$, March, 1879.

Enumeration of 57 species collected by H. W. Harkness. Describes $16 \mathrm{new}$, and one species from New York. See also COOKE and HLRKNEsS.
+ 139. - Mycographia seu Icones Fungorum. Figures of fungi from all parts of the world, drawn and illustrated by M. C. Cooke. 4\%. Vol. I. Pl. 113. Fig. 406. Discomycetes. London. 1879.

Of this work only one rolume has been issued. It includes 6 parts, which appeared as follows: Oct. 1875, April, 1876 , Oct. 1876, April, 1877, Jan. 1878, Feb. 1879. There is a sys. tematic and an alphabetic index. It includes a large number of colored plates of American Discomycetes, with deseriptive text. Deseriptions of the new and little-known species given also in Iledwigia, XVI. 142-144; 147-154; XVII. 53-55.

+ 140. - Some exotic fungi. Grev. VII.XIV.

The following are North American references: VII. 95, Mareh, 1879, description of Diplodia gossypina;-XII. 37, 38, Sept. 1883, 4 speeies from New IIampshire, Ohio, South Carolina, and Texas; - XII. 85, March, 1884, Polyporus pocula ;-XIII. 6 , Sept. 1884 , Puccinia Ipomeae; South Carolina ; 90, March, 1886, Leptothyrium Liriodendri and Phoma cerasina from South Carolina.

+ I41. — On Peniophora. Grev. VIII. 17-21. Pl. 122-125. Sept. 1879.

New genus formed from species of Corticium and Stereum. Includes American forms. See no. 149.

I42. Undeseribed fungi in the Kew Herbarium. Grev. VIII. 34, 35. Sept. 1879.

Describes 2 species from Oregon and British Columbia.

+143. - Natal Fungi. Grev. VIII. 69-72.

Dec. 1879 .

Describes Uredo macrosperma, Cooke, from the United States.

+ I44. - Agaric with green spores. Grev. VIII. 53. Dec. 1879 .

Brief notice of Agaricus Morgani, Peck.

+ I45. - Reliquiae Libertianae. Grev. VIII.

81-87. Mareh, 1880.

On p. 87 describes Lasiosphaeria acicola from the Rocky Mountains.

+ 146. - New York Fungi. Grev. VIII. 117119. March, 1880.

Notices of 31 species collected by W. R. Gerard, 7 described as new. Sce also notice in Rev. MYyc. II. 98.

147. - The genus Ravenelia. Jour. Roy. Micr. Soc. III. 384-389 (1-6). Pl. 11. Read 14 April, 1880.

General account of the genus, including $R$. glandulaeformis and $R$. sessilis of the United States.

+ I48. - Observations on Peziza. Grev. VIII. 129-143. Pl. 131-133. June, 1880.

Incidental reference to American species.

+ I49. - On IIymenochaete and its allies. Grev. VIII. 145-150. June, 1880.

Enumeration of species, including American, with descrip. tions of setae. Also gives additional species of Peniophora. See no. 141 .

+150. - The Perisporiaceae of Saccardo's Sylloge Fungorum. Grev. XI. 35-38. Sept. 1882.

Gives his views with regard to Erysiphella and Pleochaete, with notes of 12 American species, principally Asterinae and Mrelialae.

+ 151. - Re-appearance of Cycioderma. Grev. XI. 95, 96. Pl. 160. Fig. 10. March, 1883.

Describes Cycloderma Ohiensis, Cooke and Morgan, with figures, and states that Mfilleria herbatica, Peck, is Testicu. laria Cyperi, Klotzsch. See KI OTzSCH, J. F.

1 152. North American Fungi. Grev. XI. 106-111. March, 1883.

Enumerates 44 species from Maine, Ohio, California, the greater part from South Carolina and Georgia, in Ravenel's Fungi Americani Exsiccati; 27 described as new. See same title above, no. 129. 
Cooke, Mordecai Cubitt.

153. (n) Iylaria and its allies. Grev. XI. 8194. Mareh, 1883.

A revision of species of Tylaria in Saccardo's Sylloge. Gives letrils of spores of sone North American species, with figures on pl. 162-165 of vol. XI. and 166-171 of vol. XII. See 110. 155.

154. - On Sphaerella and its allies. Britten's Jour. Bot. XXI. 67-71, March, 1883; 106110, April, 1s83 ; 136-139, May, 1883.

Includes notes or descriptions of about 50 species of the Uniced States, some from IIerb. Schweinitz and Ravenel's Fungi Americani Fxsiecati.

155. - Mypoxylon and its allies. Grev. TI. 121-140. June, 1883.

Revision of species in Saccardo's Sylloge. Contimuation of no. 153. Details of spores of in number of North American species, with description of 6 species. See next title.

\&56. Nummularia and its allies. Grev. XII. 1-8. Sept. 1883.

Coutinuation of papers on Fylaria and Mypoxylon. See abore. Notes on sereral North Amerieau species, with descriptions of three Sehweinitzian species.

157. - New American fungi. Grev. XII. 22-33. Sept. 1883.

Pyrenomycetes imperfectae and ITyphomycetes in Fungi Americani Exsiccati, 94 new species, a new genus, Sphaerellonsis, notes on other species, aud deseriptions of 2 Californian species, Torula fusoidea and Camptoum cuspi. datum.

i58. - The genus Anthostoma. Grev. XII. 49-53. Dec. 1883 .

Revision of this genus in Saccardo's Sylloge, including notes ou 16 Ameriean species.

159. _- The structure and affinity of Sphaeria pocula, Schweinitz. Jour. Linn. Soc. XX. 508511. Pl. 47. 1884. Read 17 Jan. 1884.

Species referred to Polyporus.

I60. - Notes on IIypocreaceae. Grev. XII. 77-83, 100, 101. March and June, 1884.

leriew of species in Saceardo's Sylloge, II., including details of 26 American species, with descriptions of several new species.

tr6r. - Synopsis Pyrenomycetum. Grev. XII. 102-113, June, 1884; - XIII. 8-16, Sept. 1884; 41-45, Dec. 1S8t; 61-72, March, 1885; 100-109, June, 1885 ; - IIV. 14-17, Sept. 1885; 46-56, 1)ec. 1885 -93-97, March, 1886.

Cieneral revision of the order as given in Saccardo's Sylloge, with numerous notes on $A$ merican species.

I62. - Sphaeriaceae imperfectae cognitae. Grev. XIII. 37-40. Dec. 1884.

Review of this group in Saccarlo's Sylloge, $\Pi$., with additional details of 47 species from the schweinitz Herbarium, in part by W. C. Stevenson, and descriptions of 2 specics omitted by saccarlo.

r63. - Praceursores ad monographiam Polypororum. Grev. XIII. 80-87, March, 1885; 11t-119, June, 1885; - XIV. 17-21, Sept. 1885 ; 77-87, Marel, 1886; 109-115, June, 1886;-XV. 19-27, Sept. $1886 ;$ 50-60, Dec. 1886 .

Finuneration of all known species of Polyporus, with occasional notes on syuonymy. XV.19-27, contitius descriptions of 13 American species. Sec no. 134.

if $\mathbf{6 4}$ - A new Stereum from North Carolina. Jour. Myc. I. 130. Oct. 1885.

Descriptions of S. caroliniense.

of 15 . Valsa vitis, again. Grev. XIV. 4446. Dec. 1855 .

Full smonymy given for Diatmpe (Valsaria) vilicola, Scluw., ralsu (Eutypellu) ritis, Schw., and $V$. viligera, Cooke.

and Berkeley, M. J.

166. Fungi : their nature and uses. Edited by Rev. M. J. Berkeley. 8. pp. 299. Fig. 109.
New York. 1875. The International Scientific Series.

IIas incidental references to American species. A general treatise on the structure and properties of fungi, including substance of notes on edible fungi of the United States by $\mathbf{M}$. A. Curtis. Sce CurTis, M. A., and Porcher, F. P.

\section{and Ellis, J. B.}

\& 167. New Jersey Fungi. Grev. IV. 178-180, pl. 68, June, 1876 ; - V. 30-35, pl. 75, Sept. 1876 ; $49-55$, pl. 80,81 , Dec. 1876 ; 89-95, March, 1877 ; -VI. 1-18, pl. 95, 96, Sept. 187т; 81-96, pl. 99, 100, March, 1878;-VII. 4-10, Sept. 1878; 3742 , 1ec. 1878 ; - VIII. 11-16, Sept. 1879 ; - IX. 103, March, 1881.

Enumeration of a large number of species, with notes and deseriptions of not far from $300 \mathrm{new}$ species. There appears to be no reference to fig. 2 and 8 of pl. 81 .

\section{and Harkness, H. W.}

+ 168. Californian Fungi. Grev. IX. 6-9, Sept. 1880; 81-87, Mareh, 1881;-XII. S3, 84, March, 1884; 92-97, June, 1884;-XIII. 16-21, Sept. 1884; 111-114, June, 1885; - XIV. 8-10, Sept. 1885 .

Enumerates a large number of species, of which the greater part, alout 200, are described as new Collected by IIarkhess, and species mentioned again in Bull. Cal. Acad. Sci. See no. 170. See Rev. Mryc., A pril, 1881, p. 44. For remarlss on genus ITarknessia, see Winter, $G$.

$+169$

IX. 127-130. June, 1881.

Notices of 42 species, of which 18 are described as new. Names of new species given in Rev. Myc., July, 1881, p. 67 .

$\$ 170$

Fungi of the Pacific Coast. Bull. Cal. Acad. Sci. I. 13-10. Feb. 1881.

List of 91 species previously described in Grevillen, IX. 6-9, 81-87, griving loosts and localities. Also 2 species described by Ellis aud IIarkness in Torr. Bull. VIII. 51.

and Peck, C. H.

+171. Pezizae Americanae. Grev. I. 5-7. Pl. 1. July, 1872.

Notices and descriptions of 12 species, 6 of which are new ; six colored figures.

172. — - The Erysiphei of the United States. Trimen's Jour. Bot. X. (n. s. I.) 11-1t. Jan. 1872.

Enumeration of 26 species and 13 inquirendac, with descriptions of some of the species. Descriptions also given in Hedroigia, XI. 74, 75 .

173. - Supplementary notes on the Erysiphei of the United States. Trimen's Jour. Bot. X. (n. s. I.) 170-172. June, 1872.

Notes on 14 species, including Uncinula Americana, Jlowe, and one other new species; 6 species and 1 variety not included in last-named paper.

Corda, August Karl Joseph. Reichenberg, 22 Oet. 1809. tLost at sea Sept. 1849.

174. Anleitung zum studium der mycologie nebst kritischer besehreibung aller bekannten gattungen, und einer kurzen geschichte der systematik. 8\%. CXXII. 223. Pl. 1-8. Prague. $18 \pm 2$.

Some of the illustrations given in this work are taken from Bose's Némoire, q. v. Descriptions of Americau genera are given. There is also an index of autloo's and works on mycology, including some relating to North America.

* Icones Fungorum hucusque cognitorum. $f^{\circ} .6$ vol. Prague. 1837-1854. II as figures of many species found aiso in this country, but not specially credited to the United States. A considerable part of the edition on the first five rolumes was destroyed in the great fire at Prague, and complete sets of the original edition are now rery rare. The remaining copies of vol. VI. were purchased by R. Friedländer \& Solun, of Berlin, who have reproduced the first five volumes by photo-lithography, and most copies now offered for sale have only tlic sixth volume of the original edition. 
Cornu, Maxime. Orléans, France, 16 July, 1843. 175. Note sur le Ptychogaster* albus, Corda. Bull. Soc. Bot. France, XXIII. 359-363. 10 Nov. 1876 .

Ias notes on the structure of Pilucre Petersii, B. and C.

I76. - Présence du Podisoma JuniperiSabinae sur le Juniperus Virginiana, et sur divers autres génévriers. Bull. Soc. Bot. France, XXV. 122-125. 12 April, 1878.

Acconnt of species of Podisoma which oceur on $\%$. Firginiana in France, with notes on a comparison of some A merican with European speeimens.

177. - Enumération des Péronosporées de France. Bull. Soc. Bot. France, XXV. 293-300. 13 Dec. 1878.

Notes the occurrence of Peronospora basidiophora in the United States.

178. - Le charbon de l'oignon ordinaire (Allium Cepa), maladic nouvelle, originaire d'Amérique, causée par une Ustilaginée (Urocystis Cepulae, Farlow). Comptes Rendus, LXXXIX. $51-53(1-3) .7 \mathrm{July}, 1879$.

Includea uotes ou this species in the United States.

179. —_ Maladies nouvelles pour l'Europe à propos d'une Ustilaginée (Urocystis Cepulae) parasite sur loignon ordinaire. Bull. Soc. Bot. France, XXVI. (I. u. s.) 263-267. $11 \mathrm{July}, 1879$.

Additional remarks on subject of last title. Continued in next title.

180. - Observations sur la maladie des oignons (Urocystis Cepulae). Bull. Soc. Bot. France, XXVII. (II. n. s.) 39-42. 13 Feb. 1880.

I8r. Notes sur quelques maladies des plantes. Bull. Soc. Bot. France, XXVIII. (III. 11. s.) 143-146. May, 1881.

Notes the occurrence of the American Caeoma luminatum in France.

I82. Observations sur le Phylloxera et sur les parasitaires de la vigue. Etude sur les Péronosporées. $4^{\circ}$. pp.91. Pl. 1-5. Paris. 1882.

Contains a full bibliography of the subject, with numerous extracts from works ou Americ'un fungi.

183. - Sur quelques Ustilaginées nouvelles ou peu connues. Ann. Sci. Nat. G ser. XV. 269 296. Pl. 14-16. June, 1883.

Has accounts of the structure of American species of Testicularia, Cintractia, and Doassunsiu, with figures. See also Bull. Soc. Bot. Frunce, XXX. 130-133. March, 1883.

Coulter, John Merle. Ningpo, China, 20 Nov. 1851. See Botanical Bulletin. See Haxden, F. V. Sé Porter, T. C.

+ 184. - Uromyces Lespedezae. Bot. Gaz. (Bot. Bull.) I. 20. Mareh, 1876.

Synonymy of species given in note from C. $\Pi$. Peck.

< 85 . A large puff-ball. Bot. Gaz. VI. 290. Nov. 1881.

Note on a Lycoperdon giganteum, $58 \times 32$ inches in circumference.

Coulter, (M.) Stanley. Ningpo, China, 2 June, 1853. See Botanical Gazette.

Cragin, Francis Whittemore. Greenfield, N. H., 4 Sept. 1858.

ir6. First contribution to the catalogue of the Fymenomycetes and Gasteromycetes of Kansas. Bull. Washburn Coll. Laboratory Nat. IIist. Topeka, Kan. I. 19-28, Sept. 1884; 33-42, pl. 1, Feb. 1885.

Part I. gives an enumeration of 139 IIymenomycetes, 3 new species by Cragin, the others determinel mostly by J. B. Ellis, A. P. Morgan, and C. H. Peck. Part II., Gasteromycetes, including 15rrogastres, 49 speries nanjed, 8 new, with plate of Phalloidei. New species given in Jour. Mryc. I. 28. Rev. in Am. Nat. XIX. 399.
$+187 .-$ Second contribution to the catalogue of the IIymenomycetes and Gasteromycetes of liansas. Bull. Washburn Coll. Laboratory Nat. Hist. I. 65-67. Ja11. 1885 .

Coutinuation of the above. Twenty species cnumerated, onc new, all IIymenomycetes. Sec Jour. MYyc. I. 58.

+I88. - A contribution to the knowledge of the lower fungi of Kansas. Bull. Washburn Coll. Laboratory Nat. Hist. 1. 67-72. Feb. 1885.

Enumeration of 69 species, principally Ascomycetes, $2 \mathrm{new}$ species. See Jour. MYyc. I. 47.

$+\mathbf{r} 8$. - A new genus and species of Tremelline Fungus. Bull. Washburn Coll. Laboratory Nat. Hist. I. 82. Feb. 1885.

Deseribes a new genus, Ceracea. See Jour. DIyc. I. 58.

Currey, Frederick. Eltham, Kent, England, 19 Aug. 1819 . †Blackheath, 8 Sept. 1881.

rgo. Synopsis of the fructification of the Compound Sphaeriae of the Hookerian Herbarium. Trans. Iinn. Soc. XXII. 257-287. P1. 45-49. 1859. Read 4 March, 1858.

See following titles.

Igr. - Synopsis of the fructification of the Simple Sphaeriae of the Hookerian Herbarium. Trans. Linn. Soc. XXII. 313-335. Pl. 57-59. 1859. Read 5 May, 1859.

See next title.

192. - Supplementary observations on the Sphaeriae of the Hookerian Herbarium. Trans. Linn. Soc. XXV. 239-262. 1866. Read 15 June, 1865.

In the three papers just mentioned there is an account of the spores of a considerable number of species from the Schweinitz herbarium, with figures of some of them.

- and Hanbury, 10.

193. Remarks on Sclerotium stipitatum, Pachyma Cocos, and some similar productions. Trans. Linn. Soc. XXIII. 93-97. Pl. 9, 10. 1860. Read 3 May, 1860.

An account of Tnckahoe, with notice of prerious works on the subject.

Curtis, Edward. See Brlungs, J. S.

Curtis, Moses Ashley. Stockbridge, Mass., May 11, 1808. †Hillsborough, N. C., April 10, 1872. See Berkfley, M. J. See Dubr, J. E. See Porcher, F. P. See Rarentel, H. IV., Exsiecati in Supplement. See Sprague, C. J. See Tuckerman, $\mathbf{E}$.

- 194. Contributions to the mycology of North America. Am. Jour. Sci. Arts, 2 ser. VI. 349-353, and 444. Nov. 1848 .

A brief account of works relating to North American fungi up to date, followed by an enumeration of 30 species, 10 deseribed as new. The species are, with one exeeption, from North and Sonth Carolina, and most of them are repeated in Berkeley's "Notices of North Ameriean Fungi." See no. 42, above. For contiuuation of this paper, see BERkELEr and Curtis, no. 47 , above.

195. - Geological and natural history survey of North Carolina. Part 3, Botany ; containing a catalogue of the indigenous and naturalized plants of the state. $8^{\circ}$. pp. 156 . Raleigh. 1867.

Gives a list, pp. 83-154, of 2392 fungi, edible species in italies. This list is the most extensive up to date, with the exception of Schweinitz's Synopsis. Many of the names expear here for the first time in print, and the descriptions, appear here Borkeley and Curtis, were given by Berkeley in the Notices of North American Fungi, q. v. Four species ounitted from the list are given on a supplementary unnum. bered page. A few species named bave never been described. The list of edible fungi is given by F. P. Porcher in "Fungi, edible and poisonous," q. v.. See also Rept. U. S. Dept. Agr. for 1876, p. 79. 
Curtis, Dloses Ashley.

Ig6. On the esculent fungi of America. Jour. Roy. Hort. Soc. II. new ser. 71-79. 1870. Also Gard. Chron.9 Oct. 1869 . p. 1066.

Gives in a letter to $13 \mathrm{crkel} y$ the results of experiments on a number of species, especially from Carolim, with remarks on tiste und value as food. Substance of this article to be found in "Funit, their mature and uses," p. 88 et seq. See CoOKF and BFAKELEY.

V. 13. In Am. Jour. Sci. Arts, XLII. 2 ser. p. 129, it is stated that Curtis is abont to pnblisl a work on cdible fungi, but the contenulated work was never issued.

\section{and Borlroloy, M. J}

197. United States Exploring Expedition during the years 1s38-1542, under the command of Charles Wilkes, U.S.N. Vol. XV1I. Botany. 4\%. Philadelphia. 1862. 1874. Fungi. pp. 195-203. Pl. 1.

Thirly-one species named, of which 3 are from Oregon: Conthorellus uurantiacus, Polyporus rellereus var. and Butrerer phalloides. For other fungi of Wilkes' Expedition. see BERKEIEY and CUlitis, no. 48, above. Extras distributed iil 1.66:. Volume issued 1874.

Day, David Fisher. Buffalo, 11 June, 1829. I 98 . A catalogue of the native and naturalized plants of the city of Buffalo and its vicinity. Bull. Soc. Nat. Sci. Buffalo, IV. 65-152, 1882; 153-290, 1883. Reprint. $8^{\circ}$. pp. 215 , with cliart. Buffalo. 1883.

The fungi, pp. 17t-235 (110-171), were determined by C. II. Peck from suecimens collected by $G$. W. Clinton; 869 species are enumerated, with their habitats and, in some cases, stnonymy and critical notes. Some of tlie species previously mentioned in Reports $N$. I. Stale Museum Nat. Wist. Sce PECK, C. II.

Domotrio, Carl Hermann. Eisenberg, Saxony, 24 Dec. 18t5. Sce Winter, G.

r99. Department of Agricalture of the

United States. See Agricultural Reports.

Desmazières, Jean Baptiste Henri Joseph. 1796. +Lambersart, near Lille, 23 June, 1862. See Berkeley, M. J.

Desvaux, Nicaise Augustin. Poitiers, 28 Aug. 1784. +Bellerue, near Angers, $12 \mathrm{July,} 1856$. See Persoon, C. H.

200. Observations sur quelques genres à établir dans la famille des champignons. Desvaux's Journal de Botanique, II. 88-105. Paris. 1809.

On p. 94 the genus Calostoma is described, founded on Scleroderma calostoma, P., Mitremyces cinnabarinus, Schw.

Duby, Jean Etienne. Geneva, 1798. †Genera, 24 Nov. 1885.

201. Mémoire sur la tribu des Hystérinées de la famille des IIypoxylées (Pyrénomycètes). $4^{\circ}$. pp. 58. Pl. 1, 2. Geneva. 1561. Ext. Mém. Soc. Phys. et II ist. Nat. XVI. 15-70. Pl. 1, 2. Genera. $181 ; 2$.

Includes descrintions of 19 American species, 8 new principally from collections of M. A. Curtis, H. WV. Ravenel, and Sehweinitz, with figures of some of them. For revision of spccies in this work, sec REHM, H., and BrLlings, J.S. title no. $63^{\mathrm{b}}$.

Dadley, Plimmon Henry. Freedom, Portage Co., O., 21 May, 1843.

202. Fungi inducing deeay in timber. Trans. Acad. Sci. New York, V. 110-118. Jan. 1886.

Fires a list of 17 species, Mymenomyceles, known by the writer to cause rotting of timber in the Eastern States, Lentirus lepideus being the most destructive. Also notes on the action of other specics named.

203. Fungi which cause decay in timber. Jour. N. Y. Micros. Soc. II. 36, 37. Feb. 1886.

Account of the growth of Lentinus lepideus.

204. Woods and their destructive fungi. Pop. Sci. Monthly, XXIX. 433-44t, 604-617. Fig. 23. Aug.-Sept. 1896.

Account of ohservations on the astion of fungi on timber and the remedlica, with descriptions and figures of a number of apecies, principally Polyporti.
Earle, Franklin Sumner. Dright, Grundy

Co., IIl., 4 Sept. 1856.

+ 205. Notes on the North American forms of Podosphaera. Bot. Gaz. IX. 24-26. Feb. 1884.

Account of different forms referred to $P$. Oxyacanthae (DC.).

205a. - Fungoid diseases of the strawberry. Rept. Kansas State IIort. Soc. XIV. 133-137. Topeka. 1885. Also Rept. Am. Hort. Soc. for 1884.

See next title.

+ 206. - Fungoid diseases of the strawberry. Fruit-Growers' Jour. Cobden, Ill. 29 Jan. 1885. Also Trans. Miss. Valley Hort. Soc. III. 47-54.

Account of Ramularia Tulasnei, Gloeosporium Poten. illae, and a new Bacillus.

Eaton, Amos. Chatlam, Columbia Co., N. Y., 17 May, 1776. †Troy, N. Y., 10 May, 1842.

207. A Manual of Botany for the Nortlern States, comprising generic deseriptions of all phaenogamous and eryptogamous plants to the north of Virginia, hitherto described; with references to the natural orders of Linnaeus and Jussieu. $12^{\circ}$. pp. 164. Albany. 1817.

This volume, which has on the title-page "By the mem. bers of the botanical class in Williams College, from a manuscript system compiled by the anthor of Richard's Botanical Dictionary," is the first edition of the following work.

208. - A Manual of Botany for the Northern and Middle States. Part 1, containing generic descriptions of the plants to the north of Virginia, with references to the natural orders of Linnaeus and Jussieu. Part 2, containing descriptions of the indigenous plants, which are well defined and established; and of the cultirated exotics. $12^{\circ}$. Albany. 1817. pp. 524. 2d ed. 1818; 3d ed. 1822 ; 4 th ed. $1824 ; 5$ th ed. $1829 ; 6$ th ed. 1833 ; 7 th ed. $1836 ; 8$ th ed. 1840 .

The 1st edition bas 3 pages, with 22 genera of fungi, each illustrated by a single species, compiled by the anthor mostly from Turton and Linnaeus. The $2 \mathrm{~d}$ edition has the same genera, pp. 117-119 of part 1 , but they are repeated alpbabeticaliy in the second part, and sereral species described under them, including some not given by Muhlenberg. The generic characters of fungi of this edition are said to be taken from Linnacus. In the $3 \mathrm{~d}$ edition, 54 genera of fungi are given from Persoon's Srnopsis, and the list of speeics corresponds with Muhlenherg's Catalogue. Editions 4 and 5 have the same fungi. The 6 tli edition has the genera of fungi, pp. 6-13, part 2, rcarranged from Loudon on Fries's system, the species the same as before, with some additions. In editions 7 and 8 , fungi the same as before.

209. - Observations on the Boletus igniarius, showing its analogy to animal substances in closing its several parts. Am. Jour. Sci. Arts, VI. 177. Jan. 1823.

Account of the union of cut surfaces in this species.

Edwards, A. M.

f-210. Note oll fungi. Torr. Bull. II. 39, 40. Oct. 1871 .

Mention of 5 Uredineae from New York, determined by M. C. Cooke.

Ehronberg, Christian Gottfried. Delitzsch, 19 April, 1795. †Berlin, 27 June, 1876.

2Ix. Fungos a viro clarissimo Adalberto de Chamisso sub auspiciis Romanzoffianis in itinere circa tcrrarum globum collectos enumeravit novosque descripsit et pinxit Dr. C. G. Ehrenberg. Horae Physic. Berolin. pp. 79-104. Pl. 17-20. fo. Bonn. 1820.

Includes descriptions of 7 species from Unalaschka, 2 from 8t. Lawrence Island, 4 new, with figures.

Ellis, Job Bicknell. Potsdam, N. Y., 21 Jan. 1829. See Britton, N. L. See Crarin, F. IV. See Cooke, M. C. See Journal Mrcology. Sec Macote, J. Sce Peck, C. H. See SaCCARDo, P. A. 
Ellis, Job Bicknell.

+ 212. New species of fungi found at Newfield, N. J. Torr. Bull. V. 45, 46, Nov. 1874; VI. 75-77, Feb. 1876.

Deseriptions of 16 species.

+ 213. - South Jersey fungi. Torr. Bull.

VI. 106-109, 133-135. Sept. 1876-Feb. 1877.

Continuation of paper by C. II. Peek, Torr. Bull. VI. 13,

14. Characters of 29 new species, new name proposed for Boletus squamulosus, and note on synonymy of Sphaeronema nigripes.

+214. - Rediscovery of a lost Sphaeria.

Torr. Bull. VI. 231, 232. May, 1878.

Reports the discovery of $S$. barbirostris, Duf., in New Jersey.

2I5. - On the variability of Sphaeria Quercuum, Schw. Proc. Acad. Nat. Sci. Philadelphia. 1879. pp. 66-70.

Twentr different species are considered to be the same as $S$. Quercuum, and the name Melogramma fuliginosum is proposed to designate them all. See review by $\mathrm{M}$. C. Cooke in Grev. VIII. 35, 36, and reply by J. B. Ellis in Proc. Acad. Nat. Soi. Philadelphia, 1879, pp. 381, 382, and Grev. VIII. 143-14, with supplementary note by Cooke.

+216. - A new Sphaeria on grapes. Torr. Bull. VII. 90, 91. Aug. 1880.

Describes $S$. Bidroellii, which is thought to be connected with Phoma uvicola.

† 217. - Development of Sphaeria Solidaginis, Schw. Torr. Bull. VIII. 29. March, 1881.

States that Coleosporium Solidaginis is a form of Dothidea Solidaginis.

+ 2r8. — New species of North American fungi. Torr. Bull. VIII. 64-66, 73-75, 89-91, June, July, August, 1881 ; - IX. 18-20, 73, 74, 98, 99, 111, 112, 133, 134, Féb., June, Aug., Slpt., Nor. $1882 ;-\mathrm{X} .52-54$, Nay, 1883.

Characters of 92 species, principally from New Jersey, some distributed in Torth American Fungi Exs. Also 10 speeies from Utah, and a few from Maine, New York, etc. In two of the parts the title reads "New North American Fungi."

+ 2rg. New Ascomycetous fungi. Torr.

Bull. VIXI. 123-125. Nov. 1881.

Characters of 13 species collected in Pleasant Valley, $U t a b$, by S. J. Harkness.

† 220. - Diatrype disciformis var. Magnoliae. Am. Nat. XVI. 238, 239. March, 1882.

The variety separated as a species under the name $D$. tremellophora.

t22r. - New fung1. Am. Nat. XVI. 810,

811. Oct. 1882 .

Describes 7 species of Septoria and Cercospora from Lex. ington, $\mathrm{Ky}$.

+ 222. - New species of North American fungi. Am. Nat. XVII. 192-196, 316-319, Feb., March, 1883.

Descriptions of 35 Ascomycetes, principally from New Jersey and Iowa.

t2 223. - Large fungi. Am. Nat. XVII. 1064.

Oct. 1888 .

Note on large Hydnum septentrionale, Trametes suaveolens, etc.

+224 . - Notes on Gymnosporangium and Roestelia. Am Nat. XVI1. 1281. Dec. 1883.

+225 . Notes on fungi. Am. Nat. XVIII. 530, 531, 721, 722. May, June, 1884.

Notes on peculiarities of a number of Polypori.

+226. Note on Sphaerella polystigma, Ell. and Everl. Torr. Bull. XI. 120. Oct.1884. Notes a septum in the spores.

+227 . Microsphaera fulva-fulcra, Cooke. Jour. Myc. I. 83. June, 1885.

The no. 323, North American Fungi Exs., and 3045,

Fungi Eur., are Podosphaera, probably P. minor, Howe.
+228 . Notes on Polyporus. Jour. Myc.

II. 5, 6. Jan. 1886 .

Observations on $P$. frondosus, $P$. flavo-virens, $P$. depen. dens, and $P$. Ellisii.

-229. Notes on some published species of fungi. Jour. Myc. II. 43, 44. April, 1886.

Notes on 5 species. See next title.

230. Uncinula polychaeta, B. and C. Jour. Myc. II. 52, 53. May, 1886.

A comparison of this species with $U$. Lynchii, Speg. See also Jour. iryc. If. 43.

231. - Phosphorescent fungi. Jour. Myc.

II. 70, 71. June, 1886 .

Panus stypticus said to be phosphorescent.

- and Everhart, B. M.

$\div$ 232. New species of fungi. Torr. Bull. $\mathbf{X}$. $76,77,89,90,97,98,117,118$. July-Oct.
1883.

Describes 29 species, mostly Ascomycetes, the greater part from Pennsylvania aud New Jersey.

233. - New (species of) North American fungi, Torr. Bull. XI. 17, 18, 41, 42, 73-75. Hob., A pril, July, 1854.

Describes 24 species from New Jersey, Iowa, Illinois, Canada, and California.

+234. - New species of fungi from Washington Territory, collected by W. N. Suksdorf during the summer and fall of 1883. Bull. Washburn Coll. Laboratory Nat. Hist. I. 3-6. Sept. 1884.

Descriptions of 16 species of different orders.

+235 . - Enumeration of the North American Cercosporae, with descriptions of the species. Jour. Mryc. I. 17-24, 33-40, 49-56, 6167. Feb.-May, 1885.

Deseriptions of 116 species, with list of host-plants. See no. 246.

†236. - New fungi. Jour. Myc. I. 4244, 140, 141, 148-154. March, Nov., Dec: 1885 .

Descriptions of 42 species; a new genus, Fellermania. See also Hedwigia, XXV. 108-110.

- 237. - - On Ramularia obovata, Fuckel. Jour. Myc I. 69, 70. May, 1885.

Critical note on some forms referred to this species, some of which are placed in a new species $R$. decipiens.

+238. —_ North American species of Ramularia, with descriptions of the species. Jour. Myc. I. 73-83. June, 1885.

Descriptions of 41 species, with list of host-plants. See no. 211.

+239. Canadian Fungi. Jour. Ifyc.

I. 85-87. July, 1885 .

List of 34 species collected by Prof. John Macoun, 5 described as new.

$\div 240$. - New species of fungi. Jour. Myc. I. 88-93. July, 1885.

Descriptions of 23 new species from Dakota, Oregon, Utah, New Jersey, and other States.

¿24I. - Supplementary notes on Ramularia. Jour. MYyc. I. 102. Aug. 1885.

Note on $R$. macrospora $\nabla$. Senecionis and $R$. crypta.

+242. —_ - The North American species of Gloeosporium. Jour. Myc. I. 109-119. Sept. 1885.

Forty-seven species, with list of hosts.

-243. - - A new genus of Pyrenomycetes. Jour. Myc. I. 128, 129 . Oct. 1885.

Description of Hypsotheca, with 3 species.

+244 . — - North American species of Cylindrosporium. Jour. Myc. I. 126, 128 . Oct. 1885 .

Descriptions of 8 species, with list of host-plants. 
Ellis, J. B., and Everhart, B. M.

245. Finngi of the Greeley Expedition. Bot. Gaz. X.366. Oct. 1885. Also Jour. Myc. I. 141. Nov. 1885.

Deseriptinn of Puccinia Cheiranthi on C.pygmaeus from Grimell Land.

\section{$\therefore 246$.}

Supplementary enumeration of the Cercosporae. Jour. Myc. II. 1, 2. Jan. 1886.

Gires 8 ndditional species, 5 of them new.

247. — Synopsis of the Nortl Amer1can IIypocreaceae, with descriptions of the species. Jоur. Myc. II. 28-3i, 49-51, 61-69, 73-80, 97-99, 109-111, 121-125, 133-137; - III. 1-6. March, 1886-Jan. 1887.

Full descriptions of 161 species, with host index.

248. - New species of fungi from various localities. Jour. Myc. II. $37-42,87-89$, 99-104. A pril-Sept. 1886.

Fifty-four species, mostly from Louisiana. One new genus, Coscinaria.

249. - Eellermania, Ell. and Everh. Jour. Myc. II. 111 . Oct. 1886.

The genus emended and two new species described.

- and Markness, H. W.

250. Some new species of North American fungi.

Torr. Bull. VIII. 26-28, 51-52. March-May, 1851 .

Descriptions of 11 species, 2 from California, the rest from New Jersey and Peunsylrania.

1-251. - New Californian fungi. Bull. Cal. Acad. Sci. I. 26-29 (6-9). Feb. 1884.

Descriptions of 8 species, all but 1 Uredineae.

- and Holway, E. W.

252. New fungi from Iowa. Jour. Mryc. I. 4-6.

Jan. 1885.

Descriptions of 15 species.

- and Kellerman, W. A.

253. New species of North American fungi. Am. Nat. IVII. 116t-1166. Nov. 1883.

Fourteen species, principally from Fairfeld Co., Ohio.

$+254 .-$ Kansas Fungi. Torr. Bull.

XI. 114-116, 121-123. Ogt., Nov. 1s8t.

Descriptions of 26 species, the greater part Cercosporae.

\% 255. New Kansas Fungi. Jour. Myc.

I. 24, Jan. 1885 ; - II. 3, 4, Jan. 1886.

Deseriptions of 20 new species. See species in Medwigia, XXIV. 126, 127, 172-1it.

of 256. — Two new species of Cylindrosporium. Jour. Myc. II. 81. July, 1886.

Descriptions of C. Tradescantiue and C. angustifolium.

- and Martin, G.

257. Some new species of Sphaeriaceous fungi.

Am. Nat. XVI. 809,810 . Oct. 1882.

Describes 5 species, 4 of them on Sabal sermulata in Florida.

258. - New species of North Américan fungi. Am. Nat. XVI. 1001-1004, Dec. 1882 ; - XVIII. 1147-1148, 1264, Nov., Dec.1584; XIX. 76, 77, Jav. 1885 .

Descriptions of 41 species, the greater part from Florida.

f 259. —_ New Elorida Fungi. Am. Nat. XVII. 1283-1285, Dec. 1883 ; - XVIII. 69, 70, 188-190, Jan., Feb. 1884. Describes 22 species Collected near Green Cove Sṕring,
Florida.

260. New Florida Fungi.

Jour.

3fyc. I. 97-101. A11g. 1885.

Nintes nurl deseriptions of 15 specics, some from New York, New Jersey, anl Lake Supcrior. Descriptious also gireu in Hedrcigia, XXV. $40-44$.
$+26 \mathrm{r}$. N New Fungi. Jour. Myc. II. 128, 130 . Nov. 1886.

Descriptions of 11 species, principally Phyllostictae, from different localities.

Endlicher, Stephan Ladislaus. Pressburg, 24 June, 1804. †Vienna, 28 March, 1848.

262. Genera Plantarum secundum ordines naturales disposita. $4^{\circ}$. pp. LX. 1483. Vienna. 1836-1840.

Includes descriptions of American genera of fungi taken from original sources. Nothing of importance in supplements.

Engolmann, George. Frankfort-on-the-Main, 2 Feb. 1809. +St. Louis, 4 Feb. 1884. See de Bary, A. See Goethe, R.

263. - Diseases of grapes. Trans. Acad. Sci. St. Louis, II. 165, 166. Read 16 Sept. 1861 .

The report of proceedings of this meeting contains some remarks on Peronospora viticola and the black rot, said to be caused by Naemaspora ampelicida. See also de BARY, A. Also given in full in Am. Pomol. Soc. Session 1879, pp. 41, 42 .

264. — Oak and grape fungi. Trans. Acad. Sci. St. Louis, III. cexv., cexvi. 1878. Read 5 June, 1876.

Notes on Phoma viticola, Depazea Labruscae, and a new species, Septoria Querci, the latter not described.

265. - Illustrated descriptive catalogue of American grape vines. A grape-growers' manual. $4^{\circ}$. pp. 153. St. Louis. 1883. $3 \mathrm{~d} \mathrm{ed.}$

The so-ealled Bushberg Catalogue of Bush \& Son \& Meiss. ner, of which earlier editions appeared in 1869 and 1875. Has on pp. 47-18, a notice of diseases of the grape vine by Engelmann, including Peronospora viticola, Oidium, and Phoma uvicola. Not in earlier editions which, like $3 d$ ed., have general notices of grape diseases by the editors.

Everhart, Benjamin Matlack. West Whiteland, near West Chester, Pa., 24 April, 1818. See Ert.s, J. B. See Exsiccati in Supplement.

Farlow, "William Gilson. Boston, Mass., 17 Dec, 1844.

266. The Potato Rot. Bull. Bussey Inst. I. 319338. Fig. 7. 1875.

A popular account of potato rot, lettuce mould, etc.

267. - On a disease of Olive and Orange Trees, occurring in California in the spring and summer of 1875 . Bull. Bussey Inst. I. $404-414$. Pl. 1. March, 1876.

Detailed account of Capnodium Citri and the injury done by it, with notes on synonymy. Rev. in Pacific Rural Prcss, XXV. 208. 10 Mareh, 1883. See no. 270.

268. - On the American grape-vine mildew. Bull. Bussey Inst. I. 415-429. Pl. 2, 3. March, 1876.

Account of the development of Peronospora vilicola, followed by is synopsis of the Peronosporeae of the United States, in which 9 speeies are described. Rev. in Jour. Roy.
Horl. Soc. V. 2 ser. 68-76. See no. 270 .

+ 269. - List of fungi found in the vicinity of Boston. Bull. Bussey Inst. I. 430-439, March, 1876 ; - II. 224-252, Jan. 1878.

Part 1 gives an enumeration of 242 species, with localities and hosts. Part 2, an enumeration of 121 additional species, with eritical notes on Chytridineae, one new opecies described, Uredineae, Gasteromycetes, and other orders, besides a supplementary note with descriptions of Ilender. sonia Curtisii, Berk., IT. notilis, B. and C., and II. pubens, B. and C., fiom the Curtis herbarium. See no. 270 .

270. The Black Knot. Bull. Bussey Inst. I. 440-45t. Pl. 4-6. March, 1876.

Detailed account of the anatomy and action of Sphaeria morbosa, Schw. Rev. in North Am. Entomol. I.13-15. This paper, bound with no. 267 and 268 and the first part of no. 269, was issued as a separate pamphlet, with original paging,
March, 1876 . 
Farlow, William Gilson.

27x. Onion Smut, an essay presented to the Massachusetts Society for Promoting Agriculture, pp. 15, pl. 1, and fig. 5. Boston. 1877. Extr. 24th Rept. Mass. Board Agr. for 1876. Part 2. pp. 164-176.

Account of Urocystis Cepulae, and damage done by it in New England, with a note on Pupulasporte sepedonioides. Rev. in Grevillea, V. 158; and Gard. Chron. 1877. pp. 441 and 634 .

- 272. - Notes on some common diseases caused by fungi. Bull. Bussey Inst. I1. 106-114. PI. 1.1877.

Notes on Sphaeria morbosa, Urocystis Cepulae, ete., with aecount of Uncinula spiralis and its action, with figures. Rev. in Jour. Roy. Hort. Soc. V. new ser. 69-71. Plate also given in Rural New - Yorker. 30 Jan. 1886.

273. — Diseases of fruit-bearing trees. 25th Rept. Mass. Board Agr. for 1877. pp. 218-233 (1-16). Fig. 7. 1878 .

Popular account of blaek-knot of plums, grape moulds, ete., with figurcs from author's previous papers.

274. - On the synonymy of some species of Uredineae. Proc. Amer. Acad. Arts and Sci. Boston, XIII. (V.n. s.) 251, 252 (262, 263). 1878. Read Feb. 1878.

Notes on noinenelature of a Uromyces on Brizopyrum, Ramularia on Rumex, and a Puccinia on Malvastrum, in California. By a misprint, pages of reprint differ from original.

275. - Diseases of forest trees. Trans. Mass. Hort. Soc. for 1879. Part 1. pp. 44-46. 1879 .

Abstract of popular lecture. Substance given in Bot. Gaz. IV. 214.

276. - The Gymnosporangia or cedar apples of the United States. Anniversary Memoirs Soc. Nat. Hist. Boston. pp. 1-38. Pl. 1, 2. 1880.

Description of the Gymnosporangia and Roesteliae of the United States, with aceount of eultures made to show the connection of the species of the two genera. One hundred reprints issued. As paper is hard to obtain, consult following reviews. $X$ Bot. Gaz. VI. $209 ; \times A m$. Nat. XV. 471; XTorr Bull. VIII. 71 \% Grevillea, IX. 138.

+ 277. Unusual habitat of a Coprinus. Torr. Bull. VIII. 67. June, 1881.

Notes the occurrence of $C$. stercorarius on water contain ing algae.

+278 . Notes on Gymnosporangia. Torr.

Bull. VIII. 85-87. Aug. 1881.

New loealities of Gymnosporangia and Calyptospora.

+279 . Notes on some species in the $3 \mathrm{~d}$ and 11 th centuries of Ellis's North American Fungi. Proc. Am. Acad. Arts and Sci. Boston, XVIII. (X.n. s.) 65-85. 1883. Issued July, 1883.

Critical notes on several Peronosporae, Uredineae, and Exoasci of this series, with deseriptions of 7 new species, one from Bermuda, preceded by gencral remarks on nomenclature of Uredineae. Rev, in Bot. Gaz. VIII. 302.

+280 . - Note on Phallus togatus, Kalchb. Bot. Gaz. VIII. 258, 259. July, 1883.

Notes on the nomenclature of the Dictyophorae of the United States. $P$. togatus, Kalchbr., referred to $P$. duplicatus, Bose. See KalchbrenNer, $\mathbf{K}$.

+ 28r. Notes on some Ustilagineae of the United States. Bot. Gaz. VIII. 271-278 (1-7). Aug. 1883.

General notes on Ustilagineae of the United States, with synopsis of species of the genus Entyloma in the United States, with descriptions of $4 \mathrm{new}$ speeies, and description of Doassansia Epilobri.

1 282. - Additional note on Ustilagineae. Bot. Gaz. VIII. 318. Oct. 1883.

Notes on the Doassansia and other species mentioned in last-named paper.
- 283. - Enumeration of the Peronosporeae of the United States. Bot. Gaz. VIII. 305-315, 327337. Oct., Nov. 1883.

Descriptions and synonymy of 35 species known in this country, 5 new species, with list of hosts. See 10.285.

+ 284. - Notes on the cryptogamic flora of the White Mountains. Appalachia, III. 232-251. Jan. 1884.

Enumeration of 108 fungi, pp. 237-251, 8 new species deseribed, including 3 given also in Bot. Gaz. VIII. $275-277$. Critieal notes on several of the speeies, iucluding an aceount of the Peridermia of the White Mountains. pp. 239-243. Rev. in Rev. Myc. Vr. 129.

+ 285. - Additions to the Peronosporeae of the United States. Bot. Gaz. IX. 37-40. March, 1884.

Two species and one rariety new to the United States, with new hosts for 13 species enumerated in paper above. See no. 283 .

286. - Maladies des morues sèches. Rev. Myc. VI. 197-199. Pl.49. Fig. 1,2. July, 1884.

Description of Sarcina Aforrhuae and Oidium (Torula) pulvinatum, with figure. See also foot-note in Rev. $M y y c$. VII. 17, for correction.

287. - Notes on a fungus parasitic on species of Potamogeton. Trans. Field-Naturalists' Club, Ottawa, II. 127-129 (1-3). 1884.

Notes on Doassansia Farlowii received from Dr. J. Fletcher.

+ 288. - Notes on fungi. Bot. Gaz. X. 219221. Feb. 1885.

Remarks, with corrections of statements in papers here numbered 279 and 281.

- 289. - Notes on some species of Gymnosporangium and Chrysomyxa of the United States. Proc. Am. Acad. Arts and Sci. Boston, XX. (XII. n. s.) 311-323. 1885. Read 11 Feb. 1885.

Summary of cultures of spores of Gymnosporangia made by author up to date, with additional remarks on the Perider. mia and Chrysomyxae of the White Mountains and other localities. Rev. in Jour. Myc. I. 45.

290. The Synchytria of the United States. Bot. Gaz. X. 235-245. Pl. 4. March, 1885.

Synopsis of species in the United States, two of them new.

$+29 \pi$. Notes on some injurious fungi of California. Bot. Gaz. X. 346-348, Oct. 1885 ; and Proc. Am. Ass. Adv. Sci. XXXIV. 300-303. Also in Proc. Soc. Prom. Agr. Sci. 1885. pp. 29-31.

Account of Peronospora Myoscyami and Puccinia Mal. vastri in California.

292. —_ Vegetable parasites of codfish. Bull. U. S. Fish Comm. VI. 1-4. Fig. 2. 1886.

Summary of papers on the subject, including fungi from paper no. 286, with additional notes.

4-293. - Development of Roesteliae from Gymmosporangia. Bot. Gaz. XI. 189, 190. July, 1886.

Preliminary report. See next title.

+294 . - The development of the Gymnosporangia of the United States. Bot. Gaz. XI. 234-241. Sept. 1886.

Paper read at the meeting of the Am. Ass. Adv. Bei. at Buffalo, N. Y., Aug. 1886, giving results of cultures made by B. D. Halsted and R. Thaxter, etc. Abstr. in Proc. Am. A Adv. Sci. XXXV. 254. The paper by Thaxter, not here included, issued Jan. 1887, in Proc. Am. Acad. Boston.

295. - On a supposed disease of roses caused by a fungus. Proc. 7 th Ann. Meeting Soc. Prom. Agr. Sci. pp. 24, 25. Columbus, O. 1886.

Read at the meeting at Buffilo in August; issued in November. Leptothyrium chromospermum, Peck, on roses, said to be the spores of a Pilobolus, and Gymnosporium harknessioides, Eltis and Holway, considered to be spores of some ascomycetous fungus. 
Fnrlow, William Gilson.

296. Puccinia Malracenrum, Mont., in Massachusetts. Hot. Gaz. XI. 309, 310. Nor. 1886.

livports the discorery of this species on dlthaea rosea at Beverly, Mass.

\section{Fiold, Martin.}

297. On the origin of ergot. Am. Jour. Sci. A\%s, IX. 359-362. June, 1825.

l.ctter. with notes, on ergot in Vermont. Of little value at the present diay.

\section{Fisch, C.}

298. Fintwickelungsgeschichte ron Doassansia Sagittariae. Ber. Deutsch. Bot. Gesell. II. 405416. 1'1. 10. Oct. 1854.

Includes a reference to $D$. Epilobii of the United States.

Fischor, Eduard.

299. Beitrag zur kentniss der gattung Graphiola. Bot. Zeit. XILI. 745-756, 761-773, 777-788, 793801. 1'1. 6. Nov. 1883.

Includes accounts, with figures, of $G$. Phoenicis, G. congestr, and ${ }_{r}$. compressa, the latter new, from United States.

300. — Versuch einer systematischen Uebersicht uiber die bisher bekannten Phalloideen. Jahrbuch des Bot. Gart. und Bot. Mus. zu Berlin, IV. 1-92. Pl. 1. 1886.

General aceount of this group followed by full descriptions, including species of the United States. Rev: in Am. Nat. XX. 804.1886.

Fischer do Waldhoim, Alexandre.

301. Beitrïge zur biologie und entwickelungsgeschichte der Ustilagineen. Pringsheim Jarhb. VII. 61-144. Pl. 7-12. 1869.

Does not refer specially to speeies of the United States. Giren here because an English translation of the work was giren in Trans. N. 1. State Agr. Soc. for 1870. pp. 280-354. ก!. 1-6. Albany". $18 \% 1$.

302. - Aperçu systématique des Ustilaginées, leurs plantes nourricières et la localisation de leurs spores. $4^{\circ}$. pp. 51. Paris. 1877.

Paper presented at the International Botanical Congress at Amsterdam. Preliminary account of species published in full is Ann. Sci. Nat. Bot. See no. 304 .

303. - lievue des plantes nourricières des Ustilaginées. $8^{\circ}$. pp. 20. Moscow. 1877. Extr. Bull. Soc. Imp. Nat. LII. 312-331. Moscow. 1877.

Taken from work named abore, with emendations.

304. — Les Ustilaginées et leurs plantes nourricières. Ann. Sci. Nat. Bot. 6 ser. IV. 190276. 1876. (1877.)

General account of the order, followed by descriptions of all known species, including American forms, with host-index and index of orgins at tacked by these fungi. Also in Polish, with additional synonymy "Les Ustilaginées, esquisse monographie," part 1, pp. 15, 1877; part 2, pp. 131, 1873. Warsaw.

Forster, John Reinhold. Dirschau, 22 Oct. 1729. +IIalle, 9 Dec. 1798.

305. Travels through that part of North America formerly called Louisiana by Mr. Bossu, Captain of tle French marines. Translated from the French by John TReinhold Forster, F.A.S. Illustrated, with notes relative chiefly to Natural History. To which is adder by the translator a Bystematic catalogue of all the known plants of Finglish North America, or, a Flora Americae Septentrionalis, together with an abstract of the most useful and necessary articles contained in Peter Loefling's travels through Spain and Cumana in South America. $\quad 8^{\circ}$. Vol. I., II. pp. $407,432$. London. 1771 .

On II. p. 69, is a list of 6 fungi from Tirginia and Carolina. The catalogue of plants was also issued separately.

306. Fortieth Prrallol Expodition, Fungi of. See Billixis, J. S., and Frost, C. C.
Fotvler, James. Bartibog, Miramichi, N. B., 17 July, 1829.

307. List of New Brunswick plants. Ann. Rept. Secretary Agr., Province New Brunswick, for 1878. Appendix B. pp. 35-63. Fredericton, N. B. 1879.

On p. 63 a list of 55 fungi, with hosts and localities. Also in Elucational Circular, no. 9. Fredericton. 1880.

Fries, Flias Magnus. Femsjö, Smoland, 15 Ang. 1794. tUpsal, 8 Feb. 1878.

308. Observationes Mycologicae. $12^{\circ}$. Vol. I. pp. 230. Pl. 1-4. 1815. - Vol. II. pp. 372. Pl. 5-8. 1818. Copenhagen.

Notes on a small number of Schweinitzian species.

+ 309. Systema Mycologicum, sistens fungorum ordines, genera et species, huc usque cognitas, quas ad normam methodi naturalis determinavit, disposuit atque descripsit Elias Fries. $12^{\circ}$. Vol. I. pp. 520. Lund. 1821. - Vol. II. 2 parts. pp. 620. lund. 1822-23. - Vol. III. pp. 524, with Index, pp. 202, of genera, species, and synonyms in the Systema Mycologicum and Elenchus Fungorum. Greifswald. 1832.

Includes descriptions and revisions of a considerable num. ber of American fungi, especially species of Schweinitz and Muhlenberg. See no. 311 .

310. - Systema orbis regetabilis primas lineas novae constructionis periclitatur Elias Fries. Pars 1. Plantae Homonemeae. 12\% pp. 374. Lund. 1825 .

Has references to genera and species of Bose and of Schweinitz's Syn. Fung. Car. Sup.

† 3II. - Elenchus Fungorum, sistens commentarium in Systema Mycologicum. 12. Vol. I. pp. 238. Vol. II. pp. 154. Greifswald. 1828.

This is the title given to the whole work in copies seen. There is a secondary title for each part, same as title herc given, no. 309, to which is added "Supplementa roluminis primi-sceundi. 1830." This work is in fact a supplement to rols. I. and II. of the Systema DIycologicum, and the con. tents are indexed in vol. III. of the Systema, published two years later.

312. - Gonera Hymenomycetum. 8. pp. 17. Upsal. 1836.

Has notes on Thelophora sistotremoides and $T$. pedicellata of Schweinitz.

313. - Synopsis generis Lentinorum. $8^{\circ}$. pp. 15. Upsal. 1836.

Includes descriptions of 10 species from the United States, among them Aguricus chama, Bosc, referred to Lentinus.

+ 314. - Epicrisis systematis mycologici, seu synopsis IIymenomycetum. $8^{\circ}$. pp. 608. Upsal. $1836-1838$.

Includes descriptions and revisions of American species.

315. — Summa regetabilium Scandinaviae, seu enumeratio systematica et critica plantarum quum cotyledonearum tum nemearum inter mare occidentale et album inter Eidoram et Nordkap lactenus lectarum indicuta simul distributione geographica. $8^{\circ}$. 2 parts. pp. viii. 572. 18461849. Stockholm and Leipsic.

In the second part is an enumeration and generic rerision of fungi, pp. 261-525,563,564, including a considerable number ot revisions of American genera, principally Schweinitzian.

3r6. - Novae Symbolae Mycologicae in peregrinis terris a botanicis Danicis collectae. Nova Acta. Sci. Upsal, I. 17-136. 1851.

Includes notes on or descriptions of 54 species from the United States, mostly collected by M. A. Curtis and Schweinitz, some by II. W. Ravenel and Liudblom, and a number from the herbarium of $\mathrm{E}$. Tuckerman.

- 317. - Novarum symbolarum mycologicarum mantissa. Nova Acta. Sci. Upsal, I. 225231. 1851.

Continuation of previous paper, including 20 species principally from Mlexico and St. Thomas, W. K., but none cred. ited to the United States. 
Fries, Elias Magnus.

+3 18. Queletia, novum Lycoperdacearum genus. Accedit nova Gyromitriae specics, Oefv. $K l$. Vetensk. Akad. Förhand. XXVIII. 171-174. Pl. 4. 1871.

The Jforchella Caroliniana of Bosc, referred to Gyromitra.

Frost, Charles Christopher. Brattleboro, Vt., 11 Nov. 1805. †Brattleboro, 16 March, 1880. See Billings, J. S. See Peck, C. H. See TuCKerman, E.

319. - Further enumeration of New England Fungi. Proc. Soc. Nat. Hist. Boston, XII. 77-81. 1869. Read 20 May, 1868.

Continuation of paper by C. J. Spraguc, l. c. V. 325 , and VI. 315. A list of 263 species, without localities, some of the species not described at the time, and the names, apparently manuscript names, given by M. A. Curtis.

+320. - Catalogue of Boleti of New England, with descriptions of new species. Bull. Soc. Nat. Sci. Buffalo, II. 100-105. June, 1874.

Enumeration of 47 species, 21 described as new by the antlior. Also one species by J. L. Russell and one of Berkeley and Curtis. See also Peck's 29th Rept. p. 45, and 38th Rept. p. 110.

N. B. The paper by this anthor, "Catalogue of crypto. gamous or flowerless plants of Vermont," in Arehives of Science, Newport, Vt., I. - III. does not include fungi.

Fuckel, Leopold. Ostrich-on-the-Rhine. †Vienna, 8 May, 1876. See Bororden, H. F.

Gerard, William Ruggles. Newburgh, N. Y., 29 March, 1841. See Bulletin Torrey Club. -321. New species of fungi. Torr. Bull. IV. 47,48 , Oct. 1873 ; 1. c. 64 , Dec. 1873 ; - V. 26, 27, June, 1874 ; 1. c. 39,40 , Sept. $1874 ;-V I .31,32$, April, 1875; 1. c. 77, 78, Feb. 1876.

Description of 48 new species, principally from Poughkeepsie, N.Y. Some of the species attributed to Cooke and Gerard. The species in parts 1,3, and 5 also given in Hedvoigia, XIII. 28, 29,111,112; XIV.188-190. See also no.324.

322. - White mildews or blights. Proc. Soc. Nat. Sci. Poughkeepsie, I. 2-16. PI. 1-4. 8 Feb. 1875.

General account of classification of fungi, with plate, including figure of Hysterium magnosporium, Gerard, followed by detailed account of Erysiphei, with figures of 28 species and deseription of Microsphaera Van Bruntiana, Gerard. Plate 1 of reprint is colored.

+323. - Oct. 1876 .

States, on the authority of C. V. Riley, that Rhytisnua Solidaginis, Schw., and R. Asteris, Schw., are insect galls.

324. Descriptions of new fungi. Proc. Soc. Nat. Sci. Poughkeepsie, I. 85-87. Read

1 Nov. 1876.

Descriptions of 11 species, 7 previously described in Torr. Bull. VI. 77, 78.

+ 325. - A truffle new to the North American flora. Torr. Bull. VI. 276. Nov. 1878.

Reports the discovery of Tuber dryophilum in Staten Island; also notes on distribution of trnffles in the United States. Also given in Proc. Soc. Nat. Sci. Poughkeepsie, 20 Nov. 1878, pp. 18, 19.

326. - The Saprolegnia ferax. Proc. Soc. Nat. Sci. Poughkeepsie. 18 Dec. 1878. pp. 25-28. +327 . A new fungus. Torr. Bull. VII. 8-11. Pl. 1, 2. Jan. 1880.

Description and figure of Simblum mubescens, followed by a "List of United States Phalloidei," in which 13 species are enumerated. See Rev. IYyc. II. 93. Pl. 6. Fig.1.

+ 328. Additions to the U. S. Phalloidei. Torr. Bull. VII. 29, 30. March, 1880.

Supplementary notes on last-named paper, with remarks on some species of Rafinesque.

+ 329. - Correlation between the odor of the Phalloids and their relative frequency. Torr.

Bull. VII. 30-33. March, 1880.
+330 . The preservation of pileate fungi for the herbarium. Torr. Bull. VIII. 14-16. Feb. 1881. Description of several methods. See also Torr. Bull. VIII. 32 et seq., for account of G. Herpell's method.

+33r. Some fungi from New Mexico. Torr. Bull. VIII. 34. March, 1881.

Enumeration of 5 species, one described as new, Aecidium Rusbyi.

+ 332. - Reliquiae Rafinesquianae. Torr. Bull. XII. 37, 38. April, 1885.

Includes notes on 31 species of fungi in Rafinesque's "Seleet new plants of North America," of which there is a set of the proof of unpublished plates in the library of the New York Academy of Science.

N. B. Species of new fungi by W. R. Gerard are included in Peek's 24th, 25th, and 26th Reports. Title no. 120 includes a letter from Gerard.

Goethe, Robert.

333. Mittheilungen über den schwarzen Brenner. pp. 22. Berlin and Leipsic. 1878.

Original not seen. Au austract is given in English in Proc. Am. Pomol. Soc. Session 1879, pp. 44-46, entitled "Abstract of a treatise on the anthracnose (Sphaceloma ampelinum), which includes references to Engelmann's paper on grape rots.

Goodale, George Lincoln. Saco, Me., 3 Aug. 1839. See American Naturalist.

Gore, James Howard. Frederick Co., Virginia, 18 Sept. 1856.

334. Tuckahoe or Indian bread. Rept. Smithson. Inst. for 1881. pp. 687-701 (1-13). Fig. 5. Washington. 1883.

Account of properties and structure of tuckahoe, with sum. mary of writings on the subject.

Gray, George Robert.

335. Notices of insects that are known to form the bases of fungoid parasites. $4^{\circ}$. pp. 22 . Pl. 6 . 1858.

Privately printed by the author. No place of publication given on title-page, but probahly London. A general summary, with full account of literature of the subject, including American references and good plates reproduced from various sources.

Greeley Expedition, Fungi of. See Elurs, J. B., and Everitart, B. M.

t 336. Grevillea. A monthly (quarterly) record of cryptogamic botany and its literature. Edited by M. C. Cooke. $8^{\circ}$. Illustr. Vol. I. $-\mathrm{XV}$. July, 1872-Jan. 1887. London. To be continued.

Issued in monthly parts, from July, 1872-June, 1874, vols. I., II. From Sept. 1874 to present time issued quarterly. Includes all orders of cryptogams. Titles of papers on American fungi in this journal given here under authors' names. Reviews by the editor.

Gronovius, Jan Fredrik. 1690. †1762.

337. Flora Virginica exhibens plantas quas $V$. C. Johannes Clayton in Virginia observavit atque collegit. Easdem methodo sexuali disposuit, ad genera propria retulit, nominibus specificis insignivit et minus cognitas descripsit Joh. Fred. Gronovius. 8. Part 1, pp. 128, and index. 1739. Part 2, pp. 129-206, preface and index. Leyden. 1743.

Part 1, p. 128, gives a list of 3 fungi. Part 2, p. 205, 7 fun Ei, including Tuckahoe. In the $2 d$ edition of this work, nublished in $1762, \mathrm{pp} .176$, index and map, $4^{\circ}$, is a list of 20 fungi, pp. 174-176.

Hagen, Hermann August. Königsberg, Prussia, $30 \mathrm{May}, 1817$.

338. Destruction of obnoxious insects, Phylloxera, potato-bectle, cotton-worm, Colorado grasshopper, and greenhouse pests, by application of the yeast fungus. $8^{\circ}$. pp. 11. Cambridge. 1879.

A paper reprinted, with slimht modifications, from Cana. dian Entomologist, XI. 110-114. Recommends on application of yeast for destroying insects, and gives the results of application in certain cases. 
Hagen, Ilermanu August.

5. 339. On the destruction of obnoxious insects by yeast. Canadian Entomologist, XII. 81-83. May, 1880.

Supplementary to last-named paper, with notes on the botinical relations of Entomophtharae.

340. Cordyceps Ravenelii on the larrae of Phyllophaga. Canadian Entomologist, XII. 89. May, 1880.

341. - Experiments with yeast in destroying insects. Canadian Entomologist, XIV. 38, 39. Feb. 1882.

Gives a letter from Thomas $\Pi$. IIart, relating his success in applying yeast, from which certain conclusions are drawn.

\section{Hallier, Erust.}

342. Coniathecium Stilesiamm. Rept. N. I. State Agr. Soc. for 1867, XXVII. part 2, 1149, 1150.

A letter from Hallier, including an account of this fungus, supposed to be the caluse of Texas cattle fever. Also a letter froun Hallier on p. 1146 .

Hnlsey, Abraham.

343. Remarks on certain entozoical fungi. Ann. Lyc. Nat. Ilist. New York, I. 125, 126. April, 1824.

Notice of exotic forms and brief reference to Isaria Sphingum, Schw.

Malsted, Byron David. Venice, Cayuga Co., N. Y., 7 June, 1852.

344. Our common moulds. Pop. Sci. Month. IX. 398-409. Fig. 13. Aug. 1876 .

345. - Injurious and other fungi. Trans. Mass. Hort. Soc. for 1877. Part 1. pp. 28-44.

Pl. 1-3. Read 27Jan. 187\%.

Brief account of several common diseases of fungi, wheat bliglit, grape mould, black knot, etc.

346. - Fungi injurions to farmers. Regis. ter of Rural Affairs, VIII. 181-187. Fig. 5. Also in Rept. Connecticut Board Agr. for 1881.

347. The white mildews. Am. Pomolog.

Soc. Session 1883, pp. 87-89.

An account of several species of Perisporiaceae and the harm done by them.

348. - How fungi live in winter. Pop. Sei. Month. XXVI. 611-620. Fig. 1-12. March, 1885. Account of resting condition of several species.

449. A new Iowa Aecidium. Jour. Myc. II. 52. May, 1886.

Description of Aecidium Phrymae.

350. - Gymmosporangium macropus on Pirus coronaria. Bot. Gaz. XI. 190, 191. July, 1886.

Account of open-air cultures made at Ames, Iowa, to show the development of the aecidia of this species. See also paper entitled "Thic wild crab apple" in Country Gentleman, 21 Oct. 1586.

35x. An interesting Peronospora. Bot. Gaz. XI. 272. Pl. 8. Fig. a. b. Oct. 1886.

$\Lambda$ ccount of $P$. graminicola in Iowa.

The following papers from the American Agriculturist, New York, shoultl also be noted: "The American gripe millew," 1879, p. 348, fig. 5; also in Michigun Pomol. Rep. 187, p. 134; - "What are fungi?" 1879, p. 268, fig. 4; "The Wheat Rust," 1879, p. 301, fig. 6;-"Ergot," 1879, p. 399, fic. 8; - "Apple-leaf fungus," 1879, p. 468 , fig. 5 ; "White Mildews," 1879, p. 508, fig. 4; - "Blark Knot," 1890, p. 22, fife. 6;- "Onion Smut," 1850, p. 104, fig. 3; "Isttuen Mould," 1890 , p. 149, fig. $3 ;$ - "Elderberry fonmua," 1890, p. 234, fig. 1; “The Corn smut," 1880, p. 475, fig. 4 ; - "Wheat, oat, ant barley smut," 1881, p. 40t, fig. 3 .

Manbury. Daniel. 11 Sept. 1825. fLondon, 24 March, 1875. See Ccriney, F.

Marloness, Harvey Wilson. Pelham, Mass., 25 May, 1821. See Cookf, M. C. See Eruls, J. B. See Pullips, IV. See Plowright, C. B.
352. A Foe to the Lumberman. Pacific Rural Press, XVII. 49. Fig. 2. 25 Jan. 1879.

Account of a fungus, Daedalia vorax, which attacks the wood of Alies Dougiasii.

353. - Fungi injurious to fruit-trees. Ann. Rept. State Board Hort. California, 1883. pp. 51$59(1-8)$.

General account of diseases of plants caused by fungi, with notices of Peridermium Harknessii, Duedalea vorrx, Sphaeria morbosa, Capnodium Cilri, Phyllosticta circum. sciss $\alpha$, and other fungi.

$+354 .-$ New species of Califorman Fungi. Bull. Cal. Acad. Sci. I. 29-47. Feb. 1884.

Descriptions of 4 new genera, Camposporium, Troposporizm Theclospora, Cleistosoma, and 71 new species.

$+355 .-$ Fungi of the Pacific Coast. Bull. Cal. Acad. Sci. I. 159-176, pl. 1, Feb. 1885 ; 1. c. 256-271 (1-16), 19 Nov. 1885.

Part 1 gives an enumeration of 192 species, with hosts and habitats, and descriptions of 10 new species and 2 new genera, Dieranidion and Geopora, witl plate of Lycoperdon sculp. tum. Part 2 is an enumeration of 171 species, with losts, localities, and notes, including one new species, Secotium nubigenum, followed by a list of 50 species from the IIarkness herbarium, previously described in Grevillea, XIII. 111-114; and XIV. 8-10. See no. 168. See Rer. AYyc. VII. 253.

N. B. The paper by this author on "White Mildew," read before the S:tn Franeisco Micros. Soc., 5 April, 1877, was not formally published, but distributed privately by the author. In this patper an account is given of pycnidia in Erysiphe Graminis. See also reviews of this paper by M. C. Cooke in Grerillea, V. 154, and Gardeners' Chronicle, 1877, pp. $4 \pm 1$ and 634 .

\section{and Moore, J. P.}

+356 . Catalogue of the Pacific Coast Fungi. Published under the direction of the California Academy of Sciences. $8^{\circ}$. pp. 46 . Read 2 Feb. 1880 .

An enumeration of 812 species of fungi from California, with hosts and localities, and 2 from the Saudwich Islands. One new species, Agaricus tridens, is described. This Catalogue was issued separately, and did not appear in any volume of the bulletins of the California Academy. Rev. in Torr. Bull. VII. 58, and Rev, BYye. VII. 253.

Hayden, Ferdinand Vandeveer. Westfield, Mass., 7 Sept. 1829.

357. Sixtl Annual Report of the United States Geological Survey of the 'Territories, embracing portions of Montana, Idalio, Wyoming and Utah, being a report of progress of the explorations for the year $1872.8^{\circ}$. pp. 814. Washington. 1873. Botany by J. M. Coulter. pp. 74.7-792.

Includes on last page a list of 10 fungi, with descriptions of two new species, Peziza vulcanalis and Sphaeria Coulleri,
by C. H. Peck.

358. Hedwigia. Ein notizblatt für kryptogamisclie studien. Vol. I.-XXV. May, 1852Jan. 1887. To be continued. $8^{\circ}$. Dresden. Illustr.

Vol. I. extends from 1852-1857, and rol. II. from 1858-1863. Since that date there hiss been a volume annually. Until Nov. 1878, the editor was L. Rabenliorst, and since then $G$. Winter. With the exception of tlie first two volumes the numbers appeared montlily until 1855, when the journal was enlarged and the numbers issued bi-monthly. Includes all orders of cryptogams. Papers on American fungi, which are given here under authors' names, hardly go back farther than 1873.

Henry, Aimé. See Nees yox Esendeck, T. F. L.

Herald, Voyage of. See Seeman, $B$.

Hesse, $\mathrm{R}$.

359. Mikroscopische unterscheidungsmerkmale der typischen Lycoperdaceen genera. Pringhsheim Jahrb. X. 383-398. Pl. 28, 29. 1876. Gives a figure of capillitium of aritremyces lutescens,
Schw. 
Hine, Frauk Brooks. Berlin, Erie Co., O., 24 April, 1853.

36o. Observations on several forms of Saprolegnieae. Am. Quart. Micros. Jour. I. 18-28, 136146 (1-20). Pl. 4-7. Oct. 1878-Jan. 1879.

Ientions 3 species of Achlya and onc Saprolegnia, two epecies nunamed, and describes a new species, Mlonoblepha. ris lateralis.

Hitchcock, Edward. Deerfield, Mass., 24 May, 1793. †Amherst, Mass., 27 Feb. 1864.

36r. Physiology of the Gyropodium coccineum. Am. Jour. Sci. Arts, IX. 56-60. Pl. 3. June, 1825. Acconnt of the peculiarities of Mitremyces cinnabarinus, Schw., with colored figures.

362. - Catalogue of the plants growing without cultivation in the vicinity of Amherst College. $8^{\circ}$. pp. 64. Amherst. 1829.

A list of all plants found within 40 or 50 miles of Amherst, including, on pp. 58-64, 49 genera and 170 species of fungi, the determinations apparcntly obtained from Prof. John Torrey, ancl indirectly from Nuhlenberg and Scliweinitz. The only copy of this rare work which we have examined was without title-page, and the title here given was taken from the reference in Tuckerman's Catalogne of plants within 30 miles of Amberst. Sce TuckenMan, E.

363. - Catalogues of the animals and plants of Massachusetts. $8^{\circ}$. pp. 142. Amherst. 1835.

Taken from "Report on the geology, mineralogy, botany, and zoölogy of Massachusetts." Amljerst. 1835, pu. 525-652. On pp. 125-129 (645-649) is a list of fungi, alphabetically arranged, same as in last title, with 6 additional species, all but one of which, sphaeria Hypoxylon, are also given in first edition of the Report, Amherst, 1833.

\section{Hoffmann, Hermann.}

364. Index Fungorum, sistens icones et specimina sicca nuperis temporibus edita; adjectis synonymis auctorc Hermann Hoffmann. Indicis mycologici editio aucta. $8^{\circ}$. pp. vi. 153 . Leipsic. 1863.

A general index of figures and exsiccati of fungi, including Imerican specics. This work is an enlarged edition of a pamphlet distributed as a supplement to the Botanische Zeitung for 1860.

Holway, Edward Willett (Dorland). Adrian, Mich., 13 Feb. 1853. See Ellis, J. B. +365. Fungi on Anemone nemorosa. Bot. Gaz. V. 77. July, 1880.

List of 6 species found on this host.

366. - Sound of discharging ascospores. Bot. Gaz. VIII. 160. Jan. 1883.

Note on the sound produced by the discharge of spores in Peziza pubida.

+367 . - Gymnosporium harknessioides, Ell. and Hol. Jour. Myc. II. 52. May, 1886.

This species said to be merely spores of some Sordaria, Chaetomium or allied fungus.

Howe, Elliot Calvin. Jamaica, Windham Co., Vt., 11 Feb. 1828.

+368. New Fungi. Torr. Bull. V. 3-4, 42, 43, Jan., Oct. 1874; - VI. 30, 31, April, 1875.

Deseriptions of 17 species from New York. The parts of this serics arc numbered 1,2, and 4. Part 3 appear's never to have been published. Species of part 4 also given in Hedroigia, XIV. 188.

+369 . - Agaricus (Tricholoma) Peckii, Howe.

Torr. Bull. VI. 66. Dec. 1875.

Characters of the species.

N.B. The descriptions of Uncinula Americana and $U$. luculenta by this author were not formally published, but were printed oll sheets of note paper and distributed among botanists of lis acquaintance. Puccinia Peckiana, by this author, is in Peck's 23d Report, p. 57.

Hnse, William W.

370. The diseases of tobacco, or some inquiries into the causes, the appearance, and the preventives of tobacco-mould. $8^{\circ}$. pp. 36 . New York. 1857.

Has a few woodcuts of fungi of slight value.
James, Joseph Francis. Cincinnati, 8 Feb. 1857.

37r. Catalogue of the flowering plants, ferns, and fungi growing in the vicinity of Cincinnati. Jour. Soc. Nat. Hist. Cincinnati, II. 42-68 (1-27). April, 1879.

On pp. 22-27, a list of 319 fungi, taken from Lea's Cata. logue. Sec no. 381 .

Johnson, Asa Emery. Co., N. Y., 16 March, 1825.

Bridgwater, Oneida

372. The nyycological flora of Minnesota. Bull. Acad. Nat. Sci. Ninnesota, I. 203-302, 325-344. $1877,1878$.

First part has an enumeration of 559 species, with habi. tats, descriptions of two new Agarici, and of most of the genera, precederl by general notes on Agaricini. List of all species but one given also in 5th Ann. Rept. Geol. and Nrte. II ist. Survey, Ninnesota, pp. 66-87. St. Paul. 1877. The second part las enumeration of 83 species, 13 of them de. scribed as new.

+373. Journal of Mycology. Vol. I.-II. 1885, 1886. To be continued. Monthly. $8^{\circ}$. Manhattan, Kansas. Edited by WV. A. Kellerman, J. B. Ellis, and B. MI. Everhart.

Treats of North American fungi, except a few notices of lichens. Fach volume has an index of gener:i, species, and host plants. Articles given here under authors' names.

Kalchbronnex, Károly (Charles). 1806. †TVallendorf, Inungary, 5 June, 1886. See $R_{A} U$, E. A.

374. Plalloidei novi vel minus cogniti. Természettudományok Köréböl. Magyar Tudományas Akad. (Mem. Hungarian Acad.) X. 1-23. Pl. 1-3. Budapest. 1880.

In Hungarian, followed by Latin translation. Includes, on pp. 17, 18, a bricf list of North American Phalloidei. Reprint only seen

375. - Gasteromycetes novi vel minus cog. niti. Természettudományok Köréböl. Magyar Tudományas Akad. (Mem. Hungarian Acad.) XIII. 1-10. Pl. 1-5. Budapest. 1883.

Hungarian and Latin. Description of Phallus togatus, with colored figure. Pl. 1. Sce no. 280.

Kellerman, William Ashbrook. Ashville, O., 1 May, 1850. See Ellis, J. B. See Jouryal of MYCOLOGY.

* 376. A partial list of the Kansas parasitic fungi, together with tlicir host-plants. Bull. Washburn Coll. Lab. Nat. Hist. I. 72-81, Feb. 1885, and Trans 16 \& 17 Ann. Meetings Kansas Acad. Sci. IX. 79-86. 'Topeka. 1885. Read Nov. 1884.

Enumeration of 181 species, and an index of host plants. One species described as new, the other new species described in Am. Nat. Nov. 1883, Torr. Bull. Oct. 1884, and Jour. Mryc. Jan. 1885. See notice in Jour. Mryc. I. 71.

$37^{\mathrm{a}}$. - Some parasitic fungi that infest orchards and gardens. Rept Kansas State Hort. Soc. for 1884, XIV. 111-115. Topeka. 1885.

A popular account of plum blight, peach curl, grape mildew, and strawberry leaf blight.

KIotzsch, Johann Friedrich. See BerkereY, M. J. Wittenberg, 9 June, 1805. †Berlin, 5 Nov. 1860 .

377. Mycologische berichtigungen zu der nach. gelassenen Sowerbyschen sammlung so wic $\mathrm{zu}$ den wenigen im Linnéschen Herbarium vorhandenen pilzen, nebst aufstellung einiger ausländischen gattungen und arten. Limnaea, VII. 193-204. Pl. 8-10. 1832.

Includes descriptions of Favolus Canadensis and Testic. ularia Cyperi, the latter figured.

378. - Fungi exotici e collectionibus Britannorum. Limnaea, VIII. 478-490. Pl. 11, 12. 1833.

Includes enumeration of 34 species from North America, seren described as new, inostly collected by Sir J. Rich. ardson. 
Koernicke, Friedrich.

379. Mykologische Beiträge. Medwigia, XV. $178-186$, 1)ec. $1876 ;-$ IVI. 1-3, 17-31, 33-40, Jan.-March, 1877.

Ineludes notes on United States Uredineae and Ustila. ginerse, with description of the new genus Hamaspora, founded on Podisoma Ellisii, Berk.

Kunze, Gustav. Leipsic, 4 Oct. 1793. †Leipsic, 30 April, 1851 .

\section{- and Schmidt, Johann Carl.}

380. Mrkologische Hufte, nebst einem allgemeinbotanischen anzeiger. $8^{\circ}$. Vol. I. pp. xvi. 109, and some unnumbered supplementary pages. Pl. 2. Leipsic. 1817. - Vol. II. pp. xii. 176. Pl. 2. Leipsic. 1823.

A collection of short papers by the editors, with three papers by Ehrenberg, Fries, aud Nees. Vol. I. 45-52, pl. 2, fig. 22, "I3eselureibung der, gattung Solenarium, Spreugel," by Kunze, gires an account of S. Afuehlenbergii (Glonium stellutuml) from Pennsylvania. Other papers do not treat of American fungi, althongh Sphaeria sarmentorum is recorded by Fries on Yenispermum Canadense on II. p. 52.

Lea, Thomas Gibson. IVilmington, Del., 14 Dec. 1785. +Waynesville, O., 30 Sept. 1844. See Berreley, M. J.

381. Catalogue of plants, native and naturalized, collected in the vicinity of Cincinnati, O., during the years 1834-1841. $8^{\circ}$. pp. iv. 77. Philadelphia. 1849 .

Tbis cataloguc was printed under the direction of Isaac I.ca, after thic death of bis brother, whose collections were placed in the hands of W. S. Sullivant for publication, the fungi having been defermined by Rev. M. J. Berkeley. On pl. $45-i \bar{i}$ is a list of 319 species of fungi, with localities and dates of collection. In foot-notes are given observations on some of the species, and descriptions of $53 \mathrm{new}$ speeies by Berkeley, some of which were previously deseribed in "Decades of fungi"; see title no. 31. A few figures also given in 13erkeley's Introduction; see title no. 38. Original copies are rare, but a reprint of the notes and descriptions of the present list will be found under the title "Descriptions of new species of fungi, collected in the ricinity of Cincinnati," Jour. Soc. Nat. Hist. Cincinnat, V. 197-217 (1-21). Dec. 15s2. See also title no. 371. Rev. in Am. Jour. Sci. Arts, VIII. new ser, 302. 1849.

Leggett, W. H. See Bulletin Torrey Clob.

Loidy, Joseph. Philadelphia, 9 Sept. 1823.

382. On the existence of Entophyta in healthy animals as a natural condition. Proc. Acad. Nat. Sci. Philadelphia, IV. 225-233. 9 Oct. 1849.

Account of regetable parasites, $p$ p. 225-229, including deseriptions of the new genera Enterobrus, Cladophytum and Arthromilus.

383. - Deseriptions of new genera and species of Entophyta. Proc. Acad. Nat. Sir. Philadelplia, IV. 249, 250. 25 Dec. 1849.

Descriptions of the new genera Corynocladus and Crypto. desma, and uew speeies of Enterobrus and Cladophylum.

384. - Entophyta in bodies of animals. Proc. Acad. Nat. Sci. Philadelphia, V. 7, 8. 12 Feo. 1850.

Remarks on Mucor mucedo, Achlya prolifera, and some unnamed species found in insects and cray-fish.

385. - Entophytes in insects and myriapoils. Proc. Acad. Nat. Sci. Philadelphia, V. 8, 9. 19 lieb. 1850 .

IRemarks on species of Enteroumis, ctc.

386. Descriptions of new Entophyta growing within animals. Proc. Acad. Nat. Sci. Pliladclplia, V. 35, 36. 30 April, 1850.

Description of one species of Arthromitus and tro of a new genus, Eccrina.

387. - lingus in a dead molc-cricket. Proc. Acad. Nat. Sci. Philadelplia, V. 20t, 210, 211. 20 Mas-3 June, 1851.

Notes on : 1 unnamed fungus attacking Gryliotalp Americana, and general remarks ou parasitic discases of ingects.
388. Fungi in insects. Proc. Acad. Nat. Sci. Philadelphia, V. 235. 1 July, 1851.

Notes on a fungus in the abdomen of Cicada septemdecim, and one on a lamellicorn larra.

38g. — A flora and fauna within living animals. Smithsonian Contrib. to Knowledge, V. 1-67. P1. 1-10. April, 1853.

A detailed account, pp. 17-40, of forms mentioned in papers whose titles are given above, with plates. MIost of the species would be placed at the present day in Schizomy. cetes. Also on pp. 51-53, accounts of other fung1, including that whicl attacks Cicada septendecim.

39o. - Fungus parasite on a mouse. Proc. Acad. Nat. Sci. Philadelphia, pp. 260, 261. 22 April, 1873.

Describes a Torula or Didium on a mouse caught in a hospital. Note also by Dr. Le Conte, who attributed a cutaneous eruption on his own person to infection from a similarly diseased mouse.

39r. - On a fungus in a flamingo. Proc. Acad. Nat. Sci. Philadelphia, pp. 11, 12. Fig. 1. 19 Jan. 1875.

Finds an Aspergillus, which may be A. nigrescens, in lungs of a flamingo which was probably killed by the fungus.

392. - Ant infected with a fungus. Proc. Acad. Nat. Sci. Philadelphia, p. 9. 1 Jan. 1884.

Note on an undescribed fungus attacking Camponotus Pennsylvanicus.

Lesquereux, I.eo. Neuchâtel, 18 Nov. 1805.

393. Les Rhizomorpha du nouveau monde. Rev. Myc. III. 74. July, 1881.

Note on $R$. subcorticalis and malformed Agaricini from mines.

Léveillé, Joseph Henri. Crux-Ia-Ville, 28 May, 1796. †Paris, 3 Feb. 1870.

394. Champignons exotiques. Ann. Sci. Nat. Bot. 3 ser. II. 167-221; III. 38-71. $1844,1845$.

Contains notices of 6 spccics from North America.

395. — Descriptions des champignons de l'herbier du Muséum de Paris. Ann. Sci. Nat. Bot. 3 ser. V. 111-167, 249-304. 1846.

Notes on 14 species of North America, of which 7 are described as new.

Link, Heinrich Friedrich. Hildesheim, 2 Feb. 1767. †Berlin, 1 Jan. 1851.

396. Caroli a Linné Species Plantarum exhibentes plantas rite cognitas ad genera relatas cum differentiis specificis, nominibus trivialibus, synonymis selectis, locis natalibus secundum systema sexuale digestas. Editio quarta, post Reichardianam quinta adjectis vegetabilibus hueusque cognitis olim curante Carolo Ludovico Willdenow, continuata a H. F. Link. Tomus, VI. Part 1. 8. pp. xv. 162. Berlin. 1824. - Part 2. pp. vi. 128. Berlin. 1825.

The sccond part of vol. VI. contains Gymnomycetes (Ure. dineae, etc.), including species from Schwcinitz, revised by I.ink.

Linnaeus, Carolus. See LiNk, H. F. See Sprenget, C.

Macbride, James.

397. Some account of the Lycoperdon solidum of the Flora Virginica, the Lycoperdon cervinum of Walter. Trans. Linn. Soc.XII. 368-371. 1818. Read 3 June, 1817.

General account of tuckahoe.

Macoun, John. Maralin, Co. Down, Ireland, 17 April, 1832.

398. Edible and poisonous fungi. Trans. FicldNaturalists' Club, Ottawa, II. 62-67. Read 31 Jan. 1884.

$\Lambda$ general address on the subject before the Society, with exhibitiou of plates. 
Macoun, Jolin.

399. List of plants collected on the coasts of Labrador, Hudson's Strait and Bay, by liobert Bell, in 1884. Report of Progress of Geol. and Nat. Hist. Survey and Museum of Canada for 18821884. Appendix DD. pp. 38-47. Montreal. 1885.

Includes on p. 47 a list of 10 fungi, named by J. B. Ellis.

400. Manual of the natural history, geology, and physies of Greenland and the neighboring regions; prepared for the use of the Aretic Expedition of 1875, under the direction of the Arctic Committee of the Royal Society, and edited by Prof. 'T. Rupert Jones, F.R.S., F.G.S., \&c., together with instruetions suggested by the Aretic Committee of the Royal Society for the use of the expedition. $8^{\circ}$. London. 1875. pp. 1-86 and 1-782.

The first part of this work contains general direetions for collecting and observing. The second part, pp. 1-600. gives a summary of papers on A retie plants and animals, witl lists of species. For referenees to fungi in this work, see BERKELEx, M. J., BoNonden, H. F., Fucles, L., and SMith, TV. G.

Martin, George. Claymont, Delaw are Co., Pa., 29 April, 1827. †West Chester, Pa., 28 Oct. 1886. See Lillis, J. B.

40r. Synopsis of the North American species of Asterina, Dimerosporium, and Meliola. Jour. Myc. I. 133-139, 14t-148. Nov., Dec. 1885.

Deseriptions of 31 Asterinae, with host index, 6 Dimerosporia, and 7 Meliolae.

+ 402. The Phyllostictas of North America. Jour. Nyc. II. 13-20, 25-27. Feb., March, 1886.

Deseriptions and notes on 70 species, with list of host-plants.

Martin, Lillie J.

+403 . A botanical study of the mite gall found on the black walnut. Am. Nat. XIX. 136-140.

Pl. 4-6. Feb. 1885.

The Erineum anomalum of Sehweinitz shown to be an insect-gall. Abstract given in Proc. Am. Ass. Adv. Sci. XXXIII. 507, 508 .

Massachusetts, Province Laws of.

404. An Act to prevent damage to English grain arising from Barberry Bushes. Provinee laws of Massachusetts, 1736-1761, pp. 153, 154. Anno Regini Regis Georgii II., Vicesimo Octavo, Chap. X. Published 13 Jan. 1755.

Text of an act requiring the cutting down of barberry bushes in order to prevent the rust in grain. See Am. Jour. Sci. Arts, XXV. 3 ser. 315, 316, April, 1883.

Meehan, Thomas. Potter's Bar, near Barnet, Hertforlshire, England, 21 March, 1826.

405. Note on a fungoid root parasite. Proc Acad. Nat. Sci. Philadelphia, p. 41t. 2 Dec. 1873.

States that the Norway epruce sufters from some mycelium which attaeks the roots.

406. - Change of habit through fungoid agency. Proc. Acad. Nat. Sci. Philadelphia, p. 146.22 Sept. 1874 .

Notes the change in habit of Euphorbiae attacked by an Aecidium.

407. - Influence of nutrition on form. Proc.

Acad. Nat. Sci. Philadelphia, p. 329. 20 July, 1875.

Notes change in habit of Portulaca attacked by a fungus.

408. - Fungi and fruit diseases: are they cause or concomitant? Am. Pomol. Soc., Session 1875 , pp. 51-54.

Notes of several diseases caused by fungi. States that he has seen Roestelia cancellata on apples.

409. - Note on Phallus foetidus. Proc. Acad. Nat. Sci. Philadelphia, pp. 194, 195. 3 Oct. 1876. Reports this species at Germantown, Pa.

410. - Formation of crow's-nest branches in the cherry tree. Proc. Acad. Nat. Sci. Philadelphia. p. 273.1 June, 1886.

Notes the occurrence of Exonscus Wiesneri on escaped cherry trees, near Germantown, $\mathrm{Pa}$
Merrill, F. J. H. See BulletiN Torrey Club.

4r. Michelia, commentarium mycologicae italicae, curante P. A. Saccardo. $8^{\circ}$. Vol. I. pp. 619. June, 1877-Sept. 1879. - Vol. II. pp. 682. 25 April, 1880-1 Dec. 1882. Padua.

Published at irregular intervals. Devoted exclusircly to fungi. 'Two rolumes have appeared up to present datc. Includes some notices of Uuited States fungi. Sce SACCARDo, P. A.

Mitchell, Samuel L.

412. Views of the process in nature by wlich, under particular circumstances, vegetables grow on the bodies of living animals. Am. Jour. Sci. Arts, XII. 21-28. June, 1827.

A letter to A. P. De Candolle, eontaining remarks on fungi which attack insects, with notes on forms knowu to the writer in the United States, espceially that on Melolontha.

Montagne, (Jean François) Camille. Vaudoy, France, 15 Feb. 1784. †Paris, 5 Jan. 1866. See Beriseley, M. J.

4r3. Troisième centurie de plantes cellulaires exotiques nouvelles. Décades 5-8. Ann. Sci. Nat. Bot. 2 ser. XVIII. 241-282. 1842.

Has descriptions of Polyporus Sullivanlii and Clavaria albipes from Ohio.

4r4. - Quatrième centurie ditto. Décades 8-10. Ann. Sci. Nat. Bot. 2 ser. XX. 352-379. Pl. 15, 16. 1843.

Ineludes descriptions of $4 \mathrm{new}$ species from the United Stiltes.

415. - Sixième centurie ditto. Décades 8-10. Ann. Sci. Nat. Bot. 3 ser. XII. 285-320. 1849.

Has emendation of the genus Lichenopsis, $\mathrm{Schw}$, and notes on Scorias spongiosa and Sphaeria lycopodina from Newfoundland.

416. Huitième centurie ditto. Décades 6-7. Ann. Sci. Nat. Bot. 4 ser. VIII. 285-310. 1857. - Décades 9, 10, 1. c. XII. 167-192. 1859.

First part has notice of Graphium Berkeleyi from the United States. The sccond, Sphatria Desprauxii from Newfoundland

4r7. - Neurième centurie ditto. Décades 1, 2. Ann. Sci. Nat. Bot. 4 ser. XIV. 16i-185. Pl. 10, 11. 1860.

Includes description of Agaricus Tagetes, Berk. and Mont., from Ohio.

4 I8. iconibusque illustratas, nune ad diagnosim reductas, nonnullasque novas interjectas, ordine systematico disposuit J. F. Cam. Montagne, M.D. $8^{\circ}$. pp. 498. Paris. 1856 .

Besides a summary of species, including Ameriean, described by Montagne in various places, there are deseriptions of 62 new species and 2 new varieties from the United States, 17 of them aseribed to Berkeley and Montagne. The greater part of the species colleeted in Ohio by W.S. Sullivant, who sent figures and specimens to Montagne.

Moore, Justin Payson. Augusta, Me., 1841. Sec Harkxess, W. H. See Punllips, IV.

Morgan, Andrew Price. Centretille, near Dayton, O., 27 Oet. 1836. See Cragin, F. W. 4rg. The study of fungi. Bot. Gaz. II. 104, 105. May, 1877 .

Urges the study and gives some statistics of United States fungi.

4I9a. - Flora of the Miami Valley, Ohio. 12. pp. 68. Dayton. 1878.

On np. 59, 60 is a list of 37 snecies of fungi. The last 8 pages are entitled supplement for $18 \pi$, and include 42 additional species of fungi on $\mathrm{pp} .67,68$. 
Morgnn, Andrew Price.

420. Agaricus Morgani, Peck. Bot. Gaz. IV. $208,209 . \quad$ Sept. 1879 .

Ailditional notes on this species.

421. - A new Polyporus. Bot. Gaz. VII.

135, 136. Nor. 1852.

Description of I'olyporus reniformis from Ohin.

422. - Kientucky fungi. Bot. Gaz. VIII.

$156,157$. Jan. 1883 .

Fillumeration of 27 species, all but one, Irymenomyceles, with criticnl notes on several of them.

423. - The Nortl American Geasters. Am. Art. XVI1I. 963-970. Oct. 1884.

Descriptions of 10 species, with figures. Sce also Jour. IIyc. I. 11-13.

424. The mycologie flora of the Miami Valley, O. Jour. Soc. Nat. Hist. Cincinnati, VI. 5t-81, 1). 2-5, April, 1883; 97-117, July, 1883; 173-199, pl. 8, 9, Oct. 1883 ; - VII. 5-10, pl. 1, April, 18st; - VIII. 91-111, pl. 1, July, 1885; 168-174, Oct. 1885 ; - IX. 1-8, April, 1886.

Contzins full descriptions of the Agaricini and Polyporei of the region, with critical notes and colored figures of some Eperies. Twelve speciey are deseribed as new. 'The last four pages of reprint of the fourth part of this series do not quite correspousl to pages of the original.

\section{Morgan, L. V.}

1. 425. Discharging ascospores, Bot.Gaz. VIII. 246. June, 1883 .

Notes the found in discharge of spores of Peziza coccinea.

Mueller, liarl. See Sculecutexda, 1). F. L.

Muhlenberg, Henry (Gottlilf Heinrich Ernst). T756. tLancaster, Pa., 24 June, 1817. See IULIX, TV.

426. Index Florae Lancastriensis. Trans. Am. Phil. Soc. Philadelphia, III. 157-184. 1793.

Includes an enumeration of 8 genera and 25 species of fungi on 1.154.

427. - Supplementum Indicis Florae Lancastriensis. Transs. Am. Phil. Soc. Philadelphia, IV. 235-242. 1799.

Inclules an cnumeration of 109 fungi, in the determination of which the author apparently followed to a great extent the works of batsch and Sclueflet.

428. — Catalogus Plantarum Amerieac Septentrionalis, huc usque cognitarum indigenarum et cicurum; or a catalogue of the hitherto known native and naturalized plants of North America, arranged according to the sexual system of Linnaeus. $8^{\circ}$. pl. 112. Lancaster, Pa. 1813. $2 \mathrm{~d}$ edition, 1818.

4 bare list, pp. 103-106, of 201 funci, belonging to 52 gerera, arranged arecording to Persoon's Symopsis. Authoritjes not quoterl. The 21 edition has 1 Xylome, 8 Tremellue, and 23 claraviae in addition to the above. The prefice of 1 st edition states that the Catalorne was written in 1809 , printing lecku in Nor. 1512, and finished in July, 1813, and that nearly all the cryptogans were collecter in Pennsylvania. In the preparation of the 2 d colition the author was ipparently aided by solawiuitz. For brict refereses to fungi collected by Minlenberz, see Fures, E., Kuxze, G., and Sprenaed, C. sice 110.618 .

Murray, Dennis. See Sprague, C. J.

Naturnlist. See AnERICAN.

Noes von Esenbeck, Christian Gottfried. Reichenherg, near Erbach, 14 Feb. 1776. †Breslatu, 16 March, 1858.

429. Dis system der pilze und schwaimme. Ein versuch 4\% pp. 334. Pl. 44. Fig. 366. Würzhurg. 1816 .

Tnelucles fienrea taken from Bose's Mrimoire. Copies of

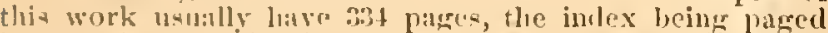
incorreetly, allel the date of jistle being 1516 and 1817 on

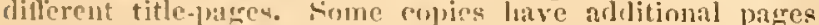
numberel 1-86, iontaining lescriptions of the figures. The title stated that there are $4 i$ plites, but there are only 44 Dlates, with 3 fin figures, besides in illuminated title-page and a few charts. Fot to be confounded with next title.
Nees von Esenbeck, Theodor Fricdrich Ludwig. Reichenberg, $29 \mathrm{July,} \mathrm{1787.} \mathrm{+Hyères,}$ 12 Dec. 1837.

And Honry, A., and Bail, Th.

430. Das system der pilze. Durch beschreibungen und abbildungen erlaütert. $8^{\circ}$. Part 1. pp. vi. 74. Pl. 1-12. Bonn. 1837. - Part 2. pp. iv. 110. Pl. 13-38. Bonn. 1838.

Descriptions of genera of fungi, with illustrations taken from various sources, ineluding Bose's MIémoire and Schweinitz's Syn. Fung. Car. The tirst part by Nees and Henry; the second by Nees, Henry, and Bail.

Nuttall, Thomas. Settle, Yorkshire, England, 1786. +Preston, Lancashire, England, 10 Sept. 1859.

431. Collections towards a flora of the Territory of Arkansas. Trans. Am. Phil. Soc. Philadelphia, V. new ser. 139-203. 1837. Read 4 April, 1834.

Has on p. 139 names of 3 species of Fuligo and 3 species of Trichia from Arkansas.

Olney, Stephen Thayer. Burrillville, R. I., 15 Feb. 1812. †rovidence, $27 \mathrm{July,} 1878$.

432. Rhode Island plants, or additions and emenclations to the catalogue of plants published by the Providenee Franklin Society in March, 1845. Proc. Franklin Soc. Providence, I. 1-2t. April, 1846.

Ientious Rhizomorpha fragilis, var. compressa, on p. 24.

Palmor, Julius Auboineau. Boston, I March, 1840 .

433. 'Toadstool-eating. Pop. Sci. Month. XI. 93-100. May, 1877.

Popular article without botanieal interest.

$433^{a}$. - Empoisonnement par les champignons. Moniteur Scientifique, IX. 3 ser. 99-102. Feb. 1879.

General account of the aetion of poisonous fungi, cspecially Amanitue.

For papers by this anthor on the toxicoloricil action of fungi, see the following: "Poisonous Mushrooms," Mredical and Surgical Journal, Boston, 6 Nov. 1879, and 20 Sept. 1853; "Toadstool poisoning," 1. c. 28 Aur. 1879; "Mlushroom or Toatstool Poisoning," Medical and, Surgical lie. porter, Philadelphia, 20 Sept. 1879; also article on "Mush. rooms and Toadstools" in the Annual Cyclopaedio, D. Appleton \& Co., New York. 1886.

434. - Muslurooms of America, edible and poisonous. Boston. 1885 .

A series of cliarts, issned also in smaller form, of which there lave alleady appeared twelve colored plates, representing 29 species, with 4 pages of general direetions. With each plate is a short description of the species and directions for cooking. Rev, in Jour. Myc. I. 107, and Science, VI. 277.

Pamme1, Louis Herman. La Crosse, Wis., I9 Ápril, 1862.

434 $4^{\mathrm{a}}$. Apple scab and leaf blight. Prairie Farmer. 14 Nov. 1885.

434 b. Smut on Timothy-grass. Prairie Farmer. 31 July, 1886.

Summary of paper by Trelease, no. 609, with figures.

Parker, E. H.

435. Fungus-eating. Proc. Soc. Nat. Sci. Pouglkeepsic, I. 70-84. 1 Nov. 1875.

General notes on the sulject, followed by a list of 47 edible spccies found near Poughkeepsie.

Parker, George Howard. Philadelphia, 23 Dee. 1864.

436. On the morphology of Ravenelia glandulaeformis. Proc. Am. Acad. Arts and Sci. Boston, XIII. 205-219. Pl. 1, 2. Sept. 1886.

Account of the formation of the spores in this speeies, with motes on the spores of sereral other species of the genus, including notice of $R$. Indica on Acacia from Mexico. 
Parry, Charles Christopher. Gloucestershire, England, 28 August, 1823.

437. Botanical Observations in Western Wyoming, with notices of rare plants and descriptions of new species collected on the route of the Northwestern Wyoming Expedition under Capt. W. A. Jones. $8^{\circ}$ pp. 25 . Salem, Mass. 1874. Extr. Am. Nat. VIII. 9-14, 102-108, 175-180, 211-215. 1874.

Has on the last page descriptions of Aecidium Psoraleae and Aec. Parryi, by C. II. Peck.

Peck, Charles Horton. Sandlake, Rensselaer Co., N. Y., 30 March, 1833. See Cooks, M. C. See Cragin, F. W. See DaY, D. F. See HaYnen, F. V. See Parri, C. C. See Porter, 'T. C. See Tuckerman, E.

N. B. The reports of the botanist here given form parts of general reports made to the Regents by the Curator of the State Museum. The report for each year is transmitted to the Legislature and afterwards published. The reports of the botanist include plants of all orders, althougl the greater portion relates to fungi. With exceptions noted below, the reports are all arranged on the following plan: An introdne. tion, giving statistics of species mentioned, followed by gen. eral remarks not quoted here, except in cases where fungi are specially noted; a list of species of which specimens have been mounted, not quoted here; a list of plants collected, pages where fungi are named given here; a list of specimens obtained by contribution and exeliange, not given here; a list of species growing spontaneously in the State, and not previously reported, with localities, habitats, and descriptions of new species, fungi given here; new stations of rare plants, remarkable varieties and observations, not given liere, except in a few cases of special interest; followed in some reports by special reports, synopses of diflicult genera, etc., given here. The separate copies of the reports distributed by the botanist lave the original paging.

438. - Twenty-second annual report of the Regents of the University of the State of New York, on the condition of the State Cabinet of Natural History and the historical and antiquarian collection annexed thereto. Albany. 1869. D. Report of the Botanist. pp. 25-106.

Fungi collected, pp. 39, 40; not before reported, pp. 76$101 ; 173$ species; and list of 33 edible fungi, p. 51. This report for 1868 was issued in $\mathbf{1 8 6 9}$.

+439. - Twenty-third Report ditto for 1869 , issued 1872. pp. 27-135. Pl. 1-6.

Fungi collected, pp. 39-42; fungi on summit of Mt. Marey, 2 species, p. 44; list of 15 edible fungi, p. 49 ; not before reported, pp. 52-65; 67 new speeies. Synopsis of New T $^{r}$ ork Agaricini, pp. 66-127. Symopsis of New York Boleti, pp. 127-133. Deseriptions of new Agarici of this report repeated in Grevilled, I. 2-1, 17-19; and Iledwigia, XI. 148-151. List of edible fungi given in Grevillea, I. 15.

t440. Twenty-fourth Report ditto for 1870 , issued 1872. pp. 41-10s. Pl. 1-4.

Fungi collected, pp. 49-52; edible fungi, 12 species, p. 52; not betore reported, pp. 59-100; 90 new species, 2 new gener: Key to New York Clavariae and Aecidia,pp. 104-107. IIostlist of Aecidia, p. 108.

441. - Twenty-fifth Report ditto for 1871 , issued Sept. 1873. pp. 57-123. Pl. 1, 2.

Fungi collected, pp. 63-65; not before reported, pp. $72-$ 106; 98 new species, one new genus. Synopsis of New York Pucciniae, pp. 110-123; spores figured on pl. 2.

+442. - Twenty-sixth Report ditto for 1872, issued April, 1874. pp. 35-91.

Fungi collected, pp. 43-45; not before reported, pp. 49-87; 111 new species which lrad appeared previously in "Descriptions of new species of fungi" in Bull. Soc. Nal. Sci. Buffalo, I. 41-72. See no. 458.

443. - Twenty-seventh Report ditto for 1873 , issued 1875. pp. 73-116. Pl. 1, 2.

Introduction has notes on the edible qualities of Agaricus naucinus and Coprinus micaceus, on diseases of spruces, Peridermium decolorans, Uredo luminata, and direetions for collecting fungi. Fungi collected, pp. 83-85; not before reported, pp. 92-111; 45 new species; key to New York Ustilagines, p. 115.
+ 444. Twenty-eighth Report ditto for 1874 , issued 1876. pp. 31-88. Pl. 1,2.

Fungi collected, pp. 40-42; not before reported, pp. 4S-S2; 83 new species, 1 new genus.

+ 445. - Twenty-ninth Report ditto for 1875 , issued 1878. pp. 29-82. Pl. 1, 2.

Fungi collected, pp. 34, 35; not before reported, pp. 38-63, 78 new species. Also, pl) $63-65$, descriptions of 12 species previously reported without deseriptions; pp. 66-71 notes on 19 other species, one described as new; and pp. 71-82, list of parasitic fungi of New Y ork and their hosts, including Uredinerte, Coniomycetes, Perisporiaceae, ete. Rev, in Bot. Gaz. IV. 125, 126, under title "Fungoid friends and foes"; and Rev. Myc. I. 92, 93.

446. Thirticth Report ditto for 1876, issued Sept. 1878. pp. 23-78. Pl. 1, 2.

Fungi collected, pp. 28, 29; not before reported, pp. 37-67, 82 new species; remarks and observations, $P l .70-77$, including synonymy of speeies of Lenzites and supplement to list of parasitic fungi in 29th Report. See Rev. Dfyc. I. 92-93. 4 447. Thirty-first Report ditto for 1877, issued 1879. pp. 19-60.

Introduction has notes on a fungus attacking Cicrda septendecin, Sphaeropsis DHatorum in apples, and several fungi on Abies balsumea. Fungi collected, pp. 26, 27; not before reported, pp. 31-51; 66 new species, new genera Mitleria and Mussospora; observations on fungi, pp. 54-60 including list of New York Myxurnycetes, arranged aceording to Rostafinski, and keys to Helcellne mul Yylarice of New Iork. Rev. in Torr. Bull. VI. 363, and Rer. NIyc. II. 48, 49.

+448 . — Thirty-second Report ditto for 1878 .

This report was transmittel to the Legrislature, but was never published except as a public document. A small num. ber of copies, however, were printed in 1856 , but withont the plates which accompanied the report. The copies, which are very rape, have pp. 17-72, with descriptions of 80 new species, 23 Agaricini, 16 Discomycetes, 9 Pyrenomycetes, and 32 others, keys to New lork species of Puxitli, Cr'aterelli, and the Dapetes tribe of Lactarii (see 3sth Report), and an account of New York species of Lycoperdon, and a description of a new genus, Glomerularia. Most of these hive since been published in other places.

449. Thirty-third Report ditto for 1879 , issued Oct. 1883. pp. 11-49. Pl. 1, 2.

Fungi collected, pp. 14, 15; not before reported, pp. 18-34; 70 new species, one new genus; synopsis of New York Amanitae, pp. 38-49. Rev. in Rev. DIyc. VI. 58, 59.

4 450. Thirty-fourth Report ditto for 1880 , issued Oct. 1883 . pp. 24-58. Pl. 1-4.

Introduction has a popular account of fungi on maize, Ramularia Fragariae, Mucor inclequalis, Fusicladium dendriticum, Oidium fructigenum, sphaeropsis Mrilorum, etc. Fungi collected, pp. 39, 40; not before reported, p $\overline{\mathbf{p}}$. 42-533; 39 new species, critical notes on fungi, pp. 57, 58. Rev. in Rev. Myc. VI. 59.

th 45. Thirty-fifth Report ditto for 1881, issued 1885. pp. 125-164.

Fungi collected, p. 129; not before reported, pp. 131-145; 41 new speeies, 2 by C. C. Frost, one new gemns; syopsis of New York Lepiotae, pp. 150-164. Rev, in Rev. Myc. VII. 254. 4 452. — Thirty-sixth Report ditto for 1882, issued 1885. pp. 29-49.

Synopsis of New York Psalliotae, 41-19. See Rev. Mryc. VII. 254.

453. — Thirty-seventh Report ditto for 1883.

This report was transmitted to the Legislature, but never pnblished. pp. 63-68 were struck off, but the borly of the report is nowhere in print. According to the table of eontents, $p .65$, the report contained a monograph of New York Paxilli, Cantharelli, and Craterelli, and an arrangement of New York Pyrenomycetes on Saceardo's system.

f 454. — Thirty-eighth Report ditto for 1884 , issued 1885. pp. 77-138. Pl. 1-3.

Fungi not before reported, pr. 83-106; 62 new species, one new genus; synopsis of New York Laclurii, pp. 111-133; synopsis New York Plulei, pp. 133-138. Rev. in Rev. Myc. VIII. 120.

455. — Fungi. Trans. Albany Inst. VI. 209-226. 1870. Read 15 Feb. 1870.

General notice of fungi, their uses and the larm done by them, with an account of some American works on fungi and brief notes on the black knot. 
Pock, Cliarles IIorton.

456. lieport of the second class in the second department. Botany. Trans. Albany Inst. VII. 3j-4:3, 1s6-204. 1872. Rearl 17 Jan. 1871, and (F Fel. 1572. - VIII. 152-166. 1876. Read 18 Mirch, 1873.

Potices of recent works on botany. Second part lass also su neconnt of freputhobium pusillum and an extended account of the black linnt, Sphrieria morluosn. See Jour.

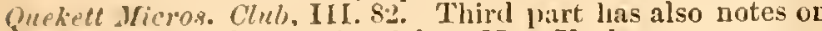
Exobasilium and other fungri from New York.

457. - Symopsis of New York Uncinulae.

Trans. Albamy Inst. VII. 213-217 (1-7), with plate. 1872. Real 20 Feb. 1872.

Deseriptions of 7 species, 5 ncw, nne by Gerard, with a note oll 2 speries of E. C. Howe. Rev. in Grev. I. 62.

458. - Descriptions of new species of fungi. Bull. Soc. Nat. Sci. 13nffalo, I. 41-72. July, 1873.

Characters of 96 IIymenomycetes, 11 Gasteramycetes and Mryromycetes, 18 Con iomycetes, 6 Hyphomycetes, and $11 \mathrm{As}$ comyceles. Also in 26th Report. See above.

459. — Fern Fungus. Torr. Bull. IV. 41.

Sept. 1873.

Note on Dolhiden Pleridis.

460. - Two new fungi from New Jersey. Torr. Thull. V. 2, 3. Jan. 1874.

Deseription of Prolomyces Mlartindalei and Roestelia tounsformans.

461. - New fungi from New Jersey. Torr.

Bull. VI. 13, 14. Feb. 1875.

Deseription of 4 species collected by J. B. Ellis.

462. The Black Spruce. Trans. Albany

Inst. VIII. 283-301. 1s76. Read 4 May, 1875.

Includes observations on Peridermium decolorans on Alies nigra.

1463. - Two new fungi. Torr. Bull. VI. 77. Feb. 1876

Description of Lycoperdon Warnei, Illinois, and Septoria Besseyi, Iowa.

464. A new fungus from Pennsylvania.

Tor\%. Bull. VI. 135, 136. Feb. 1577.

Description of Pestalozzia Stevensonii.

4465. Colorado Fungi. Bot. Gaz. III.

34, 35. April, 1878 .

Deseriptions of 11 species collected by T. S. Brandegce.

466. - United States species of Lycoperdon.

Trans. Albany Inst. IX. 285-318 (1-34). 1879.

Real 4 F cb. 1879 .

Full descriptions of 23 species. Rev. in Torr Bull. VI. 31); Bot. Guz. IV. 175; Rev. Jyc. I. 133.

467. - New species of fungi. Bot. Gaz. IV. 126-128, 137-139, 169-171, 216-219, 230, 231. Feb., Mirch, June, Oct., Nov. 1879.-V. 33-36. March, 1880. - VI. 226-228, 239-241, 274-277. Juse, July, Oet. 1881. - VII. 43-45, 54-57. A pril, May, 1882.

Descriptions of 10.5 speeies of different orders from rarious parts of the country, especially the Western States and Territories. Sce same title below.

468. - Plants of the summit of Mt. Marey. Alimondack Survey. Verplanck Colvin, Supt. Tth Report. Albany. 1880. Botany, pp. 401412.

I.ist of 7 fungi on $p .405$.

\section{9.}

I'olyporus volvalus, Pk., and its varietics. Torr. Bull. VII. 102-105. Fig. 3. Oct. $18 s 0$

Gires characters of the typical form and 3 rarieties.

470. Two new species of fungi. Torr. Bull. VITI. 49-.jL. 1'. T. NIay, 1881.

Dracrijution ind figures of Ascomysetella quercina, and

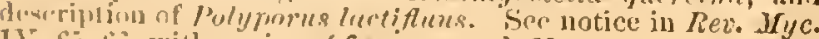
11. Giv, b.j, will copies of figures on pl. 25. t 47 I.

Fungi in wrong genera.

Torr. Bull.

IX. 1-4. PI. 9. Jan. 1882 .

Describes the geuus Physalacria, founded on Mfitrula inflata, Schw., and Secotium Warnei.

+472 . - New species of fungi. Torr. Bull. IX. 61, 62. PI. 24. May, 1882. - X. 73-75. Pl. 35. July, 1883. - XI. 26-28, 49, 50. March, May, 1884. - XII. 33-36. PI. 49. April, 1885.

First part describes and figures the genera Physarella and Caliciopsis. Part 2 gives 11 speries, with plate of Boletus DIorgani. Part 3 has 9 species, with new genus, Afyriadoporus. Part 4, 9 species, principally Uredineae from Utah, Washington Terr. and Ohio. Part 5, principally Ureatineae; 11 species from Arizona and New Mexico.

+ 473. - An imperfectly described Phalloid. Torr. Bull. IX. 123, 124. Pl. 25. Oct. 1882.

Noteg on the structure of Phallus Ravenelii, B. and C.

t 474. - A new Fern Rust. Torr. Bull. X. 62. June, 1883 .

Description of Caeoma Cheilanthis from Arizona.

t- 475. - A new genus of Sphaeriaceous Fungi. Torr. Bull. X. 127, 128. Dec. 1883.

Describes the genus Neopeckia of Saccardo, and gives details of N. Coulteri.

476. — Edible Puff-balls.

man. 1885. p. 901. Albany.

Notes on the edible properties of puff-balls, especially Lycoperdon giganteum, L. cyuthiforme, and L. caelatum.

477. - The smooth Agaric (Ag. naucinoides, Pk.). Country Gentleman. 1886. p. 833. Albany.

Account of edible properties of Ag. naucinoides, and com. parison of that species with $A g$. phalloides, with figures of both.

N. B. Besides the above, there is a considerable number of notes on fungi by this author in the Country Gentleman, mostly answer's to correspondents about speeies sent for determination.

Penhallow, David Pearce. Kittery Point, Me., 25 May, 1854.

478. Yellows in peach trees. Trans. Mass. Hort. Soc. 1882, 124-130. Fig. 6. Boston. 1882.

Includes on pp. 129,130, notes on fungi found in disensed trees, Bispora monilioides, Pleospora herbarum, DFacrosporium sarcinula, and Ascomyces deformans.

479. - Peach yellows. Rept. Houghton Far'm Experiment Station, 3 ser. no. 2, pp. 25-42, Pl. 4, 1882. New York (1883).

An account of the disease, including some notes on fungi, pp. 34-35, none of which is eonsidered by the anthor to be the true cause of the disease. The subject is continued in an appendix, l.c. ser. 3, no. 3, pp. 53-64, 1853, which, however, does not include notes on fungi.

480. - Diseases of plants. Pop. Sci. Month. XXV. 385-396. July, 1884.

Refers ineidentally to the action of fungi in disease.

48r. - Plants in their relation to disease. Rept. Kansas State IIort. Soc. for 1884, XIV. 125-133. Topeka. 1885. Also in Trans. Am. Hort. Soc. III. 167.

Subject treated mainly from a elsemical standpoint, without reference to speeial fungi.

N. B. The following papers by this author do not inelude specific notices of fungi, but relate to listological and physiological matters: "Notes on some of the struetural nnd pliysiological peculiarities incident to the diseases of firuits." Proc. Am. Ass. Ad". Sci. XXXI. 519-521. "Peach yellows." Country Gentleman, XLVIII. 700. 30 August, 1893.

Porsoon, Christian Hendrik. Capetown, 1755. †Paris, 17 Feb. 1837. See Desvaux, N. A.

482. Mémoire sur les Vesse-loups ou Lycoperdon. Desvaux's Journal de Botanique, II. 1-31. I'l. 1, 2. Paris. 1809.

Srlemiterma calostoma (Mitremyces cinnabarinus) dcscribed and figurcd. 
Persoon, Christian Hendrik.

483. Observations de M. Persoon et création du nouveau genre Gyrocephalus. Hém. Soc. Linn. Paris, III. 75-78. -1825. P.

The Tremella stipitata of Bosc referred to G. Carolinensis,

The "Synopsis methodica fungorum" of this antlor forms the basis of classification of the earlier Anerican catalogues of fungi, as those of Muhlenberg and Torrey, but the work itself lias no special American references.

Phillips, William. Presteign, Radnorshire, Wales, 4 May, 1822.

+484 . Discomycetes from California. Grev. V. 35, 36. Sept. 1876.

Nanes and habitats of 22 species collected by II. W. Harkmess.

$+485 .-$ Fungi of California and the Sierra Nevada Mountains. Grev. V. 113-118. Pl. 8789. March, 1877.

Notices of 66 species collected by H. W. IIarkuess and J. P. Moore, 14 deseribed as new. See Rev. Bfyc. I. 29.

+486 . Fungi of Califormia. Grev. VII. 20-23. Sept. 1878 .

Notices of 50 species collected by $\mathrm{II}$. W. IIarkness and J. P. Moore, 10 Discomycetes described as new.

487. - On a new speeies of ITelvella. Trans. Linn. Soc. I. 2 ser. 423. Pl. 48. 1880. Read 5 June, 1879.

Description and plate of ITelvella Californica. Reprint not paged. Sec also Jour. Bot. 2 ser. VIII. 287.

488. - A revision of the genus Vibrissea. Trans. Linn. Soc. II. 2 ser. 1-10. Pl. 1, 2. Read 20 .Jan. 1881. Issued Dec. 1881.

Includes descriptions of 3 Ameriean species, 1 new, $V$. turbinata, with 2 figures. Species given in Ledwigia, XXII. 23.

- and Harkness, H. IV.

+489 . Fungi of California. Bull. Cal. Acad. Sci.

I. 21-25 (1-5). Feb. 1884 .

Descriptions of 20 new species and 1 new variety of Discomycetes collected in 1882 .

+ 490. - Discomycetes of California.

Grev. XIII. 22, 23. Sept. 1884.

Descriptions of 10 new speeies from California, and Phacidium nigrum, Cooke, from Georgia.

Phippen, George Dean. Salem, Mass.,

13 April, 1815.

491. Report on the onion disease. Proc. Essex Inst. Salem, Mass. II. 211-215. 1862. Read 10 July, 1857.

Includes notes on a disease apparently caused by Urocystis Cepulae.

Pirotta, Romualdo. Pavia, 7 Feb. 1853.

492. I funghi parassiti dei vitigni. $8 . \quad$ pp. 96.

Pl. 4. Milan. 1877. Extr. Arch. Trienn. Lab. Bot. Critt. Pavia, II. 129-225. Pi. 10-13. Milan. 1879.

An account, with full descriptions, of all fungi which attack species of Vitis, including 37 species of the United States. Rev. in Grevillea, VI. 147-150. Ste no. 133.

Planchon, J. E.

$492^{\text {a }}$. Les vignes Américaines, leur culture, leur résistance au Phylloxera et leur avenir en lurope. 8. pp. 240. Montpellier and Paris. 1875.

IIas on p. 20 bricf refereuce to Peronospora viticola; on p. 24 to Erysiphe mors-uvae and E. necatrix; and on p. 54 a notice of grape-1ot.

Plowright, Charles Bagge. King's Lynn, England, 3 April, 1849.

493. Some remarks upon Sphaeria (Gibbera) morlosa, Schw. Month. Micros. Jour. XIII. 209, 210. London. May, 1875.

Notes on the conidia and aseospores of this species, with review of papers on the black knot by C. II. Pcck and Thomas Taylur.
494. Californian Fungi. Grev. V. 74.

Dec. 1876.

Notices of 24 species collected by II. W. IIarkness, two described as new.

+ 495. - Califormian Sphaeriae. Grev. VII. 71-74. Dec. 1878.

Notices of 39 species of I'yrenomycetes collected by II. W. Harkness and J. P. Moore, 10 described as new. For cmendations of this paper, see Grev. VIII. 73.

and Harkness, 11. W.

496. New spccies of Californian fungi. Bull. Cal. Acad. Sci. I. 26 (6). Feb. 1884.

Description of 2 new Nectriae.

Plukenet, Teonard. 1642. †London. 1706.

497. Plyytographia sive stirpium illustriorum et minus coguitarum icones, tabulis aeneis sumnat diligentiâ elaboratac; quarum unaquaeque titulis descriptoriis ex notis suis propriis et characteristicis desumptis insignita; ab aliis ejusdem sortis facile discriminatur. $4^{\circ}$. London. Part 1 , preface, pl. 1-72, 1691. Part 2, pl. 73-113; appendix, pl. 114-117; appendix altera, pl. 118-120, 1691 . Part 3, pl. 121-239; appendix, pl. 240-250, 1692. Part 4, pl. 251-328, 1696.

A collection of uncolorerl figures, witl brief Latin descriptions at the foot of each plate. See next title.

498. - Almagestum botanicum sire Plytographiae Plukenctiauae onomasticon methodo gyntheticâ digestum, exhibens stirpium exoticarum, rariorum, novarumque nomina, quae descriptionis locum supplere possunt. 4\% pp. 402. London. 1696.

Descriptions of plants, including those figured in the Phytographia, alphabctically arranged. In the Phytographia, pl. 116, fig. 7, and pl. 184, figs. 4-10, represent fungi from Virginia, and the descriptions are also given in the Alma. gestum, pp. 162-164. P. 116, fig. 7, is cridently a Pilobolus. Pl. 184, fig. 4, may be a Calocera. Fig. 5 is apparently Mitremyces; fig. 6, perlaps Cynophallus crninus; fig. 7 is unrecognizable; fig. 8, appatrently Lycoperdon cyathiforme; fig. 9 is a Cyathus, and fig. 10 probably llericium IIystrix.

Porcher, Francis Peyre. St. Joln's, Berkley, S. C., 14 Dec. 1825 .

499. On the medicinal and toxicological properties of the cryptogamic plants of the United States. Trans. Am. Med. Ass. VII. 167-284. $8^{\circ}$. 1854.

Contains on pp. 211-284 a general account of the properties of fungi, followed by a list of species, with their properties, compiled from various sources.

500. - The nedicinal, poisonous, and dietetic properties of the cryptogamic plants of the United States. 8?. pp. 126. Newryork. 1854.

Reprint of last papcr, with index.

501. - Resources of the Southitrit fields and forests, medical, economical, and agricultural. Being also a medical botany of the Confecterate States; with practical information on the useful properties of the trees, plants, and shrubs. $8^{\circ}$. pp. 601. Charleston, 1863.

An account of the edible mushroom and its culture, and notes on tuckahoe given on pp. 594-599.

502. - A reference handbook of the medical sciences, embracing the entire range of scientific and practical medicine and allied science. $4^{\circ}$. New York. Wm. Wood \& Co.

In the $3 d$ volume of this work, 1886 , is an article, by F. P. Porclier, on "Fungi edible and poisonous," pp. 264-284, figs. $1355-1376, \mathrm{pl} .12,13$. A general treatise on elible and poisonous species, with illustrations taken fiom different sources, especially Palyer and Valmr, followed by a deseriptive list of species found in the United States, including a letter from II. W. Ravenel, 266-26i, anil numerous extracts from an nupublished paper of $\mathrm{M}$. $\Lambda$. Curtis on edible fungi. Plate 12 lias 15 colored figures of erlible species, by C. I Cnrtis, not previonsly published; and pl. 13, 6 figures of poisonous species tilken from Cordier and .J. A. Palmer. At the end of the article is a list of erlible or useful species taken from Curtis's Catalogne Plants North Carolina, q. $\nabla$. 
Porter, Thomas Conrad. Alexandria, Huntinglon Co., l'enu., 22 Jan. 1822.

- and Coulter, J. M.

503. Synopsis of the flora of Colorado. Misc. Publ. no. 4, Dept. Interior, U. S. Geol. and Geog. Survey of the Temitories. F. V. Hayden, U. S. Geologist, in charge. $8^{\circ}$. pp. 180 . Washington, 1874 .

On pp. 163, 16t, is an account of 8 fungi by C. II. Pcck, two of then described as new.

Prontiss, Albert Nelson. Cazenovia, N.Y., 22 Мау, 1836.

$503^{n}$. lnff-balls and their kindred. Cornell Era, IV. 179-181. 8 Dec. 1871.

A popular account of fungi, with description of a large speciuen of Lycoperdon giganteum.

$503^{\mathrm{b}}$. - Destruction of obnoxious insects by means of fungoid growths. Am. Nat. XIV. $5 \%$ ij-581, 630-635. Aug., Sept. 1880.

lieport on a number of experiments with regard to the action of yeust in destroying inseets, from which the autloor concludes that yeist has very little value as a means for destroying inseets. Substanee of this paper also given in $A \mathrm{~m}$. Entomol. and Bot. I1I. 269.

Prillienx, Edouard Ernest. Paris, 11 Jan. 1829. 504. Quelques mots sur le rot des vignes Américaines et l'anthracuose des vignes françaises. Bull. Soc. Bot. France, XXVII. 34-38. 13 Feb. 1880.

Includes notes on Phoma uvicola, considered by the author to be distinet from the fungus of anthricnose. The paper is followed by remarks of Dr. M. Comu, whose views are differcat from those of the :uthor.

505. - Cause du rot des raisins en Amérique. Comptes rendus, XCV. 605 . 2 Oct. 1882.

Notes on Peronospora vitico'a in America, and on the trouble eaused by a species of Phoma.

Provanchor, L. (Abbé). See Thuemen, F. von.

Rabenhorst, (Gottlicb) Ludwig. Treuenbrietzen, 22 March, 1806. †Meissen, 24 April, 1851. See Henwigia. See Exsiccati in supplement. See Winter, G.

Rafinesque-Schmaltz, Constantin Samuel Galata, near Constantinople, 22 Oct. 1783. †Phila. delphia, 1840. See Geraro, W. R.

506. Précis des décourertes et travaux somiologiques de Mr. C. S. Rafinesque-Schmaltz entre 1800 et 18I4, ou choix raisonné de ses principales décourertes en zoologie et en botanique pour servir d'introduction it ses ouvrages futurs. $12^{\circ}$. pp. 65. I'alermo. 1814.

On pp. 49-52 are deseriptions of 18 species of fungi and five new genera from the United States, principally Delaware and New Jersey.

507. Florula Ludoviciana; or, a Hora of the State of Louisiana. 'Translated, revised, and improved from the Frencl of C. C. Robin, by C. S. Rafinesque. $8^{\circ}$. pp. 178. New York. 1817.

On p. 12 is a notice of 7 fungi taken ostensibly from work of C. C. Rolin, sce no. 532, one of them called a new species, Morchellu odoritit.

508. l'rollrome des nouveaux genres de plantes olservés en 1817 et 1818 tans l'intérieur les litats-Unis d'Amérique. Isis, 1820, part 1 , $236-244$.

IIas on $\mathrm{n} .213$ descriptions of three new genera of fungi, Rimella, Endoconia, and Gemmularia, from the United Stites.

509. - Míclical Flora, or manual of the melical hotany of the United States of North America, containing a selection of above 100 ficures aul descriptions of medical plants, with threir names, qualities, properties, history, ete., and notes or remarks on nearly 500 equiralent substitutes. $8^{\text {o }}$ Philadelphia. Vol. I. pp. 268 pl. 52. 1828. - Vol. II. pp. 276, pl. 48. 1830.

In volume 11. references to Agaricus, Amanita, Boletus, ete., of no value.

Ran, Eugene Abraham. Bethlehem, Pa., 22 July, $18 \pm 8$.

+5 50. A new Phallus. Bot. Gaz. VIII. 223, 224. Pl. 4. May, 1883.

Aceount of a species found at Bethlehem, Pa., with a de. seription in Latin of Phallus togatus by Kalchbrenner. Sce nos. 280 and 375.

Ravaz, I. See VIAlA, P.

Ravonol, Henry William. St. John's Parish, Berkley Co., S. C., 19 May, 1814. See Berkelex, M. J. See Cooke, M. C. See Porcher, F. P. See'Inuenen von F. Sce Exsiccati in supplement.

5II. - Contributions to the cryptogamic botany of Soutl Carolina. Med. Jour. and Rev. Charleston, IV. 428-433. July, 1849. - V. 324327. May, 1850.--VI. 190-199. March, 1851.

Part 1 has mosses and hepaties, part 2 lieliens, and part 3 fungi. A list of 169 IIymenomycetes, with habitats and notes in some cases on edible qualities. In in foot-note is an account of the fairy rings of Agaricus Achimenes, B. and C.

512. Report on the fungi of Texas. Rept. Commissioner Agr. on diseases of Cattle in United States. $4^{\circ}$. pp. 171-174. Washington. 1871.

General acrount of fungi collected in Texas, with statistics of fungi of that region.

513. - A list of the more common natire and naturalized plants of South Carolina. In South Carolina. Resources and Population, Institutions and Industries. Published by the State Board of Agriculture of South Carolina. 8. $\mathrm{pp}$. 312-359. Charleston. 1883.

On pp. 353-356 is a list of 35 species of fungi, of which the popular and seientific names are given, but no deseriptions.

N. B. A paper on "Edible Mushrooms of this country" was read by this author before the Aiken Vine-(irowing and Ilort. Ass. abont the year 1862-3, and was printed in the newspapers of Cliarleston. The patper gave a popular ac. count of the unode of identi fying enmmon erlible mushrooms, Agaricus campestris, A. amygdalinus, and some others.

Ray, John. Black Notley, Eng., 29 Nov. 1628. +17 Jan. 1705.

$513^{\mathrm{a}}$. Historia Plantarum, etc. fo. Vol. I.III. London. 1686-1704.

Title here given in an abbreviated form. The only refer. ences to United States fungi in this work are the species collected by $J$. Banister in Virginia in 1680 , which were previously deseribed and figured by Plukenet, q. v. The Pilobolus mentioned in title 498 is first given by Ray in rol. II. 1926, and is the only fungus in the list of speeies collected by Banister. The other fungi of Banister are in the third volume of Ray, pp. 20, 25.

Roos, Max.

514. Die rostpilzformen der deutschen Coniferen zusammengestellt und beschricben von Dr. Max Rees. $4^{\circ}$ pp. 70. Pl. 2. Halle. 1869. Extr. Abhandl. Naturf. Gesells. Halle, XI. 49-118. Pl. 1, 2. 1870.

IIas a note on Gymnosporangium macropus.

Bohm, Heinrich. Ederlicimnear Nördlingen, Ba varia, 20 Oct. 1828. See Exsiccati in supplement 5I5. Note on Peziza calycina, Schum. Grev. IV. 169. June, 1876.

Inelndes a deseription of a new spceies, Peziza Ellisiana, scnt from New Jersey by J. B. Illis. See no. 118.

5r6. - Bemerkungen über (cinige) Ascomyceten. Hedwigia, XVIIT. 113-115, Ang. 1879; 161-169, Nov. 1879. - XXI.'130-139, Sept. 1882 ; 145-148, Oct. 1882.

Part 1 has no $\Lambda$ meriean references. Part 2, p. 168, has a note on Dillymosphaeria grumata from New Jersey. Part 3 has notes on 19 American species, most of them from Ellis's North Americ:in Fungi. Part 4, p. 146, has a note on Fal. saria cincta, also from Ellis. 
Rehm, Heinrich.

517. Revision der Hysterineen im herb. Duby. Hedwigia, XXV. 137-155, 173-202 (1-48). Aug.Oct. 1886.

Includes notes on North American IIysteriaceae with a review of species described in work here numbered 201.

518. — Ascomyceten. In getrockneten exemplaren lierausgegeben von Dr. Med. Rehm. Fasc. 1-11. 26th Bericht Naturhistor. Vereins, Augsburg. 1881. pp. 1-132.

This number of the Berichte gives diagnoses of new specie and critical notes on others found in the first eleven fascicnli of the collection of dried Ascomycetes issued by the author. For an account of this collection and list of American species which it contains, sce SUPPLEMENT. Descriptions of new species of first 11 faseiculi are repeated in Hedroigia, XXI. 35-48 and 55-64. Notes on species in later fasciculi are given in IIedwigin as follows: Fasc. 12, vol. XX. 33-12, 49-54; fase. 13, XXI. 65-75, 81-86; - fasc. 14, XX11. 33-11, 52-61; fisc. 15, XXIII. 49-57, 69-77; - fasc. 16, XXIV. 7-17, 66-72; - fasc. 17, XXIV. 225-246.

5rg. Revue Mycologique, recueil trimestriel illustré consaeré ł̀ l'étude des champignons. $8^{\circ}$. Vol. I.-VIII. Jan. 1879-Jan. 1887. To be contimued. Toulouse. Edited by C. Roumeguère.

Includes papers on lichens as well as fungi. Papers on North Ameriean fungi given here tunder anthors' names. Reriews by the editor. In rol. 1II. the numbers are paged independently.

Rex, George A. Chestnut Hill, near Philadelphia, 28 April, 1845.

+520. Syphoptychium Casparyi, Rostfk. Bot. Gaz. IX. 176. Oct. 1884.

Notes the discovery of this species in New York State.

+521 . The banded-spore Trichias. Jour. Myc. II. 85-87. Aug. 1886.

Notes on the distribution of Trichia chrysosperma, $T$. affinis, and $T$. Jackii, and allied forms, in the United States.

Rich, C. W. L.

522. Large Fungi. Am. Nat. XVIII. 68-69.

Jan. 1884.

Reports finding a IIydnum septentrionale weighing twelve pounds.

Rich, Oliver $\mathrm{O}$.

523. A Synopsis of the genera of American plants according to the latest improvements of the Linnaean System. $8^{\circ}$. pp. 167. Georgetown, D. C. 1814 .

Describes, pp. 141-144, 17 genera of fungi.

Richardson, (Sir) John. Dumfries, Scotland, 1787. tGrasmere, England, 5 June, 1865.

$5^{23^{a}}$. Botanical appendix to Capt. Franklin's narrative of a journey to the shores of the Polar Sea. 4\%. pp. 40. PI. 4. London. 1823.

On p. 35 is a list of 19 fungi.

Riloy. Charles Valentine. London, England, 12 Sept. 1843.

+ 524. The periodical Cicada. Rept. State Entomologist, Missouri, I. 18-42. Jefferson City. 1869.

On p. 26 remarks on the enemies of the Cicad $\alpha$, with note by $D r$. W. D. Ilartman on a greenish powdery fungus found in this Cicada.

525. - The white grub. Rept. State Entomologist, Missouri, I. 156-159. Fig. 88-89. Jefferson City. 1869.

On p. 158, fig. 89 , are notes and a figure of a Cordyceps on the larva of the May-bectle.

t 526. - Fungus on wild plums. Am. Entom. and Bot. II. 308. Fig. 190.' Sept. 1870.

Figure of a distortion of shoots of Prunus Americana from Mississippi, supposed to be caused by a fungus.

527. - The unarlorned Tiphia, or white grub parasite, Tiphia inornata, Say. Rept. State
Entomologist, Missouri, VI. 123-126. Fig. 31, 35. Jefferson City. 1874.

Account of a Cordyceps which grows on this insect, with figure, which is also given in Scientific American, $25 \mathrm{M}$ ay, 1875 .

528. The white grub fungus. Am. Entomologist, III. 137-140. Fig. 53-55. June, 1880.

Account and figures of Tormbia Ravenelii, B. and C., with summary of previous writings on the subject. Sec also RURAL WorLd, 12 June, 1875, for account of same fungus there called $T$. elongata.

4529. - Reports of experiments, chiefly with kerosene, upon insects injuriously affecting the orange tree and the eotton plant, made under the direetion of the Entomologist. U.S. Dept. $A g r$. Division of Entomology, Bulletin No. 1. $8^{\circ}$. pp. 62. Washington. 1883.

On p. 25 is a notice of a fungoid growth on Parlatoria and Mrytilaspis in a report made by Joseph Voyle.

- 530. Reports of olsservations on the Rocky Mountain locust and the chinch bug, together with extracts from the correspondence of the Division on miscellaneous insects. $U$. S. Dept. Agr. Division of Entomology, Bull. 2. $8^{\circ}$ pp. 36. Washington. 1883.

On p. 30, a notice of Scorias spongiosa on honey dew.

531. _- The mildews of the grape vine. An effectual remedy for Peronospora. Rural NewYorker, XLV. 72, 87. 30 Jan.-6 Feb. 1886.

Accounts of Uncimula spiralis and Peronospora viticola and the means of destroying them. Issued separately on a folio sheet.

Other papers by this anthor, in which fungi are noticed withont reference to special American species, are "Wheat rust and barberry rust," Am. Entom. and Bot. II. 162; and "Fungus foes,"' 1. c. III. 297.

Robin, Charles (Philippe). Jasseron, France, 4 June, 1821.

$53 \mathrm{I}^{\mathrm{a}}$. Histoire naturelle des végétaux parasites qui eroissent sur l'honme et sur les animaux vivants. 8. pp. 702. Atlas. PI. 15. Paris. 1853.

Includes references to Schweinitz's species of Isaria, and species described by Leidy.

Robin, Claude C.

532. Voyages dans l'intérieur de la Lonisiane, de la Floride Oecidentale, et dans les Isles de la Martinique et de Saint-Domingue pendant les années 1802, 1803, 1804, 1805 et 1806. Suivis de la Flore Louisianaise. $8^{\circ}$. Vol. I.-III. Paris. 1807.

The Flore Louisianaise in vol. III. pp. 311-525, forms the basis of Rafinesque's Florula Ludoviciana, q.. . Some rather vague references to fungi on $\mathrm{pp}$. 325-328.

Rose, Joseph Nelson. Dunlapsville, Union Co. Ind., 11 Jan. 1862.

t. 533. Mildews of Indiana. Bot. Gaz. XI. 60-63. March, 1886.

Enumeration of 12 species of Perisporiaceae, with hosts, localities, and notes.

Rostafinski, Joseph Thomas. Warsaw, 14 Aug. 1850 .

533 ${ }^{\mathrm{a}}$. Śluzowee (Mycetozoa). Monografia. $4^{\mathrm{o}}$. pp. 432. Pl. 13. Fig. 1-242. Paris. 1875. Supplement 1. pp. 42. Fig. 243-246. 1876.

A work comprising descriptions of all known species of this order, ineluding notes and revisions of American species. The figures of the first 12 plates are repioduced in the "MIyxomycetes of Great Britain," by M. C. Cooke, London, 1877, a work which gives also translations of Rostafinski's descriptions of British species. Sec also title no. 124 and 447.

Roumegnère, Casimir. Toulouse, 15 Aug. 1828. See Revue Mrcologique. See Exsiccati in supplement. 
Russell, Tohn Lewis. Salem, Mass., 2 Dec. 1803. †Salem, 7 June, 1873.

534. Contributions to the cryptogamic flora of Essex County. Proc. Essex Inst. Salem, Mass., I. $191-194.1856$.

Notes on Dothidea (Asteroma) pomigena, Phragmotrichum Chailletii, and sphaeronema sultite.

535. - The smaller fungi. Am. Nat. II. 561-570, fig. 2; 623-638, fig. 5. Jan., Feb. $18(i 9)$.

(renersl account of moulds, blights, etc., with figures, being a review of "Rinst, smut, Mildew, and Mould," by M. C. Conke. See also article by this author on Muslizonms, a review of Cooke's "I'lun and Easy Account of Britisly Fungi," in Am. Nut. II. -292-303. Also "Potato Mould," Am. Vat. II. 463-166. These papers do not contain special Americ:un references, and state ouly general facts.

Saccardo, Pietro Andrea. Treviso, 23 April, 1845. See Mirnelis.

536. — Fungi Italici autographice delineati. 4\%. lig. 1500. With index. Padua. May, 1877 - May, 1886 .

Isaned in 39 fasciculi, with an index of snecies in last fiaciculus. The following are the numbers of plates repreEentiur Uniterl Stutes species: $683,684,686,687,777,781,789$, $792,81 \bar{i}, 849,866,932,959,111 \bar{i}, 1358,1362,1409,1440$.

537. — Finumeratio Pyrenomycetum Hypocreaceorum hucusque cognitorum systemate carpolorico dispositorum. Nichelia, I. 277-325. July, 1878 .

Iucludes notes on 58 species of the United States.

538. Conspectus generum fungorum Italiae inferiorum nempe ad Sphaeropsideas, Melnnconiens et Iyphomyceteas pertinentium systemite sporologico dispositorum. Mfichelia, II. 1-33. April, 1850 .

Includes descriptions of Pyricularia and Ellisiella, new Americul gener:ı.

539. — Fungorum Fxtra-Europaeorum pugillus. Michelia, II. 136-149. April, 1880.

Notes on 44 Nortl American species, 21 described as new.

540. - Fungi Veneti novi vel critici. Series 3. Hedwigia, XIV. 68-76, May, 1875; and Michelia, I. $416-452$, Nov. 1878. - Series 11. Michelia, II. 15t-176, April, 1880.

Saries 3 laas note on Mrassaria epilenca, B. and C. Series II has it note on Credo Aspidiotus, Peek.

54I. - Fungi Gallici. Series 2. Michelia, II. 39-13.), April, 1880. — Series 4, 1. c. 583-648, 1)ec. 18.52 .

Firit part has a note on Tromyces Enphorbiae, C. and P., on 1.45 . The sccond part has in addenda a description of Grinnlaria eurotivides from $N \mathrm{ew}$ Jersey.

572. - Fungi aliquot Extra-Europaei. Michelia, II. 372-377. March, 1881.

Notices of 22 species from the United States, 6 deseribed : $11 \%$ w, rollected mosily by J. 13. Ellis in New Jersey. Rev. in Ree. SYyc. Ill. April, Issi.

543. — Fungi Toreali-Americani. Michelia, II. I) $54-592$. Dec. 1882 .

Notea on 103 species, 53 descrilsed as new, 5 new genera from the Uniter States, collected prineipally by J. B. Ellis. Noat of the specics ascribed to siccardo and Ellis.

544. - Genera Pyrenomycetum schematice delineata a 1'. A. Saccarlo. Illustratio arlcommodata ad usum Sylloges Pyrenomy cetum ejusdem auctoris. $8^{\circ}$. pp. 6. J1. 1-14. I'adua. Nov. 1883. A series of figures illnstrating the genera of the first two rolumes of Saceardo's Sylloge. Iier. in Am. Nat. XVIII. 137 .

545. - Conspectus generum Discomycetum hucusque cognitorum. Bot. Centralblatt, XVIII. $21: 3-220,247-256(1-16) .1884$.

Incluile descriptions of North American genera. See An.
546. Miscellanea mycologica. Att. Real. Istit. Veneto. II. (6 ser.) $435-463$ (1-29). 1584.

Includes deseriptions of one new genus and 3 new species from the United States, two of which are credited to Saccardo and Winter. See next number.

\section{and Berlese, $A$. N.}

547. Miscellanea mycologica. Att. Real. Istit. Venet. III. (6 ser.) 711-742 (1-32). Pl. 8-11. 1855 .

Includes ennmeration of 19 species from the United States, 9 species and 2 genera deseribed as new. Figures of 5 species. See also Jour. Mrc. I. 95 and 105 . See last number.

\section{and Berlese, A. N., and Voglino, P.}

548. Sylloge Fungorum omnium hucusque cognitorum. $8^{\circ}$. Vol. I.-IV. Padua. 1882-1856. 'To be continued.

Vol. I. Prefatio V.-viii. Bibliotheca AIycologica, ix.xix. Sylloge Pyrenomycetum, pp. 768. 13 June, 1882.

Vol. II. Sylloge Pyrenomycetum, pp. 815; Addenda, rol. I., i.--]v.; Addenda, vol. II. lvi.-lxix; Index, species, and genera of vol. I. and II. pp. 77. 13 June, 1883.

Vol.III. Sylloge Sphaeropsidearum et Irelanconieamu, pp. 860. 15 Dec. 1884.

Vol. IV. Sylloge IIyphomycetum, pp. 807. 10 April, 1886. Additamenta ad volumina I.-IV. curantibus A. N. Berlese et P. Voglino. pp. 484. 31 Dec. 1886. To be continued.

A work including descriptions of all known species of the orders named above. For reviews of American species in this work, see CoOKE, M. C. Also reviews in Am. Nat. and Jour. Myc. and Hedwigia, XXIV. 97

Salmon, D. E.

549. The nature, chemical composition, and action of ergot. U. S. Dept. Agr. First Ann. Rept. Bureau Animal Industry for 1884. pp. $195-203$.

Included in a report on "Enzootics of Ergotism" is a chapter with title liere given, which has a botanical account of ergot, with a plate giving the gross appearance of the fungus, and a plate, taken from Tulasne, of the microseopic structure.

Saunders, TVillian.

550. Cultural Remarks. Rept. U. S. Dept. Agr. for 1883. pp. 182-196. Washington. 1883.

Has some remarks on the conditions which affect diseases of grapes.

Schlechtendal, voN, Diedrich Franz Leonard. Xanten, duchy of Cleves, 27 Nov. 1794. †Halle, 12 Oct. 1866.

551. Eine neue Phalloidee, nebst bemerkungen über die ganze familie derselben. Limnaea, XXXI. 101-194. Pl. 1. 1860.

Includos observations on Phallus duplicatus, Bose, and some other species which have been said by some writers to occur in the United States.

- and Mueller, K.

552. Mitremyces Junghuhnii, eine neuc art, beschrieben und abgebildet von v. Schlechtendal und K. Müller. Bot. Zeit. II. 401-404. Pl. 3, B. 7 June, 1844.

Includes notes on Mritremyces lutescens of thie United States.

Schmidt, Johann Carl. Bernstadt, Oberlausitz, 6 April, 1793.' †Berme, 2 Dec. 1850. See Kúuze, G.

Schrenk, Joseph.

×53. Notes on 'Tuckahoe. Tor Bull. XI. 1-5. Pl. 43. Jan. 1884.

Gives listol ogical and chemical structure of tnckalıoe, with consideration of the mycelium found in this substance.

Schroeter, Julius.

554. Ueber einige amerikanische Uredinecn. Iledwigia, XIV. 161-172, 177-182. Nov., Dec. 1875.

Proposes a new genus, Tropyxis, founded on Puccinia Amorplice, B. and C., and gives notes on synonymy and distribution of 35 other species. 
Schulzer von Müggenburg, Steplan.

555. Berichtigung. Ileduigia, XXII.

March, 1883.

IIas a note on Secotium Warnei, Peck, which author consillers to be tlic same as $S$. acuminatum, Tul.

Schwaegrichen, Christian Friedrich. I.eipsic, Sept, 1775. †Leipsic, 2 May, 1853. See Scmwe1NITz, L. D.

Schweinitz, de Lewis David. Bethlehem, Pa., 13 Feb. 1780. †Bethlehem, 8 Feb. 1834. See Berieley, M. J., and Curtis, M. A. See Curry, F. See Dury, J. E. Sec Fries, E. Sec Stevtexsor, IV. C.

556. - Description of a number of new Allerican species of Sphaeriae Jour. Acad. Nat. Sci. Philadelphia, V. 3-16. Pl. 1,2. 15 Feb. 1825.

Describes 3 new species of Cordyceps, 2 of Irypoxylon, 2 of Poronia, and 9 other Spha eriacece, with figures of 11 species. This paper is repeated in substance, without figures, iu $\mathrm{Lin}$ naea, Litteratur-Bericht für $1829, \mathrm{pp} .44-18$. Species also included in next paper. See also Coore, M. C., title no. 159.

557. - Synopsis fungorum Carolinac Superioris secundum observationes Lndovici Davidis de Schweinitz. Edita a D. F. Schwaegrichen. Schriften der Naturforschenden Gesellschaft. Leipsic. $4^{\circ}$. I. 20-131 (1-105). Pl. 1, 2. 1822.

The first paper of importance on fungi written by an Amerie:n; 1373 species are enumerated, many of them describel as new, and a few new genera. No index either to the original or the separate erlition. See also next title. For early review of this work, see FlokA, VI. part 2, pp. 65-84, 1823.

558. — Synopsis fungorum in America Boreali media degentium. Secundum observationes Ludovici Daridis de Schweinitz. Communicated to the American Philosophical Society, Philadelplia, 15 April, 1831. 4\%. Trans. Am. Phil. Soc. (n. s.) IV. 141-316. Pl. 19. 1834.

A revision and extension of last-named paper, including 3098 species, principally from Pennsylvania : many of them new, and several new genera. The inclex gives the species alphabetically, not including those preriously given in Fung. Car. Sup., without references to pages. For eommentaries on this paper, see Berkeley and Curis, Cooke, M. C., Grev. XIII. 37-40.

Scribmer, Frank Lamson (changed from Franklin Pierce Lamson), Salem, Mass., 19 April, 1551. + 559. Fungous diseases of plants. Rept. U. S. Dept. Agr. for 1885. pp. 76-87. Washington. 1886.

Includes account of com smut, Rocstelia on apples, grape moulds, several diseases caused by Perisporiaceae and Peronosporeae, and honey-dew. Pl. 17 of this report, which accompanies the present paper, belongs to a paper by Pearson, which was priuted in Special Bulletin No. 2, Botanical Division, issued Jan. 1887.

- 560. - Botanical characters of the Black Rot. Physalospora Bidwellii, Sacc. Bot. Gaz. XI. 297-302. Pl. 9. Nov. 1886.

An account of the structure and development of Phome uricold, with references to Sphaeria Bidoelliz, Ellis, and review of paper on the subjeet by Viala and Ravaz.

56r. - Black Rot. - Physalospora Bidwellii (Ell.) Sacc. Proc. Seventh Ann. Neeting Soc. Prom. Agr. Sci. Buffalo, 1886. pp. 82-88. Columbus, O. Nov. 1886. Also Colman's Rural World. 30 Dec. 1886.

The substance of last-named paper, with notes on remedies and on Gloeosporium ampelophagum.

t562. Notes on the orange-leaf scab. Torr. Bull. XIII. 181-183. Oct. 1886.

Account of the disease, with a consideration of the reme. dies. Has a reference to Fusarium sarcocleroum, Desm., found on the leaves. This paper was read at the Botanical Ulub of the Am. Ass. Adv. Sci. Buffilo, Aug. 18S6, and an bstract is given in Bot. Gaz. XI. 246, Sept. 1856.
Seaman, William Henry. New Iork City, 1 Nov. 1837 .

563. Edible fungi. Field and Forest, I. 71. Jan. 1876.

Gives a list of 27 species eaten in Washington.

N. B. The paper by this author, "Description of a new fungus on the leares of the pear tree," Blastema tridens, Zabriskic, Morthiera Mespili, Fuckel var., was reat at the 25th meeting $\mathrm{Am}$. Ass. Adv. Sci. Buffalo, Aug. 1576, but was not published.

$563^{a}$. - Some remarks on fungi considered as insecticides. Am. Entomologist, III. 40, 41 . Feb. 1880.

Sedgwick, William Thompson. TVest IIartford, Ct., 29 Dec. 1855.

564. On fermentation. Lectures delivered to the employés of the Baltimore \& Ohio R. R. $8^{\circ}$. pp. 51-73. Fig. 1-10. Baltimore. 1882.

A popular lecture on the growth and action of yeasts and bacteria.

and Duggan, J. R.

564a. Pleomorphism in Penicillium. Johns ITopkins University Circulars, II. no. 21 . p. 50. Feb. 1883.

A preliminary communication giving an account of the change in habit undergone by Penicillium whein growing submerged in it saccharine fluid.

Seeman, Berthold. Hanover, 28 Feb. 1825. †Javali, Nicaragua, 10 Oct. 1871.

565. The botany of the voyage of II. M. S. Herald, under the command of Captain Henry licllett, R.N., C.B., during the year 1845-51. Fdited by Berthold Seeman. 4\%. pp. 483. I'l. 100. London. 1852-1857.

On p. 49 Dothidea betulina, forma nana, is recorded in Western Esquimaux Laud.

\section{Senex. (Pseudonym.)}

$5^{6} 5^{a}$. Essay on smut in wheat. Mrassachusetts Agricultural Repository and Journal, V. 134-150. 1818.

General remarks of no interest exeept for the date when written.?

Seymour, Arthur Bliss. Moline, Ill., 3 Jan. 1859. +566. Puccinia heterospora, B. and C. Bot. Gaz. VIII. 357, 358. Dec. 1883.

The Puccinia Thuaitesii of Winter stated to be the same as $P$. heterospora, $\mathrm{B}$. and $\mathrm{C}$., to which species Cromices pulcherrimus, B. and C., is also referred.

566. - Curl leaf in peach trees. Country Gentleman. 25 June, 1885.

Account of Exoascus deformans, with figure.

567. - Distribution of Puccinia heterospora. Jour. Myc. I. 94. July, 1885.

Gjves 11 additional hosts for this species from the Gray herbarium.

$567^{\circ}$. The apple tree rust. New England Homestead. 5 June, 1886 ; with 4 figures.

A popular account of Gymnosporangium macropus and Aecidium pyratum, Schw., witl figures.

568. A Palm disease. Am. Florist.

1 Sept. 1886 ; with 3 figures.

A popular account of Graphiola Phoenicis.

$568^{\mathrm{a}}$. - Some fungus diseases of small fruits. Rept. State Ilort. Soc. Minnesota, for 1886, IIV. 213-221 (1-S). Fig. 1-6.

Acconnts of the orange rust of blackberries, witlr ficure, taken from Burrill, F'usisporium Rubi, with figure, from Earle, Septoric Ribis, and some other diseases of raspberries and blackberries.

Seynes, DE Jules. Lyons, France, 16 Jan. 1833. 569. Recherches pour servir à l'histoirc naturelle des végćtaux inférieurs. I. Des Fistulines. 4\%. pp. v. 71 . Pl. 7.

Inclucies descriptions and figures of $F$. spathulata, B. and C., and $F$. pallida, B. and $K$., besides geveral account of Fistulincie. 
Seyuēs, JiE Tules.

* 570. Un Agaricus ascophorus, Peck. Grev. III. 169,170 . June, 1875.

Description of the cystidia and basidiospores in this enceies, followed ly sonic olsservations of Berkeley on the sume species taken from Gard. Chron. of 17 April, 1875.

Shocut, John Iouis Edward William.

57r. Flora Carolinaeensis; or a historieal, medieal, and econonieal display of the vegetable kinglom, aceording to the Linnaean or sexual system of bulany. 8\% pp. 579. Charleston. 1806.

The plants are arranged alphahetically, and some genera of fingi are giren, with areounts of some of the species. The work appears to be a compilation of no value at the present day. The second volume wats never published.

Smith, Worthington George.

572. Florula Discoana: Contributions to the plivto-greograplyy of Greenland, within the parallels of $68^{\circ}$ and $70^{\circ}$ north latitude. By Dr. Robert Brown. Trans. Bot. Soc. Edinburgh, IX. $430-$ $465(1-37)$. $1867-68$.

Ias a list of 6 species, determined by W. G. Smith. Re. peated in Arctic Manual, part 2, p. 283.

Sommers, John. St. Joln's, Newfoundland, 29 March, $18+3$.

573. Nova Scotian fungi. Trans. Nora Scotia Inst. Sci. V. 188-192, 247-253, 332-333. Halifax. 1882. Read 26 Jan., 10 Dec. 1880, and Dec. 1881.

Enumeration of 144 speeies, all but a few Basidiomycetes, with hosts, localities, and notes in some cases. Some of the species determined by C. II. Peek.

Spalding, Volney Morgan. East Bloomfield, N. I., 29 Jan. $18+9$.

574. Ustilago Maydis and related species. 12", pp. 16, fig. 7. Extr. Therapeutic Gazette, V. 121-125. 15 April 1881.

Account of the derelolmment of $U$. Mraydis, with notes on the cermination of the spores in several other species of the order.

Spegazzini, Carlo.

575. Iungi Argentini additis nonnullis Brasiliensibus Muntevideensibusque. Pugillus quartus. $8^{\circ}$, pp. 138, pl. 1. Buenos Ayres. Extr. Anal. Soc. Cient. Argent. XII. 13-30, 63-82, 97-117, 174-189, 208-227, 241-258; XIII. 11-35, 60-64. July, 1881 -Fels. 1882.

In XII. $101(41,42)$, there are giren in a foot-note full de. seriptions of some Meliolne of the United States. On XII. $225(90)$ is a description or the new genus, diarenelulu, with a species on Subal from Florida, la. gaimestillensis. On XIII. 2I (120), Ihurknessia Euculypti, Cooke, is referred to II. uromycoides, Sper.

Sprague, Charles James. Boston, 16 Jan. 1823. 576. Contributions to New Ingland myeology. Proc. Soc. Nat. Ifist. Boston, V. 325-331. 1856. Rearl 5 March, 1856. - VI. 315-321. 1859. Read 6 Jan. 1558.

A list of 692 species collected in Massachusetts by Mr. Spraguc and Demis Mlurray, it fow by JI. A. Curtis and J. J. liusscll, and some from Counecticut by C. Wright. The eperies determined ly M. $\Lambda$. Curtis, some not described until later l'apers by Berkeley and Curtis.

577. - List of cryptogamous plants collected at Lake Superior by Dr. S. Kneeland. J'roc. Soc. Nret. Ilist. Boston, VI. 296. 1859. Reall 28 Nov. 1857 .

Jist of 8 species of fungi, 2 lielıens, and one moss determined by C. J. Sprague.

Sprengel, Tíurt (Curtius). Bollekow, near Anklam, 3 Aug. 1766. tIlalle, 15 Mareh, 1833.

578. J'lantarum minus cognitarum pugillus primus. 12? pl. 66. Halle. 1813.

On po fis is a deseription of Solenarium byssoideum from Penusylrania, collected by Muhleuberg.
579. - - Systema regetabilium (Caroli Linnaei). Editio decima sexta curante Curtio Sprengel. $8^{\circ}$. Vol. I.-V. Göttingen. 1825-1828.

Fungi are ineluded in vol. IV. 376-580, 1827. Includes species of Schweinitz and Bosc, with descriptions of $\Lambda$ meri. can species, some referred to new genera.

Stevenson, William Clark, Jr. Philadelphia, 7 Dee. 1848.

580. Additions to Mr. Cooke's paper on "The Talsei of the United States." Proc. Acad. Nat. Sci. Philadelphia, pp. 86-88. April, 1878.

Gives details of spores of 38 species from the Scbweinitz herbalinm. Supplementary to paper by M. C. Cooke. See no. 123 .

Streinz, Wenecslaus Maternus.

581. Nomenclator fungorum exhibens ordine alphabetico nomina tan generica quam specifica ac synonyma a seriptoribus de scientia botanica fungis imposita. $8^{\circ}$. pp. viii. 735. Vienna. 1862.

A general index of generil and species of fungi, including American, and a bibliography of works on fungi.

Sturtevant, Edward Lewis. Boston, 23 Jan. 1842.

582. A list of edible fungi. Trans. Mass. Mort. Soc. for 1881. pp. 322-348. Boston. 1881.

An enumeration of a large number of species compiled from various sources, the American authorities being Curtis and Harkness and Moore.

Sullivant, William Starling. Franklinton, O., 15 Jan. 1803. +Columbus, O., 30 April, 1873. See Lea, T. G. See Montagne, J. F. C.

Taylor, Thomas.

583. Certain fungi parasitic on plants. Month. Micros. Jour. Eondon (XII. 118-125. P1. 95-97. March, 1875).

Notes on black-knot and Erysiphe Tuckeritaken from Rept. U. S. Dept. Agr. for 1873, pp. 196-200; and Rept. 1874, pp. $174-17 \%$.

584. Oidium Tuckeri, a fungus of the grapevine. Am. Month. Micr. Jour. V. 5-7. 'Jan, 1884. This species said to be the eonidial form of $U$. spiralis. Includes quotation from letter of Berkeley in Gard. Chron. Rev in Grev. XII. 81.

$\times 585$. Elible mushrooms of the United States. Rept. U. S. Dept. Agr. for 1S85. pp. 100108. Pl. 2. Washington. 1886.

Account of 12 speeies, with colored figures.

N.B. For other papers by this author, see Mficroscopic Observations in Reports $U$. $S$. Department of Agriculture prior to 1876 , as follows: $1871, \mathrm{pp}$. 110-122, pl. 4-17, " Report on fungoid diseases of plants," grape mildews, lilac mildew, diseases of pear, and peach-trees. - 1872, pp. 188-203, fig. 27-38, pear-bliglit, onion-smut, peach-yellows, and potatorot. -1873 , pp. 183-210, figs. 11-21, hawthorn-blight, potatorot (also in Monthly Report Deparlment of Agriculture, June, 1874), blaek-knot, orange blight, and apple-rot. - 1874, Pp. 161-178, pl: 1-5, cranberry-rot, black-knot (also in Ifonthly Report for Nov. and Dec.), and Oidium Tuckeri. - 1875, pp. 193-206, pl. 15, cranberry-rot and black-knot. -1876, pp. 74-81, mushrooms. - Also, "Puecinia on paper," in the Lens, I. 170-171; " The yellows of the peach," 1. c. II. 36-38; "Potato blight and rot," 1 . e. II. 152-157.

Thuemen, vov Felix. Dresden, 1839. See Exsiccati in supplement.

$\times$ 586. New speeies of Amcrican fungi. Tor Bull. VI. 95. June, 1876.

Characters of 3 species and one rariety.

ч 587. - Contributions à la flore mycologique de la province de Québec. Naturaliste Canadien, X. 8-10. Jan. 1878 .

Paper introluced into an artiele, "Nos cliampignons," by the Albe I'rovincher. Enumeration of 25 species, one new species, Gnomonia Provancheriana, and one new variety described.

$\times 588$ New species of North American

Uredinci. Torr. Bull. VI. 215, 216. Mareh, 1878.

Descriptions of 6 species. 
Thuemen, ron Felix.

589. Fungorum Americanorum triginta species novae. Flora, IXI. (XXXVI. new ser.) 177-184. 21 April, 1878.

Descriptions of one new genus, Thuemenia, and 28 new species collected prineipally by II. W. Ravenel in South Carolina. One species is credited to Rehm and one to Cooke.

590. - Die pilze des weinstockes. Mono graphische bearbeitung der sämmtlichen bisher bekannten, auf den arten der gattung Vitis, L., vorkommenden pilze. $8^{\circ}$ pp. xx. 225. Pl. 5 . Vienna. 1878.

Includes descriptions of Ameriean viticolous fungi, several speeies described as new. New species of this work are repeated in Hedicigia, XVIII. 118-124. Rev, in Grev. VI. 147 , and Bot. Zeit. XXXVII. 60.

$\times$ 59r. - Iyphomycetes nonnulli novi Americani. Rev. Myc. I. 58-61. A pril, 1879.

Descriptions of 13 species collected by $\mathbf{H}$. W. Ravenel.

592. - Melampsora salicina, der weiden rost. Iledwigia, XVIIT. 76-79. May, 1879.

Extr. Mitheil. aus dem forstl. Versuchswesen Oesterreichs. II.

Original not seen. Seven species made of the original species, 4 of which belong to the United States. See also Rev. Mryc. I. 136.

$\times$ 593. - A brief contribution to the mycological flora of the United States. Torr. Bull. VI. 350, 351. Oct. 1879.

Notes on 8 species, of which 2 are described as new.

594. — Fungi pomicoli. Monographische besclireibung der auf den obstfrücliten der gemässigten climata vorkommenden pilze. $8^{\circ}$. pp. xii. 143. Pl. 3. Vienna. 1879.

Includes descriptions of $\Lambda$ merican species.

595. - Die blasenrost-pilze der Coniferen. Monographie der gattung Peridermium, Lév. Mittheil. aus dem forstl. Versuchswesen Oesterreichs. II. part 3, pp. 297-323. $4^{\circ}$. Vienna. 1880.

Includes descriptions of and comments on American species of the genus.

$\times$ 596. - Two undescribed North American species of Septoria. Bot. Gaz. V. 122-123. Oct. 1880.

Characters of S. Albaniensis and S. querceli.

Torrey Botanical Club. Sce Buldetrix of.

Torrey, Jolın. New York, 15 August, 1796. †New York, 10 March, 1873.

597. A catalogue of plants growing spontaneously within thirty miles of the city of New York. $8^{\circ}$. pp. 100. Albany. 1819.

On pp. $87-89$ is a list of 92 fungi arranged according to Persoon, specific authorities not given.

598. — Catalogue of Nortl Ameriaan genera of plants, arranged according to the orders of Lindley's Introduction to the natural system of botany; with the number of species belonging to each genus as far as they are at present determined. $8^{\circ}$. pp. 22 . New York. 1831.

This was originally printed as an appenrlix to the American edition of Lindley's Introduction. Fungi arc given on pp. 12, 13. The names of genera only giren with the number of supposed species of each genus, in all 3101.

Trelease, William. Mt. Vernon, N. Y., 22 Feb. 1857.

$\times$ 599. Notes on the relations of two Cecidomyians to fungi. Psyche, IV. 195-200. August, 1884.

Account of Rhytisma Solidaginis, Schw., R. bifrons, Schw., and $R$. Asteris, Schw., which, author thinks, are probably primarily insect-galls.

600. - The Philadelphia rose rot. Gard. Monthly. pp. 211, 212, with figure. July, 1884.

An account of Peronospora sparsa, and the means for destroying it. 6or. - The onion mold. First Ann. Ript. Wisconsin Agr. Exp. Station for 1883. 1p. 3844. Fig. 1-5. Madison, 1884 .

An account of Peronospora Schleideniana, De Bary, with notes on its occurrence in Wiseonsin.

602. — The apple-scab and leaf-blight. (Fusicladium dendriticum, Wallroth). First Ann. Rept. Wisconsin Agr. Exp. Station for 1883. pp. 45-56. Fig. 6-9. Madisoll. 1884.

Account of the fungus and detals of the ravages cansed by it in Wisconsin. See $434^{*}$.

603. - Preliminary list of the parasitic fungi of Wisconsin. Trans. Wisconsin Acad. Sci. Arts, VI. 106-144 (1-40). 1886.

An enumcration of 271 species, with hosts, localitics, and critical notes, including descriptions of 16 uew specics, onc of them eredited to Peck and one to Farlow, followed hy a lostindex. New speeies given in Jour. Hyc. I. 13-15. The reprint was issued early in Nov. 185t, the volume of transactions in July, 1886.

604. — The spot disease of strawberry leaves (Ramularia Tulasnei, Sace.) Second Ann. Rept. Wisconsin Agr. Exp. Station for 1884. pp. 4758. Fig. 1-3. Madison. 1885.

Account of the fungus and its distribution in Wisconsin.

1605. - Heteroecismal Uredineae. Jour.

Myc. I. 25, 26. Feb. 1885.

A summary of 29 species.

606. - The genus Cintractia. Torr. Bull. XII. 69, 70. Pl. 50. July, 1885.

Account of the structure of Ustilago Junci, Schw., which is referred to the genus Cintractia of Cornu.

607. - The wax-bean fungus. Cultivator and Country Gentleman. p. 800 . Fig. 1. Oct. 1885. Account, with figures, of Gloeosporium Lindemuchianum.

608. The grape-rot. Trans. Miss. Talley Hort. Soc. II. 223-227. Also in Trans. Wisconsin State Hort. Soc. XV. 190-198, with addition of fig. 1-9.

Includes notices of Peronospora vilicola and Phoma uricola, with figures. The pagination of reprint from IVis. Trans. is changed a little from original.

6o9. - The smut of Timothy. Rept. U. S. Dept. Agr. for 1885. pp. 87, 88. Pl. 18. Washington. 1886.

An aecount of Tilletia striaeformis, Westd., with notes on some Ustilagineae which may prove injurious to cattle. See $434^{\mathrm{b}}$.

6ro. - A yellow opium mould (Eurotium Aspergillus-glaucus). Contrib. Dept. Pharmacy, Univ. of Wisconsin. No. 2. pp. 5-9. Fig. 1-8. 1886. Reprint without pagination.

Notes on Eurotiun Aspergillus-glaucus.

Troviranns, Ludolf Christian. Sept. 1779. †Bonn, 6 May, 1864.

6rr. Ueber die Pietra Fungaja und ein rerwandtes gebilde aus den Vereinigten Staaten. Verhandl. Naturhistor. Verein der preuss, Rhein. und Westphal. VI. 281-289. Pl. 12. 1849.

Includes an accomt, with figure, of what is apparently a monstrosity of some Polyporus, eollected by Lieut. IIacomb at Grand Traverse Bay, Lake Michigan.

Tuckerman, Edward. Boston, 7 Dec. 1817. †Amherst, Mass., 15 March, 1886. See Fries, E. M.

- and Frost, C. C.

6I2. A catalogue of plants growing without cultivation within thirty miles of Anlherst College. $8^{\circ}$. pp. vi. 98. Amlierst, 1875.

The fungi by Frost, pp. 62-97, nclurle a list of over 1100 species, with babitats. In the determination of the species the author was aided by M. A. Curtis, and some of the names represent species published later in Berkeley's Notices of North American Fungi (see title 42), and some are manuscript names of Curtis and Frost, which were never published. A number of species are referred to C. I. Perk, and nuh. lished by him elsewhere. See Peck, C. H. The names in this catalogue are frequently misspelled. 
Tulasne, Charles. Langeais, 5 Sept. 1816. †Hyeres, 28 Aug. 1884.

Tulasne, Louis René. Azay-le-Rideau, France, 12 Sept. $181 \%$. †Ilyères, 22 1)ec. 1885.

6r3. - L. R. Observations sur l'organisation des Trémellinées. Ann. Sci. Nat. Bot. 3 ser. XIX. 193-231 (1-39). 1'l. 10-13. 1853.

Includes a brief reference to Podisoma maeropus, Schr.

6r4. - Mémoire sur l'ergot des Glumacées. Ann. Sci. Nat. Bot. 3 ser. XX. 5-56. PI. 1-4. 1853.

IIas rery bricf referenees to American authors on the subject.

$614^{\mathrm{A}}$. - L. and C. R. Recherches sur l'organisation et le mode de fructification des chamjignons de la tribu des Nidulariées, suivies d'un essili monographiquc. Ann. Sci. Nat. Bot. 3 ser. I. 41-107. Pl. 3-8. 1844 .

Includes descriptions of United States speeies, one new species figured.

6r5. — Mémoire sur les Ustilaginées comparées aux Urédinées. Ann. Sci. Nat. Bot. 3 ser. VII. 12-127. Pl. 2-7. 1847.

Inelurles obserrations on Testicularia and Ustilago Schwei. nitzii (Credo Zeae, Schw.), from the United States.

616. —— Fungi Hypogaei. Histoire et monographie des champignons IIypogés. Folio. up. xix. 222. Pl. 22, 9 colored. Paris. 1851. 2d edition, pp. xxiv. 222. P1. 21. Paris. 1863?

Of the first edition of this sumptuous work only 100 copies were printed, and each copy was numbered. A second edition was afterwards issued same as the first in all respects, except that there is another prefiee. The date of issue of second edition is uncertain. The title-page bears the date 1553 , but the preface of seeond edition is dated June, 1862 . Probably the 18.33 of title-page should be 1863 . This work includes deseriptions of American genera of Bose and Schweinitz, as Arachnion, Iy/perviza, ete.

6r7. — - Selecta fungorum carpologia, ea documenta et icones potissimum exhibens quae varia fructuum et scminum genera in eodem fungo simul aut vicissim adesse demonstrent. Folio. Vol. I. xxviii. 242. Pl. 5. Paris, 1861. - Vol. II. xix. 319. Pl. 34. 1863. - Vol. III. xvi. 221. Pl. 22. 1565.

First rolume includes Erysiphei, the seeond Fylariei, Falsei, and Sphaeriei, and the third Nectriei Phacidiei, and Pezizei. 'This elassic work does not treat specially of A merican fungi, but there are many critical notes and references to species of this country scattered through the text.

\section{Tully, William.}

$6 r 8$. Reduction of all the genera of plants contained in the Catalogus Plantarum Americae Septentrionalis of the late Dr. Muhlenberg to the natural families of Mr. J) .Jussicu's system. For the use of the gentlemen who attended the course of elcmentary and philosophical botany in Philadelphia in 1815. Philadelphia. 1815.

II as an enumeration of 47 genera of fungi, p. 5, ineluding Erineum.

United States. Department of Agriculture. See AgRictltural Relolits.

- Geological Surreys. Sce Brumrgs, J. S. Sce Hatder, F. V. See Parry, C. C. See P'orter, T. C.

Ventenat, Ftienne Pierre. Limoges, 1 March, 1757. +I'aris, 1: $\Lambda$ ug. 1808.

6rg. Jissertation sur le genre Phallus. Mém. de l'Instit. Nat. (Classe Sci. Math. et Phys.) I. 503-523. PI. 7. Paris. 1798.

Includes an account of Phallus indusiatus from Guiana, witl figures, to which species some United States specimens have since been referred, but no mention made of United States specimens by this author.
Viala, Pierre, and Ravaz, L.

620. Mémoire sur une nouvelle maladie de la vigne. Le Black Rot (pourriture noire). $8^{\circ}$. pp. 62. Pl. 4 . Montpellier. 1886.

Gives notes on strueture and synonymy of Phoma uvicola, B. and C., and Sphacria Bidwellii, Ėlis.

621. — - Sur de nouvelles espèces du genre Phoma se développant sur les fruits de la vigne. Bull. Soc. Bot. France, XXXIII. Ixi.lxvii. 1886. Not issued until 10 Jan. 1887.

Includes details of Phoma uvicola, B. and C.

Vize, John Edward. London, Eng., 7 March, 1831.

622. Californian Fungi. Giev. V. 109-111. March, 1877. - VII. 11-13. Sept. 1878.

Notices of 66 speeies collected by H. W. Harkness, of which 21 are described as new.

Voglino, Pietro. Turin, 23 March, 1864.

623. Sul genere Pestalozzia saggio monografico. Atti. Soc. Voneto-Trentina Sci. Nat. IX. 209-243 (1-37). Pl. 8-10. 1885. Issued 1886.

A deseription of all known species of the genus, being a revision of the species given in the third volume of Saccardo's Sylloge. Besides the revised descriptions, there are also figures of some North American species.

Voyle, Joseph. See Riley, C. V.

Waltor, Thomas. Hampshire, England, 1740. +Carolina, 1788 .

624. Flora Caroliniana secundum systema vegetabilium perillustris Linnaei digesta: characteres essentiales naturalesve et differentias veras exlibens: cum emendationibus numerosis: descriptionum antea evulgatarum : adumbrationes stirpium plus mille continens, nec non generibus novis non paucis, speciebus plurimis novisque, ornata. $8^{\circ}$. pp. 263. Pl. 1. London. 1788.

Deseribes, pp. 260-263, 7 Agarici, 5 Boléti, 1 Hydnum, 1 Phallus, 1 Clavaria, 4 Lycoperdon, and 1 unnamed species.

Walsh, Benjamin Dann. Frowe, Worcestershire, England, 21 Sept. 1808. †Rock Island, Ill., 18 Nov, 1869.

× 625. Black Knot. Practical Entomologist, I. 48-51. 26 March, 1866. - II. 63. March, 1867.

Arguments to show that this disease is caused by a fungus and not by an insect.

626. - A plant growing out of an insect. Practical Entomologist, II. 116. Aug. 1867.

Reports a fungus growing out of a May-bug in Iowa.

- and Riley, C. V.

626". Fungoid growths. Am. Ent. I. 91, 92. Jan. 1869.

Notice of the white grub fungus, with remarks by W. H. Edwards.

Watson, Screno. East Windsor Hill, Conn., 1 Dec. 1826. See Brulrings, J. L.

Watt, David, A. P.

627. A provisional catalogue of Canadian Cryptogams. Canadian Naturalist and Geologist, II. new series, 390-40t. Oct. 1865 .

On pp. $390-392$ is a list of 152 species of fungi from the collections of Dr. W. P. Maclaggan, determined by Berkeley, and of $D$. A. Watt, determined by Curtis. The species are arranged alphabetieally, and names of authorities are not given.

Wilkes Expedition. See CurTrs, M. A., and Berkeley, M. J.

Wingate, Harold. Philadelphia, 25 August, 1852 .

628. A new genus of Myxomycetes. Jour. Myc. II. 125,126 , with figure. Nov. 1886 .

Charaeters of the new genus Orthotricha and description of 0 . microcephala. 
Winter, Georg. Leipsic, 1 Oct. 1848. See Exsiccat in supplement. See Hedwigia.

+629. Bemerkungen ueber einige Uredineen. Hedwigia, XIX. 17-29. Feb. 1880.

The Uredo Aspidiotus of Peck referred to $U$. Filicum.

63о. - Eine neue Chrysomyxa. Bot. Centralblatt, V. 250, 251. Feb. 1881 .

The Uredo pirolata, Koernicke, from New York, referred to Chrysomyxa.

+631 . Ueber die gattung Har-knessia, Cooke. Hedwigia, XXII. 19-21. Feb. 1883.

Gives notes on $I T$. Eucalypti, Cooke, and other exotic species.

× 632. - New North American Fungi. Torr.

Bull. X. 7, 49, 50. Jan., May, 1883.

First part has descriptions of Sorosporium Ellisii, Uslilago Vilfue, and Gonatobotrys maculicola, also given in Hedwigia, XXII. 1, 2. Second part has 5 species from Illinois and Kentucky, also given in next title.

+633. - Fungi nonnulli novi. Hedwigia,

XXII. 1-3. Jan. 1883.

Includes 3 species from the United States. See last title.

-634. - Ueber einige Nordamerikanische pilze. Hedwigia, XXII. 67-72, 129-131. May, Sept. 1883.

First part has deseriptions of 8 new species, 5 of them same as in last title, besides notes on Ascomycetella and several Uredineae. Second part has descriptions of 2 new species, and notes on Entyloma Physalidis and Puccinia Thwaitesi.

635. Mycologische Notizen. Iledwigia, XXII. 7-9. Jan. 1884.

IIas notes on Puccinia heterospora, Enlyloma Physalidis, and Cercospora fusco-virens.

636. - New North American Fungi. Jour. Myc. I. 101, 102. Aug. 1885.

Descriptions of Sphaerella Eariana, Fusicladium effusum, Darluca interseminata, and Doassunsia decipiens.

× 637. — Fungi novi Missourienses. Jour. Myc. I. 121-126. Oct. 1885.

Characters of 25 new species collected by C. H. Demetrio, same as in title no. 640 .

638. — - Fungi exotici III. IIedwigia, XXV. 92-104. June, 1886.

Notes the occurrence of Arthrosporium parasilicum, Winter, in Florida.

639. - Pilze : in Dr. I. Rabenhorst's Kryptogamen-Flora von Deutschland, Oesterreich und der Schweiz. Zweite Auflage. $8^{\circ}$. Band I. Erste Abtheilung, pp. 924, and index. Schizomy- cetes, Saccharomycetes, and Basidiomycetes. Leipsic. 1884. - Zweite Abtheilung, pp. 880. Pyrenomycetes. To be continued.

This well-known work is issued in parts, the first of which appeared in 1881. The complete first volume has the date 1854. Of the second volume, there had appeared prior to 1887,800 pages, and the volume is not yet completed. The work is illustrated with numerons woodcuts of the genera and one colored cliart. Oceasional references are made to American writers in the synonymy. and Demetrio, C. H.

† 640. Beiträge zur pilzflora von Missouri. Series I. IIedwigia, XXIV. 177-214 (1-37). Oct. 1885.

An enumeration of 350 species, with host-plants and sy. nonymy in some cases, 25 of the species described as new, one attributed to Bresadola. See title no. 637.

Woronin, Max.

641. Beitrag zur kenntniss der Ustilagineen. $4^{\circ}$. pp. 35. Pl. 4, partly colored. Frankfort. 1882.

This paper forms the fifth part of "Beitraege zur Mor. phologie und Physiologic der Pilze," by De Bary and Woronin. Sorosporium Astragali, Peck, and S. Desmodii, Peck, are referred to the genus Thecaphora.

Wyman, Jeffries. Chelmsford, Mass., 11 Aug. 1814. †Bethlehem, N. H., 4 Scpt. 1874. and Berkeley, M. J.

642. On a minute fungus, Podisoma macropus, growing on Juniperus Virginiana in North America, by Dr. Wyman in a letter addressed to Sir IV.J. Hooker; with some additional remarks by the Rev. M. J. Berkeley. London Jour. Bot. IV. 315-319. Pl. 12. Fig. 6. 1845.

A letter, with an account of gross and microscopic characters of the common cedar apple of New England, with figure of the fungus, Podisoma mucropus, and a note by Berkeley on its botanical relations.

Zabriskie, Jeremiah Lott. Flatbush, L. I., 3 Feb. 1835.

643. A catcrpillar fungus from New Zealand and some related species of the United States. Jour. N. Y. Micr. Soc. I. 89-94. Fig. 1-6. April, 1885.

Includes descriptions and figures of Tommbia militaris, $T$. Ravenelii, and T. clavulata of the United States.

644. — Fruit of the fungus Uncinula flexuosa, Peck, on leaves of the horse-chestnut. Jour. N. Y. Micr. Soc. II. 144, 145 . Dec. 1886.

645. — Fruit of the fungus Microsphaera extensa. Jour. N. Y. Micr. Soc. II. 149, 150. Dec. 1886. 


\section{ADDENDA.}

Bail, Theodor Sec Nefs von Esenbeck, T. F. L.

Bischoff, Gottlieb Wilhelm. Durkheim, 1797. tIleidelherg, 11 sept. 1854.

646. Lelirbuch der botanik. $8^{\circ}$. Vol. I.-III., and supplement. Stuttgart. 1S34-1St0.

lortion of "Naturgeschichte der drei liciche." The plates which belong to this work form it separate quarto, the index heing in vol. II. Pl. 7 , fig. 173 , represents Ifitremyces cinnabrerinus.

Brondol, F. $\Lambda$ dd to title no. 76 : List of species alvo given in the Pharmacist, XV. 263-268, 291299. July, Aug. 1882.

Catesby, Mark. 1680. fLondon, 23 Dee. 1749.

647. The natural history of Carolina, Florida, and the Baliama Islands. fo. Vol. I., II. London. 1731-43. $2 \mathrm{~d}$ ed. $1754.3 \mathrm{~d}$ ed. 1771.

Vol. I. pl. 36, hi:1s a figure ealled "fungoides capitulo intorto, Toal stool," not recognizable.

Copeland, Robert Morris.

648. Edible fungi. Atlantic Monthly, XXXT. 223-229. Feb. 1573.

Popular article on the subject, without spccial refercnce to $\Lambda$ nnerican species.

Crozier, Arthur Alger. Georgetown, Ottawa Co., Mich., 22 Sept. 1556.

649. Notes on black-knot. Bot. Gaz. X. 368, 369 , Sept. 1885; and Jour. Myc. I. 142.

Remarks on the date of the ripening of the spores in this speries.

Duggan, J. R. See SEDGmick, W. T.

Hubbard, Henry Guernsey. Detroit, Mich., $6 \mathrm{May}, 1850$

$\times$ 650. Insects affecting the orange. Report on the inscets affecting the culture of the orange and other plants of the Citrus family, with practical suggestions for their control or extermination, made, under the direction of the Entomologist, by H. G. Hubbard. U. S. Dept. Agr. Division of Entomology. 8․ pp. x. 227. Pl. 14. Washington. 1885 .

In the introduetion, pp. 1-4, pl. 2, is a notice of die-back, bark-fungus, foot-rot, and smut, due to the growth of fungi.

Osborn, Herbert.

×651. An epidemic disease of Caloptenus differentialis. Am. Nat. XVII. 1286, 1287. Dee. 1883.

Aceount of a disease eansed by Entomophthora Calopteni, Bessey, with a note by C. V. Riley. Paper first real before the lowa $\Lambda$ eademy of Science, 27 Sept. 1883. See title no. 63, where p. 1286, which belongs to present title, is incorrectly cited.

Ott, Isaac. Northampton Co., Pa., 30 Nov. 1847.

652. Poisonous mushrooms. Jour. of Nerrous and Mental Diseases, IV. 48-53. Chicago. Jan. 1877.

A general account of the toxicological action of fungi, in cluding experiments on the action of muscarin, especially those of Smiedeberg.

\section{Pech.}

653. Catalogue of the United States plants in the Department of Agrieulture.

The above is the title of a very rare pampllet found in a few libraries. It has no value except as a literary and botanieal curiosity. The autlor was at one time employed as botanist at the United States Agrienltnral Department, and prepared a list of plants, pp $2 \pi$, which was distributed as a cirenlar, but was never printed in any government publieation. It is said to have been suppressed in consequence of the very numerons errors it eontains. On pp. 26, 27 is a list of 72 fungi, anparently from Missouri, 31 of them said to be nerv species. There is no title-page, and the date, which is not printed but written, is Nor. 1,1866 .

\section{CORRIGENDUM.}

In place of the note following title no. 174, substitute the following title : -

174. Icones Fungorum lueusque cognitorum. Ablildungen der pilze und sehwaemme. fo. Vol. I.-VI. I'rague. 1837-1854.

$\Lambda$ work witl firures and descriptions of many snecies of fungi. Vol. VI., lublished after Corda's death, was cdited by J. B. Zobel, and contains a small number of species from North America. A considerable part of the edition of the first five volumes was destroyed in the great fire at Prague, and eomplete sets of the original edition are now very rare. The remaining copies of vol. VI. were purchased by $\mathbf{R}$ Friedlaender \& Solın, of Berlin, who have reproduced the first five volumes by photo-lithography, and most eopies now offered for sale have only the sixth volume of the origin:l edition. 


\section{SUPPLEMEN'T.}

\section{FUNGI EXSICCATI AM.-SEPTENTRIONALIS, A LIST OF PUBLISIIED SETS OF DRIED FUNGI,} WIICH INCLUDE SPECINENS FROM TIE UNITED STATES.

Fungi Caroliniani Exsiccati. Fungi of Carolina, illustrated by natural specinens of tlic species by H. W. Ravenel. Fasc. 1, 1852; 2 $1853 ; 3,4,1855 ; 5,1860$. Charleston, S. C.

Each fasciculus contaius 100 specimens, bonnd in quarto with a systematic index; and 5 contains an alphabetical index of the whole series. Each faseienlus is numbered separately. Only 30 copies of this series were issued and complete sets are now very rare. The specimens were mostly collected by Ravenel himself, and M. A. Curtis aided in the determination of species. For notices of this work, see Am. Jour. Sci. Arts, XVI. 2 ser. 129; XVII. 285; XY. 284; XXIII. 439; and Charleston Med. Jour. and Iev. VII. 690 et seq.

Fungi Americani Exsiccati. H. W. Ravenel and M. C. Cooke. Cent. 1-8. 1878-1882.

This work includes fungi collected by Ravenel principally in South Carolina, Georgia, and Texas. The speeimens were determined by Cooke and issued in centuries on loose slyeets of quarto size, $t$ specimens on each sheet, similar in form to Fungi Britannici, $2 d$ edition. About 30 sets were issued, the place of publication being London. The centuries were issued two at a time, as follows: 1 and 2,$1878 ; 3$ and 4,1879 . 5 and 6,$1881 ; 7$ and 8,1882 . There are no inderes accompanying the centuries, but an enumeration of the species, with comments and descriptions of the new species in this series, will be found as follows: Grevilled, VI. 129-146; VII. 32-35, 43-54; XI. 106-111; XII. 22-33;-Uedwigiu, XVII. 37-40;-Britten's sour. Bot. XXI. 67-71, 106-110, 136-139;-Jour. Roy. Hort. Soc. V. new serics, 92. See titles here given, nos. 129, 135, 154 .

North American Fungi. Edited and published by J. B. Ellis. Newfield, N. J. Cent. 115. $1878-1885$.

Second Series. Edited and published by J. B. Ellis and B. M. Everhart. Cent. 16, 17. Newfield. 1886. 'To be continued.

This series contains specimens bound in quasto form, with an index to each century. There is also an "Alphabetical Index of Cent. 1-10," 7 pages, prepared by W. C. Stevenson, an "Alphabetical Index of Cent. 1-15," compiled by B. MI. Everhart, and an "Index of habitats" of Cent. 1-10, compiled and published by IV. C. Sterenson. Two centuries lave been issued annually, and the edition, which consists of 50 copies, lias up to present date all been sold.

Cent. $3,11,15$ contain principally Uredineae, Ustilagineae, and Peronosporece. For review, see title no. 279. Cent. 1, 5, 9 are principally Ascomycetes. Cent. 12 and 17 largely Septorice, Phyllostictae, and their allies. Cent. 13 and 16, Cercosporae, icamulaviue, and related genera. See reviews as follows: IIedroigia, XIX.124; XX. 100; XXI.110; XXII. 124; XXV. 44;-Am. Nut. XV. $652 ;$ XVII. $873 ;-.70 u 0$. Myc. I. 83. See title no. 22. Notices of this series, with lists of new species, also given in current numbers of Revue $M y$ cologique.

Herbarium Vivum Mycologicum sistens fungorum per totam Germaniam erescentium collectionem perfectain.

This series of fungi exsiccati includes 20 centuries, of which the first was issued at Berlin in 1832 , by .J. F. Klotzsel. A notice of the first fasciculus appeared in Linnaeu, LittercuturBericht für 1833, p. 17. Two parts were issued by Klotzsel, but the remaining parts were issued by $\mathrm{L}$. Rabenhorst nuder the title "Klotzschii ITerbarium vivum mycologicum," etc. Dresden. 1842-1855. For notice of Rabenhorst in connection witl this series, see Linnaer, $18+2$, pp. 333, 334. The centuries issued by Rabenhorst are bound in quarto covers. There appeared two indexes: "Index in Klotzschii IIerbarium vivum mycologicum," Cent. 9; and "Index alpha. beticus et conspectus systematicus in Klotzselii herbarium mycologicum," curante Ludovico Rabenhorst, pp. 12, 1851, including the first 14 centuries. Complete copies of this series are very rare, and very fow lave the first three centuries complete, since, apparently, the specimens of the first two centuries it least liarl the specimens loose aud not bound like the later centuries.

Klotaschii Herbarium Vivum Mycologicum, etc. Editio nova, cura Dr. I. Rabenhorst. Cent. 1-8. Dresden. 1855-1858.

A continuation of the previous series and similar in form. An index on a loose sheet witl each century.

N. B. At some subsequent period what purported to be sets of this series were sold by Rabenlorst, but the speeies do not correspond to those of the oricinal issue. The titlepages of these spurious sets bear dates from 1845 to 1559 , without any regular order, and are evidently nothing but old titles left over from this and the precelling series.

Fungi Europaei Exsiccati. Klotzschii herbarii vivi mycologici continuatio. Editio nova. Series secunda. Cent. 1-36. Dresden. 18591886. To be continued.

A continnation of the preceding and of the same form. Previous to Cent. 27 there were no indexes, since then an alphabetical index bound with each ecntury. This series was edited by Rabenliorst until his death and since then, beginning with Cent. 27, the editor has been G. Winter. At tlie same date the title was changed to "Fungi Europaei et Extraeuropalei." With Cent. 21, the numbering of the titlepages began again with 1 , the number of the whole series being also retained; and it is by this latter number that the centuries are designated. Although the title-pages bear the name Dresden, the later centuries have in reality been issued from Leipsic. The number of copies issued is somewhat over 100.

N.B. What is sometimes offered for sale as Klotzsch's Ilerbarium vivum includes only the Fungi Europaei. In many sets Cent. 26 , which was in preparation at the time of Rabenhorst's deatl but not issued until later, is either wanting or imperfect. The only part of the whole series which contains American specimens is that from Cent. 27 on. The following notices of the centuries in which American num. bers occur may be consulted in Iledwigia. Cent. 27,4 species from United States, rol. XXI. 5-12; Cent. 28 and 29, 8 species, vol. XXII. 9-15; Cent. 30, 37 species, vol. XXII. 173-176, 180-185; Cent. 31 and 32, 53 species, vol. XXIII. 164-175; Cent. 33 and 34 , SS species, vol. XXIV. 252-264; Cent. 35 and 36,99 species, vol. XXV.257-263; XXV $1.24-35$.

Ascomyceten. In getrockneten exemplaren herausgegeben von Dr. Med. Relim. Fasc. 1-17. Nos. 1-850. Regensburg. 1873-1885. To bo contiuued.

Each fasciculns of this series is composed of 50 specimens contained in loose envelopes or boxes, and only 25 copies svere issued. The following numbers are from the United States, collected principally hy J. B. Ellis : 269, 287, 303, 307, $310,311,314,315,316,318,325,329$, 337, 338, 347, 360, 361, 363, $364,369,381,394,423,431,438,441,446,447,464,465,473,522$, $609,770,822,845,846,849$

For notes on species in this collection, see title no. 518 . Also, see Winter, "Diagnosen and Notizen zu Relum's Ascompceten," in Flora, LV. (XXX.) 508-511, 523-527, and 542-544. Also, Grevillea, II. 157, V. 37, etc. Eummeration of species in the earlier fasciculi are given in Flora, 1873 , 1874,1875 .

Mycotheca Universalis cura F. de Thucmen. Cent. 1-23. 1875-1884.

A collection of specimens in envelopes attaclied to loose slieets witl quarto covers. Cent. 1-5 were issued at Bairentl 6-18 at Klosterneuburs, 19-22 at Vienna, and 2:3 at Görz. There was an anpliabetical index of the species in Cent. 1-12, $8^{\circ}, \mathrm{pp} .35$, issuerl in 1879 .

There are specimens from the United States in each of the centuries, in all 310 speeies, and to some of their lishels are 
suldel deacriptions am uotes, which aro also given in Flora under the lumliug "Diamosen zu 'l']uemen's Mreotheca

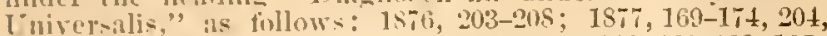

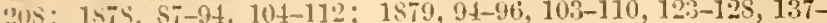
139 ? $1580,310,3.20,323-332 ; 1851,237-239,251-255,260-272$ y-303. Siee notice of Cent. 1 and 2 in Torr. Bull. TI. 4745, and of Cent. 18 and later parts in liee. Myyc. 1879 et seq., Alil. Itat. XVI. 321.

Herbarinm Mycologicum Oeconomicum. Die fiir die land-, forst-und lauswirthschaft, den gartenlonu und die industric schädlichen resp. niit\%lichen pilze in getrockneten exemplaren. Edited hy F. von 'Thuenen. Fase. 1-15. Nos. 1750. 'l'eplit\%, Baireuth, Klosterneuburg. 1872-1880.

II have not seen this collection, which includes a small mumber of species from the United States, colleeted by Ellis, Peck, and liarenel.
Fungi Gallici Exsiccati. Edited by C. Roumeguère. Cent. 1-40. Tonlouse. 18791886. To be continued.

This collection includes specimens attached, generally in envelopes, to loose sheets with quarto covers. There is an "Index alphabétique des renres, espèces et stnonymes des Fungr Galliei Exsicenti, Cent. 1-25, 1879-1883," taken from Revie Mycologique, $V$. 137-164. July, 1883. The following numbers of this collection are from the Uniter States : 2006 , $2097,2327,2330,2390,2429,2453,2465,3412,3413,3414,3421$, $3470,3478,3595,3839,3840,3841,3842,3845,3859,3860,3861$ $3862,3864,3865,3866,3867,3873,3876,3877,3879,3881,3882$, $3921,3926,3927,3961,3976,3977,3994,4000$. Notices of the species in this collection are given in the current numbers of the Rerue. Those of the numbers from the United States are as foHlows: IV. 96-105; V. 6-30; VII. 215-224; VIII. 14$23,190-200$; IX. 19-29. 


\title{
Lilurary of llarbard Jintibersite.
}

\section{BibLIOGRAPHiCAL CONTRIBUTIONS.}

\author{
EDITED BY JUSTIN WINSOR,
}

LIBRARIAN.

\section{No. 31.}

(SUpplemential, to No. 25.)

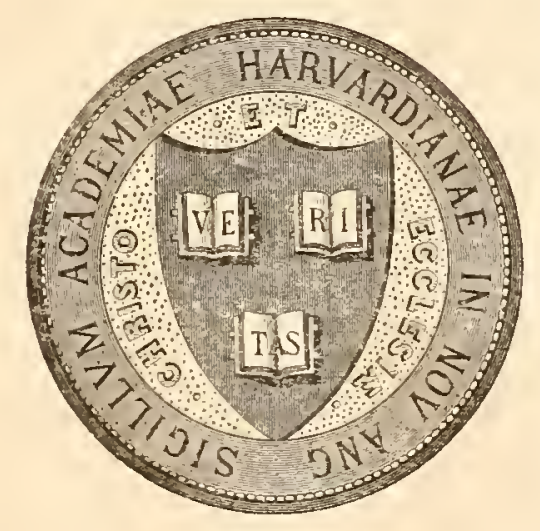

A SUPPLEMENTAL LIST OF WORKS ON NORTH AMERICAN FUNGI. BY W. G. FARLOW.

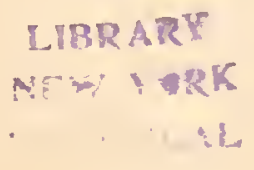

CAMBRIDGE, MASS. :

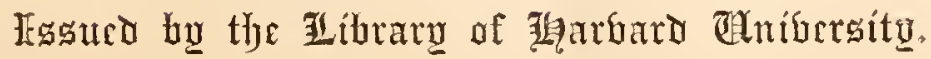




\section{Already issued or in preparation:}

A Star prefixed indicates they are not yct ready.

\section{VOL. I.}

1. Edvard S. IIolden. Index-Catalogue of Books and Memoirs on the Transits of Mercury.

2. Justin Wixsor. Shakespeare's Poems: a Bibliography of the E:rlier Editions.

3. Cirarles Eliot Norton. Principal books relating to the Life and Works of Michelangelo, with Notes.

4. Justix Winsor. Pietas et Gratulatio. An Inquiry into the authorship of the several pieces.

5. LIST OF APPARATUS in different Laboratories of the United States, available for Scientific Researches involving Accurate Measurements.

6. Tile Collection of Books and Autograpis, be. queathed to II arvard College Library, by the II onor. abic Charles Sumner.

7. Willias C. Laxe. The Dante Collections in the IJarvard College and Boston I"ublic Libraries.

S. CaleNdar of the Arthur Lee Manuscripts in IIarvard College Library.

9. George Lincoln Goodale. The Floras of different countries.

10. Justin Wissor. Halliwelliana: a Bibliography of the Publications of James Orchard IIalliwell.Phillipps.
II. Samuez H. Scudder. The Entomological Libraries of the United States.

12. First List of the Publications of Harvard University and its Officers. is $70-1$ SSO.

13. Samuel H. Scudder. A Bibliography of Fossil Insects.

14. William H. Tillinghast. Notes on the Historical IIydrograplyy of the Handkerchief Shoal in the Bahamas.

15. J. D. Whitney. List of American Authors in Geology and Palæontology.

16. Richard Biss. Classified Index to the Maps in Petermann's Geogra!hische Mitheilungen. IS55ISSr.

17. Richard Bliss. Classified Index to the Maps in the Royal Geographical Society's Publications. ${ }^{1} S_{30-}$ ISS3.

IS. Justin IVinsor. The Bibliograplyy of Ptolemy's Geograpliy.

19. Justin Winsor. 'The Kohl Collection of Early Maps.

20. WilliaM C. Lane. Index to Recent Reference Lists, no. 1. ${ }_{1} S_{4}-1 S_{5}$.

\section{VOL. II.}

21. SECOND List OF THE l'UBlications of llarvard University and its Officers. ISSO-1SS5.

*22. Justix Wissor. Calendar of the Sparks Manuscripts in II arvard College Library.

23. Willan H. Tilinghast. Third List of the Publications of Ilarvard University and its Oflicers. ISS51856.

24. Willian C. Lave. Index to Recent Reference I.ists, no. 2. $1 S \$ 5-1 S S 6$.

25. W. G. Harlow and Wiblan Thelease. I is tof Works on North American Fungi.

26. Whtias C. Iasie. The Carlyle Collection.
27. Andrew McI. (Mavis. A few notes on the Records of IIarvard College.

2S. Wilimam H. Tillinginast. Fourth List of Publications of IIarvard University and its Officers. ISS6iSST.

29. William C. Lane. Index to Recent Reference Lists, no. 3. ISS7.

30. Smelley"s "SKylark," a Facsimile of the original manuscript.

31. W. G. Jarlow. Supplemental List of Works on North American Fungi. 


\title{
SUPPLEMENTARY LIST OF WORKS ON NORTH AMERICAN FUNGI.
}

\author{
BY W. G. FARLOW.
}

The present list is a continuation of Bibliographical Contributions no. 25, which included the titles of works on the fungi of the United States and the northern parts of North America published prior to 1887. The plan followerl is the same as that of the paper jnst mentioned, the object being to give additions and corrections to be made to the list of works previously published with the addition of titles of works which appeared in 1857. The original list with the present supplement is is complete a presentation of the snbject as the writer is able to make, and as he does not propose to continue the work further it is to be hoped that some other person or persons will undertake the task hereafter.

CAMBRIDGE, March 1, 1888 .

W. G. FARLOW,

\section{LIST OF WORKS ISSUED BEFORE 1887.}

\author{
ADDENDA AND CORRIGENDA.
}

No. 15. Add: also in Studies from the Biolog. Lab. Johns Hopkins University, Session 1877-78, no. 1. Baltimore. 1879.

After no. 22 insert the following title:-

Barbeck, IVilliam.

22. Microscopical fungi infesting our cereals. Am. Nat. XIII. 612-620. Oct. 1879 .

General notes on ergot, smuts, rusts and blights.

No. 31. Add to note: no. 109, Leotia elegans, Berk. London Jour. Bot. V. $6(5)$.

No. 49. For 7 species, read 8 species.

No. 69. For pp. 174-190, read pp. 156-170.

No. 121. For p. 277 read 273.

No. I25. Add: See also Gard. Chron. VII. 634.

$1877, \mathrm{I}$.

After no. 145 insert the following :-

Cooke, Mordecai Cubitt.

I45 ${ }^{\mathrm{a}}$. The sub-genus Coniophora. Grev. VIII.

88-89. March, 1880.

Includes notes on four American species.

No. I96. This paper is reproduced in "Mushroom Culture" by IV. Robinson, London, 1870, pp. 145-160.

After no. 200 insert the following :-

Dowoy, Chester. Sheffield, Mass., 25 Oct. 1784. † Rochester, N. Y., 13 Dec. 1867.

200". A history of the county of Berkshire, Massachusetts; in two parts, the first bcing a general view of the county; the second, an account of the several towns. By gentlemen in the county, clergymen and laymen. sm. $8^{\circ}$. pp. 468 , with 2 maps and several illustrations. Pittsfield, Mass. 1829 .

Part one of this work includes "Catalogue of Plants fonnd in the County of Berkshire," pp. 43-86, by Rev. Chester Dewey. On pp. 85-86 is a list of 9 genera and 13 species of fungi. Authorities of names not given.

To Fischer, Eduard, add: Born at Berne, Switzerland, 16 June, 1861.

After no. 265 add the following llote:-

The papers marked nos. 263. 264, and 265, are reproduced in The Botanical Works of the late George Engelmann. Collected for Henry Shaw, Esq. Edited by William Tre. lease and Asa Gray. Cambridge. 1887. no. 263, 265, and that part of 264 relating to grape fungi are on pp. $430-432$. That part of 464 relating to oak fungi is on p. 410 .
No. 300. Add: Rev. in Hedw. XXVI. 113.

After no. 336 insert the following:-

Griffith, Robert Eglesfeld. †Philadelphia, Pa., 26 Jnne, 1850.

$36^{\mathrm{a}}$. Medical botany, or descriptions of the more important plants used in medicine, with their history, properties, and mode of administration. 8. pp. 70t. Philadelphia. 1847.

The clapter" on Fungales, pp. 674-679, figs, 326-330, gives an account of several species of fungi, but there is no special reference to American species.

No. 372. For 13 of them, read 14 of them.

No. 374. 'To pp. 17, 18, add p. 16. Rev. Torr. Bull. VIII. 95.

After no. 380 insert the following :-

Lamarck DE, Jean Baptiste Antoine Pierre Monnet. Bazentin, France, 1 Aug. 1744. †Paris, 18 Dec. 1829.

$380^{a}$. Encyclopédie méthodique, Botanique. Paris. 1783-1817.

In supplement V. of this work, Paris, 1817, pp. 475, 476, are descriptions of Lycoperdon cyathiforme, L. transversarium, L. heterogeneum, L. callostoma, Onygena decorticata, from North America. See titles no. 73 and 200.

After no 425 insert the following :-

Morison, Robert. Aberdeen, 1620. †London, 10 Nov. 1683.

$425^{\natural}$. Plantarum historiae universalis Oxoniensis Pars tertia seu herbarum distributio nova, etc.

In this work, p. 6+2, London, 1699, is a reference to a "fungus crepitus lupi" from Virginia. The species cannot now be recognized.

After no. 498 insert the following:-

Porcher, Francis Peyre.

498. Medicinal plants of South Carolina indigenous and introduced. Trans. Am. Hed. Ass. II. $683-862.1849$.

The only reference to fungi in this work is a brief note on Amanita muscaria, pp. 853,854 .

No. 5or. Add : 2d edition in 1869 , fungi on pp. $694-699$.

After no. 505 insert the three following titles:-

Provancher, L. (Abbé).

$505^{\natural}$. Un champignon remarquable. Naturaliste Canadien, XVI. 50-58, fig. 56. Oct. 1886.

Account of Phalles impudicus and its place among fungi. 
Rafinesque-Schmaltz, C. S.

$505^{b}$. 1'rospectus of Mr. Rafinesque-Schnaltz's two intended works on North American botany; the first on the new genera and species of plants discorered by himself, and the second on the natural history of the funguses, or musliroom-tribe of America. Medical Repository. Second Hexade. r: 350-356, New York, 1808.

On lu. 355, 356, is an cummeration of 17 new genera with brief romitis and nanes of some of the species included wulle the genera. The author states that he "shall describe in this work nearly $\$ 50$ species or varieties of American mushrooms, of which one halt will be new orders, and most of them elucidated by plates."

$505^{c}$. Iissential generic and specific characters of some new genusses and species of plants observed in the United States of Aneriea in 1803 and 180t. By Mr. C. G. Rafinesque-Schmaltz. In a communication to Dr. Mitchell, dated Palermo, Sept. 1, 1807. Medical Repository. Second Hexade. V. 356-363, New York, 1808.

On p). 362, 363, are descriptions of 20 new species of fungi, principally l'ezizae and Clacariue. Neither this paper nor the preceding have any scientific ralue.

After no. $523^{\mathrm{B}}$ insert the following : -

Richardson, (Sir) John.

$523^{\mathrm{b}}$. Remarks on the climate and regetable productions of the Hudson's Bay countries. Edinburgh Phil. .Tour. XII. 197-231. April, 1825. Also reprint, pp. 35.

In a table on p. 224 is a list of 12 genera of fungi with the the number of species growing in the ITudson Bay country.

After no. 508 insert the following:-

Sahagun, Bernardino De.

508 . Historia universal de las cosas de Nueva España.

This work, whose anthor lived about the 16th century, is giren in Fingsborough's Antiquities of Mexico. Vol. VII. London. 1831. In the 11th book, 7th chapter, pp. 369-394, is an account of plants, including some fungi. According to Mr. WT. R. Geraid, who has called our attention to this title, this is probably the earliest account of North American fungi. There are bricf descriptions with Aztec names of 12 species, one of which appears to be Lycoperdon giganteum and another probably Agaricus muscarius.

After no. 528 insert the following :-

Riley, Charles Valentine. The cabbage Plusia. Rept. U. S. Dept. Agr. for 1883, pp. 119-122.

On p. 121 is a notice of a disease of Plusia Brassicae caused by Batrytis Rileyi Farlow, of which a description is given.

After Schmidt, J. C., insert the following :-

Schoepf, Johann David. Wunsiedel, 8 March, 1752. +Anspach, 10 Sept. 1800.

$552^{\mathrm{a}}$. Materia medica americana, potissimum regni vegetabilis. Erlangen. 1787. 8\% pp. 170.

On p. 159 is an account of Agaricns coriacens, Ag. Auricula.judae, Lycoperdon Tuber, and L. Bovista, taken from Gronorius's Flora Virginica, 2d ed. See title no. 337. In this work the author's name is given Schoepf but in other morks it is Schoepfl:
No. 563. In the note, Blastema should be Blastesis. The statement that this species was not published is incorrect. See "A fungus on the leaves of pear trees," by W. H. Seaman, in Am. Nonth. Micr. Jour. III. 169, 170. Sept. 1882.

To Seymour, A. B., add : See Burrill, T. J.

After no. $5^{68^{\mathrm{a}}}$ insert the following:--

Seymour, Arthur Bliss.

$568^{\mathrm{b}}$. Rusts of pomaceous fruits.

Farmer, p. 648, fig. 1-4. 2 Oct. 1886.

Prairie

An account of the Gymmosporangia and Roesteliae of the Eastern United States, including a letter from J. G. Vaughn concerning forms observed in Marion Co., Ill. Same in sub. stance as no. $5^{6} 7^{2}$.

After no. 598 insert the following:-

Tournefort, Joseph Pitton DE. Aix, Provence, 5 June, 1656. †Paris, 28 Dec. 1708.

598 . Institutiones rei herbariae. Elitio altera, etc. Vol. III. pp. 697. Pl. 476. Paris, 1700.

On pp. 560,563, 565, 666, are references to 5 fungi from America, 2 of them taken from Plukenet and one from Mo. rison. The species are also giren in the third edition on the same pages, except 666 .

After no. $6 \mathrm{r}$ insert the following:-

Tuckerman, Edward.

6rin. Note on Geaster quadrifidus. An. Jour. Sci. and Arts, XXXVI. 380. July, 1839.

Brief note stating that he has found this species in Cam. bridge, Mass.

After Wilkes Expedition insert the following :-

Williams, Stephen W.

$627^{\mathrm{a}}$. Report on the indigenous medical botany of Massachusetts. Trans. Am. Med. Ass. II. 863927. 1849 .

On p. 924, under the heading "Nushrooms," is note on Helvella amara and Boletus Laricis, of no value.

In Supplement, p. 35, 19th line from bottom, instead of "See title no. 22 " read "See title no. 227."

The statement mnder Fungr Europaer ExsicCATI that "the only part of the series which contains American specimens is that from Cent. 27 on" is incorrect. In the appendix to Cent. 9 of the Herbarium Vrvum Mrcologicum is Stereum fasciatum from North America.

The attention of mycologists should be called to the Contribution to American Bibliography of Insect Diseases in Psyche, V. 15-20, 45, 46, JanApril, 1888, which gives a number of references to titles, partly botanical and partly entomological, which cannot well be introduced into the present list. 


\section{LIST OF WORKS PUBLISHED IN 1887.}

F. I.

Arthur, Josepli Charles. See also Scribner, +654 . Report of the Botanist to the New York Agricultural Experiment Station. 5 th Ann. Rept. N. Y. Agr. Exp. Station for 1886. pp. 275-315, with 10 figures. Elmira, N. Y. 1887. Extras distributed 30 Jan. 1887.

Besides articles on pear blight this report contains the following articles on diseases caused by fungi : Rotting of tomatoes; Disease of clorer-leaf weevil, with note on peculiarity of the germination of the spores of Entomophthorc Phyto. nomi (also in Bot. Gaz. xii. 72); Strawberry nildew, Sphaerotheca Castagnei, Lev.; Plum-leaf fungus, Septoria cera$\sin \alpha$, Peck, with figmres.

On title-page of reprint the report is said to be for $\mathbf{1 8 8 7}$, an error tor 1886. A corrected second edition of reprint was issued in Oct. 1857, paged 259-296, snbstance the same as iu first reprint, to which is added "Smut in oats," by C. S. Plumb, pp. 124-130, and "Hyposulphite of soda is a fuugieide," by Emmett S. Goff, pp. 173, 174. Rev. Am. Nat. XXI. 380.

+655 . - Remarks at session of the Botanical Club of the A. A. A. S., 15 Aug, 18s7. Torr. Bull. XIV. 207, 208. Sept. 1887.

Notes the occurrence of black-knot on Prunus maritima, and a Cintractia on Cyperus Grayii on Staten Island.

\section{- and Holway, E. IV. D.}

656. Fungi: in Geological and Natural History Survey of Minnesota. Bulletin no. 3. Report on botanical work in Minnesota for the year 1886 . $8^{\circ}$. pp. 56. St. Paul. Distributed Oct. 1, 1887. The fungi, pp. 26-31, Basidiomycetes, Uredineae, and Ustilagineae, pp. 32-36, Pyrenomycetes, Hetrellacene, Perisporiaceae, and Peronosporeae, and pp. 39, 40, Nyxomycetes and Chytridineae, were mainly determined by Arthur and Holway, some by C. H. Peck and J. B. Ellis. A list of 233 species of fungi, with localities and habitats, inclucling descriptions of 3 new species by Arthur and IIolway and 7 by Ellis and Holway.

Berlese, Augusto Napoleone.

657. Fungi Moricolae. Iconografia e descrizione dei funghi parassiti del gelso. Fasc. IV. 8. Padua. 20 Aug. 1887.

This work is a series of octavo plates, in part colored, illustrating the fungi which grow upon species of Morus. In fasc. 4 is figured the American species, Massaria epileuca, B. and C. Of the previous fasciculi, no. 2, issned 22 April, 1855 , is the only one containing special American species. In that fisciculus are figures of Lophidium fenestrale, Sacc., and IIysterographium Nori, Rehm.

Bessey, Charles Edwin.

+658. The growth of Tulostoma mammosum. Am. Nat. XXI. 665-666. July, 1887.

Account of the stipe and the peridium in this speeies.

-659. - Ash-rust again. Am. Nat. XXI. 666. July, 1887.

Notes the rarity of Aecidium Fraxini, S., in Nebraska in 1887. See title no. 68 .

See, also, The Use of English names for Fungi by this author in Am. Nat. Narch, 1887.

\section{Brendel, Frederick.}

66o. Flora Peoriana, the vegetation in the climate of Middle Illinois. $8^{\circ}$ pp. 89. Peoria, Ill. 1887.

On pp. 68, 69, is a list of 109 species of fungi without notes. This paper is not a translation of no. 76 , as might be snpposed from the title itself, but is different in several respects.

Britton, Nathaniel Lord.

66r. Remarks on fungi. Proc. Natural Science Ass. of Staten Island.

In the proceedings of 9 April, 1887, Dr. Britton made re marks on fungi collecterl by members of the Association during the past two years. General notice of Aqarici, Polypori, etc. Report on octavo sheet, without paging.

The title of last paper is "Strncture of certain Timber-Tics. Behaviour and Canscs of their Decay in the Road-Bed." Printed and a few copies struck off in 1887 bnt not generally issued until 1888
Burrill, Thomas Jonathan, and Earle, F. S. +662 . Parasitic fungi of Illinois. Part II. Bull. Illinois State Lab. Nat. Hist. II. 387-432, with 8 figures. Peoria, Ill.

Issued in July, 1887. General account of the order with figures of the genera found in Illinois, together with a key to genera, followed by descriptions of 29 species, with full synonymy and critical notes. There is a systematic index and index of hosts. Rev. Torr. Bull. XIV.193. Sept. 1887. Bot. Gaz. XII. 303 . Dee. 1887.

\section{Calkins, IVilliam Wirt.}

+663 . Notes on Florida fungi. no. 10. Tour. Myc. III. 7 , Jan. ; no. 11, 1. c. 33, 34, March; no. 12, l. c. 46 , April; no. 13,1 . c. 58,59 , May; no. 14 , 1. c. 70 , June; no. 16 , 1. c. 82 , July, 1887.

Continued from title no. Ioo. Part 15 is not given, proba. bly by typographical error.

Cobb, N.A.

$663^{a}$. A list of plants found growing wild within thirty miles of Amherst. $8^{\circ}$ pp. 51. Northhampton, Mass. 1887.

On pp. 31-48 is given a list of fungi, including lichens. The fungi proper are in the main those given by C. C. Frost in Tuckerman's Catalogne. See title no. 612.

Cooke, Mordecai Cubitt.

4 664. Synopsis Pyrenomycetum. Grev. XV.8086, 122-125; XVI. 16-19, 50-56. March, June, Sept., Dec. 1887.

Continuation of title no. r6r. Inclndes notes on a number of N. Am. species of Byssosphaeria, Cucurbitaria, Lasios. phaeria, Venturia, Chaetomium, Rosellinia, Sordaria, etc-

665. - New species of Ravenelia. Grev. XV. 112. June, 1887. Jour. Myc. III. 83.

Description of $R$. verrucosa, Cke. and Ellis from Mexico. 666. - Some exotic fungi. Giev. XVI. 15, 16, 25, 26. Sept., Dec. 1887.

IIas descriptions of Sphaeronema tenuirostris, Cucurbi. taria Ravenelii, and Cylindrocolla quercina, from the United States.

$\div 667$. - Australasian fungi. Grer. XVI. 30-33. Dec. 1887.

Bovista ovalispora Che. \& Mass. is said to occur in South Carolina.

$667^{\mathrm{a}}$. - New British fungi. Grev. XVI. 42-49, Dec. 1887.

Also includes deseription of Bovista ovalispora and Lycoperdon Cookei, Mass.

Craig, John. Lakefield, Province of Quebec, Canada, 27 April, 1864. See Halsted, B. D.

- 668. Observations on corn and corn smut. Bull. Iowa Agr. Coll. Nov. 1886, p. 16. Issued 1887.

The smut found to be most abundant in the fruit which has a north-east exposure.

Cuboni, J. See Saccardo, P. A.

DieteI, $P$.

668. Beitrage zur morphologie und biologie der Uredineen. Bot. Centralblatt. XXXII. 54-56, $84-91,118-121,152-156,182-186,217-220,246-$ 250, Pl. 1, Oct., Nov. 1888.

Has incidental references to a number of American species.

Dudley, Plimmon Henry.

6б. Polyporus sangwineus, Jour, N. Y. Micr. Soc. III. 29, 30. July, 1887.

Note ot the occurrence of this species, and species of $T_{r} \cdot a$ metes and Lenzites, on the isthmus of Pauama. 
670. C Chamaccyparis sphaeroidea, Spach; white cedar, and its fungus, Agaricus campanclla, Jatsch. Jour. 1. 1. Micr. Soc. III. 30-34. Fig. 1. July, 1887 .

Y. B. Sec also other naners by this author in Jour. $N$. I. Mic\%. snc. III. 40, 41, July, 18sï, notes on Merulius lacrymens, and in Jept. of Agr. Forestry, Bult. no. I. pp. 39, 40, $42,44,4 \pi, 45,50,52$, where a list of fungi found on six species of wond nsed for railload ties is given.

Dudley, William Russell. Guilford, Conn., 1 March. 1849. Sec Merrx, M.

Earle, Franklin Sumner. See also Burrill, T. .J.

671. Pear blight and root-rot. Prairie Farmer, p. 86. 5 Feb. 1887 .

The rnot-rot is said to be cansed by Polypomes versicolor, gurl an account is giren of its prevalence in Illiwois.

672. - Pear leaf-blight and scab. Prairie Farmer, p. 102. 12 Feb. 1887.

A popular account of ITorthiera Lespili and Fusicladium pyrinum.

673. - Pear discases cansed by fungi. Trans. Illinois Ilont. Soc. XX. 167-171. 1887.

Substance same as in the last two papers.

Ellis, Job Bicknell. Sce Artuur, J. C., title no. 656, Laxglois, A. B., and Martin, G.

4.674. Terfezia Leonis, 'T'ul. - Tuber niveum (Desf.). Jour. Myc. 1I1. 10. Jan. 1887.

Reports the discovery of this species in Lonisiaua by Rev. A. B. Langlois, and rives notes on its microscopical characters and edible properties.

f 675. - Authority in nomenclature. Jour. Myc. III. 33. March, 1887.

Objects to the use of the name Physalospora Bidwellii, Sacc., by Scribner, in paper, title no. 560 , aud thinks that the name of Ellis should be added in parenthesis.

+ 676. - Melanconis dasycarpa, E. \& K. Jour.

Mycr. III. 118 . Oct. 1887.

The author suspects that this species is not different from Melanconis Everhartii, Ell.

- 677. — Tricotheciumgriscum, Cooke. (Pyricularia, Sacc.) Jour. Myc. III. 126. Nov. 1887.

This species is reported to be associated with Phyllachora graminis on Muhlenbergir. A new var. leptosperma, E. \& $\mathrm{K}$., is mentioned on Paspalum setaceum.

_- and Everhart, B. MI.

678. Additions to Cercospora, Gloeosporium, and Cylindrosporium. Jour. HIyc. III. 13-22. Feb. 1887 .

Descriptions of 40 new species of Cercospora, 3 of Gloeasporium, and 4 of Cylindrosporium, with notes on a few other species. G. aridum is referred to Ellis and Ifolway. This paper is a supplement to titles 242,244 , and 246 .

679. - New species of Ustilagineae and Uredineae. Jour. Hyc. III. 55-57. May, 1887 .

Descriptions of 11 species, most of them from New Mexico, Arizona, and Washington Territory.

1680 . - Symopsis of the North American species of Mylaria and Poronia. Jour. Myc.

I1 I. 97-102, 109-113. Sept., Oct. 1887.

Descriptions of 30 species, with notes.

68ז. Adritions to Iypocreaceae. Jour. Myc. 11I. 113-116. Oct. 1887 .

Account of 9 species, of which 3 are described as new.

682. — - New species of fungi from various localitics. Jour. Myc. III. 116-118, 127-130. Oct., Nov. 1887.

Descriptions of 24 species, 7 of them Gloposporia.

- and Kellerman, W. A.

+ 683. New Kansas fungi. Jour. Myc. III. 102105. Sept. 1887 .

Descriptions of 1.5 spccies, principally fungi imperfecti. sice next title.
684. - New species of fungi from Kansas. Jour. Myc. III. 126, 127. Nov. 1887.

Descriptions of 6 species. See also title abore.

Everhart, Benjamin Matlack. See EurIs, J. B.

Fairman, Charles E.

4-685. Vermicularia phlogina Fairman. Bot. Gaz. XII. 67. March, 1887.

Characters of species given.

Farlow, William Giìson. See Klebahw, H.

686. Vegetable parasites and evolution. Bot. Gaz. XII. 173-189. Aug. 1887.

Includes a few statistics of fungi of the United States.

687. - Aecidinm on Juniperus Virginiana. Bot. Gaz. XII. 205-207. Sept. 1887. Also in Rev. Mycol. X. 32-34.

Description of Aecidium Bermudianum on Tuniperus Bermudiana in Bermuda, and on $J$. Virginiana in Missis. sippi. On p. 207, Roestelia lacerata should be $R$. hyalina.

Galloway, Beverly Thomas. Callaway Co. Missouri, 16 Oct. 1862.

\& 688. Celery leaf blight (Cercospora Apii, Fres.). Bot. Gaz. X1I. 66, 67. March, 1887.

Reports damage done by this species in Missouri.

Goff, Emmett, S. See Arthur, J. C., title no. 654.

Halstod, Byron David.

689. Bulletin of the Iowa Agricnltural College from the botanical department. Nov. $1886.8^{\circ}$. pp. 66. Issued 1887 .

Includes sereral articles on fungi by the editor. Germination of ergot from the wild rye, pp. 19-21. Notes upon the Peronosporeae for $1886, \mathrm{pp} .53,54$. Notes on 10 species from Iowa and Colorado. Notes upon the Ustilaginear, pp. 54, 55. The ash-leaf Rust, Aecidium Frarini, Schw., p. 55. The clover mould, pp. 55, 56, fongus not named. Fungi of Forest Trees, pp. 56, 57. Colorado Fungi, pp. 57-59; account of 25 species, principally Uredineae collected in July, 1886; 2 new species, Tuberculina Lupini, Farlow, and umamed Aecidium on Abies Douglasii. Relation between cedarapples and the leaf-rust on the wild crab apple; a full account of experiments briefly described in tltle 110.350 . See also Craig, J., and Hitchicock, A. S.

69o. - A new Uromyces. Jour. Myc. III. 138. Dec. 1887.

Description of Uromyces digitatus on Leersia Firginica from Iowa.

Harkness, Harrey Wilson.

+69 r. Fungi of the Pacific coast. Part5. Bull. Cal. Acad. Sci. II. no. 7, 438-447. Issued June 16,1887 .

An enumeration of 76 species, with their hosts, localities, and notes. Tluree of the species described as new.

Harvey, Francis LcRoy. Ithaca, N. Y., $22 \mathrm{Apr}$. 1850 .

+ 692. Proliferous fungi.

with figure. Nov, $188 \%$.

Notice, with figure, of a monstrosity of Lactarius cinereus, Peck

Hitchcock, Albert Spear. Owasso, Mich. 4 Sept. 1865.

693. A partial list of Iowa powdery mildews. Bull. Iowa Agr. Coll. pp. 64-66. Nov. 1886. Issued 1887.

Notes on 20 species of Perisporiaceae found in Iowa. See title no. 689 .

Holway, Edward Willet (Dorland). See ARTuur, J. C., and Ellis J. B., and Everhart, B. M. Humphrey, James Ellis. North Weymouth, Mass., 5 Allg. 1862.

+694 . The preparation of agarics for the herbarium. Bot. Gaz. XII. 271-273. Nov. 1887.

Practical directions for drying and pressing.

Kollerman, William Ashbrook. See ElıIs, J. B. 
Klebahn, II.

695. Beobachtungen und streitfragen neber die blasenroste. Abhandl. Naturwiss. Ver. Bremen, X. 145-155. Pl. 1 .

Ineludes brief notes by Farlow on the distribution of $\mathrm{Cr}^{\mathrm{O}}$ nartium and Peridermium in the Uuited States.

Knowles, Etta (Marietta) Laughridge.

6g6. The curl of peach leaves; a study of the abnormal structure induced by Exoascus deformans. Bot. Gaz. XII. 216-218. Pl. 13. Sept. 1887.

Notes on the histolngical characters of the distortions.

Lagerheim, G. (Nils Gustaf). Stockholm, 18 Oct. 1860 .

697. Mykologiska Bidrag. III. Ueber einige auf Rubus arcticus L. vorkommende parasitische pilze. Botanista Notiser, 60-67. Fig. 1-3. 1887.

Notes the occurrence of Puccinia Peckiana, Howe, in Lapland, and gives comparisons with Amcrican forms of the species, with figures.

Langlois, Auguste Barthélemy. Chavanay, Loire, France, 24 Apr. 1832.

te 698. A new Volutella. Jour. Myc. III. 57. May, 1887.

Description of $V$. Ellisii from Louisiana.

699. - Catalogue provisoire des plantes phanerogames et cryptogames de la Basse Louisiane. $8^{\circ}$. pp. 35. Saint Etienne.

On pp. $2 \pi-35$ is a list of more than 600 species of fungi determined by J. B. Ellis. Localities and habitats are not given. Rev. in Torr. Bull. XIV. 239, and Rev. Myc. IX. 201.

Ludwig, Friedrich. 24 Oct. 1851.

700. Einiges ueber rostpilze. Centralblatt fiir Bacteriologie und Parasitenkunde, I. 690-693. June, 1887.

Ineludes references to Puccinia Peckiana, P. Afalvastri, $P$. heterospora, and Gloeosporium Lindemuthiamm in the United States summarized from previous papers by Lagerheim, Farlow, Seymour, Burrill, and Ellis and Everhart.

Mancini, V. See SAccardo, P.A.

Martin, George.

40r. Enumeration and description of the Septorias of North America. Jour. Myc. III. 37-41, 49-53, 61-69, 73-82, 85-94. April-Aug. 1887.

Descriptions of 188 species of Septoria, 158 by this autlior, the remainder, together with 8 species of Phloeosporr, 20 of Rhabdospora, and 8 of Phlyctaen $\alpha$, by the editors of Journal of Mycology. Also an index of species and host-plants.

Massee, George Scampston, Yorkshire, England, 20 Dec. 1850 .

702. A monograph of the genus Lycoperdon (Tournef.), Fr. Jour. Roy. Nicr. Soc. VII. 701727 (1-27). Pl. 12, 13. Read 8 June, 1887.

Includes descriptions of 25 speeies from United States, 3 of them new, with figures of some of the species.

703. - - On Gasterolichenes: a new type of the group Lichenes. Philos. Trans. Royal Soc. London. Vol. CLXXVIII. 305-309. Pl. 25. Read 16 June, 1887.

Includes an account, with figures, of Trichocoma laevispora from South Carolina, which is here referred to lichens.

- 704. - Revision of Polysaccum.

Grev.

XVI. 27-29. Dec. 1887.

Ineludes 2 species from the United States.

Merry, Martha. Phoenix, N. Y., 10 Aug. 1864. +705 . The identity of Podosphaera minor, Howe, and Microsphaera fulvo-fulcra, Cooke. Bot. Gaz. XII. 189-191. Pl. 11. Aug. 1887.

Account of the examination of specimens of the two specie ; from different loealities to show their identity. Followed by a supplementary note hy W. R. Dudley.
Morgan, Andrew Price.

706. The mycologic flora of the Miami Valley, Oliio. Jour. Soc. Nat. Hist. Cincinnati. X. 718. March, 1887.

Continnation of title no. 424, including descriptions of IIydnei. Two species, IIydnum casearium and II. alboviride, described as new.

4t 707. - North American Agarics.-The subgenus Amanita. Jour. Myc. III. 25-33. March, 1887.

Descriptions of 28 species, with analstic key and critical notes.

4708. - The genus Geaster. Am. Nat. XXI. 1026-1029. Fig. 1, 2. Nov. 1887.

A review of De 'Toni's monograph, see title no. 730, with descriptions of two new specics, $G$. campesiris and $G$. delicatus, from Nebraska, with figures.

Pearson, Alexander W. See Scribrer, F. L.

Peck, Charles Horton. See Arthur, J. C., title no. 656 .

409. Notes on the Boleti of the United States. Jour. Myc. III. 53-55. May, 1887.

Critical notes on a number of American specics. $B$. Russellii, Frost, and $B$. Morgani, Pk., form a group, Laceripedes, peeuliar to this country. Name of $B$. robustus, Frost, changed to $B$. eximius, and description given.

A. 7 Io. — Thirty-ninth Report for 1885, issued Sept. 1587. pp. 30-73. Pl. 1,2. Continued from title no. 454 .

Fungi not before reported, pp. 38-53, 37 new species; remarks on fungi, pp. 57, 58; synopsis of New York species of Pleurolus, Claudopus and Crepidotus, pp. 58-73.

I 7rI. - Contributions to the botany of the State of New York. Bull. N. Y. State Muserm Nat. Hist. I. no. 2. May, 1887. pp. 66. Pl. 1, 2.

Contains the following: "Descriptions of new species ot New York fungi," pp. 5-24, giving descriptions of 14 IIymenomycetes from thc unpublished 37 th Report (sce title no. 453), and 40 species from 32nd Report (see title no. 448); "Additions to the flora of the State of New York in 1883, with remarks and observations," pp. 25-28, including notes on fungi taken from 37th Report; New York speeies of Paxillus, pp. 29-33, New York speeies of Cantharellus, pp. 34-43, New York species of Craterellus, pp. 44-48, all from 37th Report; names of New York Pyrenomycetous Fungi, pp. 49-56, also from 37th Report; and New York species of viseid Boleli, pp. 57-66. Although this paper bears the date May, 1857, it was apparently not distributed until the following January.

Penzig, Otto.

7г2. Studi botanici sugli agrumi e sulle piante affini. Memoria premiata dal R. Ministero d'Agricoltura, con un atlante in folio. Ministero $d i$ agricoltura, industria e commercio. Direzione generale dell' agricoltura. Annali di agricoltura. 1887. Rome, 1887. Text, pp. vi.590. $8^{\circ}$. Atlas, pl. 58. Folio.

The plates of fungi in this work are reproduced from Saccardo's Fung. Ital. Del. (see title no. 536). The text contains references to American fungi on pp. 323, 325, 349, 379, and 390 .

Plumb, C. S. See Arthur, J. C., title no. 654. Provancher, L. (Abbé).

+ 7r3. Le Phallus et la Morille. Naturaliste Canadien, XVI. 115-119. Feb. 1887.

A reply to questions concerning the nomenclature of the fungus described in the $N$ aturaliste for Oct. 1886. See no. 505.

Ravaz, Louis Etienne. Saint Roman, Isère, France, $10 \mathrm{May}, 1863$. See VIAxA, P.

Ravenel, Henry William. †Aiken, S. C. 17 July, 1887.

Riley, Charles Valentine.

7I4. Fungus disease of the web-worm. Rept. of the Entomologist in Rept. U. S. Dept. Agr. for 1886. pp. 527-529. Washington, 1887.

Gives an account of the growth of Bolrylis Bassiana on web-worms bred in Washingtou. 
Robinson, Benjamin Lincoln. Bloomington, Ill., 8 Nor. 1864.

+ 7r5. Notes on the genus Taphrina. Annals of Botany, I. 163-176. Nov. 1887.

A general aecount of the structure of the genus followed by a syopsis of 8 species of the United States, with critieal notes on structure and distribution.

Saccardo, Pictro Andrea.

+ 7r6. Sylloge Fingorm omnium hucusque cognitorum. Vol. V. Srlloge Hymenomycetum omnium hncusque cognitorum digessit P. A. Saccardo collaborantibus J. Cuboni et V. Mancini. Vol. I. Agaricineae. 8\%. 11.1146. Padua. 28 May, 1887. Continnation of title no. 548 , ineluding all known North American species.

Scribner, Frank Lamson.

7r7. Report on the fungus diseases of the grape rine. Dept. of Agr., Botanical Division. Bulletin no. 2. Section of Plant Pathology. Washington. 1886. Issued Jan. 188\%. 8\% pp. 136. Pl. 7 , in part colored, and 4 figures.

Report eontains full aecount of Downy Mildew, Peronosyora viticola, Powdery Mildew, Uncinula spiralis, the 13lick-1ot, Physalospora Bidwelliz, Antbraenose, Sphaceloma ampelinum, Grape-leaf Blight, Cercospora viticola, and Grape-leaf Spot, Phrllosticta Labruscae. Appendix A fires syopsis by Irwin F. Smith of replies to circular on rot and nildew. Appeudix $B$ has remarks on rot and mildew by Col. A. W. Pearson. Rer. Torr. Bull. XIV. 38.

718. - Ejection of ascospores from the asc ${ }^{\mathbf{i}}$ of Physalospora Bidwellii. Torr. Bull. XIV. 207. Sept. 188\%.

A note of remirks before the Botanical Club of the A.A.A.S., stating that the spores of this speeies were thrown from 10 to $15 \mathrm{~mm}$.

719. White Rot of grapes. Colman's Rural World. 27 Oct. 1887.

An aecount of the discovery by Prof. P. Viala of Coniothecium Diplodiella, Speg., on grapes at Neosho, Missouri.

$719^{2}$. _ Grape leaf-spot disease and blackrot. Colman's Rural World. St. Louis. 29 Nov. 1887.

Phyllosticta Labruscae, Thm., is stated to be a foliicolous form of Phoma ruricola, B. \& C.

Arthur, J. C., and Trelease, W.

720. Report of the Mycological Section. Rept. U. S. Dept. Agr. for 1886 . pp. 95-138. Pl. 1-8, besides 3 maps and diagram. Washington. 1887.

Contains the following papers by F. L. Srribner: The dowuy mildew, Peronospora viticola, p. 96-105, pl. I.; The pow lery mildew, Encinula spiralis, pp. 105-109, pl. II.; Black-rot, Physalospora Bidwellii, pp. 109-112, pl. III.; Anthracnose, Sphaceloma ampelinum, 112-115, 131, pl. IV.; pl. V.; Celery-leaf blight, Cercospora apii, pp. 117-120, Orange-leaf scab, pp. 120-121, pl. VI.; The potato-rot, Phy. tophthora infestans, pp. 121-124, pl. VII. See also TreLEAsE, W. This Report also includes a paper by J. C. Arthul, on the Pear Blight, Wicrococcus amyloromes.

-72r. and Viala, P. Le Greeneria fuliginea, nouvelle forme de rot des fruits de la vigne, observéc en Amérique. Comptes Rendus. $12 \mathrm{Sept.}$ 1887. Vol. CV. $473,474(1,2)$.

Substance same as next title.

722. On a new fungus disease of the vine, Greeneria fuliginea. Agricultural Science, I. 210-211, Sept. 1887, and Proc. Soc. Prom. Agr. Sci. Sth reeting. pp. 74-76. Oct. 1887.

Aecount of a new genus and species of Sphaeropsideae found on grapes in North Carolina. Rev. Jour. Myc. III. 119. See Colman's Rural World, 13 Oct. 1887.

Seymour, Arthur Bliss.

723. Urchard Riusts. Trans. Am. Ifort. Soc. $1 T^{+} .15-160(1-8)$. 1 ig. 1-4. Indianapolis. 1886. licprint issucd $7 \mathrm{dpr}$. 1887.

An account of riseases caused hy species of Gymnosporangium in the Lnited States. Rev. Centrallutt Bacteriol.
I. $22-230$.
724. - The hollyhock disease. Am. Florist, II. 512,513 , with figure. 1 Aug. 1887 .

Account of Puccinia Ifalvaceamm with notes on its occurrence in Massaehusetts.

725. A discase of the mignonette. Am. Florist, III. 38.1 Sept. 1887.

Aecount with figures of an unnamed fungus destroying mignonette at Champaign, Ill.

726. - Character of the injuries produced by parasitic fungi upon their host-plants. $A m$. Nat. XXI. 1114-1117. Dec. 1887.

General aceount of the injuries and distortions produced by parasitic fungi on plants.

Smith, Erwin F. Gilbert's Mills, Oswego Co., N. Y., 21 June, 1854. See Scribner, F. L.

Somers, John.

727. Additions to the list of Nova Scotian fungi. Proc. and Trans. Nora Scotian Inst. Nat. Sci. VI. 286-288. 1886. Issued 1887.

Enumeration of 23 speeies, principally IIymenomycetes, with localities and some notes.

Thaxter, Roland. Newton, Mass., 28 Aug. 1858.

1728. On certain cultures of Gymnosporangium, with notes on their Roesteliae. Proc. Am. Acad. Arts and Sci. Boston. XXII. 259-269. Separate edition issued Jan. 1887. Also in Centralblatt fuir Bacteriologie und Parasitenkunde, I. $429-434,457-460$.

Account of enltures showing the eonneetion of $G$. conicum with $R$. cornuta, $G$. clavipes and $R$. aurantiaca, $G$. clavariaeforme and $R$. lacerata, $G$. macropus with $R$. pyrata, $G$. biseptatum with $R$. botryapites, and eritical notes on the eharaeters of other speeies. Rer. Jour. A

Thomas, Friedrich A. TV.

729. Synchytrium cupulatum, n. sp. Bot. Centralblatt, XXIX. 19-22. Jan. 1887.

Has note on the Ameriean form of $S$. Nyosotides var. Potentillae, which author thinks should probably be referred to his new speeies, S. cupulatum.

Toni de, Giovanni Battista. Venice, 2 Jan. 1864. 730. Revisio monographica generis Geasteris Mich. e tribu Gasteromycetum. Rev. Nyc. IX. 61-77, 125-133. Pl. 62, 63. April, July, 1887.

Bibliography of the genus with deseriptions of known spe. eies, ineluding 17 speeies from the' United States, of whicl some are figured. There is also an author's edition of 25 pages and 2 plates. See title no. 708 .

Trelease, William. See also Scribrer, F. L., title no. 720 .

5 73I. A spot disease of orchard grass, Scolecotrichum graminis. Rept. U. S. Dept. Agr. for 1886. Mycological section, pp. 129-131. Pl. VIII. Washington. 1887.

Account of the appearance of this fungus in Wisconsin.

732. Fungi injurious to grasses and clovers. From Beal's Grasses of North America, pp. 413431. Fig. 159-175.

A popular aeeount of the more eommon, Ustilagineae, Uredineae, Ascomycetes, Peronosporeae, etc., whieh attack forage plants in the United States, with brief notes on some allied fungi of Europe. Rev. Centralblatt Bacteriol., I. 726.

Viala, Pierre. Montpellier, France, 24 Sept. 1859. See also Scribner, F. L.

733. Les maladies de la vigne. Deuxième édition ornée de 5 planches en chromo et 200 figures dans le texte, avec une étude des appareils de traitement par M. Paul Ferrouillat. $8^{\circ}$. pp. 462. Montpellier and Paris. 1887.

$A$ general treaties on fungi which eause diseases of vines, inclucling referenees to fungi found both in Europe and Ameriea, and to a few speeial $A$ merican species, as Uncinula spiralis, Phoma uvarum, B. and C., ete. Tibrissea (Roesleria) hypogaea, Thum. and Pass. is said, on the authority of Planehon, to occur in Missouri. Rev. in Bot. Gaz. XIII. 45. 
734. - Le whitc-rot ou rot blanc (Coniothyrium diplodiella) aux Etats-Unis d'Amérique. Comptes Rendus, CV. 624, 625. 10 Oct. 1887.

Account of the diseovery of this fungus on grapes in Missonri.

Viala, Pierre, and Ravaz, L.

735. La inélanose. $8^{\circ}$ pp. 18. Pl. 3. Montpellier.

One of the series, "Recherches sur les maladies de la vigne" issued in the Bibliotheque du Progrès Agricole et Viticole. Account of a disease caused by a fungus, Septoria rampelina, first described from America by Berkeley and Curtis. Substance of this paper also given in Viala's Maladies de la vigne. See above.

Wettstein, Richard rox.

736. Ueber Helotium Willkommii (Hart.) und einige ihm nahe stehende IIelotium-Arten. Bot. Centralblatt, XXXI. 285-287, 317-321.

Includes a deseription of Peziza Ellisiana, Relum, which is referred to Helotium.

White, James Clarke.

737. Dermatitis venenata: an account of the action of external irritants upon the skin. $8^{\circ}$. pp. 216. Boston. 1887.

On p. 99 is a note in relation to Ustilago hypodites and its occurrence in the United States.

Wiuter, Georg. †Connewitz, near Leipsic, 16 Aug. 1887.

th 738. Exotische Pilze, IV. Iledwigia, XXVI. 6-18. Jan. 1887.

On p. 8 is a reference to Coleosporium Baccharidis, Cke. \& Hark.
739. - Repertorium. Rabenhorstii Fungi Europaei ct Extraeuropaci Exsiccati cura Dr. G. Winter. Centuria, 35 et 36 . Hedwigia, XXVI. 24-35. Jan. 1887.

IIas notes on 7 speeies from the United States.

\section{FUNGI EXSICCATI.}

Norti American Fungi. Ellis, J. B., and Fverlıart, B. M., cditors. Cent. 18, 19. Newfield, N. J. Issued April, 1887.

Cent. 18 is composed largely of species of Cercospora and Septoria and Cent. 19 of Uredineae. Rev. in Rev. Myc. IX. 120 and Am. Nat. XXI.379.

Fungi Europaei et Extraeuropaei. G. Winter, cilitor.

Cent. 35 and 36 , the last that have appeared, were issued late in 1886 , but not generally received in this country until 1887. Reviews of these Centuries given in Re\%. Mryc. IX. 121 and Jour. Myc. III. 35, where some corrigenda of Ameriean specimens are given. See title 110. 739.

Fungi Galici Exsiccati. C. Roumeguère, editor.

Cent. 41-44 appeared during 1887, the labels of the last three Centuries having the heading Fungi selecti exsiccati. The following are the numbers of specimens from the United States : 4032, 4010, 4094, 4095, 4097 and 4315. 













$$
(1, y)(6,3)
$$

3) $100 \times$ (

ma (c)

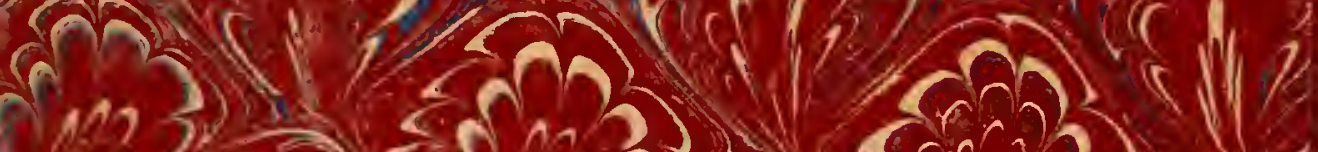

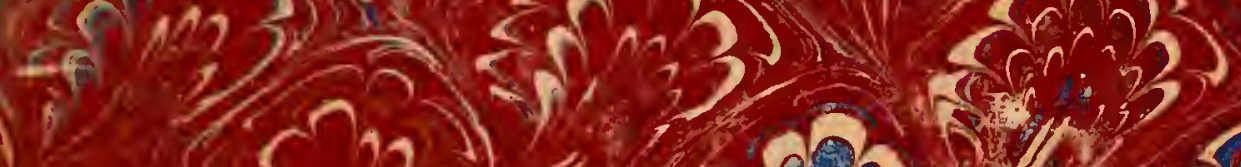
(1)

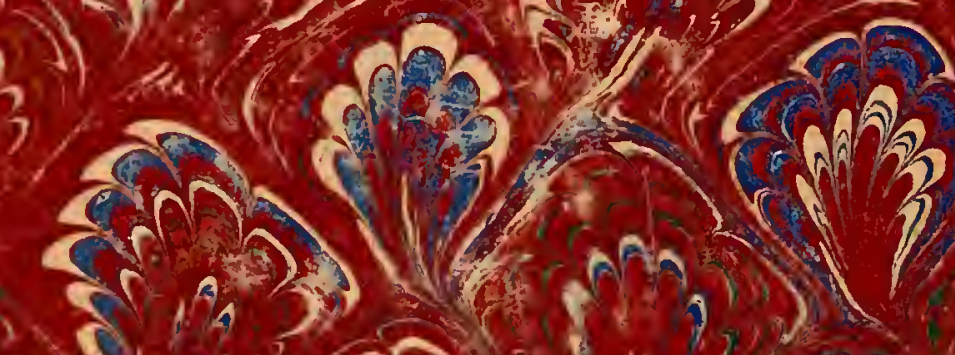

a(c) 3$)^{2}$

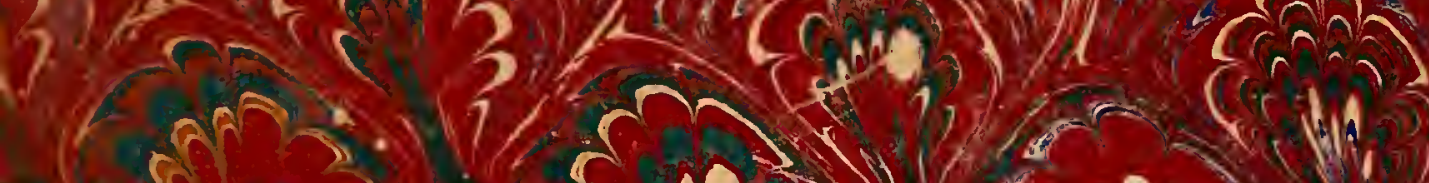

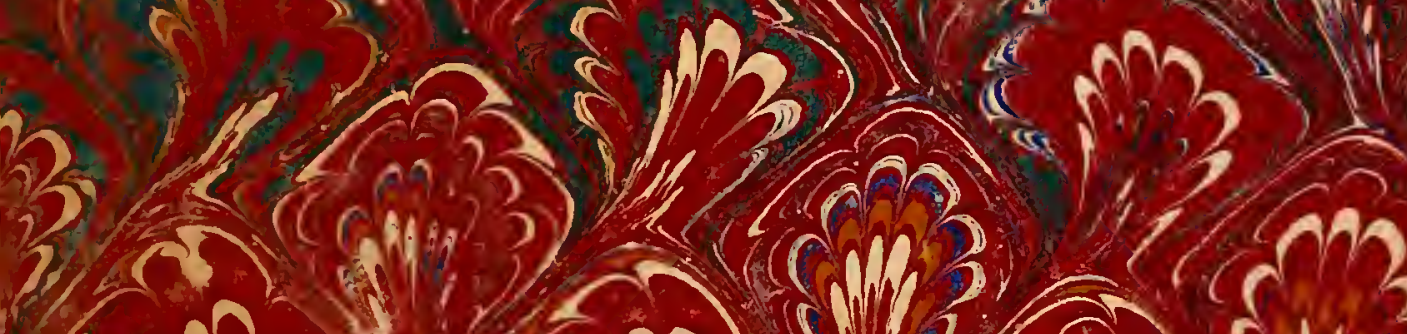
1) (n) $3(192)\}$ (c)

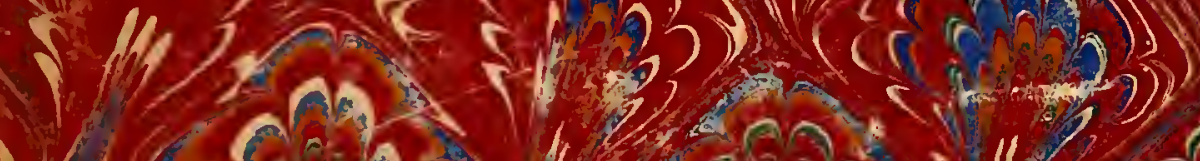

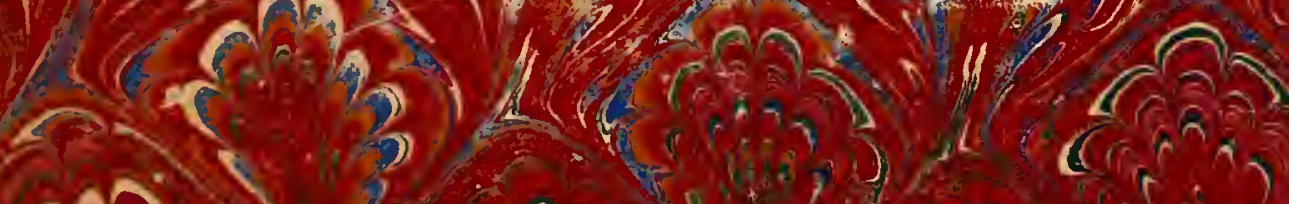

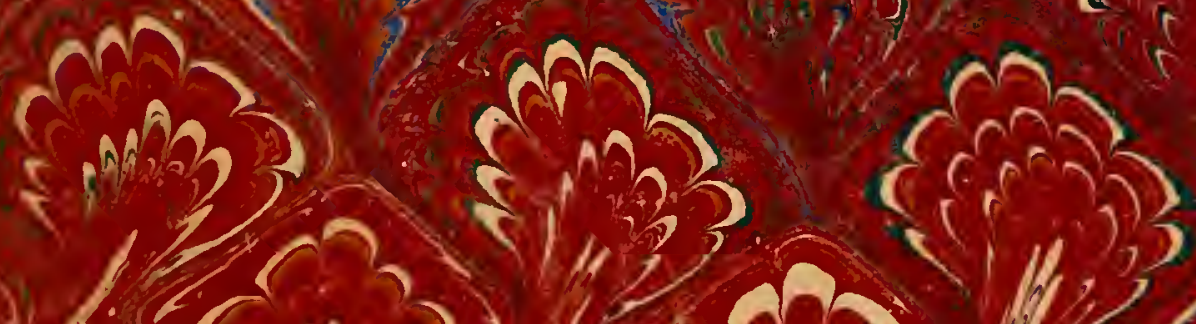

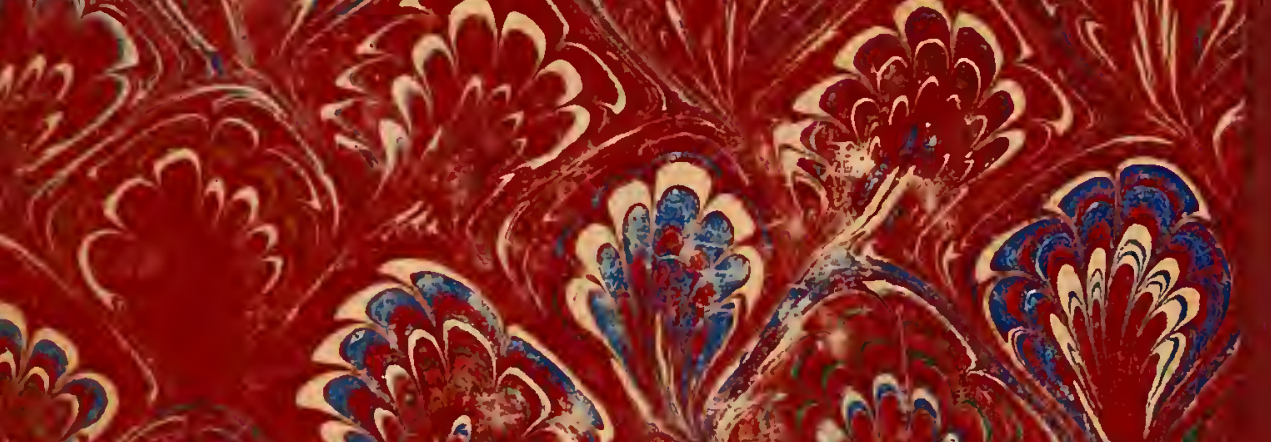
कर (M)

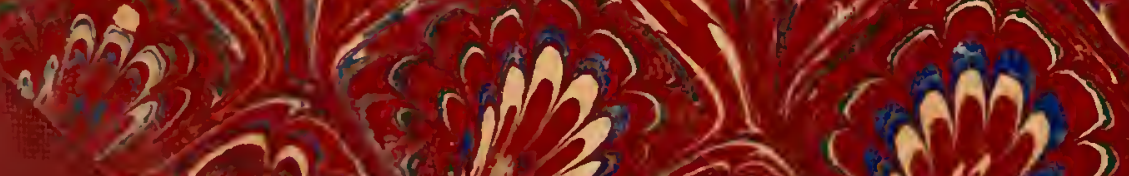
no Non 3. 